\title{
WEST VIRGINIA \\ GEOLOGICAL SURVEY
}




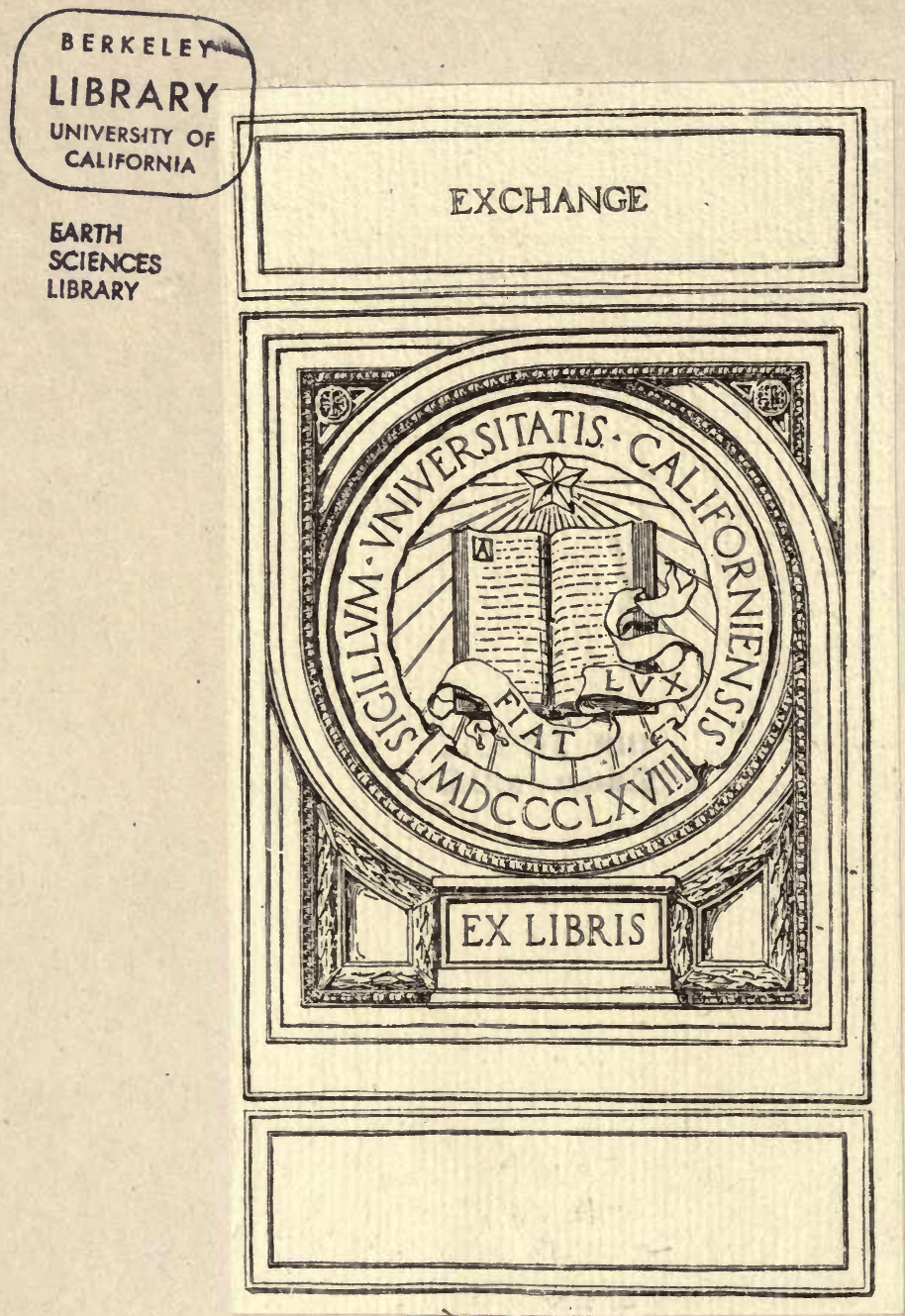










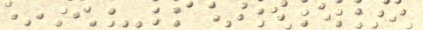




\section{axy 145 $3 x$}

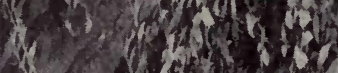

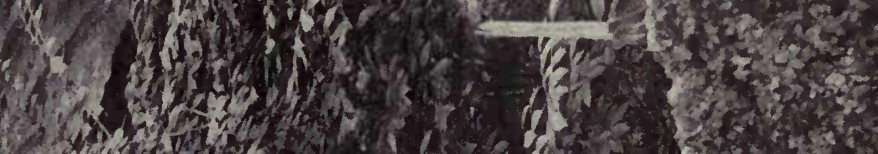

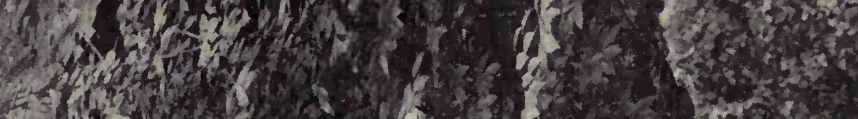

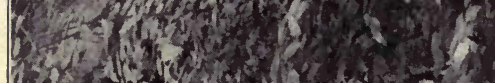

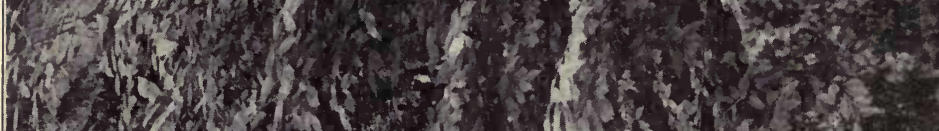

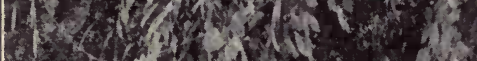

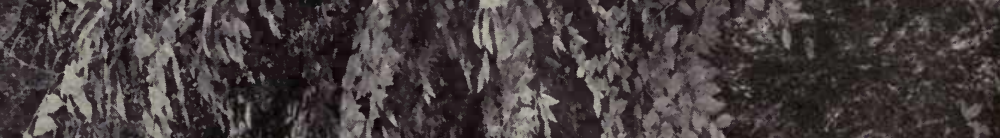

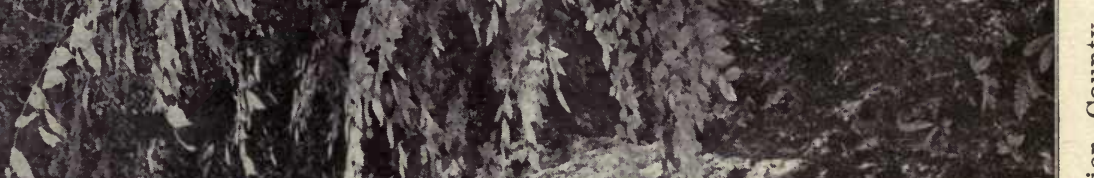

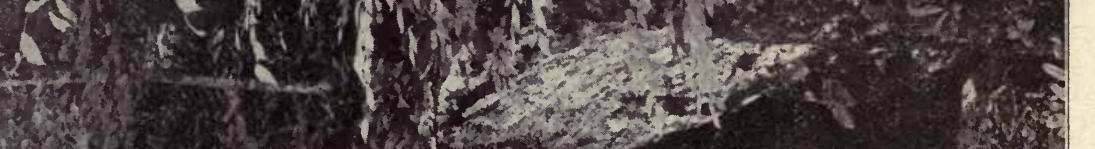

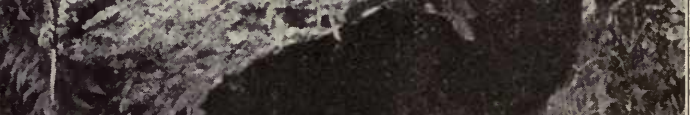

吾

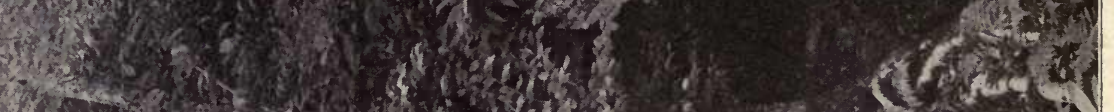
61

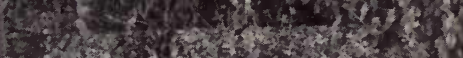

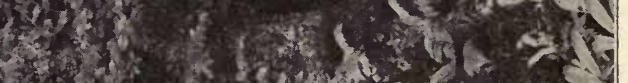
G.

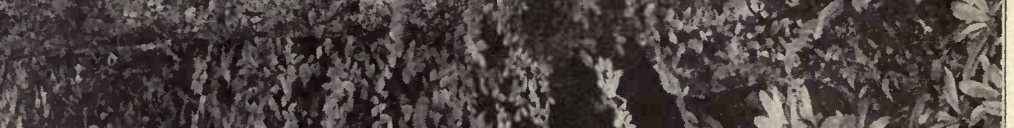

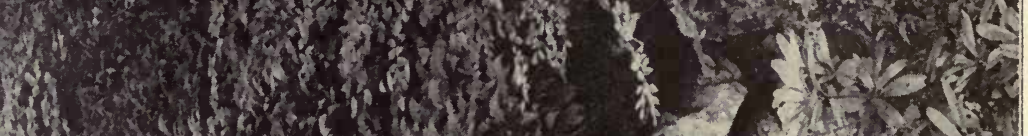
1. ito

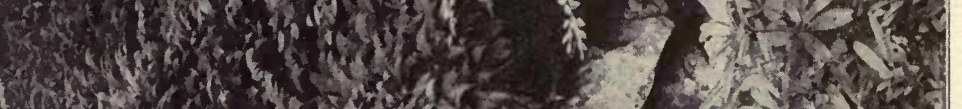

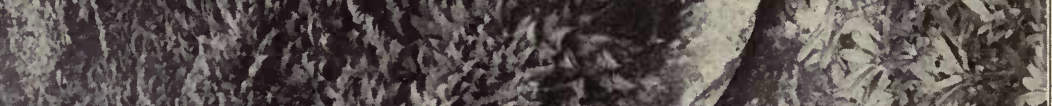
H.t.

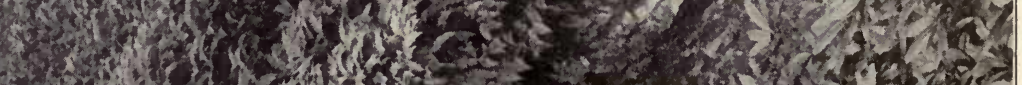

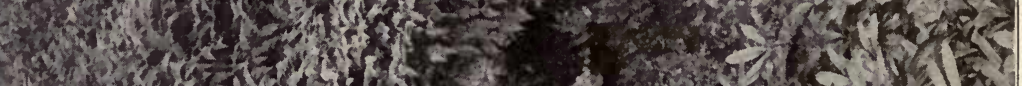

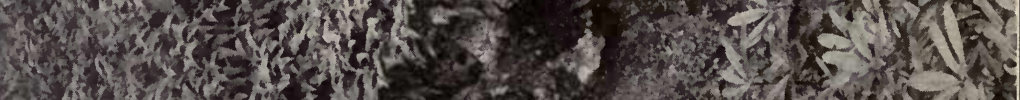

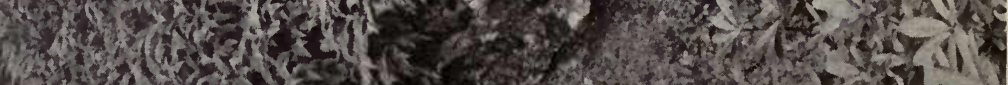

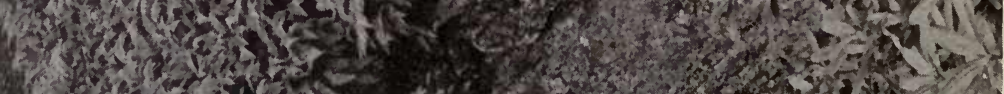

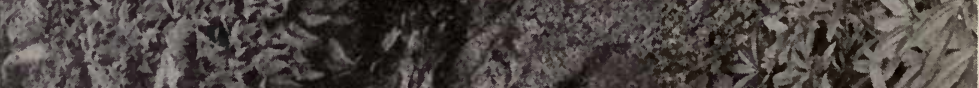

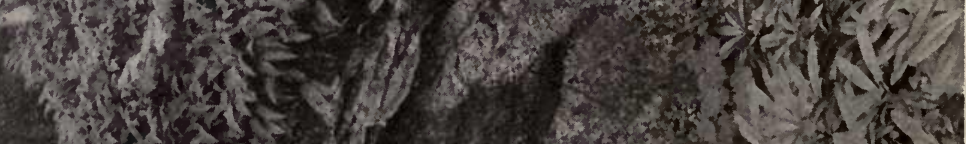

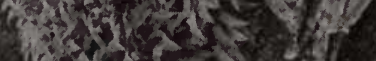

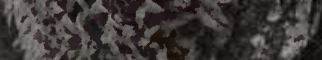

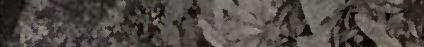

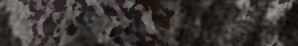

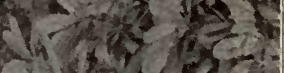
aindrey 


\title{
WEST VIRGINIA
}

\section{GEOLOGICAL SURVEY}

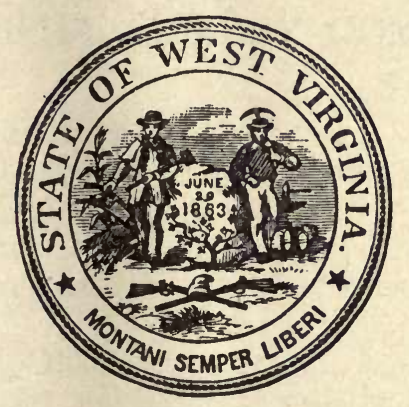

\section{PART I.}

The Living Flora of West Virginia

\author{
By \\ C. F. MILLSPAUGH
}

PART II.

The Fossil Flora of West Virginia

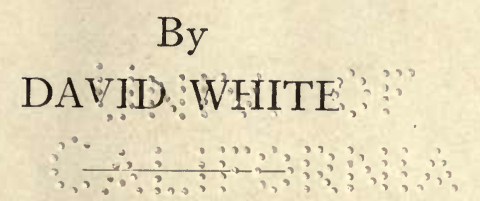

I. C. WHITE, State Geologist. 
$A 4$
$V, 5 A$

EARTH SCIENCES LIBRARY

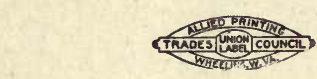

WHEELING NEWS LITHO. CO. WHEELING, W. VA.

1913

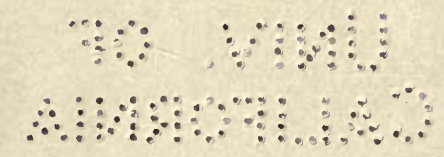




\section{GEOLOGICAL SURVEY COMMISSION.}

HENRY D. HATFIELD ...................... President GOVERNOR OF WEST VIRGINIA.

E. L. LONG .........................Vice President TREASURER OF WEST VIRGINIA.

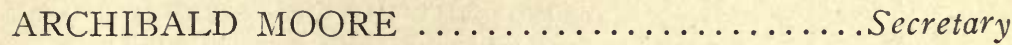
PRESIDENT, STATE BOARD OF AGRICULTURE.

THOMAS E. HODGES..................... Tredsurer PRESIDENT, WEST VIRGINIA UNIVERSITY.

E. DWIGHT SANDERSON .............Executive Officer DIRECTOR, STATE AGRICULTURAL EXPERIMENT STATION.

\section{STATE BOARD OF CONTROL.}

JAMES S. LAKIN ......................President

E. B. STEPHENSON....................... Treasurer JOHN A. SHEPPARD.............................. 


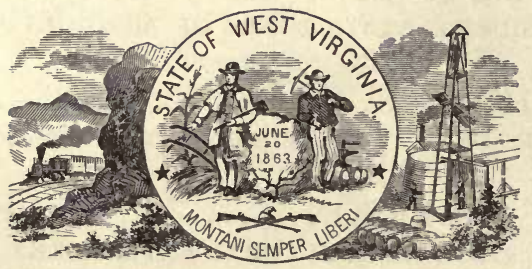




\section{SCIENTIFIC STAFF.}

I. C. WHITE ................................ Geologist SUPERINTENDENT OF THE SURVEY.

RAY V. HENNEN.................. Assistant Geologist

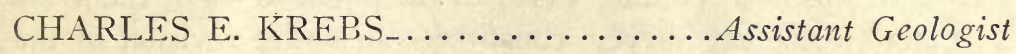

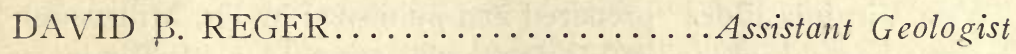
D. D. TEETS, JR....................... As Asistant BERT H. HITE........................ Chemist

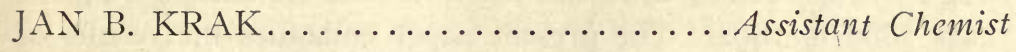
EARL M. HENNEN........................... Chief JENKIN L. WILLIAMS.................... 


\section{LETTER OF TRANSMITTAL.}

To His Excellency, Hon. Henry D. Hatfield, Governor of West Virginia, and President of the West Virginia Geological Survey Commission :

SIR: I have the honor to transmit herewith the very interesting work of Dr. C. F. Millspaugh, Curator of Botany in the Field Museum of Natural History, Chicago, Ill., on the Living Flora of West Virginia, and also the very complete work of Dr. David White, Associate Curator of Paleobotany in the Smithsonian Institution, and Chief Geologist U. S. G. Survey, Washington, D. C., on the Fossil Flora of West Virginia.

The very exhaustive paper of Dr. Millspaugh which forms Part I. of this new Volume V(A) of the publications of the West Virginia Geological Survey, constitutes an entire revision of the "West Virginia Flora" prepared and published by Dr. Millspaugh in 1896 , as a revision of his first publication of a "Preliminary Catalogue of the Flora of West Virginia," I89I, published by the West Virginia Agricultural Experiment Station when Dr. Millspaugh was connected with that Institution as Botanist during the years 1889 to 1892 . The present paper embodies the results of a much wider and later study not only by Dr. Millspaugh through the works and collections of other authors, but also the results of Dr. John L. Sheldon's (Professor of Botany, W. Va. University) recent studies communicated freely to Dr. Millspaugh for use in this publication, so that a very large addition to the West Virginia Flora is thus made known to the world by this "labor of love" on the part of Dr. Millspaugh, for which all those interested in the botany of the State will be deeply grateful.

Dr. David White's paper on the Fossil Flora of West Virginia which constitutes Part II. of this volume is the first publication of its kind made by the State giving a complete list of the known fossil plants, or the Flora that covered the land and peat swamps of West Virginia millions of years ago when the vegetable deposits which now form our coal beds were in process of 
accumulation. These plants are of course all extinct at the present time, and only a few of their diminutive relatives and descendants still survive in our ferns, rushes, and club-mosses, since the Carboniferous Flora herein described lived before the day of flowering plants, birds and mammals had dawned upon the earth. Dr. David White, one of the most distinguished of paleobotanists, has listed these interesting fossil remains in the relation which they sustain to the well-known Coal beds of the State, and hence as the fossil plants of each great coal horizon contain in their entirety some types and facies not found in connection with any other geological horizon, the publication in question cannot fail to prove of great economic value as an aid in the correlation of our numerous important coal horizons. The preparation of each part of this volume by the authors of the same has required an immense amount of careful and painstaking labor, all of which has been generously donated to the State Geological Survey by the distinguished authors to whom not only the Survey but all the people of the State are under lasting obligations for their disinterested and most valuable services.

This volume together with Volume $\mathrm{V}$ on Forestry and Wood Industries of the State by A. B. Brooks, published under date of February Ist, I9II, will furnish a very fair account of the plant life of the State, and in due time it is hoped finally to add a volume on the animal life of the State, as provided in the scheme of general publications contemplated by the Survey.

Very respectfully,

I. C. White, State Geologist.

Morgantown, W. Va., June Ist, I9I3. 


\section{CONTENTS.}

Members of the Geological Survey Commission and State Board of Control

Members of Scientific Staff

III

Letter of Transmittal

Table of Contents

\section{PART I.}

The Living Flora of West Virginia

Botanical Field Work of Previous Authors_-_-_-_-_-_-_-_-_ 2-13

Special Features of the West Virginia Flora_-_-___-___-__-_-_ 14-18

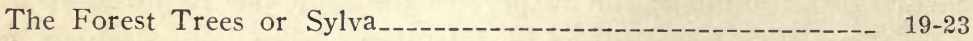

Systematic Catalogue -_-

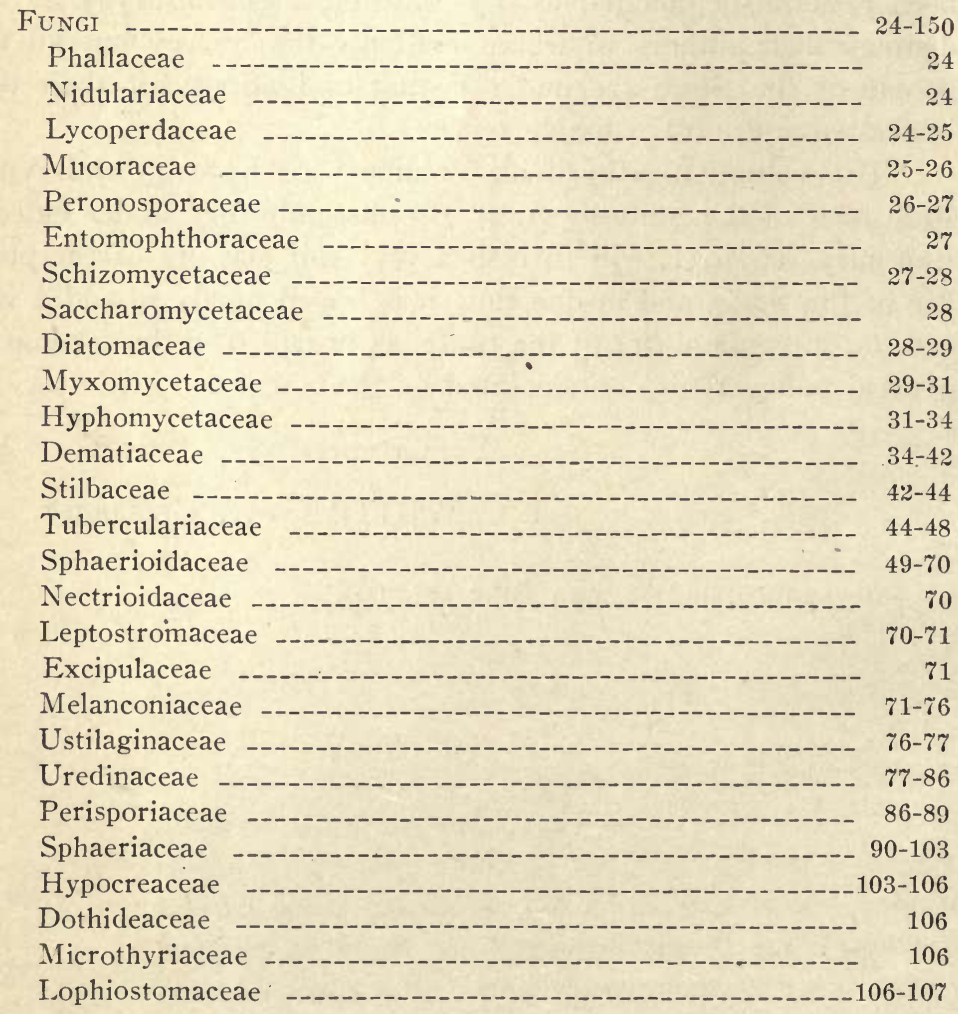


Helvellaceae -

Pezizaceae -_-

Ascobolaceae -

Dermateaceae _.

Bulgariaceae -_-

Stictaceae -_- 117

Phacidiaceae -

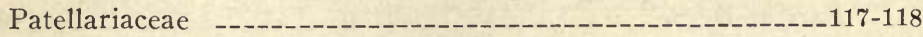

Gymnoascaceae _-_-_-_- 118

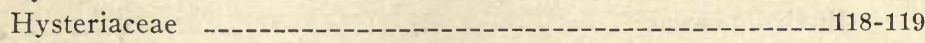

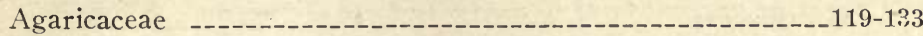

Polyporaceae -

Hydnaceae --_-

Thelephoraceae -_-_-_-_-_-_-_-

Clavariaceae -

LICHENES -_-

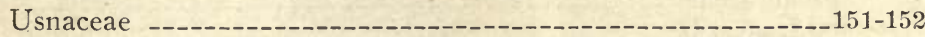

Parmeliaceae _._. 152

Physciaceae --_-_- 153

Umbilicariaceae -

Peltigeraceae -

Pannariaceae -

Collemaceae -_-

Lecanoraceae -_-

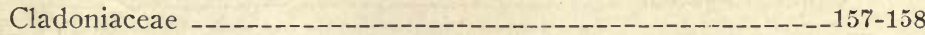

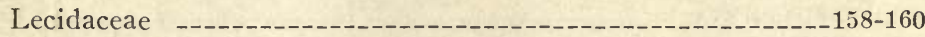

Graphidaceae -._-_-_-_-161

Caliciaceae _-_-_- 161

Verrucariaceae -

Trypetheliaceae -_-

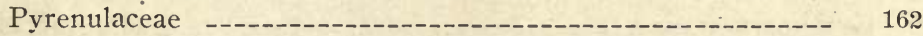

HePaticae -

Ricciaceae --_-- 163

Marchantiaceae _-_-_-_-_-163-164

Jungermanniaceae -_-_-_-_-_-_-_-_-_-_-_-_-_-_-_-_-_-_164-171

(Anacrogynae) -

(Acrogynae) -

Anthocerotaceae --_-_-

Sphagnaceae --_-_-_-_- 172

Andreaeaceae _...- 172

MUSCI -- -

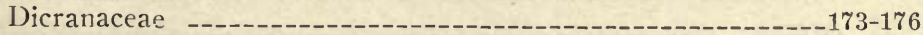

Leucobryaceae _-_-_-_-_-_- 176

Fissidentaceae -

Encalyptaceae _. 
Pottiaceae -

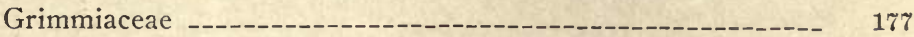

Orthotrichaceae _._.

Funariaceae -_-

Bryaceae --_.

Mniaceae _-_- 180

Aulacomniaceae _-

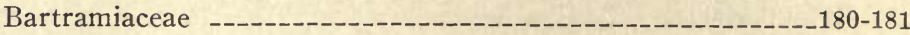

Weberaceae _-_._. 181

Buxbaumiaceae _-_-_-_-_- 181

Georgiaceae -

Polytrichiaceae _-

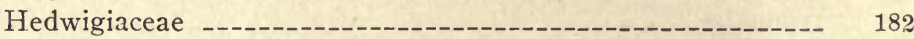

Fontinalaceae -

Climaciaceae -

Leucodontaceae _._- 183

Neckeraceae -

Fabroniaceae - 183

Entodontaceae -

Hookeriaceae -..-_-_-_- 184

Leskeaceae -_-

Hypnacae --_-_-_-_-

Sematophyllaceae _-_-_-_-_-_-_- 189

Brachytheciaceae _-_._-189-190

Ophioglossaceae - - 190

Pteridorhyta _-

Filices -

Equisetaceae -

Lycopodiaceae - -

Selaginellaceae _- 198

GYM NOSPERMAE _-

Pinaceae -

Taxaceae _-_-_-_-_-_-_- 201

Typhaceae -_- 201

Sparganiaceae - 202

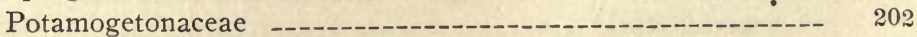

Scheuchzeriaceae -

Alismaceae --

Vallisneriaceae --_- 203

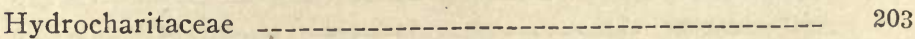

Gramineae -

Cyperaceae -_-

Araceae -

Lemnaceae _- 224

Commelinaceae -

Juncaceae _- 
Melanthaceae -

Liliaceae _...

Convallariaceae -

Smilaceae -- -

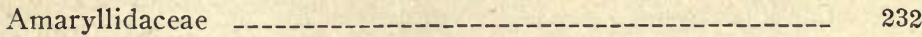

Dioscoreaceae -_-

Iridaceae _- 233

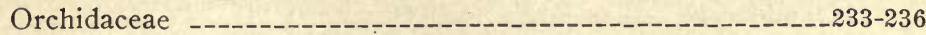

Saururaceae -_-

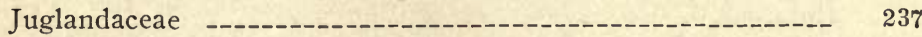

Salicaceae -_-

Myricaceae -_- 239

Betulaceae -

Fagaceae -

Ulmaceae -_- 242

Moraceae _-_- 242

Urticaceae -_.

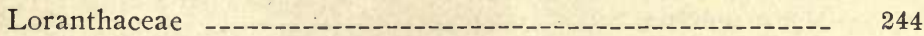

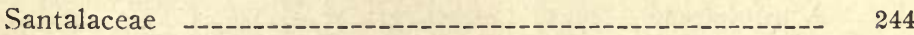

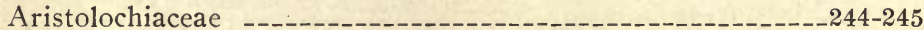

Polygonaceae -

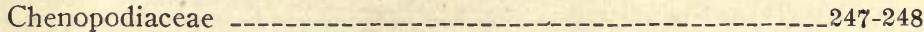

Amaranthaceae

Phytolaccaceae -

Aizoaceae -_-

Portulaceae -

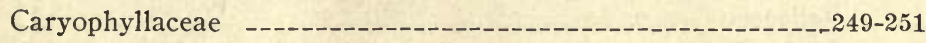

Nymphaeaceae --_-_-_-_-_-_-_-_-_-_-_-_-_-_-_-_-_-_-_-_-_- 252

Magnoliaceae -_-

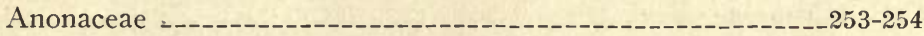

Ranunculaceae

Berberidaceae -

-Menispermaceae --_-_-_-_-

Calycanthaceae _.

Lauraceae -_-

Papaveraceae . -

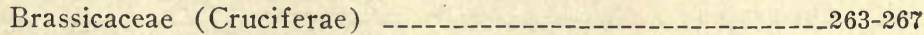

Capparidaceae -

Droseraceae _- 267

Podostemonaceae _._-

Crassulaceae --_- 268

Penthoraceae - - 268

Parnassiaceae _. 268

Saxifragaceae

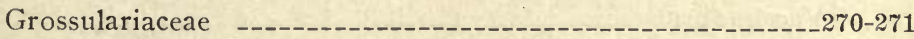

Hamamelidaceae _._- 271 
Platanaceae -

Rosaceae --_-1-

Pomaceae --_

Drupaceae _-_- 282

Caesalpinaceae --_-_-

Papilionaceae -

Gerianiaceae -_-

Oxalidaceae -

Linaceae -

Rutaceae --

Simarubaceae _-

Polygalaceae --_-

Euphorbiaceae --_-_-

Callitrichaceae --

Empetraceae --

Buxaceae -_-

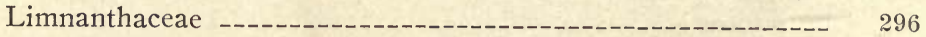

Anacardiaceae -_-

Aquifoliaceae -

Celastraceae --

Staphyleaceae --_-_- 299

Aceraceae -.

Sapindaceae -_-

Balsaminaceae -_- 301

Rhamnaceae -_-

Vitaceae -_.

Tiliaceae -_- 303

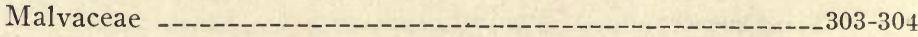

Hypericaceae -

Cistaceae -

Violaceae -

Passifloraceae _. 309

Cactaceae -

Thymelaeaceae _-_ 309

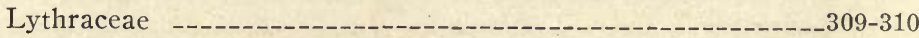

Melastomaceae _-_._- 310

Onagraceae --_-

Araliaceae -_-

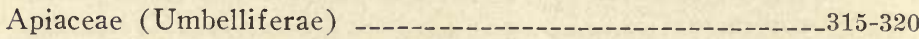

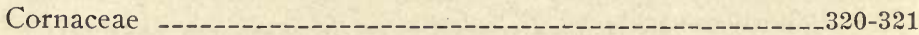

Clethraceae -_- 321

Pyrolaceae - -

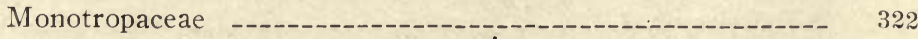

Ericaceae - -

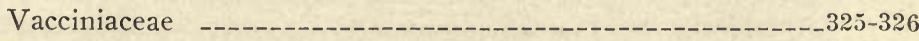

Diapensiaceae _-

Primulaceae _._. 
Ebenaceae - -

Styracaceae -

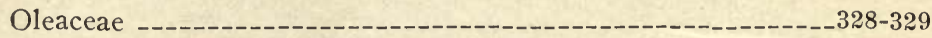

Loganiaceae -_- 329

Gentianaceae - 330

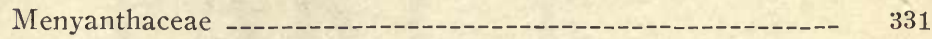

Apocynaceae -.-_- 331

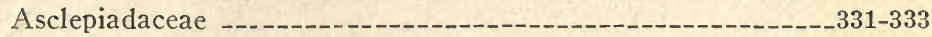

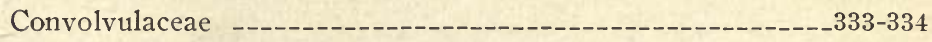

Cuscutaceae -

Polemoniaceae -

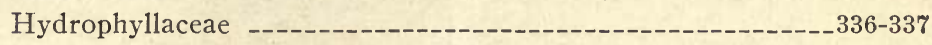

Boraginaceae - -

Verbenaceae -_- 340

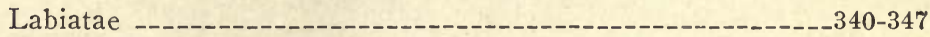

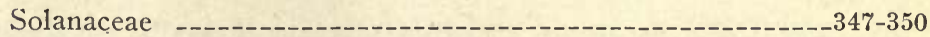

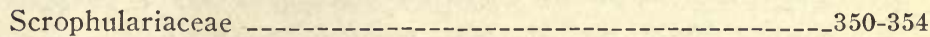

Lentibulariaceae -_-_-_-_-_-_- 35 5

Orobanchaceae --_-_-_.-_-_-_- 355

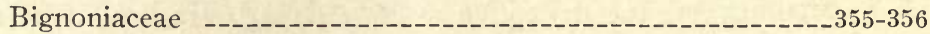

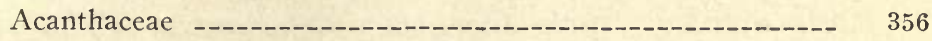

Phrymaceae - -

Plantaginaceae -

Rubiaceae -_-

Caprifoliaceae -

Valerianaceae - 362

Dipsacaceae - -

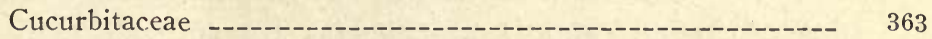

¿ampanulaceae -_-

Cichoriaceae -_-

Ambrosiaceae -_- 369

Carduaceae -

PART II.

The Fossil Flora of West Virginia

Index to Part I. $390-453$

Summary of Part 1

Index to Part II. 



\section{PART I.}

\section{The Living Flora of West Virginia}

\section{By C. F. MILLSPAUGH}

The State of West Virginia comprises about 24, I70 square miles of territory lying between $37^{\circ} 30^{\prime}$ and $40^{\circ} 30^{\prime}$ north latitude, and $0^{\circ} 45^{\prime}$ and $5^{\circ} 30^{\prime}$ west longitude from Washington. Its outline of varied boundaries has become a synonym for irregularity, as a glance at the accompanying map will show; it might also comprise the topography, suggesting as it does an immense field over which a gigantic plow has left a confused maze of deep and irregular furrows. This topographical condition is mainly due to the great number of mountain ranges and a vast network of rapid streams, that, rising in the higher altitudes of the eastern and southern borders, pass in varied and tortuous courses through the State, to augment the Ohio on the west and northwest, and the Potomac on the northeast.

Along the low, as well as the lofty mountain ranges, there is comparatively little tableland, and in the wedge-like valleys there is a like absence of extensive bottoms, except along Tygart's Valley River in Randolph County, the Great Kanawha and tine Ohio. Although there are many mountain glades, some nearly dry and others swampy, plainly indicating their late occupancy by small lakes, there is today neither pond nor lake within the limits of the State, and very little if any stagnant water.

As the major portion of the State lies west of the Alleghanies, the climate is much like that of western Pennsylvania, partaking little indeed of that southern atmosphere that we are wont to associate with the name Virginia.

The prevailing soil of the hills and valleys is stiff clay, and sandy and clayey alluvium, over which there is in general but 
little loam. The clay of the more open steep hillsides is so unctuous and unstable that frequent landslides occur during Spring, sometimes of great extent. This subsidence renders the valley streams muddy throughout the year. The rocks are principally sandstone and limestone, with some outcroppings of shales on the northeastern heights. The special features of very fertile and quite sterile soils, varied altitude and the vast areas of primitive forests, yield a flora of great variety, often widely differing at points only a few miles apart.

The amount of exploration necessary to gain a full knowledge of the flora under these conditions becomes an arduous undertaking, though the interest in searching an almost virgin field is so deep as to greatly lighten the labor.

\section{BOTANICAL FIELD WORK IN THE STATE.}

That several of the early Pennsylvanian, Virginian and North Carolinian Botanists, including Peter Kalm, John Fraser, John Clayton, Thomas Nuttall, John Mitchell and others, collected within the eastern borders of the State is reasonably certain, though I have not been able, so far, to establish their localities with any degree of certainty. There is great difficulty in locating West Virginian stations in the field work of the very early Botanists from the fact that most of their notes and labels read simply "Virginia." Previous to I784 Virginia extended indefinitely from the Atlantic to any point beyond the Ohio River, while the border lines of West Virginia were not established until 1863. Then, too, the country was so thinly settlemented and localities so uncertainly named that ascribing definite geographic place to collected plants was impossible to the knapsack traveller.

The following chronologic tabulation gives all the information that I have been able to compile upon the field work of botanists within the State.

(1) Andre Michaux, the renowned French Botanist (who traveled extensively in North Carolina, Virginia, Kentucky and Illinois; even North into Canada and South into Florida and the Bahamas); made many journeys, under conditions of great hardship and no little danger, along the out confines of the mountain borders of West Virginia; especially in the New River region of southwestern Virginia. He is credited by one of his biographers with field work in the contiguous West 
Virginia counties, but his carefully and explicitly kept Journal does not bear out such a conclusion. He did however collect at Harpers Ferry, Charlestown and Summit Point, in Jefferson County, and at Wheeling, Buffalo Point, and the mouth of the Little Kanawha River, on his journey to Kentucky and Illinois in 1793. His collections are in the Herbarium of the Museum of Paris.

(2) Matthias $\mathrm{KIN}_{\mathrm{N}}$, a German nurseryman and collector, took up his residence in Philadelphia and from there made many extensive excursions for the purpose of collecting living plants and seeds for horticultural purposes. During these trips he also preserved plants for herbarium material. About the year 1800 he collected extensively from Cumberland, Md., through the Tygarts River Valley in Randolph County. His exact localities are in doubt as his labels are eccentrically written in "Pennsylvania Dutch." The prime set of his collections of dried plants is in the Royal Botanical Garden, Berlin.

(3) Frederick Pursh, a noted German Botanist (born in Russia) established himself in Philadelphia in 1799. From there he made many important botanical explorations. His most interesting collections were made in the vicinity of Harpers Ferry, Jefferson County; and in Greenbrier and Monroe Counties in the neighborhood of White Sulphur and Sweet Springs in 1805. His prime collection is in the Herbarium of the Royal Botanical Gardens at Kew, London.

(4) (Constantine Samuel) Rafinesque (Schmaltz); the eccentric Franco-German naturalisi (Born in Turkey), immigrated to Philadelphia in 1802 . In 1818 , on his way to Kentucky, he collected plants at Wheeling, Williamstown and Point Pleasant: and on his return walked from Wheeling to Kenilworth through Ohio, Brooke and Hancock Counties, collecting throughout the route. In 1819 he collected afoot along the Potomac River trom Harpers Ferry to Cumberland, Md., through Jefferson, Berkeley, Morgan and Hampshire Counties. In 1825 he again collected in Ohio County from Valley Grove to Wheeling; and later, starting from Cumberland, he followed the South Branch of the Potomac from its mouth through Hampshire, Hardy, and Pendleton Counties to the South Fork Mountains, where he "collected many fine plants." From here he crossed the county and the mountains to Virginia. In 1832 he again collected in Jefferson County in the neighborhood of Harpers Ferry. While his collections were undoubtedly large, and very valuable, their numerical strength is unknown as his private herbarium has never been found. Some of his duplicates are in the herbaria of the University of Pennsylvania and the Philadelphia Academy of Sciences.

(4a) Dr. William E. A. Arkin of Baltimore, Maryland, collected in the neighborhood of Harpers Ferry, Jefferson County, about the year 1832. The extent and disposition of his collections is at present unknown. I have seen but one specimen of his, that in Dr. Torrey's herbarium, New York Botanical Garden.

(5) Gray, Carey and Constable. Dr. Asa Gray, John Carey and John Constable, on their collecting trip to the mountains of 
Southwestern Virginia and North Carolina in 1841, passed through Jefferson County from Harpers Ferry to the Virginia line. Although West Virginia plants are here and there credited to them it is certain that they did not collect within the borders of the State.

(6) Gray and Sullivant. Dr. Asa Gray and W(illiam) S(tarling) Sullivant collected through. the Allegheny mountains from Maryland to Georgia in 1843. While I am unable to outline their route with exactness, yet it is certain that they collected along the old Stauniton Pike, from Tygarts Valley through the Cheat Mountains, via Cheat Bridge and Travelers Repose; along the upper reaches of the Kanawha River; and at Big Spring in Mercer County. Just how much material they collected in the State there is no way of determining. Their plants are in the Gray Herbarium of the Cambridge Botanical Gardens, Cambridge, Mass.

(7) I(SAAC) F(ARWELL) HoltoN collected extensively from Charleston, Kanawha County, to Hawks Nest, Fayette County, in October, 1849. The full number of plants collected by him is unknown: 50 are in the Herbarium of the Field Museum of Natural History. His private Herbarium was deposited in that of Columbia University and is now at the New York Botanical Garden.

(8) Prof. W (illiam) H(enry) Brewer, while Professor of Botany at Yale, in 1860, made a collection of plants in Ohio County in the vicinity of Wheeling, and Brooke County near Bethany. The amount of material he secured is unknown to me; it is deposited in the Herbarium of Yale University.

(9) W(illiam) M(ARrioti) Canby, an extensive Botanical collector late of Wilmington, Del., made a small collection in. Taylor County, near Grafton, in 1868. The details of his collecting in the State I have not been able to determine. His plants are deposited in the Herbarium of the New York College of Pharmacy.

(10) J. S. Merriam made a collection of plants in Jefferson County in the vicinity of Harpers Ferry in 1871. The extent of his collecting I have not yet been able to determine. His specimens are deposited in the U. S. National Herbarium, Washington, D. C.

(11) Dr. T( імотнy) F (IELd) Allen, of New York, collected in July 1872 , on Kates Mountain and at other points in the neighborhood of White Sulphur Springs. His plants are deposited in the Herbarium of the New York Botanical Garden. (See also Allen and Britton).

(12) J (OSEPH) F(RANCIS) JAMES, of Ohio, spent about two weeks in 1877, investigating the Flora of the region of the Guyandotte and Great Kanawha Rivers from Huntington to Kanawha Falls and the Hawks Nest; and the region of Loup Creek, in Fayette County. The amount of material that he collected is not known to me. His plants are in the Gray Herbarium and the Cryptogamic Herbarium at Harvard University. 
(13) E. RICHARDSON collected in Ohio County in the vicinity of Wheel111g, in 1877 and 1879 ; and the neighborhood of Bethany, Brooke County, in 1878. The cxtent of his collections is not known to me at this writing. His plants are deposited in the U. S. National Herbarium, Washington, D. C.

(14) Mertz \& Guttenberg. Profs. H. N. Mertz and Gustav Guttenberg, of Wheeling and Pittsburg, collected extensively from 1877 to 1888 through all the northern counties of the State from Wheeling to Harpers Ferry. They published, in 1878, "A Check List of the Plants of West Virginia" in which they enumerated 590 species. Their collections are deposited in the herbarium of the Carnegie Museum, Pittsburg.

(15) Mertz \& Jones. Prof. H. N. Mertz and Miss Hattie Jones made a comprehensive collection of the plants of Cranberry Summit, Preston County, in 1878. Their specimens are preserved in the herbarium of the Carnegie Museum, Pittsburg.

(16) Prof. Cha(rle)s R(eid) Barnes, late professor of Physiological Botany, University of Chicago, spent a short time, in June, 1879, collecting along the Kanawha River from Charleston to Gauley Bridge, principally in the neighborhood of Charleston. His collections are in the Herbarium of Wabash College. (See his "Notes from West Virginia" in Botanical Gazette Vol. 4:181-2, 1879).

(17) Prof. John M (erle) Coulter, Professor of Botany, University of Chicago, conducted, while Professor of Biology at Wabash College, Indiana, an excursion of his class in Geology, to the mining regions of southern West Virginia, in 1879. On this trip he collected a few interesting plants from Charleston, Kanawha County, to Gauley Bridge, Fayette County. His specimens are deposited in the herbarium of Wabash College.

(18) Captain John Donnell-Smith spent his summers, from 1879 to 1882, at Oakland, Md., from whence he made occasional short trips to various points in West Virginia, especially those reached by the Baltimore \& Ohio Railroad. During these excursions he collected such plants as appeared to be of special interest, particularly at Tygarts Valley, Mannington and Grafton. His plants are in his private herbarium now deposited in the U. S. National Herbarium, Washington, D. C.

(19) C(yrus) G(Uernsey) Pringle, the noted botanical collector, late of Charlotte, Vt., made a small but interesting collection at White Sulphur Springs, in Greenbrier County, and in the neighborhood of Ronceverte, in Fayette County, in 1880. The exact number of his specimens is not known to me at this writing; they are deposited in his private herbarium now at the University of Vermont.

(20) Porter \& Redfield. Prof. Tho(ma)s C(onrad) Porter and Mr. J (ohn) H(oward) Redfield collected from White Sulphur Springs, Greenbrier County, to Hawks Nest, Fayette County, in 1880. The numerical strength of their separate collections I have not been able to determine. Prof. Porter's plants are in the herbarium of Lafayette College, Easton, $\mathrm{Pa}$; those of $\mathrm{Mr}$. 
Redfield in the herbarium of the Philadelphia Academy of Sciences.

(21) Dr. Aug (ustine) D(awson) Selby, Botanist of the Ohio Agricultural Experiment Station, collected plants in 1885 to 1887 in his neighborhood while Superintendent of Schools at Huntington, Cabell County. He also extended his plant observations up the Kanawha River as far as Kanawha Falls in Fayette County. His plants are in his private herbarium.

(22) S(amuel) B (oardman) Brown, Professor of Geology, West Virginia University, formerly Principal of the Normal School at Glenville, collected a large number of plants in Berkeley and Gilmer counties from 1885 to 1890 . Of these he preserved about 100 specimens which are still in his possession.

(23) (Charles) David White, Geologist of the U. S. Geological Survey, while engaged in paleobotanical collection in various portions of the State from 1886 to 1894 , collected a few recent plants, principally ferns; these are deposited in the U. S. National Herbarium, Washington, D. C.

(24) Miss Verona Mapel, then Preceptress of the High School at Glenville, Gilmer County, collected largely in her immediate neighborhood from 1888 to 1901 . Her material, consisting of about 355 species (not including common weeds nor the grasses and sedges), is deposited in the herbarium of the school mentioned.

(25) Dr. Rosencrans Workman, a Physician at Bayard, Mineral County, collected a representative series of the flowering plants of his immediate neighborhood from 1888 to 1891 . His material (which he loaned me for examination in 1891) is in his private possession.

(26) Winfield E. Hill, notes in "Garden and Forest" (Vol. 3: 182-183, 1890), a few plants from Fairview, Hancock County, observed in 1889. Whether he has preserved a collection of plants from his region I am unable to determine at this writing.

(27) Dr. H (amilton) McS(parkin) Gamble, a Physician at Moorefield, Hardy County, did considerable herbalizing in connection with zoological field work in the valley of the South Branch of the Potomac River, and its water shed in Hardy, Grant, Mineral and Hampshire counties from 1889 to 1910 . He donated his plant collection of 157 species (in 1891) to the herbarium of the West Virginia Agricultural Experiment Station, at Morgantown, where it is now deposited.

(28) M(erton) B(enway) Waite, Pathologist, Bureau of Plant Industry, U. S. Department of Agriculture, spent about a week, in 1889, in Fayette and Greenbrier Counties, in the collection of parasitic fungi. He secured about 300 numbers at Kanawha Falls and about 200 at White Sulphur Springs. As his plants were distributed in the herbarium of the Bureau, unlisted, they are unfortunately unavailable for this publication. In 1911 he collected a few numbers of like material in Berkeley County, near Gerrardstown; and Morgan County, at Sleepy Creek, Paw Paw and Hancock. His plants are deposited, as above indicated, in the herbarium of the U. S. Bureau of Plant Industry. 
(28a) W(ALTER) B(RUCE) NutTer, an attorney-at-law of Buckhannon, collected for his personal pleasure in Upshur, Lewis, Gilmer and Randolph Counties from 1890 to 1897. His herbarium of about 700 specimens is in his personal possession.

(29) Dr. J ( $\mathrm{OHN}$ ) K (UNKel) SMall, of the New York Botanical Garden, spent about a week in the neighborhood of Cumberland, Maryland, with special reference to collecting in the contiguous Pennsylvania field in 1890. During this work he made several trips across the Potomac River into Mineral County, where he collected about 50 specialized plants; these are now in the herbarium of the New York Botanical Garden. (See also Small \&. Brown).

(30) Mrs. N. L. Britton (E. G. Britton) collected a number of mosses in August, 1890, in the neighborhood of White Sulphur Springs, Greenbrier County. These are deposited in the Moss Herbarium of the New York Botanical Garden.

(31) Dr. C(harles) F (rederick) Millspaugh, Curator of Botany, Field

- Museum of Natural History, Chicago, began in 1890 a systematic survey of the plant life of the State. In the work he visited several localities in nearly every county. On one trip he drove through Wood, Wirt, Calhoun, Gilmer, Lewis, Upshur, Randolph, Webster, Nicholas, Fayette, Kanawha, Putnam and Jackson counties collecting leisurely throughout. During his field work, covering two seasons, he collected 1580 numbers; these are preserved in the herbarium at the West Virginia Experiment Station with more or less full duplicate sets in the herbarium of the New York Botanical Garden and that of the Field Museum of Natural History. (See his "Preliminary Catalogue of the Flora of West Virginia 1891" and "Flora of West Virginia 1896").

(32) Dr. N (athaniel) L (oRd) Britton collected one day in August, 1890 , in the neighborhood of White Sulphur Springs, securing about 40 interesting numbers; he again collected the same region in 1898, obtaining about the same number of specimens. Both of these collections are deposited in the herbarium of the New York Botanical Garden. (See Allen \& Britton).

(33) Mr. L (awrence) W (Illiam) Nuttall, then a mine owner at Nuttallburg, Fayette County, spent most of his spare time from business duties, from 1890 to 1898 , in botanical field and laboratory work on the Flora of his neighborhood. His large collections, mostly of fungi, are in his private herbarium though a considerable duplicate series including his types in Lichenes are in the herbarium of the Field Museum of Natural History. His types in Fungi are in the Ellis Herbarium, now at the New York Botanical Garden. (See Millspaugh \& Nuttall, "Flora of West Virginia").

(34) Small \& Brown. Hon. Addison Brown and Dr. J. K. Small spent about a week, in July, 1892, in Greenbrier County, in an explora. tion of Kates Mountain and the vicinity of White Sulphur Springs. The resulting material, about 150 plants, is now in the herbarium of the New York Botanical Garden.

(35) Dr. A (NDREW) D(ELMAR) Hopkins, Forest Entomologist of the U. S. Department of Agriculture, has taken special interest in col- 
lecting unusual plants during his many trips in various portions of the State, from 1892 to 1902 , in pursuit of his entomological studies. His plants are deposited in the herbarium of the West Virginia Agricultural Experiment Station, with duplicates in the Herbarium of the Field Museum of Natural History.

(36) Prof. A (mos) A (rTHur) Heller, of the University of Nevada, did considerable plant collection on Kates Mountain and the neighborhood of White Sulphur Springs in Greenbrier County in 1893. A set of his plants is deposited in the herbarium of the Field Museum of Natural History with the balance of his private herbarium.

(37) L(eE) C(Leveland) Corbett, while engaged in forest field work for the West Virginia Agricultural Experiment Station, collected a number of plants from 1894 to 1896 ; these are i1 the herbarium of the Station.

(38) W(Illiam) Mc(Cally) Pollock, while a student at the University of West Virginia, collected largely, from 1893 to 1897, in Lewis, Upshur, Preston, Pocahontas, Taylor and Wood connties, and from 1898 to 1899 in Monongalia County. The full number of plants he secured amounted to about 1700 specimens. His prime series was accidentally destroyed. Sets of over a hundred duplicates are in the herbaria of the Field Museum of Natural History, New York Botanical Garden, and U. S. National Museum.

(39) W (Illiam) E(aRle) Rumsey, of the Department of Entomology, University of West Virginia, collected a considerable number of plants in various parts of the State while engaged in entsmological field work, from 1894 to 1899. His plants are in the herbarium of the West Virginia Agricultural Experiment Station

(40) Allen \& Britton. Drs. T. F. Allen and Nathaniel L. Britton collected for one full day in May, 1897, in the neighborhood of White Sulphur Springs, Greenbrier County. They secured from 40 to 50 species of especial interest, which were deposited in the herbarium of Columbia University, now a part of the herbarium of the New York Botanical Garden. (See also 1872 T. F. Allen, and 1890 N. L. Britton).

(41) Rev. A. Boutlou, of Fairmont, Marion County, spent considerable of his spare time, from 1897 to 1907, in botanizing in his immediate neighborhood and in Taylor, Monongalia and Preston Counties. His collections are in his private possession though many of his interesting finds are represented in the herbarium of the Field Museum of Natural History.

(42) Prof. Edw (ard) L(eE) Greene, of the U. S. National Herbarium, has spent a day or so once or twice each year, from 1897 to July, 1912, in Jefferson County, in the vicinity of Harpers Ferry. His collecting in this region has been of a highly discriminating character, confined to selecting only such plants as appeared to differ from the usual form. His specimens are in the U. S. National Herbarium, with a few particularly interesting duplicates in the herbarium of the Field Museum of Natural History. 
(43; Edward S(trieby) Steele spent the latter half of August and the first half of September, 1898, collecting, with the aid of Mrs. Steele, in the neighborhood of Aurora, Preston County. He secured about 325 numbers, the first set of which he deposited in the U. S. National Herbarium.

In 1903 , and again in 1905 , he collected near Old Sweet Springs, Monroe County; in 1906 he spent one day at White Sulphur Springs, Greenbrier County, searching particularly for plants of the genus Laciniaria. In 1910, while collecting in Garret County, Maryland, he utilized a small part of his time gathering specimens across the line in Grant County, W. Va.; this he also did in 1911, in Hardy County, while occupied principally on the Virginia slopes of North Mountain, near Orkney Springs. Those later collections are also deposited in the U. S. National Herbarium, Washington.

(44) Pollard \& Maxon. Charles L(ouis) Pollard and William R(alph) Maxon collected during the latter part of August, 1899, in Fayette County, near Quinnimont and in Summers County, near Lowell. Of the 125 numbers they secured about 30 were new to the Flora of the State as known at that date. Their plants are deposited in the U. S. National Herbarium, Washington, D. C. (See "Proceedings of the Biological Society of Washington, Vol. $14: 161-163)$.

(45) E(DWARD) L (yMAN) Morris, Curator, Brooklyn Institute Museum, while engaged in field work for the U. S. Fish Commission in 1900 , made a collection of plants in that region of the State lying south of the New, Greenbrier and Kanawha Rivers in Summers, Monroe, Mercer, McDowell, Raleigh and Wyoming Counties. His collection of 397 numbers was made in July and August of a particularly dry season; it is deposited in the National Herbarium, Washington, D. C. (See his "Some Plants of West Virginia" in the Bulletin of the Biological Society of Washington, Vol. $13: 171-182$ (1900).

(46) Henry C(Urtis) Beardslee spent the Summer of 1900 collecting principally fleshy fungi, in the neighborhood of Brookside, Preston County. His collections, numbering about 600 specimens, are in his private herbarium at Asheville, N. C. (See his "Notes on the Boleti of West Virginia" in "Torreya." Vol. I:37-39).

(47) Captain K. D. Waliker, of Fairmont, West Virginia, contributed a few plants of his collecting to the West Virginia Experiment Station, in 1891. They are from Little Falls, Monongalia County, and are deposited in the herbarium of the Station.

(48) C(URtis) G(ates) Lloyd, of the Lloyd Herbarium, Cincinnati, Ohio, collected for about a fortnight at Eglon, Preston County, in 1901. His specimens, principally fungi, are preserved in the Lloyd Herbarium, Cincinnati, Ohio.

(49) Prof. W (illiam) A (shbrook) Kellerman, late professor of Botany in the University of Ohio, collected, principally fungi, at Durbin and Marlinton, Pocahontas County, in August, 1902. I am unfortunately unable to establish the extent of or locate his specimens at this time. 
(50) Prof. A (Lbert) S(PEAR) HitchCock, Agrostologist of the U. S. Department of Agriculture, made small but discriminative collections, mostly of grasses, in the neighborhood of Morgantown, Monongalia County, in 1902, and Harpers Ferry, in Jefferson, in 1905. His plants are in the herbarium of the Department in Washington.

(51) F(RED) E(RNEST) BRooks, of the U. S. Bureau of Entomology, traveled extensively throughout the State engaged in field work for the Bureau, from 1902 to 1912. During that work he collected such plants as appeared of particular interest. His plant collection is deposited in the herbaria of Prof. John L. Sheldon and the West Virginia Agricultural Experiment Station; both at Morgantown.

(52) Kenneth K(ent) Mackenzie, EsQR., collected, from August 27th to September 9th, 1903, in Greenbrier County, in the vicinity of White Sulphur Springs and Kates Mountain. He secured 187 numbers of interesting plants, among which is his new genus in the parsley family (Pseudotaenidia). His prime set of plants is in his private herbarium and a full series of duplicates in that of the New York Botanical Garden.

(53) C(harles) L. Boynton, of the Biltmore Herbarium, collected, during the month of June, 1903, at Huntington, Kenova, Milton, St. Albans, Saltes, Charleston, Eastbank, Gauley Bridge, Thurmond, Hinton and White Sulphur Springs; revisiting the latter place in October. In May and September 1904 he again collected in the neighborhood of White Sulphur Springs and Tuckahoe. His total gatherings amounted to 295 plant numbers, the prime set being deposited in the Biltmore Herbarium.

(54) Dr. A (LBERT) LeRoy Andrews, while connected with the West Virginia University from 1903 to 1904 , collected in many localities in Monongalia and Preston Counties. He specialized in the Bryophytes. His plants are principally in the herbaria of the Experiment Station at Morgantown and at Williams College, and in his private herbarium. (See his "Additions to the Bryophytic Flora of West Virginia", in "The Bryologist", Vol. 8:63-65 1905).

(55) John L(ewis) Sheldon, Professor of Botany and Bacteriology at the University of West Virginia, has made discriminative and specialized collections in the State, covering principally the counties of Monongalia, Preston, Greenbrier, Monroe, Pocahontas, Berkeley, Jefferson, Randolph, and all those along the Ohio River, from 1903 to 1912 . His field work has added a large number of species to the previously known Flora; these are indicated in the text of this work. Prof. Sheldon's prime set of 4454 numbers is deposited in his private herbarium at Morgantown, where it is freely utilized by his classes at the University. His collections consist largely of cryptogams. (See "The Bryologist," Vol. X:80-84; Vol. XIII :64-65 for his "Additional West Virginia Hepaticae," and "Species of Hepaticae Known to Occur in West Virginia.")

Dr. Sheldon has contributed to this Flora full lists of al! the fungi of his herbarium that have passed under the critical examination of specialists in the various groups. He has also 
sent in for examination unusual species of his flowering plants and in other ways assisted materially in this compilation.

(56) The following names that appear now and then upon the labels of plants in the herbarium of Prof. Sheldon are either those of his students or incidental correspondents. As one or more of their few plants may find publication or stray into some other herbarium, I have considered it best to make the following record of the names:
Alderson, R. W.
Allen, E. A.
Fram, T. J.
Moore, S. W.
Atwood, Horace
Bacon, Nathaniel
Gibson, H. B.
Grose, E. R.
Groves, H. D.
Nolan, A. W.
Bargerhuff, A.
Beale, J. M.
Behrens, Ed.
Bender, I. L.
Bonner, C. M.
Boord, J.
Bowers, J. F.
Brake, W. E.
Britton, L. S.
Brooks, C. L.
Hall, William McG. Post, A. Lee
Pollock, J. F.
$\begin{array}{ll}\text { Hartley, Carl P. } & \text { Sheldon, Clara } \\ \text { Heavner, M. M. } & \text { Shimer, S. S. }\end{array}$
Heck, A. T.
Showers, George E.
Henson, Miss A. MShull, C. L.
Hodges, Charles E. Shunk, I. V. D.
Hoff, E. B.
Smaltz, R. B.
Holman, Lucien
Smith, N. S.
Humphreys, H. C. Staats, Miss Georgia L.
Brooks, Earle A
Hunter, Glenn
Brooks, Len
Carroll, H. D.
Chappell, A. M.
Hurley, J. J.
Johnson, R. W.
Johnson, T. C.
Keister, John D.
Cooper, H. E.
Cross, E. L.
Crow, C. S.
Kline, C. S.
Lambert, F. B.
Stalnaker, J. J.
Curtis, A. W.
Cutright, Frank
Larkin, Finney L.
Lewis, C. C.
Lewis, E. M.
Docherty, S. W.
Lindsley, Miss
Dodd, D. R.
Lively, E. L.
Starcher, G. C.
Stewart, T. W.
Stewart, R. H.
Stewart, W. R. P.
Stutzman, John
Summers, T. B.
Dumire, Mrs. George
McCutcheon,
Swank, G. L.
Swisher, J. A. H.
Thatcher, J. N.
Thompson, S. M.
Emmart, L. P.
Martin, C. S.
Fenton, Winnifred B. Maxwell, W. B.
Fidler, J. Z.
Merrick, C. D.
Fleshman, H. F.
Moffatt, B. H.
Fling, Miss Eva M.
Moore, Lewis $M$.
Vanorsdale, M. A.

(57) Dr. J (ESSE) M (ORE) GReenman, Assistant Curator of the Department of Botany, Field Museum of Natural History, spent the month of September, 1904, collecting at the following stations: Parsons and Hendricks in Tucker County; Dry Fork, Horton, Spruce Knob, Elkins, Read, Whitmar, Glady, Harman, and Huttonsville, in Randolph County; and Travelers Repose in Pocahontas County. The prime set of his collection, consisting of about 400 numbers (1500) specimens, is in the Gray Herbarium, Cambridge, Mass.; and a nearly full duplicate set in the herbarium of the Field Museum of Natural History.

(58) Albert H (Anford) Moore. of Cambridge, Mass., collected with Dr. J. M. Greenman in 1904, over the same route and gathering substantially the same species. His collection is in his private herbarium. 
(59) Frank F(ITCh) Grout, while connected with the West Virginia Geological Survey as Chemist, collected a number of plants in various parts of the State, from 1904 to 1906, especially parasitic fungi. His collections are in the herbarium of Prof. Sheldon and that of the West Virginia Agricultural Experiment Station.

(60) Huron H(erbert) Smith, Assistant in Dendrology at the Field Museum of Natural History, collected, in 1908, 246 numbers in Randolph, Webster, Nicholas, Lewis, Upshur and Wood Counties. His species, most of which are indicated in the following catalogue, are deposited in the herbarium of the Museum.

(61) O(tro) E(mery) Jennings, Botanist at the Carnegie Museum, Pittsburg, made a collection of plants in Monongalia County along the Monongahela River from Lock 9 to Morgantown, Mont Chateau and Coopers Rocks, in 1909. His specimens are deposited in the herbarium of the Carnegie Museum.

(62) W(illiam) W(ebster) Eggleston collected from 1909 to 1911 with a view of securing all possible material in the Pomaceae gathering also a considerable number of other plants that appeared to be of particular interest. He collected his numbers 4317-4372 at White Sulphur Springs in 1909; 5514-5551 at Gauley Bridge in 1909 ; 5552-5562 near Thurmond 1909 ; 5563-5587 near Hinton and 5588-5593 at White Sulphur Springs the same year. In 1911 he collected again at White Sulphur Springs (77617767). His material is deposited in the herbarium of the New York Botanical Garden.

(63) Mr. A(lonzo) B (excher) Brooks, while occupied in amassing data for Volume V, of this Survey, collected a considerable number of particularly interesting plants, from 1909 to 1911 , especially of the Glade Regions of the northern and northeastern sections of the State. The material that he preserved is deposited in the herbarium of the West Virginia University, at Morgantown. (See "West Virginia Geological Survey, Vol. $\mathrm{V}, 1911)$.

(64) I have seen labels, bearing the following names, upon plants collected in recent years in the State; but have not yet been able to secure details as to the extent of the collections they appear to indicate:

Brown, William $\mathrm{H}$.

Hayes, N. S.

Hinzman, W. W.

McCartney, $\mathrm{H}$.

Morse. 


\section{INDEX TO COLLECTORS.}

(The numbers following the names refer to those of the preceding paragraphs.)

Aikin, W. E. A. 4a

Alderson, R. W. 56

Allen, E. A. 56

Allen, T. F. 11, 40

Andrews, A. LeRoy 54

Atwood, Horace 56

Bacon, N. 56

Bargerhuff, A. 56

Barnes, C. R. 16

Beale, J. M. 56

Beardslee, H. C. 46

Behrens, Ed. 56

Bender, I. L. 56

Bonner, C. M. 56

Boord, J. 56

Boutelou, A. 41

Bowers, J. F. 56

Boynton, C. L. 53

Brake, W. E. 56

Brewer, W. H. 8

Britton, E. G. 30

Britton, L. S. 56

Britton, N. L. 32,40

Brooks, A. B. 63

Brooks, C. L. 56

Brooks, E. A. 56

Brooks, E. E. 51

Brooks, L. 56

Brown, Ad. 34

Brown, S. B. 22

Brown, W. H. 64

Canby, W. M. 9

Carroll, H. D. 56

Chappell, A. W. 56

Cooper, H. E. 56

Corbett, L. C. 37

Coulter, J. M. 17

Cross, E. L. 56

Crow, C. S. 56

Curtis, A. W. 56

Cutright, F. 56

Docherty, S. W. 56

Dodd, D. R. 56

Dunmire, Mrs. G. 56

Eggleston, W. W. 62

Emmart, L. P. 56

Fenton, W. B. 56

Fidler, J. Z. 56

Fleshman, H. F. 56

Fling, E. M. 56

Fram, T. J. 56

Gamble, H. McS. 27

Gibson, H. B. 56

Gray, Asa 5, 6

Greene, E. L. 42
Greenman, J. M. 57.

Grose, E. R. 56

Grout, F. F. 59

Groves, H. D. 56

Guttenberg, G. 14

Hall, W. McG. 56

Hartley, C. P. 56

Hayes, N. S. 64

Heavner, M. M. 56

Heck, A. T. 56

Heller, A. A. 36

Henson, A. M. 56

Hill, W. E. 26

Hinzman, W. W. 64

Hitchcock, A. S. 50

Hodges, C. E. 56

Hoff, E. B. 56

Holman, L. 56

Holton, I. F. 7

Hopkins, A. D. 35

Humphreys, H. C. 56

Hunter, G. 56

Hurley, J. J. 56

James, J. F. 12

Jennings, O. E. 61

Johnson, R. W. 56

Johnson, T. C. 56

Jones, H. 15

Keister, J. D. 56

Kellerman, W. A. 49

Kin, M. 2

Kine, C. S. 56

Lambert, F. B. 56

Larkin, F. L. 56

Lewis, C. C. 56

Lewis, E. M. 56

Lindsley, Miss 56

Lively, E. L. 56

Lloyd, C. G. 48

McCartney, H. 64

McCutcheon, R. P. 56

Mackenzie, K. K. 52

Mapel, V. 24

Martin, C. S. 56

Maxon, W. R. 44

Maxwell, W. B. 56

Merriam, J. S. 10

Merrick, C. D. 56

Mertz, H. N. 14, 15

Michaux, A. 1

Millspaugh, C. F. 31

Moffatt, B. H. 56

Moore, A. H. 58

Moore, L. M. 56

Moore, S. W. 56
Morris, E. L. 45

Morse 64

Nolan, A. W. 56

Nuttall, L. W. 33

Nutter, W. B. 28 a

Pollard, C. L. 44

Pollock, J. F. 56

Pollock, W. M. 38

Porter, T. C. 20

Portmess, E. T. 56

Post, A. L. 56

Pringle, C. G. 19

Pursh, F. 3

Rafinesque, C. S. 4

Redfield, J. H. 20

Richardson, E. 13

Rumsey, W. E. 39

Selby, A. D. 21

Sheldon, C. F. 56

Sheldon, J. L. 55

Shimer, S. S. 56

Showers, G. E. 56

Shull, C. L. 56

Shunk, I. V. D. 56

Small, J. K. 29, 34

Smaltz, R. B. 56

Smith, H. H. 60

Smith, J. D. 18

Smith, N. S. 56

Staats, G. L. 56

Stalnaker, J. J. 56

Starcher, G. C. 56

Steele, E. S. 43

Stewart, J. W. 56

Stewart, R. H. 56

Stewart, W. R. P. ${ }^{56}$

Stutzman, J. 56

Sullivan, W. S. 6

Summers, T. B. 56

Swank, G. L. 56

Swisher, J. A. H. 56

Thatcher, J. N. 56

Thompson, S. M. 56

Vanorsdale, M. A. 56

Waite, M. B., 28

Walker, K. D. 47

Warden, J. M. 56

Welton, C. B. 56

White, C. S. 56

White, D. 23

Wilcox, C. S. 56

Wolfe, E. O. 56

Wood, A. N. 56

Workman, R. 25 
As in the preliminary catalogue, all species that have been described from known West Virginia types are republished in full.

\section{SPECIAL FEATURES OF THE FLORA.}

The distribution of plants within the boundaries of the State is wonderfully comprehensive. Canada places representatives of her boreal flora upon its Alleghanian mountain tops, some that have even passed by the States of New York, New Jersey, Pennvania, Delaware and Maryland in the transit; the Southern States contribute to its flora through the influence of the mysterious New River on the southeast; the great trunk lines of railway, as well as the open condition of the western border line along the Ohio River, give entrance to individuals of a migratory character from the Western Plains; and from some not readily accountable reason many Eastern forms, and even coast line species stray within its limits.

Of the noteworthy species we connect in Anemone trifolia, L., Canby's and Curtiss' Virginian stations with Knipe's Pennsylvanian, and that in a direct and sequential manner through the State. Our elevations for this species range from 850 to 2,300 feet. Trautvetteria Carolinensis (Walt.), Vail, is to be found along all such mountain rills as are deeply shaded, at altitudes above I,O0o. We extend the Manual distribution of Caltha palustris, L., southward nearly one degree, by finding it quite plentiful in the mountains of Randolph County, where Isopyrum trifoliatum (L.) Britt., keeps it company. Helleborus viridis, L., has its most western station on the north branch or the Potomac River, in Hardy County, whence it was first reported to Dr. Gray by Dr. Gamble, of Moorefield. Aconitum uncinatum, L., is at home all along the banks of Cheat River, at altitudes varying from 780 to 3,550 feet. Both species of Actaea are to be met with on the higher Alleghanies, where Cimicifuga Americana, $\mathrm{Mx}$., is the principal representative of the tribe.

Magnolia Fraseri, Walt., is a striking vernal feature of the whole Alleghanian region, while tripetala and acuminata are common. Berberis Canadensis, Mill. becomes a veritable weed in many fields in the southern portion of the State, vying with Papaver dubium, L., and Glaucium Glaucium (L.), Karst, in the 
northeastern section. Bicuculla eximina (L.), Millsp., we have found only upon the highest peaks of the Alleghanies.

Of the rambling crucifers, Sisymbrium Thaliana (L.) Celak, finds a home in Fayette County, and Lepidium campestre (L.), $\mathrm{R}$. Br., is the worst and most prevalent weed in the cultivated portions of the northeastern section.

Nearly all the violets lend their beauty to the adornment of the woods and meadows; Viola pedata, var. bicolor, Pursh., with flowers as large as the cultivated pansy and fully as beautiful, is frequent among the Devonian shales of the northeast; Viola primulaefolia, L., comes in from the coast as far as four degrees; and Viola hastata, Mx., is quite plentiful in the southern section.

Although Sida hermaphrodita (L.), Rusby, has not been found in the Alleghanies as yet, it is not at all rare along the New and Great Kanawha Rivers, from Quinnimont to the Ohio, and thence down that river to the limits of the State. Hibiscus Moscheutos, L., is found not only along the bottoms of the Great Kanawha, but also in the northeastern part of the State, while H. Trionum, L., is a quite common weed in gardens.

All of the native species of Aesculus, together with the var. hybrida of octandra, are found in the State. Ailanthus glandulosus is becoming a most troublesome weed in many sections, especially in the northern counties.

Among the Leguminals we have re-discovered Astragalus Carolinianus, L., which from our specimens is considered by Professor Britton to be indistinct from A. Canadensis, L., and as it has priority of publication, the latter well-known name becomes a synonym. Another important discovery in this genus is that $A$. distortus, $\mathrm{T}$. and G., habits the Devonian shales of Hardy County, the only station known for the species east of the Mississippi valley. This species is here associated with Opuntia polyacantha, Haw, in great quantity, giving this peculiar spot in the Alleghanies much the appearance of an arid waste in Arizona. Stylosanthes hamata (L.), Britt., here ventures farther east than has heretofore been supposed, and finds congenial soil along New River in Fayette County. Lespedeza striata (Thunb.), H. \& A., spreads profusely throughout the southwestern portion of the State. A new clover, Trifolium 
Virginicum, Small, has been discovered in Greenbrier County, by Mr. J. K. Small.

The roses are striking in the many new forms they produce: Rubus Millspaughi, Britt., is so profuse in the mountains of Pocahontas and Pendleton Counties that, according to the moun. taineers and hunters, it is upon it that the bears depend principally for fattening food prior to hibernation. Rubus Canadensis roribaccus, Baily, the Leucretia dewberry, came originally from Randolph County. Another peculiar Rubus ( $R$. Columbianus Millsp., has 5 to 7 -incised leaves of striking character. A new Spirea (S. Virginiana, Britt.), grows plentifully within half a mile of the University at Morgantown. Mercer County in the southern section of the State, presents a wonderful array of Crategi: C. spathulata. C. cordata, C. apiifolia, C. coccinea, $C$. tomentosa, C. punctata, C. Crus-galli, C. flava and its variety pubescens, and $C$. uniflora were all found during one day's botanizing in this section.

Of the Calycanths we have all, even the two species recorded "Virginia doubtful" in the Manual.

Of the saxifrages we have notably, Astilbe decandra, Don., Saxifraga erosa, Pursh., Boykinia aconitifolia, Nutt., Heuchera villosa, Mx., and $H$. Americana, L., and even the Laboradorian Ribes prostratum, L' Her.

Sedum Pulchellum, Nevii, ternatum, telephioides, and telephium, are with us. The beautiful Liquidambar Styraciflua extends limitedly down the Gauley and Great Kanawha. The Onagraceæ yield a new form in Ludwegia alternifolia, L., var. linearifolia, Britt. The purple and yellow passion-flowers ( $\mathrm{P}$. lutea, L., and, incarnata, L.) grace the thickets.

Passing many minor forms, the Compositæ gives us Elephantopus Carolinianus, Willd., and tomentosus, L. (called, as a weed, "The Devil's Grandmother") ; Eupatorium, coelestinum, I., profuse; Solidago Curtisii, S. rupestris, and Riddellii, Silphium perfoliatum, L.; Rudbeckia speciosa, Wend.; Helianthus grosseserratus, Mart., $H$. doronicoides, Lam., and $H$. laevigatus, T. and G.; Verbesina Virginica, L.; Cacalia suaveolens, reniformis, and atriplicifolia; Cnicus Virginianus, and pumilus, Torr.; Cichorium Intybus, L.; Tragopogon porrifolius, L.; Hieracium Canadense, 
$\mathrm{Mx}$., and longipilum, Torr. (extending both); and Chondrilla juncea, L., called, as a weed, "Naked-weed, Skeleton-weed."

The interesting bell-worts are: the white form of Lobelia syphilitica, L., and the rare Campanula divaricata, $\mathrm{Mx}$.

Of the rarer heaths Schollera erythrocarpa, Mx., grows on the highest peak of the Alleghanies, alt. 4,800 ft.; Chiogenes hispidula at the Falls of Blackwater; Menziesia globularis Salisb., Clethra acuminata, Mx., Moneses grandiflora, Salisb., and all the Rhododendrons except Rhodora and Lapponicum; even the rare $R$. canescens ( $\mathrm{Mx}$.), Porter, being found along the Cacapon River.

Naumbergia thyrsiflora is found in Upshur County and Mohrodendron Carolinum (called Shittim-wood) is plentiful along the Gauley and New Rivers. Polemonium Van-Bruntiae, Britt., comes south to our flora, as well as all the Hydrophyllums; together with Phacelia Purshii, Buck, and parviflora, Pursh.

The beautiful morning-glories, Impomoea coccinea, hederacea, purpurea, and pandurata, are all too plentiful as weeds here; and Cuscuta glomerata, Gronovii, and Epithymum, have been found sparingly. Physalis viscosa, L., steals away from "near the coast" and is found along the Ohio River, keeping company with Lycium vulgare, Dun., and Physalodes Physaloides; Gaertn.

The notable Scrophularias are: Collinsia verna, Nutt., Chelone obliqua, L., and Pentstemon canescens. Of the mints we have notably; Koellia verticilata, clinopodioides, pycanthemoides, and montana. The other mints worthy of remark are: Meehania cordata, Clinopodium vulgare, Scutellaria saxatilis, serrata, incana, parvula, and nervosa; Marrubium vulgare, Galeopsis tetrahit, and Stachys palustris and cordata.

Of the ten Euphorbias the most notable are E. Darlingtonia and E. Glyptosperma, var., pubescens, Engl., the latter not having been previously found east of Iowa as far as we can learn.

The presence of Quercus ilicifolia, Wang, in Hardy County. extends the Manual distribution southeastward; and the southing of $Q$. macrocarpa, Mx. is also extended by several stations in the State.

As to the conifers, we have about 270,000 acres of Picea Mariana, a few representatives of Abies balsamea, Thuya occi- 
dentalis, and several species of Pinus, as well as a few scant growths of Tarus Minor.

Among the sedges the principal item of interest is the rediscovery in Fayette County of what was doubtless the origina! type station of Carex Fraseri, And.

Of the Equisetaceae the most notable form so far found is E. laevigatum, Braun., gathered in the southernmost part of the State thus extending its distribution southeastward.

Of the Filices, the rarer forms found with us are: Polypodium polypodioides; Pellaea atropurpurea in great quantities in the southern section; Asplenium pinnatifidum, montanum (plentiful) and angustifolium; Dryopteris Goldieana, and marginalis Cystopteris bulbifera; Dicksonia punctilobula; and strange to say on the summit of Spruce Knob at an altitude of 4,800 ft. Dryopteris fragrans, in such great quantity that it is cut and stacked for fodder, this species being greatly relished by cattle.

Lycopodium lucidulum, L., annotinum, L., obscurum and its var. dendroideum, L., clavatum, and L. complanatum are all found in the forests of black spruce along the Alleghanies.

In the mosses, hepatics, and lichens, but little collecting has so far been done, no systemic searches having been made for specimens in these classes of plants. In the search for hepatics incidental to other exploration, in Mercer County, the dry bald face of a large limestone cave yielded a new species in Plagiochila Virginica Evans, as well as a rarity in the eastern flora of the United States, Radula Xalapensis, Mont. Among the mosses we have been rewarded in our itinerant work by finding two new forms Dicranodontium Virginicus, Britt. m. and D. Millspaughi Britt. m., as well as numerous noteworthy species.

Beside these unique forms, we report many species from our region that have not been before credited to the flora of North America, include many hitherto unpublished asci and spore measurements of species otherwise well described, and have transferred many not before well understood.

The host plants have proven also to be of special interest in that many of them yield certain species for the first time in the mycologic literature of this country, and many others pose as altogether new to Host Indices. 
THE SYLVA.

The State is very happily located for the growth of forests, being in the favored belt of temperature between about $37^{\circ}$ and $4 \mathrm{I}^{\circ}$. north latitude. Within its boundaries trending northeast and southwest, thus opening the country to the damp, warm winds from the Gulf, are numerous ranges of the great Appalachian, or Atlantic highlands, but by far the larger portion of the State lies on the westward slope of these mountains in the trans-Appalachian belt, the waters of which chiefly run northwestward and southwestward to the Ohio River. The altitude of the country descends from the Backbone or Alleghany range of the Appalachians, from an altitude of from 2,500 and 4,800 feet to 500 at the southwestern corner of the State on the Ohio, at Kenova, and about 600 on the same river at Wheeling. The altitude of the eastern corner of the State at Harper's Ferry is 272 feet; thus the range of altitudes in the State is from 272 feet to about 4,800 , giving a climatic range of 3,728 feet, or the equivalent of about $16^{\circ}$ of latitude; consequently West Virginia has extensive areas of adaptability for every variety of forest growth that is found within the limits of the northern States east of the Rocky Mountains.

The most elevated portion of the State is the great eastern border of the ridgy plateau from which the trans-Appalachian country descends, a territory some 200 miles in length from the headwaters of the Big Sandy to those of the North Branch Potomac, this region is in the main from 2,500 to 4,800 feet in altitude, and furnishes a congenial home to the black spruce, the white pine, and other evergreen trees peculiar to northern latitudes.

West Virginia has a greater amount of hardwood timber in its forests than any other State in the Union. A thorough examination convinces us that nearly or quite two-thirds of the State remains uncleared, and of this about a million and a half acres is still in virgin forests where the ax of man has never found its way, and where magnificent specimens of forest growth stand thickly side by side and reach a towering height, no finer view of standing timber may be had within the confines of the Union. These splendid forests covering over twenty-three thousand square miles yield nearly every species found in the north. 
Here trees grow to such size that ordinary methods will not suffice to handle them, and are frequently so densely compact that the light of day scarce penetrates their shade, and pathways must be cut before the ax men can find room to work.

The following list of the trees of the State, giving the diameter of trunks and width of board yield, is that of the State exhibit in the Forestry Building at the World's Columbian Exposition, 1893. The specimens were all gathered, by the author, during the winter of $1892-3$.

Ash (white), Fraximus Americana. Logs 40 in., 62 in., 93 in., planks 27 and 33 in., finished boards, plain, 20 and 21 in., mottled, 9,13 and 16 in., figured 9 and 13 in.

Ash (black), F. nigra.

Ash (mountain), Sorbus Americana. Trunk 8 in.

Ash (prickly), Xanthoxylum Americanum. Trunk 12 in.

Alder (mountain), Alnus viridis. Trunk 4 in.

Beech, Fagus atropunicea. Trunks 24 in., 27 in. and 38 in., plank 27 in., finished boards 27 and $3 \mathrm{I}$ in., quartered boards ? and 9 in.

Beech (water), Carpinus Caroliniana. Trunk Io in.

Birch (red), Betula le'nta. Trunks 34 in., 93 in., 6r in., 48 in., planks 15 and 30 in., finished boards 12 and 16 in., figured boards $8, \mathrm{I} 3$, and 14 in.

Birch (yellow), Betula lutca. Trunks 34 in., 36 in., 42 in., 49 in., plank 26 in.

Birch (hybrid), Betula lenta $\times$ lutea. Trunk 2 in.

Basswood (white), Tilia Americana. Trunks 32 in., 36 in., 43 in., $5 \mathrm{I}$ in., plank 28 in., finished board 16 in.

Basswood (yellow), Tilia heterophylla. Trunk 26 in.

Buckeye, Aesculus glabra. Trunk 29 in.

Buckeye (purple), Ae. octandra hybrida. Trunk 29 in.

Box Elder, Acer Negundo. Trunk 27 in.

Butternut, Juglans cinerea. Trunk 12 in.

Balsam Fir, Abies balsamea. Trunk 13 in.

Chestnut, Castanea dentata. Trunks 53 in., 40 in., 62 in., 78 in., plank 40 in., finished boards 26 in.

Cherry (wild), Prunus serotina. Trunks 36 in., 39 in., 48 in,. 53 in., plank 27 in., finished boards 6 and 18 in., blistered I3, figured 6 to 19 in., curly 9 to 21 in. 
Cherry (wild), Prunus Pennsylvanica. Trunk I4 in.

Cucumber, Magnolia acuminata. Trunk 35 in., plank 26 in.

Cottonwood, Populus moniiifera. Trunk 26 in.

Crab (wild), Pyrus coronaria. Trunk 8 in.

Dogwood, Cornus florida. Trunks 7 in., 13 in., 28 in.

Elm (White), Ulmus Americana. Trunks 23 in., 42 in., $6 \mathrm{I}$ in.

Elm (red, slippery), Ulmus pubescens. Trunk 16 in.

Fringe tree, Chionanthus Virginica. Trunk 3 in.

Gum (black), Nyssa sylvatica. Trunk 36 in., plank 27 in.

Gum (sweet), Liquidambar styraciflua. Block I9 in.

Grape (Fox), Vitis Labrusca. Trunk 22 in., branches 18 in.

Hackberry, Celtis occidentalis. Trunk I8 in.

Haw (black), Viburnum prunifolium. Trunk 5 in.

Hemlock, Tsuga Canadensis. Trunk 42 in., plank 30 in., finished boards 12 and 19 in.

Hercules Club, Aralia spinosa. Trunk 5 in.

Hickory (bitter), Hicoria minima. Trunks 9 and $\mathrm{x} 2$ in.

Hickory (red), Hicoria glabra. Trunks II and I7 in.

Hickory (shagbark), Hicoria ovata. Trunk 27 in., planks I4 and 18 in.

Holly (white), Ilex opaca. Trunk Io in.

Holly (mountain), Ilex monticola. Trunk 6 in.

Ironwood, Ostrya Virginiana. Trunks I2 in., 24 in., 38 in.

Juniper, Juniperus Virginiana. Trunk 7 in.

Laurel, Kalmia latifolia. Trunk 8 in., root 24 in.

Locust (yellow), Robinia Psendacacia. Trunks I8 in., 24 in., 38 in.

Locust (honey), Gleditchia triacanthos. Trunks I4 in., 28 in., 30 in., in thorn to in.

Magnolia, Magnolia tripetala. Trunk ${ }_{5} 5$ in.

Magnolia (ear-leaf), Magnolia Fraseri. Trunk 12 in.

Mulberry, Morus rubra. Trunks 8 and II in.

Mulberry (paper), Papyrius Papyrifera. Trunk 9 in.

Maple (blistered), Acer saccharum, Marsh. Trunk 26 in., boards 9 and $I I$ in.

Maple (sugar), Acer saccharum, Marsh. Trunks 30 in., 40 in., 48 in., plank 28 in., finished boards 29 in., curly boards I3 to 16 in. 
Maple (black), Acer nigrum. Trunk r6 in.

Maple (white), Acer saccharum L. Trunks 35 in., 49 in., plank 26 in., birdseye boards Io to 12 in.

Maple (red), Acer rubrum. Trunk 14 in.

Maple (striped), Acer Pennsylvanicum. Trunk 6 in.

Osage Orange, Toxylon pomiferum. Trunk 4 in.

Oak (black), Quercus velutina. Trunks Io and 27 in., finished board II in.

Oak (Spanish), Quercus digitata. Trunks I3 and 18 in.

Oak (swamp), Quercus palustris. Trunk $\mathrm{I}_{5}$ in.

Oak (laurel), Quercus imbricaria. Trunk to in.

Oak (chestnut), Quercus Prinus. Trunks I4 in., 27 in., 36 in., finished board $2 \mathrm{I}$ in.

Oak (black jack), Quercus nigra. Trunks 18 in., 23 in.

Oak (white), Quercus alba. Trunks 50 in., 28 in., 32 in.. $6 \mathrm{I}$ in., plank 37 in., boards (quartered) 5 to $\mathrm{I} 3$ in., quartered curly io in.

Oak (red), Quercus rubra. Trunks 42 in., 54 in., 84 in., plank 44 in.

Persimmon, Diospyros Virginiana. Trunk 12 in.

Poplar (yellow), Liriodendron Tulipifera. Trunk 48 in., trunk "wheels" $24,36,48,60,74,90$, and $\mathrm{I} 38$ in.; the tree from which the last wheel came cut 25,000 feet of merchantable boards, plank 36 and 57 in., cube 36 in., finished boards 27 to 46 in.

Pine (white), Pinus strobus. Trunk 36 in.

Pine (yellow), Pinus echinata. Trunk 28 in.

Pine (pitch), Pinus rigida. Trunk 16 in.

Papaw, Asimina triloba. Trunk 8 in.

Rhododendron, Rhododendron maximum. Trunk 6 in.

Red Bud, Cercis Canadensis. Trunks $5^{\mathrm{T} / 2}$ and 9 in.

Sycamore, Platanus occidentalis. Trunks 52 in., 36 in., 40 in., finished board quartered 33 in.

Sassafras, Sassafras Sassafras. Trunk 22 in., finished board "wavy" 18 in.

Silver Bell, Mohrodendron Carolinum. Trunk 8 in.

Sourwood, Oxydendron arboreum. Trunk $\mathrm{I}_{5}$ in.

Spruce (black), Picea Mariana. Trunks 31, 34, and 49 in., planks 8 and 40 in., finished boards 18 and 20 in.

Spicewood, Benzoin Benzoin. Trunk 2 in. 
Sumach, Rhus typhina. Trunk 8 in.

Service, Amalanchier Canadensis. Trunks 8 and 9 in.

Thorn, Crategus coccinea. Trunk Io in.

Tree of Heaven, Ailanthus glandulosus. Trunks 8 in., 24 in., 38 in.

Witch Hazel, Hamamelis Virginica. Trunks 4 and 5 in.

Walnut (black) Juglans nigra. Trunks 30 and 36 in., plank 29 in., finished boards "wavy" 20 in., "figured" $2 \mathrm{I}$ in.

Willow (black), Salix nigra. Trunk 12 in. 


\section{CATALOGUE.*}

\section{Fungi.}

P H A L L A C E AE.

ITHYPHALLUS Fries.

I. IMPUDICUS (Linn.) Fr.

In the juvenile, egg stage, June I5, 1893, Oct. 28, 1893, we find the veil under pileus about one-third its length and part of it encircling the stipe about the middle (Nuttall, 983).

\section{N I D U L A R I A C E AE.}

\section{CRUCIBULUM Tul.}

C. vulgare Tul.

On sticks and leaves, open woods, and on old cotton cloth, July 6, I893 (Nuttall, I079).

\section{Y C O P E R D A C E AE.}

TYLOSTOMA Pers.

T. mammosum (Mich.) Fr.

On light soil on rock, March 24, I893 (Nuttall, 880).

\section{MITREMYCES Nees.}

M. Lutescens Schn.

On wet mossy banks, alt. 2,000 ft., March 25, I893 (Nuttall, 88I).

GEASTER Mich.

G. mammosus Chev.

On earth, Short Creek, July 6, I893 (Nuttall, I080).

G. Hygrometricus Pers.

Along Horsepen Creek, McDowell County, July 30August I, I900 (Morris, I I05a).

*Unless otherwise stated, all of Nuttall's numbers in the fungi are from Fayette Co., near Nuttallburg. 
BOVISTA Dill.

B. LEPIDOPhora (E. \& E.) De Ton.

In grass on lawn, Sept. 5 , I893. Clavate $8 \mathrm{~cm}$. high, I2 $\mathrm{cm}$. broad, sterile base $3.5 \mathrm{~cm}$. thick, white cuticle $1.5 \mathrm{~mm}$. thick, gleba pale yellow (Nuttall I I93).

B. PILA Berk. \& Curt.

Free on open ground. Monongalia Co., near Morgantown (Millspaugh).

\section{LYCOPERDON Tourn.}

L. GEmmatum Batsch.

On ground in woods, alt. 2,000 ft., Aug. 24, I893 ( Nuttall, I I 80 ).

L. FURFURACEUM Schæff.

In grass on lawn, Sept. 3, I893. Capillitium thicker than the spores, branches few, 2.5 to $4 \mathrm{~cm}$. in diameter, outer coat pure while (Nuttall, II88).

L. PEDiCELlatum Peck.

On moss on ground in pine woods, alt. 2,000 ft., March I0, I893. Pedicels $20 \mu$ long (Nuttall, 864).

L. PYRIFORME Schæff.

Under bark of Quercus palustris, Monongalia Co., near Morgantown and near Little Falls (Millssaugh) on rotten wood, Feb. 2, 1893 (Nuttall, 832).

\section{SCLERODERMA Pers.}

S. vUlgare Hornem.

On chips of Picea Mariana, Tucker Co. Falls of Blackwater (Millspaugh). Grant Co., on dead logs, Otter Fork of Cheat; and Monongalia Co., Tibbs Run, plentiful on clay of a path (Millspaugh). In laurel thickets, July 28, I893. Spores Io $\mu$ (Nuttall, II25).

S. Bovista Fr.

On ground, March 21, I893 (Nuttall, 876).

BOVISTELLA Morg.

B. Ohioense (Ell. \& Morg.) Morg.

In grass on lawn, June 26, I893 (Nuttall, 1003).

\section{U COR A CE AE.}

\section{MUCOR Mich.}

M. Mucedo Linn.

On open canned fruit left standing (Millspaugh). On dead dry fruit (Asimina triloba, Oct. I, I895 (Nuttall, ı866. 754). 
SPORODINIA Link.

S. Aspergillus (Scop.) Schroet.

On dead Agaricus and Boletus, Sept. 10, 1893 (Nuttall, I I96).

PER O N O S POR A CEAE.

\section{CYSTOPUS Lev.}

C. Candidus (Pers.) Lev.

On living leaves of Dentaria diphylla, Monongalia Co., at Little Falls (Millspangh). On Brassica nigra, July 8, I893 (Nuttall, 1082). On Sisymbrium officinale; Raphanus sativus and Lepidium Virginicum (Sheldon, 686, I 577, 3020, 3291 ; I792, 3035; 4457).

C. Ipomoeae-Panduratae (Schw.) Stev. \& Sw.

On Ipomoea pandurata (Sheldon, 701, 706, 784, 919, 1956, 3I 59, 3246).

C. Tragopogonis (Pers.) Schroet.

On Senecio aureus and Ambrosia artemisiaefolia (Sheldon, 2960, 3080).

C. Portulacae (DC) Lev.

On Portulacca oleracea, July 8, I893 (Nuttall, 1083).

C. Bliti (Biv.) DeB.

On Amaranthus retroflexus, July 20, 1893. Spores 18 to $22 \times 15$ to $18 \mu$ (Nuttall, I6I5).

\section{PLASMOPARA Schroet.}

P. viticola (B. \& C.) Berl. \& De Ton.

On fruit of Vitis Labrusca, Monongalia Co., near Morgantown (Millspaugh).

P. Cubensis (B. \& C.) Humphrey.

On cucumbers, Mason, near Elwell, and on Musk Melons, Ohio, near Elm Grove (Sheldon, I018, 2009, 2097).

P. OBDUCENS Schroet.

On Impatiens sp. (Sheldon, 2297).

P. Geranil (Pk.) Berl. \& DeToni.

On Geranium maculatum (Sheldon, 651, 2967).

P. RIBICOLA Schroet.

On Ribes sp. (Wild gooseberry) (Sheldon, 2446).

P. Halstedir (Farlow) Berl.

On Ambrosia trifida (Sheldon, 2957).

PHYTOPHTHORA DeBary.

P. Infestans (Mont.) DeB.

On living leaves and tubers Solanum tuberosum, Monongalia Co., near Morgantown (Millspaugh). 
BREMIA Regel.

B. Lactucae Regel.

On Lactucca Canadensis and L. hirsuta, June I4, I894

(Nuttall, 1549). On Lactuca sativa (Sheldon, 53).

P. obovata Bon.

PERONOSPORA Corda.

On living leaves Spergula arvensis, Preston Co., near

Terra Alta (Millspaugh).

P. Arthuri Farlow.

On Onagra biennis (Sheldon, 2324).

P. Corydalis DeBy.

On Corydalis flavula (Sheldon, 3996).

P. Parasitica (Pers.) DeBy.

On Lepidium Virginicum and Cardamine Pennsylvanica (Sheldon, 55, 630, 1330).

E N T O M O P H T H O R A E AE.

\section{EMPUSA Cohn.}

E. muscae (Fr.) Cohn.

On Musca domestica, Monongalia Co., at Morgantown (Millspangh). Very prevalent on a species of Tachina found on maple leaves in great number in 1892 at Morgantown (Millspaugh).

E. GRYLLI Fr.

On tufted caterpillars, on grasshoppers, and on the house fly, Monongalia Co., at Morgantown (Millspaugh).

\section{S C H I Z O Y Y E T A CE AE.}

\section{BACCILLIUS Cohn.}

B. TUBERCulosis Koch.

In sputa of consumptive (Millspangh).

B. ACIDI-LACTICI (Zopf) Schroet.

In soured Milk (Millspaugh).

B. subTilis (Ehrenb.) Cohn.

In infusion of hay and on exposed boiled potato (Millspaugh).

B. Ulna Cohn.

On exposed coagulated egg albumen (Millspangh).

B. AMYlovorus: (Burrill) Schroet.

(Microccus Burrill).

Berkeley: on Malus Malus fruits at Gerrardstown (Waite). On fruit of Pyrus communis, Monongalia Co., near Morgantown (Millspaugh). 
S. undula (Muell) Ehrenb.

SPIRILLUM Ehrenb.

In infusion of hay (Millspaugh).

BACTERIUM Ehrenb.

B. Lineola (O. F. Muell) Cohn.

In infusion of radish (Millspaugh).

B. Termo (O. F. Muell.) Ehrenb.

In various decomposing organic substances (Millspaugh).

MICROCOCCUS (Hall.) Cohn.

M. Crepusculum (Ehrenb.) Cohn.

Found associated with Bacterium Termo in decomposing vegetable infusions (Millspaugh).

M. aurantiacus, Cohn.

Caught on sterilized potato in laboratory (Millspaugh). M. Luteus Cohn.

Caught on sterilized potaoto in laboratory (Millspaugh). STREPTOCOCCUS Billr.

S. Pyogenes (Rosenb.) Zopf.

Micrococcus septicus, Cohn. In blood of dead calf (Millspaugh).

S. UREAE (Cohn.) Trev.

Micrococcus ureae, Cohn. In decomposing urine (Millspaugh).

SACCHAROM Y CE T A CEAE.

SACCHAROMYCES Meyen.

S. Cerevisiae Meyen.

In Pasteur's liquid left uncorked in laboratory (Millspaugh).

S. MYCODERMA Reess.

On same liquid as above at same date (Millspaugh).

D I A T O M A CE AE.

Cymbella gastroides, Kuetz.

Cymbella turgida (Grun.) Greg.

Stauroneis Phoenicentron Ehrb.

Navicula viridis, Kuetz.

Navicula major, Kuetz.

Navicula NobILIS (Ehrb.) Kuetz.

NAVICULA RHOMBOIdES, Ehrb.

Navicula boREalis (Ehrb.) Kuetz. 
NaVicula trinodis, Lewis.

Achnantes lanceolata, Breb.

SynEDRA ULNA (Nitzsch.) Ehrb.

Nitzschia Amphioxys intermedia, Grun.

M YXO M Y E T A CEAE.

CERATIOMYXA Schroet.

C. Fruticulosa (Muell.) MacB. (Det. O. F. Cook.)

Along Delashmeet Creek, Mercer County, altitude 2,090 feet, July 25, Igoo (Morris, 946).

\section{PHYSARUM Pers.}

P. CITRINUm Schum.

On moss, alt. 2,000 feet, Aug. 2, I893 (Nuttall, i 160).

P. PULCHERRIMUM B. \& R.

On dead wood, July 25, I893 (Nuttall, II33).

P. PSitTACINUm Ditm.

On fruit cones of Magnolia Fraseri, alt. 2,000 ft., Aug. I2, I893 (Nuttall, I165).

P. SINUOSUM (Bull.) Rost.

On bark of Aralia spinosa, Aug. 16, I893 (Nuttall, II54)

P. Rufipes (A. \& S.) Morgan. (Determined by O. F. Cook.)

Along Tugg Creek, Hinton, Summers County, July Io, 1900 (Morris, 945).

\section{LEOCARPUS Link.}

L. FRAGILIS (Dicks.)

On Aspidium spinulosum and twigs of Tsuga Canadensis, Nov. 25, I895 (Nuttall, 1888).

\section{TILMADOCHE Fr.}

T. Nutans (Pers.) Rost.

On dead Asparagus leaves, and dead limbs Magnolia Fraseri, Nov. 4, I893 (Nuttall, 1264).

T. GYROCEPHALA (Mont.) Rost.

On dry bark of Hicoria ovata, living leaves of Hydrangea, etc., Short Creek, alt. I,300 ft., Aug. 2I, I893 (Nuttall, I I68).

T. VIRIDIS (Gmel.)?

On dead limbs Magnolia Fraseri, Sept., I895 (Nuttall, I856, 744?)

DIDYMIUM Schrad.

D. souamulosum (Alb. \& Schw.) Fr.

On new timber in mine near the entrance, Sept., 1893 (Nuttall, I342). 
D. LEucopoda (Bull.) Rost.

DIACHEA Fries.

On dead wood and leaves in damp, shady place, June 23. I893 (Nuttall, 998).

LAMPRODERMA Rost.

L. violaceum (Fr.) Rost.

On very rotten wet log, Short Creek, alt. I,300 ft., Oct. 25, I893 (Nuttall, 1255).

COMATRICHA P. Hoyer.

C. Friesiana (DeB.) Rost.

On rotten log, Nov. 6, I893 (Nuttall, 1237).

\section{STREMONITIS Gled.}

S. FUSCA Roth.

On rotten log, and on Hydnum sp., April 7, I893 ( Nuttall, 889 ).

S. FERRUGINEA Ehrh.

On log, Keeney's Creek, June 9, I893 (Nuttall, 973).

S. Smithil MacB. (Det. MarBride.)

Summers, along Tugg Creek near Hinton (Morris 945).

TUBULINA Pers.

T. CYLINDRicA (Bull.) DeC.

On dead log. Spores brown, rough (reticulate?) approx. $6 \mu$ diameter, Oct. 20, I893 (Nuttall, 1230).

\section{LINDBLADIA Fries.}

L. EFFUSA (Ehr.) Rost.

Forms large patches on dead Tsuga Canadensis at Short Creek Cliff and Masterson's Glade, July I5, I893 (Nuttall. II 30$)$.

C. Aurantiaca Schrad.

CRIBRARIA Pers.

On dead wet log, July 25, I895 (Nuttall, I826, 712).

R. LyCOPERDON Bull.

RETICULARIA Bull.

On dead log, Short Creek, April 6, 1893 (Nuttall, 888). ARCYRIA Hall.

A. Punicea Pers.

On rotten stump, June I 5, I893 (Nuttall, 979).

A. CINERA (Bull.) Schum. 
On damp dead wood and weeds in shade, July 24,1893 (Nuttall, III8).

A. NUtans (Bull.) Grev.

On dead log, Short Creek. Spores $7.5 \mu$, July 24, I893 (Nuttall, III9).

P. Flavida Peck.

\section{PERICHAENA Fries.}

On bark of dead Magnolia Fraseri. Fayette, near Nuttallburg.(Nuttall).

\section{LYCOGALA Mich.}

L. EPIDENDRON Buxb.

On wet dead logs, June I5, I893 (Nuttall, 995). On Magnolia Fraseri, Nov. 1895.

L. Conicum Pers. (Det. O. F. Cook.)

Summers, along Tugg Creek, near Hinton (Morris, 947).

T. FAllax Pers.

TRICHIA Hall.

On side of dead log, Oct. I9, I893. Rich reddish-brown color when fresh, shining pale-brown when dry; elaters long and slender pointed, spores Io to $12.5 \mu$ (Nuttall, I200).

T. Chrysosperma (Bull.) DeC.

On decayed wood, Monongalia Co., near Morgantown (Millspangh). On dead logs, Feb. 8, I893 (Nuttall, 842). T. PROXIMELLA Karst.

On Liquidambar styraciflua, Feb. 6, I893 (Nuttall, 836).

HEMIARCYRIA Rost.

H. RUBIFormis (Pers.) Rost.

Under bark of Fraxinus Americana and Quercus alba, Monongalia Co., near Morgantown (Millspaugh). On bark of dead Quercus sp. Feb. 13, I893 (Nuttall, 843).

H. clavata (Pers.) Rost.

Under bark of wet decaying log Quercus alba. Monongalia Co., near Morgantown (Millspaugh). On dead log, June I 5, I893 (Nuttall, 83I).

H. stipata Schw.

On dead wet limbs of Magnolia Fraseri, Sept. I6, I895 (Nuttall, I847).

H Y P H O M CE T A C E AE.

OOSPORA Wallr.

O. fasciculata (Berk.) Sacc.

On decaying orange, Jan. I5, I893 (Nuttall, I I34). 
M. Aureo-Fulva C. \& E.

\section{MONILIA Pers.}

On under side of dead log Liquidambar styraciflua, Aug. 29, I893 (Nuttall, II86).

M. FRUCTIGENA Pers.

On ripe fruit Prunus cerasus cult. Monongalia Co., near Morgantown (Millspaugh). On fruit of Prumus domestica cult. and Amygdalus Persica cult., June 28, 1893 (Nuttall, I006).

\section{POLYSCYTALUM Riess.}

P. SERICEUM Sacc.

On Quercus Prinus, Nov., I893 (Nuttall, I335, 293).

\section{OIDIUM Link.}

O. ERYSIPHOIDES Fr.

On Zizia cordata, Eupatorium purpureum and Rubus odoratus, Nov. 18, 1893 (Nuttall, 1266, 210).

O. LEUCOCONIUM Desm.

On leaves of Rosa cult., Cabell Co., near Huntington (Millspaugh).

O. MONILIOIDES Link.

On living leaves Poa pratensis, Preston Co., near Terra Alta (Millspaugh). On same host, May 30, I894 (Nuttall, I5I2).

BOTRYOSPORIUM Corda.

B. PUlchrum Corda.

On Lactuca Canadensis, Aug. 23, I895. Tips of branches inflated. Spores $7.5 \times 2.5 \mu$ (Nuttall, 1844, 735).

TRICHODERMA Pers.

T. LIGNORUM (Tode) Harz.

Under bark rotten Hicoria ovata Short Creek, alt. I,250 ft., Dec. 8, I893 (Nuttall, I299, 248).

\section{ASPERGILLUS Mich.}

A. GLAucus (Linn.) Link.

On Crategus sp., April 30, 1894 (Nuttall, I474).

A. glaucus oblongisporus E. \& W. Field. Mus. Bot., i :88 (I896).

Found on Lachnocladium semivestitum after neglect in plant press ( Nuttall, I871, 760).

This is either a new species or a distinct variety of A glaucus. The smooth oblong-elliptical conidia are quite different from the usual form. Spores 5 to $7.5 \times 2.5$ to $3 \mu$. 
A. FLAVUS Lk.

Also found on Lachnoladium semivestitum neglected in plant press (Nuttall, I877, 760).

Conidia 4 to $6 \mu$ diameter.

\section{STERIGMATOCYSTIS Cram.}

S. NIGRA v. Tiegh.

On dried peach in garden, Aug. I5, I893 (Nuttall, I I70).

PENCILLIUM Link.

P. GLAUCUM Link.

On Hydnum sp. and Polyporus varians, Aug. I6, I893 (Nuttall, I164, I36).

BOTRYTIS Mich.

B. olivacea E. \& E. Field Mus. Bot., I :88 (I896).

Type habitat: On dead log. Short Creek, alt. I, $800 \mathrm{ft}$, Feb. 26, I894 (Nuttall, discov. I4I I, 38I). Fungi Columbiana, 593. N. A. F., 3187.

Forming thin olivaceous patches 2 to $4 \mathrm{~cm}$. in extent, composed of olive-brown, septate threads, about $4 \mu$ thick, and dichotomously or oppositely branched above, the short ( I5 to $25 \mu$ ) branches sub-attenuated above, and bearing the obovate or elliptical, olive-brown, 6 to $8 \times 4$ to $4.5 \mu$ conidia at their tips.

B. torta E. \& E., Proc. Phila. Acad., I894, 375.

Type habitat: On dead leaves Carex Fraseri, Dec. Io. I83 (Nuttall, discov. I248, 257).

Hyphæ simple, sparingly branched, twisted above as in $B$ streptothrix or in Streptothrix atra B. \& C., brown, 80 to IOO $\times 3$ to $4 \mu$, forming numerous small brownish-black tufts, effused or gregarious, on both sides of the leaf. Conidia elliptical brown, 5 to $6.5 \times 3$ to $3.5 \mu$.

Differs from $B$. streptothrix (C. \& E.) in its much smaller conidia and more dwarfish growth.

B. vULGARIS Fr.

On leaves Magnolia Fraseri, alt. 2,000 ft., July I2, I893; on burs of Xanthium Canadense, Nov. I, I893; on pedicels of Ipomaea pandurata, Feb. 17, 1894; on decaying Brassica oleracea, Jan. I8, I894; on Cicuta maculata, March I8, I894 (Nuttall, i I I6).

VERTICILLIUM Nees.

V. osteophilum E. \& E., Field. Mus. Bot., i :89 ( 1896 ).

Type habitat: On jaw bone of calf, in woods, Nov. 27, I894 (Nuttall, discov. I761, 647). 
Prostrate sterile hyphæ yellowish-brown, distantly septate, rough, 6 to $7 \mu$ diameter; fertile hyphæ loosely cespitose: erect septate, smooth, yellowish-hyaline, 7 to i $5 \mu$ diameter. the primary branches alternate, and issuing at right angles, bearing opposite or ternately-verticillate branches, the ultimate division $\mathrm{I} 2$ to $\mathrm{I} 5 \times 4 \mu 3$ to 4 in a terminal verticil, with tips often curved and bearing I to 4 terminal, globose, hyaline, 3.5 to $4 \mu$; conidia.

V. PUNiCEum (Cke \& E.) Grev.

On dead sticks, June, I893 (Nuttall, I I I5).

\section{TRICHOTHECIUM Link.}

T. Roseum (Pers.) Link.

On dead dried peach on ground; on decaying leaves Ilex verticillata, Sept. I4, I893 (Nuttall, I I99).

\section{CEPHALOTHECIUM Corda.}

C. Roseum Corda.

On old Polyporus pergamenus, Short Creek, alt. I,300 ft., Nov. 10, I893 (Nuttall, 1260, 205).

D. DENDRoides (Bull.) Fr.

\section{DACTYLIUM Nees.}

On Polystictus versicolor, and apparently checking its development. Oct. I2, I893 (Nuttall, I206). Spores $28 \times 10 \mu$.

\section{RAMULARIA Ung.}

R. Celastri El1. \& Mart.

On leaves of Celastrus scandens near the ground. Oct. I I, I894. Conidia I5 to $28 \mu$ (Nuttall, I700).

R. Tulasnei Sacc.

On Fragaria cult., Oct. 27, I894 (Nuttall, I724).

R. TARAXACI Karst

On Taraxacum Taraxacum, Oct. 20, I894 (Nuttall, I722).

D E M A T I A C E AE.

CONIOSPORIUM Link.

C. HARKnessioides (Ell. \& Holl.) Sacc.

On Rumex acetosella, Oct. 20, 1894 (Nuttall, г727, 613).

\section{TORULA Pers.}

T. dimidiata Penz

On Rhus hirta, alt. I,300 ft., Nov. 21, 1893 (Nuttall, 1273, 220). 
T. HERBARUM Link.

On Asparagus officinalis, Nov. I, 1893 (Nuttall, I240, I86).

\section{PERICONIA Bon.}

P. PYCNOSPORA Fres.

On Parthenocissus quinquefolia, Feb. 20, 1894. Conidia I 2.5 to I $5 \mu$ diameter. On Rubus odoratus. On Polymnia Uvedalia. On Phytolacca decandra, hyphæ 600 x I 5 to I6 $\mu$ (Nuttall, I413). On Polymnia Uvedalia, spores 12.5 to I5 $\mu$ Nov. 16, I894 (Nuttall, I743).

\section{GONATOBOTRYUM Sacc.}

G. maculiocolum (Wint.) Sacc.

On young sprouts Hamamelis Virginica I to 2 feet high; Short Creek, alt. I,850 ft., Aug. 15, I894 (Nuttall, I656).

S. ATRA B. \& C.

\section{STREPTOTHRIX Corda.}

On dead limbs on ground. Conidia 6 to $8 \times 4.5$ to $5 \mu$, Dec. 16, I893 (Nuttall, I329).

\section{ZYGODESMUS Corda.}

Z. GRAMINICOLA E. \& E.

On Carex Fraseri, spores $7.5 \mu$ Feb. II, I894 (Nuttall, I374, 334).

Z. PANnosus B. \& C.

On charred bark, May 28, I894 (Nuttall, I 531, 502).

Z. TILIACEUS E. \& E.

On bark of dead Magnolia Fraseri, Fayette, near Nuttallburg (Nuttall.)

\section{STACHYLIDIUM Link.}

S. caricinum E. \& E., Proc. Phila. Acad., I894, 377.

Type habitat: On dead leaves Carex Frascri Feb. II, I894 (Nuttall, discov. I375. 355).

Hyphæ fasciculate brown, septate 600 to $700 \times 3 \mu$ simple or occasionally forked above towards the tip, with short cylindrical hyaline branches opposite or in whorls of three, bearing at their tips the elliptical, hyaline 4 to $5 \times 1.5$ to $2 \mu$ conidia, collected into a globose head Io to I $2 \mu$ diameter.

FUSICLADIUM Bonord.

F. DENDRITICUM (Waller.) Fckl.

On living Pyrus Malus leaves and fruit. Monongalia Co., near Morgantown (Millspaugh). 
F. Virginiense E. \& E. sp. nov?

Type habitat: On living leaves Aster infirmus, June 29, I894 (Nuttall, discov. I593, 54I).

\section{POLYTHRINCIUM Kunze \& Schm.}

P. Trifolir Kunze.

On Trifolium repens, July 4, I894 (Nuttall, I588)

\section{CLADOSPORIUM Link.}

C. herbarum (Pers.) Link.

On Polygonatum biflorum, Menispermum Canadense, and leaves of Rhus hirta, June 28, I894 (Nuttall, I564).

C. HeRbarum FASCiculare Corda.

On Reseda cult., Jan. 28, I894. Conidia 15 to $18 \times 6$ to $7.5 \mu$ I to 2 septate (Nuttall, I354).

C. NIgrellum E. \& E., Proc. Phila. Acad., I893, 463.

TyPe haBITAt: On inner bark of Robinia Pseudacacia railroad ties, Keeney's Creek, Oct. I893 (Nuttall, discov. I227, I72).

Hyphæ densely tufted, septate, sub-equal, I 50 to $200 \times 5$ to $6 \mu$, tufts effused, subconfluent, forming a black, velvety coat extending over the surface of the bark indefinitely, with the same habit as Macrosporium nigrellum C. \& E. Conidia smoky-hyaline, becoming pale brown, variable in size, the smaller ones ovate, continuous or uniseptate, 6 to $8 \times 5 \mu$, the larger ones oblong-elliptical or sub-cylindrical, 2 to 3 septate, I 2 to $15 \times 5$ to $6 \mu$.

C. Triostei Peck.

On living leaves Triosteum perfoliatum, alt. I,200 ft., July 26, I894 (Nuttall, 1824).

C. ePiphyllum (Pers..) Mart.

On dying leaves Robinia Pseudacacia, July 31, I893 (Nuttall, II39).

C. CORYNITRICHUM E. \& E.

On leaves of Magnolia Fraseri. Fayette, near Nuttallburg (Nuttall).

C. $\mathrm{sp}$.

On dead decorticated limb Magnolia Fraseri, Sept., 1895 (Nuttall, 1853, 739).

A most beautiful velvety black form.

C. EPIMyces Cooke.

On pileus Polyporus varians, Jan. 25, 1894 (Nuttall, I34I). 
CLASTEROSPORIUM Sz.

C. cornutum E. \& E., Field Mus. Bot., I:92 (1896).

Type habitat: On decaying wood, Oct. 21, I895 (Nuttall, discov. 1883).

Hyphæ prostrate, septate, branched, brown, about $6 \mu$ diameter, effused in black velvety patches I to $2 \mathrm{~cm}$. across. Conidia in pairs or threes, horn-shaped, Io to 14 septate. IOO to $225 \times$ I4 $\mu$, broadest below, gradually tapering above to an obtuse point, curving outward at the base, rising and spreading out above like the horns of an ox. The conidia are but slightly constricted at the septa and are sessile on the hyphæ, appearing at first as a simple nodule or tubercle on the side of the thread.

Allied to C. Hirudo Sacc., but that has solitary multiseptate ( 55 to 65$)$ conidia and evanescent hyphæ.

C. sigmoideum E. \& E., Bull. Torr. Club, $24: 472$ (1897).

Hyphæ effused, crooked, septate at intervals of about $15 \mu$, forming an olive-black stratum on the bark for many $\mathrm{cm}$. in extent, subcespitose, $300-400 \times$ ( $6-7 \mu$; conidia broad-fusoid, sigmoid (ends curved in opposite directions), 4 (exceptionally 5) septate and slightly constricted at the septa, intermediate cells brown, end cells hyaline, 40-70 x 12-I5 $\mu$, mostly subtruncate above.

On dead limbs of Castanca. Fayette, near Nuttallburg, March, I896 (Nuttall, 819).

\section{HELMINTHOSPORIUM Link.}

H. PERSistens Cooke.

On branches of Acer in pine woods, alt. 2,000 ft., March 9, 1894. Conidia 75 to $150 \times$ Io to i $5 \mu$ (Nuttall, I421, 392). H. MACROCARPon Grev.

On decorticated limbs Platanus occidentalis. Oct, 6, I895 (Nuttall, I887). On Magnolia Fraseri, Nov., I895.

H. Folliculatum Corda.

On Zea Mays, Aug. 2, I895 (Nuttall, 1835).

H. attenuatum Peck \& Cooke.

On dead log, Short Creek, alt. I,800 ft., June 10, 1894 (Nuttall, I547).

H. SEPTEMSEPTATUm Peck.

On Magnolia Fraseri, June 20, 1894 (Nuttall, 1574, 534). H. BRACHypus E. \& E., Field Mus. Bot., I :92 ( 1896).

Type habitat: On dry wood of old log. Oct. 8, 1895 (Nuttall, discov. 1873, 757).

Effused in brownish-black patches of several centimeters in extent, velutinous, thin. Prostrate hyphæ only sparingly branched, obscurely septate, crooked; fertile hyphæ ces- 
pitose, erect, 40 to $50 \times 4$ to $6 \mu, 2$ to 3 septate, abruptly constricted at the tips and subtruncate. Conidia terminal, oblong-fusoid, 5-septate brown, not constricted at the septa, 30 to $40 \times$ I 2 to $14 \mu$, with a short ( 8 to Io $\mu$ ) obconical, hyaline or pale-brown, persistent pedicel, which is sometimes prolonged into a hyaline thread as long as the spore. This prolongation may be something of the nature of a pith or medulla drawn out of the supporting hypha and remaining attached to the pedicel.

H. FUSIForme Corda.

On old barrel staves. Fayette, near Nuttallburg (Nuttall).

\section{CERCOSPORA Fres.}

C. Armoraciae Sacc.

On Roripa Armoracia. Conidia $175 \times 5 \mu$, Aug. 26, I894 (Nuttall, I687, 593).

C. Caulophylli Peck.

On living leaves Caulophyllum thalictroides, Grant Co., near Bayard (Millspaugh).

C. Violae Sacc.

On Viola obliqua, Aug. Io, I895 (Nuttall, I84I, 73I).

C. GRANUliformis Ell. \& Hol.

On living leaves Viola obliqua, June 28, I894 (Nuttall, I 565 ).

C. oculata E. \& K.

On leaves Virnonia Noveboracensis, Aug. 6, I894 (Nuttall, $\mathrm{r} 682$ ).

C. Vernoniae E. \& K.

On leaves Vernonia Noveboracensis, Oct. Io, 1894 (Nuttall, r698).

C. омpHaCODES E1l. \& Hol.

On Phlox amoena, July 14, I894 (Nuttall, I605).

C. Diantherae E. \& K.

On leaves of Dianthera Americana. Oct. 23, 1895 (Nuttall, 1879).

C. Avicularis Wint.

On living leaves Polygonum aviculare, July 5, I895 (Nuttall, I8I 5).

C. Dubia (Riess) Wint.

On Chenopodium album viride, July 4, I894 (Nuttall, I 587).

C. Beticola Sacc.

On leaves Beta vulgaris, Oct. 4, I894 (Nuttall, I692).

C. Boehmeriae Peck.

On leaves Boehmeria cylindrica, Oct. 5, 1894 (Nuttall, I694). 
C. Acalyphae Peck.

On leaves Acalypha Virginica, Oct. 3, I894 (Nuttall, I69I).

C. Ampelopsidis Peck.

On Parthenocissus quinquefolia. Conidia $+40 \times 3 \mu$, June 30, I894. (Nuttall, I 578).

C. Menispermi E. \& H.

On leaves Menispermum Canadense. Conidia $+77 \times 5 \mu$ June 26, I894 (Nuttall, I 562)

C. Ageratoides E. \& E.

On Eupatorium ageratoides, Oct. I4, I894 (Nuttall, I706).

C. Anthelmintica Atk.

On living leaves Chenopodium anthelminticum, Oct. I5, I894 (Nuttall, I713, 601)

C. Kalmiae E. \& E.

On leaves Kalmia latifolia, Aug. I5, I893 (Nuttall, , 167).

C. Oenotherae E. \& E., Proc. Phila. Acad., 1894, 380.

Type habitat: On leaves Onagra biennis, Oct., I894 (Nuttall, discov. I704, 599)

Spots irregular, mostly elongated, grayish-brown, subangular, 3 to $5 \times 2$ to $3 \mathrm{~mm}$. subconfluent. Hyphæ amphigenous, sub-hyaline, continuous or faintly $\mathrm{I}$ to 2 septate, I 5 to $20 \times 3 \mu$ in minute scattered tufts, few in a tuft, spreading subundulate. Conidia linear or only slightly attenuated above, smoky-hyaline, nucleate and faintly 3 to 5 or more septate, 25 to $80 \times 2$ to $2.5 \mu$ straight or only slightly curved.

C. Chronanthi E. \& E., Field Mus. Bot., I :94 ( I896).

TyPE habitat: On living leaves Chionanthus Virginica, Sept., I895, (Nuttall, discov. 1852, 738).

Spots variable in shape, subindefinite, dark brown, grayish above, 2 to $4 \mathrm{~mm}$. diameter. Hyphæ epiphyllous, cespitose, 75 to $150 \times 3.5$ to $5 \mu$, brown, septate, and subgeniculate or subundulate above. Conidia fusoid or clavate, becoming brown, 3 to 5 -septate, 30 to $60 \times 4$ to $4.5 \mu$.

C. septorioides E. \& E. Field Mus. Bot., i :94 ( I896).

TyPe habitat: On leaves Rubus Canadensis, Oct. I6, I894 (Nuttall, discov. I725, 610).

Spots dirty-brown, irregular, subangular, more or less limited by the veinlets, 2 to $3 \mathrm{~mm}$. diameter, definite, bui without any differently colored border. Hyphæ epiphyllous, cespitose on a small tubercular base, simple, sub-entire, continuous, brownish, 20 to $25 \times 3 \mu$. Conidia cylindrical, slightly curved, hyaline, nucleate, 35 to $63 \times 2$ to $2.5 \mu$.

Differs from $C$. Rubi Sacc. in its narrower conidia without septa, and its shorter hyphæ. 
C. Rubi Sacc. 676).

On leaves Rubus Canadensis, Nov., 1894 (Nuttall, I785,

C. LEPAZEOIdes (Desm.) Sacc.

On Sambucus Canadensis, July 26, I894 (Nuttall, I626).

C. Smilacis Thum.

On living leaves Smilax rotundifolia, Monongalia Co., near Camp Eden (Millspaugh). On Smilax rotundifolia (Nuttall, I 573, 533).

C. Citrullina Cooke.

On living leaves of the Watermelon, Mason, near Point Pleasant and Elwell (Sheldon).

C. Smilacina Sacc.

On leaves Smilax glauca, Aug. 26, I894 (Nuttall, I653, 573).

C. COLUMnaris E. \& E.

On Phaseolus vulgaris cult. Oct. I3, I895 (Nuttall, I870).

\section{SPORODESMIUM Link.}

S. TORULOIDES E. \& E.

On dead twigs Cormus florida, June Io, I894 (Nuttall, S. $\stackrel{\text { I 548, 5I4). }}{\text { sp. }}$

Type Habitat: On Tsuga Canadensis, Feb. 3, I894 (Nuttall, discov. 33I).

S. MORIFORME Peck.

On Opulaster opulifolius, May 12, I894. Spores 25 to $40 \times 25 \mu$ (Nuttall, I 503, 483).

S. Aurantiacum B. \& C.

On a dead stick, Short Creek, July 25, I893 (Nuttall. II 32, II 5).

S. CONCINNUM Berk.

On wet dead sticks in association with Clavaria mucida, Oct. and Nov., I893. Conidia II 2 to II $5 \times 30$ to $32 \mu$; 65 to $100 \times 30 \mu$ (Nuttall, 1233).

\section{SPEIRA Corda.}

S. MINOR Sacc.

On Pimus Virginiana, Dec. I5, I894. Conidia $30 \times$ 10 $\mu$ (Nuttall, I775, 664).

\section{MACROSPORIUM Fr.}

M. commune Rabh.

On dead stems of garden Asparagus officinalis, conidia + $40 \times$ I $5 \mu$, Nov. I, I893 (Nuttall, I222). On petioles of 
Aralia spinosa, conidia $75 \times 20 \mu$ (Nuttall, 358). On Rume.r. obtusifolius, Rhus radicans and Cicuta maculata? (Nuttall).

M. - sp.

Type habitat: On dead stems Polymnia Uvedalia, Nov. 3, 1893 (Nuttall, discov. I736, 623).

M. Abutilonis Speg.

On Abutilon Abutilon, July 21, I895 (Nuttall, 1836).

M. - sp.

Type habitat: On decayed fruit Diospyros Virginiana, April 25, I895 (Nuttall, discov. 1825, 711).

M. Asclepiadeum Cooke?

On Asclepias Syriaca, Nov. 3, I894 (Nuttall, 1730).

M. CAUDATUM C. \& E.

On dead flowers of Yucca cult., Sept. 5, 1894 (Nuttall. I680, 587). On stems of Phytolacca decandra, conidia 88 $\mathrm{x} 20 \mu$.

M. Saponariae Peck.

On Saponaria officinalis, Oct. 6, I894 (Nuttall, 1695).

M. olivaceum E. \& E., Proc. Phila. Acad., I894, 383.

Type habitat: Parasitic on Sphaeropsis Asiminae on dead limbs of Asimina triloba, March, I894 (Nuttall, discov. I4I8, 388).

Forms a light olive velutinous coat over the pustules of the Sphaeropsis. Hyphæ tufted, yellowish brown (under the microscope), septate, erect, nearly straight or subundulate, 80 to $100 \times 4$ to $5 \mu$. Conidia obovate or obpiriform, 3 to 5 -septate and muriform, brown, 24 to $38 \times$ I 5 to $20 \mu$ terminal, sessile. Conidia also occur subcubical or subglobose I 5 to $20 \mu$ diameter with 2 septæ crossing each other at right angles.

M. AntenNaeforme B. \& C.

On Celtis occidentalis, Aug. II, I894 (Nuttall, 1652). Conidia $+95 \times 15 \mu$.

M. - sp.

Type habitat: On pods of Datura Stramonium, Feb. Io, I894 (Nuttall, discov. I369, 326).

M. Томато Cooke?

On ripe fruit Lycopersicum esculentum cult. Spores shorter than described. Sept. 9, 1893 (Nuttall, i 195). Same host Monongalia Co., at Morgantown, prevalent r891 (Millspaugh).

M. MAYDIS C. \& E.

On leaves Zea May's cult., with conidia smaller than de. scribed, Nov., I893 (Nuttatl, I334, 292). 


\section{TRICHAEGUM Corda.}

T. nodulosum E. \& E., Proc. Phila. Acad., I894, 385.

Type habitat: On dead leaves Carex Fraseri, Feb. II, I894 (Nuttall, discov. I373, 333).

Erumpent, tufted, becoming subeffused, black, tufts gregarious, forming subvelutinous patches 2 to $4 \mathrm{~mm}$. across, or when standing singly the hyphæ and conidia forming a compact mass .5 to I $\mathrm{mm}$. diameter, and resembling somewhat the sorus of a Puccinia. Hyphæ simple sparingly fasciculate, brown, septate, often swollen at the septa, about $4 \mu$ thick and 200 to $300 \mu$ long. Conidia near the base of the hyphæ, at first elliptical, yellowish-hyaline, uniseptate, 8 to IO $\mathrm{x} 6$ to $7 \mu$, soon becoming 4 to 6 -septate, muriform and opaque, Io to $25 \mu$ diameter, subglobose, obovate, or elliptical.

\section{SEPTOSPORIUM Corda.}

S. Equiseti Peck, Rep. State Bot. N. Y., I892, 25.

Type habitat: Tips of living leaves Equisetum arvense, Doddridge Co., near Center Point, and Monongalia Co., on College campus, Morgantown (Millspaugh, discov. I89I).

Hyphæ forming minute tufts, the fertile very short, bearing acrogenous spores, the sterile longer, septate, colored; spores elliptical, usually with three transverse septæ and one or two longitudinal ones, colored, .001 in. long, .005 in. broad.

\section{ALTERNARIA Nees.}

A. Brassicae nigrescens Pegl.

On Musk-melons, Ohio, near Elm Grove (Sheldon).

\section{SARCINELLA Sacc.}

S. HETEROSPORA Sacc.

On Cercis Canadensis, Oct. 16, I894 (Nuttall, 1726, 610).

\section{STILBUM Tode.}

S. MAgNum Peck.

In cracks of bark, Nov. 6, I893. Spores $2.5 \times 1.25 \mu$ (Nuttall, 1278, 225).

S. Flavipes Peck.

On dead driftwood Platcnus occidentalis, Dec. 12, 1894 ( Nuttall, I767).

S. ERYTHROCEPHALUM Ditm.

On dung of Rabbit, Jan. 18, I894 (Nuttall, I345, 304). 
S. vULGaRE Tode?

On fallen dead leaves, Short Creek, July 25, I893 ( Nuttall, II 37, I 20).

\section{PILACRE Fries.}

P. GRacilipes E. \& E., Proc. Phila. Acad., r894, 386.

Type habitat: On rotten wood. Short Creek, alt. I,750 ft., Dec. I6, 1893 (Nuttall, discov. 1219, 274).

Scattered, stem slender, white-pruinose, $3 \mathrm{~mm}$. long, .25 to $.33 \mathrm{~mm}$. thick. Head hemispherical, olivaceous, about I mm. diameter. Fertile hyphæ hyaline, ciichotomously branched, 2 to $2.5 \mu$ thick, bearing the conidia laterally. Conidia globose or subglobose, yellow-brown under the microscope, 4 to $5.5 \mu$ diameter.

Smaller and of a more slender growth than P. Petersii B. \& C.

P. Petersii B. \& C.

On Acer saccharum and Ilex opaca, Feb. IO, I894 (Nuttall, I37I). On Magnolia Fraseri, Nov., I895.

\section{ISARIA Pers.}

I. Virginiensis E. \& E. Proc. Phila. Acad., I893, 465.

Type Habitat: On the young stroma of Hypoxylon rubiginosum? June 30, I893 (Nuttall, discov. I I09, 95, I02).

Stromata gregarious, simple, slender-clavate, 1.5 to $2 \mathrm{~mm}$. high. Yellowish-white, obtuse and subcapitate at the apex, curved, often decumbent, clothed nearly to the base with spreading, hyaline, dendroid, 1.5 to $2 \mu$ branching hyphæ (sporophores) 40 to $45 \times 2.5 \mu$ their tips often toothed and bearing I to 4 elliptical or ovate, hyaline $3.5 \times 2.5 \mu$ conidia.

Analogous to I. umbrina Pers (Institale acariforme Fr.), but differs in several respects.

I. Clavata Ditm.

On dead sticks on ground, June, I893 (Nuttall, II IO).

$$
\text { CERATIUM A. \& S. }
$$

C. Hydnoides (Jacq.) Alb. \& Schw.

On rotten log, June I0, 1893 (Nuttall, 976).

\section{SPOROCYBE Fr.}

S. Rhois (B. \& C.) Sacc.

On Rhus copallina, March 24 , 1894 , spores $7.5 \times 2$ to $2.5 \mu$. On Rhus hirta, March 28, I894, spores Io x $2.5 \mu$ (Nuttall, I430).

S. Azaleae (Peck) Sacc.

On capsules of Rhododendron maximum, Dec. I2, I893. 
Conidia varying from globular through elliptical to cylindrical, dark brown, outer coat easily ruptured, Io $\mathrm{x}$ IO, $8 \times 5$, I $2 \times 5$, I $5 \times 7.5 \mu$ (Nuttall, I307).

\section{T U B E R C U L A R A C E AE.}

\section{TUBERCULARIA Tode.}

T. vulgaris Tode.

On twigs, Feb. 23, I893. On Robinia Psendacacia, Dec. I, I893. On Aralia spinosa, Dec. 13, I893. On Sambucus pubens. On Acer Negundo, April 20, I894 (Nuttall, 942) On limbs of dead Rhus hirta, Monongalia Co., near Morgan* town, I89I (Millspaugh).

T. hamata E. \& E., Proc. Phila. Acad., I894, 386.

Type habitat: On dead limbs of Celtis occidentalis. Feb. 2, I894 (Nuttall, discov. I357, 313).

Depressed hemispherical, umbonate, soon becoming black, .5 to $1.5 \mathrm{~mm}$. diameter, Conidia oblong, slightly curved, hyaline, 5 to $8 \times 1.5$ to $2 \mu$ on slender simple sporophores 30 to $40 \mu$ long, incurved or involute at the tips.

T.

On Asimina triloba, Feb. 22, I894. Flesh colored spores I8 to $22 \times 3 \mu$ (Nuttall, I39I, 36r).

T. $-\mathrm{sp}$.

Conidia of Nectria verrucosa. On dead twigs Morus rubra July 25, I895 (Nuttall, I827, 715).

T Sambuci Corda.

On Sambucus pubens, Feb. 26, I894 (Nuttall, I409).

T. Celastri Schw.

On Celastrus scandens, April 25, I895. Spores 5 to $6 \times 2 \mu$ (Nuttall, I8I I).

\section{DENDRODOCHIUM Bon.}

D. AFfine Sacc.

On bark wet dead stumps, Oct. 24, I893 (Nuttall, I229, I74).

D. RUBELlum Microsporum Sacc.

On dead limb Magnolia acuminata, Aug. 16, 1894, Short Creek, alt. I,oon ft. On Liriodendron Tulipifera (Nuttall, т659).

\section{TUBERCULINA Sacc.}

T. PERSicina (Ditm.) Sacc.

Parasitic on Uredo (Cæoma) nitens, that on Rubus Bailey. anus, June IO, I894 (Nuttall, I557, 527). 
ILLOSPORIUM Mart

I. Caespitosum E. \& E., Proc. Phila. Acad., i894, 385.

Type habitat: On rotten log, Nov. I5, I893 (Nuttall. discov. I286, 23I).

Sporodochia globose, Ioo to I Io $\mu$ diameter, cespitose, forming tufts about I mm. diameter. Hyphæ 6 to $8 \mu$ thick, branched, the branches curved or tortuous. Conidia globose or ovate 6 to $12 \mu$ diameter.

Differs from I coccinellum Cke. in its color, and larger cespitose sporodochia.

I. malifoliorum, J. L. Sheldon, Torreya, 8:I39-4I (igo8).

Spots suborbicular, or coalescing and becoming irregular, brown or sometimes mottled with gray and with a small gray spot near the center, 5 to $15 \mathrm{~mm}$. in diameter; sporodochia hypophyllous, minute, gelatinous, yellowish-amber and blackening, subspherical when moist ( $150 \mu$ ), becoming disc-shaped or irregular when dry (6o to $100 \mu)$; sporophores branched; conidia hyaline, oblong, I x 3.5 to $4 \mu$.

On leaves of Malus in various parts of the State (Sheldon). Greenbrier, near White Sulphur Springs (Waite).

\section{HYMENULA Fr.}

H. cerealis E. \& E., Proc. Phila. Acad., r894, 386.

TyPe haBITAT: On wheat straw Triticum sp. brought in from Painesville, Ohio, May 24, I894 (Nuttall, discov. I 520, 495).

Sporodochia gelatinous, orbicular, yellowish-amber color becoming darker, at first sub-pulvinate, becoming depressed or flattened, .5 to $.75 \mathrm{~mm}$. diameter. Basidia slender, 25 to $30 \times 1.25 \mu$ simple or oftener branched. The branches erect. Conidia hyaline, oblong, minute, 3 to $4 \mathrm{X}$ I to I.25 $\mu$.

\section{CYLINDROCOLLA, Bon.}

C. Dendroctoni Peck, in Millsp. Flora, .W. Va., I892, 5 I6.

Type навітат: On dead insects, Dendroctonus frontalis, beneath the bark of pine. Hampshire Co., near Romney, (Millspaugh).

The insects are probably killed by this fungus, as they lie dead in their burrows in the inner bark of the tree (Pinus Virginiana).

Sporodochia minute, forming irregular masses, soft, somewhat waxy, white or whitish; sporophores slender, abundantly branched above, often compacted below into a short stem-like base, spores catenulate, short cylindrical, subtruncate, colorless, .000I6 to .0002 in. long, .00008 to .000I in. broad. 
On some of the insects there is a cottony or flocculent mass of white mycelium interwoven in a somewhat reticulate manner, and collected in strings or bundles. It bears no fruit, but is probably a luxuriant growth of the mycelium of this fungus.

Occasionally the fungus seems to spread from the insect to bark immediately adjacent to it.

C. flagellaris E. \& E. Field Mus. Bot., i :IOO (I896).

Type habitat: On dead stem of Helianthus decapetalus, Dec. 3. I894 (Nuttall. discov. I762, 650).

(C. lactea S. \& E. partly, Sacc. No. 3550.)

Sporodochia gregarious, subglobose, subhyaline and gelatinous when fresh, lens-shaped, with a thin spreading margin, orbicular, .33 to $.50 \mathrm{~mm}$ diameter, yellowish-amber colo: (whitish in the center) when dry. Sporophores densely fasciculate, flagelliform 2 to 3 times dichotomously branched, 80 to Ioo $\mu$ long, separating into the short cylindrical, hyaline, 4 to $5 \times \mathrm{I} \mu$ conidia.

This was included by Saccardo in Michelia II. p. 581, in

- Cylindrocolla lactea, S. \& E. (on rotten wood of Kalmia), but differs in the shape and color of the sporodochia and the sporophores only 2 to 3 times dichotomously branch. $C$. lactea retains its white color when dry, and resembles young Lasiosphaeria ovina.

\section{SCORIOMYCES Ell \& Sacc.}

S. Cragini E. \& S.

Under loose bark of dead Hicoria sp., Short Creek, alt. I,250 ft., Nov. 21, 1893 (Nuttall, 1272, 219).

\section{VOLUTELLA Tode.}

V. ciliata (A \& S.) Fr.

On decaying leaves of Prunus cult. in grass, June 20, I893 (Nuttall, IоIо).

\section{BACTRIDIUM Kunze.}

B. FLAVUM K. \& S.

Under decaying bark of Quercus alba, Monongalia C.o., near Morgantown (Millspaugh).

\section{HELISCUS Sacc.}

H. Lugdunensis Sacc.

On dead limbs of Ilex opaca, thrown in wet places one year previously, March I6, I894; sporodochia $+2 \mathrm{~mm}$. diameter, conidia 30 to $33 \times 5 \mu($ Nuttall, 1427,400$)$. 
FUSARIUM Link.

F. CULMORUM Smith.

On heads of ripe living wheat. Monongalia Co., Laurel Point (Millspaugh).

"This specimen combines the characters of a number of so-called species, making it difficult to say which it really is. Probably they are all forms of one species." Professor Peck (in letter).

F. SARCochroum (Desm.) Sacc.

On Acer Negundo, April 24, I894 (Nuttall, I482, 462).

F. Aleurinum E. \& E., Bull. Torr. Club, $24: 476$ ( I897).

Sporodochia compact, subtuberculiform-effused and subconfluent, reddish-orange, mycelium white; fertile hyphæ erect, Imuch branched, branches erect; conidia terminal, fusoid, slightly curved, continuous or faintly I-3-septate. nucleate, 35 to $45 \times 2.5$ to $3 \mu$.

On wheat flour spilt on the ground and left exposed four months. Fayette, near Nuttallburg (Nuttall).

F. Oxydendri E. \& E. Bull. Torr. Club 24:477 (1897).

Sporodochia tuberculiform, about I $\mathrm{mm}$. diameter, slatecolor, subcartilaginous, truncate or concave above, erumpent through, and closely surrounded by the ruptured epidermis ; hyphæ branched, hyaline, nucleolate (olivaceous in the mass); conidia arcuate, nucleate, continuous (as far as seen), 40 to $60 \times 2.5$ to $3 \mu$.

Allied to F. Schreveinitzii Ell. \& Hark., but that has conidia oblong, obtuse, 20 to $30 \times 6 \mu$.

On Oxydendron arboreum. Fayette, near Nuttallburg, March, I896 (Nuttall, 827).

F. ROSEUM Link.

On follicles of Asclepias Syriaca, May 3I, I894. Conidia $55 \times 4 \mu+6$-septate (Nuttall, I530, 503).

F. ROSEUM - Var. nov?

On dead fruit of Diospyros Virginiana, Aug., I895 (Nuttall, I83I, 72I).

F. Solani Mart.

Found associated with "black rot" on Tomato fruits that have fallen to the ground. Monongalia Co., at Morgantown, I89I (Millspaugh).

F.

On stems of Asparagus officinalis, Oct. 28, I893. Color, light-pink. Conidia oblong to obovate 7.5 to Io $\times 2.5 \mu$ (Nuttall, 1239, I248, 185). 


\section{MICROCERA Desm.}

M. erumpens E. \& E., Proc. Phila. Acad., I894, 386.

Type habitat: On dead limbs of Tsuga Canadensis, March, I895 (Nuttall, discov. I398, 371).

Sporodochia scattered, depressed-globose, $.5 \mathrm{~mm}$. diameter, at first covered by the epidermis, raising it into little whitish pustules, then erumpent and closely embraced by the ruptured epidermis, at first orange-red, then becoming nearly black, and finally leaving subcupuliform cavities in the bark, when dry. Conidia falcate to fusiform, multinucleate, and finally three or more septate, 60 to $83 \times 3$ to 4.5 $\mu$ hyaline, narrowing to a slender point at each end, borne on short sporophores ( 20 to $35 \mu$ ), which are more or less branched above.

Differs from $M$. coccophila Desm. in the shape of the sporodochia and their subcuticular origin.

(The additional description, incorporated in this relation of the specific characters, is by E. \& E.)

\section{EPICOCCUM Link.}

E purpurascens Ehrenb.

On cardboard box in grass, April I3, I894 (Nuttall, I453).

E. Neglectum Desm.

On living leaves of Avena sativa and Catalpa Catalpa, Monongalia Co., at Morgantown, i89I (Millspangh).

E. Duriaeanum Mont.

Underside of outer bark of Robinia Psendacacia, Nov. Io, I893. (Nuttall, I285, 229).

\section{EPIDOCHIUM Fries.}

E. Melanochlorum Desm.?

On Carex Fraseri, Feb. II, I894 (Nuttall, I376, 337).

TRIMMATOSTROMA Corda.

T. Americana Thum.

On Salix nigra, March 21, I894 (Nuttall, I437, 410).

\section{HYPHELIA Fries.}

H. TERRESTRIS Fr.

On damp ground. June I8, I893 (Nuttall, IoI6). 
SPHAEROPSIDEAE.

\section{S P H AE R I O I A C E AE.}

\section{PHYLLOSTICTA Pers.}

P. Paviae E. \& E.

On Aesculus octandra hybrida and Hamamelis Virginica, June I0, I894 (Nuttall, I539). (P. sphaeropsidea E. E E.)

P. CARyigena Sacc.

P. Caryac E. \& E. On living leaves of Hicoria ovata and H. microcarpa, June 30, I894 (Nuttall, I580).

P. Celtidis E. \& K.

On living leaves of Celtis occidentalis, Oct. Io, I894 (Nuttall, I7 I5, 603).

P. Hamamelidis Pk.

On living leaves of Hamamelis Virginica, June Io, I894 (Nuttall, I556).

P. Catalpae E. \& M.

On living leaves of Catalpa Catalpa, July 25, I895 (Nuttall, I623).

P. Sanguinariae Wint.

On living leaves of Sanguinaria Canadensis, June 29, I894 (Nuttall, 1567).

P. Ipomoeae E. \& K.

On Ipomoea pandurata, Sept. I9, 1895. Spores 5 to IO $\mathrm{x} 2$ to $2.5 \mu$ (Nuttall, I86I, 749).

P. solitaria E. \& E.

On Malus Malus and coronaria, Monongalia, near Morgantown (Sheldon). Berkeley, on Malus Malus at Gerrardstown (Waite).

P. HAyNALDI Roum.

On Ilex verticillata. Oct. 5,1895 . Spores 5 to $8 \times 2.5$ to $3 \mu$ (Nuttall, 1872, 756).

P. Ribis E. \& E. Field Mus. Bot., I :102 (1896).

Type habitat: On leaves of Ribes cult. Oct. 21, I895 (Nuttall, discov., I882, 768).

Spots large, irregular in shape, .5 to $\mathrm{I} \mathrm{cm}$. or often confluent along the margin of the leaf for $2 \mathrm{~cm}$., rusty brown, becoming whitish or grayish, and mostly zonate, definite, but without any differently colored border. Perithecia epiphyllous, scattered, I50 to $200 \mu$ diameter, the apex prominent and black. Sporules oblong-elliptical, hyaline, granular and nucleate, I 5 to $22 \times 6$ to $9 \mu$.

Distinguished from $P$. ribicola Fr., and $P$. Grossulariae Sacc. by its much larger sporules. 
P. Althaeina Sacc. all.)

On Abutilon Avicennae. Fayette, near Nuttallburg (Nutt-

P. Galacis (Cke).*

On leaves of Galax aphylla, May 4, I894 (Nuttall, I486, 474).

P. AMPELOPSIDis E. \& M.

On Parthenocissus quinquefolia, June I8, I893 (Nuttall, IOI4).

P. ASIMinae E. \& K.

On living leaves Asimina triloba, Monongalia Co. near Camp Eden, I89I (Millspangh). On same host June I, I894, spores 8 to Io $\mathrm{x} 6 \mu$ (Nuttall, I 5 I 7 ).

P. Quercus-prini E. \& E., Proc. Phila. Acad., i894, 356.

Type habitat: On leaves Quercus Prinus, June 29, I894 (Nuttall, discov. I 594, 542).

Spots orbicular, rusty brown, definite, with a very narrow border, 2 to $3 \mathrm{~mm}$. diameter. Perithecia epiphyllous, scattered, erumpent, 90 to I IO $\mu$ diameter. Sporules ovate or oblong, hyaline, mostly a little curved, 5 to $6.5 \times 2$ to $2.5 \mu$.

This comes very near Ph. Ludoviciana $\mathrm{E}$. \& E., but in that species the spots are larger, perithecia more prominent below, and sporules rather larger. Ph. marginalis E. \& E. also has similar sporules, but the perithecia are hypophyllous.

P. macrospora E. \& E., Proc. Phila. Acad., I894, 355.

TyPe habitat: On leaves of Liriodendron Tulipifera, Aug. 7, i894 (Nuttall, discov. 1654, 574).

Spots few, suborbicular, ferruginous-gray, 3 to $5 \mathrm{~mm}$. diameter, with a narrow, raised, darker border. Sporules oblong elliptical hyaline, nucleate, 15 to 30 (mostly 20 to 25) $\mathrm{x} 6$ to $7 \mu$.

P. Pirina Sacc.

On Malus Malus, Greenbrier, at White Sulphur Springs (Waite, 7I6).

P. Rosae Desm.

On Rosa humilis. Spores 6 to $7.5 \times 2.5 \mu$ Sept. I8, 1895 (Nuttall, I863).

P. gentianicola (DC).

On leaves of Gentiana Andreresii, Sept. 19, 1895. Spores oval, $7.5 \times 5 \mu$ (Nuttall, I857).

P. Rhoicola E. \& E., Proc. Phila. Acad., i894, 356.

Type Habitat: Rare, on leaves Rhus radicans. Aug. I8, I894 (Nuttall, discov. I668, 567 in part).

Spots 4 to $6 \mathrm{~mm}$. diameter, deciduous, gray1sh-white, with

*Phoma Galacis Cke., Messrs. Ellis \& Everhart decide from our specimens that this species belongs in Phyllosticta. 
a narrow black border. Perithecia epiphyllous, scattered, Ioo to I Io $\mu$ diameter, convex-prominent above, visible also below. Sporules narrow-elliptical, hyaline, 2 -nucleate, 5 to 6 $\mathrm{x} 2$ to $3 \mu$.

Differs from P. toxica, E. \& M., in larger deciduous spots with a narrow border, and its narrow-elliptical sporules.

P. Lindericola E. \& E., Proc. Phila. Acad., I894, 354.

Type habitat: On leaves Benzoin Benzoin, Aug. 26, I894 (Nuttal, discov. I688, 593).

Spots various; punctiform and minute without any definite border, often irregular in shape I $\mathrm{mm}$. to I or $\mathrm{I} .5 \mathrm{~cm}$. diameter, or marginal, forming a narrow strip along the edge of the leaf for half its length, pale brown, nearly the color of weather-beaten wood, with a dark (almost black) border. Perithecia epiphyllous, hemispherical, Ioo to $120 \mu$ diameter, covered by the blackened epidermis which is raised into pustules barely pierced at the apex. Sporules oblong-elliptical, hyaline, 4 to $7 \times 2$ to $3 \mu$.

This differs in almost every respect from $\mathrm{Ph}$. Linderae E. \& E.

P. Smilacis subeffusa E. \& E.

On Smilax rotundifolia, Sept. 21, I894 (Nuttall, I7or, 594). Perithecia scattered over surface of dead dry leaveş of the host.

P. Oxydendri E. \& E., Field Mus. Bot., I : IO4 (1896).

Type habitat: On leaves of Oxydendron arboreum, Oct. I0, I894. (Nuttall, discov. I717, 605).

Spots suborbicular or elliptical, reddish-gray, 3 to $8 \mathrm{~mm}$. diameter, with a narrow, slightly raised dark red border, beyond which the leaf is generally shaded reddish-purple. Perithecia epiphyllous, subapplanate, , oo to $120 \mu$ diameter. Sporules oblong-elliptical, 2-nucleate, hyaline, 7 to $8 \times 2.5$ to $3 \mu$. The spots finally become of a lighter, dirty-white color.

P. Celastri E. \& E., Field Mus. Bot., I :IO4 (i896).

Type habitat: On leaves Celastrus scandens, Oct., I894 (Nuttall, discov. I718, 606).

Spots mostly marginal, 8.5 to $\mathrm{I} \mathrm{cm}$. diameter, ferruginous, definite, with a narrow, darker border. Perithecia epiphyllous, subapplanate, I $50 \mu$ diameter. Sporules oblong, 7 to Io $\mathrm{X} 2$ to $2.5 \mu$ hyaline, 2 -nucleate.

P. globifera E. \& E., Field Mus. Bot., I :I04 (1896).

Type habitat: On leaves Cormus florida, Oct. 24, I894 (Nuttall, discov. I728, 615).

Spots marginal, light-brown, continuous, extending across the tip of the leaf and partly down each side, 5 to $\mathrm{I} \mathrm{cm}$. wide, separated from the green part of the leaf by a narrow, purple margin. Perithecia amphigenous, discoid, black, IoO 
to I $2 \mu$ diameter. Sporules globose or short-elliptical, hyaline, granular, 6 to $8 \times 5$ to $6 \mu$.

P. Negundinis Sacc.

On Acer Negundo, July 5, I894. Sporules 5 to $10 \times 3$ to $7 \cdot 5 \mu$ (Nuittall, I631, 560, 561).

P. $\longrightarrow$ sp.

On Geum Canadense, Oct. 24, I894 (Nuttall, I733). Spores irregular 4 to $7.5 \times$ I.5 to $2.5 \mu$ 2-nucleate.

P. ACERICOLA E. \& E.

On living leaves Acer saccharinum, Putnam Co., near Buffalo, I89I (Millspaugh). On leaves A. rubrum, June 24, I894 (Nuttall, I 56r).

P. Xanthorrhizae E. \& Nuttall, Field Mus. Bot., i :I05 (i8g6).

Type habitat: On leaves Xanthorrhiza apiifolia, Nov. 3, I893 (Nuttall, discov. I738, 625).

Spots subelliptical, dirty-white in the center with a dark shaded margin, $3 \mathrm{~mm}$. to $\mathrm{I} \mathrm{cm}$. long $\times 2$ to $8 \mathrm{~mm}$. wide, finally more or less deciduous. Perithecia seated ' on the white part of the spots, epiphyllous, subdiscoid, perforated above, 60 to $75 \mu$ diameter. Sporules oblong-elliptical, hyaline, 2-nucleate, 5 to $6 \times 2.5$ to $3 \mu$.

Accompanied by a Macrosporium on the same spots.

P. - sp.

On leaves Rubus Canadensis, Nov. 3, I894. Sporules variable in form, $5 \times 1.25$ to $2 \mu$ (Nuttall, I747, 627).

P. CERCIDicola E. \& E., Field Mus. Bot., i : IO5 ( I896).

Type habitat: On leaves Cercis Canadensis, Nov. 21, I894 (Nuttall, discov. I75I).

Spots suborbicular, .5 to $\mathrm{I} \mathrm{cm}$. diameter, rusty-brown, becoming lighter, paler below, margin narrow reddish-purple. Perithecia epiphyllous, convex, suberumpent, but covered (except the apex) by the epidermis, I Io to I50 $\mu$ diameter. Sportules oblong, slightly narrowed and rounded or obtusely pointed at the ends, hyaline, 2 to 3 -nucleate, 15 to $20 \times 6$ to $7 \mu$.

Differs in the character of the spots, and in the presence of a perithecium, from Gloeosporium Cercidis, E. \& E.

P. Araliae E. \& E., Proc. Phila. Acad., I894, 355.

Type habitat : On le̊aves Aralia spinosa, Sept. 20, I895 (Nuttall, discov. I703, 598 588?).

Spots suborbicular, light-brown with the margin a little darker, 5 to $\mathrm{I} \mathrm{cm}$. diameter. Perithecia epiphyllous, somewhat flattened, IOo to I $50 \mu$ diameter. Sporules oblongelliptical, hyaline, 2 -nucleate, Io to ${ }^{5} \times 5$ to $6 \mu$.

P. Sassafras Cooke.

On leaves Sassafras Sassafras, June 28, I894. Sporules 5 to $7 \times 2$ to $3 \mu$ (Nuttall, I 566). 
P. Sambuci Desm.

On leaves Sambucus Canadensis, July 20, I894 (Nuttall, I6I4).

P. LABRUSCAE Thúm.

On living leaves Vitis cult. (Concord), Wood Co., near Lockhart's Run, i891 (Millspangh). On leaves of $V$. oestivalis and rupestris, June 29, I894 (Nuttall, I 569).

P. CORNICOLA (D. C.) Rabh.

P. Cookei Sacc.

On Cormus florida, Nov. 5, 1894 (Nuttall, 1748, 638).

On leaves Magnolia acuminata, Aug. 9, 1894. Sporules I 5 to $20 \times 6$ to $7.5 \mu$ (Nuttall, 1655,577 ).

P. Tiliae Sacc.

On leaves Tilia Amcricana, Aug. Io, I894. Sporules 7 to $8 \times 2.5$ to $3 \mu$ (Nuttall, 1672, 579).

P. Chionanthi Thum.

On Chionanthus Virginica, Aug. I0, 1895. Spots darker than in type (from Portugal). Spores round or oval, 5 $\mu$ or $7.5 \times 5 \mu$ (Nuttall, 1833,724$)$.

P. LIRIODENDRICA Sacc.

On leaves Liriodendron Tulipifera, June 16 and Oct. 29, I894. Sporules 7.5 to Io $\times 2$ to $3 \mu$ (Nuttall, I 57 I). $P$. Liriodendri, Cke.

P. Ulmi West?

On leaves Ulmus pubescens, July 9, 1894. Sporules + I6 x $7.5 \mu$ (Nuttall, I631, 563).

P. PHOMIFORMIS Sacc.

On leaves Quercus Prinus and Q. alba, July 4, I894. Sporules 15 to $30 \times 6$ to $8 \mu$ (Nuttall, I 585 ).

P. Castaneae E. \& E., Proc. Phila. Acad., I894, 357.

Type habitat: On leaves Castanea pumila (not on Castanea vesca as originally published), July 26, I894 (Nuttall, discov. 1639, 570).

Spots orbicular, rust-color, with narrow dark-shaded, margin. Perithecia epiphyllous, scattered on the spots, dark, semierumpent, Ioo $\mu$ diameter, often collapsing. Sporules oblong, hyaline, 5 to $7 \times 2$ to $2.5 \mu$.

P. LAPpae Sacc.

On Arctium Lappa, July 20, I894 (Nuttall, I613).

P. Dioscorean Cooke.

On leaves Dioscorea villosa, June 29, I894 (Nuttall, I570).

P. Opaca E. \& E. Field Mus. Bot., I :106 ( 1896).

Type habitat: On leaves of Ilex opaca. Oct. 5, I895 (Nuttall, discov., I88r).

Spots mostly marginal or terminal, of irregular shape, I and $2 \mathrm{~cm}$. across, dirty-white, with a raised subferruginous narrow border. Perithecia evenly scattered, subepidermal, 
sublenticular, black, raising the epidermis into pustules, 200 $\mu$ diameter. Sportules elliptical, hyaline, $3 \times 1.5 \mu$.

Differs from P. Ilicis E. \& E. only in its minute sporules.

\section{PHOMA Fries.}

P. $\longrightarrow$ sp.

On Opulaster opulifolius, May 7, 1894 (Nuttall, I5Io, 494). Spores 7 to $10 \times 2.5$ to $3 \mu$.

P. negundinicola Ramicola E. \& E., Proc. Phila. Acad., I894, 357.

Type habitat: On dead twigs Acer Negundo, April, I894 (Nuttall, discov. I483, 465, 466?).

Perithecia evenly but not thickly scattered, globose, small, .2 to $.33 \mathrm{~mm}$. diameter, covered by the slightly ruptured epidermis, but distinctly prominent. Sporules oblong, elliptical, or ovate hyaline, with a single large nucleus, io to I $3 \times 4$ to $5.5 \mu$.

Differs from the typical form, on the dead peduncles, in its broader sporules, the former being but 2.5 to $3 \mu$.

P. Pennsylvanica E. \& E., Proc. Phila. Acad., I894, 357.

Type habitat: On dead limbs Acer Pennsylvanicum, Feb. 2, I894 (Nuttall, discov. 1361, 318).

Perithecia numerous, evenly scattered, subepidermal, .33 to $.5 \mathrm{~mm}$. diameter, whitish inside, raising the epidermis into minute pustules, but scarcely rupturing it. Sporules subglobose, 6 to $7.5 \mu$ diameter, nearly hyaline.

P. Asclepiadea E. \& E., Field Mus. Bot., i :Io7 (1896).

Type habitat: On dead stems of Asclepias Syriaca, Oct. I0, I894 (Nuttall, discov. I7I7, 604).

Perithecia thickly, but evenly scattered, subelliptical, I Io to $\mathrm{I} 2 \mathrm{O} \mu$ in the longer diameter, subcuticular, covered by the blackened epidermis, which is raised into minute pustules but scarcely ruptured. Sporules oblong-elliptical, 2-nucleate, hyaline, 5 to $6 \times 2$ to $2.5 \mu$. On curved (almost hooked) basidia 12 to I $5 \mu$ long.

Differs from the next form, which occurs with it on the same stems, in its larger perithecia and sporules and its curved basidia.

P.

On dead stems Asclepias Syriaca, Oct. Io, I894 (Nuttall).

P. melaleuca B. \& C.

On petioles Aralia spinosa, April 25, I895 (Nuttall, I8Io)

P. Leucostoma Lev.

On Opulaster opulifolius, May I3, I894. Sporules 2.5 to $4 \times$ I $\mu$ (Nuttall, I 506, 487). 
P. mixta B. \& C.

On fire-killed Liriodcndron Tulipifera, Oct. 16, 1894. Sporules appendiculate at each end, 2 -nucleate, 7 to $10 \times 2$ to 3 $\mu$, oblong fusoid; the accompanying filiform process hooked at the apex $20 \times .75$ to I.5 $\mu$ (Nuttall, I709).

P. SCAERA Sacc. 434).

On Platanus occidentalis, March 30, I894 (Nuttall, I459,

P. obscurans E. \& E., Proc. Phila. Acad., I894, 357.

Type habitat: On leaves of Fragaria cult., July 8, I894 (Nuttall, discov. I600, 554).

Spots 5 to $8 \mathrm{~mm}$. diameter, with a ferruginous center and broad purple border, paler below. Perithecia few, scattered, convex prominent. Sporules oblong-elliptical, hyaline 2-nucleate, 4.5 to $5.5 \times 1.5$ to $2 \mu$. Basidia simple, lanceolatefusoid, 8 to $12 \times 1.5 \mu$.

Phyllosticta fragaricola Desm. has similar sporules, but the spots are much smaller, with a white center. The basidia also indicate Phoma and not Phyllosticta.

P. Ilicicola (C. \& E.) Sacc.

On leaves of Ilex opaca, June I8, I893 (Nuttall, IoI7).

P. Spiraeae Desm?

On Opulaster opulifolius, May I3, I894. Sporules Io to I3 $32 \mu$ (Nuttall, I 508, 489).

P. HERBARUm West.

On Onagra biennis, March I8, I894 (Nuttall, I435, 406).

P. Phytolaccae B. \& C.

On stems Phytolacca decandra, July 31, I893 (Nuttall, I I 40).

P. Pedunculi E. \& E., Proc. Phila. Acad., I894, 357.

Type habitat: On old peduncles Magnolia Fraseri, Jan., I894 (Nuttall, discov. I339, 297).

Perithecia scattered, flattish pustuliform, .5 to $.75 \mathrm{~mm}$. diameter, with a prominent papilliform ostiolum. Sporules fusoid-oblong, hyaline, 2 to 3 nucleate, 7 to I I $\times 2$ to $2.5 \mu$ ends acute.

Differs from Stagonospora pedunculi in its larger, flatter perithecia and fusoid-oblong (not cylindrical), rather shorter sporules.

P. uvicola B. \& C.

On Vitis cult. (Concord), July 4, I893 (Nuttall, I I08, 94). P. Nyssaecarpa Cooke.

On dead limbs Nyssa aquatica, April I5, I895 (Nuttall, I809, 696). Sporules Io x $3 \mu$.

P. SAMARARUm Desm.

On samaras Acer Pennsylvanicum, Feb. 2, I894 (Nuttall, I363, 321). 
P. Smilacina (Peck) Sacc.

On Smilax rotundifolia, Aug. I7, I894. Sporules about I8 $\times 6$ to $7.5 \mu$ (Nuttall, I660).

\section{APOSPHAERIA Berk.}

A. pezizoides E. \& E., Proc. Phila. Acad., I894, 358.

Type HaBitat: On decorticated trunks of Platanus occidentalis, Salix nigra falcata, Betula nigra, Fraxinus sp., and Liquidambar Styraciflua, on abrasions made by driftwood and ice, where it seems to grow exclusively and always below high-water mark, April, I894 (Nuttall, discov. I 458,432 ).

Perithecia erumpent-superficial, seriate-aggregate, hemispherical, about $.75 \mathrm{~mm}$. diameter, with a distinct papilliform ostiolum, at length collapsing and pezizoid or discoid. Sporules elliptical or ovoid, 4 to $6 \times 2.5 \mu$ ends obtuse.

\section{DENDROPHOMA Sacc.}

D. Therryana Sacc. \& Roum.

On Platanus occidentalis, April I9, I894 (Nuttall, I463).

\section{SPHAERONAEMA Fries.}

S. Physocarpi E. \& E., Proc. Phila. Acad., I894, 358.

Type habitat: On dead stems Opulaster opulifolius, May, I894 (Nuttall, discov. I504, 484).

Perithecia gregarious, erumpent-superficial, hemispherical, I $50 \mu$ diameter, with a straight, stout beak 90 to II $5 \mu$ long. Sporules narrow-elliptical, hyaline, 2 to 3 -nucleate, 7 to 9 $\mathrm{x} 2.5 \mu$.

S. infuscans E. \& E. Field Mus. Bot., I : Iog (I896).

TYPE HABITAT: On dead dry wood Juglans cinerea., Dec. 5, I894 (Nuttall, discov: I766, 66I).

Perithecia gregarious, erumpent-superficial, hemispherical, brownish-black, about $.25 \mathrm{~mm}$. diameter, with a short cylindrical $(.33 \mathrm{~mm}$.), shining-black, obtuse, perforated ostiolum. Sporules abundant, elliptical, continuous, hyaline 3 to $4 \times$ I. $5 \mu$ on basidia simple or branched below, about I2 $\mu$ long and $.5 \mu$ thick at the base, narrowed above.

The surface of the wood is blackened.

S. ACERINum Peck.

On Acer rubrum, June I2, I894 (Nuttall, I543).

S. CORNEUM C. \& E.

On Onagra biennis, Jan. 21, I895 (Nuttall, I79I, 682 in part). 
S. Magnoliae Peck.

On Magnolia acuminata, Short Creek, alt. I,ooo ft., Aug. I6, I894 (Nuttall, I658). Sporules Io x $6 \mu$ nucleus large.

\section{SIROCOCCUS Preuss.}

S. Halesiae E. \& E., Proc., Phila. Acad., I894, 358.

On dead limbs Mohrodendron Carolinum (Halesia tetraptera) Feb. 26, I894 (Nuttall, discov. I407, 377, 376?)

Perithecia scattered, or 2 to 3 -cornate, erumpent, subglobose about $.33 \mathrm{~mm}$. diameter, black, with a minute papilliform ostiolum. Sporules subglobose, about $3 \mu$ diameter, greenish-hyaline, 3 to 6-concatenate, the terminal sporule oblong and paler. Chains simple or sparingly branched, arising directly from the proliferous layer without any distinct basidia, collected in stellate clusters.

\section{VERMICULARIA Fries.}

V. PHLOGINA Fairm.

On dead leaves Phlox amoena, Sept. I5, I894 (Nuttall, I606).

V. COMPACTA C. \& E.

On Rubus odoratus, May 30, I894; sporules 20 to $25 \mathrm{x}$ $30 \mu$ (Nuttall, I5I3). On Caulophyllum thalictroides (552).

V. PETIOLORUM Schw.

On Aralia spinosa, and on Robinia Psendacacia?. Feb. I7, I894 ( Nuttall, I388, 356).

V. ToxicA E. \& E., sp. nov?

On Rhus radicans, Aug. I8, I894 (Nuttall, I669, 569).

V. Dematium (Pers.) Fr.

On Cassia Marilandica, Oct. 28, I893 (Nutall, I25I, I93).

On Heuchera Americana.

V. Dematium var.

On Onagra biennis, March 9, I894 (Nuttall, I434, 405). V. Arctil Schw.

On dead leaves and petioles Arctium Lappa, on ground. July 2I, I894. Sporules fusiform slightly curved + or $20 \times 3 \mu$. Setæ 35 to $+125 \times 3$ to $5 \mu$ (Nuttall, 16I7).

V. subeffigurata Schw.

On fruit peduncles Magnolia Fraseri in association with Stagonospora pedunculi E. \& E., July 25, i893 (Nuttall, I 136$)$.

V. SUbEFFigurata scapincola Schw.

Bases of dead leaves Yucca filamentosa cult., Feb. I9 1894. Sporules I8 $\times 2.5 \mu$, largest setæ $200 \mu$ (Nuttall, I367). V. Trautvetteriae Nuttall, sp. nov.

Type habitat: On dead leaves Trautvetteria Carolinensis, July I5, I894 (Nuttall, discov. I633). 
Perithecia very small, scattered. Sporules curved, sharp pointed, 18 to $25 \times 2.5$ to $3 \mu$. Setæ $+100 \times 10 \mu$.

V. Liliacearum West.

On Asparagus officinalis, Vagnera racemosa, Clintonia umbellulata and Iris cristata? Nov. I, I893 (Nuttall, I24I, I88).

\section{DOTHIORELLA Sacc.}

D. Asiminae E. \& E., Field Mus. Bot., i :I io (I896).

On Asimina triloba, Feb. 2, I894 (Nuttall, I392, 362). Sporules 5 to $7 \times 2$ to $3 \mu$ oval or oblong, some of them slightly curved. Perithecia suberumpent, seriate, $.25 \mathrm{~mm}$. diameter, white inside and out, ovoid, in series of 3 to 5 , raising the epidermis into short ridges split along the top so as to expose the pallid white apices of the peritnecia. Sporules oval or elliptical, hyaline, 5 to $7 \times 2$ to $3 \mu$.

D. Minor E. \& E., Field Mus. Bot., i :I Io ( I896).

Type habitat: On dead limbs Liriodendron Tulipifera April I I, 1895 (Nuttall, discov. I804, 691).

Perithecia seriate-subconfluent, .5 to $.75 \mathrm{~mm}$. diameter, irregularly ruptured above, white inside, erumpent, splitting the epidermis into short ( 2 to $4 \mathrm{~mm}$.) longitudinal cracks. Sporules elliptical, hyaline continuous, 5 to $7.5 \times 3$ to $3.5 \mu$. Differs from D. Liriodendri, Cke. in its much smaller sporules.

D. GLandulosa (Cooke) Sacc?

On Robinia Pseudacacia, March I4, I894 (Nuttall, I423, 395).

\section{FUSICOCCUM Corda.}

F. Ilicinum E. \& E., Proc. Phila. Acad., I894, 359.

Type habitat: On dying, transplanted, Ilex opaca (transplanted Dec. 28, I893), May 24, I894 (Nuttall, discov. I 52 I, 496).

Stromata cortical, convex, about I or $1.5 \mathrm{~mm}$. diameter, multilocular, whitish inside, the tuberculiform apex pierced with a single pore, rupturing the epidermis and slightly raising it. Sporules fusoid, hyaline, nucleate 15 to $22 \times 2.5$ to $3 \mu$.

F. nervicolum E. \& E, Bull Torr. Club, 25:509 (1898).

Stromata mostly on the midrib and nerves of the leaf, elliptical, 400-60o $\mu$. long, subcutaneous, black, convex, the subconoid apex erumpent: sporules narrow-elliptical, hyaline, subacute, 2-nucleate, 7-10 X 2.5-3 $\mu$.

On old fallen leaves of Magnolia Fraseri, Nuttallburg, May, I898 (Nuttall, 940). 
CYTOSPORELLA Sacc.

C. carnea E. \& E., Bull., Torr. Club, 24:287 (I897).

Stroma at first tuberculo-hemispherical and covered by the epidermis, soon erumpent through the transversely or laciniately ruptured epidermis, brown outside, white and of firm consistence within (except the central portion), multilocular cells light-colored; sporules elliptical, hyaline, continuous, $5-7 \times 2.5-3 \mu$.

The stroma is about $\mathrm{I} .5 \mathrm{~mm}$. wide and I $\mathrm{mm}$. high and finally shrinks away from the ruptured epidermis and then is more or less distinctly flesh-colored.

On dead limbs of Castanea. Fayette: near Nuttaliburg, March, I8g6 (Nuttall).

CYTOSPORA Ehrenb.

C. Celtidis E. \& E., Proc. Phila. Acad., i894, 360.

Type habitat: On dead limbs Celtis occidentalis, Feb. 2, I894 (Nuttall, discov. I358, 314).

Stroma valsoid, flat, thin, $\mathrm{I} .5$ to $2 \mathrm{~mm}$. diameter, only penetrating the surface of the bark, multilocular, gray inside, raising the bark into small pustules and finally rupturing it, cells representing perithecia. Sporules allantoid, 6 to $7 \times$ I to $1.5 \mu$.

C. Halesiae E. \& E., Proc. Phila. Acad., i894, 36i.

Type habitat: On dead limbs of Mohrodendron Carolinum (Halesia tetraptera), June 7, I894 (Nuttall, discov. I 540).

Stromata convex-conical, sunk in the bark, orbicular, about I $\mathrm{mm}$. diameter, white inside, unilocular, the inner surface of the cavity lined with simple straight basidia about I $5 \mu$ long, bearing the oblong-fusoid, hyaline, 2-nucleate, straight. 5 to $7 \times$ I to I.5 $\mu$ sporules, which are expelled through a single orifice perforating the raised epidermis.

This probably is the spermogonial stage of Diaporthe Halesiae or D. tetrapterae, both of which are found in company with it.

C. Leucostoma (Pers.) Sacc.

On cultivate̊d Prumus domestica and Amygdalus Persica, Dec. I2, I894 (Nuttall, I769).

C. exasperans E. \& E., Proc. Phila. Acad., i 894, 360.

Type habitat: On dead limbs Acer Pennsylvanicum, Feb. 2, 1894, Short Creek, alt. I,300 ft. (Nuttall, discov. I366).

Stroma buried in the bark, orbicular, about I mm. diameter, 4 to 6-celled (at length one-celled), prolonged above 
into a stout, conical beak opening by a single round pore at the apex. Sporules oblong or allantoid, hyaline, 5 to $7 \mathrm{x}$ I. $25 \mu$.

C.

On Chionanthus Virginica, March 28, I894 (Nuttall, $1455,427)$.

C. tumulosa E. \& E. Bull. Torr. Club, $24: 288$ (I897).

Stroma cylindric-conical, erumpent, brown, lighter inside. about I mm. broad, the upper erumpent part .75 $\mathrm{mm}$. high, sporigerous cells perithecioid, whitish, globose, sunk in the lower part of the stroma; sporules allantoid, hyaline, curved, $6-8 \times 1.5 \mu$.

On dead buds of Magnolia Fraseri. . Fayette: near Nuttallburg, March, I8g6 (Nuttall, 808).

C. Persicae Schw.

On Amygdalus Persica cult. "Early Rivers," June 30 , I893 (Nuttall, I 106).

C.

On Cercis Canadensis, April Io, I894 (Nuttall, I47I, 447). Spores 5 to $6 \times .75 \mu$.

C. Rhoina Fr.

On Rhus hirta, June 20, I894. Sporules 5 to $8 \mu$. (Nuttall, I 55 I, 636).

C. Caryigena E. \& E., Proc. Phila. Acad., I894, 359.

Type habitat: On dead limbs Hicoria ovata, May 3I, I894 (Nuttall, discov. I524, 513).

Stroma cortical, convex, 1.5 to $2 \mathrm{~mm}$. diameter, slatyblack, 4 to 6-celled, cells yellowish-white, subglobose, small. Sporules allantoid, hyaline 3.5 to $4.5 \times .75$ to I $\mu$.

(Spermogonia of Valsa caryigena, B. \& C.)?

C. Rhois-hirtae Nuttall, Field Mus. Bot., i :II2 (I896).

On Rhus hirta, June Io, I894 (Nuttall, I 533, 635).

The form is clearly distinct from $C$. Rhoina Fr. Cirrus reddish yellow. Perithecia very prominent. Spores 8 to IO $\mathrm{x} 2$ to $2.5 \mu$.

C. Sassafras E. \& E. Field Mus. Bot., i ili2 (i896).

Type habitat: On fire-killed twigs of Sassafras Sassafras, Dec. 20, I894 (Nuttall, discov. I766, 666). Jamaica, Long Island (F. C. Stewart).

Stromata thickly scattered, conical, rising from an orbicular base about I mm. diameter, the black, shining apices erumpent and roughening the bark, at first multilocular, becoming unilocular. Sporules allantoid, hyaline, only slightly curved, 4 to $5 \times$ I $\mu$ borne on simple, straight basidia about I2 $\mu$ long. 
C. Betulina Ehr?

On Betula nigra, Feb. 20, I894. Cirrus yellowish, sporules, 4 to $6 \times$ I $\mu$ (Nuttall, I396, 367).

C. Salicis (Corda) Rabenh?

On Salix nigra, March 22, I894 (Nuttall, 1429).

C. Platani Fckl. 396).

On Platanus occidentalis, March I4, I894 (Nuttall, I424,

C. LeUcosperma (Pers) Fr.

On Carpinus Caroliniana, Nov. I7, I893. Sporules $5 \times$ I $\mu$ (Nuttall, I257).

C. CERATOPHORA Sacc.

On Fraxinus Pennsylvanica, Oct. I7, I893 (Nuttall, I234, I63).

C. Curreyi Sacc?

On Pinus Virginiana, Dec. I3, 1844; on Tsuga Canadensis, April I5, I895 (Nuttall, I771, 697).

C. Coccinea (Reb.) Fr.

On Robinia Pseudacacia, Feb. 2, 1894. Sporules $3 \times .75$ $\mu$ (Nuttall, I355).

C. - Spermogonia of Valsa goniotoma Sz.? On fire-killed Liquidambar Styraciflua, Oct. 21, I895 (Nuttall, I884).

C. ORTHOSPORA B. \& C.?

On Robinia Pseudacacia, April 20, 1894. Sporules irregu-

lar in shape I2 to I $8 \times 2.5$ to $3 \mu$ (Nuttall, I478, 458).

C. CARPHOSPERMA Fr.

On Asimina triloba, Nov. 5, I893 (Nuttall, I292, 237).

\section{SPHAEROPSIS Lev.}

S. Linderae Peck.

On dead twigs Benzoin Benzoin, April 8, I895 (Nuttall, I795).

S. Asiminae E. \& E., Proc., Phila. Acad., i894, 36i.

Type habitat: On dead limbs Asimina triloba, Feb. 24, I894 (Nuttall, discov. I 397, 370).

Perithecia scattered or subseriate, buried in the bark which is raised into little pustules over them and soon ruptured. Perithecia small $(.25 \mathrm{~mm}$.) thick-walled, with an obscurely papilliform ostiolum. Sporules oblong-elliptical, brown, obtuse, I 8 to $22 \times 8$ to Io $\mu$.

S. Asiminae fructigena E. \& E. Field Mus. Bot., i ili3 (i8g6).

Type habitat : On old dried-up fruits of Asimina triloba; Oct. 3, I895 (Nuttall, discov. I87 I, 755).

A fructigenous form of $S$. Asiminae E. \& E. Perithecia smaller than in the species (IIO to I $40 \mu$ ) and more abundant. Sporules a little larger ( 20 to $24 \times 9$ to $12 \mu$ ). 
S. Ampelopsidis C. \& E. ${ }^{\bullet}$

On Parthenocissus quinquefolia, March 30, I894. Sporules 18 to $20 \mathrm{x}$ IO to I2 $\mu$ (Nuttall, I460, 437).

S. Cerasina Peck.

On Prunus serotina, alt. I,200 ft., Nov. 22, I 894 (Nuttall, I746).

S. Physocarpi E. \& E., Proc. Phila. Acad., I894, 36r.

Type haвitat: On dead Opulaster opulifolius, May 2, I894 (Nuttall, discov. I 502, 482, 486).

Perithecia scattered, $.33 \mathrm{~mm}$. diameter, buried in the inner bark, with their apices and pipilliform ostiola erumpent, white inside (at first), sometimes, as in the preceding species 2 to 3 confluent. Sporules varying from short-elliptical 12 to I $5 \mathrm{x}$ Io $\mu$ to oblong or obovate-elliptical I $_{5}$ to $20 \mathrm{x}$ Io $\mu$.

Differs from S. Nielliae E. \& E. in its buried perithecia and much larger sporules.

S. Celastrina Peck.

On branches Celastrus scandens, April 27, I895 (Nuttall, I8I2).

S. Malorum Berk.

Berkeley: On Malus Malus at Gerrardstown (Waite).

S. Oenotherae E. \& E., Field Mus. Bot., i :I I4 ( I896).

Type habitat: On dead stems Onagra biennis, Jan. 2I, I895 (Nuttall, discov. I792, 683).

Perithecia scattered, erumpent, superficial, globose, 200 to $225 \mu$ diameter, with a papilliform ostiolum, occasionally subcollapsing above. Sporules oblong-elliptical, pale brown, 20 to $25 \times$ IO to $I 2 \mu$ on stout basidia shorter than the sporules. There are indications that the sporules become finally uniseptate.

S. Sumachi (Schw.) C. \& E.

On branches Rhus hirta, March 29, I894. Sporules 20 to $26 \times 8$ to $12.5 \mu$ (Nuttall, I 439 ).

S. Sassafras C. \& E.

On Sassafras Sassafras, April Io, 1895. Sporules $23 \mathrm{x}$ Iо $\mu$ (Nuttall, I80 I, 688).

S. Phomatella Peck.

On Fraxinus Americana, March 3I, I894 (Nuttall, I44I). S. Caryae C. \& E.

On hickory barrel-hoop, April 9, I894. Sporules I8 to $25 \times 8$ to I I $\mu$ (Nuttall, I448).

S. Ipomoeae E. \& E., Proc. Phila. Acad., I894, 362.

Type habitat: On dead peduncles of Ipomoea pandurata, Dec. 9, I893 (Nuttall, discov. I305, 249).

Perithecia scattered, ovate-globose, 250 to $300 \mu$ diameter, the upper part prominent and closely covered by the shining 
black epidermis. Sporules elliptical, brown, 18 to $23 \times$ Io to I $3 \mu$ on pedicels of about the same length as the sporules.

S. Menispermi Peck.

On Menispermum Canadensis, Jan. I7, I894 (Nuttall, I343, 302).

\section{CONIOTHYRIUM Corda.}

C. Fuckelii Sacc.

On Parthenocissus quinquefolia, sporules globular, 2 to $2.5 \mu$ March 3I, I894 (Nuttall, I442).

C. Concentricum (Desm.) Sacc. ?

On leaves Yucca filamentosa, Sept. I, I894 (Nuttall, II 87 ).

C. Pirina (Sacc.) J. L. Sheldon.

(Phyllosticta Sacc.)

On leaves Malus, Greenbrier: at Blue Sulphur Springs (Nuttall, I624.) On same, Monongalia: near Morgantown (Sheldon).

\section{HAPLOSPORELLA Sperg.}

H. Celtidis E. \& E., Proc. Phila. Acad., i894, 362.

Type habitat: On dead limbs Celtis occidentalis, Feb. 2, I894 (Nuttall, discov. I359, 3I5).

Perithecia mostly in valsoid clusters of 3 to Io, small, 200 $\mu$ white inside, slightly sunk in the inner bark, their papilliform ostiola rupturing the epidermis. Sporules elliptical, brown, continuous, I 8 to $22 \times$ Io to $12 \mu$ on basidia of about the same length as the sporules.

H. Araliae E. \& E., Proc. Phila. Acad., i894, 362:

Type habitat: On dead limbs Aralia spinosa, Feb. 26, I894 (Nuttall, discov. I406, 375).

Stromata seriate-connate, erumpent through longitudinal cracks in the bark, and extending from 4 to $5 \mathrm{~mm}$. to 2 or more centimetres. Perithecia ovate-globose, buried in the black, subcarbonaceous stroma, 3 to 6 in each single stroma, about $.33 \mathrm{~mm}$. diameter. Sporules elliptical, brown, 20 to $25 \mathrm{x}$ IO to I2 $\mu$.

This may be the pycnidia of Botryosphaeria fuliginosa (M. \& N.).

\section{DIPLODIA Fries.}

D. LiRIODENDRI Peck.

On fire killed Liriodendron Tulipifera, Oct. I6, I894 (Nuttall, I7IO).

D. Salicina Lev.

On dead twigs Sali. nigra, March 25, 1894 (Nuttall, I457). 
D. Cercidis E. \& E., Proc. Phila. Acad., I894, 363.

Type habitat: On dead limbs Cercis Canadensis, April 9, I894 (Nuttall, discov. I475, 449).

Perithecia subseriate, globose, .33 to $.5 \mathrm{~mm}$. diameter, slightly sunk in the inner bark and splitting the epidermis with short longitudinal clefts. Sporules elliptical, 20 to $23 \times$ Io to $15 \mu$ on stout basidia, uniseptate.

D. infuscans E. \& E., Proc. Phila. Acad., I894, 363.

Type habitat: On bark of dead limbs Fraxinus Americana, April 27, 1894 (Nuttall, discov. I492, 459).

Perithecia ovate-globose, small, i Io to I50 $\mu$ diametei, thickly scattered, blackening both the outer and inner surface of the bark. Ostiolum not conspicuous, obscurely papilliform. Sporules oblong-elliptical, I2 to I $5 \times 8$ to Io $\mu$ scarcely constricted.

In $D$. inquinans West, the sporules and perithecia are larger, and the bark is not blackened within.

D. Atrata (Desm.) Sacc.

On dead limbs Acer Negundo, April 20, I894 (Nuttall, 1465).

D. caryigena E. \& E., Proc. Phila. Acad., I894, 363.

Type Habitat: On dead limbs Hicoria ovata, May 3I, I894 (Nuttall, discov. I 525). Also Canada (Dearness.)

Perithecia subseriate, sunk in the inner bark, covered by the epidermis, which is raised into pustules and ruptured, about $.5 \mathrm{~mm}$. diameter, black. Sporules elliptical, brown, uniseptate, scarcely constricted, I 5 to $20 \times 8$ to IO $\mu$ (Pycnidia of Valsa caryigena B. \& C.).

D. Viticola Desm.

On Vitis, March 26, I894. No septum visible in sporules (Nuttall, I443, 412).

D. ILICICOLA Desm.?

On Ilex opaca, Dec. 20, I894. Sporules 22 to $25 \mathrm{x}$ II to I3 $\mu$ (Nuttall, I777, 667).

D. Rubi Fr.

On Rubus cult., Feb. I9, I895. Sporules $20 \times 8$ to I2 $\mu$ (Nuttall, I789).

D. INQUINANS West.

On Fraxinus Americana, April 27, I894 (Nuttall, I49I, 459).

D. Juglandis Fr.

On Juglans cinerea, April 9, I894 (Nuttall, I449).

D. Maydis (Berk.) Sacc.

On Zea Mays, Aug. 10, I895. Spores $30 \times 4 \mu$ (Nuttall, I840, 729). 
D. paraphysata E. \& E.? Bull. Torr. Club, $24: 288$ (i897).

Perithecia subcuticular, depressed-globose, pilose-tomentose, black (white inside), 300-400 $\mu$ diam. with papilliform osteolum raising the epidermis into pustules and finally rupturing it, thickly scattered, often $2-3$ sub-confluent; sporules ovate-elliptical, hyaline at first, then brown and uniseptate but not constricted, $22-27 \times$ I2-I $5 \mu$, accompanied by stout branching paraphyses IOO-I IO $\mu$ long by $1.5-2 \mu$ thick and borne on stout basidia mostly shorter than the sporules.

On bark of Tilia, in company with Malanconis tiliacea. Fayette: near Nuttallburg, March, 1896. (Nuttall, 832).

\section{BOTRYODIPLODIA Sacc.}

B. Acerina E. \& E., Proc. Phila. Acad., i894, 363.

Type habitat: On dead limbs Acer Pennsylvanicum, Feb. 2, I894 (Nuttall, discov. 1362, 319).

Perithecia erumpent in botryoidal clusters, often seriately confluent for i or more $\mathrm{cm}$., about $.5 \mathrm{~mm}$. diameter, white inside, flattish above, with a broad papilliform ostiolum. Sporules elliptical, brown, uniseptate, 20 to $25 \times 12$ to I $5 \mu$ on basidia of about the same length.

B. $\longrightarrow$ sp.

Stylosporus stage ? of Pseudovalsa sigmoidea (C. \& E.) On dead limbs Quercus Prinus, with that species. Spores brown, I-septate $25 \times 12.5 \mu$ (Nuttall, I829).

ASCOCHYTA Lib.

A. Clematidina Thum.

On Clematis Virginiana, Aug. Io, I895 (Nuttall, I842, 733).

\section{ACTINONEMA Fries.}

A Rosae (Lib.) Fr.

On Rosa cult., June 27, I894 (Nuttall, I563).

\section{HENDERSONIA Berk.}

H. Lirella Cooke.

On Salix nigra falcata, April 23, I894 (Nuttall, I480, 460). Sporules I5 x $7.5 \mu$.

H. Desmazieri Mont.

On bark of limbs Platanus occidentalis, April 24, I89.1. Sporules $+40 \times 22 \mu($ Nuttall, 1466).

\section{STAGONOSPORA Sacc.}

S. petiolorum E: \& E., Proc. Phila. Acad., I894, 365.

Type habitat: On dead petioles Aralia spinosa, Feb. I7, I894 (Nuttall, discov. I389, 357). 
Perithecia scattered, innate, small, slightly prominent and covered by the shining, blackened epidermis, I 50 to $250 \mu$ diameter, mostly sub-elliptical. Sporules oblong, hyaline, nucleate, becoming one or more septate, I 2 to $20 \times 2$ to $5 \mu$. S. pedunculi E. \& E., Proc. Phila. Acad., I893, 457.

Type habitat: On old fruit peduncles Magnolia Fraseri (not Liriodendron Tulipifera, loc. cit.). Short Creek, July 25, I893 (Nuttall, discov. I I35, I I9).

Perithecia gregarious, pustuliform, about $.33 \mathrm{~mm}$. diameter, covered by the blackened epidermis. Sporules cylindrical, I 8 to $22 \times 2$ to $2.5 \mu$, multinucleate, hyaline, straight. S. Physocarpi E. \& E., Proc. Phila. Acad., I 894, 365.

Type habitat: On dead stems and limbs Opulaster opulifolius, May. 12, I894 (Nuttall discov. I 505, 485).

Perithecia scattered, depressed-hemispherical; 200 to $250 \mu$ diameter, sunk in the bark, with the upper part prominent but covered by the epidermis, which is pierced by the papilliform ostiolum, white inside. Sporules linear, multiseptate, hyaline 25 to $35 \times 3$ to $4 \mu$.

S. collapsa (C. \& E.) Sacc.

On Acer saccharinum, Jan. 26, I894. Sporules 15 to $23 \mathrm{x}$ $3 \mu$ (Nuttall, I353, 309).

S. $\stackrel{3 \mu \text { (Np. }}{\text { Ond }}$.

On Chionanthus Virginica (Nuttall, 1462, 440). Spores $+25 \times 7 \mu 4$-septate. This may prove to be only a stylosporus stage of some Diothidaceous species-Ellis.

GLOMERELLA S. \& Von S.

G. Rufomaculans (Berk.) S. \& von S. Berkeley: on Malus Malus fruit at Gerrardstown (Waite).

CAMAROSPORUM Schulz.

C. Linderae E. \& E., Field Mus. Bot., I :i i7 (I896).

Type habitat: On dead limbs Benzoin Benzoin, April i 5 , I895 (Nuttall, discov. I808, 694).

Perithecia scattered, semierumpent $.75 \mathrm{~mm}$. diameter, the upper part, except the papilliform, erumpent ostiolum, covered by the closely adherent epidermis, which is raised into distinct, hemispherical pustules. Sporules oblong, 3-septate with I to 2 cells divided by a longitudinal septum, brown, obtuse, not constricted I2 to I8 (mostly I2 to I 5 ) $\times 5$ to $7 \mu$. The perithecia are of a firm consistence and slaty-black inside

SEPTORIA Fries.

S. Tecomae E. \& E., Proc. Phila. Acad., I894, 367.

Type habitat: On leaves Tecoma radicans, Aug. II, I894 (Nuttall, discov. I67I, 580). 
Spots light brown (wood color), irregular in shape, small 1.5 to $2 \mathrm{~mm}$., inconspicuous and indistinctly margined. Perithecia immersed, small $(65$ to $70 \mu)$ barely visible with a lens. Sporules 40 to $50 \times 2$ to $2.5 \mu$, not strongly curved, mucleate, hyaline.

S. Brassicae E. \& E., Field Mus. Bot., i il i7 (i896).

Type habitat: On leaves Brassica nigra, Nov. 26, 1894 (Nuttall, discov. 1759, 646).

Spots suborbicular or subangular, 3 to $4 \mathrm{~mm}$. diameter, dull white with a narrow, darker and sometimes slightly raised border. Perithecia epiphyllous, numerous, sublenticular pale brown, coarsely cellular, broadly perforated above, IoO to II $5 \mu$ diameter. Sporules numerous, curved, obtuse, continuous, hyaline, 25 to $45 \times 2$ to $3 \mu$.

Closely allied to S. Sisymbrii E11., but that has the spots greenish at first and never becoming more than whitish and the sporules I to 3 -septate.

S. RuBi West.

On living leaves Rubus Canadensis, Wood Co., Lockhart's Run, I891 (Millspaugh). On same host July 26, I894. (Nuttall, I627).

S. Corni-Maris Sacc.

On Cormus florida, alt. I,700 ft., July I9, I894 (Nuttall. I612).

S. CORNICOLA Desmz.

On Cormus alternifolia, Sewell Valley, Aug. 6, I894 (Nuttall, 1647).

S. kalmiaecola (Schw.) B. \& C.

On living leaves Kalmia latifolia, I89r, Monongalia Co., at Camp Eden (Millspaugh).

S. ochroleuca B. \& C.

On leaves Castanea dentata, June 30, I894. Maximum number of perithecia in a single macula 20 (Nuttall, I579). S. Microsperma Peck.

On Betula lenta, alt. I,200 ft., Oct .24, I894. Sporules + I8 $8.25 \mu$ (Nuttall, I722).

S. Polymniae E. \& E., Proc. Phila. Acad., i894, 368.

Type habitat: On leaves Polymnia Uvedalia, June 29. I894 (Nuttall, discov. I595, 543).

Spots, scattered, angular, limited by the veinlets, 2 to 4 $\mathrm{mm}$. diameter, dirty green. Perithecia epiphyllous, minute, $75 \mu$ diameter, scattered, innate, inconspicuous. Sporules filiform, continuous, 35 to $50 \mathrm{x}$ I to $\mathrm{I} .25 \mu$.

S. Trautvetteriá E. \& E., Proc. Phila. Acad., I894, 368.

Type habitat: On Trautvetteria Carolinensis, July 20. I894 (Nuttall, discov. 1632, 564).

Spots irregular, subangular, partly limited by the veinlets 
of the leaf, often elongated and acute at one end, brownishblack, with an irregularly shaped white center, which is well defined, angular, 3 to $5 \mathrm{~mm}$. in the longer diameter. Perithecia epiphyllous, but also visible below, small $(65$ to $75 \mu)$, scattered, dark. Sporules abundant, nearly straight or slightly curved, continuous, 22 to $30 \times 2 \mu$.

Differs from $S$. Anemonis Desm. in its broad, darkmargined white-centered spots, and rather longer and thicker sporules.

S. hyalina E. \& E., Proc. Phila. Acad., I894, 368.

Type haвiтat: On Viola primulaefolia, July $26, \quad$ I 894 (Nuttall, discov. I64I, 572). Also on $V$. blanda, Michigan (Hicks) ; and on $V$. lanceolata, Massachusetts (Miss Clarke).

Spots minute (.5 to I $\mathrm{mm}$.) white, with a dark purpleshaded border. Perithecia punctiform, black, epiphyllous, subglobose, 65 to $75 \mu$ diameter, not abundant. Sporules filiform, nearly straight, or slightly curved, hyaline, nucleate, not visibly septate, 20 to 40 (mostly 25 to 35 ) x I to $1.25 \mu$.

S. Violae West, has yellowish-brown perithecia on pale zonate spots with a reddish-brown border.

S. $\triangle$ CICULOSA E. \& E.

On Fragaria cult., Nov. 3, I894. Sporules 15 to $25 \times .75 \mu$ (Nuttall, I73I).

S. Helianthi E. \& K.

On Helianthus decapetalus, Oct. 29, I894. Sporules $50 x$ $2 \mu$ (Nuttall, I723).

S. Leptostachyae E. \& K.

On leaves Phryma Leptostachya, July 4, I894. Sporules 20 to $25 \times$ I $\mu$ (Nuttall, I 584).

S. Nolitangere Thum.

On Impatiens biflora, Aug. 5, I894. Sporules 20 to $25 \mathrm{x}$ I.5 to $2 \mu$ (Nuttall, r649).

S. Violae West.

On Viola sagittata, June 30, I893. Sporules $+25 \times 1.25$ $\mu$ (Nuttall, I577).

S. Saxifragae Pass.

On Heuchera Americana, June I0, I894. Sporules 15 to $20 \times 2.5$ to $3 \mu$ (Nuttall, $1544,52 \mathrm{I})$.

S. Lobeliae Peck.

On Lobelia cardinalis, June 22, I894 (Nuttall, I559).

S. Scrophulariae Peck.

On Scrophularia Marilandica, June I0, I894. Sporules 40 to $50 \mu$ long (Nuttall, I538).

S. Verbenae Rob. \& Desm.

On leaves Verbena urticacfolia, Jefferson Co., near Shenandoah Junction (Millspaugh). 
S. psilostega E. \& M.

On Galium circaezans, July I4, I894 (Nuttall, 1604).

S. NABALI B. \& C.?

On Prenanthes serpentaria, June 8, I894 (Nuttall, I536).

S. ATRO-PÜRPUREA Peck.

On Aster cordifolius, Nov. 24, I894. Sporules +35 to $55 \times$ I to $\mathrm{I} .5 \mu$ (Nuttall, I757, 644).

S. Polygonorum Desm.

On Polygonum Hydropiper, June 8, 1894. Sporules 25 to $40 \times 1.5 \mu$ (Nuttall, I 537).

S. Smilacinae E. \& M.

On Vagnera racemosa (Smilacina), Aug. I4, I894. Maculæ suffused with the marginal color late in season (Nuttall, 1252).

RHABDOSPORA Mont.

R. Kalmiarum (Schw.)*

Sphaeria Kalmianum Schw. On Kalmia latifolia, Aug. 26, I894 (Nuttall, 1685, 590).

PHLYCTAENA Mont \& Desm.

P. VAgabunda Desm.

On Vernonia Noveboracensis, Phytolacca decandra and

Onagra biennis, March 18, I894 (Nuttall, 1436, 408).

P. SEPTORIOIDES Sacc.

On Phytolacca decandra, May 25, I894 (Nuttall, I498).

P. Ipomoeae E. \& E., Proc. Phila. Acad., I894, 369.

Type habitat: On calyx lobes Ipomoea pandurata, Dec.

9, I894 (Nuttall, discov. I305, 250).

Perithecia scattered, subcuticular, 75 to $80 \mu$ diameter, covered by the blackened, slightly raised epidermis. Sporules linear, hyaline, curved above, I 5 to $20 \times 1.25 \mu$.

P. ARcuata Berk.

On Onagra biennis, March 18, I894. Sporules 30 to $35 \mathrm{x}$ $.75 \mu$ (Nuttall, I433, 403).

GELATINOSPORUM Peck.

G. Betulinum Peck.

On Betula lenta, April 12, 1895. Sporules subfiliform nearly semicircular, 32 to $40 \times 2.5 \mu$ (Nuttall, 1797).

\section{SPHAEROGRAPHUM Sacc.}

S. HyStricinum (Ell.) Sacc.

On Azalea viscosa, May 4, I894. Sporules narrowly arcuate-falcate $34 \times 3 \mu$ (Nuttall, I 501, 48I).

*Transferred to Rhabdospora by E. \& E. 
S. Fraxini (Peck) Sacc.

On dead limbs Fraxinus Americana, Short Creek, alt. I,300 ft., July 16, I894. Sporules 50 to $60 \times 2.5 \mu$ (Nuttall, I609).

NECT R I O D A C E AE.

ASCHERSONIA Mont.

A. $\longrightarrow \mathrm{sp}$. 687).

On Cornus florida. Spores 3 to $5 \times .75 \mu$ (Nuttall, I8oo,

LE P T O T R OM A CEAE.

LEPTOTHYRIUM Kunze \& Schm.

L. PYRI Sacc.

Berkeley: On Malus Malus at Gerrardstown (Waite).

L. $\longrightarrow$ sp.

On living leaves Ilex opaca, Aug., I894 (Nuttall, I679, 584). Maculæ white.

L. LIRIODENDRI Cke.

On dead fallen leaves Liriodendron Tulipifera, April I7, I895 (Nuttall, I805).

L. DRYINUM Sacc.

On living leaves Quercus rubra, alt. I,300 ft., June 29. I894 (Nuttall, I 592, 540).

L. Castaneae (Spr.) Sacc.

On fallen leaves Castanea dentata, April I7, I895 (Nuttall. I806).

L. PETIOLORUM — var.

On dead leaves Magnolia Fraseri, April I I, I895. Sporules 5 × I $\mu$ (Nuttall, I802, 689).

L? FORAminulatum Sacc. \& Ell.

On fallen leaves Ilex opaca, Aug. 16, i894. Sporules $25 \mathrm{x}$ $3 \mu$ (Nuttall, 1657).

L. vUlgare (Fr.) Sacc.

On Dioscorea villosa, on Aralia spinosa, and on Onagra biennis. Sporules 5 to $7 \times .75 \mu$ on each host, Feb. 8, I894 (Nuttall, 1368, 323).

\section{SACIDIUM Nees.}

S. Vitis E. \& E., Field Mus. Bot., I :I2I ( I896).

Type навітат: On $V$ itis cordifolia, Nov. 6, i893 (Nuttall, I254, I98). Fungi Columbiani 290, N. A. F., 3067.

Perithecia scattered; scutellate not perforated, I80 to 220 $\mu$ diameter. Sporules oblong-cylindrical, straight or curved, I 5 to $20 \times 4$ to $5 \mu$, filled with small nuclei. 
In the specimens of Sacidium viticolum, B. \& C. in Rav. $\mathrm{F}$. Am. the sporules are ovate or elliptical, 6 to $7 \times 5 \mu$, and the perithecia are only 75 to $100 \mu$ diameter.

D. Artoceras (Tode) Fr.

\section{DISCOSIA Lib.}

On Castanea dentata, Oct. 20, I893. On Epigaea repens, Jan. I8, I894. On Betula lutea, Fraxinus Pennsylvanica, Magnolia Frascri, Cornus florida, Sassafras Sassafras, and Cephalanthus occidentalis (Nuttall, I228, I73).

D. maCUlicola Ger.

On living leaves Disporum lanuginosum, Grant Co., near Bayard (Millspangh). On Smilax rotundifolia and Oxydendron arboreum, July 8, I894 (Nuttall, I 598).

D. rugulosa B. \& C.

Parasitic on upper surface of the maculæ of Phyllosticta caryigena, July 2, 1894 (Nuttall, 1583).

E. maculatum Lev.

\section{ENTOMOSPORIUM Lev.}

On living leaves and fruits Pyrus communis cult., Monongalia Co., Morgantown (Millspaugh).

LEPTOSTROMELLA Sacc.

L. Filicina (B. \& C.) Sacc.

On Dryopteris spinulosa, May 4, I894 (Nuttall, I487).

$$
\text { E X C I P U L A E A E. }
$$

DINEMASPORIUM Lev.

D. Hispidúlum (Schrad.) Sacc.

On Asimina triloba, March 9, I894. Sporules I4 to I8 x 2 to $2.3 \mu$ (Nuttall, i 422,394$)$.

MEJ.A N CON I A CEA E.

H. Rubi (West).

\section{HAINESIA Ell. \& Sacc.}

On leaves of Rubus cult. Oct. 28, I895 (Nuttall, I880).

GLOEOSPORIUM Desm. \& Mont.

G. sp.

On leaves of Cercis Canadensis (Nuttall, 985). Sporules $18 \times 7.5,+20 \times 7.5,23 \times 7,25 \times 6 \mu$.

G. Sanguinariae E. \& E., Proc. Phila. Acad., i894, 37 I.

Type habitat: On leaves Sanguinaria Canadensis, July 8, I894 (Nuttall, discov. I601, 555). 
Spots yellow, oblong or irregular, 3 to $5 \mathrm{~mm}$. diameter, situated near the apex of the leaf which is more or less uniformly blackened. Acervuli epiphyllous, numerous, innate, yellow and conspicuous. Conidia oblong, hyaline, continuous, mostly a little curved, 8 to $15 \times 3.5$ to $5.5 \mu$.

G. ARIDUM E. \& H.

On Fraxinus Pennsylvanica, June Io, I894 (Nuttall, I545. 5 I8).

G. Rumicis E. \& E., Field Mus. Bot., I :I22 (1896).

Type habitat: On leaves Rumex obtusifolius, Oct. 27 , I894 (Nuttall, discov. I729, 6I7).

Spots reddish-brown, lighter in the center, more or less distinctly zonate, 3 to $4 \mathrm{~mm}$. diameter, margin darker. Acervuli innate, small. Sporules erumpent above, oblong, hyaline, continuous, 5 to $8 \times 2$ to $2.5 \mu$.

The spots resemble those of Ovularia obliqua Cke., on Ramularia decipiens E. \& E.

G. Alni E. \& E., Field Mus. Bot., I :123 (1896).

Type habitat: On living leaves Alnus rugosa, Nov. 24, I894 ( Nuttall, discov. I737, 624).

Spots dirty brown, suborbicular, indistinctly zonate, .5 to I cm. diameter, paler in the center, border concolorous, rather indefinite; acervuli epiphyllous, numerous, small, roo to I 20 $\mu$ diameter, chestnut color, becoming darker ; conidia oblongelliptical, I2 to $16 \times 6$ to $8 \mu$.

Differs from G. cylindrospermum Bon., on the same host, in its much broader conidia, and from G. rubicolum E. \& E. in the different character of the spots and rather broader conidia.

G. Nervisequum (Fckl.) Sacc.

On leaves Platanus occidentalis, June Io, I894: Sporules I 2.5 to $18 \times 4$ to $6 \mu$ (Nuttall, I 555, 526).

G. Robergei Desm.

On Carpinus Caroliniana; July I6, I894 (Nuttall, I608).

G. Betularum Ell. \& Mart.

On Betula nigra, sporules Io x $7 \mu$, July I6, I894 (Nuttall, 1607).

G. CYLINDROSPERMUM Bon.

On leaves of Alnus rugosa. Spores 8 to $10 \times 2$ to $2.5 \mu$ (Nuttall, I875).

G. venetum Speg.

On living leaves of Rubus strigosus, Sept. 19, I895 (Nuttall, 1859).

G. Rubicolum E. \& E., Field Mus. Bot., i :I23 (I896).

Type habitat: On leaves of Rubus strigosus (Nuttall, discov. 747).

Spots at first numerous, angular, small and yellowish or 
reddish-yellow, soon confluent forming large, brown, dry, dead spots of irregular shape and indefinite outline, occupying a large part of the leaf. Acervuli not numerous, scattered, epiphyllous, prominent, resembling perithecia; conidia oblong-elliptical, I2 to $16 \times 6$ to $7 \mu$.

Differs from G. Rubi E. \& E. in its larger, fewer, black acervuli and broader conidia, which also are about twice as large as in G. Venetum Speg.

\section{MYXOSPORIUM Link.}

M. Lutem E. \& E., Proc. Phila. Acad., I893, 458.

Type habitat: On bark Hicoria ovata, April to Tuly, I893 (Nuttall, discov. IOI 5, 79). E. \& E. N. A. F. 2953. Fungi Columbiani 150.

Stroma globose-conical, light yellow, .75 to $\mathrm{I} \mathrm{mm}$. diameter, slightly sunk in the inner bark, unilocular and opening by a single pore. The surface of the inner bark around this pore is of a pale slate color, the colored portion definitely limited by a black line so as to form an irregular circle about $2 \mathrm{~mm}$. across, but this line does not penetrate the bark. Sporules navicular-oblong, hyaline, obtuse, ro to II $\mathrm{x} 4$ to $5 \mu$ with I to 2 large nuclei. Basidia slender-cylindrical I 5 to $20 \times 1.5 \mu$. Mass of exuded sporules flesh color.

M. Rhois (B. \& C.) Sacc.

On fire killed Rhus hirta, June 20, I895 (Nuttall, I575, $537)$.

M. platanicolum E. \& E., Proc. Phila. Acad., I894, 372.

Type habitat: On limbs Platanus occidentalis, April 24. I894 (Nuttall, discov. I485, 467).

Acervuli subcutaneous, vesiculoid, pale, I mm. diameter, raising the ruptured epidermis into pustules but not erumpent. Sporules oval or oblong-ovate, hyaline, nucleate at first, Io to $12 \times 5$ to $6 \mu$, on stout basidia.

M. seriatum E. \& E., Proc. Phila. Acad., I894, 372.

Type habitat : On bark of Acer sp., June, I894 (Nuttall, discov. I 552, 523).

Nuclei pallid, orbicular, about I $\mathrm{mm}$. diameter, seated on the surface of the inner bark; surrounded by a thin layer of smoky colored radiating hyphæ, from the inner extremities of which the botuliform or oblong, 6 to $8 \times 2$ to $2.5 \mu$ hyaline conidia are produced. The nuclei are seriately arranged, and the pale flesh-colored; flattish cirrhi are erumpent through narrow, longitudinal cracks in the bark. 
COLLETOTRICHUM Corda.

C. Lindemuthianum (S. \& M.) Scrib.

On pods of Phaseolus cult. "Wax, Butter Bean." Monungalia Co., near Morgantown, I89I (Millspaugh).

C. $\mathrm{sp}$.

On Sassafras Sassafras, Oct. I5, I894. Sporules 8 to I8 $\mathrm{x} 4$ to $7 \mu$ (Nuttall, I7I4, 602).

C. lineola Corda.

On Zea Mays, Aug. 10, I895 (Nuttall, 1834, 726).

C. lagenarium (Pass.) Ell. \& Halst.

On watermelon, Monongalia, near Morgantown (Sheldon).

\section{CYLINDROSPORIUM Ung.}

C. Crataegi E. \& E., Proc. Phila. Acad., I894, 372.

Type habitat: On leaves Crataegus sp., July 26, 1894 (Nuttall, discov. I640, 57I).

Leaves more or less mottled with rusty red, at.length uniformly of this same color. Acervuli innate, erumpent on both sides, and whitening the surface of the leaf with abundantly discharged conidia, which are 75 to $100 \times 3$ to $3.5 \mu$, nearly straight or more or less undulate and curved, nucleate, and faintly 3 to 5 septate.

C. Toxicodendri (Curtis) E. \& E. Proc. Phila. Acad., i893, 460.

On leaves Rhus radicans, June 29, I894. Sporules, largest, $50 \times 3 \mu$ (Nuttall, I568).

\section{LIBERTELLA Desm.}

L. FAGINEA Desm.

On bark of dead Fagus atropunicea, Grant Co., near Bayard (Millspaugh). On dead Prunus sp. cult. Aug. Io, I895. Cirrus bright red. Sporules variously curved is to. 25 x I $\mu$ (Nuttall, I843, 734).

L. $\mathrm{sp}$.

On felled Robinia Pscudacacia, April 4, I894. Sporules I 5 to $20 \times .75$ to I $\mu$ (Nuttall, I470, 446).

L. ACERINA Westend.

On Acer rubrum or saccharinum, April 4, I894. Sporules hyaline, I 5 to I8 x I $\mu$ (Nuttall, I445).

\section{MELANCONIUM Link.}

M. PALlidum Peck.

On dead limbs Hicoria ovata. Sporules $+20 \times 5 \mu$. May 3I, I894 (Nuttall, I5I4).

M. oBlongum Berk.

On dead limbs Juglans cinerea. Sporules $20 \times$ Io to $2 \mu$, Dec. 5, I893 (Nuttall, I29I, 236). 
M. BICOLOR Nees.

On Betula nigra, May 2, I894 (Nuttall, I489).

\section{THYRSIDIUM Mont.}

T. hedericolum Carpini Sacc.

On' dead shoots Carpinus Caroliniana, May 2, I894 (Nutt: all, I486).

\section{MARSONIA Fisch.}

M. Juglandis (Lib.) Sacc.

On Juglans cinerea, June 22, I894 (Nuttall, I560).

M. Martini Sacc. \& Ell.

On Quercus alba, velutina, et Prinus, Aug. I8, I894 (Nuttall, I674, 583).

\section{CORYNEUM Nees.}

C. cupulatum E. \& E., Proc. Phila. Acad., I894, 374.

Type habitat: On dead limbs Tsuga Canadensis, Short Creek, Dec. I6, I893 (Nuttall, discov. I317, 272).

Erumpent superficial. Acervuli tuberculiform, black, I to I. $5 \mathrm{~mm}$. diameter, hollowed out so as to be cup-shaped above. Conidia clavate, sessile, 6 to 9 septate, brown, 60 to $80 \mathrm{x}$ I 2 to I $5 \mu$.

C. pustulatum Peck.

On dead limbs Castanea dentata, Dec. 7, I893 (Nuttall, I 383$)$.

\section{PESTALOZZIA DeNot.}

P. $\mathrm{sp}$.

On leaves Geum Canadense, Oct. 24, I894 (Nuttall, I734). Sporules $20 \times 6 \mu$. Setæ 5 to I2 $\mu$ long.

P. FUNEREA Desm.

On Ipomoea pandurata, Sept. I9, I895. Spores 23 to $25 \mathrm{x}$ 6 to $7.5 \mu$ (Nuttall, 1862,750$)$.

P. Guepini Desm.

On leaves Rhododendron maximum, R. Catawbiense and Azalia viscosa. Spores 18 to $20 \times 8 \mu$, Aug. 2, I894 (Nutt all, I I49).

P. JEFFERISII Ell?

On Opulaster opulifolius, sporules Io to I $5 \times 4$ to 5 and $25 \times 5 \mu$, May 7 , I894 (Nuttall, I 509).

P. Toxica E. \& E., Proc. Phila. Acad., I894, 374.

Type habitat: On leaves Rhus radicans, Aug. I8, I894 (Nuttall, 1670, 567).

Spots and perithecia as in Phyllosticta rhoicola E. \& E. Sporules clavate-oblong, 4 -septate, I2 to $\mathrm{I} 5 \times 4$ to $5 \mu, 3$ 
intermediate cells pale brown, end cells short, conical, hyaline, the upper cell with a crest of 3 short, spreading hyaline bristles 6 to $7 \mu$ long. Distance between the two extreme cells I $2 \mu$, pedicels shorter than the spores.

S. Castaneae Lib?

\section{STEGANOSPORIUM Corda.}

On Castanea dentata, Nov. 14, I893 (Nuttall, I275, 221). S. PIRIForme (Hoffm.) Corda.

'On dying Acer rubrum, June 12, I894 (Nuttall, I553). S. MURICATUM Bon.

On dead limbs Betula nigra in drift-wood along river, Oct. 2 I, I895. Spores 35 to $50 \times 15$ to $18 \mu$ (Nuttall, 1878 ).

\section{U S T I L A G I N A CEA E.}

\section{USTILAGO Pers.}

U. TRITICI (Pers.) Rostr.

On living leaves Triticum vulgare. Clinton, North Am. Flora, $7: 8$.

U. Utriculosa (Nees) Tul.

On Polygonum Pennsylvanicum. Clinton, North Am. Flora, $7: 22$. (Sheldon, 2230).

U. Anomala J. Kunze.

On Plygonum scandens. Clinton, North Am. Flora, 7 :22.

U. Avenae (Pers.) Jens. (U. segetum of Flora).

On living heads of wheat and oats, Monongalia Co., near Morgantown; Lewis Co., near Alum Bridge; Taylor Co., near Thornton (Millspaugh).

U. TRICUSPIDIS, Ell \& Gall.

On Sieglingia seslerioides. Clinton, North Am. Flora, $7: 13$.

U. ZEAE (Beckm.) Unger.

(U. Maydis Corda).

On living ears and tassels of sweet corn, prevalent in Monongalia Co., I89I ; near Morgantown (Millspaugh). On garden corn, July 31, I894 (Nuttall, 1636).

U. Laevis (Kell. \& Swing.) Magn. (U. avenae laevis, Kell \& Swing.).

On oats, Sewell Mountain, alt. 2,600 ft., Aug. 23, I894 (Nuttall, 1678).

U. PERENNANS Rostr.

On Arrhenatherum elatius (Sheldon, 2361).

U. Cesati F. de W.

On Panicum sanguinale (Sheldon, 9ro). 
SPHACELOTHECA DeBary.

S. Hydropiperis (Schum.) DeBary.

On Polygonum sagittatum. Clinton, North Am. Flora, $7: 30$.

TILLETIA Tul.

T. STRIAEFORMIS (West) Wint.

On Phleum pratense, Poa pratensis and Agrostis alba (Sheldon, 5I, I 589, I 380, 3556, 507).

T. Tritici (Bjerk.) Wint.

Ustilago tritici, C. Bauhin. On living leaves of wheat, Monongalia Co., near Morgantown (Millspaugh).

\section{ENTYLOMA DeBy.}

E. Floerkeae Holw.

On Floerkea proserpinacoides (Sheldon, 3549).

E. Menispermi Pass.

On Menispermum Canadense (Sheldon, 1050).

GRAPHIOLA Poit.

G. Phoenicis (Moug.) Poit.

On a species of cultivated Phoenix (Sheldon, 757).

UROCYSTIS Rabenh.

U. Anemones (Pers.) Wint.

On living leaves and under stem cuticle of Actaea alba,

Monongalia Co., near Morgantown (Millspaugh).

U. CARcinodes (B. \& C.) Fisch. de Waldh.

On Actea alba. Clinton, North Am. Flora, 7:57.

U. Agropyri (Preuss) Schroet.

On Elymus? sp. (Sheldon, 2952).

\section{UREDIN A C E A E.}

\section{NIGREDO Roussel.}

N. pedatata (Schw.) Arthur.

On Andropogon Virginicus (Sheldon, 2768, 2234, 3248, 3292). Arthur, North Am. Flora, 7 :223. On Viola, Monongalia, Morgantown (Sheldon, 3249, 3579, 3580).

N. Silphit (Burrill) Arthur.

On Juncus tenuis. Arthur, North Am. Flora, $7: 239$.

N. Junci-effusi (Sydow) Arthur.

On Juncus effusus. Arthur, North Am. Flora, $7: 240$. On same (Sheldon, I102, 2436), and on J. tenuis (Sheldon. 2305, 2349, 3195). 
N. houstoniata (Schw.) J. Sheldon. (Aecidium Houstonianum Schw.).

On Houstonia caerulea, Monongalia, near Morgantown (Millspangh). On Houstonia longifolia, Fayette, near Nuttallburg (Nuttall, I518). On Sisyrinchium graminoides, Monongalia, near Morgantown (Sheldon, 2328, 2346). On H. coerulea (Sheldon, I3, 38, 39, 539). On H. purpurea (Sheldon, 2924).

N. Polygoni (Pers.) Arthur. (Uromyces Polygoni Fckl.).

On Polygonum erectum, Fayette, near Nuttallburg (Nuttall).

N. Lespedezae-procumbentis (Schw.) Arthur. (Uromyces Lespedezae $\mathrm{Pk}$.).

On Lespedeza violacea, Monogalia, near Morgantown (Millspaugh). On L. frutescens, Arthur, North Am. Flora, $7: 248$ (Sheldon).

N. Hedysari-paniculati (Schw.) Arthur. (Uromyces Farl.).

On living leaves Meibomia canescens, Mason, near Point Pleasant (Millspaugh). On leaves M. paniculata, Fayette, near Nuttallburg (Nuttall, I6Io). On several Meibomias (Sheldon).

N. fallens (Desmaz.) Arthur.

On Trifolium pratense, Arthur, North Am. Flora, $7: 255$ (Sheldon, 2330).

N. Trifoli (Hedw. f.) Arthur. (Uromyces Trifolii Lev.).

On living leaves Trifolium pratense, Mason, near Point Pleasant (Millspaugh). On Trifolium, Fayette, near Nuttallburg (Nuttall, I604). On T. hybridum and repens, Arthur, North Am. Flora, $7: 255$ (Sheldon, 641, 1686, 3233, $323 \dot{2})$.

N. Medicaginis (Pass.) Arthur.

On Medicago lupulina, Arthur, North Am. Flora, $7: 256$ (Sheldon, 1982).

N. appendiculata (Pers.) Arthur. (Uromyces appendiculatus Fr.).

On living leaves of Phaseolus vulgaris, Monongalia, near Morgantown (Millspaugh). On P. polystachus and Strophostyles pauciflora, Arthur, North Am. Flora, $7: 258$. On Phaseolus helvolus (Post, I733). On Ph. polystachus (She!don, 4212).

N. Proeminens (DC.) Arthur.

On Poinsettia dentata, Ohio, near Wheeling (Millspaugh). On same (Sheldon, 93I, 26I7), and on Chamaesyce Preslii (932, I035). 
N. Hyperici-frondosa (Schw.) Arthur. (Uromyces Hyperici Curt.).

On Hypericum mutilum, Fayette, at Rupert's (Nuttall, I625).

N. Hower (Peck) Arthur. (Uromyces Hozvei Peck).

On Asclepias Syriaca, Fayette, near Nuttallburg (Nuttall, I869). Summers, near Lowell (Pollard \& Maxon, I30).

On $A$. amplexicaulis, incarnata, and on Vincetoxicum gonocarpos, Arthur, North Am. Flora, $7: 265$. On same (Sheldon, I0I 5, I961, 1976); on Asclepias obtusifolius (Sheldon, I934); on $A$. incarnata (Sheldon, 3182).

N. Spermacoces (Schw.) Arthur.

On Diodia teres, Arthur, North Am. Flora, 7:267 (Sheldon, 2028).

N. Caladir (Schw.) Arthur. (Uromyces Caladii Farl.).

On Arisaema triphyllum, Fayette, near Nuttallburg (Nuttall, I5II). On $A$. dracontium (Sheldon, I321, 1620).

N. CARYophyllina (Schrank) Arthur.

On Dianthus caryophyllus (Sheldon, 34).

N. Rhyncosporae (Ellis) Arthur.

On Rhyncospora glomerata (Sheldon, 2688).

N. Plumbaria (Peck) Arthur.

On Onagra biennis (Shcldon, 2323).

\section{UROMYCES Link.}

U. Terebinthi (DC.)

On living leaves Rhus radicans, Oct. I7, I895 (Nuttall, I876).

U. EFFusus Arthur. don).

On Juncus effusus, Monongalia, near Morgantown (Shel-

U. Silphir (Lyd.) Arthur.

On Juncus tenuis, Monongalia, near Morgantown (Sheldonj.

\section{MELAMPSORA Cast.}

M. FARINosa (Pers.) Schroet.

On Salix nigrn, Oct. I7, I893. Spores at first hyaline $(22 \times$ I $3 \mu)$ but the granules finally turn yellow $(22 \mu)$, and perfectly fill the spore $(25 \times$ I $8 \mu)$ (Nuttall, IIO7).

P. Asparagi (DC.).

\section{PUCCINIA Pers.}

On Asparagus officinalis (Sheldon, 918).

P. Caricis-Asteris Arthur.

On Aster sps. (Sheldon, 2959, 3559). 
P. Chrysanthemi Roze.

On Chrysanthemum chinensis (Sheldon, I5).

P. Circaeae Pers.

On Circaea lutetiana and C. alpina (Sheldon, 769, I770, I889, 3477, 3864; Grout, I702).

P. Cyperi Arthur.

On Cyperus strigosus (Sheldon, 774, I709).

P. Dayi Clinton.

On Steironema ciliatum (Sheldon, 3I57, Bonner, 360I).

P. Eatoniae Arthur.

On Eatonia Pennsylvanica, Ranunculus abortivus and $R$. micranthus (Sheldon, 3235; 8, 3242; 4445).

P. Eleocharidis Arthur.

On Eleocharis ovata (Sheldon,, 46, 3176).

P. emaculata Schw.

On Panicum capillare (Sheldon, II55, I245).

P. GRAMinis Pers.

On Berberis Canadensis (F. E. Brooks). On Phleum pratense, Agrostis alba and Triticum vulgare (Sheldon, 3007, 3173; 1247, 1246; 719).

P. Impatientis Arthur.

On Impatien's aurea and Elymus sp. (Sheldon, 7, 653, 2306, 2330).

P. Investita Schw.

On Gnaphalium polycephalum (Sheldon, 2014, 2125, $22 \mathrm{IO})$.

P. LATERIPES Berk. \& Rav.

On Ruellia ciliosa (Sheldon, 927, 3127).

P. Lobeliae Ger.

On Lobelia syphilitica (Sheldon, 3801).

P. Polygoni-Amphibii Pers.

On Geranium maculatum, Polygonum convolvulus, $P$. dumetorum and $P$. hydropiperoides (Sheldon, 3238, 4I I3: 736, I942; 925, I I $30 ; 4257$ ).

P. RECEDENS Syd.

On Senecio aureus (Heck, 2908).

P. Podophylli Schwein.

On Podophyllum peltatum, May 3I, I894 (Nuttall, I5I5).

P. TENUIS Burrill.

On Eupatorium ageratoides, June I, I894 (Nuttall, I 519).

P. Asteris Duby.

On Aster cordifolius, Nov. 24, 1894 (Nuttall, 1758, 645). On Aster macrophyllus (Sheldon).

P. Helianthi Sz.

On Helianthus decapetalus, Sept. I9, I895 (Nuttall, I85I). On Helianthus annuus (Sheldon, I966, 2150). 
P. Violae (Schum.) DC.

On Viola hastata, æcidial stage; æecidia $18 \times 25 \mu$. A species of small reddish caterpillar found feeding on the spores, April 30, 1893. Uredo stage on Viola rotundifolia or $V$. blanda, April 3o, 1893 (Nuttall, 906). On Viola striata, rostrata, villosa, and blanda (Sheldon, 1902, 4200; 1394; 23; 3060, 3478).

P. Convolvuli (Pers.) Cast.

On Convolvulus repens. Aecidium May 3, Uredo June I4, Teleuto, Oct. 4, I894; all on same vine (Nuttall, I5I6). On Convolvulus Sepium (Sheldon, 926, 2337, 2657, 3158).

P. Convolvuli — var.

On dead stems of Convolvulus repens, Feb. I9, 1895 Nuttall, I793).

P. Pimpinellae (Strauss) Link.

On living leaves Osmorrhiza Clavtonii, Monongalia Co. near Morgantown (Millspaugh). On Washingtonia longistylis (Sheldon, 36).

P. Menthae Pers.

On Cunila origanoides, Nov. 27, I894. Teleutospores 28 x $20 \mu$, not yet verruculose (Nuttall, I753). On Monarda didyma; Mentha spicata; Clinopodium vulgare; Koellia verticillata; Monarda media; $M$. fistulosa; Blephilia hirsuta; and Koellia mutica (Sheldon, 2434; 3804; II95; II24; I039; I860; I785; I839).

P. Coronata Corda.

On Holcus lanatus, Uredo. June 30, 1893; teleuto. Nov. I, I893, on same specimen. Length of pedicel 5 to $7.5 \mu$ very thick, corona $+38 \mu$ broad, teeth $\mathrm{I} 2.5 \mu$, teleutospores 50 × I5 $\mu$ (Nuttall, 1265 ). On Avena sativa (Sheldon. 32, $747,1852)$.

P. Rurigo-vera (DC.) Wint.

On leaves Triticum vlugare, Wood Co., near Kanawha Station (Millspaugh). On Elymus hirsutiglumis and on Secale cereale (Sheldon, 3I98; I607, I7II).

P. CARICIS (Schum.) Reb.

On Carex sp. July Io, I894, uredospores and teleutospores (Nuttall, I 590).

•P. Hieracir (Schum.) Mart.

P. flosculosorum Roehl. On living leaves Carduus lanceolatus, Mason Co., near Point Pleasant (Millspaugh). On Taraxacum Taraxacum, Oct. 20, I894 (Nuttall, I72I).

P. suaveolens (Pers.) Rostr.

On living leaves Carduus lanceolatus, Wood Co., near Kanawha Station (Millspaugh). 
P. Sorghi Schwein.

Uredo, and teleutospores on Zea Mays, Sept. 7, I894 (Nuttall, I676).

P. Conoclinir Seymour.

On Eupatorium coelestinum, Sept. I8, I895 (Nuttall. I849).

P. Anemones-Virginiana Sz.

On Anemone Virginiana, Sept. I8, I895 (Nuttall, 1850).

P. Heucherae Sz.

P. Spreta Pk., P. Tiarella B. \& C. On Tiarella cordifolia. Oct. 2I, I895 (Nuttall, I886).

P. Smilacis Schwein.

Uredospores on Smilax glauca, Oct. I4, I894 (Nuttall, I708).

P. Sambuci (Schw.) Arthur.

On Sambucus Canadensis, Carex Asa-Grayi and C. Frankii (Sheldon, 40, 639, I479; 2073; 739, I264).

P. Saxifragae Schlect.

On Saxifraga Virginiensis (Sheldon 3).

P. Veratri Niessl.

On Veratrum viride (Sheldon, I499).

P. Vernoniae Schw.

On Vernonia sp. (Sheldon, 33, 3183).

P. Windsoriae Schw.

On Sieglingia sesleroides (Sheldon, 1959, 2194, 2754).

P. Xanthil Schw.

On Ambrosia trifida, A. artemisiaefolia and Xanthium. Canadense (Sheldon, 731; 734; 792, 1022).

P. malvacearum Mont.

On Malva rotundifolia and Althea rosea (Sheldon, I344, I6I I, 2215; I365, I406, 2123). On Malva sylvestris (F. E. Brooks, 3202).

P. Mariae-Wilsoni Clinton.

On Claytonia Virginica (Sheldon, I 309).

P. Muhlenbergiae A. \& H.

On Muhlenbergia diffusa and M. mexicanus (Sheldon, I267, 2012; 4199).

P. oBscura Schroet.

On Juncoides campestre (Sheldon).

P. ALBIPERIDIA Arthur.

On Ribes cynosbati (Johnson, 4298).

P. orbicula P. \& C.

On Prenanthes altissima (Sheldon, 2498).

P. PERminuta Arthur.

On Agrostis perinnans, Pocahontas, near Durbin (Kellerman). 
P. Poarum Niessl.

On Poa pratensis (Sheldon, I3I9).

SYNCHYTRIUM DeBy. \& Wor.

S. DECIPIENS Farlow.

On Falcata comosa (Sheldon, 655, I384, 1438, 335I).

\section{PUCCINIASTRUM Otth.}

P. Hydrangeae (B. \& C.) Arth.

On Hydrangea arborescens. Arthur, North Am. Flora, 7 :106. (Sheldon, 4, 2130).

P. Agrimoniae (Schw.) Tranz.

On Agrimonia mollis and parvitlora. Arthur, North Am. Flora, 7 :Io6. (Sheldon, 771, I016, 2122, and $A$. parviflora 2138). On Agrimonia gyrosepala (Sheldon, 3855).

P. minimum (Schw.) Arth.

On Menziesia pilosa. Arthur, North Am. Flora, 7 :Iog.

P. pustulatum (Pers.) Dietl.

On Epilobium angustifolium and E. coloratum (Sheldon, $2430,3803,3850)$.

GYMNOSPORANGIUM, Hedw.

G. Clavarifforme (Jacq.) Rees.

Aecidiospores on Crataegus sp., Aug. 2, I894 (Nuttall, 1637). On various species of Crataegus (Sheldon, I107, 27, I774, I023, 2021, 1096, 21 19; Thatcher, I714; Grout, I707; F. E. Brooks, I427).

G. Juniperinum (Linn.) Fr.

Teleutospores on Juniperus Virginiana, April 27, I894 ( Nuttall, I473).

G. JUniperi-Virginianae Schw.

(G. macropus Link).

Mercer: near Princeton, on Juniperus Virginiana (Millspaugh), in other localities on same (Sheldon). Aecidia on Malus coronaria at Fayette: near Nuttallburg (Nuttall, II57). On twigs, leaves and fruit of Malus Malus and $M$ coronaria (Sheldon). Berkeley: on Malus Malus at Gerrardstown (Waite).

G. CORNICULANS Kern.

On Amelanchier Canadensis, Greenbrier : near Fort Spring (Sheldon).

G. GLobosum Farl.

On Crataegus macracantha and punctata, and on Juniperus Virginiana. Arthur, North Am. Flora, 7 :205-6 (Sheldon 2707, F. E. Brooks 2298).

G. Nidus-Avis Thaxter

On Amelanchier Canadensis (Sheldon 2699, 2717). 
G. Germinale (Schw.) Kern.

On fruits of Crataegus punctata from Spruce Knob, Pendleton County, (Greenman, 257).

PHRAGMIDIUM Link.

P. Potentillae (Pers.) Karst.

On Potentilla Canadensis, uredospores, Monongalia Co., near Morgantown (Millspaugh). On P. Canadensis, uredo. March I 5, I894, teleutospores, Oct. 5, I894 (Nuttall, I400?).

P. SUBCORTICIUM (Schrank.) Wint.

On Rosa humilis, June I8, I893 (Nuttall, I008). On Rosa sp. (Sheldon 420I).

P. Rubi-odorati Dietl.

On Rubus odoratus. Arthur, North Am. Flora, $7: 166$. (Grout.125I).

P. AMERICANUM Dietl.

On a cultivated Rose. Arthur, Nortli Am. Flora, 7:167. P. Disciflorum (Tode) J. F. James.

On a cultivated Rose. Arthur, North Am. Flora, $7: 1$;2. (Sheldon 1991, 2135, 2139; F. E. Brooks 6).

\section{KUEHNEOLA Magn.}

K. Oztusa (Strauss) Arthur.

On Potentilla Canadensis. Arthur, North Am. Flora, 7 : 185 .

K. Uredinis (Link) Arthur.

On Rubus Allegheniensis and Canadensis, Arthur, North Am. Flora, 7:186. On Rubus hispidus (Sheldon 3476, 3829). On $R$. Canadensis $1250,1258,2435,2762,3835$. On R. strigosus (Shimer 2740).

\section{COLEOSPORIUM Lev.}

C. Senecionis (Schum.) Fr.

On living leaves of Pinus echinata (æcidia), Wood Co., near Lockhart's Run (Millspaugh).

C. Sonchi (Pers.) Lev.

On living leaves Vernonia Noveboracense, Mason Co., near Point Pleasant (Millspaugh). On Aster cordifolius, Dec. 3, I894 (Nuttall, i760).

C. Impomoeae (Schwein) Burrill.

On Ipomoea pandurata, Oct. I5, I894. Spores $85 \times 23 \mu$ (Nuttall, I720, 608).

C. Vernoniae B. \& C.

On Vernonia Noveboracense at Rupert's, Sept. 25, 1894. Spores $100 \times 30 \mu$ (Nuttall, I702, 595). 
C. Campanulae (Pers.) Lev.

On Campanula Americana.. Arthur, North Am. Flora, 7 :89. (Sheldon 2954, Grout I700, Post I796). .

C. Helianthi (Schw.) Arthur.

On Helianthus sp. (Sheldon, 3184).

C. Solidagrnis (Schwein.) Thum.

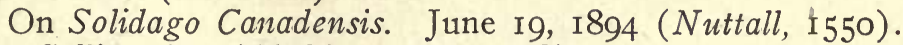
On Callistephus (Sheldon, 2024, 2028).

\section{AECIDIUM Pers.}

Ae. Impatientis Schwein.

On Impatiens biflora, June 21, I894 (Nuttall, I558).

Ae. Ludwigiae E. \& E.

On Ludwigia alternifolia (Sheldon, 2344).

Ae. Solidaginis Schw.

On Solidago lanceolata (Sheldon, I840).

Ae. Ilicinum E. \& E.? Bull. Torr. Club, $24: 284$. (I897).

Spots amphigenous, purplish-black above, dirty-orange below, indefinite. Aecidia hypophyllous, mostly only one in the center of a spot, hemispherical or tuberculiform and closed at first, then laciniately dehiscent and margin coarsely toothed; æcidiospores irregular in shape, globose, ovate, elliptical or subangular, I8-22 $\mu$ in the longer diameter, epispore more or less wrinkled or tuberculate-roughened.

On living leaves of Ilex opaca. Fayette: near Nuttallburg, April, I896 (Nuttall, 839).

\section{PERIDERMIUM Lev.}

P. PeckII Thum.

On living leaves Tsuga Canadensis, Pocahontas Co., near Traveler's Repose (Millspaugh).

P. Balsameum Peck.

Under surface living leaves Abies balsamea, Randolph Co., Shades-of-Death (Millspangh).

U. Agrimoniae (DC.) Schroet.

\section{UREDO Pers.}

On Agrimonia striata, July Io, I894 (Nuttall, I591).

U. Bigelowi (Thum.) Arthur.

On Salix nigra. Arthur, North Am. Flora, 7 :Ior.

U. Medusae (Thum.) Arthur.

On Populus grandidentata (Fenton, 4255). On P. deltoides and candicans (Sheldon, 2747, 2746). 
GYMNOCONIA Lagerh.

G. INTERSTITIALIS (Schlecht) Lagerh.

(Caeoma nitens).

On Rubus occidentalis (Sheldon, 25, 502). On R. procumbens (24), on $R$. Canadensis (48, 2433). On living leaves Rubus hispidus, Monongalia Co., near Morgantown (Millspaugh). On Rubus villosus, May 2, I893 (Nuttall, 907).

RAVENELIA Berk.

R. ePIPHylla (Schw.) Dietl.

On Cracca Virginiana. Arthur, North Am. Flora, 7 :142.

PILEOLARIA Cast.

P. ToxicodendRI (Berk. \& Rav.) Arthur.

On Rhus radicans. Arthur, North Am. Flora, 7:I48.

TRANZSCHELIA Arthur.

T. punctata (Pers $)$ ) Arthur.

On Hepatica acutá. Arthur, North Am. Flora, 7:I5I.

POLYTHELIS Arthur.

P. FUSCA (Pers.) Arth. (Puccinia Anemones Pers.)

On Anemone quinquefolia, Randolph: at Sugar Creek (A. B. Brooks).

PER I S P O R A CE AE.

PODOSPHAERA Kunze.

P. Oxyacanthae (DC.) DeBy.

On living leaves of Prunus cult., Crataegus oxyacantha and Diospyros Virginiana, Monogalia Co., near Morgantown (Millspangh). On Prunus serotina and Crataegus, Monongalia, near Morgantown; Preston, near Cranesville; and Berkeley, near Martinsburg (Sheldon). On Cydonia vulgaris and Spiraea salicifolia (Sheldon, 1085, 1845).

P. Leucotricha (E. \& E.) Salm.

On Malus Malus (Sheldon, 2755, 2758).

P. tridactyla (Wallr.) DeBy.

On living leaves of Prunus cult., Cabell Co., near Huntington (Millspaugh).

SPHAEROTHECA Lev.

S. Humuli (DC.) Burrill.

On. living leaves Agrimonia striata, Preston Co., near Terra Alta (Millspaugh). 
S. Humuli fuliginea (Schlecht.) Salmon.

On Erechthites hieracifolia and Bidens frondosa (Sheldon, II98, 3138).

j. PANNOSA (Waller) Lev.

Monongalia, near Morgantown, on Crimson Rambler rose (Starcher, 3200).

5. Mors-Uvae (Schw.) B. \& C.

On cultivated Gooseberry (F. E. Brooks, 3288).

ذ. Castagnei Lev.

On living leaves Erechtites hieracifolia, Nov. II, I894. Asci $78 \times 58 \mu$ spores $+20 \times$ I $2 \mu$ (Nuttall, I740).

S. Mali (DuBy.) E. \& E.

Berkeley: on Malus Malus at Gerrardstown (Waite).

PHYLLACTINIA Lev.

P. CORYlea (Pers.) Karst.

(P. suffulta Sacc.).

On living leaves Castanea dentata, Oct., I893 (Nuttall I3I5), and on Magnolia Fraseri. On Polycodium stamineum, Greenbrier, at White Sulphur Springs (Waite).

U. Ampelopsidis Peck.

\section{UNCINULA Lev.}

On cultivated grapes, Monongalia: near Morgantown (Millspaugh). On leaves of Parthenocissus quinquefolia, Fayette: near Nuttallburg (Nuttall, I683).

U. MACROSPORA Peck.

On Ulmus Americana, Greenbrier: near Ronceverte; and Mason; near Point Pleasant (Sheldon, II39, I935). On juglans nigra; Syringa vulgaris; Euonymus atropurpureus; Betula sp.; and Viburnum sp. (Sheldon, 3144; 956, 2010; 742,3135 ; 1225; 1083).

U. GENICULATA Gerard.

On Morus rubra, Monongalia: near Morgantown (Sheidon, $3487)$.

U. PARVula Ck. \& Peck.

On Celtis, Berkeley: near Martinsburg (Sheldon, 1986).

U. CIRCINATA Ck. \& Peck.

On Acer saccharum, Monongalia: near Morgantown (Grout, I26I).

U. NECATOR (Schw.) Burr.

(U. Americana Howe).

On leaves of Vitis cordifolia, Fayette: near Nuttallburg (Nuttall, i684). On Vitis sps. (Sheldon, 61, 953, 21 10, 2II4, 2206, 3I3I).

MICROSPHAERA Lev.

M. Alni (Wallr.) Salmon.

(M. erineophila $\mathrm{Pk}$.)

On leaves of Castanea dentata, Fayette: near Nuttallburg 
(Nuttall, I69, I218). On leaves of Fagus, same location and collector (I773). On Betula, Monongalia: at Cheat View; and on Quercus, Preston: at Rohr (Sheldon, I223, I224).

M. Grossulariae (Wallr.) Lev.

On leaves Sambucus Canadensis, Fayette: near Nuttallburg (Nuttall, 1693). On same Preston: near Cranesville (Sheldon).

M. elevata Burr. and Bess.

On leaves of Catalpa Catalpa, alt. 2,200 ft. Oct. 2, I894 (Nuttall, I69o).

M. Russellit Clint.

On Oxalis stricta, Aug. 9, I694 (Nuttall, 1650).

M. VaccinniI Schw.

On Epigaea repens, Oct. I893. Threads slender, sharply pointed, somewhat rough, asci 5 -spored, 50 to $65 \times 30$ to 40 $\mu$, spores I8 to $20 \times$ IO to I I $\mu$ (Nuttall, I255).

M. Quercina (Schw.) Burr.

On Quercus palustris, at Rupert's, Aug. 26, I894 (Nuttall, I686, 59I).

M. Alni-Vaccinit.

On Vaćcinium sps. (Sheldon, 1222, I226, 2015, 2684).

M. DIFFusA Ck. \& Pk.

On Symphoricarpos vulgaris and Desmodium sp. (Shel. don, 2667, 3485).

M. Euphorbiae (Pk.) B. \& C.

On Tithymalopsis corollata (Sheldon, I I09, I946, 2030).

ERYSIPHE (Hedw.) DC.

E. Cichoracearum (DC.) Burr.

On Xanthium Canadense, Nov. I, I893 (Nuttall, I223), and on Eupatorium purpureum.. On cultivated Dahlia; on Phlox paniculata; Taraxacum sp.; Achillea millefolium; Aster sps.; Verbesina alternifolia; Vernonia sp.; Plantago sp. ; Ambrosia trifida and artemesiaefolia; Lappula Virginianum; Helianthus annuus and Eupatorium perfoliatum (Sheldon, 4254; 3529, 3532, I652, 21 I8; 3482; 31 34; 3488; 3I45; 353I ; II4I, 3I25, 3I4I ; 59; II74, 2248, 3I72, 3483; 723; 31 33; 56; I967; 1257).

E. Communis (Wall.) Fr.

On Eupatorium ageratoides, Oct. I4, I894 (Nuttall, I707). E. Graminis DC.

On living leaves Poa pratensis, Preston Co., near Terra Alta (Millspaugh). On Triticum vulgare; Dactylis glomerata; Secale cereale and Poa compressa (Sheldon, 3002; I638; 1606; 3224). 
E. SP.

Upshur: near Buckhannon, on Micrampelis lobata, Sept. I2, 1905 (Sheldon). There is a strong probability of this being E. Polygoni upon a peculiar host!

E. LIRIODENDRI Schw.

On Liriodendron Tulipifera in deep shade, Short Creek, alt. I, I50 ft., Oct. 9, I894 (Nuttall, I697).

E. AGgregata (Peck) Farlow.

On Alnus, Greenbrier: near Durbin (Sheldon, ir6o, 3787).

E. Polygoni DC.

On Liriodendron tulipifera, Monongalia, near Morgantown (Sheldon).

The following are in the Sheldon Herbarium and collected by him unless otherwise indicated: On Onagra biennis (3533), Trifolium procumbens $(3253,3268$, Starcher 3218), $T$. pratense (3522), T. hybridum (3534), T. stoloniferum (35I3), Cuphaea viscosissima (Grose 348I ; Johnson 2233), Brassica napus (3523), Pisum sativum (3070, E. E. Broolis I649), Anemone sp. (Grout 1262), Falcata comosa (308I), Ranunculus abortivus (1202), and Polygonum sps. (737, 935).

EUROTIUM Link.

E. HERBARIORUM (Wigg) Link.

On damp leaves Liriodendron Tulipifera neglected in plant press, Sept. I, I894 (Nuttall, I68I).

APIOSPORIUM Kuntze.

A. Pelliculosum (B. \& Rav.). (Capnodium B. \& Rav.).

Tucker: on Prunus sp. (Plum), at Parsons (Swisher, 23I4).

A. Leemingit E. \& E.

ASTERINA Lev.

On Galax aphylla, May 4, I894 (Nuttall, I493, 475).

DIMEROSPORIUM Fckl.

D. Galactis E. \& E.

On leaves of Galax apyhlla in conjunction with the last species above, May 4, I894 (Nuttall, I 576, 475).

D. Collinsir (Schw.) Thum.

On leaves Amalanchier Canadensis, Glade Creek, May 4, I894 (Nuttall, I490, 472). On Carpinus Caroliniana, Kegley, Mercer County, July 27, Igoo (Morris, 1078).

S. spongiosa (Schw.) Fr.

SCORIAS Fries.

On living Fagus atropunicea, at Rupert's July 26, I894. Spores 16 to $18 \times 5 \mu$ (Nuttall, I634). 


\section{ANTHOSTOMA Nitschke.}

A. Discincola (Schw.) Sacc.

On Crabapple, Monongalia: Morgantown (Sheldon).

\section{VALSA Fries.}

V. CERATOPHORA Tul.

On fire killed Hicoria minima, Oct. 17, I894. On Acer saccharinum, L. Asci $38 \times 5 \mu$, spores 7.5 to Io $\times 1.5$ to I.75 $\mu$ (Nuttall, I7II).

V. Diospyri E. \& E. Proc. Phil., Acad., 340 (1894).

Type habitat: On dead limbs Diospyros Virginiana, Dec. 10, I893 (Nuttall, discov. I308, 253).

Stroma consisting of the slightly blackened substance of the bark, convex, about $2 \mathrm{~mm}$. diameter, not circumscribed. Perithecia 4 to 10 in a stroma, subglobose, .25 to $.33 \mathrm{~mm}$. diameter, necks converging and united above in a small, black disk which perforates the pustuliform-elevated epidermis, but does not rise above it. Ostiola short, conic-cylindrical, with a smooth, round opening crowded and finally obliterating the disk. Asci clavate-lanceolate, p. sp. $25 \times 5 \mu$, 8 -spored, paraphysate. Sporidia allantoid, hyaline, 8 to Io $\mathrm{v}$ I.5 to $2 \mu$. Spermogonia (Cytispora), stroma multilocular gray inside, opening by a single, central pore. Spermatia allantoid, hyaline, moderately curved, 4 to $5 \times 1.25 \mu$.

V. etherialis E. \& E. Proc. Phila. Acad., 34I (I894).

Type habitat: On dead limbs of Acer rubrum, Feb., I894 (Nuttall, discov. I304, 373).

Stromata cortical, thickly scattered, convex I to $1.5 \mathrm{~mm}$. diameter. Perithecia 6 to I2 together, circinate, buried in the unaltered substance of the bark, small, I50 to $250 \mu$ diameter their short necks terminating in an erumpent, compact fascicle of obtuse, black, slightly umbilicate ostiola closely embraced by the epidermis and scarcely rising above it. Asci (p. sp.) fusoid, 15 to $22 \times 4$ to $4.5 \mu$, stipitate, 8spored. Spordia biseriate, allantoid, hyaline, curved, slender, 5 to $6 \times$ about I $\mu$. When well developed, the epidermis is raised into subdiscoid pustules in which the slight protuberances indicate the position of the subjacent perithecia.

$V$. delicatula C. \& E. has fewer, larger perithecia and broader sporidia. $V$. microstora Cke. \& Plowr. has also larger perithecia and yellowish sporidia, and the ostiola are more or less distinctly sulcate, indicating its close relationship to Eutypella. In $V$. etherialis the spordia both in and out of the asci are perfectly hyaline. 
V. CORONAta (Hoffm.) Fr.

On young, fire-killed Castanea dentata, Nov. I4, 1893 (Nuttall, I263, 209).

V. albopuncta E. \& E., Field Mus. Bot., I :I33 (I896).

Type habitat: On dead limbs Liriodendron Tulipifera, Oct., 1894 (Nuttall, discov. 1712, 600).

Stromato minute (.5 to $7.5 \mathrm{~mm}$.), subseriate, included in the thick epidermis and not penetrating the inner bark, white throughout, and without any distinct circumscribing line. Perithecia 3 to 6 in a stroma, pale slate-color, I IO to I $30 \mu$ diameter; necks very slender, terminating in minute, subglobose, slate-colored ostiola taridly erumpent around the margin of the minute, snow-white, pulverulent disk. Asci clavate cylindrical, $50 \times 6 \mu$, short-stipitate, obtuse above, paraphysate? Spordia biseriate, allantoid, hyaline, moderately curved, 6 to $9 \times \mathrm{r} .5$ to $2 \mu$.

A beautiful species. The lines of snow-white disks, which barely perforate the ruptured epidermis and are closely surrounded by its torn and slightly raised margin, present a very neat appearance.

V. Abietis Fr.

On Tsuga Canadensis Short Creek, alt. I, I00 ft., Feb. 2, I893 (Nuttall, I349).

V. Vitis (Schw.) Fck1.

On Vitis sp., March 26, I894 (Nuttall, I432).

V. PRAESTANS B. \& C.

On dead twigs Nyssa aquatica, Nov. I4, 1893 (Nuttall, 1247). Spores Io to $12 \times 3$ to $4 \mu$.

V. NySSAE Cooke.

On fire killed Nyssa aquatica, Nov. I4, I893 (Nuttall. I689).

V. Subclypeata C. \& Peck.

On young fire killed Sassafras Sassafras, Nov. 14, 1893 (Nuttall, 1262, 209).

V. Ambiens (Pers.) Fr.

On Asimina triloba, Cornus fiorida and Aralia spinısa, Feb. I8, I894 (Nuttall, I383).

V. Chionanthi E. \& E., Proc., Phila. Acad., 340 (I894).

Type habitat: On dead limbs of Chionanthus Virginica, March, I894 (Nuttall, discov. I456, 228).

Perithecia 4 to Io, globose, .25 to $.33 \mathrm{~mm}$. diameter, buried in the unchanged substance of the bark, with convergent necks, terminating in short-cylindrical, obtuse, perforated ostiola erumpent in a close fascicle perforating and slightly raising the bark. Asci clavate, p. sp. 40 to $45 \times 8$ to Io $\mu$, 8-spored, paraphysate? Spordia allantoid, hyaline, 12 to 15 $\mathrm{x} 3.5$ to $4.5 \mu$, biseriate above. 
Spermogonia (Cytispora Chionanthi E. \& E.) buried in the bark, flask-shaped, .5 to $.75 \mu$ diameter, multilocular, the cells soon confluent, the apex erumpent and perforated by a single pore. Sporules allantoid, 4 to $6 \times \mathrm{I}$ to $\mathrm{I} .25 \mu$, borne on basidia branched above, the branches erect, straight, nucleate, 7 to io $\mu$ long.

V. PAUperata C. \& E.

On Acer rubrum, March 26, I894 (Nuttall, I444, 423).

V. Leucostoma (Pers.) Fr.

On peach tree, Dec. 2, I894 (Nuttall, I768).

V. Linderae Peck.

On Benzoin Benzoin, April I I, I895 (Nuttall, I796).

\section{EUTYPELLA Nits.}

E. densissima E. \& E., Proc. Phila. Acad., 34I (I894).

Type habitat: On dead limbs Aralia spinosa, Feb., I894 (Nuttall, 363).

Stromata scattered, cortical, depressed-conical, 2 to $3 \mathrm{~mm}$. diameter, not circumscribed, but staining the bark olive-gray. Perithecia numerous, often 50 to 70 in a stroma, 100 to 120 $\mu$ diameter, closely packed, their slender necks terminating in obtusely conical, 4 cleft, black, densely crowded ostiola erumpent in a brown disk surrounded by the ruptured epidermis. The disk is soon obliterated, so that only the crowded, black, subshining ostiola are seen. Asci clavatefusoid, p. sp. 25 to $30 \times 4 \mu$. Sporidia biseriate, allantoid, hyaline, moderately curved, 8 to Io X I.5 to $2 \mu$.

E. RUGiella (C. \& E.) Sacc.

On Acer rubrum, May 4, I893 (Nuttall, 921).

E. Stellulata (Fr.) Sacc.

On Robinia Pseudacacia, March 29, I893 (Nuttall, 33). On Ailanthus, Monongalia: near Morgantown (Sheldon).

E. Platini Sz.

On Platanus occidentalis in drift. Spores 7.5 to $8 \times 1.5$ to $2 \mu$, Oct. $2 \mathrm{I}, \mathrm{I} 895$ (Nuttall, I885).

\section{EUTYPA Tul.}

E. spinosa (Pers.) Tul.

On dry dead logs, June 29, r893 (Nuttall, II05).

E. imilliaria (Fr.) Sacc.

On river drift wood, April 20, I894. Asci I $50 \times 6 \mu$ spores Io $\mathrm{x} 2 \mu$ (Nuttall, I477).

DIATRYPE Fr.

D. Stigma (Hoffm.) Fr.

On Castanea dentata, April I5, I895 (Nuttall, I799). 
D. platystoma (Schw.) Berk.

On Magnolia Fraseri, Feb. 8, 1894 (Nuttall, 1380, 342).

D. VIrescens (Schw.) Cooke.

On Quercus, Monongalia: near Morgantown (Sheldon).

\section{DIATRYPELLA C. \& DeNot.}

D. VERRUCIFORMIS (Ehrh.) Nits.

On Alnus rugosa, Nov. 20, 1893 (Nuttall, I258).

\section{ROSELLINIA DeNot.}

R. AQUila (Fr.) DeNot.

On dead limbs, March .21, I893 (Nuttall, 919, I9).

R. CORTICUm (Schw). Sacc.

On dead oak, alt. r,800 ft., Short Creek. Spores 25 to $30 \mathrm{x}$ io to $12.5 \mu$. Dec. I6, I893 (Nuttall, I3IO).

R. Subiculata (Schw.) Sacc.

On dead dry Robinia Pseudacacia, July 15, I893. Perithecia clustered or connate, each 3 to $5 \mu$, black, ostiola papillate. Spores 9 to $I 2.5 \times 5$ to $6 \mu$ somewhat inequilateral, elliptical, colored (Nuttall, I I29).

R. PUlveracea (Ehrh.) Fckl.

On dead wood, Nov. I893. Spores Io to $15 \times 7$ to $9 \mu$ (Nuttall, 1338). Monongalia, on hickory log near Uffington; on Platanus near Marilla (Sheldon).

R. millegrana (Schw.) Sacc.

On dead Platanus occidentalis, Aug. 2, I893 (Nuttall, II6I).

R. ABIETINA TRICHOTA C. \& Ell.

On Pinus Virginiana, Jan. 7, 1895 (Nuttall, I784, 675).

\section{BOMBARDIA Fr.}

B. Fasciculata Fr.

On wet dead limb, Miagnolia Fraseri, on ground, Sept. I6, I895 (Nuttall, I830).

\section{ANTHOSTOMA Nits.}

\section{A. microecium E. \& E., Proc. Phila. Acad., 344 ( I894).}

Type habitat: On dead limbs Asimina triloba, Feb. I2, I894 (Nuttall, discov. I377).

Stroma cortical, faintly circumscribed, I to $1.5 \mathrm{~mm}$. diameter, orbicular, convex. Perithecia 4 to 8 in a stroma, globose, minute ( 200 to $250 \mu$ ), circinate, necks slender, short, converging, with the minute papilliform ostiola erumpent in a small, black, hemispherical disk, which barely pierces the pustuliform-elevated epidermis, and is closely embraced by it. Asci cylindrical, 80 to I Io $\times 8$ to Io $\mu$, paraphysate 
8-spored. Sporidia uniseriate, elliptical, brown, continuous, 2-nucleate, 12 to $14 \times 6$ to $7 \mu$.

Distinguished by its very small stroma and perithecia.

A. microplacum (B. \& C.) Sacc.

On Sassafras Sassafras, alt. I,800 ft., March 2I, I893 (Nuttall, 9I5).

\section{XYLARIA Hill.}

X. POlymorpha (Pers.) Grev.

On dead logs, April 7, i893 (Nuttall, 890).

X. CORNIFORMIS Fr.

On rotten limb, Sept. 9, I893. On Magnolia Fraseri, Nov., I895 (Nuttall, I I94).

X. Hypoxylon (Linn.) Grev.

On dead log, March Io, I893 (Nuttall, 807).

X. Flabelliformis (Schw.) B. \& C.

On Carpinus Caroliniana, May I3, 1893 (Nuttall, 957).

X. Cornu-Damae (Schw.) Berk.

On wet rotten log, alt. 2,000 ft., Aug. 24, I893. Spores 20 to 2 I $\times 4.5 \mu$ (Nuttall, II79).

\section{USTULINA Tul.}

U. vUlgaris Tul.

On dead and rotting stumps, March 8, I893 (Nuttall, 954).

\section{HYPOXYLON Bull.}

H. Atroviride E. \& E., Proc. Phila. Acad., 346 (I894).

Type habitat: On bark of dead tree, Betula or Quercus, Dec., I893 (Nuttall, discov. I320, 275).

.Stroma pulvinate, I to $\mathrm{I} .5 \mathrm{~cm}$. across, and about $4 \mathrm{~mm}$. thick, orbicular, covered above with a thin $(.5 \mathrm{~mm}$.) carbonaceous crust, which is soon covered by a dark green layer of the ejected spores, laterally and internally dirty-umber color. Perithecia (which constitute the entire inner substance of the stroma) ovate compressed, and including the long, stout neck, about $3 \mathrm{~mm}$. long and I $\mathrm{mm}$. broad below. Ostiola papilliform, soon covered and obscured by the ejected sporidia. Asci cylindrical Ioo x 3.5 to $4 \mu$ (p. sp. 40 to 45 long), paraphysate, 8 -spored. Sporidia uniseriate, oblong-elliptical, pale brown under the microscope; 2-nucleate, 4.5 to $5.5 \times 2 \mu$.

This is a well-marked species. The substance of the stroma, except the superficial carbonaceous layer, is friable. and in this respect as well as the color resembles $H$. Petersii B. \& C., from which, however, in other respects it is very distinct. 
H. Coccineum Bull.

On Aralia spinosa, alt. I,300 ft., Aug. I8, I893 (Nuttall, II73, I40).

H. Nuttallit E. \& E., Proc. Phila. Acad., 346 (I894).

Type HABItAT: On bark of dead Magnolia Fraseri at Glade Creek, May, I894 (Nuttall, discov. I494, 477).

Stromata gregarious, subconfluent, depressed-hemispherical or strongly convex, 3 to $6 \mathrm{~mm}$. diameter, purplishblack, mammillose. Perithecia small (about $.25 \mathrm{~mm}$.) scattered irregularly through the stroma, which is rather soft and brownish within. Ostiola crowning the mammillose projections on the surface of the stroma, papilliform, soon deciduous, leaving a round perforation. The asci in the spec. examined had disappeared. Sporidia brown, oblongelliptical, sub-inequilateral, 7 to $8 \times 3$ to $4 \mu$ (exceptionally $9 \times 5 \mu)$.

H. Howeianum Peck.

On decorticated Magnolia Fraseri, alt. I,800 ft., Sept. I2, I893 (Nuttall, I203, I 58).

H. Fuscum (Pers.) Fr.

On dead and decorticated Acer saccharum, Grant Co., near Bayard (Millspaugh). On dead Alnus rugosa, Oct. 3I, I893. Spores I $\times 6$ to $7 \mu$ (Nuttall, I22I).

H. MUlTiforme Fr.

On dead Betula sp., alt. I,800 ft., Short Creek, Aug. II, I893 (Nuttall, I I47).

H. perforatum (Schw.) Fr.

On dead twig, Feb. 3, I894. On Ilex opaca, Dec. 28, I894 (Nuttall, I35I). Spores Io x $5 \mu$. On Magnolia Fraseri, Nov., 1895 .

H. Rubiginosum (Pers.) Fr.

On dead hard wood of Robinia Pseudacacia, Feb. 25, I893 (Nuttall, 949, 39).

H. Sassafras (Schw.) Berk.

On Sassafras Sassafras, March 21, I893. Spores 8 to I2 x 3 to $4 \mu$ (Nuttall, 9r 7$)$.

H. Stigmateum Cooke.

On living Quercus sp., originating under the outer bark which it pushes off, or at times remains attached at onc edge and stands away like a lid. Asci, total length, I50 $\mathrm{x}$ Io $\mu$, fertile portion $\mathrm{I} 20 \mathrm{x}$ Iо $\mu$, spores black, elliptical, I6 to $20 \times 6$ to Io $\mu$. Feb. 2, I894 (Nuttall, I350).

H. Colliculosum (Schw.) Nits.

On Rhododendron maximum, alt. I,80o ft., Short Creek, Dec. I6, I893 (Nuttall, I330, 289). 
DALDINIA DeNot.

D. Concentrica (Bolt.) C. \& DeN.

On bark of dying Acer sp., April I5, I893 (Nuttall, 905).

On Betula, Monongalia, at Coopers Rock (Sheldon).

D. vernicosa (Schw.) C. \& DeN.

On dead trees, March, I895 (Nuttall, I790).

\section{NUMMULARIA Tul.}

N. Discreta (Schw.) E. \& E.

Berkeley, on Malus Malus at Gerrardstown (Waite).

N. punctulata (B. \& Rav.) Sacc.

On dead branch of Quercus sp., Aug. 16, 1893 (Nuttall, I I 5 I).

\section{GNOMONIA Ces. \& DeNot.}

G. Ulmea (Sacc.) Thum.

On dead leaves of Ulmus. Summers, near Lowell (Pollard \& Maxon, I3I). On Ulmus Americana, Wayne, near Ceredo (Sheldon).

\section{GNOMONIELLA Sacc.}

G. Coryli (Batsch.) Sacc.

On Corylus Americana at Rupert's, Aug. 5, I894 (Nuttall, I647).

G. Fimbriata (Pers.) Saccardo.

On Carpinus, Greenbrier, near Durbin (Sheldon).

\section{PHYSALOSPORA Niessl.}

P. Ilicis (Schw.) Sacc.

On dead leaves of Ilex opaca, July 21, I894. Asci 7.5 to Io $\mu$ (Nuttall, I6I6). Monongalia, near Morgantown (Sheldon).

P. Dracaenae Sheldon. don).

On Dracaena fragrans, cultivated at Morgantown (Shel-

TRICHOSPHAERIA Fckl.

T. PUlChriseta Peck

On dry firm splinters of dead log, alt. I,80o ft., Short Creek, March 6, I894 (Nuttall, I4I2, 382).

BOTRYOSPHAERIA Ces. \& DeNot.

B. Quercuum (Schw.) Sacc.

On Quercus sp., Vitis sp., Pinus Virginiana, Opulaster opulifolius, and Parthenocissus quinquefolia. Perithecia 2 to Io (Nuttall, 925, I4). 
B. Quercuum - var.

B. Araliae Curtis.

On Aralia spinosa, Feb. 24, 1894. Spores 20 to $30 \times 8$ to Io $\mu$ (Nuttall, I395).

B. PYRIOSPORA (Ellis) Sacc.

On Chionanthus Virginica, March 28, 1894. Asci, fertile portion, $75 \times 15 \mu$ (Nuttall, I438).

B. Hibisci forma.

On Celastrus scandens, April 27, I895 (Nuttall, I8I9, 707). Spores 26 to $35 \times 6$ to $7.5 \mu$.

C. compta (Tul.) Sacc.

On dead limbs of Fagus atropunicea, Nov. I7, I893. Asci, fertile portion, IOO $\mathrm{x}$ I 2.5 to I $5 \mu$, spores granular $22 \times$ Io $\mu$ (Nuttall, 1256).

SPHAERELLA Ces. \& DeNot.

S. MACUliformis (Pers.) Awd.

On leaves of Castanea dentata on ground, Dec. I3, I893 (Nuttall, I3I4).

S. infuscans E. \& E., Bull. Torr. Club, $25: 504$ ( I898).

Perithecia gregarious, minute, covered by the thin epidermis which is raised into minute pustules and blackened continuously for one or more $\mathrm{cm}$. in extent; ostiolum conicpapilliform, erumpent, asci oblong-cylindrical, 35 to $45 \mathrm{x}$ 7 to $8 \mu$, sporidia biseriate, fusoid, slightly curved, hyaline. nucleate, becoming faintly uniseptate, but scarcely constricted.

Differs from the description and figures of $S$. petiolicola (Desm.) in its longer fusoid sporidia.

On old petioles of Liriodendron tulipifera, Nuttallburg, May, I898 (Nuttall, 937).

S. CONICOLA Sacc.

On dead cones of Tsuga Canadensis, Dec. I6, I893. Spores IO $\times 3 \mu$ (Nuttall, I3I I).

S. Gaultheriae C. \& P.

On Gaultheria procumbens, June 8, I894 (Nuttall, I534).

S. NySSAECOLA Cooke?

On Nysa aquatica, Oct. Io, I894 (immature) (Nuttall, I719, 607).

DIDYMELLA Sacc.

D. Physocarpi E. \& E., Proc. Phila. Acad., I894, 335.

Type habitat: On Opulaster opulifolius, May i2, I894 (Nuttall, I507, 488). 
Perithecia gregarious, covered by the pustuliform epidermis, about .25 mm. diameter, white inside, depressed-globose, the papilliform ostiolum barely penetrating the epidermis. Asci clavate-cylindrical, short-stipitate, 85 to $90 \mathrm{x}$ IO to $\mathrm{I} 2$ $\mu$, paraphysate, 8-spored. Sporidia biseriate, fusoid, 4nucleate, uniseptate, constricted at the septum, hyaline, I9 to $22 \times 4$ to $5 \mu$, mostly a little curved.

M. MODONIA Tul.

\section{MELANCONIS Tul.}

On Castanea dentata, Nov. I4, I893 (Nuttall, I274, 221 ). M. Tiliacea (E11.) E. \& E.

On bark of Tilia, in company with Diplodia paraphysata E. \& E., Fayette, near Nuttallburg (Nuttall). Monongalia, at Morgantown (Sheldon).

HERCOSPORA Tul.

H. Tiliae (Fr.) Tul.

On Tilia heterophylla, Feb. I6, I894. Spores $18 \times 7.5 \mu$ (Nuttall, I834, 346).

\section{DIAPORTHE Nits.}

D. Gladioli E. \& E.?

On Caulophyllum thalictroides, July 8, I894. Asci $38 \times 5$ to $7 \mu$, spores septate, 4 nucleate, about Io $\times 3 \mu$ (Nuttall, I603, 553).

D. Araliae E. \& E., Proc. Phila. Acad., 339 (i894).

Type habitat: On Aralia spinosa, Feb. 2, I894 (Nuttall, discov. 1356,312$)$.

Stroma buried in the wood and circumscribed by a penetrating, black line, elliptical, about $5 \times 4 \mathrm{~mm}$. Perithecia buried in the stroma, few ( 6 to $\mathrm{so}$ ) globose, .5 to $.75 \mathrm{~mm}$. diameter, their short-cylindrical ostiola projecting from a black, tubercular disk seated on the surface of the wood and perforating the pustuliform-elevated epidermis, but scarcely rising above it. Asci clavate-cylindrical, 40 to $45 \times 5$ to $6 \mu$, paraphysate, 8-spored. Sporidia biseriate, oblong, 4-nucleate, becoming uniseptate and slightly constricted, hyaline, obtuse, I 2 to $13 \times 2.5$ to $3 \mu$.

Allied to and resembling D. ocularia C. \& E.

D. (Chorostate) Halesiae E. \& E., Proc. Phila. Acad., 339 ( 1894$)$.

Type habitat: On dead limbs of Mohrodendron Caro. linum, May, I894 (Nuttall, discov. I54I).

Perithecia 4 to 8 , loosely circinate. $.75 \mathrm{~mm}$. horn-color inside, becoming nearly black, sunk in the wood, necks converging with their obtuse, smooth, hemispherical ostiola 
erumpent in a close fascicle, closely surrounded by. the ruptured epidermis. Asci slender, 55 to $60 \times 6$ to $7 \mu$, shortstipitate. Sporidia sub-biseriate, fusoid-oblong, hyaline, 2 to 4-nucleate, becoming uniseptate and slightly constricted, straight, I 2 to $15 \times 2.5$ to $3 \mu$.

There is no distinct circumscribing line around the stroma, but the inner surface of the bark is uniformly blackened.

D. (Chorostate) Tetrapterae E. \& E., Proc. Phila. Acad., 339 ( 1894$)$.

Type habitat: On dead limbs Mohrodendron Carolinum, May. I894 (Nuttall, discov. I542).

Stroma cortical, 1.5 to $2 \mathrm{~mm}$. diameter, surrounded by a black line which does not penetrate the wood. Perithecia 4 to 12 , circinate, $5 \mathrm{~mm}$. diameter, sunk to the wood and leaving their impress on its surface but not penetrating it. Ostiola obtusely rounded and perforated, erumpent in a conlpact fascicle and closely surrounded by the ruptured epidermis. Asci clavate-cylindrical, 75 to $80 \times$ I $2 \mu$. Sporidia biseriate, oblong-fusoid, slightly curved, subobtuse, hyaline, uniseptate and constricted at the septum, each cell with a large nucleus, 9 to $22 \times 5$ to $7 \mu$.

Differs from $D$. Halesiae E. \& E., in its smaller perithecia, not sunk in the wood, and its much larger sporidia.

D. (Chorostate) Aceris Fckl.

On Acer saccharinum, L., Jan. 26, I894 (Nuttall, 1739).

D. Hydrangeae E. \& E., Field Mus. Bot., i :140 ( I896).

TyPe HABITAT: On dead stems Hydrangea arborescens, Nov., I894 (Nuttall, discov. 1756, 64I).

Perithecia scattered, ovate-globose, 350 to $380 \mu$ diameter, sunk in the wood, which is not at all discolored. Ostiola stout, erumpent, short-cylindrical, or conic-cylindrical, the apex papilliform. Asci (p. sp.) oblong-cylindrical, 5o to 60 x $8 \mu, 8$-spored, obscurely paraphysate. Sporidia biseriate. oblong-elliptical, hyaline, uniseptate, slightly constructed, I2 to $\mathrm{I} 5 \times 3$ to $4 \mu$, ends subacute.

This may be the Sphaeria spoken of by Schweinitz as found by him on limbs of Hydrangea, in company with his Sphaeria (Teichospora) Hydrangeae.

D. (Chorostate) cercophora.(Ellis.) Sacc.

On Ilex opaca, Dec. 23, I893, March I 5, I894 (Nuttall, I333).

D. (Chorostate) oncostoma (Du B.) Fckl.

On Robinia Pseudacacia, April 7, I894. Spores I7 to I9 $\mathrm{x}$ 4 to $5 \mu$ (Nuttall, I446).

D. (Chorostate) sociata (C. \& E.) Sacc.

On Benzoin Benzoin, April I3, I895. Helminthospores 75 to $100 \times$ I $5 \mu$. 7 -septate (Nuttall, I798). 
D. (Chorostate) obscura (Peck) Sacc.

On Rubus villosus, Feb. I3, I893 (Nuttall, I313, 260).

D. (Euporthe) aculeata (Schw.) Sacc.

On Phytolacca decandra, Nov. I4, I894. Asci 40 to $55 \mathrm{x}$ 6 to $7.5 \mu$. Spores 12.5 to $18 \times 3$ to $3.5 \mu$ (Nuttall, I754, $639)$.

D. (Tetrastaga) rostellata (Fr.) Nits.

On Rubus odoratus, May 31, 1894 (Nuttall, 1528, 560).

\section{AMPHISPHAERIA Ces. \& DeNot.}

A., Pinicola Rehm.

On Pinus rigida at Glade Creek, and on Pinus Virginiana. May 4, I894. Spores very variable +35 x $12 \mu$ (Nuttall, I499, 480).

VALSARIA Ces. \& DeNot.

V. exasperans (Ger.) Sacc.

On Juglans cinerea, Nov. 3, I893 (Nuttall, 1260, 202). On same, Monongalia, near Morgantown (Sheldon).

\section{MASSARIA DeNot.}

M. Magnoliae E. \& E., Field Mus. Bot., I :I4I ( I896).

Type habitat: On bark of dead limbs Magnolia acuminata, April 25, 1895 (Nuttall, discov. 1818, 700, 705).

Perithecia scattered, buried in the bark, depressed-globose, .75 to $\mathrm{I} .25 \mathrm{~mm}$. diameter, the subconical ostiolum piercing but not perceptibly raising the bark. Asci broad oblong, p. sp. I 50 to $200 \times 55$ to $65 \mu$ with a very short, nodular stipe, 8-spored, paraphysate. Sporidia crowded-biseriate, oblong or clavate-oblong, 3 to 5-septate, mostly 3-septate, when young, hyaline at first, finally deep brown and then with only one distinct septum, though often I to 4 other faint septa can be seen. There is a distinct constriction at the main septum, which is a little below the middle of the spore, but none at the other faint septa which are often wanting. The sporidia measure 65 to $80 \times 20$ to $25 \mu$, larger than in $M$. foedans (Tode), which has sporidia about $50 \times 20 \mu, 3^{-}$ septate and constricted at all the septa, besides being more distinctly narrowed below.

\section{LEPTOSPHAERIA (Pers.) DeNot.}

\section{Doliolum (Pers.) DeNot.}

On Polymnia Uvedalia and Helianthus decapetalus, Nov. 16, I894. Perithecia covered by outer bark. Asci, fertile part I IO $\times 6 \mu$, stipitate, spores overlapping at ends. Spores $22 \times 4 \mu, 23 \times 4.5 \mu, 25 \times 5 \mu, 27 \times 5 \mu, 30 \times 5 \mu$; 3 -septate, 
nucleate, constricted at septum when mature; straight, onesided, or curved (Nuttall, I744).

L. VAGABUnda Sacc.

On dead stems Hydrangea arborescens, Nov. 21, I894.

Asci 125 to I5O $\times 7.5 \mu$ total length (Nuttall, I745).

L. ogilviensis (B. \& Br.) Ces. \& DeNot.

On Cimicifuga racemosa, July 4, I894 (Nuttall, I586).

L. Clavigera (C. \& E.) Sacc.

On old stems Phytolacca decandra, Nov. I4, I894 (Nuttall, I74I). Spores $+43 \times 6 \mu$.

L. ORTHOGRAMmA (B. \& C.)

On Zea Mays, Aug. Io, I895 (Nuttall, 1832, 722).

\section{MELANOMMA Nits \& Fckl.}

M. Pulvis-pyrius (Pers.) FckI.

On decorticated Magnolia Fraseri, alt. I,80o ft., Short Creek, Sept. I0, I893. Spores I5 x $3.5 \mu$ (Nuttall, I200).

\section{TREMATOSPHAERIA Fckl.}

T. vitigena E. \& E., Field Mus. Bot., I :I42 ( I896).

Type habitat: On old, decaying wood of Vitis rupestris. April, I894 (Nuttall, discov. 454).

Perithecia gregarious, sunk in the wood all except the obtuse, convex apex, .5 to $.75 \mathrm{~mm}$. diameter, depressedglobose, the buried part rather thin-walled, the erumpent. convex apex thick, solid, like the stromatic shield of Clypeosphaeria. Ostiolum papilliform, soon perforated. Asci clavate-cylindrical, paraphysate, 8-spored, 86 to Io0 $x$ Io to I2 $\mu$. Sporidia sub-biseriate, fusoid-oblong, 3 -septate, and constricted, subacute, pale-brown, 20 to $25 \times 6$ to $7 \mu$.

PSEUDOVALSA Ces. \& DeNot.

P. SIgmoidea (C. \& E.)

On dead limbs Quercus Prinus. Spores 50 to $88 \times 5$ to $7 \mu$ (Nuttall, I828, 7I7).

\section{LASIOSPHAERIA Ces. \& DeNot}

L. ovina (Pers.) Ces. \& DeNot.

On Juglans cinerea, and parasitic on Hypoxylon, Short Creek, alt. I,800 ft., Dec. I6, I893. Spores 50 to $60 \times 6$ to $7.5 \mu$. Asci I35 x $12.5 \mu$ (Nuttall, I322).

\section{ACANTHOSTIGMA DeNot.}

A. decastylum (Cooke) Sacc.

On dead log, June i8, I893 (Nuttall, roI8). 
HERPOTRICHIA Fckl.

H. Diffusa (Schw.) E. \& E.

On dead twig, Sept. Io, I893 (Nuttall, II97). On Morus rubra, at Marilla (Sheldon).

\section{CALOSPORA Sacc.}

C. Aculeans (Schw.) Sacc.

On Rhus hirta and copallina, March 29, 1894. Spores I 5 to I7 $\times 3 \mu$, aculeate (Nuttall, I440).

C. Rhoina (C. \& Ell.) Sacc.

On Rhus hirta, Dec: 9, I893. Spores I5 to $18 \times 5$ ? $\mu$ hyaline, 4-nucleate, uniseptate constricted at septum. (Nuttall, 1348, 306).

PYRENOPHORA Fr.

P. Calvescens (Fr.) Sacc.?

On Chenopodium anthelminticum, Aug. Io, I895 (Nuttall. $1845,736)$.

\section{TEICHOSPORA Fckl.}

T. NITIDA E. \& E.

On Rubus villosus, Apr. 25, 1895. Asci 75 x 12? $\mu$. Spores 20 to $23 \times 9$ to $10 \mu$ (Nuttall, 1817, 706).

\section{CARYOSPORA DeNot.}

C. putaminum (Schw.) DeNot.

On Hickorynut, Monongalia, Morgantown (Sheldon).

\section{PLEOSPHAERIA Speg.}

P. Corticola E. \& E., Proc. Phila. Acad., i894, 332.

Type habitat: On bark of Pinus rigida, Glade Creek, June 24, I894 (Nuttall, discov. I 582).

Perithecia scattered, superficial, ovate, 300 to $400 \mu$ diameter, carbonaceo-membranaceous, pilose-strigose, hairs IoO to $200 \times 5$ to $6 \mu$ soon opaque, very faintly and sparingly septate, here and there collected into closely compacted fas. cicles resembling stout bristles. Ostiolum papilliform, obtuse. Asci oblong-cylindrical, abruptly short-stipitate, 80 to IOO $\times 20$ to $25 \mu$. Paraphysate? Sporidia crowded, acutely elliptical, nearly hyaline at first, becoming olive-brown and 5 to 7 septate and muriform, but not constricted.

Comes near P. strigosa Sacc., but perithecia rather smaller, not depressed, and hairs fasciculate.

P. - sp.

On Tecoma radicans, Feb. 20, I894 (Nuttall, I4I4, 385). 
CUCURBITARIA Gray.

C. Fraxini E. \& E.

On Fraximus Americana, Apr. 27, 1894 (Nuttall, 1523).

C. elongata (Fr.) Grev.

On Robinia Pseudacacia, Jan. I5, 1894 (Nuttall, I783).

OPHIOBOLUS Riess.

O. PORPhyrogonus (Tode) Sacc.

On Vernonia Noveboracensis, Apr. 24, I895 (Nuttall, I820, 702).

O. acuminatus (Sow.)

On Zea Mays, Aug. I0, 1895 (Nuttall, 1839, 728).

O. Fulgidus (C. \&.P.) Sacc.

On Vernonia Noveboracensis, Apr. 24, I895 (Nuttall, I82I, 703).

CRYPTOSPORA Tul.

C. Femoralis (Peck) Sacc.

On Almus rugosa, Feb. 25, I894. Asci 60 to $75 \times 12.5$ to $15 \mu$. Spores 50 to $65 \times 3.5 \mu$ in middle and enlarging to $5 \mu$ at each end (Nuttall, I3I8).

C. cinctula (C. \& P.) Sacc.?

On Castanea dentata, Dec. 8, I893. Asci about $75 \times 7.5$ $\mu$. Spores 60 to $75 \times 4 \mu$, hyaline, nucleate, curved, often slightly thickened toward one end (Nuttall, I296, 245).

C. tRICHOSPORA (C. \& P.) Sacc.?

On Quercus sp., Feb., I894. Spores + 50? $\mu$ (Nuttall. I450).

H Y P O R E A C E A E.

HYPOMYCES Fr.

H. Rosellus (Alb. \& Schw.) Tul.?

On Hymenocetum on Magnolia Fraseri, March 6, 1894 (Nuttall, I417, 387).

H. aurantius (Pers.) Tul.

On Stereum sp. Sept. 3, I893 (Nuttall, I I98). Short Creek, alt. I,800 ft.

\section{CREONECTRIA Seaver.}

(`. purpuren (L.) Seaver. (Nectria cinnabarina Fr.).

On Aralia spinosa, March 5, I894. Asci $75 \times 7.5 \mu$. Spores 20 to $23 \times 4$ to $5 \mu$ (Nuttall, I405, 374). On Acer saccharum, Monongalia: near Morgantown (Sheldon). 
C. coccinea (Pers.) Seaver. (Nectria coccinea Fr., ditissima Tul.).

On bark of dead Magnolia Fraseri, March, I894. Asci $65 \times 7.5 \mu$. Spores Io to I5 $\times 3.5$ to $6 \mu$ (Nuttall, I $472,45 \mathrm{I})$.

On dead and fallen Robinia Pseudacacia, on Magnolia Fraseri, Oct. I7, I893 (Nuttall, I I 5, I62).

\section{NECTRIA Fries.}

N. Peziza (Tode) Fries.

(N. vulpina E. \& E.).

On bark of dead Ulmus pubescens? Oct. I7, I894. Spores Iо $\mathrm{x} 5 \mu$, I-septate, 2-guttulate (Nuttall, I2I6, I64).

N. EPISPHAERIA (Tod.) Fr.

On dead fallen twigs, Aug. I5, I893 (Nuttall, II75, I43). On Fagus, Monongalia: near Morgantown (Sheldon).

\section{APONECTRIA Sacc.}

A. inaurata (B. \& Br.) Sacc.

On Rhus copallina, Mar. 24, I894 (Nuttall, I43I). Microspores $3 \times 1 \mu$. Spores Io to I $2.5 \times 3 \mu$.

\section{HYPOCREA Fr.}

II. LENTA (Tode) Berk. \& Br. (H. Schreinitzii Sacc.). On an Oak log. Boone: near Lick Run (Sheldon).

H. PATElla Cooke \& Peck.

On dead wood, Fayette: near Nuttallburg ( Nuttall, 75).

H. Virginiensis E. \& E. Proc. Phila. Acad., 442 (I893).

TyPe habitat: On leaves of Rhododendron maximum, Aug. I2, I893 (Nuttall, discov. I163).

Epiphyllous, stroma carnose, scattered, sub-hemispherical or depressed-turbinate, I to $2 \mathrm{~mm}$. diameter, of a yellowish gray color, subtruncate above and obscurely margined (when mature). Perithecia buried in the stroma, ovate, membranaceous, IIO to I5O $\mu$ diameter. Ostiola hemispherical, prominent, black, broadly perforated and sometimes collapsed. Asci clavate-cylindrical, p. sp. 40 to $45 \times 7$ to $8 \mu$, short-stipitate, filiform-paraphysate, 8 -spored. Sporidia biseriate, oblong-fusoid, yellowish-hyaline, uniseptate, slightly constricted at the septum, obtusely pointed at the ends, IO to I $2 \times 3$ to $3.5 \mu$.

$H$. viridans B. \& C. seems to differ in its hidden ostiola (ostiolis latitantibus).

This species is said by Seaver to be a discomycete.

H. tenerrima E. \& E., Proc. Phila. Acad., 442 (i893).

Type habitat: Incrusting moss, leaves, twigs, living Gaultheria procumbens, etc. July, I893 (Nuttall, discov. II38, I23). 
Perithecia gregarious, minute (.16 mm.), clothed (except the black apex) with a thin white tomentum, seated on a thin snow-white, tomentose-arachnoid subiculum. Asci cylindrical, $40 \times 3 \mu$, without paraphyses, 8-spored. Sporidia uniseriate, oblong, obtuse, hyaline, 5 to $6 \times 2 \mu$, with a single nucleus in the centre (becoming uniseptate)?

This is closely allied to $H$. subcarnea E. \& E., but differs in the color of the subiculum, the black apex of the perithecia, the narrower asci, and rather longer sporidia. Possibly Nectria Rexiana Ellis.

PODOSTROMA Karst.

P. alutaceum (Pers.) Atk.

On decaying organic matter on the ground. Seaver, North Am. Flora, $3: 36$.

HYPOCREOPSIS Karst.

H. LICHENOIDES (Tode) Seaver.

(H. riccioidea Karst.).

On Kalmia latifolia, alt. 2,000 ft., Aug. 24, I893. Spores apparently moniliform (Nuttall, I I78).

GIBBERELLA Sacc.

G. PUlicaris (Fries) Sacc.

(G. Saubinetii Sacc.).

On Asparagus officinalis, Nov. I, 1893 (Nuttall, I243, I90).

\section{SCOLEONECTRIA Seaver.}

S. Scoleosperma (Bref.) Seaver.

On Pinus Strobus. Monongalia: at Morgantown (Sheldon).

BROOMELLA Sacc.

B. Ravenelir (Berk.) Sacc.

On Acer rubrum, April 4, 1894 (Nuttall, I461, 439).

THYRONECTRIA Sacc.

T. DENigrata (Wint.) Seaver.

(Pleonectria Wint.).

On Gleditsia triacanthos, Monongalia: near Morgan. town (Sheldon).

T. Missouriensis (E. \& E.) Seaver. (Pleonectria E. \& E.). On dead stick, Fayette: near Nuttallburg (Nuttall, 87I).

\section{CORDYCEPS Fries.}

C. MiLITARIS (Linn.) Link.

In laurel thicket on ground, Aug. I2, I893, Keeney's Creek (Nuttall, I205, I6I). 
C. PARAsitica (Willd.) Seaver. (C. ophioglossoides Link). On ground in wet woods, Aug. 24, 1893 (Nuttall, I172).

\section{DOTH I E A C E E.}

\section{PHYLLACHORA Nits.}

I". Graminis (Pers.) Fckl.

On living leaves of Hystrix Hystrix, Fayette Co.: near Nuttallburg (Millspaugh). On Elymus Canadensis, Oct. 28 , I893 (Nuttall, I250, I92).

i. CARICIS (Fr.) Sacc.?

On Korycarpus diandrus, March I, I894 (immature) (Nuttall, I402, 238).

P. POMAGENA Sacc.

Berkeley, on Malus Malus at Gerrardstown (Waite).

$$
\text { DOTHIDELLA Speg. }
$$

L. Ulmae (Sz.) E. \& E.

On Ulmus pubescens, Nov. 5, I894 (Nuttall, 1749, 629)

\section{PLOWRIGHTIA Sacc.}

P. morbosa (Schw.) Sacc.

On limbs of cultivated Plum and Cherry, Monongalia Co., near Morgantown (Millspaugh). On Prunus serotina, Pocahontas: Cranberry Mountain (Sheldon).

$$
\text { M I CR O T H Y R I A CE A E. }
$$

MYICOPORON Speg.

M. Smilacis (De Not) Sacc.

On Smilax sp. April 12, I895 (Nuttall, I807).

$$
\text { LOPHIOSTOM A CEA E. }
$$

\section{LOPHIOTREMA Sacc.}

L. Nucula (Fr.) Sacc.

On dead heartwood of Liquidambar Styraciflua, April 20 , I894 (Nuttall, I479, 459).

\section{LOPHIDIUM Sacr.}

L. nitidum E. \& E., Proc. Phila. Acad., 333 (i894).

Type habitat: On Vitis cult. Feb. 2, 1894 (Nuttall, discov. I336, 295).

Perithecia scattered, semi-erumpent, small (200 to $250 \mu$ diameter) black and shining, subhemispherical, the flattened base immersed in the bark. Ostiolum compressed, thin, 
narrow, sometimes obsolete. Asci cylindrical, short ( 8 to Io $\mu$ ), stipitate, about $100 \times$ Io to I2 $\mu$, paraphysate, 8spored. Sporidia uniseriate, oblong-elliptical, yellow-brown, 5 to 7 -septate with a longitudinal septum running through two or more cells, I9 to $22 \times 8$ to Io $\mu$, ends mostly rounded and obtuse, not at all or only slightly constricted in the middle.

L. Compressum (Pers.) Sacc.

On Salix nigra falcata, April 23, I894. Asci 70 to 100 $\mathrm{x}$ I $3 \mu$. Spores 25 to $30 \times 8$ to Io $\mu$ (Nuttall, I48I, 46I).

\section{H E L V E L L A C A E.}

\section{MORCHELLA Dill.}

M. esculenta (Linn.) Pers.

Along an old road among grasses and leaves under'an oak. April 18, I893 (Nuttall, 897). (Sheldon, 2308, 2842, 3229).

M. ANGUSticeps Peck.

On ground, Short Creek, April 18, 1893. Spores 25 to $28 \times$ I $5 \mu$ (Nuttall, 895).

M.

On ground. Short Creek, $5 \mathrm{~cm}$. high, with smooth spores $28 \times$ I7 $\mu$, April I8, I893 (Nuttall, 896).

\section{GYROMITRA Fr.}

G. CURTIPES Fr.

On ground below cliffs, Short Creek. Asci, fertile part $50 \mathrm{x}$ I6 to $\mathrm{I} 8 \mu$. Total length $350 \mu$. Spores 22 to $30 \mathrm{x}$ Io $\mu$, 2-nucleate (Nuttall, I8I3).

\section{HELVELLA Linn.}

H. MACRopus (Pers.) Karst?

On ground in woods at base of cliffs, July I5, I893 (Nuttall, 1097).

H. CRISPA (Scop.) Fries.

(Sheldon, 2098).

M. Phalloides (Bull.) Chev.

\section{MITRULA Fr.}

In water on leaves of Quercus Primus, etc., spores to 20 x $3 \mu$. Masterson's Glade, June I I, I893 (Nuttall, 935). On decaying leaves in a gutter (Sheldon, 2923).

M. Lutescens B. \& C.

On ground and on dead logs in deep woods, alt. 2,000 ft. (Nuttall, I024). 
S. Clavata (Schæf.) Sacc.?

In woods among dead leaves, stipe dark brown, spores short $25 \mu$, July IO, I893 (Nuttall, 1087).

S. velutipes C. \& F.

Among mosses on a decaying log (Sheldon, 3437).

\section{VIBRISSEA Fr.}

V. TRUnCORUm (A. \& S.) Fr.

On wet dead sticks, alt. I,80o ft., June I5, r893 (Nuttall. 99I). On wood in running water (Sheldon, 2986, 2922).

\section{PEZ I Z A C A E.}

RHIZINA Fr.

R. Inflata (Schaef.) Quel.

On sandstone rock in sandy loam. Blackish-brown, spreading, 2 to $9 \mathrm{~cm}$. $x$ I to $3 \mathrm{~cm}$., convex, loosely attached to the soil by strong rootlets from all parts of the underside, margin strongly rolled inward. Asci 375 ? to $4 \mathrm{I} 5 \times \mathrm{I} 2.5 \mu$, spores hyaline, apiculate, large, $40 \mathrm{x}$ ro $\mu$. Keeney's Creek, Nov. 3, I893 (Nuttall, I224).

\section{GEOPYXIS Pers.}

G. Nebulosa Cooke.

On dead wet wood at low and high altitudes, Aug. Io, I893 (Nuttall, II77). On a decaying log (Sheldon, 2453, $2455,3425,3467)$.

G. CARBONARIA A. \& S.

On ground upon which logs had been burned the previous autumn, April 18, I893 (Nuttall, 908).

G. CUPULARIS Linn.

(Sheldon, 3469).

\section{PEZIZA Dill.}

P. aurantia Pers.

On wet and freshly crushed sandstone ballast, and on filling of sandstone cut on railroad. Spores curiously rough and apiculate at both ends. Nov. 3, I893 (Nuttall, 1225). On soil (Sheldon, 1239, 3239).

P. BADIA Pers.

On ground, Short Creek, April 18, 1893 (Nuttall, 922).

P. REPANDA Wahl.

On earth and decaying wood (Sheldon, 3519, 2909, 2844). P. vesiculosa Bull.

On earth in a greenhouse (Sheldon, $3 \mathrm{II} 7$ ). 


\section{OTIDEA Pers.}

O. ONOTICA OCHRACEA Fr.

On ground and on rotten Tsuga Canadensis, July I4, 1893. Asci I75 $\times$ io $\mu$ total length, spores I I to I $3 \times 7.5 \mu$, nuclei 2 (Nuttall, rog8).

\section{HUMARIA Fr.}

H. ECHinosPerma Peck.

In soil of ditch, July 20, I894 (Nuttall, 1630).

H. CESTRICA E. \& E.

On earth (Sheldon, 3104).

H. Gerardir Cke.

On earth in woods (Sheldon, 3468).

H. AVANEOSA Bull.?

On wood ashes (Sheldon, 2945).

\section{SARCOSCYPHA Fr.}

S. occidentalis (L. \& S.) Boud.

On various dead twigs and on soil (Sheldon, I4I9, 2987, 3250, 3013).

S. RUFUM (L. \& S.) Rehm.

On wood (Sheldon, 646).

S. coccinea Jacq.

On sticks, on ground in damp shady place, March 20, r893 (Nuttall, 852). On decaying wood (Sheldon, 2789, 283I).

S. FLOCCOSA Schw.

On a stick in a laurel thicket, alt. 2,000 ft., July I2, I893 (Nuttall, I I I2).

CIBORIA Fuckel.

C. Juglandis Preuss?

On hicknry shucks (Sheldon, 30I6).

PLICARIELLA Sacc.

P. Miniata (Crouan) Lindau.

On earth (Sheldon, 2796).

P. SCABRosa (Sacc.).

On decaying log (Sheldon, 2459).

(Phaeopeziza Sacc.).

\section{LACHNEA Fr,}

L. SCUTELlata, Linn.

On rotting log Fagus atropunicea and under bark of wet log Quercus alba, Monongalia Co., near Morgantown (Millspaugh). On rotten wood in old drift of mine, and on bare sandy ground among pebbles, Short Creek, May 
I I-I7, I893 (Nuttall, 936). On decaying wood, leaves, etc. (Sheldon, 557, I 556, 2061, 1042, 3364, 2888, 2449, 2887 , $337 \mathrm{I}, 2930$, I4I3).

L. UMBRARUM Fr.

On open bare sandy soil, Short Creek, May, I893 (Nuttall, IIO7).

L. HIRTA Schum.

On floor of cave, July 25, I893 (Nuttall, I I27).

L. vitellina Pers.

On dead log. Asci 235 x I5 $\mu$, Short Creek, July 2, I893 (Nuttall, III4).

L. erinacea (L. \& S.) Sacc.

On underside of log Qucrcus alba, Monongalia Co., near Morgantown (Millspaugh). On decaying logs (Sheldon. 2060, 3187, 3399, 3401).

\section{SCLEROTINIA Fuckel.}

S. FRUCTIGENA Rehm.

Monongalia: near Morgantown, on a fallen Peach (Sheldon, 2832). Conidial stage on Plum, Peach, Raspberry and Apple (Sheldon).

S. Tuberosa (Hedw.) Fckl.

On earth (Sheldon, 3179).

S. TRIFOLIORUM Eriks.

On ground in open woods, May 18, I893 (Nuttall, 960).

$$
\text { GEOGLOSSUM Pers. }
$$

G. GLABRUM Pers.

On soil (Sheldon, 2454).

\section{GORGONICEPS Karst.}

G. Guernisaci Sacc.

On decaying wood (Sheldon, 2866).

\section{HELOTIUM Fr.}

H. Lacteum E. \& E., Proc. Phila. Acad., I45 (I893).

On bare log, Dec. I6, I893. Pure white, stipitate, plane .4 to $.6 \mathrm{~mm}$. asci clavate 95 to $\mathrm{r}$ Io $\times 7.5 \mu$. Spores curved I6 to $25 \times 3$ to $4 \mu$, nuclei 4 , paraphyses slender, Short Creek near the falls (Nuttall, I3I8).

H. IAERBARUM (Pers.) Fr.

On Polymnia Uvedalia, Nov. I5, I894. Cups 6 to $12 \mathrm{~mm}$. diameter, stipes I to $2 \mathrm{~mm}$. long. Asci 55 to $75 \times 7.5$ to Io $\mu$. Spores 12 to $15 \times 2.5 \mu$ (Nuttall, I755).

H. Confluens Schw.

On dead logs, alt. I,800 ft. Oct. 20, I893 (Nuttall, I2I3). 
H. CITRINUm (Hedw.) Fr.

On wet dead log. Short Creek, alt. I,800 ft. Sept. I2, I893 (Nuttall, I20I).

H. castaneum Sacc.

On leaves of Rhododendron maximum. Short Creek, alt.

I,800 ft. Sept. I2, I893 (Nuttall, I206).

H. ephiphyllum (Pers.) Fr.

On decaying leaves, twigs, etc. (Sheldon, 3428).

H. PHyLlophilum Desm.

On decaying leaves (Sheldon, 3363).

H. Fructigenum Rehm.

On dead wood, leaves, chestnut bur and acorn (Sheldon, $3470)$.

H. FRATERNum Peck.

(Sheldon, 3377).

PHIALEA Fr.

P. scutula (Pers.) Gill.

On decaying herbaceous stems (Sheldon, 3514).

P. cyathoides (Bull.) Gill.

(Sheldon, 3544).

P. virgultorum (Vahl.) Sacc.

On old grape twigs on wet ground. Asci $105 \times 7.5 \mu$. Spores $18 \times 4 \mu$. Nov. I, I894 (Nuttall, I735). (Sheldon, I080).

\section{PSEUDOHELOTIUM Fckl.}

P. Fibrisedum (B. \& C.) Sacc.

On wet dead wood of Juglans cinerea, Dec. 5, I894. More than $2 \mathrm{~mm}$. diameter, margin ciliate, red-maculate; asci oblong short-stipitate, total length $65 \times 7.5 \mu$ (approx.). paraphyses filiform. Spores hyaline I-septate (?) oblong, straight or slightly curved, I2 to I8 × $3 \mu$ (Nuttall, I764).

\section{CYATHICULA De Not.}

C. QUisquiliaris E. \& E., Proc. Phila. Acad., 45I (I893).

Type habitat: On dead racemes of Quercus Primus, on dead leaves, on rock in woods, April 2, I893 (Nuttall, discov. 94I).

Gregarious, sessile, I to $1.5 \mathrm{~mm}$. diameter, cup-shaped, disk pale with a tinge of brick color when dry, dull, dirtywhite and purpuraceo-squamulose outside, margin distinctly cleft-toothed and incurved when dry. Asci cylindrical, subsessile, 55 to $65 \times 6$ to $7 \mu$, with filiform paraphyses scarcely thickened above. Sporidia uniseriate, or sub-biseriate above, elliptical, hyaline, continuous, 6 to $8 \times 3$ to $4 \mu$. 


\section{CHLOROSPLENIUM Fr.}

C. Aeruginosum (Oeder) De N.

On loose core inside dead tree, Nov. 6, 1893. Disk more brilliant than margin in light and when young, in shadow varying to pure white. Asci over $105 \times 7.5 \mu$ total length, spores guttate $12.5 \times 3 \mu$ (Nuttall, 1235). On decaying log (Sheldon, 2063).

C. VERSIFORME (Pers.) Karst.

On rotten mossy log, Short Creek, Oct. 28, I893 ( Nuttall. I238). On decaying log (Sheldon, 3045).

C. Schweinitzin Fr.

Top of oak log, Dec. I0, I893. Yellow in spring, green in autumn and winter (Nuttall, 1276).

M. Dehnir (Rabenh.) Karst.

On Potentilla Monspeliensis, July 21, I804 (Nuttall, 16r8).

M. Finastri (C. \& P.) Sacc.

On Pinus Virginiana, Dec. 3, I894 (Nuttall, I763).

M. Cinerea (Batsch.) Karst.

Peziza cinerea Batsch. On decayed log, Monongalia Co., near Morgantown (Millspaugh). On a decaying Black Walnut log (Sheldon, 3369).

PYRENOPFZIZA Fuckel.

P. lacerata (C. \& P.) Sacc.

On Rubus odoratus, May 3 I, I894. Asci 45 to $50 \times 5$ to $6 \mu$. Spores 8 to $12.5 \times 2$ to $2.5 \mu$ (Nuttall, I529). On Rubus odoratus (Sheldon, 549).

\section{PSEUDOPEZIZA Fuckel.}

P. Trifolir (Biv. \& Bernh.) Fck1.

On Trifolium pratense (Sheldon, 1596).

P. Medicaginis (Lib.) Sacc.

On Medicago sativa (Sheldon, 1605).

\section{TAPESIA Pers.}

T. SAnguinea (Pers.) Fckl.

On dead log, and on Magnolia Fraseri and Juglans cinerea, June Io, I894 (Nuttall, I 546).

T. FUsCA (Pers.) Fckl.

On rotten burned log Tsuga Canadensis; on dead rotting $\log$ Juglans cinera, alt. I,300 ft. Asci 45 to $92 \times 495 \mu$. Spores 8 to Io $\times 2 \mu$. Short Creek, Feb. 2, I894 (Nuttall, I 365$)$. 
T. subiculata (Schw.) Sacc.

On wet sticks, June I 5 and Oct. 25, I893. Spores large. Io $\times 3 \mu$ (Nuttall, 992).

\section{LACHNELLA Fr.}

L. Virginica E. \& E., Proc. Phila. Acad. 349 (i894).

Type habitat: On decaying wood of Magnolia Fraseri, Feb., I894 (Nuttall, discov. I410, 380).

Gregarious, sessile, .75 to $1.2 \mathrm{~mm}$. diameter. Disk pallid, concave, nearly closed when dry, margin fringed with brown septate hairs, IOO to I $25 \times 3 \mu$, outside clothed with shorter reddish-brown hairs. Asci about $50 \times 5 \mu$, clavate-sylindrical, short stipitate, paraphysate, 8 -spored. Sporidia sub-biseriate, oblong, 6 to $8 \times 1.5$ to $2.5 \mu$.

\section{TRICHOPEZIZA Fuckel.}

T. - - sp.

Torn end of firm dead tree, Short Creek, alt. I,80o ft. Dec. I6, I893 (Nuttall, I326).

T. Albo-Lutea (Pers.) Sacc.?

On rotten log, Short Creek, June I8, I893, stipitate (Nuttall, IOI3).

T. Capitata (Peck.) Sacc.

On leaves Quercus palustris on ground in woods, alt. 2,000 ft., June I8, I893. At Rupert's, July 26, I894 (Nuttall, IOI I).

DASYSCYPHA Fr.

D. virginea (Batsch) Fckl.

On dead sticks on wet cliff, and on wet dead wood of Juglans cinerea, June I2, I893 (Nuttall, 84I).

D. Luteola (Cun.) Sacc.

On a stick (Hartley, 2974).

D. Ellisiana (Rehm.) Sacc.

On Pinus rigida, top of Cavendish cliff, April 8, I894 (Nuttall, I447).

\section{HELOTIELLA Sacc.}

H. Nuttalli E. \& E.. Proc. Phila. Acad., 35I (I894).

Type habitat: On wet shady side of rotten log, March I5, I894 (Nuttall, discov. I425, 397).

Ascomata gregarious, sessile, flat-hemispherical, .5 to I mm. diameter, cup-shaped when fresh, contracted and subspherical when dry with only a small round apical opening, orange color throughout, disk watery-orange, outside and fringed margin paler. Substance fibrous, the fibers prolonged 
above so as to make a marginal fringe of pale yellow, roughish straight hairs. Asci clavate-cylindrical, sessile, curved, 50 to $60 \times 5$ to $6 \mu$. Paraphyses filiform, hardly thickened above, with a series of orange-colored, globose nuclei. Sporidia biseriate, oblong, hyaline, obtuse, uniseptate, but not constricted, 8 to $10 \times 2.5$ to $3 \mu$. The outside of the ascomata is slightly hairy so that the species might be referred to Solcnpeziza if that is really worthy of generic distinction.

\section{DERMATELLA Karst.}

D. viticola E. \& E., Proc. Phil. Acad., 352 (1894).

Type habitat: On dead shoots of Vitis (cult.) Nov. 1893 (Nuttall, discov. I337, 295).

Sessile, convex-discoid and pale when moist, concave and flesh-color when dry, and then scarcely projecting above the surface of the bark. .50 to $.75 \mathrm{~mm}$. diameter. Asci clavatecylindrical, about 70 x $12 \mu$, with filiform paraphyses, 8spored, sub-sessile. Sporidia sub-biseriate, oblong elliptical, hyaline, I to 3 -septate ( 3 to 4 -nucleate at first), I 5 to $18 \times 6$ to $6.5 \mu$.

\section{BELONIDIUM Mont.}

B. AURelia (Pers.) DeNot.

Peziza aurelia, Pers. On a dead leaf in rotton log, Monongalia Co., at Little Falls (Millspangh).

\section{ERINELLA Sacc.}

E. Miniopsis (E11.) Sacc.

On wet rotten wood, May, I893 (Nuttall, 934).

E. On Acer spicat

On Acer spicatum (Sheldon, 2560).

\section{A S C O B O L A C E A E.}

\section{ASCOBOLUS, Pers.}

A. Furfuraceus Pers.

On old dung of cow, June 8, r893 (Nuttall, 974).

A. CARbonarius Karst.

On bare spots where logs were burned by forest fire the previous winter, associated with Geopyxis carbonaria. It appears that only the prolonged burning of large logs makes proper habitat for this species, as it does not grow where the fire was light. May 20, I893 (Nuttall, 926).

ASCOPHANUS Boud.

A. GRANUliformis ( $\mathrm{Cr}$.) Boud. ?

On dung of cow, Dec., I893 (Nuttall, I340, 298). 
A. Carneus (Pers.) Boud.

On dung of cow, Oct. IO, I893 (Nuttall, I249, I82).

DER M A T E A E A E.

\section{URNULA Fr.}

U. CRATERIUM (Schw.) Fr.

On dead twigs on ground, Short Creek, April I5, I893 (Nuttall, 898). On soil (Sheldon 70), and on dead Oak branch lying on the ground in a wood (McCutcheon, 3205).

\section{DERMATEA Fr.}

D. Rubi (Lib.) Rehm.

On Amelanchier Canadensis (F. E. Brooks, 944).

D. Chionanthi E. \& E.?

Macrostylospores of this species? on Chionanthus Virginica, March 25, I894 (Nuttall, I454, 426).

D. LOBATA Ellis.

On the maculæ of Pestalozzia Guepinii on leaves of Rhododendron maximum. Asci $\mathrm{I} 30 \mathrm{x} \mathrm{I} 2.5 \mu$ spores $\mathrm{I} 5 \times 8 \mu$. Aug. I5, I895 (Nuttall, I162, I32).

\section{TYMPANIS Tode.}

T. Oxydendri E. \& E., Proc. Phila. Acad., 352 (i894).

Type habitat: On dead limbs of Oxydendron arboreum, Dec. 12, 1893 (Nuttall discov., I296, 258).

Cespitose, 3 to 5 together, erumpent, at length deciduous, black .5 to $.75 \mathrm{~mm}$. diameter, contracted below into a short thick stipe, margin subundulate. Asci cylindrical r Io to I 30 x 15 to $18 \mu$ including the short stipe. Paraphyses slender, abundant, Sporidia numerous, minute, oblong, hyaline or yellowish 2.5 to $3 \times$ I $\mu$. Allied to $T$. fasciculata, but asci larger and sporidia smaller.

T. CONSPERSA Fries.

On Apple trees (Sheldon, 85, 5I I, 909).

C. Aceris (Hazsl.) Sacc.

\section{CENANGELLA Sacc.}

On bark of dying Acer rubrum, June Io, I894. Sporidia I $5 \times 6 \mu$ (Nuttall, I554).

\section{SCLERODERRIS Fr.}

S. Rhabarbarina (Berk.) E. \& E.

On Rubus Baileyanus, Dec. 8, I893, Short Creek. Asci total length 63 to $75 \times 17$ to $21 \mu$, 8-spored, clavate, very 
short stipitate. Spores 20 to $27 \times 6$ to $7.5 \mu$, nucleate, becoming clearly 3 -septate and rather one-sided (Nuttall, 1294, 242).

S. PALlidula (Cooke) Sacc.

On dead branches and bark of Acer saccharinum, L., Feb. 26 , I894. On Oxydendron arboreum. Sporidia 20 to $22 \mathrm{x}$ $7 \mu$ (Nuttall, I408, 665).

\section{B U L G A R I A C E AE.}

L. i.ubrica (Scop.) Pers.

LEOTIA Hill.

On ground, alt. I,800 ft., Short Creek, Aug. I4, I893 (Nuttall, II50). On earth (Sheldon, 3049, 3462, 3463).

\section{ORBILIA Fr.}

O. RUBEI.LA (Pers.)

On decorticated log, Liriodendron Tulipifera, Sept. 19, I895 (Nuttall, I858, 748).

O. vinosa (A. \& S.) Karst.

On Poria spissa, Nov. 6, I893 (Nuttail, 1261).

O. Luteo-Rubella (Nyl.) Karst.

On decaying wood (Sheldon, 3047).

O. occulta (Rehm) Sacc.

On dead log, Aug. 20, I893 (Nuttall, I202).

O. Coccinella (Somm.) Karst.

On dead log Juglans cinerea, Short Creek, July 25, I8.93 (Nuttall, I I34, 663).

O. Xanthostigma Fr.

On decorticated limbs Magnolia Fraseri, Sept. I895 (Nuttall, I855, 742, 743).

BULGARIA Fr.

B. inguinans (Pers.) Fr.

In clefts of bark of trees and railroad ties, Oct. IO, I893 (Nuttall, I210).

\section{SARCOSMA Casp.}

S. RUFUm (L. \& S.) Rehm.

On dead Beech branches in woods (Sheldon, 3279).

C. URnalis (Nyl.) Sacc.

CORYNE Tul,

On rotting log Fagus atropunicea; under bark of Quercus alba log, Monongalia Co., near Morgantown (Millspaugh). On wet mossy logs, alt. I,800 ft. and over, Oct. IO, I893 (Nuttall, 1212, 167). 
C. sarcoides (Jacq.) Tul.

On decaying log (Sheldon, I4I4, 3I86, 3I89).

HOLWAYA Sacc.

H. орніовоLus (Ellis) Sacc.

On bark of dead Magnolia Fraseri, alt. 1,800 ft., Dec. I6, I893, Short Creek (Nuttall, I323, 279).

S T I C T A C E AE.

\section{PROPOLIS Fr.}

P. Faginea (Schrad.) Karst.

On Juglans cinerea and on old board of Liriodendron Tulipifera, Dec. 5, I894 (Nuttall, I765, 660). On Liquidamber Styraciflua.

P. LeONis (Tul.) Rehm.

On bark of Pinus rigida and Virginiana, June 3I, I894 (Nuttall, I58I).

S. Fimbriata Schw.

\section{STICTIS Pers.}

On strobiles of Pinus Virginiana, alt. 2,000 ft., March, I894 (Nuttall, I468, 443). Sporidia 5 to $7 \times 1.5 \mu$.

$$
\text { P H A C I D I C E AE. }
$$

RHYTISMA (Pers.) Fr.

R. punctatum (Pers.) Fr.

On leaves of Acer Pennsylvanicum, alt. 2,00o ft., Oct. 22, I893 (Nuttall, I2I7, I68). On Acer spicatum, Randolph: near Cheat Bridge (Sheldon).

R. DECOLORANS Fr.

On Xolisma ligustrina, at Rupert's, July 26, I894 (Nuttall, I638, 569).

R. ACERINUM (Pers.) Fries.

On Acer rubrum, Preston : near Rohr, and Randolph: near Cheat Bridge; on Acer saccharum, Monongalia : near Morgantown (Sheldon).

R. PRINI Schwein.

On leaves of Ilex verticillata, at Rupert's, Sept. 7, I894 (Nuttail, 1677).

\section{P A T E L L A I A C E AE.}

D. Commutata Fckl.

\section{DURELLA Tul.}

On dead log, Short Creek, Dec. I6, I893. (Nuttall, I328) 
LECANIDION Rabenh.

L. Atratum (Hedw.) Rabenh.

On Aralia spinosa, Feb. I7, 1894. Sporidia $50 \times 12.5 \mu$ (Nuttall, I390, 359).

L. Hamamelidis (Peck) Sacc.

On bark of Hamamelis Virginica. Sporidia I to 3-septate when mature. Dec. I8, I894 (Nuttall, I772).

\section{G Y M N O A S C A C E AE.}

\section{EXOASCUS Fckl.}

E. DEFormans (Berk.) Fckl.

Taphrina deformans Tul. On leaves of Amygdalus Persica, Jefferson Co., near Charlestown (Millspaugh).

E. Pruni Fckl.

Taphrina Pruni Tul. On fruits of Prunus domestica. Monongalia Co., at Morgantown (Millspaugh).

H Y S TER I A C E AE.

GLONIUM Muehl.

G. stellatum Muehl.

On rotten oak plank, and on Sassafras Sassafras, July 28, I893 (Nuttall I I28). On wood fence, Dellslow; on Juglans nigra, Preston, near Albright (Sheldon).

G. PARvulum (Ger.) Sacc.

On fence rails Castanea dentata, April 20, I894 ( Nuttall, I496).

G. SIMULANS Ger.

On old fence rails, alt. 2,000 ft., March I6, I894. Asci $75 \times 7.5$ to $10 \mu$. Spores I 3 to I $8 \times 5 \mu$. On Junglans cinerea, asci $65 \times$ Io $\mu$; spores I2 to I $5 \times 4$ to $5 \mu$ (Nuttall. I 4I5).

\section{HYSTERIUM Tod.}

H. Kalmiae Schw.?

On dead Kalmia latifolia, Nov., I893 (Nuttall, I287, 232).

HYSTEROGRAPHIUM Corda.

H. Insidens (Schw.) Sacc.

On old fence rails, April 6, I894. Largest spores $43 \times 7.5$ $\mu$, I I-septate (Nuttall, I452).

H. subrugosum (C. \& E.) Sacc.

On old fence rails, May 26, I894. Spores 33 to $35 \times 12.5$ $\mu$ (Nuttall, I522).

H. MorI (Schw.) Rehm.

On old fence rails, April 6, I894 (Nuttall, I45I). 
H. Desmazieri DuBy.

On living leaves of Pinus echinata. Wood Co., near Lockhart's Run (Millspaugh).

H. VIRGULTORUM DC.

On Rubus Baileyanus, Dec. 5, I893 (Nuttall, 1293).

H. Commune (Fr.) Duby.

On Hydrangea arborescens, Nov. 3, I893 (Nuttall, I259)

On old petioles Aralia spinosa (Nuttall, I830, 719).

\section{LOPHODERMIUM Chev.}

L. RHODODENDRI (Schw.)

On Rhododendron maximum, Sept. 4, I893 (Nuttall, I 192). Boone: near Lick Run (Sheldon.)

L. Pinastri (Schrad.) Chev.

On leaves of Pinus rigida, Short Creek, Aug. 2; 1893 (Nuttall, I I 58).

L. culmigenum (Fr.) Karst.

On straw brought in from Ohio, May 24, I894. Measurements of asci and spores prove to be the same as those of L. arundinaceum (Schrad.) Chev. (Nuttall, I496).

OSTROPA Fr.

O. CINerea (Pers.) Fr.?

Issuing from cracks in a decorticated log, May 3I, I894 (Nuttall, I 526).

A G A R I C A CEAE.

AMANITA Pers.

A. VERnA Fr.

On ground in open woods, July I I, I893. Spores round, $7.5 \mu$ (Nuttull, 964).

A. MUSCARIA Linn.

Rooted on buried limb of Betula lenta, Grant Co., near Bayard (Millspaugh).

An ecarunculate form, apparently of this species, on leaf mold in deep woods, same locality.

A. Muscaria alba Peck.

On ground in laurel thicket, Short Creek, alt. 2,00o ft.

Has the odor of buttermilk. Spores $9 \times 6 \mu$ (Nuttall, II3I). A. Flavo-RUBEns Berk.

On ground in woods, Short Creek, July 6, I893 (Nuttall, 1078).

A. polypyramis B. \& C.

Among bushes, alt. 2,400 ft., July 6, I893. Largest stipes 
I $5 \mathrm{~cm}$. long, thick, heavy and deep in ground. Pileus $\mathrm{I}_{5}$ $\mathrm{cm}$. broad. Spores Io x $6 \mu$ (Nuttall, I IOI).

A. Caesarea Scop.

Preston: common near Brookside (Beardslee).

A. phalloides Fr.

Preston: near Brookside (Beardslee).

A. Frostin Peck.

Preston: near Brookside (Beardslee).

A. verna Bull.

Preston: very common near Brookside (Beardslee).

A. STROBILIFORMis Vitt.

Preston: near Brookside (Beardslee).

A. solitaria Bull.

Preston: near Brookside. Specimens and photographs secured illustrate both this and the preceding species as they are understood in the United States. They are listed here in spite of the fact that their status is unsatisfactory. Most of our A. solitaria is referred, by European Mycologists, to A. echinocephala Vitt.-Beardslee.

A. Vittadini Fr.

Preston: near Brookside. I believe this simply a well marked form of A. echinocephala Vitt. My specimens and photographs have been carefully verified and show the species, such as it is, to be certainly West Virginian.Beardslee.

A. Rubescens Pers.

Preston: near Brookside (Beardslee).

A. SPRETA Peck.

Preston: near Brookside. A. cinerea Bres. is identical with this although our plants much exceed the limits set by Bresadola. It is extremely variable, a pure white form being especially noteworthy.

A. SPRETA PARVA Beardslee. var. nov.

Pileus I to $\mathrm{I} .5$ in. broad, thin, almost membraneous, deeply sulcate-striate. Stipe slender, about 2 in. tall. Annulus me-. dian. Spores, color and volva as in A. spreta.

An interesting form, found growing in colonies, in gravel high up on the mountain near Brookside, Preston County. Both Peck and Bresadola are inclined to consider it a distinct species.

LEPIOTA (P. Browne) S. F. Gray.

L. PROCERA Scop.

Preston: near Brookside (Bcardslee).

L. NAUCina Fr.

Preston: near Brookside (Beardslee). 
L. Americana Peck.

Preston: near Brookside (Beardslee).

L. Cristata (Bolt.) Quel.

Preston: near Brookside (Beardslee).

L. acutisouamosa Weim.

Preston: near Brookside (Beardslee).

L. CLYPEOLARIA Bull.

Preston: near Brookside (Beardslee).

L. metulaespora B. \& Br.

Preston: near Brookside (Beardslee).

L. amianthina Scop.

Preston: near Brookside (Beardslee).

\section{POLYMYCES Batt.}

P. CINEREus Batt.

(Agaricus melleus Vahl.)

Armillaria mellea Quel. Preston: near Brookside (Beards. lee).

AMANITOPSIS Roze.

A. Nivalis (Grev.) Sacc.

In loam on rocks in laurel tangle, June 26, I893 (Nuttall. I0O4).

A. strangulata Fr.

Preston: near Brookside (Beardslee).

VAGINATA (Nees) S. F. Gray.

V. Vaginata (Bull.) Merrill.

Preston: near Brookside (Beardslee).

AMANITELLA Earle.

A. farinosa (Schw.) Earle.

Preston: near Brookside (Beardslee).

(Amanita farinosa Schw.)

TRICHOLOMA Fr.

T. Fumoso-Luteum Peck?

On unknown substance, June I5, 1893, Short Creek (Nuttall, 990).

T. AURANTIA Schaeff.

Preston: near Brookside. This is certainly T. Peckii

Howe. Our plant does not agree rigidly with the description

but it corresponds exactly with the plant as it grows in

Sweden, where I have collected it.-Beardslee.

T. GRAVE Peck.

Preston: near Brookside (Beardslee).

MONOMYCES Batt.

M. RUtilens (Shaeff.) Batt. (Tricholoma rutilens Schaeff.) Preston: near Brookside (Beardslee). 


\section{CLITOCYBE Fr.}

C. Illudens Schwein.

On old stumps in clusters of about eight, dark and deep rich orange color throughout, $+20 \mathrm{~cm} . \mathrm{x}+\mathrm{I} 2 \mathrm{~cm}$. broad stipe $+25 \mathrm{~mm}$. thick. Spores $+6 \mu$ diameter, uneven. July r4, r893 (Nuttall, ro93).

C. Laccata Scop.

Common in wet sand, bank of Short Creek, alt. 2,000 ft., July 28, I893. Spores Io $\mu$ diameter, echinate (Nuttall, II24).

C. NebUlaris Batsch.

Preston: near Brookside (Beardslee).

C. ODORA Bull.

Preston: near Brookside (Beardslee).

C. cerussata Fries.

Preston: near Brookside (Beardslee).

C. Candicans Pers.

Preston: near Brookside (Beardslee).

C. Dealbata Sow.

Preston: near Brookside (Beardslee).

C. INFUndibuliformis Schaeff.

Preston: near Brookside (Beardslee).

C. CYATHIFORMIS Fries.

Preston: near Brookside (Beardslee).

C. platyphylla Fr.

COLLYBIA Fr.

On mold and moss on trunk of tree, Short Creek, June I5, I893 (Nuttall, 98I).

C. CONIGENOIDES Ellis.

On last year's cones Magnolia Fraseri, Sept. I8, I895 (Nuttall, I848).

C. DRYOPHILA Bull.

In moss on log in deep woods, Grant Co., near Bayard (Millspaugh).

C. maculata A. \& S.

Preston: near Brookside (Beardsiee).

C. Butyracea Bull.

Preston: near Brookside (Beardslee).

C. Confluens Pers.

Preston: near Brookside (Beardslee).

C. Cirrihata Schum.

Preston: near Brookside (Beardslee).

CRINIPELLUS Pat.

C. Stipitaria (Fries.) Pat.

(Collybia stipitaria Fr.).

Preston: near Brookside (Beardslee). 
COLLYBIDIUM Earle.

C. velutipes (Curt.) Earle.

(Collybia velutipes Curt.)

About roots of rotting stumps, Jan. 3I, I895 (Nuttall, 830). Preston: near Brookside (Beardslee).

GYMNOPUS Rouss.

G. RADICATA (Relh.) Rouss.

(Collybia radicata Relh.).

On leaf mold in rich woods, Monongalia Co., near Morgantown (Millspaugh). May I7, I893 (Nuttall, 980). Preston: near Brookside (Beardslee).

\section{MYCENA Fr.}

M.

White. On mossy stump, July 4, I893 (Nuttall, Iо19).

M. galericulata Scop.

In rich woods under oak twig, and oak bark on log, Monongalia Co., near Morgantown (Millspaugh). On damp dead logs, May 7, I893 (Nuttall, 930).

M. ePIPTERygia Scop.

On moss-covered log and in clefts of bark, Feb. 28, I893 (Nuttall, 948).

M. RUBRo-MARginata Fries.

Preston: on Hemlock cones near Brookside (Beardslee). M. PURA Pers.

Preston: near Brookside (Beardslee).

M. FILopes Bull.

Preston: near Brookside (Beardslee).

M. haematopa Pers.

Preston: near Brookside (Beardslee).

M. galopa Pers.

Preston: near Brookside (Beardslee).

M. vUlgaris Pers.

Preston: near Brookside (Beardslee).

M. tenerrima Berk.

Preston: near Brookside (Beardslee).

M. CORTICOLA Schum.

Preston: near Brookside (Beardslee).

O. campanella Batsch.

OMPHALIA Fr.

On leaf mold at base of Castanea dentata, Preston Co., near Terra Alta (Millspaugh). On rotten log in thicket, June I8, I893 (Nuttall, I009).

O. CHRysophylla Fries.

Preston: near Brookside (Beardslee).

O. stellata Fries.

Preston: near Brookside (Beardslee). 
O. FIBULA Fries.

Preston: near Brookside (Beardslee).

P. CORTicatus Fr.?

$$
\text { PLEUROTUS Fr. }
$$

On fallen Acer sp. Gills not anastomosing, July 28, I893 (Nuttall, II23).

P. SAPIDUS Kalchbr.

On dead log, Feb. 2, 1893 (Nuttall, 850).

P. SEROTINUS Schrad.

On dead trunks, Nov. 20, 1893 (Nuttall, 1267).

P. CYPHELLIFORMIS Berk.

On dead stems of Polymnia Uvedalia, Nov. I5, I894 (Nuttall, 1742).

P. NIGER Fr.

On bark of dead tree, May 13, I893 (Nuttall, 953).

P. PETALOIDES Fries.

Preston: near Brookside (Beardslee).

$$
\text { HYGROPHORUS Fr. }
$$

H. Nitidus B. \& C.

On decayed log, May I4, I893 (Nuttall, 968).

H. PRATENSIS Fries.

Preston: near Brookside (Beardslee).

H. virgineus Fries.

Preston: near Brookside (Beardslee).

H. Laetus Fries.

Preston: near Brookside (Beardslee).

H. CERACEUS Fries.

Preston: near Brookside (Beardslee).

H. coccineus Fries.

Preston: near Brookside (Beardslee).

H. miniatus Fries.

Preston: near Brookside (Beardslee).

H. Conicus Fries.

Preston: near Brookside (Beardslee).

H. Psittacinus Fries.

Preston: near Brookside (Beardslee).

\section{LACTARIA Pers.}

L. CILICIOIDES Fries.

On border of woods and swamp, July 4, I893 (Nuttall, IO2O).

L. piperata (L.) Pers.

Preston: near Brookside (Beardslee).

L. Pergamena (Sw.) Fries.

Preston: neat Brookside (Beardslee). 
L. GLAucescens (Cross1.) Beardslee. comb.nov. (LactariusCross1.)

Preston: rather rare near Brookside but doubtless occurs in the southern mountains quite generally. This species was observed, studied, and noted under a temporary name. The peculiar glaucous-green color assumed by the latex was different from anything before observed. My plants should certainly be referred to this species, which has since been published. It has possibly been confused with L. vellereus which it resembles.

L. VELLERA Fries

Preston: near Brookside (Beardslee).

L. INSULSA Fries.

Preston: near Brookside (Beardslee).

L. TRIVALIS Fries.

Preston: near Brookside (Beardslee).

L. Deliciosa (L.) Fries.

Preston: near Brookside (Beardslee).

L. Indigo (Schw.) Fries.

Preston: near Brookside (Beardslee).

L. Plinthogala (Otto) Burl. (Lactarius fuliginosus Fr.)

Preston: near Brookside (Beardslee).

L. Lactiflua (L.) Burl. (Lactarius volemus Fr.)

Preston: near Brookside (Beardslee).

L. HYDROPIPEROIDES Berk. \& Curt.

Preston: near Brookside (Beardslee).

L. subdulcis (Pers.) Fries.

Preston: near Brookside (Beardslee).

R. EMETICA Fr.?

\section{RUSSULA Pers.}

In woods, July 4, I893 (Nuttall, IO2I).

R. NIGRICANS Fries.

Preston: near Brookside (Beardslee).

R. ADUSTA Fries.

Preston: near Brookside (Beardslee).

R. DELICA Fries.

Preston: near Brookside (Beardslee).

R. FURCATA Fries.

Preston: near Brookside (Beardslee).

R. FRAGILIS Fries.

Preston: near Brookside (Beardslee).

R. viRESCENS Fries.

Preston: near Brookside (Beardslee). 


\section{CANTHARELLUS Adans.}

C. MINOR Peck?

On ground in woods, alt. 2,000 ft., July 4, I893 (Nuttall, IO23).

C. WrightiI Berk?

Wet sand of river bank in shade, July I8, I893. Whole plant light red except gills, which are little deeper red than salmon-color, gills decurrent, veined between, pileus more convex than plane, and depressed edge wavy, rather tough Largest $4 \mathrm{~cm}$. broad, stem $5 \mathrm{~cm}$. high, $5 \mathrm{~mm}$. thick. Spores. 7.5 to $8.5 \times 3.5$ to $5 \mu$, apparently rough and guttulate inward (Nuttall, I IO4).

C. TUBAEFORMis Fr.?

On rotten wood in woods, alt. 2,00o ft., July 4, I89.3 (Nuttall, 1022).

C. Floccosus Schw.

On open bank of stream in laurel thicket, alt. 2,000 ft., June 20, I893. Largest squamules $.75 \mathrm{~cm}$. long, thick and triangular, forming a cone which closes the funnel below the top an inch or more. Spores $15 \times 7.5 \mu$ (Nuttall, 992).

C. CIBARIUS Fries.

Preston: near Brookside (Beardslee).

C. aurantiacus Fries.

Preston: near Brookside (Beardslee).

C. INFUNDIBULIFormis Fries.

Preston: near Brookside (Beardslee).

C. Cinnabarinus Fries.

Preston: near Brookside (Beardslee).

\section{NYCTALIS Fr.}

N. ASTEROPHORA Fr.

On dead Agaric in deep damp woods, July I4, I893. Stars $20 \mu$ diameter (Nuttall, Iog6).

\section{MARASMIUS Fr.}

M. Semihirtipes Peck.

On dead leaves, sticks, etc., Short Creek, May I4, I893 (Nuttall, 967).

M. ERYTHROPUS Fries.

Preston: near Brookside (Beardslee).

M. SCORODONIUS Fries.

Preston: near Brookside (Beardslee).

M. Foetidus Fries.

Preston: near Brookside (Beardslee).

M. ANDRosaceus Fries.

Preston: near Brookside (Bcardslee). 
M. SPLANCHNOIDES Fries.

Preston: near Brookside (Beardslee).

M. PERFORANS Fries.

Preston: near Brookside (Beardslee).

M. Instititus Fries.

Preston: near Brookside (Beardslee).

M. epIPHyllus Fries.

Preston: near Brookside (Beardslee).

M. opacus B. \& C.

Common on dead branches Rhododendron maximum, Grant Co., near Bayard (Millspaugh).

M. Campanulatus Peck.

On dead leaves and sticks in damp woods, Short Creek, alt. I,300 ft., Aug. I6, I893 (Nuttall, II53).

M. rotula (Scop.) Fr.

On dead.limb of Betula lenta, Grant Co., near Bayard (Millspaugh). On stick in open woods, June 8, 1893 (Nuttall, 972).

COLLYBIOPSIS (Schroet.) Earle.

C. Ramealis (Fr.) Earle.

(Marasmius ramealis $\mathrm{Fr}$.)

Very common on twigs and leaves in laurel thickets, June I 5, I893 (Nuttall, 994).

SCORTEUS Earle.

S. OREADES (Fries) Earle.

(Marasmius oreades Fr.)

Preston: near Brookside (Beardslee).

TAPINIA (Fries) Karst.

T. PANuoldes (Fries). (Paxillus panuoides Fr.)

Preston: near Brookside (Beardslee).

\section{LENTODIUM Morg.}

L. squamosum (Huds.) Murrill.

Preston: near Brookside (Beardslee).

\section{LENTINUS Fr.}

L. STRIGOSUS Fr.

On dead log Betula lutea, Grant Co., near Bayard (Millspaugh). On dead logs, common, May 6, I893 (Nuttall, 904).

L. CRINITUS (L.) Fries.

On Acer saccharum, Monongalia, near Lick Run (Sheldon).

PANUS Fr.

P. Stipticus (Bull.) Fr.

On failen dead limb, Jan. 29, I893 (Nuttall, 950). 
P. DORSALIS Bosc.

On Tsuga Canadensis, Sept., I893 (Nuttall, I253, I96).

P. CONChAtus Fries.

Preston: near Brookside (Beardslee).

\section{TROGIA Fr.}

T. CRISPA (Pers.) Fr.

On various dead dry logs and limbs, March 2I, I893 (Nuttall, 875).

\section{LENZITES.}

L. Petulina (Linn.) Fr.

On logs, Sept. 6, i893 (Nuttall, 837). Monongalia : Lick Run (Sheldon).

L. Abietina (Bull.) Fr.

On dead branches Tsuga Canadensis, alt. I,600 ft., Dec. I 5, I893 (Nuttall, I3OI).

L. CORRUGATA Klot.

On Castanea dentata, alt. I,800 ft., Dec. I9, I893 (Nuttall, I302).

\section{GLOEOPHYLI_UM Karst.}

G. hirsutum (Schaeff.) Murrill. (Lenzites sepiaria Fr.).

On Pinus Virginiana, Monongalia, near Dellslow and on Fraxinus, near Ringgold; on spruce log, Pocahontas, Spruce Mountain, alt. 4,000 ft. (Sheldon). On decorticated stumps Picea Mariana, Tucker Co., near Falls of Blackwater (Millspaugh). Co.

A resupinate form on dead logs Tsuga Canadensis Grant

G. TRABEum (Pers.) Murrill.

On Acer saccharum, Monongalia : near Lick Run and Morgantown (Sheldon).

\section{SCHIZOPHYLLUM Fr.}

S. COMMUNE Fr.

On bark log Quercus alba, Grant Co., near Bayard. On dead twig Pyrus Malus, Monongalia Co., near Morgantown (Millspaugh). On dead logs, Jan. 30, I893 (Nuttall, 827). S. Alneum (L.) Schroet.

On Acer, Pocahontas: Cranberry Glades (Sheldon).

\section{VOLVARIA Fr.}

V. вомbycina (Pers.) Fr.

On dead insect sp.? Monongalia Co., near Morgantown (Millspaugh). 
V. Hypopita Fries.

Preston: near Brookside. This is the only Volvaria I have detected in West Virginia. It is the plant commonly referred to $\mathrm{V}$. pubescentipes $\mathrm{Pk}$. My specimens have been verified by Bresadola and I an confident they are correct.Beardslee.

\section{PLUTEUS Fries.}

P. CERvinus (Schaeff.) Quel.

Preston: near Brookside (Beardslee).

P. ADMIRABILIS Peck.

Preston: near Brookside (Beardslee).

P. Longistriatus Peck.

Preston: near Brookside (Beardslee).

\section{ENTOLOMA (Fries) Quel.}

E. SERICELLA Fries.

Preston: near Brookside (Beardslee).

E. RHODOPOLIA Fries.

Preston: near Brookside (Beardslee).

E. STRICTIOR Peck.

Preston: near Brookside (Beardslee).

E. SERICEA Bull.

Preston: near Brookside (Beardslee).

\section{CLITOPILUS Fr.}

C. ABORTIVUS Fr.

Wet woods, alt. I,800 ft., aborted specimens in quantity, Sept. 4, I893 (Nuttall, IIgo).

C. PRUNUlus Scop.

Preston: common near Brookside (Beardslee).

C. orcella Bull.

Preston: common near Brookside (Beardslee).

$$
\text { NOLANEA (Fries) Quel. }
$$

N. Pascua Pers.

Preston: near Brookside (Beardslee).

N. MAmmosa Linn.

Preston: near Brookside (Beardslee).

$$
\text { LEPTONIA (Fries) Quel. }
$$

L. Serrulata Pers.

Preston: near Brookside (Beardslee).

L. ASPERELla Fries.

Preston: near Brookside (Beardslee). 
CLAUDOPUS (W. G. Smith) Gillet.

C. variabilis Pers.

Preston: near Brookside (Beardslee).

C. Byssisedus Pers.

Preston: near Brookside (Beardslee).

PHOLIOTA (Fries) Quel.

P. EREBIA Fries.

Preston: near Brookside (Beardslee).

P. Candicans (Bull.) Schroet.

Preston: near Brookside (Beardslee).

(P. praecox Pers.).

P. ADIPOSA Fries.

Preston: near Brookside (Beardslee).

P. mutabilis Schaeff.

Preston: near Brookside (Beardslee).

ROZITES Karst.

R. caperata (Pers.) Karst.

(Pholiota caperata Pers.).

Preston: common near Brookside (Beardslee)..

$$
\text { FLAMMULA (Fries) Quel. }
$$

F. FLAVIDA Schaeff.

Preston: near Brookside (Beardslee).

$$
\text { NAUCORIA (Fries) Quel. }
$$

N. Festiva Fries.

Preston: near Brookside. A photograph of this curious plant was secured and seems to be correctly placed. The glutinous pileus and long rooting stipe are very markedBeardslee.

N. SEmiorbicularis (Bull.) Quel. ( . pediades Fr.).

Preston: common in lawns near Brookside (Beardslee).

CONOCYBE Fayod.

C. TENER (Schaeff.) Fayod. (Galera tener Schaeff.).

Preston: near Brookside (Beardslee).

C. Hypnorum (Batsch.) Murrill. (Galera hypnorum Batsch.).

Preston: near Brookside (Beardslee).

TUBARIA (W. G. Smith) Gillet.

T. FURfuracea Pers.

Preston: near Brookside (Beardslee).

CREPIDOTUS (Nees.) S. F. Gray.

C. CALOLEPis Fries.

Preston: near Brookside. This is the plant commonly 
referred to $C$. fulvotomentosus Peck. Fries' description does not well apply to our plant, but the Swedish plant referred to this species is identical with ours. I found, in Sweden, specimens nearly three times the dimensions given by Fries and differing in no way from specimens collected in West Virginia and in North Carolina-Beardslee.

C. applanatus Pers.

Preston: near Brookside (Beardslee).

C. malachius B. \& C.

Preston: near Brookside (Beardslee).

C. putrigenus B. \& C.

Preston: near Brookside (Beardslee).

C. versutus Peck

Preston: near Brookside (Beardslee).

C. Herbarum Peck.

Preston: near Brookside (Beardslee).

CORTINARIUS (Pers.) Roussel.

C. CAerulescens Fries.

Preston: near Brookside (Beardslee).

C. PURpurascens Fries.

Preston: near Brookside (Beardslee).

C. Rodes B. \& C.

Preston: near Brookside (Beardslee).

C. violaceus Fries.

Preston: near Brookside (Bcardslee).

C. Pholideus Fries.

Preston: near Brookside (Beardslee).

C. cinnamomeus Fries.

Preston: near Brookside (Beardslee).

C. Tokvus Fries.

Preston: near Brookside (Beardslee).

C. armillatus Fries.

Preston: near Brookside (Beardslee).

PAXILLUS Fries.

P. involutus (Batsch) Fries.

Preston: near Brookside (Beardslee).

P. atramentosus Fries.

Preston: near Brookside (Beardslee).

VENENARIUS Earle.

V. MUSCARIUS (Fries) Earle.

(Agaricus muscarius Fr.)

AGARICUS Linn.

A. Rodmani Peck.

On ground, June 28, I893 (Nuttall, 1005). 
A. CAM PESTER Linn.

On lawn, June 24, I893 (Nuttall, 1002).

A. SILV ICOLA Vitt.

In woods, Short Creek, alt. I, 50 ft. (Nuttall, I699). Spores 5 to $7 \times 3$ to $4 \mu$.

A. SILvaticus Schæff.

In woods, Short Creek, July 2, I893 (Nuttall, III7).

A. ARVENSIS Linn.

Preston: near Brookside (Beardslee).

A. Placomyces Peck.

Preston: near Brookside (Beardslee).

STROPHARIA Fr.

S. STERCORARIA Fr.

On decaying vegetable matter, Preston Co., near Terra Alta (Millspaugh).

S. Semiglobata (Batsch.) Quel.

Preston: near Brookside (Beardslee).

HYPHOLOMA Fr.

H. sublateritium Schæff.

Under bark log Fraxinus Americana, Monongalia Co., near Morgantown (Millspaugh).

H. ELAEODES Fr.?

On sticks on ground, May I8, 1893 (Nuttall, 858).

H. FASCICULARIS Huds.

Preston: near Brookside (Beardslee).

H. LACHRYMABUNdA Fries.

Preston: near Brookside. The plants referred here, with some misgivings, were found growing in large masses comprised of a great number of individuals, resembling in number and scaly appearance Cooke's figure of H. storea Fr. I have never seen it since-Beardslee.

H. Candolleana Fries.

Preston: near Brookside (Beardslee).

H. appendiculatum (Bull.) Quel.

Preston: near Brookside (Beardslee).

C. variegatus Peck?

\section{COPRINUS Pers.}

On stick in shady place, Aug. II, I893 (Nuttall, II43).

C. Micaceus (Bull) Fr.

On bases of stumps in woods, July 8, I893 (Nuttall, IO8I).

C. Domesticus (Pers.) Fr.

In woods on mixture of manure and leaf mold. June 29, I893 (Nuttall, I007). 
C. Semilanatus Peck?

In heavily manured asparagus bed in garden, Apr. 29, I893 (Nuttall, 903).

C. ePHEMERUs Fr.

On horse dung, July 2, I893 (Nuttall, III3).

C. Angulatus Peck.

On baked soil where log had been burned, with Peziza carbonaria, May 8, I893 (Nuttall, 932).

P. Campanulatus Linn.

PANAEOLUS Fr.

On cow dung in deep coniferous woods, Grant Co., near Bayard (Millspaugh). Plentiful on manured lawn, May 8, I893, (Nuttall, 933).

P. SOLIDIPES Peck.

Preston: near Brookside (Beardslee).

P. Disseminata Pers.?

PSATHYRELLA Karst.

In rotten mold about the base of a stump, a mass about I meter containing perhaps thousands of specimens. Specimens 3 to $5 \mathrm{~cm}$. high, thin, fleshless. Pileus $\mathrm{I} \mathrm{cm}$. broad, $8 \mathrm{~mm}$. high, slightly umbonate, sulcate striate, mealy, gray. stems white, smooth I mm. thick, at first slightly furfuraceous. Largest stems $6 \mathrm{~cm}$., broadest pileus $18 \mathrm{~mm}$. Spores 6 to Iо $\times 3$ to $5 \mu$ (Nuttall, I I89). Sept. 3, I893.

\section{POL Y PORA CEA E.}

\section{GYROPORUS Quel.}

G. cyanescens (Bull.) Quel. (Boletus cyanescens Bull.).

Bare soil and on clay banks along roads. Preston, near Brookside (Beardslee).

\section{TYLOPILUS Karst.}

T. GRACILIS (Peck) P. Henn.

Preston, near Brookside (Beardslee).

(Boletus gracilis Peck).

T. Alboaster (Schw.) Murrill. (Boletus nigrellus Peck).

Preston, near Brookside (Beardslee).

CERIOMYCES Batt.

C. Chromapes (Frost) Murrill. (Boletus chromapes Frost).

Preston: abundant near Brookside (Beardslee).

C. Auriflammeus (B. \& C.) Murrill. (Boletus auriflammeus B. \& C.)

In dry gravel high on the mountain sides, Preston: nea: Brookside (Beardslee). 
C. PARAsiticus (Bull.) Murrill. (Boletus parasiticus Bull.). On Scleroderma vulgare in wet woods, Fayette: near Nuttallburg (Nuttall, I I9I).

C. eximius (Peck) Murrill. (Boletus eximius Peck).

Hillsides, Preston: near Brookside (Beardslee).

C. CRassus Batt.

(Boletus edalis Bull).

Old field under Rhus copallina, pileus $\mathrm{I} 5 \mathrm{x}$ Io $\mathrm{cm}$., stipe $4 \times 5 \mathrm{~cm}$. Spores Io to I $4 \times 3$ to $4 \mu$, Fayette, near Nuttallburg (Nuttall, 990). Preston: rarely seen at Brookside.

C. CRassus SeParans Peck. (Boletus separans Peck).

Hillsides, Preston: near Brookside (Beardslee).

C. AfFinis (Peck) Murrill. (Boletus affinis Peck).

Preston: abundant everywhere about Brookside (Beardslee).

C. Speciosus (Frost) Murrill. (Boletus speciosus Frost).

On ground in woods, alt. 2,00o ft., Fayette: near Nuttallburg (Nuttall, I099).

C. BICOLOR (Peck) Murrill. (Boletus bicolor Peck).

Common along sandy roads, Preston: near Brookside (Beardslee).

C. Communis (Bull.) Murrill. (Boletus chrysentron Fr.)

In deep woods, Fayette: near Nuttallburg (Nuttall, 987)

\section{PULVEROBOLETUS Murrill.}

P. Ravenelin (B. \& C.) Murrill. (Boletus Ravenelii Berk. \& Curt.).

Preston: near Brookside (Beardslee).

STROBILOMYCES Berk.

S. strobilaceus (Scop.) Berk.

Shady fence row, alt. 2,300 ft. Spores about io $\mu$ round. Fayette: near Nuttallburg (Nuttall, I IOO).

\section{FISTULINA Bull.}

F. hepatica Fr.

On dead stumps, alt. 2,000 ft. Fayette: near Nuttallburg (Nuttall, I IO2).

\section{POLYPORUS (Mich.) Paulet.}

P. Polyporus (Retz.) Murrill. (P. brumalis Fr.).

On dead logs, Fayette: near Nuttallburg (Nuttall, 1835).

P. Fissus Berk.

On Acer. Pocahontas: Cranberry Glades (Sheldon).

P. arcularius (Batsch) Fries.

On rotten logs, largest specimens $4 \mathrm{~cm}$. broad with stipe $2.3 \mathrm{~cm}$. Fayette: near Nuttallburg (Nuttall, 929). 
P. elegans (Bull.) Fries.

( $P$. varius of Am. Auth.).

On dead logs, specimens thin, tough, $20 \mathrm{~cm}$. broad, Fayette: near Nuttallburg (Nuttall, 840).

\section{BJERIKANDERA Karst.}

B. Adusta (Willd.) Karst.

(Polyporus adustus Fr.).

On dead branch Rhus hirta, and in decayed stunip Hicoria ovata, Monongalia: near Morgantown (Millspaugh); on dead log, Star City; on dead oak, near Randall (Sheldon). On Willow, Ohio: near Moundsville (Sheldon).

\section{PORODISCULUS Karst.}

P. pendulus (Schw.) Murrill. (Polyporus pocula B. \& C.).

On dead Rhus copallina, Fayette: near Glade Creek (Nuttall, 478, I495).

\section{ISCHNODERMA Karst.}

I. Fuliginosum (Scop.) Murrill. (Polyporus resinosus Fr.). On dead log, Fayette: near Nuttallburg (Nuttall, 879).

\section{FUSCOSPORIA Murrill.}

F. víticola (Schw.) Murrill.

On Quercus and Robinia, Monongalia: near Morgantown (Sheldon).

\section{IRPICIPORUS Murrill.}

I. Mollis (Berk. \& Curt.) Murrill.

On Acer saccharum, Monongalia: near Lick Run (Sheldon).

I. LACTEUs (Fries) Murrill.

Monongalia : on Quercus at Lick Run; on Juglans cinerea at Morgantown (Sheldon).

\section{TYROMYCES Karst.}

T. CHIONEus (Fries) Karst.

On Acer, Monongalia: at Sabraton; on dead wood, Upshur: at French Creek (Sheldon).

T. undosus (Peck) Murrill.

On decaying trunks of Hemlock and Pine, Murrill, North Am. Flora, 9:34.

I. PERplexus (Peck) Murrill.

IONOTUS Karst.

On Fagus, Monongalia: near Morgantown (Sheldon). 


\section{PHAEOLUS Pat.}

P. sistotremoides (Alb. \& Schw.) Murrill. don):

On Hemlock log, Pocahontas: Cranberry Glades (Shel-

PYCNOPORUS Karst.

P. cinnabarinus (Jacq.) Karst.

Preston: on Prunus serotina, near Reedsville, on Betula near Cranesville; on dead wood, Pocahontas: Cranberry Mountain; Monongalia: near Morgantown, on Prunus serotina and on Quercus (Sheldon).

\section{GRIFOLA (Micheli) S. F. Gray.}

G. Ramosissima (Scop.) Murrill. (Polyporus umbellatus Fr.).

In dry exposed hollow of oak stump, Monongalia: near Morgantown (Millspaugh).

G. Berkeleyi (Fr.) Murrill. (Polyporus B, \&. lactifluus Pk.).

On ground in open woods, height $30 \mathrm{~cm}$. breadth $60 \mathrm{~cm}$. Fayette: near Nuttallburg (Nuttall, II I ). In dry exposed hollow of oak stump, Monongalia: near Morgantown (Millspaugh). On dead wood, Pocahontas: Cranberry Mountain (Sheldon).

\section{AURANTIPORUS Murrill.}

A. Pilotae (Schw.) Murrill. (Polyporus Pilotae Schw.).

On dead and rotten log, Fayette: Sewell Valley (Nuttall, I66I). Under side of dead log, Nuttallburg (Nuttall, I66, I214), Polyporus hypococcineus Berk.

\section{LAETIPORUS Murrill.}

L. SPeciosus (Batt.) Murrill. (Polyporus sulphureus Fr.).

On decaying oak stump, Monongalia: near Morgantown (Millspaugh).

\section{HAPALOPILUS Karst.}

H. Rutilans (Pers.) Murrill.

(Polyporus nidulans Fr.).

On dead trunk Prunus serotina, Fayette: along Short

Creek (Nuttall, 548, I 599).

H. GILvUs (Schw.) Murrill.

On Fagus, Monongalia: near Morgantown; on Acer rubrum; Upshur: French Creek (Sheldon).

H. LICNOIDES (Mont.) Murrill.

On Acer saccharum, Preston: near Albright (Sheldon). 
GANODERMA Karst.

G. Tsugae Murrill.

On Tsuga, Monongalia: at 'Tibbs Run and Sturgisson (Sheldon).

\section{PYROPOLYPORUS Murrill.}

P. igniarius (L.) Murrill.

On Betula, Pocahontás: Cranberry Glades; on Acer, Preston: near Cranesville (Shcldon). Randolph: near Pickens (H. H. Smith, 1946).

P. Robiniae Murrill.

On living Robinia, Monongalia: near Sturgisson and Mason: near Elwell (Sheldon).

P. conchatus (Pers.) Murrill.

On dead log, Monongalia: near Morgantown (Sheldon).

\section{ELFVINGIA Karst.}

E. Fomentaria (L.) Murrill. (Fomes fomentarius of Flora).

On dead Betula iutea, Grant: near Bayard (Millspaugh): on dead log, Greenbrier: near Rupert (Nuttall, I635).

E. megaloma (Lev.) Murrill.

On dead wood, Monongalia: Lick Run; on Acer saccharum, Preston, near Cranesville (Sheldon).

\section{FOMES Fr.}

F. Lucidus (Leys.) Fr.

On dead logs Tsuga Canadensis, Preston Co., near Terra Alta (Millspaugh). On old stumps in deep woods, April Io, I893, full grown in June (Nuttall, 892).

F. volvatus Peck.

On west side Pinus Virginiana, March 8, I893 (Nuttall, $870)$.

F. Ungulatus (Schaeff.) Sacc (F. pinicola of Flora).

On Tsuga, Preston: near Cranesville (Sheldon); on fallen trunks Tsuga Canadensis, Short Creek, May I, I893 (Nuttall, 924).

F. Applanatus (Pers.) Wallr.

On dead Acer saccharum and Quercus palustris, alba and rubra, Wood Co., near Kanawha Station, and McDowell Co., near Elkhorn. On dead Fraximus Americana, Grant Co., near Bayard, and Monongalia Co., near Morgantown (Millspaugh). On dead logs, Jan., I893 (Nuttall, 828).

F. RIMOSUS Berk.

On dead Robinia Pseudacacia, Monongalia Co., near Morgantown (Millspaugh). On same sp. and on Oak? alt. 2,000 ft., March 21, I893 (Nuttall, 914). 
F. Roseus (Alb. \& Schw.) Cooke. (F. carneus of Flora.)

On Tsuga, Preston: near Cranesville; on Picea rubens. Pocahontas: Cranberry Glades; on living Peach tree, Monongalia : near Washington (Sheldon). On dead, decorticated Picea Mariana, Tucker Co., near Falls of Blackwater (Millspaugh): On Pinus Virginiana, March, I893 (Nuttall, 865).

\section{POLYSTICTUS Fr.}

P. PERGAMENUS Fr.

On dry exposed oak railroad tie, Monongalia Co., near Morgantown (Millspaugh). On various dead trees, Feb. I7, I893 (Nuttall, 847).

P. cinnabarinus (Tacq.) Fr.

On dead limbs of cultivated Prunus, Monongalia Co., near Morgantown (Millspaugh). On various dead deciduous trees, Jan. 31, I893 (Nuttall, 829). Randolph: on Betula lenta near Pickens (H. H. Smith, 1848).

P. Abietinus Fr.

On fallen Tsuga Canadensis, Grant Co., near Bayard (Millspaugh). Ôn Pinus Virginiana, March 4, 1894 (Nuttall, 1420, 390).

\section{CORIOLUS Quel.}

C. Pubescens (Schum.) Murrill. (Polyporus pubescens Fr.). On dead Betula sp. Fayette: near Nuttallburg (Nuttall, 1673). On Fagus, Upshur: near French Creek, and Monongalia: near Morgantown (Sheldon).

C. abietinus (Dicks.) Quel.

On Picea rubens, Pocahontas: Cranberry Glades; on Tsuga, Monongalia: near Cooper's Rock and Lick Run (Sheldon).

C. VERSICOLOR (L.) Quel. (Polystictus versicolor Fr.).

On Juglans nigra and on Betula, Monongalia: near Morgantown; on Betula, Pocahontas: at Cranberry Glades and Preston: near Albright (Sheldon); on decorticated stump Picea Mariana, Tucker Co., near Falls of Blackwater. Under bark of log Quercus alba, Monongalia Co., near Morgantown (Millspaugh). On various dead trees, Feb., I893 (Nuttall, 834). Randolph: near Pickens on Betula (H. H. Smith, 1850).

C. PROlificans (Fries) Murrill. (Polyporus prolificans Fr.).

On Betula, Pocahontas : at Cranberry Glades, on Quercus, near Seebert; on Fagus, Preston : near Albright; on Prunus Americana, Monongalia : near Morgantown (Sheldon).

C. Nigromarginatus (Schw.) Murrill. (Polystictus hirsutus Fr.).

On Liriodendron, Monongalia : near Oliver; on dead wood 
Pocahontas : Cranberry Glades; on Betula, Monongalia : near Lick Run (Sheldon) ; on dead twig cultivated Pyrus Malus, Monongalia Co., near Morgantown. On dead Betula lutea, and on log Liriodendron Tulipifera. A form with white spores, on roots fallen Betula lenta, Grant Co., near Bayard (Millspaugh). On Fagus atropunicea, Keeney's Creek, Oct. 20, I893 (Nuttall, I2I I).

\section{CORIOLELLUS Murrill.}

C. Sepium (Berk.) Murrill. (Trametes Sepium Berk.).

On dry railroad ties, Monongalia: near Morgantown (Millspaugh), on Juglans nigra (Sheldon). Resupinate form on dead oak beams over a boiler, Fayette: near Nuttallburg (Nuttall, I300).

\section{MUCRONOPORUS E. \& E.}

M. Everhartil E. \& Gall.

On Ulmus pubescens, Nov. 6, I893 (Nuttall, I 598).

M. Gilvus (Sz.) E. \& E.

On Castanea dentata, July 20, I894 (Nuttall, II69, 558). M. Ferruginosus (Schrad.) E. \& E.

On Juglans cinerca, Aug. 29, I893 (Nuttall, I I83).

\section{PORIA Pers.}

P. vUlgaris Fr.

On various dead limbs, Feb. 2 and Nov. 20, I893 (Nutt. all, 833). A nodular form on oak ties, Nov. 5, I894 (Nuttall).

P. obducens Pers.

On underside of dead log, Feb. 8, I893 (Nuttall, I297, 246).

P. NIGRA Berk.

On side and beneath dead Hicoria? log. Effused, long and narrow, perennial (Nuttall, I268).

P. TOMENTO-CINCTA B. \& R.

Effused on bark underside of log Betula lenta, $60 \times 30 \mathrm{~cm}$ One layer taken off on Sept. I6, the same surface of the bark was found to be again covered with the species on Oct. 5, I895. (Nuttall, I865, 752). On Magnolia Fraseri, Nov., 1895 .

P. VAPORARIA Fr.

On Prunus serotina, Dec. 8, I893 (Nuttall, I295, 244). P. Tulipiferae Schw.

On Liriodendron Tulipifera, April I3, 1893 (Nuttall, $893)$. 
P. Xantha Fr.

On Pinus Virginiana, March 21, I893 (Nuttall, 913).

P. MICANS (Ehrenb.) Fr.

On burned log, Short Creek, alt. I,300 ft., Nov. 24, I893

(Nuttall, 1277, 223).

P. CORTICOLA Fr.

Underside of young dead Liriodendron Tulipifera, diffused through clefts of bark, incrusting other polypori, etc. Alt. 925 ft., Nov. 20, 1893 (Nuttall, 1280).

P. sinuosa Fr.?

Appears to be between this and Irpex obliquus, preferably this (E. \& E.). On Kalmia latifolia, Short Creek,alt. I,800 ft., Aug. I2, I893 (Nuttall, I166, I38).

F. FARINELLA Fr.

On dead log, Short Creek, alt. 1,800 ft., Sept. I2, I893 (Nuttall, 1204, 160).

T. Ohioensis Berk.

\section{TRAMETES Fr.}

On dead standing sapling, Short Creek, alt. I,800 ft., April .0, I894 (Nuttall, I467).

T. ROBINIOPHILA Murrill.

On wounds of Robinia pseudacacia, Monongalia: near Morgantown (Sheldon).

T. MOLLIS Fr.?

On drift wood, March, I893 (undeveloped) (Nuttall, I 597, 550).

T. ODORATA Wulf.

On dead limbs in a drain, July 20, I894 (Nuttall, I628, 557). Ozonium auricomum, Link., a curious tawny mycelium, supposed to be intimately connected with this species, was found here with this specimen.

\section{DAEDALEA Pers.}

D. confragosa (Bolt.) Pers.

On dead Cornus florida, Fayette: near Nuttallburg (Nuttall, I774). On Betula, Monongalia: Lick Run, and on Quercus near Star City; on Betula, Pocahontas: Cranberry Glades (Sheldon).

D. Aesculi (Schw.) Murrill.

(D. ambigua Berk.).

On dead Acer, Fayette: near Nuttallburg (Nuttall, 846).

\section{CERRENA (Micheli) S. F. Gray.}

C. UNICOLOR (Bull.) Murrill.

(Daedalea unicolor Fr.).

On dead Hicoria ovata, Fayette: near Nuttallburg (Nuttall, 247). Randolph: near Pickens, on Fagus (H. H. Smith. I829). 
M. INDURATUS Peck.

MYRIADOPORUS Peck.

Top of decayed oak stump, Monongalia Co., near Morgantown (Millspaugh). Professor Peck remarks, in a letter, on receipt of the specimens: "This is probably only an imperfect condition of Poria abducens."

\section{HEXAGONA Pollini.}

H. alveolaris (DC.) Murrill. (Favolus Europaeus Fr.).

On dead wood, Monongalia: on Dorseys Knob near Morgantown (Sheldon). On dead branch, Feb. I2, I894 (Nuttall, I360). Spores I2.5 × $3 \mu$ somewhat curved, obliquely apiculate. Feb. I7, I895 (Nuttall, I788).

G. CONCHOIDES Mont.

\section{GLOEOPORUS Mont.}

On oak chips, Monongalia Co., near Morgantown (Millspaugh). On decaying log, Monongalia: at Sabraton and on Prunus serotina at Mona : on apple, Preston: at Albright. and on dead wood, Wayne: near Ceredo (Sheldon).

\section{MERULIUS Hall.}

M. TREMELlosus Schrad.

Under bark Quercus alba log, Monongalia Co., near Morgantown (Millspaugh). On Alnus rugosa, Oct. 3I, I893 (Nuttall, I220).

M. RUBELlus Peck.

On dead Quercus alba, Short Creek, Nov. 9, I893 (Nuttall, 1244).

Also found parasitic on Stereum versicolor. In some cases the hymenium simply covers the under surface of the Stereum. In nearly all specimens observed it grows at the base of the host to which it is at least partly adherent. Largest pileus $5 \times 5 \mathrm{~cm}$., sometimes laterally connected for I $5 \mathrm{~cm}$.

N. Corium Fr.

On branches, April 27, I895 (Nuttall, I822, 699).

M. Molluscus Fr.

On dead limb Tsuga Canadensis, Short Creek, alt. I,800 ft., Feb. 8, I894 (Nuttall, I399).

M. Ambiguus Berk.

On' dead Prunus serotina, Short Creek, alt. 975 ft., Jan., I894 (Nuttall, I602).

M. Lachrymans (Jacq.) Fr.

On bottom of box in cellar, Feb. I4, I894 (Nuttall, I379, $34 \mathrm{I})$. 
M. PUlverulentus Fr.

Underside of old logs, Oct. I9, I893. Spores orange colored, nucleate, I0 $66 \mu$ (Nuttall, 1208).

\section{POROTHELIUM Fr.}

P. LACERUM Fr.

On dead trunk, Feb. I I, I894 (Nuttall, I38I, 343).

\section{SOLENIA Hoffm.}

S. OCHRACEA Hoffm.

On dry dead log, Short Creek, alt. 1,800 ft., Dec. I6, I893 (Nuttall, i327, 285).

S. villosa Pers.

On fallen dead limb of Quercus sp., Sept., I895 (Nuttall, I854, 74I).

\section{H Y D N A C E A E.}

\section{HYDNUM Linn.}

H. REPANDUM Linn.

Fayette: near Nuttallburg (Nuttall).

H. Cinnabarinum (Schw.) Fr.

Fayette: effused under bark of dry dead limb near Nuttallburg (Nuttall, 997).

\section{HERICIUM Pers.}

H. Coralloides (Scop.) Pers. (Hydnum coralloides Scop.).

Fayette: in decayed cleft on living Fagus near Nuttallburg (Nuttall, 845).

H. Erinaceus (Bull.) Pers. (Hydnum Erinaceum Bull.).

Fayette: on top of dead log and in hollow of living Fagus near Nuttallburg (Nuttall, I752). On Robinia (Sheldon).

H. CROCEum (Schw.) Banker. (Hydnum croceum Fr.)

Fayette: effused in crevices of dead log near Short Creek (Nuttall, 398, 1426).

\section{STECCHERINUM S. F. Gray.}

S. Adustum (Schw.) Banker. (Hydnum adustum Schwein.).

Fayette: on dead limbs on the ground. Nuclei at first cream colored gradually changing from perifery to center through reddish purple to dark brown. Near Nuttallburg (Nuttall, 863).

S. ochraceum (Pers.) S. F. Gray. (Hydnum ochraceum Pers.)

Underside of dead log. Stcgonotis fusca found growing on this species. Fayette, near Nuttallburg (Nuttall, 89I). 
SARCODON Quelet.

S. IMBRICATUS ${ }^{\circ}$ (L.) Karst.

(Hydnum imbricatum L.).

Fayette: in oak woods near Nutallburg (Nuttall, 988).

\section{HYDNELLUM Karsten.}

H. velutinum (Fries.) Banker. (Hydnum velutinum Fr.).

Fayette: near Nuttallburg (Nuttall, 880).

H. Nuttalli Banker, Mem. Torr. Bot. Club, I2:I55 (I906).

Plant terrestrial, mesopodous, medium size; pileus obconic, deeply depressed to infundibuliform, somewhat round, thickest at the center, thinning uniformly to the margin, 4 to $6 \mathrm{~cm}$. wide, I $\mathrm{cm}$. or less thick near the center; surface subeven, radiately subrugose or fibrillose when fresh, grayish umber; margin thin, acute, coarsely plicate or fluted, deflexed, curled when dried, subfertile with short teeth; substance spongy-tomentose in upper half of pileus, thickest at the center, umber, lower part of pileus harder more compact darker of uniform thickness about I mm., continuous with hard central core of stem; stem central, uneven, surrounded below by bulbous mass of spongy tomentum, $0.5 \mathrm{~cm}$. wide, $4 \mathrm{~cm}$. long, bulbous base $1.5 \mathrm{~cm}$. wide; teeth capillary more or less decurrent, dark umber to black, $7 \mathrm{~mm}$. or less long, O.I 5 to $0.25 \mathrm{~mm}$. wide, about 2 to I millimeter, longest teeth about one-fourth of the distance from the stem to the margin; spores apparently few, subtuberculate, subglobose, brownish, 4 to $5 \mu$ wide.

The plant in structure is similar to $H$. velutinum, but very distinct in the form of the pileus, in its subrugose not tomentose surface, and in the long capillary teeth.

Fayette: on ground beneath old decayed log, February I3. I893, near Nuttallburg (Nuttall, 844). Reported in Millspaugh \& Nuttall Flora as Hydnum sp.

\section{TREMELLODON Pers.}

T. gelatinosum (Scop.) Pers.

On dead Tsuga Canadensis Aug. I3, I893 (Nuttall, I I48)

\section{IRPEX Fr.}

I. LACTEUS Fr.

On dead Rhus hirta, Monongalia Co., near Morgantown (Millspaugh).

I. SINUOSUS Fr.

On dead sticks, Dec. Io, 1893 (Nuttall, I309, 255).

I. obliQuUs (Schrad.) Fr.?

On Magnolia Fraseri, Feb. 8, I894 (Nuttall, I385, 347). 
R. ORBICULARE Fr.

RADULUM Fr.

On Carpinus Caroliniana? Nov. 27, 1893 (Nuttall, I270).

R. Magnoliae B. \& C.

On Magnolia Fraseri, Feb. 9, I894, Short Creek, alt. I,800 ft. (Nuttall, I352).

PHLEBIA Fr.

P. MERISMOIDES Fr.

On mossy bark of dead log, Dec. I, I893 (Nuttall, I289). On Magnolia Fraseri, Nov., 1895.

P. RAdiata Fr.

On Alnus rugosa, Oct. 31, I893 (Nuttall, 1219).

\section{GRANDINIA Fr.}

G. GRANULOSA Fr.

On underside dead log, May 20, I893 (Nuttall, I304).

G. crustosa (Pers.) Fr.

On Rhododendron maximum, May 20, 1893. On Liquidambar Styraciflua, Oct. 2, I894 (Nuttall, 909 ident. Massee).

\section{ODONTIA Pers.}

O. FARINACEA Fr.

Underside dead Tsuga Canadensis, Nov. 9, 1893, Short Creek, alt. I,300 ft. (Nuttall, I245).

\section{NEOKNEIFFIA Sacc.}

N. SETIGeRA (Fr.)

(Kneiffia setigera Fries).

In interstices dead log, Dec., I893 (Nuttall, 1290, 23I).

TH E LE P H O R A C E A E.

\section{THELEPHORA Ehrh.}

T. Sebacea Pers.

On stem of Aster sp., damp shady woods, July 25, I893 (Nuttall, I I20).

T. PEDicellata Schwein.

On Ostrya Virginiana, Feb. 8, I894 (Nuttall, I370, 328). On Crataegus, Greenbrier: near Durbin (Sheldon).

STEREUM Pers.

S. versicolor (Sw.) Fr.

On Quercus sp. and Betula lenta, March 7, I893 (Nuttall, 856). 
S. Fasciatum Sz.

In dead stumps, alt. 2,000 ft., March 8, I893 (Nuttall. $860)$.

S. VERSICOLOR var. (Peck).

On dry oak railroad ties, Monongalia Co., near Morgantown (Millspaugh).

S. LOBATUM Fries.

On Fagus, Preston: near Albright; on Quercus alba. Upshur: near French Creek (Sheldon).

S. PURPUREUM Pers.

On stump in pine woods (Liriodendron?) Dec. 9, I893

(Nuttall, 128I, 39I).

S. HiRsutum (W.) Fr.

On log, Nov. 24, I893. Short Creek, alt. I,300 ft. (Nuttall, 1277, 224).

S. sanguinolentum (A. \& S.) Fr.

On Tsuga Canadensis, Feb. 8, I894 (Nuttall, I386, 25I)

S. SUlphuratum B. \& Rav.

On log Quercus alba, Monongalia Co., near Morgantown (Millspaugh).

S. Frustulosum (Pers.) Fr.

On log Quercus sp., Monongalia Co., near Morgantown

(Millspaugh). On dead wood, March 7, I893 (Nuttall, $857)$.

S. PINI Fr.

- On Pinus Virginiana, April 6, 1894 (Nuttall, 1469, 445).

S. OChraceo-Flavum Schw.

On small dead twigs, March 21, I894 (Nuttall, I4I6).

S. Albo-BAdium Schwein.

On dead sticks and twigs of various trees, April 27, I893 (Nuttall, 1269).

S. SERICEUM Schw.

On dead twig Betula lenta, Grant Co., near Bayard (Millspaugh). On,Rhus copallina, March 6, I894 (Nuttall, I4I9, 389).

S. Complicatum Fr.

On roots of overturned Betula lenta, Grant Co., near

Bayard; on dry oak railroad ties, Monongalia Co., near Morgantown (Millspaugh). On dead trees and on Quercus Prinus, Dec. 8, I893 (Nuttall, 867, 235). On Fagus, Preston: near Albright; on Abies or Tsuga, Pocahontas:

Cranberry Glades (Sheldon).

S. VERSIFORME B. \& C.?

On Castanea dentata, Jan. I8, I893 (Nuttall, I347, 305).

S. TRISTE B. \& C.

On Rhododendron maximum in thicket, alt. I,800 ft. Feb. 8, I894 (Nuttall, 966, 350). 
S. ACERINum Pers.

On living tree trunks, March 7, I893 (Nuttall, 858).

HYMENOCHAETE Lev.

H. Rubiginosa (Schr.) Lev.

On dead log, March, í893 (Nuttall, 9II). On Robinia pseudacacia, Monongalia: near Morgantown; on Quercus alba, Preston: near Terra Alta (Sheldon).

H. tabacina (Sow.) Lev.

On dead twigs, March, I893 (Nuttall, 910); on Acer, Pocahontas: Cranberry Glades (Sheldon).

H. CORrugata (Fr.) Lev.

On decorticated limb Betula lenta, Grant Co., near Bayard (Millspaugh). "On Magnolia Fraseri, Feb. 8, I894. Setæ $38 \times 7.5 \mu$ (Nuttall, I364).

H. UNiCOLOR B. \& C.?

On Betula lenta, on dead Kalmia latifolia? 'July I, I893 (Nuttall, I I I , 96).

C. SAlicinum Fr.

CORTICIUM Fr.

On dead Salix nigra, March. I5, I894 (Nuttall, I4OI).

C. Oakesil B. \& C. $877)$.

On living Ostrya Virginica, March 21, I893 (Nuttall.

C. GLOBIFERUM E. \& E:"

Underside of dead log in deep laurel thicket, Short Creek. .March 25, I893 (Nuttall, 940).

C. ARACH NOIDEum Berk.

On dead damp twigs in dense pile, Jan. I8, I894. Diffused for $30 \times 8 \mathrm{~cm}$. Spores 4 to $5 \times 2$ to $3 \mu$. The species when fresh has a wrinkled or crusted appearance looking very like a Merulius, but soon becomes flat and plane (Nuttall, I344, 303).

C. RADIOSUm Fr.

On dead trunk, partly beneath loose bark, Short Creek, May I8, I893 (Nuttall, 965).

C. LEVE Pers.

On Hamamelis Virginica, Dec. 20, I894 (Nuttall, I500). C. albo-flavescens E. \& E., Field Mus. Bot., i :I70 (i896).

Type, навітат: On bark of dead Tsuga Canadensis, Feb. 24, I894 (Nuttall, discov. I394).

At first orbicular, subpezizoid, I to $2 \mathrm{~mm}$. diameter, becoming more irregular in shape and $\mathrm{I} \mathrm{cm}$. or more diameter, lower stratum milk-white, consisting of loosely interwoven, branching threads, and extending out beyond the pale-olivaceous or yellowish, membranaceous hymenium so as to form 
a thin sub-fimbriate, white margin. Spores abundant, globose, with a single large nucleus, 4 to $6 \mu$ diameter, slightly colored.

The hymenium has only a slight tinge of olive and is perhaps better described as light-drab color.

C. leptaleum E. \& E., Field Mus. Bot., i :i7o (i896).

Type habitat: On underside dead sapling Magnolia Fraseri, alt. I,800 ft. April I I, I895' (Nuttall, discov. 'I803, 690).

Thin, closely adnate, grayish-white, pulverulent, soon cracking into areas 2 to $3 \mathrm{~mm}$. across, with margins incurved so as to resemble the shallow cups of some Peziza. Spores oblong, hyaline, continuous, Io to I $2 \times 4 \mu$, borne on cylindrical basidia 30 to $40 \times 6 \mu$, with two stout sporophores? There are also other smaller (immature)? sporidia, globose ( 4 to $6 \mu$ ) or elliptical, 5 to $7 \times 3$ to $3.5 \mu$. The membrane on which the hymenium stands where exposed on the incurved margin of the pezizoid areas is pale brown.

C. Petersi B. \& C.

Ọ̣ Betula sp. and Vitis sp., March 21, I893 (Nuttall, 912).

C. colliculosum B. \& C.

On dead logs in laurel thicket, Short Creek, May, I893 (Nuttall, I088).

C. PRASINUM B. \& C.

On dead, burned log, Feb. 2, I894 (Nuttall, I382, 344).

C. Lilacino-Fuscum B. \& C.

On dead limb Hicoria sp., Short Creek, alt. I,800 ft., March 5, I894 (Nuttall, 1403).

C. OCHRACEUM Fr.

On underside dead log Tsuga Canadensis, May 8, I893 (Nuttall, 93I).

C. incarnatum (Pers.) Fr.

On underside dead $\log$ and on Ulmus pubescens, April I8, I893 (Nuttall, 923).

C. LILACinum Schroet.

On firm wood of dead log, March 6, I894, Short Creek, alt. I,800 ft. (Nuttall, I427, 399).

C. SUbGiganteum Berk.

On Hicoria ovata, Dec. I4, 1893. Spores $16 \times 5 \mu$ (Nuttall, 1312, 262).

C. martianum B. \& C.

On underside of wet $\log$, Dec. I6, I893. When fresh, this species is the color of fresh blood. Short Creek, alt. I,800 ft. (Nuttall, I324, 28I).

C. scutellare B. \& C.

On Magnolia Fraseri, Sambucus Canadensis and Pinus Virginiana, Aug. I8, I893 (Nuttall, I176, I44). 
ASTEROSTOMA Massee.

A. CORTicolum Massee.

On wild vine $V i t i s$ sp., in pine woods, alt. 2,000 ft., Feb. I893 (Nuttall, 1000).

A. CERVICOLOR (B. \& C.) Massee.

On bark of living Quercus sp., Short Creek, alt. I,800 ft. (Nuttall, I 325,282 ).

\section{PENIOPHORA Cooke.}

P. Quercina (Fr.) Cooke.

On Castanea dentata, Jan. I8, I893 (Nuttall, I346, 305)

P. CINEREA (Fr.) Cooke.

On dead limb, March, I893 (Nuttall, 945, I70).

\section{CONIOPHORA DC.}

C. $\mathrm{sp}$.

Near C. submembranacea, (B. \& Br.). On Zea Mays, Aug. Io, I895 (Nuttall, I846, 737).

C. olivacea (Fr.) Karst.

In dark place underside old railroad ties, Nov. 5, 1894 (Nuttall, I750, 631).

\section{EXOBASIDIUM Woron.}

E. RHODODENDRI Cram.

Forming "cups" near the tips or margins of living leaves of Rhododendron maximum. Common in Grant and Tucker Counties. I understand from Prof. Peck that this is his first knowledge of the occurrence of this species in North America (Millspaugh).

\section{L A V A R I A C E A E.}

\section{CLAVARIA Vaill.}

C. Amethystina Bull.

On ground, June 20, I893 (Nuttall, 993)

C. pyxidata Pers.

On rotten log, June Io, I893 (Nuttall, 975).

C. Formosa Pers.

On ground in laurel thicket, and deep woods, alt. 2,00s ft., July 9, I893. Spores I2.5 x $5 \mu$ (Nuttall, 1085).

C. FlACCIDA Fr.

On leaf mold, in deep woods, Grant Co., near Bayard (Millspaugh).

C. FUSIFORMIS Sowerb.

On low ground in open woods, alt. 2,000 ft., July 9, I893 (Nuttall, 1086). 
C. JUNCEA Fr.

On leaf mold in bottom of dead hollow stump. Oct. 28, I893 (Nuttall, 1232, I79).

C. MUCIDA Pers.

On upper side dead wet logs, Oct. 20, I893 (Nuttall, I23I, I78).

C. viscosa (Pers.) Fr. CALOCERA Fr.

On bark of Magnolia Fraseri, Short Creek, alt. I,800 ft., Feb. 8, 1894. Spores 12.5 x $4 \mu$ (Nuttall, I387, 354).

\section{LACHNOCLADIUM Lev.}

L. Semivestitum B. \& C.

On dead wet limbs, alt. I,800 ft., Sept. I8, I895. Three to five inches high (Nuttall, 1867, 753).

\section{AURICULARIA Bull.}

A. Auricula (L.) Underw. (Hirneola Auricula-Judae Berk.).

On damp sticks, June I5, I893, Short Creek (Nuttall, 993).

E. gLAndulosa (Bull) Fr.

EXIDIA Fr.

On bark Juglans cinerea, alt. 2,000 ft., Feb. 20, 1893 (Nuttall, 946).

E. ALba (Huds.) Bref.

On dead log, June 23, I893 (Nuttall, 999).

\section{ULOCOLLA Bref.}

U. Foliacea (Pers.) Bref.

On dead twig, Nov. Io, 1893. Spores $+12.5 \times 6$ to 7 is (Nuttall, I28I).

CRATEROCOLLA Brefeld.

C. clavus (Cke.)

(Ombrophila Quel.)

On decaying leaves and wood (Sheldon, 2979).

T. FRONDOSA Fr.

\section{TREMELLA Dill.}

On Rhododendron maximum, Dec. I6, I893 (Nuttall, I32I).

T. AURANTIA Fr.

On bark Pinus Virginiana, alt. 2,000 ft., March 20, I893 (Nuttall, 947).

T. INTUMESCENS Sm.?

On dead limbs Hicoria sp., Short Creek, Dec. I6, I893. Spores Io to I $3 \times 3$ to $4 \mu$, curved (Nuttall, I332). 
T. VIRENS Schwein.

On dead branch Cornus florida, Aug. I7, I893 (Nuttall, I I55). Spores mostly $7 \times 5 \mu$ or round $7 \mu$ diameter.

NAEMATELIA Fr.

N. Nucleata (Schw.) Fr.

On Aralia spinosa, alt. I,300 ft., Aug. I8, I893 (Nuttall, I I74, I42). On Magnolia Fraseri.

\section{DACRYOMYCES Nees.}

D. $\mathrm{sp}$.

Of this form Mr. J. B. Ellis says: "It seems to be a Dacryomyces. I find the same thing here at Newfield, N. J., but have never been able to decide upon'it, and though I have sent it to various European mycologists, I have never received a definite opinion from them." The form occurs on oak limbs on ground, Dec. 1893 ; is of a bright orange-red color, with curved spores 7.5 to Io $\times 4 \mu$ (Nuttall, 127r, 217.)

D. Deliquescens (Bull.) Dub.

On underside dead rotten limb Pinus Strobus, Dec. I3, I894 (Nuttall, I770).

D. Syringae (Schum.)?

On dead limbs of Platanus occidentalis, Nov. IO, 1895 (Nuttall, 1889).

D. Stellatus Nees.

On clean dead wood Tsuga Canadensis, Dec. 20, 1893 (Nuttall, I33I).

D. CORTICIOIDES Ell.

On decayed Tsuga Canadensis, Short Creek, alt. I,750 ft., Dec. I6, I893. Lemon yellow $.25 \mathrm{~cm}$. to $3.5 \times 1.5 \mathrm{~cm}$. Spores hyaline, nucleate 9 to $12.5 \times 5$ to $7 \mu$ obliquely apiculate at one end (Nuttall, 13I6, 27I).

D. MINOR Peck?

On Kalmia latifolia, Short Creek, alt. I,800 ft., March, I894. Spores Io to $12 \times 4 \mu$ (Nuttall, I 532).

\section{GÚEPINIA Fr.}

G. Spathularia (Schw.) Fr.

On Quercus sp., Jan. I, I894. Spores uniseptate Io ₹s $4 \mu$ (Nuttall, I782).

HOROMYCES Bon.

H. Fragiformis Cooke. Hypsilophora fragiformis (Fr.) Lev. On bark dead Fagus atropunicea, Grant Co., near Bayard (Millspaugh). On dead wood, May I8, I893 (Nuttall, 963.) 


\section{Lichenes.}

U S N A CE AE.

RAMALINA Ach.

R. RIGIDA (Pers.) Tuck.

On trees, alt. 2,000 ft. (Nuttall, 1060).

R. CALCiCARIS FASTigiata Fr.

On trees, alt. 2,000 ft. (Nuttall, 869).

R. miniuscula Nyl.

On Acer saccharinum, alt: 2,000 ft. (Ninttall, 1696).

CETRARIA Ach.

C. Ciliaris (Ach.) Tuck.

On Pinus Virginiana, alt. I,800 ft. (Nuttall, 861).

C. Lacunosa Ach.

On Pinus Virginiana, alt. I,800 ft. (Nuttall, I053).

C. AURESCENS Tuck.

On Rhododendron maximum, alt. I,850 ft. (Nuttall, r050).

C. Islandica (-L.) Ach. Reincieer Moss.

High barren places, Wood: near Nicollet (H. H. Smith).

C. Oakesiana spinulosa Merrill ined.

On Picea rubens (Sheldon, 3699), and on Kalmia latifolia (366r).

U. barbata (L.) Fr.

USNEA Ach.

Wirt Co., on old trees, common, Burning Springs. Randolph Co., on Rhododendron maximum, common, Cheat Bridge. Mercer Co., on oak twigs, Bluefield; and elsewhere about State, common on trees, rocks and old fence rails (Millspaugh). On trees, alt. 2,000 ft. (Nuttall, 819).

U. BARBATA FLORIDA Fr.

Mercer Co., on oak chips and twigs among dead leaves at Bluefield (Millspaugh). On trees, alt. 2,00o ft. (Nuttall, 859 ).

U. TRICHODES Ach.

On spruce (Sheldon, 3693).

THELOSCHISTES Norm.

T. PARIETINUS (Linn) Norm.

On Rọinia Pseudacacia, alt. 2,000 ft. (Nuttall, II45). 
T. CONCOLOR (Dicks.) Tuck.

On Robinia Pseudacacia, alt. 2,000 ft. (Nuttall, II44).

T. CONCOLOR EFFUSA Tuckerm.

Summers: near Lowell (Pollard \& Maxon, I50).

E. Furfuracea (L.) Mann.

EVERNIA Ach.

On rock; on trunks of trees (Sheldon, 2486, I 544, 386I).

\section{P A R M EL I A C E AE.}

\section{PARMELIA Ach.}

P. Perlata (Linn.) Ach.

On Acer rubrum, and on sandstone boulders (Nuttall, 848).

P. BORRERI Turn.

On Oak, alt. i,850 ft. (Nuttall, Iо32).

P. Borreri RUdecta Tuck.

On Robinia Pseudacacia, Monongalia Co., along Falling Run (Millspaugh, I 53I).

P. COLPODES (Ach.) Nyl.

On Oak, alt. I,850 ft. (Nuttall, II4I).

P. olivacea (L.) Ach.

On Liriodendron log newly felled, Monongalia Co., Falling Run (Millspaugh, I343).

P. CAperata (L.) Ach.

Monongalia Co., on sandstone rocks, and base of Beech,

Falling Run (Millspaugh, 1283).

P. CETRATA Ach.?

Sterile, but probably referable to this species. Summers: near Lowell (Pollard \& Maxon, I46).

P. tiliacea (Hoffm.) Flk.

Summers : near Lowell (Pollard \& Maxon, I 5I).

P. PHysodes (L.) Ach.

On the bark of various trees (Sheldon, I 532, 3456, 3663, $3668)$; on roof of shed, $(3056)$.

P. CONSPERSA (Ehrh.) Ach.

On a rock (Sheldon, 2160).

P. olivaria (Ach.) Hue.

On rock (Sheldon, 3344).

P. exasperata (Ach.) DeNot.

On bark of Prunus serotina (Sheldon, 3735).

P. RUdecta Ach.

On bark of Red Oak (Sheldon, 3946). 
P H Y S C I A C E AE.

P. SOREDiATA Fr.

$$
\text { PYXINE Nyl. }
$$

On trunk of Ash (Sheldon, 3690).

\section{PHYSCIA DC.}

P. Lucomela (L.) Michx.

Mercer Co., with moss on wet limestone ledge, Beaver Spring (Millspaugh, I539).

P. CILIARIS (Linn.) DC.

On mossy sandstone rocks ( Nuttall, 1063).

P. CILIARIS CRINALIS Schær.

On moss, alt. I,850 ft. (Nuttall, 1042).

P. AQUila (Ach.) Nyl.

On rocks and on moss (Nuttall, I035).

P. STEllaris (Lirn.) Tuck.

On standstone rock (Nuttall, 872).

P. Stellakis aipolia Nyl.

On sandstone rock (Nuttall, I778).

P. LEUCOMELAENA (L.) Wain.

On bark (Sheldon, 3701).

P. speciosa (Wulf.) Nyl.

On a tree trunk with mosses (Sheldon, 3696).

P. comosa (Eschw.) Nyl.

On Red Cedar twigs (Sheldon, 2722).

$$
\text { U M B IL I C A R I A C AE. }
$$

UMBILICARIA Hoffm.

U. Dillenir Tuck.

On sandstone rocks (Nuttall, 823).

U. Pennsylvanica Hoffm.

On sandstone rocks (Nuttall, 849).

U. pustulata (Linn.) Hoffm.

On faces of sandstone cliffs (Nuttall, 855).

U. papulosa (L.) Hoffm.

On rock (Sheldon, 249I).

U. Muhlenbergit (L.) Hoffm.

On rocks at the summit of White Top Mountain (Sheldon, 2480).

PEL T I G E R A E AE.

LOBARIA Hue.

L. amplissima (Scop.) Arn.

On trunks of various trees (Sheldon, I300, 2470, 345I, $3702,3963)$. 
STICTA Schreb.

S. crocata (L.) Ach.

On bark (Sheldon, 3709).

S. HERBACEA (Huds.) Ach.

McDowell Co., on oaks, Elkhorn (Millspaugh).

S. ERosa (Eschw.) Tuck.

On trees, alt. 1,850 ft. (Nuttall, 982).

S. PUlmonaria (L.) Ach.

Wirt Co., on trunks of oaks, near Burning Springs (Mills- paugh, 327). On trees, alt. I,875 ft. (Nuttall, 824).

S. Quercizans (Michx.) Ach.

On trees and rocks (Nuttall, I057).

PELTIGERA Will.

P. polydactyla (Neck.) Hoffm.

On soil (Sheldon, 649, I I 50, I455, I 543, 2578):

P. Aphthosa (K.) Hoffm.

Monongalia Co., on rock ledge, near Cassville (Millspaugh). On rocks among mosses, alt. I,80o ft. (Nuttall, IOgO).

P. - sp. non fruct.

On Rhododendron maximum (Nuttall, 938).

P. HORIZONTALIS (Linn.) Hoffm.

On rocks among mosses, alt. I,875 ft. (Nuttall, IOgI).

P. Canina (Linn.) Hoffm.

On earth, rocks and trees ( $N$ uttall, I061.)

NEPHROMA Ach.

N. Helveticum Ach.

On rocks (Sheldon, 2721, 2991, 4067).

\section{P A N N A R I A C E AE.}

PANNARIA Delis.

P. RUBiginosA (Thunb.) Delis.

On mossy logs (Nuttall, I055).

P. LEUCOSTICTA Tuck.

On mossy logs and rocks (Nuttall, 1064).

P. Nigra (Huds.) Nyl.

On sandstone rocks (Nuttall, IO77).

$$
\text { COLLE M A C E AE. }
$$

PHYSMA Zahlbr.

P. LURIDUm Mont.

On bark (Sheldon, 3685, 3698). 
COLLEMA Hoffm.

C. myochroum; Nyl.

On Rhododendron maximum, alt. 1,875 ft. (Nuttall, 1058).

C. FlaCcidum Ach.

On a ledge (Sheldon, 3398).

LEPTOGIUM Fr.

L. Pulchellum (Ach.) Nyl.

On oak (Nuttall, ro67).

L. TREMELloides (Linn. f.) Fr.

On sandstone rock (Nuttall, I093).

L. CHLOROMELUM STELLANS Tuck.

On Quercus coccinea, alt. 2,400 ft. (Nuttall, i i46).

LE C A N O R A C E AE.

PLACODIUM DC.

P. Elegans (Link.) D.C.

On sandstone boulders at base of cliffs, alt. I,800 ft. (Nuttall, 862).

P. CERINUM (Hedw.) Naeg. \& Hepp.

Monogalia Co., on bark of Beech, Falling Run (Millspaugh, I357). On Robinia Psendacacia, alt. 2,000 ft. (Nuttall, IO3O).

P. Ferrugineum (Huds.) Hepp.

On top of wet sandstone rock (Nuttall, I092).

P..camptidium Tuck.

On trees (Nuttall, I786).

P. RUPESTRE (Scop.) Br. \& Rostr.

Fayette: near Quinnimont (Pollard \& Maxon, 155).

P. cinnabarinum (Ach.) Aug.

On rock (Sheldon, 2288).

\section{LECANORA Ach.}

L. Conrctata Ach.

On pieces of sandstone under drip of wet cliff, alt. I,800 ft. (Nuttali, I780).

L. Subfusca (Linn.) Ach.

On trees and rocks ( Nuttall, I038).

L. ATra (Huds.) Ach.

Monongalia Co., on bark Liriodendron log, newly felled, Falling Run (Millspaugh, I342). On flat exposed surface sandstone rock, same locality (I287).

L. Deplanans Nyl., Bot. Gaz.; 22:334, (1896).

Thallus glauco-cinerascens tenuis areolato-rimosus determinatus; apothecia badio-rufescens (satis diluta) inṇata sub- 
concaviuscula, latit. $0.5-0.7 \mathrm{~mm}$; spora ellipsoidea, long. 0.015-16, crass. 0.009-1.010 $\mathrm{mm}$; epithecium inspersum. In toto gelatina hymenialis fulvo-rubescens.

Videtur species e stirpe Lecanora cervina, spermatiis ellipsoideis.

On rocks in bed of creek. Fayette: Short Creek, alt. I,300 ft. (L. W. Nuttall coll. no I I26).

L. VARIA (Ehrh.) Nyl.

On trees (Nuttall, I036).

L. FALLESCENS (Linn.) Schær.

On trees and fence rails (Nuttall, 937).

L. tartarea (Linn.) Ach.

On sandstone rocks (Nuttall, 886).

L. CERVina (Pers.) Nyl.

The light colored form on wet rocks; the dark form on dry rocks (Nuttall, 854).

L. PRIVIGNa (Ach.) Nyl.

On sandstone rocks (Nuttall, IO28).

L. MURALIS VERSICOLOR Fr.

On limestone rocks (Sheldon, 2654).

L. PALlida (Schreb.) Schær.

On bark (Sheldon, 3790).

\section{RINODINA Mass.}

R. OREINA (Ach.) Mass.

On sandstone rocks at base of cliff, alt. r,800 ft. (Nuttall, 952).

R. SOPHODEs (Ach.) Nyl.

On small sandstone rocks ( $N$ uttall, IO72).

PERTUSARIA DC.

P. velata (Turn.) Nyl.

On Magnolia Fraseri and other trees (Nuttall, I044).

P. multipuncta (Turn.) Nyl.

On sandstone rocks in bed of creek (Nuttall, ro43).

P. Leioplaca (Ach.) Schær.

On Oak (Nuttall, IO4I).

P. pustulata (Ach.) Nyl.

On trees (Nuttall, 853).

P. corallina (L.) Fr.

Fayette: near Quinnimont (Pollard \& Maxon, I40).

THELOTREMA Ach.

T. Subtile Tuck.

On trees (Nuttall, 1056). 
MYRIANGIUM Mont. \& Berk.

M. Duriaei (Mont. \& Berk.) Tuck.

On dead bush (Nuttall, 1062).

\section{L A D O I A C E AE.}

EPHEBE E. Fr.

E. LANATA (L.) Wain.

(E. pubescens Fr.).

On rocks in a rill (Sheldon, 3940).

\section{CLADONIA Hoffm.}

C. Mitrula Tuck.

Monongalia Co., on an old Beech log, Falling Run (Millspaugh, I346).

C. Cariosa (Ach.) Spreng.

On earth, Fayette Co. (Nuttall, 1066).

C. pyxidata (L.) Fr.

Monongalia Co., along Falling Run, on bare sandstone rocks (Millspaugh, I28I) ; on moss in clay soil (Millspaugh, 1285) ; on decayed log (Millspaugh, I338); base of Beech in soil (Millspaugh, I282): on earth (Nuttall, I03I).

C. fimbriata (Linn.) Fr.

On an old log, Fayette Co. (Nuttall, 1059).

C. FIMBRIATA tUBAEFORMIS Fr.

On an old $\log$ (Nuttall, I059).

C. GRACILIS (L.) Nyl.

Monongalia Co., along Falling Run, on decayed log (Millspaugh, I337; among mosses on clay soil (Millspaugh, I 286).

C. verticillatia Hoffm.

On soil (Sheldon, 2464, 3027).

C. Coccifera pleurota (Fckl.) Schær.

On a ledge (Sheldon, 4I08).

C. DEFormis (L.) Hoffm.

On soil (Bowers, 2500).

C. polycarpa epiphylla Merrill ined.

On a ledge (Sheldon, 4060).

C. strepsilis (Ach.) Wain.

On soil (Sheldon, 2427).

C. Subcariosa (Nyl.) Wain.

On soil (Sheldon, I298, I299, 23II, 2785, 1990).

C. sylvatica laxiuscula Wain.

On soil (Sheldon, 3682).

C. TURGIDA (Ehrh.) Hoffm.

Growing with Polytrichum commune (Sheldon, I 558).

C. uncralis (I.) Web.

On soil (Sheldon, I23I). 
C. GRACILIS verticillata Fr.

On earth (Nuttall, 885).

C. SOBolescens Nyl.

On earth (Nuttall, 839).

C. papillaria (Ehrh.) Hoffm.

On earth (Nuttall, I068).

C. DELicata (Ehrh.) Fl.

On top of stump (Nuttall, ro65).

C. caespiticia (Pers.) Fl.

On sandstone rocks ( Nuttall, 1075).

C. FURCATA RACEMOSA Floerk.

Monongalia Co., large patches on ground under chestnuts, at Dille's; Mercer Co., same growth under oaks, near Beaver Spring (Millspaugh) on earth (Nuttall, I045).

C. RANGiferina (L.), Hoffm.

Monongalia Co., on moss, Falling Run (Millspaugh, I36I); on earth, rocks and fence rails (Nuttall, 825).

C. macilenta (Ehrh.) Hoffm.

- On rotten logs ( Nuttall, IO70).

C. pulchella Schwein.

On dead wood (Nuttall, 838).

C. CRistatella Tuck.

Monongalia Co., on an old decayed log, Falling Run

(Millspangh, I336).

C. Sylvestris L.

On the mountain between Barrenshe Creek and Dry Fork,

McDowell Co., alt. r,700 ft., Aug. 6, I900 (Morris, Ir63).

C. squamosa (Scop.) Hoffm.

Fayette: near Quinnimont (Pollard \& Maxon, I43). On

a ledge (Sheldon 4I07), on a decaying log (23I3, 3386), on soil $(2482,3345,35$ I I $)$.

C. squamosa denticollis (Hoffm.) Flk.

Fayette: near Quinnimont (Pollard \& Maxon, I36).

LE C I D A C E AE.

BAEOMYCES Pers.

B. Roseus Pers,

On ground (Nuttall, 873).

B. Aeruginosus (Scop.) DC.

Sheldon on rock (2489), on decaying stump (2468), on black spruce bark (2472):

BIATORA Fr.

B. Russula (Ach.) Mont.

On bark of tree (Nuttall, I046). 
B. VARIANs (Ach.) Tuck.

On bark of tree (Nuttall, 978).

B. uliginosa (Schrad.) Fr.

On rotten stumps (Nuttall, I054).

B. Fuliginosa Ach.

On ground (Nuttall, I089).

B. Schweinitzir Tuck.

On Rhododendron maximum (Nuttall, I052).

B. RUBELla (Ehrh.) Rab.

On mossy trunk of tree (Nuttall, I029).

B. vernalis (L.) Fr.

Sheldon on Birch bark (2475), on Picea rubens (374I), among mosses on a ledge (4059).

B. Atrogrisea (Deli.) Heff.?

On bark of Magnolia acuminata (Sheldon, 3754).

B. Laureri Hepp.

On bark of Beech (Sheldon, 2466).

'B. Russelli Tuck.

On limestone ledge (Sheldon, 2652).

B. HYPNOPHILA Turn.

On moss (Sheldon, 3962).

B. Granulosa (Ehrh.) Poetsch.

On soil (Sheldon, 2579).

B. InUNdATA Fr.

On sandstone rock (Nuttall, I074).

B. Suffusa Fr.

On bark of tree. (Nuttall, ro95).

\section{COENOGONIUM Ehrbg.}

C. interpositum Nyl.

Growing with thallus of Cladonia. Fayette: near Quinnimont (Pollard \& Maxon, I4I, sterile.)

\section{LECIDEA Ach.}

L. Tennesseensis Nyl.

On sandstone rock ( $N$ uttäll, 1073).

L. Fuliginea Ach.

On upper side of dead log (Nuttall; i 787 ).

L. Nuttalli Calk \& Nyl, Bot. Gaz., 22 :334: ( I896.)

Apothecia nigra parva; epithecium impressum; spora fusca oviformis I-septata, long. 0.0I4-I6, crass. 0.005-6 mm.; hypothecium fuscum. In toto gelatina. hymenialis vinose rubescens. 
Super thallum Ricasolia sublaevis Nyl. Fayette: near Nuttallburg (L. W. Nuttall coll. no. I78I).

L. Virginiensis Calk. \& Nyl., Bot. Gaz., 22:333, (i896).

Thallus glaucescens tenuis lævigatus rimulosus citrinoflavus; apothecia fusca aut nigra convexiuscula immarginata latit. circ. $0.5 \mathrm{~mm}$., intus medio sordida; spora oblonga incoloris, long. 0.009-0.012, crass. 0.004-6 mm.; epithecium et pars media hypothecii dilute fuscescens. In toto gelatina hymenialis coerulescens, dein theca vinose rubescens.

E stirpe videtur Lecidea sanguineo-atra, prope Lecideam delineatam Nyl.

On sandstone rock under the drip of a wet cliff. Fayette: near Nuttallburg (L. W. Nuttall coll. no. I779).

L. ERICINA Nyl.

Bark of trees (Nuttall, I047).

L. Platycarpa steriza (Ach.) Nyl.

On a rock (Sheldon, 4030).

L. SPEIREA Ach.

Fayette: near Quinnimont (Pollard \& Maxon, I34).

L. Albocoerulescens (Wulf.) Schær.

Fayette: near Quinnimont (Pollard \& Maxon, 138).

L. CRustulata Ach.

On stones (Sheldon, 4058).

BUELLIA DeNot.

B. MYriocarpa (DC.) Mudd.

On bark of trees (Nuttall, i 185).

B. Petraea (Fl.) Tuck.

On sandstone rocks (Nuttall, IO7I).

B. Colludens Nyl.

On sandstone (Sheldon, 2289).

G R A P H I D A C E AE.

OPEGRAPHA Humb.

O. varia (Pers.) Fr.

Bark of trees (Nuttall, IO34).

O. ATRA Pers.

Bark of trees (Nuttall, I033).

O. minutula Merrill ined.

On sandstone ledge (Sheldon, 3987). 
GRAPHIS Ach.

G. SCRIPTA (Linn.) Ach.

On bark of trees (Nuttall, 105I).

G. SCRIPTA RECTA Schær.

On bark of Betula lutca and of B. lenta (Nuttall, I026).

G. Elegans (Sm.) Ach.

On bark of trees, Fayette Co. (Nuttall, I039).

G. DENDRITICA Ach.

On bark of Betula lenta, etc. (Nuttall, IO37).

\section{ARTHONIA Ach.}

A. CINNABARINA Wallr.

Bark of tree (Nuttall, 939).

A. Aleuromela Nyl., Bot. Gaz., 22 :334. (i896.)

Thallus albus subfarinaceus chrysogonidicus tenuissimus; apothecia subrotundata vel oblonga, prominula, latit. 0.4-0.5 mm.; spora oblongo-oviformis parte inferiore attenuata, I-septata, long. 0.0IO-I I, crass. $0.003 \mathrm{~mm}$. In toto gelatina hymenialis cœrulescens, dein obscurata.

Thallus detritus subaureus, $\mathrm{CaCl}$ vix reagens. Gonidia chroolepoidea fulvescentia.

On bark of Quercus sp. Fayette: near Nuttallburg, alt. 2,000 ft. (L. W. Nuttall, coll. no. I I82).

A. Dispersa (Schrad.) Nyl.

On bark of young Tsuga Canadensis, etc. (Nuttall, II42).

A. Astroidea Ach.

On bark of Liriodendron Tulipifera (Nuttall, I8I6).

A. PUNCTIFormis Ach.

On bark ( Nuttall, Iо76).

A. taediosa (Nyl.) Fr.

On bark (Nuttall, I069).

A. SPECtabilis F1.

On bark (Nuttall, I025).

AGYRIUM Fr.

A. RUFum (Pers.) Fr.

On smooth dead wood (Nuttall, I8I4).

\section{A L I C I A C E AE.}

\section{CALICIUM Pers.}

C. subtile Fr.

On sticks, dead leaves,. etc., under cliff (Nuttall, I226).

C. Tigillare (B. \& Br.) Sacc.

On Polyporus pergamenus, Aug. 23, I893 (Nuttall, I I59). 
VERRUCARIA Th. Fr.

V. Fuscella (Tum.) Ach.

Summers : near Lowell (Pollard \& Maron, I54).

ENDOCARPON Hedw.

E. miniatum (Linn.) Schær.

On wet cliffs (Nuttall, I049).

E. miniatum Muhglenbergir Schaer.

On rocks (Sheldon, I595).

E. Miniatum complicatum Schær.

On wet cliffs (Nuttall, i823).

T R Y P E T H E L I A C E AE.

T. vIRENS Tuck.

\section{TRYPETHELIUM Spreng.}

On Ilex opaca (Nuttall, ro40).

T. EXocanthum Tuck.

On Fagus atropunicea (Nuitall, I18I).

PYREN ULA CE AE.

PYRENULA Ach.

P. Rhyponta Ach.

On bark of Hamamelis Virginica (Nuttall, II84).

P. Nitida Ach.

On Betula lenta bark (Nuttall, 1027).

P. PACHyChEILA Tuck.

On bark of Fagus atropunicea (Nuttall, I048).

P. functella (Nyl.) Williams. (Verrucaria purictella Nyl).

Summers: near Lowell (Pollard \& Maxon, 156).

P. glabrata (Ach.) Mass.

On bark (Sheldon, 379I, not fully characteristic). 


\section{Hepaticæ.}

R I C C I A C E AE.

RICCIA Linn.

R. canaliculata Hoffm.

(Ricciella fluitans A. Br.).

The sterile, water form (syn. above). Sandy soil along edge of brook, Monongalia, near Ceredo, and on edge of small pond, near Morgantown (Sheldon, 3897, 3928).

\section{A R C H A N I A CEAE.}

REBOULIA Raddi.

R. sp.?

A sterile specimen on earth and rock, Monongalia, near Warntown (Sheldon, 363I).

CONOCEPHALUS Neck.

C. conicus (Corda) Schiffn. (Conocephalum conicum Dum.).

Monongalia: on ground and sandstone boulders in deep woods at Tibbs Run (Millspangh, I672); on rocks beside a stream near Randall, same situation north of Morgantown and on University Campus below Falling Run (Sheldon, I77, I308); also at Coopers Rock (Post, I669).

\section{LUNULARIA Adans.}

L. cruciata (L.) Dum.

In a greenhouse, Monongalia: at Morgantown (Sheldon. 294I).

\section{MARCHANTIA (L.) Raddi.}

M. FOLYMORPHA Linn.

In various situations, Monongalia: near Morgantown, between bricks of sidewalk (Millspaugh), on brick wall in Experiment Station greenhouse (Sheldon, 537); among damp mosses base of sandstone boulder, Tibbs Run (Millspaugh), on earth near the mouth of a coal mine at Richards (Sheldon). Preston: around the edge of a lime pile at Pisgah, on earth in spruce swamp near Cranesville (Sheldon, I53I, I462). Gilmer: near Glenville ( $\mathrm{Mapel})$. Fayette: near Nuttallburg (Nuttall). Randolph: on ground in a burned place at the summit of Point Mountain; Grant: in a 
similar situation near Bayard; Tucker: in a burning near Blackwater Falls (Millspaugh).

\section{J U N GER M A N N I C E A E. (Anacrogynae.)}

RICCARDIA S. F. Gray.

R. LATIFRONS Lindb.

Monongalia, a few plants growing with Scapania nemorosa on the north side of a bank at Sabraton (Sheldon, 2302).

R. mUltifida (L.) S. F. Gray. (Aneura multifida Dum.).

Grant: on wet bark and wet decorticated wood in a deep wooded ravine near Bayard (Millspangh, 2070).

R. PINGuis (L.) S. F. Gray.

Fayette: near Nuttallburg (Nuttall).

\section{METZGERIA Raddi.}

M. conjugata Lindb.

McDowell: on bark of Beech, near Elkhorn (Millspaugh, I5I3). Monongalia: on bark of twig near Morgantown (Millspaugh, I671), and at Tibbs Run (Andrews). Randolph: with mosses on tree trunks near Cheat Bridge (Sheldon, 2531). Fayette: near Nuttallburg (Nuttall).

M. CRASSIPILIS (Lindb.) Evans.

Pocahontas: on various tree trunks at Warntown, Seebert and Cranberry Glades (Sheldon, 3641, 3619, 3694).

PALLAVICINIA (S. F. G.) Steph.

P. Lyellii (Hook.) S. F. Gray.

Preston: on earth and logs among mosses near Cranes.. ville (Sheldon, 3484).

\section{PELLIA Raddi.}

P. epiphylla (L.) Corda.

Randolph: on clay near a spring at Pickens (Millspaugh, 22II), and between stones on a roadside near Cheat Bridge (Sheldon, 2523). Monongalia: on earth and rocks near a spring at Morgantown (Sheldon, 768, 1280).

P. FABRoniana Raddi.

Wayne: on bank of small stream near Ceredo (Sheldon, 3899). 
FOSSOMBRONIA Raddi.

F. Wondraczeckir (Corda) Dum.

Monongalia: on earth near Morgantown (Sheldon, 2764, 3190, 3919).

$$
\text { (Acrogynae.) }
$$

MARSUPELLA Dum.

M. emarginata (Ehrh.) Dum.

Randolph: on clay bank of a small spring at Pickens (Millspaugh), Preston: at Masontown (Andrews).

NARDIA S. F. Gray.

N. CRenulata (Smith) Lindb.

Monongalia: at a springy place near Easton (Andrewts).

Randolph: growing with Pellia epiphylla at Cheat Bridge (Sheldon).

N. CRENUliformis (Aust.) Lindb.

Monongalia: a few specimens from rocks in Tibbs Run (Andrezes).

\section{SOLENOSTOMA Mitten.}

S. Lanceolata (L.) Steph. (Jungermannia lanceolata L.). Monongalia: on rocks in Tibbs Run (Andrew's).

\section{APLOZIA Dum.}

A. Autumnalis (DC.) Schiffn. (Jamesoniella DC., Jungermannia Schraderi Mart).

Monongalia: at Tibbs Run (Andrews). Randolph: on decaying logs near Cheat Bridge (Sheldon, 25I7). Fayette: near Quinnimont (Pollard \& Maxon, II3).

J. PUMILA With.

\section{JUNGERMANNIA Auth?}

Greenbrier: on log in river at Durbin.

\section{LOPHOZIA Dum.}

L. Marchica (Nees.) Steph.

Monongalia : in a wet place on roadside near Easton ( $A n-$ drezes).

L. ventricosa (Dicks.) Dum.

Monongalia : on ledge near Coopers Rock (Shcldon, 3024).

BLEPHAROSTOMA Lindb.

B. TRICHOPhyllum (L.) Dum.

Monongalia: on ground and sandstone boulder in deep woods at Tibbs Run (Millspaugh, 1665, Andrezes). 


\section{PLAGIOCHILA Dum.}

P. Sullivantii Gottsche.

Monongalia: on earth in vicinity of Quarry Run ( $A n$ drews).

P. PORELLOIDES Lindb.

Monongalia: on sandstone boulder in Tibbs Run, Grant, in a deep ravine on wet stones and sand near Bayard (Millspaugh, I700, 2000, 2050, 204I).

P. virginica Evans, in Millsp. Prel. Cat. Flora, W. Va., 497 (I892).

Growing in wide, depressed, and intricate tufts; stems ascending from a prostrate caudex, simple or sparingly branched, sometimes geniculate and rooting at the joints, otherwise eradiculose; leaves contiguous or somewhat imbricated, widely patent, ovate or rhomboid-ovate, the dorsal margin decurrent, slightly reflexed, entire, the ventral margin plane or reflexed at base, mostly entire, the apex broad, rounded or truncate, sharply and irregularly spinulose; amphigastria none.

Stems I to $3 \mathrm{~cm}$. long, with the leaves I to $2 \mathrm{~mm}$. wide; leaves $1.2 \mathrm{~mm}$. long, $0.7 \mathrm{~mm}$. wide; spines short, acute, separated by rounded sinuses, varying in number from 2 to 3 on each leaf, usually 4 or 5 ; leaf-cells averaging 0.023 - $\mathrm{mm}$. in diameter in middle of leaf, thin-walled and scarcely thickened at the angles.

Mercer: on walls of dry limestone cave, Beaver Spring (Millspaugh, I550).

\section{CHILOCYPHUS Corda.}

C. Pallescens (Ehrh.) Dum.

Preston: near Albright, on decaying log (Sheldon, 337.3). C. polyanthus (L.) Corda.

Pocahontas: on decaying leaves and grass in Cranberry Glades (Sheldon, 3846); and Greenbrier: on decaying log at Durbin $(3766)$.

\section{LOPHOCOLEA Dum.}

L. Bidentata (L.) Dum.

Monongalia: on mossy rocks by Quarry Run (Andrcres).

L. HETEROPHYLla (Schrad.) Dum.

Monongalia: on earth near Easton (Andrezes), and at Morgantown (Sheldon, 2846). Taylor: on rocks and earth at Fettermann (Sholdon, 2535).

L. MINOR Nees.

Wayne: on rocks and earth near Ceredo (Sheldon, 3895). 
HARPANTHUS Nees.

H. scutatus (Web. \& Mohr) Spruce.

Monongalia: on ground and sandstone boulder in deep woods at Tibbs Run, and Grant: on damp dead bark near Bayard (Millspaugh, 1670; 2020, 203I).

\section{SACCOGYNA Dum.}

S. GRAveolens (Schrad.) Lindb. (Geocalyx graveolens Nees.) Monongalia: on ground and sandstone boulders in deep woods at Tibbs Run (Millspaugh, 1667; Andrews).

C. SERRIFLORA Lindb.

\section{CEPHALOZIA Dum.}

Monongalia: on rotten wood near Tibbs Run (Andrews)

C. lunulaefolia Dum.

Randolph: on decaying log at Cheat Bridge (Sheldon).

C. Virginiana Spruce.

Fayette: near Quinnimont (Pollard \& Maxon, I I5a).

C. mútiflora Spruce.

Monongalia: on ground and sandstone boulder in deep woods at Tibbs Run, and on wet hemlock log; Grant: near Bayard (Millspaugh, I662, 2080).

\section{NOWELLIA Mitt.}

N. Curvifolia (Dicks.) Mitt. (Cephalozia curvifolia Dum.). Monongalia : on sandstone boulder in deep woods at Tibbs Run, and Grant: on wet dead bark in deep woods near Bayard (Millspangh, 1663, 2021). Fayette: near Nuttallburg (Nuttall); near Quinnimont. (Pollard \& Maxon, II 5). Monongalia: on decaying wood at Tibbs Run (Andrez's). Upshur: on decaying $\log$ at French Creek, and in sime situation in Randolph: at Cheat Bridge (Sheldon, 2095, 2532).

\section{SPHENOLOBUS Lindb.}

S. Exsectus Steph. (Jungermannia exsecta Schmid.).

Grant: on wet hemlock log in deep wooded ravine (Millspaugh, 2012).

S. MichauxiI (Web.) Steph.

Monongalia: on vertical rocks at Cheat View (Andrezus).

S. minutus (Crantz) Steph.

Monongalia : on ledge near Coopers Rock (Sheldon, 3023).

PRIONOLOBUS Spruce.

P. Hellerianus (Nees) Schiffn.

Pocahontas: on decaying $\log$ at Cranberry Glades (Sheldon, 3688). 
ODONTOSCHISMA Dum.

O. prostratum (Swartz) Trevis. (O. sphagni Dum. in Flora),

Monongalia: among mosses on sandstone boulder in deep woods at Tibbs Run (Millspaugh, r664; Andrezes). Fayette: near Nuttallburg (Nuttall).

O. Denudatum (Mart.) Dum.

Monongalia: decaying stumps and logs by Tibbs Run (Andrews).

\section{KANTIA S. F. Gray.}

K. trichomanis(L.)S. F. Gray. (Calypogeia trichomanis Corda).

Tucker: on wet logs near Blackwater Falls (Millspangh, 993). Monongalia : on wet ground and on sandstone boulder in deep woods at Tibbs Run (Millspangh, I599, I666), and on earth near Morgantown (Sheldon, 2765). Randolph: on damp sand near Pickens (Millspaugh, 2207). Greenbrier: on earth at Fort Spring (Sheldon, 2728).

\section{BAZZANIA S. F. Gray.}

B. DEFlexa (Mart.) Underwood.

Monongalia: on sandstone boulder in deep woods at Tibbs Run (Millspaugh, I66I).

B. trilobata (L.) S. F. Gray.

Monongalia: on bole of tree and in wet depressions in sandstone boulder in deep woods at Tibbs Run (Millspaugh, I630, I640; Sheldon, 630); and on rocks among mosses at Dellslow (Sheldon, 2282). Grant: on wet Hemlock log near Bayard (Millspaugh, 2010). Fayette: in deep woods near Nuttallburg (Nuttall). Randolph: abundant on earth and decaying logs at Cheat Bridge, and Preston: near Cranesville (Sheldon, 2534, I 565).

B. triangularis (Schl.) Lindb.

Monongalia: on rocks by a brook near Tibbs Run ( $A n$. drews). Randolph: on decaying logs at Cheat Bridge (Sheldon, 2518).

LEPIDOZIA Dum.

L. Sylvatica Evans.

Monongalia: on ground near Tibbs Run (Andrews).

L. Reptans (L.) Dum.

Greenbrier: growing on earth near Durbin (Sheldon, $3788)$. 
HERBERTIA S. F. Gray.

H. ADUnCA (Dicks.) S. F. Gray.

Monongalia: on sandstone boulder in deep woods at Tibbs Run (Millspaugh, 1659).

\section{PTILIDIUM Nees.}

P. Pulcherrimum (Web.) Hampe.

Preston: on decaying log near Cranesville; Monongalia: on a fence rail near Morgantown, and Randolph: at Cheat Bridge (Sheldon, I536, 2830, 25I5, 2520, 2521).

\section{TRICHOCOLEA Dum.}

T. tomentella (Ehrh.) Dum.

McDowell: on roots in a rill near Elkhorn (Millspaugh, I522a). Grant: on wet sand in a deep ravine near Bayard (Millspaugh, 2040). Monongalia: on rocks in Tibbs Run (Andrezes).

\section{DIPLOPHYLLUM Dum.}

D. taxifolium (Wahl.) Dum. (Diplophylleia taxifolia Trev.).

Monongalia: on sandstone boulder in deep woods at Tibbs Run (Millspaugh, I669).

S. nemorosa (L.) Dum.

\section{SCAPANIA Dum.}

Monongalia: on damp sandstone boulder in deep woods at Tibbs Run (Millspaugh, r668), and on north side of a bank at Sabraton (Sheldon, 2318). Randolph: on clay near a spring at Pickens (Millspaugh, 2212), and on rocks in a stream at Cheat Bridge (Sheldon, 25I6). Grant: on wet hemlock log in deep woods near Bayard (Millspaugh, 20I I). Greenbrier: on rocks along stream at Fort Spring, and on limestone ledge at Durbin (Sheldon, 2726, 2526).

S. undulata (L.) Dum.

Randolph: on clay at the marge of a spring at Pickens (Millspaugh). Preston: on stones in a brook by the roadside between Cranesville and Albright, and on stones in a spring at Cranesville (Sheldon, I5I2, I524).

R. Xalapensis Mont. RADULA Nees.

Mercer: on face of a limestone cliff at Beaver Spring (Millspaugh, I55 I).

R. TENAX Lindb.

Monongalia: on sandstone boulder in deep woods at Tibbs Run (Millspaugh, 1657). Grant: on bark of living Cherry near Bayard (Millspaugh, 2060). 


\section{STEPHANIA Kuntze.}

S. complanata (L.) Kuntze. (Radula complanata Dum.). Randolph: on tree trunk at Cheat Bridge (Sheldon, 2527).

BELLINCINIA Raddi.

B. Porella (Dicks.) Kuntze.

(Porella pinnata Schw.)

Fayette: on rocks in the mist of a fall near Gauley Bridge (Millspaugh, 607).

B. Platyphylla (L.) Kuntze. (Porella platyphylla Lindb.).

Monongalia: on bark of living apple tree at Morgantown: on sandstone boulder in Tibbs Run; on tree trunks and decaying logs west of Morgantown (Millspaugh, I29I, I292, I658; (Sheldon, I620). Mercer : on oak log at Bluefield and on limestone ledge at Beaver Spring (Millspaugh, I448, I 530). Upshur: at French Creek; Preston: at Cranesville, Greenbrier: at Durbin; Randolph: at Cheat Bridge (Sheldon, 2069, I546, 2264, 2519).

\section{COLOLEJEUNEA Spruce.}

C. Bindlecomiae (Aust.) Evans.

Monongalia : on a ledge at Sturgisson, and Preston: on a decaying log at Cranesville (Shcldon, 3282, 3426).

\section{MICROLEJEUNEA Spruce.}

M. Lucens (Taylor) Evans.

Between McDowell and Tazewell: on dripping limestone along Horsepen Creek (Morris, i I $6 \mathrm{~b})$.

\section{LEJEUNEA Lindb.}

L. CAvifolia (Ehrh.) Lindb.

Monongalia: a small form growing on rocks in Tibbs Run (Andrews).

L. Cucullata N: ab E.

Randolph: ad rupes madidas in jugo Cheat Mountains (Sullivant Musci. Allegh. 65).

CHEILOLEJEUNEA Spruce.

S. PHyllobola (Nees \& Mont.) Schiffn.?

Monongalia: on Sweet birch near Coopers Rock (Sheldon, 3022).

HARPALEJEUNEA Spruce.

H. ovata (Hook.) Schiffn.

Monongalia: on stone at edge of brook at Oliver; on trunk of Sycamore growing in Quarry Run (Sheldon, 29ı6, $302 \mathrm{I})$. 
JUBULA Dum.

J. Pennsylvanica (Steph.) Evans. (Jubula Hutchinsiae Sullivantii Spr.).

Monongalia: on sandstone boulder in Tibbs Run, and McDowell: on rocks in stream at Elkhorn (Millspangh, $1655,1509)$.

FRULLANIA Raddi.

F. Asa-Grayana Mont.

Monongalia: on sandstone boulder in Tibbs Run (Millspaugh, 1654). Randolph: clinging to face of dry sandstone boulder at Pickens; at Cheat Bridge (Millspaugh, 2206; Sheldon, 2524). Preston: rather common on trees and rocks near Cranesville, and Monongalia : Lick Run; also in Greenbrier: at Durbin and Ronceverte (Sheldon, I564, I21 $4,2262,1076)$.

F. squarrosa (R. Bl. \& N.) Dum.

Upshur: at French Creek (Sheldon, 2094). Monongalia: on trees by Deckers Creek and west of Morantown ( $\mathrm{An}$ -

- drezes, Sheldon, 1259).

F. RIPARIA Hampe.

Monongalia : on trunk of Sugar maple at Lick Run (Sheldon, 2983).

F. PLANa Sulliv.

Fayette: near Nuttallburg (Nuttall).

F. EBORACENSIS Gottsche.

Monongalia: on trees near Deckers Creek, Morgantown (Andrezus). Greenbrier: on tree trunks at Durbin (Sheldon, 1074).

F. Brittonae Evans.

Monongalia: on trees near Cheat River by Ices Ferry, and Preston: near Masontown (Andrezus).

\section{ARCHILEJEUNEA Spruce.}

A. Calypeata (Schw.) Schiffn. (Lejeunea calypeata Schw.).

Monongalia: on sandstone boulder in Tibbs Run (Millspaugh, 1656). Fayette: near Nuttallburg (Nuttall). Greenbrier: on rock and base of tree along a stream at Fort Spring (Sheldon, 2720).

\section{A N T HOCEROT A CE A E.}

NOTOTHYLAS Sulliv.

N. ORBICUlaris (Schw) Sulliv.

Monongalia: edge of small pond and stream near Morgantown; Marshall: banks of the Ohio River at Mounds- 
ville; Wayne: edge of brook in sandy soil near Ceredo (Sheldon, 3518, 3921, 3888, 3900).

A. LAEvis L.

ANTHOCEROS Syn. Hep.

Monongalia: abundant along side of road and bank near Morgantown, and Greenbrier: on rocks along stream at Fort Spring (Sheldon, 67, 2725). Wvoming: on dripping limestone, along the Guyandot River below Bailyville (Morris. I 22I I).

A. punctatus L.

Monongalia: in a gutter along a roadside in Morgantown (Sheldon, 2577).

\section{S P H A G N A C E A E.}

\section{SPHAGNUM, L.}

S. IMBRicAtUm AFFine (R. \& C.) Warnst.

Monongalia: at Tibbs Run and at Lick Run; Preston: near Cranesville (Shela'on, 629, 3966, I 548).

S. Magellanicum Brid.

Preston: near Cranesville (Sheldon, 3457).

S. RECURVUM Beauv.

Preston: near Cranesville; and Pocahontas: near Cranberry Glades (Sheldon, I535, 3459, 3677, 3678).

S. cymbifolium, Ehrh.

Common in wet glades, and in deep wooded rills. Preston: Kingwood and Terra Alta. Webster: at Welsh, Long and Collett's Glades. Fayette: glade above Nuttallburg. Randolph: in the spruce forests (Millspaugh). Monongalia: Deckers Creek valley near Morgantown (Andrews). Randolph: in Blister Swamp (Moore).

S. CYMBIfolium squarrosulum Bry. Ger.

Monongalia: along Tibbs Run (Millspaugh, Andreros). S. GIRGENSOHNII Russ.

Boggy places. Pocahontas: Cranberry Glades (Sheldon). S. QUINQUEFARIUM (Braith.) Warnst.

Randolph: in Blister Swamp (Moore).

A N D R E A E A C E A E.

ANDREAEA Ehrh.

A. RUPESTRIS Hedw.

Monongalia: on rocks at summit of ridge near Cheat View (Andreres). 


\section{Musci.}

D I C R A N A E A E.

PLEURIDIUM Brid.

P. Alternifolium (Kaulf.) Rab.

Monongalia: ground on Dorseys Knob near Norgantown (Andrews). Jefferson: near Harpers Ferry (Sullizant).

\section{DITRICHUM Timm. \\ (Leptotrichum, Hampe.)}

D. Pallidum (Schreb.) Hampe. Trichostomum pallidum, Hedw.

Mercer: on ground, oak woods, Bluefield. McDowell : on clay, open woods, Elkhorn. Monongalia: on clay of open woods, Tibb's Run (Millspaugh, I458, I492, I495, I500, I598). Fayette: near Nuttallburg (Nuttall). Pocahontas. on fresh soil along a log road near Cranberry Glades (Sheldon, 3743).

D. TORTILE (Schrad.) Hampe.

Fayette: near Quinnimont (Pollard \& Maxon, I05).

\section{CERATODON, Brid.}

C. purpureus (L.) Brid.

Monongalia: on dry sandstone boulder, Morgantown; Tibb's Run (Millspaugh, I390, I633). Fayette: near Nuttallburg (Nuttall).

DICRANELLA, Schimp.

D. heteromalla (L.) Schimp.

Monongalia: on ground under rail fence, The Flats; on wet coal entrance of coal pit, Georgetown; on ground in woods, Tibb's Run (Millspaugh, I362, I378, I638). Fayette: Nuttallburg (Nuttall).

D. Rufescens (Tourn.) Schp.

Monongalia: valley of Deckers Creek near Lick Run (Andrecis).

D. VARIA (Hedw.) Schp.

Monongalia: near the road in the vicinity of Easton (Andrew's). 
RHABDOWEISIA Bry. Eu.

R. FUGAX (Hedw.) Br. \& Sch.

On ledge and roots of various trees at McKinneys Cave near Bretz, Monongalia; on a ledge near Coopers Rock, and on a sandstone ledge at Tibbs Run (Sheldon, 4r69, 3039, 4355).

\section{DICRANUM, Hedw.}

D. Flagellare Hedw.

Monongalia: on decayed oak log, Tibb's Run (Millspaugh, I593). Fayette: Nuttallburg (Nuttall).

D. scoparium (L.) Hedw.

Grant: on ground in damp woods, Bayard. Monongalia: on ground, the Flats; on decayed log, Georgetown; loc. cit., Tibb's Run. Mercer: in tufts at base of stump, Bluefield, and on decayed log (Millspaugh, I398, I382, I60I, I476, I464). Fayette: near Nuttallburg (Nuttall).

D. FUlvum Hook.

Fayette: Nuttallburg (Nuttall).

D. Drummondi Muell.

On the mountain between Barrenshe Creek and Dry Fork. McDowell County, altitude I,700 feet, August 6, Igoo (Morris, $\mathrm{I} 165)$.

D. Montanum Hedw.

Monongalia: decayed spot in a tree near Dellslow ( $A n-$ drews).

D. LONGIFolium Hedw.

Fayette: near Nuttallburg (Nuttall).

D. FUSCESCENS Turn.

Randolph: near Cheat Bridge (Sheldon, 2538).

\section{DICRANODONTIUM Br. \& Sch.}

D. Denudatum (Brid.) E. G. B. (D. Virginicus E. G. B).

Monongalia : on sandstone boulder along a woodland path, Tibb's Run (Millspaugh, I635).

Plants bright glossy green, stems matted below by a red tomentum, leafy nearly to apex, denudate roughened above, with a few leaves at summit; leaves erect or secund, straight or curled and twisted, often $5 \mathrm{~mm}$. long, narrowly subulate from a short, thick base, caducous ones with a long, slender, smooth point; persistent ones serrate, blade inflexed cells densely chlorophyllose, filled with oil globules, those of the basal angles, clear. Diœcious, the antheridia terminal in conspicuous heads, bracts brown at base, apex subulate, serrate; perichætial bracts 3 to $4 \mathrm{~mm}$. long, from a short base, suddenly subulate, dentate at apex; pedicels lateral by 
the growth of innovations, $I \frac{1}{2}$ to $2 \mathrm{~cm}$. long, pale, glossy yellow, twisted in two directions, very slender, arcuate when young, becoming erect before capsules mature. Capsule cylindric, ribbed only at the mouth, $\mathrm{I} \frac{1}{2}$ to $2 \mathrm{~mm}$. long, beak straight or curved, shorter than the capsule, peristome bright red, not deep set, teeth split unequally to middle, striolate at base, pale and granulose above, annulus none, spores small, calyptra cucullate, $2 \mathrm{~mm}$. long, beaked, entire. Maturing in summer.

Differs from European specimens of D. longirostre collected by Seringe; in the longer, paler, more slender, scarcely arcuate pedicels, longer capsules, peristome not deep set, and teeth split only to the middle, more united than figured in the Bryologia Europaea, Table 88. It may be distinguished from Campylopus Virginicus, also remarkable for its caducous leaves, by the longer, more slender subulate point. which is entire or minutely serrate and smooth on the back, by the thick base, with inflexed blades, and by the shape of the basal cells at the angles.

Monongalia, on sandstone boulder deep woods, Tibb's Run (Millspangh, I 596). Campylopus flexuosus, Sull. not Brid. D. Millspaughii E. G. B.

Plant slight yellowish green, silky, cespitose; stems matted with rufous tomentum at base, I to $3 \mathrm{~cm}$. long, a few denudate, roughened by the fragments of the slightly caducous leaves. Leaves secund or erect-spreading, 4 to $5 \mathrm{~mm}$. long, narrowly subulate from a broad base I to $1 \frac{1}{2} \mathrm{~mm}$. long becoming tubular above with inrolled margins, basal angles not auricled, filled by large hyaline cells to the base of the broad, brown vein, those of the blade oblong or square next the vein becoming spindle-shaped and prosenchymatous toward the margin, vein thick, excurrent into a dentate slender tip, rough on back. Diœcious, perichætium 5 to $7 \mathrm{~mm}$. long, bracts sheathing half their length, tapering to a long, slender, obscurely serrate tip, outer shorter, abruptly subulate, more sharply serrate; pedicels recurved. burying the capsules among the leaves, becoming erect when old, 5 to $8 \mathrm{~mm}$. long, stout and twisted in two directions; capsules pyriform-cylindric with a distinct neck, length about I $\mathrm{mm}$. without the lid, which is as long as the rest of the capsule, with a straight beak, calyptra cucullate, entire; peristome red, connivent, teeth deep set, slender, split to middle, or perforate to base, striolate below, granulose above; annulus none, mouth bordered by a dense, dark rim. Maturing in summer, old capsules persistent, not sulcate.

Differs from European specimens of $D$. longirostre in the structure of the base of the leaf, lacking the suddenly in- 
flated basal auricles; differing also in the cells above the base, teeth not split to base, occasionally only perforate. From D. Virginicus it may be distinguished by the less caducous leaves, shorter, stouter, more arcuate pedicels, smaller capsules, and longer sheathing perichætium.

Through the kindness of Dr. Robinson I have been able to compare these specimens with those collected by Sullivant on Grandfather Mt. in I843. His also are fruiting, and an excellent drawing is preserved, hence I am able to assert that the specimens are almost identical. Sullivant's showing no naked stems, but many of the leaves are caducous. Dr. Braithwaite kindly compared the West Virginia specimens with Campllopus pyriformis, sending me specimens of this and the variety Mulleri, and sketches of the bases of the leaves. It is evident that Sullivant was mistaken in referring his specimens to C. flexuosus, as they are more closely allied to Dicranodontium longirostre, var. alpinus.

\section{LE U C O B R Y C E A E.}

\section{LEUCOBRYUM Hampe.}

L. glaucum (L.) Br. \& Sch.

Monongalia: on ground in woods, The Flats (Millspaugh, r399). Fayette: Nuttallburg (Nuttall).

\section{F I S S I E N T A C E A E.}

FISSIDENS, Hedw.

F. minutulus Sulliv.

Monongalia: on rocks in Aarons Run (Sheldon, 3934).

F. obtusifolius Wils.

Monongalia : on walls of Lock No. 9, Monongahela River (Jennings).

F. ADIANTOIDES (L.) Hedw.

Monongalia: on shaly rocks under cliff, Cassville (Millspaugh, I423).

F. DECIPIENS DeNot.

Fayette: near Nuttallburg (Nuttall).

F. subbasilaris Hedw.

Summers: near Lowell (Pollard \& Maxon, II7). Upshur, on a tree trunk at French Creek (Sheldon, 2093).

\section{E N C A L Y P T A C E AE. ENCALYPTA Schreb.}

E. streptocarpa Hedw.

(Lecrsia streptocarpa Lindb.).

Mercer: face dry limestone cliff, Beaver Spring (Millspaugh, I552); near Big Spring (Sullivant). 
P O T T I A C E A E.

DESMATODON Brid.

D. Forteri James.

Jackson: on sandstone rock near Ravenswood (Shicldon, 2388).

\section{TORTULA, L.}

T. muralis (L.) Hedw. Barbula muralis (L.) Trin.

Mercer: on sandstone ledge, Beaver Spring (Millspaugh, I553).

\section{TORTELLA C. M.}

T. Humilis (Hedw.). B. caespitosa, Schwægr. Barbula humilis Hedw.

Mercer: roots of oak, Bluefield (Millspangh, I447). Fayette: Nuttallburg (Nuttall).

T. unguiculata (Huds.). Barbula unguiculata Hedw.

Greenbrier : on rocks along rill near Fort Spring; Monongalia: on Front Street curb stone and on stone pile in South Park, Morgantown (Sheldon, 2803, 205I, 2279).

T. tortuosa (L.) Limpr. Barbula tortuosa W. \& M.

Monongalia: in sand under boulder, Camp Eden (Millspaugh, I395).

\section{WEISIA Hedw.}

W. VIRIDULA Hedw.

(Mollia viridula Lindb.).

McDowell : on ground, open woods, Elkhorn (Millspaugh, I496, I497). Fayette: near Nuttallburg (Nuttall).

\section{G R I M M I A C E A E.}

GRIMMIA Ehrh.

G. apocarpa (L.) Hedw.

Monongalia : on rocks in Tibbs Run (Andrere's).

RHACOMITRIUM Brid.

R. ACiculare (L.) Brid.

Monongalia: at "Tibbs Run (Andrews).

O R T H O T R I C H A C E A E.

DRUMMONDIA Hook.

D. Clavellata Hook.

Monongalia: on a tree near Easton (Andrew's). 


\section{ORTHOTRICHUM Hedw.}

O. Porteri Aust.

Berkeley: on outcropping limestone near Martinsburg (.Sheldon, 2653).

O. Braunir Br. \& Sch. O. strangulatum, Beauv. Monongalia: on bark living apple tree, Morgantown (Millspaugh, I288).

O. Ohioense S. \& L.

Fayette: near Nuttallburg (Nuttall).

\section{ULOTA Brid.}

U. Americana (P.B.) Mitt.

(U. Hutchinsiae Schp.).

Weissia Americana Lindb. Monongalia: on dry sandstone boulder at Camp Eden (Millspaugh, r390), and on rocks in Tibbs Run (Andrews). Fayette: near Nuttallburg (Nuttall).

U. Ulophylla (Ehrh.) Broth. (Weisia ulophylla Ehrh.)

Fayette: near Nuttallburg (Nuttall).

U. Ludwigir Brid.

Greenbrier: on trunk of Birch near Durbin, and Pocahontas, at Cranberry Glades. (Sheldon, I044, 3673).

F U N A R I A CE A E.

\section{EPHEMERUM Hpe.}

E. Crassinervium (Schwgr.) Cen.

Inundated lands along the Kanawha River (Sulliz'ant).

\section{FUNARIA Schreb.}

F. Hygrometica (L.) Sibth.

Monongalia: in soil on sandstone boulder, Tibb's Run (Miilspaugh, I615, I617). Fayette: near Nuttallburg (Nuttall).

Var PATUla Br. \& Sch.

Monongalia : on rocks lining a spring, the Flats; in cinders of an old camp fire, Camp Eden (Millspaugh, I376, 1293). F. Flavicans Michx.

Monongalia: on damp sand in a "burning," Little Falls; loc. cit., Morgantown (Millspaugh, 1277, I339).

\section{APHANORRHEGMA Sull.}

A. SERratum (Hook. \& Wils.) Sulliv.

Monongalia: Roadside leading from North Front Street, and south side of Deckers Creek at the mouth of Aarons Run, Morgantown (Sheldon, 2689, 3973, 4314). 


\section{PHYSCOMITRIUM Brid.}

P. PYRIFORME (L.) Brid.

Monongalia: on top of soil of field that had been ploughed and harrowed only eight days before, Morgantown; on ground marshy spot, Dille's (Millspaugh, 1278, I403).

B R Y A C E A E.

POHLIA Hedw.

P. Nutans (Schreb.) Lindb.

On decaying wood and on soil. Monongalia: near Morgantown (4948); Preston: near Cranesville (I 525, 3458): near Terra Alta (3472); Preston: at McKinney's Cave near Bretz (4074); Preston: near Oak Park (4067, 4984) (all numbers Sheldon).

MNIOBRYUM Schimp.

M. ALbicans (Wahl.) Limpr.

Monongalia: edge of stream near Oliver (Sheldon, 292I).

\section{BRYUM L.}

B. BIMUM Schreb.

Monongalia: on shale under ledge, Cassville (Millspaugh, I424).

B. ARGENTEUM L.

Monongalia: fissures between bricks of walks, Morgantown (Millspaugh, r335). Fayette: near Nuttallburg (Nuttall).

B. INTERMEDIUM Brid.

Monongalia: near Morgantown, Middle Ferry and Lick Run (Sheldon, I67, I69, I268, 2340, 3957). Dr. Grout pronounces 2340 not typical.

B. PSEUdotriquetrum (Hedw.) Schwaeger?

Monongalia: on railroad bank at Sturgisson (Sheldon, I86).

Doubtfully referred by Dr. Grout.

B. PRoliferum (L.) Sibth. (B. roseum Schreb.)

Mercer: on roots of Oak near Bluefield; McDowell: on decayed wood at Elkhorn (Millspaugh, I449, I502), and along Horsepen Creek (Morris, I I I9).

B. CAPILLARE $\mathrm{L}$.

Monongalia: at Quarry Run (Andrews).

B. C.Aespiticium L.

Monongalia: near Dry Run (Andrew's). 


\section{LEPTOBRYUM, Wils.}

L. PYRIFORME (L.) Wils.

(Bryum pyriforme, Hedw.)

Monongalia: on sandstone boulder, Tibb's Run (Millspaugh, (1616, 1633).

\section{N I A C E A E.}

MNIUM Linn.

M. marginatum (Dicks.) Beauv.

Monongalia: on rocks near Dellslow (Sheldon, 3209).

M. AFFine Bland.

Monongalia: near Dry Run (Andrecus).

M. cuspidatum Hedw. (Astrophyllum sylvaticum Lindb).

Monongalia : on soil at Morgantown; on a dry boulder by Cheat River, and on a stone in a swampy spot at Dille's (Millspaugh, I359, I397, I 583). Fayette: near Nuttallburg (Nuttall).

M. RoSTRATUM Schrad.

(Astrophyllum rostratum Lindb.)

Grant: on wet logs at Bayard; Monongalia: on decayed wood at the Flats; McDowell: on roots in a rill near Elkhorn (Millspaugh, I377, I 523).

M. punctatum (L.) Hedw. (Astrophyllum punctatum Schreb.)

Fayette: near Nuttallburg (Nuttall).

M. HORNUM L. (Astrophyllum hornum Lindb.)

Monongalia: on sand in a rill at Tibbs Run (Millspaugh, I604.) Fayette: near Nuttallburg (Nuttall).

\section{A UL A CO M N I A CE AE.}

\section{AULACOMNIUM Schw.}

A. heterostichum Br. \& Sch. (Sphaerocephalus Britt. m.)

Monongalia: on coal, entrance to coal pit, Georgetown: hanging from sandstone boulder, Tibb's Run, Camp Eden (I392); on rocky ledge, Cassville. McDowell: on sandy bank of rill, Elkhorn (Millspangh, I3I9, I607, I414, I520). Fayette: near Nuttallburg (Nuttall).

\section{B A R T R A M I A C E A E.}

\section{BARTRAMIA Hedw.}

B. POMiformis (L.) Hedw.

Mercer: on bole dead tree, Bluefield. Monongalia: on sandstone boulder, deep woods, Tibb's Run (Millspaugh, I609). Fayette: near Nuttallburg (Nuttall). On ground, Greenbrier Co., Kate's Mountain, alt. 3.300 ft. (Small \& Vail). 
Var CRISPA (Sw.) Schimp.

Monongalia: on rock ledge, Cassville (Millspaugh, I4I7: I4I8).

P. Fontana (L.) Brid.

Mercer: on sandstone ledge in rill, Beaver Spring (Millspaugh, I 56I).

W E B E R A C E A E.

WEBERA Ehrh.

W. PROLIGERA (Lindb.) Kindb.

Monongalia: near Lick Run (Andrews).

W. sessilis (Schm.) Lindb. (Diphyscium foliosum Mohr.)

Fayette: near Nuttallburg (Nuttall).

B U X B A U M I A E A E.

B. APHYLLA Linn.

\section{BUXBAUMIA Haller}

Fayette: near Nuttallburg (Nuttall).

GE O R G I C E A E.

GEORGIA Ehrh.

G. PELlucida (L.) Rabenh.

Tetraphis pellucida, Hedw.

Monongalia: on sandstone boulder, Tibb's Run (Millspaugh, 1606, 1610, 1634).

\section{P O L Y T R I C H I A C E A E.}

\section{POGONATUM Palis.}

P. BREvicaule Beauv.

(Polytrichum tenne Menz.)

North slopes on Road Run, Wyoming County, August I2, I900 (Morris, I I76). Monongalia : roadside bank near Morgantown, and on ground at Tibb's Run (Millspaugh, г6г2). Fayette: near Nuttallburg (Nuttall).

\section{POLYTRICHUM L.}

P. COMMUNE L.

Preston: on ground in open woods, Terra Alta (Millspaugh).

P. Ohioense, Ren. \& Card. P. formosum, Sull. not Hedw.

Monongalia: on ground, Morgantown; a large form on ground Tibb's Run; an extremely small form, with minute capsules on sandstone boulder, loc. cit. Mercer: on ground 
in oak woods, Bluefield (Millspaugh, I536, I600, I6I I, 1453). Grant: on decayed logs, Bayard. Fayette: near Nuttallburg (Nuttall).

P. PILIFERUm, Schreb.

Monongalia: on bare sandstone ledge, Falling Run (Millspaugh, 1299).

\section{CATHARINAEA, Ehrh.}

C. Angustata, Brid. Atrichum angustatum, Br. \& Sch.

Monongalia, on ground in marshy spot, Morgantown. Mercer: rocks in rill, Beaver Spring (Millspaugh, 1406, 1495). Fayette: near Nuttallburg (Nuttall).

C. undulata (L.) Web. \& Mohr. Atrichum undulatum, Beauv.

Monongalia: on ground in marshy spot, Morgantown. McDowell: on roots in stream, Elkhorn (Millspaugh, I404, I522). Fayette, near Nuttallburg (Nuttall).

\section{H E D W I G I A C E A E.}

H. CILIATA Ehrh.

\section{HEDWIGIA Ehrh.}

Monongalia: on dry exposed boulders and rocks at The Flats (Millspaugh, 1400). Fayette: near Nuttallburg (Nuttall).

H. ciliata secunda Schp.

Monongalia: nicely fruited specimens with varietal characters well marked on rocks in Tibbs Run, and on boulders near Dry Run (Andrez's).

\section{F O N T I N A L C E A E. \\ FONTINALIS Dill.}

F. Dalecarlica Bry. Eu.

Monongalia: along Tibbs Run (Andrerus).

\section{L I M A C I A C. E AE. \\ CLIMACIUM W. \& M.}

C. Americanum Brid.

Monongalia: on a moist sandstone ledge near Cassville (Millspaugh, 14I3).

C. Americanum Kindbergit R. \& C.

Preston: in glades near Masontown (Andreas). 
LEUCODON T A CEAE.

L. Julaceus (Hedw.) Sull.

\section{LEUCODON, Schwaeger.}

Mercer: on limestone ledge, Beaver Springs; McDowell: on rocks in rill, Elkhorn; Monongalia: on oak log, Tibb's run (Millspaugh, I532, I 508, I 590).

L. BRACHYPUS Brid.

Grant: on wet rotten log, Bayard (Millspaugh, 937).

N E C K E R A C E AE.

NECKERA Hedw.

N. pennata (L.) Hedw.

Tucker: on tree trunks, Blackwater Falls (Millspaugh, 965). Fayette: near Nuttallburg (Nuttall).

LEPTODON Mohr.

L. trichomitrion (Hedw.) Mohr.

Monongalia: on a tree near the river at Ices Ferry; and Preston: near Masontown (Andrews).

THAMNIUM Bry. Eu.

T. Allegheniense (C.M.) Bry. Eu.

Monongalia: near Tibbs Run (Andrews).

F A B R O N I A C E AE.

\section{ANACAMPTODON Brid.}

A. SPlach NoIdEs (Frol.) Brid.

Monongalia: in a knot hole of an apple tree at Lick Run (Sheldon, 3337).

A. APICUlatus Br. \& Sch.

Pocahontas: on trunk of Beech at Cranberry Glades (Sheldon, 372I).

E N T OIO O T A CE AE.

PLATYGYRIUM Bry. Eu.

P. REPENS (Brid.) Br. \& Sch. (Entodon palatinus Lindb.)

Monongalia: on a decaying log near Morgantown (Sheldon, I75). Monongalia: on decayed log, Tibbs Run (Millspaugh, I603). Fayette: near Nuttallburg (Nuttall). 
ENTODON C. M.

E. seductrix (Hedw.) C. Muell. (Cylindrothecium Sull.). Monongalia: on bark living apple tree, Morgantuwn; on bark in oak woods, Bluefield (Millspaugh, I290, I450). Fayette: near Nuttallburg (Nuttall).

E. Cladorrhizans (Hedw.) C. Muell. (Cylindrothecium Schimp.)

Mercer: on decayed log, damp place, Bluefield (Millspaugh, 1489). Fayette: near Nuttallburg (Nuttall).

$$
\text { PYLAISIA Br. \& Sch. }
$$

P. velutina Br. \& Sch.

Monongalia: on bark living apple tree, Morgantown (Millspaugh, 1289).

P. INTRicata (Hedw.) Bry. Eu.

Monongalia: trees near Cheat River and Deckers Creel. (Andrew's).

H O K K R I A CE AE.

HOOKERIA Smith.

H. Sullivantii C.M.

Monongalia: at Tibbs Run (Andrer's).

L E S K E A C E AE.

MYURELLA Bry. Eu.

M. GRACILIS (Weinm.) Lindb.

Monongalia: on a ledge near Sabraton (Sheldon, 3283)

\section{LESKEA Hook.}

L. GRACILESCENS Hedw.

Pocahontas: on a tree trunk near Seebert (Sheldon, - 3618).

L. POLYCARPA Ehrh.

Monongalia: on trunk of Poplar near Engine Works: and Preston: on trunk of apple tree near Albright (Sheldon, 2390, 3365).

L. obSCURA Hedw.

Monongalia: base of tree near Morgantown (Andrews).

\section{THELIA Sull.}

T. hirtella (Hedw.) Sull.

McDowell: on bark living Beech, Elkhorn (Millspaugh, I493). 
T. ASPRELla (Schim.). Sull.

Mercer: on bole living Cornus florida, Beaver Spring (Millspaugh, I 535).

ANOMODON Hook. \& Tayl.

A. rostratus (Hedw.). Schimp.

Monongalia: on dry boulder, The Flats; loc. cit., Camp Eden. Mercer: on sandstone ledge in rill, Beaver Spring; on bole living Oak, Bluefield (Millspaugh, I365, I391, I 555, I456, I 536). Fayette: Nuttallburg (Nuttall); Ronceverte (Pringle).

A. attenuatus (Schreb.), Huebn.

Monongalia: on dry boulder, The Flats. Mercer: completely covering large limestone ledges in open woods, Beaver Spring (Millspaugh, I363, I367, I 531).

A. obtusifolius Br. \& Sch.

Monongalia: base of tree near Decker's Creek, Morgantown (Andrews).

\section{THUIDIUM Br. \& Sch.}

T. Virginianum Lindb.

Monongalia: on a rock near Mont Chateau (Sheldon, I 529).

T. microphyllum (Sw.) Best.

Monongalia: on a decaying log near Morgantown; Preston: along a path and roadside in the woods near McKinneys Cave, Bretz (Sheldon, 3885, 4I73, 4I76).

T. minutulum (Hedw.) Br. \& Sch.

Summers: near Lowell (Pollard \& Maxon, II8).

T. Recognitum (Hedw.) Lindb. T. delicatulum, Br. \& Sch.

Grant: on decayed logs. Bayard. Monongalia: on dry boulder, The Flats; Tibbs Run; on decayed logs, Georgetown. Mercer: on sandstone ledge, Beaver Springs; on bole dead tree; loc. cit., Bluefield (Millspaugh, I366, I608, I382, I544, I477, I5IO). Fayette: near Nuttallburg (Nuttall); near Quinnimont (Pollard \& Maxon, I I I).

T. Scitum (Beauv.) Aust.

Monongalia: on rocks along Tibbs Run (Andrews).

$$
\text { H Y P N A C AE. }
$$

\section{AMBLYSTEGIUM Br. \& Sch.}

A. Adnatum Hedw.

McDowell: on flat stone in deep woods, Elkhorn (Millspaugh, 1498). 
A. Serpens (L.) Br. \& Sch.

Monongalia: on wet rotten log, Granville; on twigs in rill, Dille's. McDowell: on pebble in deep woods, Elkhorn (Millspaugh, I298, I402, I5I9).

A. orthocladon (Beauv.) Aust. (A. serpens orthocladon Aust.)

Monongalia: on rocks lining a spring, The Flats. Mercer: on wet limestone ledge, Beaver Spring (Millspaugh, I 375, I 358 ).

A. VARIUM (Hedw.) Lindb.

(A. radicale Br. \& Sch.)

Monongalia: on rocks lining a spring, The Flats; on wet rocks in stream, Cassville. Mercer: on limestone ridge, Beaver Spring; on decayed log, Bluefield. McDowell: on top of stump in dark, deep woods, Elkhorn (Millspaugh, I 374, I42I, I 533, I488, I 536, I 52 I ).

A. IRRIGUUM (Hook. \& Wils.) Br. \& Sch.

Mercer: on sandstone ledge in rill, Beaver Spring. Monongalia: on rocks in rill, Tibbs Run (Millspaugh, I556, 1559, I 592).

A. RIPARIUM (L.) Br. \& Sch.

Monongalia: on stone in stream, Falling Rum. McDowell loc., cit. Elkhorn (Millspangh, I334, I5I2).

A. Fluviatile (Sw.) Br. \& Sch.

Fayette: near Quinnimont (Pollard \& Maxon, IIo).

A. JuRatskanum Schimp.

Monongalia: on bark of decaying $\log$ south of Morgantown (Sheldon, I352).

\section{CAMPYLIUM Mitt.}

C. Hispidulum (Brid.) Mitt.

(Hypnum hispidulum Brid.)

Mercer: on ground in oak woods near Bluefield (Millspaugh $\mathrm{I} 452$ ).

C. Chrysophyllum (Brid.) Britt.m.

Greenbrier: on the ground on Kates Mountain, alt. 3,300 ft. (Small \& V $\mathrm{Vail}$ ). Mercer: on a decayed log in damp place (Millspaugh, I490, I49I). Monongalia: on. old beech log, Morgantown; Mercer: on ground, Beaver Spring. (Millspaugh, I405, I 536).

C. TENELlum L. \& J.

Mercer: on bole of dead tree and on $\log$ in moist place near Bluefield (Millspangh, I479, 149I).

PLAGIOTHECIUM Br. \& Sch.

P. Denticulatum (L.) Bry. Eu.

Fayette: near Nuttallburg (Nuttall).

P. Denticulatum densum, Br. \& Sch.

Monongalia: on sandstone boulder, Tibbs Run (Millspaugh, 1642). 
P. Sullivantiae Schimp.

Monongalia: on sandstone boulder in deep woods, Tibbs Run (Millspaugh, 1618).

P. Striatellum (Brid.) Lindb.

Preston: on a ledge at McKinneys Cave (Sheldon, 4I72). P. micans (Sw.) Paris.

Pocahontas: on a rock at Cranberry Glades (Sheldon, $3722)$.

P? Sylvaticum Br. \& Sch.

Pocahontas: on a decaying log at Cranberry Glades (Sheldon, 3793).

P. TURFACEUM Lindb.

Preston: on a decaying log near Cranesville (Sheldon, $3452)$.

\section{HYLOCOMIUM Bry. Eu.*}

H. Rugosum (L.) DeNot.

Greenbrier: on ground on Kate's Mountain, alt. 3,300 ft. (Small \& Vail).

H. BRevirostrum (Ehrh.) Bry. Eu.

Monongalia: not uncommon in deep brook ravines near Tibbs and Quarry Runs, fruiting at the latter (Andrews).

\section{HYPNUM Linn.*}

H. patientae Lindb.

Monongalia: on wet rocks along a small stream near Morgantown (Sheldon, 2312).

H. SchreberI Willd. (Hylocomium parietinum (L.) Lindb.)

Monongalia: on ground in shade of hemlocks at Laurel Hills (Millspaugh, 1645).

H. DENTICULATUM, L.

Monongalia: on walls of dark dripping limestone cave, Cheat river; on stone in swampy place, Morgantown; on sandstone boulder, and on pebbles in stream, Tibb's Run (Millspaugh, 1405, 1614).

H. PALUSTRE.?

Monongalia: on stone in marshy spot, Morgantown; on rocks under a fall Cassville (Millspaugh, i 584, I422).

H. MOLLE, Dicks.

Monongalia: on stone in marshy place, Dille's (Millspaugh, I 584).

* These genera have been entirely revised by Brotherus, in Engler and Prantl. Having no access to an index to the species I have made no attempt to replace the species in accordance therewith. C. F. M. 
H. PROLIFERUM L.

H. splendens, Hedw.

Randolph: in dense spruce forests, where it carpets almost everything beneath the trees, Cheat Bridge. Grant: notul. idem., Bayard (Millspaugh).

H. RUtABUlum L.

Monongalia: on rocks in rill, Tibb's Run (Millspaugh, I59I).

H. Haldanianum Grev.

Fayette: near Quinnimont (Pollard \& Maxon, I I5).

H. REPTILE Rich.

Monongalia: on ground near Tibbs Run (Andrews).

H. uncinatum Hedw.

Monongalia; at Quarry Run (Andrezis).

H. TREQUetrum (L.) Br. \& Sch.

Monongalia : on ground, shade of hemlocks, Laurel Hills (Millspaugh, I649).

H. MiCROCARPUM C. Muell.

Monongalia: on bark of hemlock tree, Cheat River (Millspaugh, I389).

H. HIANS Hedw.

Mercer: on damp, decayed bark, Bluefield (Millspaugh. I840). Fayette: along Loup Creek (James).

H. DEMISSUm Wils. Rhynchostegium demissum, Br. \& Sch.

Monongalia: on stones in rill, Tibb's Run, and on wet rocks (Millspaugh, I619, I595).

H. Plumosum Huds. Brachythecium salebrosum, Br. \& Sch.

Fayette: near Nuttallburg (Nuttall).

PTILIUM DeNot.

P. CRISTA-CASTREnSe (L.) DeNot. Hypnum crista-castrense, I..

Plentiful on ground, rocks, logs, etc., in the dense spruce forests. Grant: near Bayard. Randolph: near Cheat Bridge (Millspaugh).

\section{STEREODON Mitt.}

S. IMPONENS (Hedw.) Brid.

Hypnum imponens, Hedw.

Monongalia: on decayed log, Georgetown; loc. cit., Tibb's Run. (Millspaugh, I38I, I602) Fayette: near Nuttallburg (Nuttall).

S. CUPRESSIFORMIS (L.) Brid.

$H$. cupressiforme I.

Mercer: on damp decayed log, Bluefield (Millspaugh, I487).

S. curvifolius (Hedw.) Brid. H. curvifolium Hedw.

Monongalia: on decayed oak, Little Falls; near Morgantown; near Georgeton; near Cassville; on ground, Georgetown. McDowell: on decayed log, Elkhorn. Mercer · 
loc. cit., Bluefield, and Beaver Spring (Millspaugh, 1276, I344, I380, 1420, I382, I517, I485, I491). Fayette: near Nuttallburg (Nuttall).

S. RECURvans (Schwaeger) Broth.

(Hypnum Schwaeg.).

Tucker: on decayed logs, etc., at Blackwater Falls; Mc-

Dowell: on same at Elkhorn; Monongalia: on the bole of a tree at Tibbs Run (Millspaugh, 990, 991, 992, I499, I 507.).

\section{SE M A T O PH Y L LA CEA E.}

\section{RHAPHIDOSTEGIUM DeNot.}

R. Carolinianum (C. M.) J. \& S.

Monongalia: at Tibb's Run, on rock at Quarry Run, on ledge at Burnt House (Sheldon, 553, 3031, 4236). Preston: on wet rock at McKinneys Cave, Bretz (Sheldon, 4r68).

R. Carolinianum admixtum (Sull.) J. \& S.

Monongalia: on a ledge at Burnt House (Sheldon, 4235).

R. Marylandicum (C. M.) J. \& S.

Randolph: on a rock in a small stream near Cheat Bridge (Sheldon, 2572).

R. CYlindrocarpum (C. M.) Kindb.

Monongalia: on decayed wood near Quarry Run (An-

B R A C H Y T H C I A CE A E.

BRACHYTHECIUM Bry. Eu.

B. acuminatum (Hedw.) Kindb.

On tree trunk Monongalia: near Marilla (Sheldon, I4I7).

B. plumosum (Sw.) Br. \& Sch.

Monongalia: on wet ledge along Deckers Creek opposite Marilla (Sheldon, 4003, 4999).

B. Digastrum C. M. \& K.

Monongalia: on decayed log, Lick Run (Sheldon, 3968)

B. Luteum (Brid.) Bry. Eu.

Monongalia: on ground near Quarry Run (Andrews).

B. Rivulare (Bruch.) Bry. Eu.

Monongalia: on ground near Quarry Run (Andrecus).

\section{RHYNCHOSTEGIUM Mitt.}

R. RUSCIFORME B. \& S.

Along Horsepen Creek, McDowell County, July 3I, I900 (Morris, III7). Fayette: near Quinnimont (Pollard \& Maxon, Io9).

R. Serulatum (Hedw.) Jaeg.

Monongalia: on ground about Morgantown (Andrecus). 
C. Bosci (Schw.) Grout.

CIRRIPHYLLUM Grout.

Monongalia: at Morgantown, and on ledge near Burnt House (Sheldon, 1270, 4230).

HOMALOTHECIELLA (Card.) Broth.

H. subcapillata (Hedw.) Card.

Randolph: on tree trunk near Cheat Bridge (Sheidon, 25I2).

O P H I O GLOS S A C E A E.

\section{OPHIOGLOSSUM, L.}

O. vulgatum L. Adder's Tongue.

Wet meadows and woods. Gilmer: near Glenville (Mapel).

BOTRYCHIUM, Sw.

B. ternatum (Thunb.) Sw. Moon-wort.

Dry woods. Monongalia, Marion, Preston: in Laurel hills. Grant: near Bayard (Millspaugh). Gilmer: near Glenville (Mapel). Fayette: near Nuttallburg, glades, alt. 2,000 ft (Nuttall). Preston: near Aurora (Mr. \& Mrs. Steele).

B. silaifolium Presl, (B. ternatum australe D. C. Eaton).

Grassy places. Monongalia: on the campus near Morgantown (Millspaugh).

B. Matricariae (Schrank) Spreng.

Rich opens. McDowell: near Elkhorn (Millspaugh).

B. obliguum Muhl.

Fayette: near Nuttallburg, dry opens, alt. 2,000 ft. (Nuttall). Ohio: hills back of Wheeling (Mertz \& Guttenberg). Monongalia: on the campus near Morgantown (Millspaugh).

B. DIsSeCTUm Spreng.

Glades. Fayette: near Nuttallburg, alt. 2,000 ft. (Nutt.. all). Monongalia: on the campus near Morgantown (Millspaugh).

B. Virginianum (L.) Sw. "Indicator."

Rich woods. Grant: near Bayard. Randolph: on Rich Mountains. Monongalia: Cheat River near Camp Eden. Gilmer: near Glenville. Jackson: near Ripley, where it is often called "Indicator" as its growth is thought to indicate the presence of Ginseng in the locality (Millspaugh). 


\title{
Pteridophyta.
}

\author{
F I L I E S.
}

POLYPODIUM, L.

P. vulgare L. Common Polypody.

Common on mossy rocks and in rocky woods. Kanawha: near Charleston (Barnes) ; near Coalburg (James). Gilmer: near Glenville ( $\mathrm{Mapel}$ ). Fayette: near Nuttallburg (Nuttall). Grant: near Bayard and along Buffalo Creek. Monongalia: along Cheat River. Tucker: along Beaver Creek and Blackwater. Randolph: on Rich and Cheat Mountains (Millspaugh) ; near Whitmar (Greenman, 30) ; Greenbrier . near White Sulphur Springs. Summers: near Hinton McDowell : near Elkhorn (Millspaugh).

P. vulgare deceptum Maxon, Proc. Nat. Mus., 23:628 (I90i),

Rhizoma slender, extensively creeping, covered thickly with spreading chaff; stipe 5 to 8 inches long, greenish to stramineous; laminae very dark green above, lighter below, 7 to II inches long, $2 \frac{1}{2}$ to 4 inches broad; pinnae distant from once to twice their width, broadest in the middle and tapering to an acute apex, the margin doubly crenate or occasionally nearly entire, the base broadly decurrent, veins sinuous and prominent in drying, the veinlets usually forking twice; tip of lamina long acuminate, as in $P$. falcatum; sori very large, often irregularly disposed.

Type in the U. S. National Herbarium, Smithsonian Institution, collected by E. L. Morris, No. I2I 5 , on rocks, along the Guyandotte River below Baileysville, Wyoming County, W. Va., alt. I,I00-I,250 feet, August I3-I9, I900. This fern has already been briefly characterized by Dr. Millspaugh as Polypodium vulgare forma biserrata (sic). The name biserratum being already preoccupied by a Mexican fern it becomes necessary, in referring to the West Virginian plant, to substitute a new name. In addition I would refer here Mr. Morris' I 207 collected near the type station; also Pollard \& Maxon's No. 25, collected Aug. 21, 1899, at Quinnimont, W. Va., which I have pre. viously referred tentatively to the variety acutum Moore. From acutum it differs in the narrower and more spatulate pinnae, and commonly in the double crenation, for acutum is normally with entire, or at most slightly serrulate, pinnae. 
Mr. Morris states that typical vulgare was common in the general region; from this it differs in its much greater size, its scantier foliage, and in the shape of the pinnae. There are in the National Herbarium at least two specimens, collected in West Virginia and North Carolina, which with plants collected at Great Falls, Fairfax County, Va., by William Palmer, are to be regarded as intermediate with typical oulgare, mossy boulders along the Blackwater Fork of Cheat River (Millspaugh).

P. polypodioides (Linn.) Hitch. Polypodium incanum Sw.

Rocks and tree trunks. Fayette: near Hawk's Nest (Porter) ; opposite Gauley (Eggleston, 5525). Wirt: near Burning Springs (Mertz).

\section{CHEILANTHES, Sw.}

C. Lanosa (Michx.) Watt. C. vestita (Spreng.) Sw.

Rocky places, Jefferson: near Harper's Ferry (Mertz \& Guttenberg).

C. Feei Moore.

C. lanuginosa, Nutt.

Cliffs. Fayette: near Kanawha Falls (James).

C. тоmentosa Link.

Grant: on the rocks of a high cliff at Peters Gap ( $A$. B. Brooks).

$$
\text { PELLAEA, Link. }
$$

P. Atropurpurea (L.) Link. Cliff Brake.

Dry rocks. Fayette: near Nuttallburg, rare (Nuttall). Mercer: near Beaver Spring on exposed ledges, plentiful (Millspaugh).

PTERIDIUM Scop.

P. AQuilinum (L.) Kuhn. Brake or Bracken.

Thickets and hillsides. Common throughout. Upshur: at School-house Summit. Webster: in Upper Glade (Millspaugh). Kanawha: near Coalburg and Charleston (James). Fayette: near Nuttallburg (Nuttall).

\section{ADIANTUM, L.}

A. pedatum, L. Maiden Hair Fern.

Rich moist woods. Common throughout the State (Millspaugh). Preston: near Aurora (Mr. \& Mrs. Steele).

\section{ASPLENIUM, L.}

A. Pinnatifidum Nutt. Spleenwort.

Cliffs and rocks. Jefferson: near Harper's Ferry (Merts \& Guttenberg). Monongalia: near Dellslow (Sheldon, 36r). Fayette: near Nuttallburg, in clefts of boulders, rare (Nuttall). 
A. Trichomanes, L.

Shaded cliffs. Wirt: near Burning Springs. Nicholas: along Peter Creek. Fayette: near Nuttallburg (Nuttall) Preston: near Aurora (Mr. \& Mrs. Steele); and along the Gauley River. Kanawha: near Coalburg (James). Gilmer: near Glenville. Greenbrier: near White Sulphur Springs (Millspaugh, Eggleston 5517).

A. TRICHOMANES INCISUm Moore.

Summers: hills above Hinton (Eggleston, 5565).

A. platyneuron (L.) Oakes. (A. ebenum. Ait.)

Frequent in rocky open woods. Kanawha: near Charles. ton (Barnes). Fayette: near Nuttallburg (Nuttall). Wirt: near Burning Springs (Millspaugh). Greenbrier : near White Sulphur Springs (Eggleston, 55 I5). Preston near Aurora $(M r$. \& Mrs. Steele).

A. montanum, Willd.

Cliffs and rocks. Fayette: near Hawk's Nest (Porter) (see Meehan's Monthly, Aug., I892, plate); near Nuttall. burg, alt., 2,000 ft. (Nuttall). Jefferson: near Harper's Ferry. Randolph: near Helvetia (Mertz \& Guttenberg). Grant: near Bayard. Monongalia : on boulders along Tibb's Run, plentiful (Millspaugh).

A. Angustifolium Michx.

Rich woods. Ohio: near Wheeling (Mertz \& Guttenberg). Preston: near Aurora (Mr. \& Mrs. Steele). Fayette: near Nuttallburg (Nuttall).

\section{ATHYRIUM Roth.}

A. thelypteroides (Michx.) Desv. (Asplenium Michx).

Rich woods. Kanawha: near Charleston (James). Fayette: near Nuttallburg (Nuttall). Preston: near Aurora (Mr. \& Mrs. Stcele). Jefferson: near Harper's Ferry (Mertz \& Guttenberg).

A. Filix-foemina (L.) Roth.

(Asplenium L.).

Randolph: at Glady (Greenman, 32). Moist woods. Gilmer: near Glenville (Mapel). Fayette: near Nuttallburg (Nuttall). Ohio: near Wheeling (Mertz \& Guttenberg). Tucker: near the Falls of Blackwater (Millspaugh). Preston: near Aurora (Mr. \& Mrs. Steele).

A. Filix-foemina pectinatum (Wall.)

Rich black soil. Tucker: Falls of the Blackwater (Millspaugh).

CAMPTOSORUS, Link.

C. RHizophyllus (L.), Link. Walking Fern. Walking Leaf.

Shaded rocks and conglomerate boulders. Wirt: near Burning Springs. Fayette: along the Gauley River; near 
Kanawha Falls (James). Tucker: at Blackwater Falls. Gilmer: near Glenville (Mapel). Kanawha: near Coalburg (James). Fayette: near Nuttallburg (Nuttall). Monongalia: near Morgantown (Millspaugh). Summers: near Hinton (Eggleston, 5564). Preston: near Aurora (Mr. \& Mrs. Steele).

PHEGOPTERIS, Fee.

P. Phegopteris (Linn.) Underw.

P. polypodioides, Fee.

Damp woods. Gilmer: near Glenville (Mapel). Tucker: near the Falls of Blackwater (Millspaugh).

P. hexagonoptera (Michx.) Fee.

Open woods. Gilmer: near Glenville (Mapel). Fayette: near Nuttallburg, shaded fence rows and deep woods (Nuttall). Ohio: near Wheeling (Mertz \& Guttenberg). Preston: near Aurora (Mr. \& Mrs. Steele).

P. Dryopteris (I.) Fee.

Rocky woods. Preston: near Rowlesburg (Mertz \& Guttenberg).

\section{DRYOPTERIS Adans.}

D. Thelypteris (Linn.) A. Gray. Aspidium Thelypteris (L.) Sw.

Marshy Meadows. Ohio: near Wheeling (Mertz \& Guttenberg). Preston: near Aurora (Mr. \& Mrs. Steele).

D. Noveboracensis (Linn.) A. Gray. Aspidium Noveboracense (L.) Sw.

Moist woods. Randolph: on Rich Mountain, alt. I,850 feet. (Millspaugh). Fayette: near Nuttallburg (Nuttall); near Kanawha Falls (James). Kanawha: near Charleston (James). Preston: near Aurora (Mr. \& Mrs. Steele).

D. Fragrans (Linn.) Schott. Aspidium fragrans (L.) Sw.

Opens. Pocahontas: near summit of Spruce Knob, alt. 4,800 feet, where it is cut and cured for fodder (Millspaugh).

D. Spinulosa (Retz.) Kuntze. Aspidium spinulosum (Retz.) Sw.

Damp woods. Wirt: above Burning Springs. Randolph: on Rich Mountain. Preston: near Terra Alta. McDowell: near Elkhorn (Millspaugh). Preston: near Aurora (Mr. \& Mrs. Steele). Fayette: near Nuttallburg (Nuttall). Webster: at Tater Knob (H. H. Smith, I496).

D. spinulosa intermedia (Muhl.) Underw. Aspidium spinulosum var. intermedium Eaton.

Deep rich woods throughout Grant, Tucker and Poca hontas Counties. Upshur: near Beech and Middle Fork (Millspaugh). Fayette: near Nuttallburg (Nuttall). Preston: near Aurora ( $M r$. \& Mrs. Steele). Randolph: along a mountain brook near Whitmar (Greenman, 27). 
D. spinulosa dilatata (H’offm.) Underw. Aspidium spinulosum var. dilatatum Hook.

Deep woods. Ohio: near Wheeling (Mertz \& Guttenberg).

D. Simulata Davenport, Bot. Gaz., 29:495 (I894).

Aspidium spinulosum var. Prel. Cat. Fl. W. Va., 482 (1892). Comparing my specimens from the deep, wet woods of Randolph Co. with specimens of $D$. simulata recently sent me by Professor Davenport, I find them identical. In publishing the species Professor Davenport includes it under Dryopteris as well as Aspidium, Nephrodium and Lastrea.

In deep, wet woods under Black Spruce. Randolph: neas Cheat Bridge, and Shades-of-Death (Millspaugh).

D. cristata (L.) A. Gray. Aspidium cristatum (L.) Sw.

Swampy places. Preston: near Cranberry Summit (Mert3 \& Guttenberg); near Reedsville and Terra Alta (Millspaugh) ; near Aurora (Mr. \& Mrs. Steele).

D. Goldienna (Hook.) A. Gray. Aspidium Goldieanum Hook.

Rich moist woods. Preston: near Cranberry Summit (Mertz \& Guttenberg); near Aurora (Mr. \& Mrs. Steele) Fayette : near Nuttallburg (Nuttall).

D. Filix-mas (L.) Schott. Male-fern. Aspidium Filix-mas Sw.

Rocky woods. Gilmer: near Glenville (Mapel).

D. marginalis (L.) A. Gray. Aspidium marginale Sw.

Rocky hillsides in rich woods. Tucker: near Parsons (Greenman, 28). Gilmer: near Glenville (Mapel). Kanawha: near Charleston (James). Fayette: near Nuttallburg (Nuttall). Grant: near Bayard (Millspaugh). Preston: near Aurora (Mr. \& Mrs. Steele).

\section{POLYSTICHUM Roth.}

P. Acrosticholdes (Michx.) Schott. Christmas Fern. Aspidium acrostichoides Sw.

Rocky woods. Upshur: beyond Buckhannon. Randolph: near Cricard (Millspangh). Webster: near Tater Knob (H. H. Smith, I509). Gilmer: near Glenville (Mapel). Fayette: near Nuttallburg (Nuttall). Preston: near Aurora (Mr. \& Mrs. Steele). Barbour: near Tygart Junction (Greenman, 35).

P. Acrostichoides Schweinitzir (Beck). Aspidium achrostichoides Schweinitzii (Beck.) B. S. P.

Rocky woods. Jefferson: near Harper's Ferry (Mertz \& Guttenberg). 
FILIX Adans.

F. BUlbifera (L.) Underw. Bladder Fern (Cystopteris bulbifera Bernh.).

Shaded ravines. Ohio: near Wheeling (Mertz \& Guttenberg). Fayette: near Nuttallburg, rare (Nuttall). Randolph: near Pickens (H. H. Smith, 136r).

F. FRAGilis (L.) Underw. (Cystopteris fragilis Bernh.). Shady cliffs. Fayette: near Gauley Bridge along the Kanawha (Millspaugh); near Nuttallburg (Nuttall).

\section{ONOCLEA, L.}

O. sensibilis, L. Sensitive Fern.

Moist meadows. Monongalia: the Flats. Gilmer: near Glenville (Mapel). Fayette: near Kanawha Falls (James): near Nuttallburg (Nuttall). Randolph: near Valley Head. Upshur: near Buckhannon (Millspaugh). Preston: near Aurora (Mr. \& Mrs. Steele).

WOODSIA, R. Br.

W. овтUsa (Spreng.), Torr.

Rocks and cliffs. Fayette: near Nuttallburg (Nuttall). Randolph: near Cricard (Millspaugh). Preston: near Aurora (Mr. \& Mrs. Steele).

\section{DENNSTAEDTIA Bernh.}

D. punctilobula (Michx.) Moore. Dickson's Fern (Dicksonia punctilobula Hook).

Moist shady places. Randolph: on Rich Mountains, alt. I,920 feet; Cheat mountains under Black Spruce, abundant (Millspaugh). Gilmer: near Glenville (Mapel). Preston: near Aurora (Mr. \& Mrs. Steele). Fayette: near Nuttallburg, alt. 2,400 feet (Nuttall). Pendleton: on Spruce Knob Greenman, 3I); near Kanawha Falls and Loup Creek (James). Kanawha: near Charleston (James).

\section{OSMUNDA L.}

O. REgalis, L. Royal Fern.

Swampy meadows. Upshur: near Buckhannon (Pollock). Pocahontas: near Travellers Repose (Greenman. 29). Upshur : near Randolph County line on Staunton Pike. Webster: Upper Glade. Preston: near Terra Alta and Cold Spring. Monongalia: near Camp Eden. McDowell: near Elkhorn (Millspaugh). Gilmer: near Glenville (Mapel). Fayette: near Nuttallburg (Nuttall). 
O. Claytoniana, L. Clayton's Flowering Fern.

Low grounds. Preston: near Cranberry Summit (Merts

\& Guttenberg.) Fayette: near Nuttallburg (Nuttall).

O. cinnamomea, L. Cinnamon Fern.

Marshy places. Fayette: near Nuttallburg, alt. 2,00n feet (Nuttall). Preston: near Cranberry Summit (Mertz \& Guttenberg). Randolph: along Shaver's Fork (Millspaugh); near Pickens (H.H. Smith, r392).

\section{EQUISETA CEA E.}

\section{EQUISETUM, L.}

E. arvense, L. Field Horsetail.

Moist, sandy fields. Frequent throughout the State.

E. sylvaticum, L.

Damp rich woods. Mercer: near Ada. Monongalia: near Little Falls (Millspaugh).

E. hyemale, L. Scouring Rush.

Wet wooded banks. Wirt: near Burning Springs (Millspaugh).

E. laevigatum, Braun.

Clay banks along stream. Mercer: near Ingleside. (Millspaugh).

L Y C O POD I A CEA E.

LYCOPODIUM, L.

L. LUCIDULum Michx.

Cold damp woods. Grant: near Bayard, and Tucker: near Davis (Millspaugh). Randolph: near Cheat Bridge (Millspaugh), and near Whitmar (Greenman, 22). Preston, near Aurora (Mr. \&.Mrs. Steele). Gilmer: near Glenville (Mapel). Fayette: near Nuttallburg (Nuttall).

L. ANNOTINUM L.

Cold woods. Randolph: plentiful along the west slope of Cheat Mountains near Cheat Bridge (Millspaugh). Fayette: near Nuttallburg ( Nuttall).

L. obscurum L. Ground Pine. (L. dendroideum, Michx.)

Deep, moist woods. With the last, plentiful (Millspaugh).

Randolph: near Pickens (H. H. Smith, I824).

L. clavatum L. Club Moss.

Dry woods. Common throughout the State.

L. TRISTAChyum Pursh.

Pendleton: on the western slope of Spruce Mountain (Greenman, 24). 
L. complanatum L. Trailing Christmas Green.

Deep coniferous woods. In the Alleghanies of Grant, Tucker, Randolph and Pocahontas counties (Millspaugh) Fayette: near Nuttallburg (Nuttall).

SELA G I N E L A C E A E.

SELAGINELLA, Beauv.

S. RUPESTRIS (L.) Spring.

Jefferson: near Harper's Ferry (Mertz \& Guttenberg).

S. APUS (L.) Spring

In a luxuriant mass among the grass and shrubs along the edge of an island in the Bluestone River, opposite Delashmeet Creek, Mercer County, altitude 2,080 feet, July 27, I900 (Morris, I06I). Monongalia: several stations nea: Morgantown; and Pocahontas: near Warnton (Sheldon). Barbour: near Belington (A. B. Brooks). 


\title{
Gymnospermae*
}

\section{P I N A CEAE.}

\author{
THUJA, L.
}

T. occidentalis, L. Arbor Vitæ.

Dry, rocky hills. Mineral : on Knobby Mountains. Grant: near Petersburg.

JUNIPERUS, L.

J. Communis, L. Juniper.

Dry sterile hills.. Wood: near Kanawha Station. Mineral: on Knobby Mountains. Fayette: near Nuttallburg (Nuttall).

J. Virginiana L. Red Cedar. Savin.

Wood: near Lockhart's Run and Kanawha Station (Mills. paugh), near Nicollet (H. H. Smith, 2030). Wirt: on Nigh-cut Hill. Fayette: near Crescent; Kanawha Falls (James); Nuttallburg (Nuttall). Mineral: on Knobby Mountains. Jefferson: along the Potomac. Mason: near Point Pleasant. Berkeley: near Martinsburg. Monongalia: near Morgantown. Mercer: near Ingleside. Jackson and Mason: along the Ohio River; Cabell: along the C. \& O. R. R., from Huntington, to St. Albans in Kanawha County. Jefferson Co., plentiful near Harper's Ferry, trunks 7 to 9 inches in diameter.

PINUS, L.

P. Resinosa Aiton. Pitch Pine.

Open woods. Preston: near Cranesville Glade (Rumsey).

P. Strobus L. White Pine.

Wood: near Leachtown. Wirt: near Burning Spring. Calhoun: near White Pine and Laurel Run. Pocahontas: near Sunset. Greenbrier: near Caldwell. Fayette: near Nuttallburg, probably introduced (Nuttall). Preston: near Aurora (Mr. \& Mrs. Steele).

* From here to the end of this work where no collector is mentioned the localities are those of the author. 
P. TAEDA L. Loblolly, or Old-field Pine.

Opens. Wood: near Kanawha Station. Mineral, Hampshire, and Hardy: along the table-lands.

P. RIGIDA Mill. Pitch Pine.

Kanawha: near Charleston (Barnes). Fayette: near Nuttallburg, alt. 2,000 (Nuttall). Greenbrier: near White Sulphur Springs. Mineral: on Knobby Mountains. Webster: on Tater Knob, Wood: near Nicollet (H. H. Smith. I510, 2026).

P. Pungens, Michx. f. Table Mountain Pine.

Dry rocky soils. Kanawha: near Charleston (Barnes) Mineral on Knobby Mountain. Pendleton: foot hills of Spruce Knob (A. D. Hopkins).

P. Virginiana Mill. Scrub Pine. (P. inops, Ait.).

Sterile hills. Greenbrier : near Caldwell; slopes of Kate's Mountain, alt. 3,300 ft. (Small). Fayette: near Nuttallburg, a common second growth (Nuttall). Summers: near Hinton (Eggleston, 5587). Berkeley: near Martinsburg Mercer: near Ingleside.

P. echinata Mill. Yellow Pine. (P. mitis, Michx.)

Wood: near Leachtown; near Nicollet (H. H. Smith, 2025). Randolph: near Valley Bend. Summers: near Hinton.

\section{LARIX Adans.}

L. LARICINA (DuRoi) Koch. Tamarack (L. Americana Mx.) Boggy lands. Preston: in Cranesville Glade (Rumsey).

PICEA, Link.

P. Rubens Sarg. Black Spruce. "Yew Pine.” "White Spruce,” "Spruce Pine." (Picea Mariana Prel. Cat)

In IgOI there were magnificent forests in the following regions where it grew at elevations varying from 2,500 to 4,000 feet:

Randolph County on Elk and Gauley waters..... I5,000 Randolph County on Cheat waters.......... 120,000 Randolph County on Mill Creek........... 5, 5, Randolph County on Elk Mountain........... 500 Pocahontas County on Shaver's Fork of Cheat... 20,000 Pocahontas County on the headwaters of Greenbrier River ..................... 100,000 Pocahontas County on Elk and Gauley headwaters. I00,000 Tucker County on Cheat water............. 50,000 
Mineral County .................. 25,000

Greenbrier County (by actual survey)....... 35,499

Total acreage

470,999

This estimate probably fell under the actual amount.

Summers : along Greenbrier River near Talcott. Mercer: near Ingleside (Millspaugh). Preston: near Aurora ( $M r$. \& Mrs. Steele). Randolph: near Pickens and Turkey Bone (H. H. Smith, I4I I, 2055).

\section{TSUGA, Carrier.}

T. Canadensis (L.) Carr. Hemlock. Hemlock Spruce. Abies Canadensis, Michx.

Rocky woods. Wirt: along Straight Creek. Calhoun: on Laurel Run. Nicholas: near Beaver Mills. Grant: near Bayard, abundant. Monroe: near Alderson. Preston: near Terra Alta (Millspaugh) ; near Aurora (Mr. \& Mrs. Steele). Fayette: near Nuttallburg, plentiful (Nuttall). Mercer: near Ingleside and Princeton. McDowell: near Elkhorn. Monongalia: near Uffington and Tibb's Run.

ABIES, Miller.

A. Fraseri (Pursh) Lindl. "Blister Pine." Balm-of-Gilead Fir. Balsam Fir.

Mountain Swamps. Randolph: about two miles beyond Cheat Bridge, along the Staunton Pike (Millspaugh; Sheldon, 2585).

\section{T A X A C E AE.}

TAXUS, Linn.

Taxus Canadensis Marshall. (T. baccata, Canadensis Gray).

Damp hillside woods. Marion: near the mouth of Buffalo Creek (K. D. Walker). Taylor: near Nuzums. Fayette: along Williams River. Randolph: in the Sink region Greenman, I98).

\section{T Y P H A CE A E.}

TYPHA, L.

T. latifolia, L. Cat Tail.

Ditches and swampy spots. Gilmer: near Glenville ( $M a-$ pel). Preston: near Terra Alta. Morgan: near Hancock. Monongalia: near the Dille farm. 
S P A R G A N I A CE AE.

$$
\text { SPARGANIUM, L. }
$$

S. eurycarpum, Engelm. Bur-reed.

Borders of stream and still waters. Webster: in Welch Glade. Greenbrier: near White Sulphur Springs.

S. diversifolium acaule (Beeby) Fern. \& Eam.

Pocahontas: near Travellers Repose (Greenman, 75).

$$
\text { POTA MOGETONACEAE. }
$$$$
\text { POTAMOGETON, L. }
$$

P. LONCHITES Tuck.

P. fluitans Roth.

In rivers and streams. Randolph: near Tygart's Valley River, near Huttonsville. Summers: New River: near Hin ton.

P. SPIRILlus Tuckerman.

Randolph: in the Tygarts Valley River near Huttonsville (Greenman, I32).

P. pectinatus L. (Determined by F. V. Coville).

Forming large masses on the bars of the Greenbrier River at Talcott, Summers County, altitude I,490 feet, August 24, 1900 (Morris, I342).

P. DIMORPHUS Raf.

In still water. Hardy: near Moorefield (A. B. Brooks). P. EPIHYDRUs Raf.

Preston: in a ditch near Cranesville (Sheldon 34I4 visa).

S C H E U C H Z E R I A C E E.

SCHEUCHZERIA L.

S. PALUSTRIS Linn.

Pocahontas: in Cranberry Glades (A. B. Brooks).

$$
\text { A L I S M A C E A E. }
$$

ALISMA, L. .

A. Plantago-aquatica Linn.

Wet ditches. Monongalia: near Ice's Ferry. Summers: near Hinton. Greenbrier: near White Sulphur Springs.

$$
\text { SAGITTARIA, L. }
$$

S. ARIFolia Nutt. Arrow-head.

In water or very wet places. Randolph: near Beverly. Summers: near Hinton. Greenbrier: near White Sulphur 
Springs. Wood : near Parkersburg. Marion : near Clements. Fayette: near Nuttallburg (Nuttall). Berkeley near Martinsburg.

forma ANGUSTIFolia (Engelm.) Britt.

Wet places. Putnam: near Buffalo. Mason: near Point Pleasant.

S. GRAMINEA Michx.

Summers: shores of New River near Hinton.

\section{A L L I S N E R I A C E A E.}

\section{VALLISNERIA L.}

V. SPIRALIS L. Eelgrass.

Among the pondweeds in the Greenbrier River at Talcott, Summers County, altitude I,490 feet, August 24, I900 (Morris, I34I).

\section{H Y D R O C H A R T A C E A E.}

\section{PHILOTRIA Raf.}

P. Canadensis (Michx.) Britton. Water-weed. (Elodea Canadensis Michx.)

Slow streams in slack water. Fayette: near Kanawha Falls (James). Putnam: near Buffalo (Millspaugh). Kanawha: near Charleston (Holton).

\section{GR A M I N E AE.}

\section{TRIPSACUM, L.}

T. DACTYLOIDES L.

Moist places. Fayette: near Nuttallburg (Nuttall).

\section{ANDROPOGON, L.}

\section{A. Furcatus Muhl. Beard Grass.}

Damp places. Randolph: along Tygart's Valley River near Beverly. Monongalia: along the Monongahela below Morgantown. Fayette: near Kanawha Falls. (James); near Nuttallburg (Nuttall). Summers: near Greenbrier Stockyards, and along Greenbrier River. Taylor: near Grafton. Preston: near Aurora (Mr. \& Mrs. Steele). Barbour: near Tygart Junction; and Tucker: banks of the Blackwater River near Hendricks (Greenman, 38, 86).

A. SCOPARIUS Michx. "Broom Sedge."

Dry soils. Monongalia: about Morgantown, plentiful. Webster: near Upper Glade. Mason: near Point Pleasant. 
Taylor: near Grafton. Preston: near Aurora (Mr. \& Mrs. Steele), and in every county visited.

This species, which threatens to be our most pernicious and wide-spread weed, is advancing eastward with the utmost aggressiveness. It has absolutely no fodder qualities, its nutritive ratio being only $\mathrm{I}: \mathrm{I4.50}$, and its value as a fertilizer only $\$ 2.37$ per dry ton. The method of combating this pest is as yet a mere matter of conjecture.

The plant is reported from Brooke: near Wellsburg and Fowler's. Barbour: near Overfield, Pepper and Philippi. Braxton: near Bulltown, Frametown, Tate Creek, Elmyra and Newville. Cabell : near Union Ridge and Barboursville. Clay: near Valley Fork. Doddridge: near Leopold, New Milton and Center Point. Fayette: near Fayetteville, Mountain Cove and Beets. Grant: near Medley, Petersburg and Greenland. Greenbrier: near Frankford, Trout Valley and Fort Spring. Hampshire: near Slanesville, Springfield and Three Churches. Harrison: near Clarksburg, Lost Creek, Bridgeport, Wallace, Wilsonburg, Adamsville, Good Hope and Mount Clare. Hardy: near Moorefield and Wardensville. Jefferson: near Kabletown. Jackson: near Grass - Lick, Sandy, Wilding, Belgrove, Odaville, Silverton and Kentuck. Kanawha: near Pocataligo, Blandon and Gazil. Lewis: near Vadis, Camden, Walkersville and Aberdeen, Lincoln: near Hamlin. Mercer: near Princeton and New Hope. McDowell: near Squire Jim. Monroe: near Cashmere and Johnson's X Roads. Mason: near New Haven, Grimm's Landing and.Maggie. Marion : near Canton, Farmington, Eldora, Barracksville, Hoult and Gray's Flat. Monongalia : near Morgantown. Putnam : near Hurricane, Paradise, Carpenters and Confidence. Pleasants : near New Hebron. Preston: near Masontown, Eglon and Amblersburg. Ritchie: near Ritchie C. H. Berea and Cornwallis. Randolph: near Florence and Lee Bell. Roane: near Peneil, Newton, Looneyville, Clio, Reedy and Countsville. Raleigh: near Table Rock, Egeria and Raleigh C. H. Summers : near Forest Hill, Talcott, Clayton and Indian Mills. Taylor: near Knottsville, Thornton, Grafton and Meadland. Tyler: near Wick and Long Reach. Tucker: near Texas, St. Georges and Hendricks. Upshur: near Lawrence, Overhill, Evergreen, French Creek and Hemlock. Wayne: near Adkin's Mills and Egypt. Wetzel: near New Martinsville. Wirt: near Elizabeth, Burning Springs, Reedy Ripple and Lee. Wood: near Blennerhassett and Kanawha Station. Webster: near Replete, and Welch Glade. 
A. Virginicus L.

Fayette: near Nuttallburg (Nuttall). Preston: near Aurora (Mr. \& Mrs. Steele). Barbour: near Tygart Junction (Greenman, 37).

\section{CHRYSOPOGON Trin.}

C. nutas (L.) Benth. Indian grass. Wood grass.

Fayette: near Nuttallburg (Nuttall). Barbour: near Tygart Junction (Greenman, 39).

\section{SORGHASTRUM Nash.}

S. avenaceum (Michx.) Nash.

(Chrysopogon Michx.).

Dry fields, Preston: near Aurora (Mr. \& Mrs. Steele). Fayette: near Quinnimont (Pollard \& Maxon, 36).

\section{PASPALUM Linn.}

P. mucronatum Muhl.

In wet places. Kanawha: at the Salinas (Holton).

P. Muhlenbergit Nash.

In dry or stony places. Monongalia: near Morgantown (Grout).

P. SETACEUM Michx.

Sandy soils. Monongalia: at the end of the Hogback, Decker's Creek, near Morgantown (Millspaugh).

P. LAEve Michx.

Fayette: near Nuttallburg (Nuttall). Kanawha: at Washington's Burning Springs near the Kanawha salinas (Holton).

P. LAeve PIlosum Scribn.

Wyoming: along Horse and Hound Creeks, alt. I, IOO to I,200 feet., near Baileysville (Morris, I284).

\section{SYNTHERISMA Walt.}

S. LINEARIS (Krock.) Nash.

Fields and waste places, Preston: near Aurora $(M r$. \& Mrs. Steele).

S. Sanguinalis (L.) Dulac. Crabgrass, Crowfoot grass (Panicum L.). State.

Cultivated and waste grounds, common throughout the PANICUM Linn.

P. Dichotomiflorum Michx. (P. geniculatum Ell., proliferum Lam.).

Moist grounds along streams. Monongalia: near Morgantown and Uffington (Millspaugh; Post). Preston: near 
Aurora (Steele). Randolph: at Tygart's Valley (J. D. Smith; Greenman, 8I). Tucker: near Parsons (Greenman, 54). Fayette: near Nuttallburg (Nuttall).

P. Flexile (Gattinger) Scribn. (P. capillare campestre Gatt.). Dry fields. Monroe: Peters Mountain (Steele, 28I).

P. Gattingeri Nash. (P. capillare campestre Gatt.).

Dry fields. Monongalia: on a sterile hillside up Falling Run beyond the University (Millspaugh; Sheldon, 3084). Preston: near Aurora (Stecle).

P. Philadelphicum Bernh.

Dry, open ground. Preston: near Aurora (Steele). Wyoming: near Baileysville (Morris, 1283). Monongalia: near Morgantown (Sheldon, 3083). Randolph: gravelly bed of Shavers Fork near Parsons (Greenman, 42).

P. CAI'ILlare Linn. Ticklegrass.

Dry fields. Monongalia : near Morgantown, and Greenbrier: near White Sulphur Springs (Millspaugh). Fayette: near Nuttallburg (Nuttall). Monroe: near Old Sweet Springs (Steele, 243). Preston: near Aurora (Mr. \& Mrs. Steele). Randolph: near Huttonsville and along the Blackwater River near Hendricks (Greenman, 84, 5I).

P. VIRGATUM Linn.

Moist sandy soils. Monongalia: streets of Morgantown where it seems to flourish, between the bricks of walks, the better the more it is trodden upon; and Mason: banks of the Ohio River near Point Pleasant (Millspaugh). Tucker: near Parson's (Greenman, 47). Fayette: near Nuttallburg (Nuttall). Randolph: along the Blackwater River near Hendricks (Greenman, 47).

P. AGRostoides Spreng.

Wet meadows. Monongalia: below the mouth of Falling Run near Morgantown, Kanawha: near Allen's Fork, and Upshur: near Lorentz (Millspaugh). Fayette: near Nuttallburg (Nuttall). Wyoming: at the edge of thickets along Horse and Hound Creeks, near Baileysville (Morris, 1277). Barbour: near Tygart Junction (Greenman, 57).

P. stipitatum Nash.

Moist soils. Taylor: near Grafton (Guttenberg; J. D. Smith). Monongalia: near Sabraton (Sheldon, 2689).

P. ANCEPS Michx.

Moist sandy soils. Fayette: near Nuttallburg (Nuttall). Nicholas: at Richwood (H. H. Smith, I745).

P. Depauperatum Muhl.

Dry opens. Monongalia: at Roundbottoms (Millspaugh). Fayette: near Nuttallburg (Nuttall).

P. Linearifolium Scribn.

Dry wcods. Jefferson: near Harper's Ferry (Hitchcock). 
P. MICROCARPON Muhl.

Wet woods and swampy places.' Monongalia: along the Monongahela River (Millspaugh, I98). Wyoming: near Baileysville and McDowell: near Perryville (Morris, II93, I I39).

P. NITIDUM Lam.

Moist grounds. Summers: along the edge of a meadow at Bargers Spring (Morris, 984).

P. ріснотомим Linn.

Dry places. Fayette: (Morse) near Nuttallburg (Nuttall). Wyoming: near Baileysville (Morris, II93a).

forma DEPAUPERATA Vasey.

Dry sterile fields. Monongalia: up Falling Run beyond the University (Millspaugh).

P. Barbulatum Michx.

Sterile and rocky woods. McDowell: along Dry Fork above Perryville, alt. I,200 to I,300 feet, and Wyoming: along the Guyandotte River below Baileysville, alt. I, IOO ft. (Morris, II39, II93).

P. Lucidum Ashe.

( $P$. dichotomum gracile Torrey).

Wet bottoms, usually along runs. Wood: in a swampy ditch near Kanawha Station. Wirt: in a weedy ditch near Reedy Ripple and in a spring rill in rich woods near Straight Creek. Randolph: in a cold rill in deep woods on Point Mountain, alt. 3,200 feet (Millspaugh).

P. Lindheimeri Nash.

Sterile grounds. Summers : near Bargers Spring (Morris, 984).

P. Huachucae silvicola Hitchc. \& Chase. (P. dichotomum fasciculatum Man.)

Dry open places. Monongalia and Marion: along the railroad (Millspaugh); near Morgantown and Fairmont (Hitchcock, I36-7).

P. Tennesseense Ashe.

Open moist places and borders. Monongalia and Marion: along the Monongahela River (Millspangh, 188, 197). Fayette: near Quinnimont (Pollard \& Maxon, 22).

P. villosissimum Nash.

(P. pubescens Lam.).

Damp places. Lewis: along Leading Creek (Millspaugh).

P. TSUGETORUM Nash.

Sandy woods. Jefferson: near Harper's Ferry (Hitchcock, I35).

P. polyanthes Schult.

Damp places. Wyoming: shaded banks of the Guyandot River near Baileysville, alt. I, I00 feet (Morris, I I86). 
P. Ashei Pearson.

Dry rocky woods. Monongalia: at Tibbs Run (Sheldon, 566). Fayette (Kellerman, 69or). Summers: in Oak and Beech woods near Bargers Spring (Morris, 977).

P. сомmutatum Schult.

Woods and copses. Fayette: (Kellerman, 6902).

P. clandestinum Linn. Deer Tongue Grass.

Damp meadows. Monongalia : near Morgantown (Millspaugh, Hitchcock). Preston: near Aurora (Steele). Upshur: near School House Summit (Millspangh). Fayette: near Nuttallburg (Millspangh, Kellerman). Summers: near Riffe, and Monroe: near Wolf Creek (Millspaugh).

P. LATIFolium Linn.

Rocky and sandy woods. Preston: near Aurora (Steele). Pocahontas: near Marlinton (Kellerman, 6899).

P. Boscir Poir.

(P. Porterianum Nash.)

Woodlands. Monongalia: near Little Falls, Wirt: near Burning Springs, Greenbrier: near White Sulphur Springs (Millspaugh). Fayette: near Nuttallburg (Nuttall).

ECHINOCHLOA Beauv.

E. Crus-galli (L.) Beauv.

(Panicum Linn.)

Ditches and waste grounds. Monongalia: near Morgantown, Summers : near Hinton, and Greenbrier: near White Sulphur Springs( Millspaugh). Fayette: near Nuttallburg (Nuttall).

E. Walteri (Pursh) Nash.

Ditches. Monongalia : along the railroad near Little Falls (Millspangh).

CHAETOCHLOA Scribn.

(Setaria, Beauv. I8I2.)

C. gLauca (L.) Scribn. Fox-tail Grass.

Common throughout the State, especially in stubble fields.

C. Imberbis (Poir.) Scribn.

In moist soils, Preston: near Aurora (Mr. \& Mrs. Steele).

C. VIRIDIS (L.) Scribn.

Roadsides and cultivated fields. Jackson : near Sandyville, and on Limestone Ridge.

C. Italica (L.) Scribn.

Fayette: near Nuttallburg (Nuttall). Webster: at Hacker Valley (H. H. Smith, I738).

CENCHRUS, L.

C. tRIBUloides L. Hedgehog Grass. Bur-grass.

Jefferson: near Harper's Ferry (Mertz \& Guttenberg). 
HOMALOCENCHRUS Mieg ( I768).

(Leersia, Swartz, I788.)

H. Virginicus (Willd.), Britton. White Grass. (Lecrsia Virginica, Willd.)

Wet places. Monongalia: near Beechwoods and Little Falls. Fayette: near Nuttallburg (Nuttall). Preston: near Aurora (Mr. \& Mrs. Steele). Randolph: near Huttonsville (Grecnman, 82).

H. oryzoides (L.) Poll. Rice Cut-grass. (Leersia oryzoides, Swartz.).

Wet grounds. Nicholas: in Collett's Glade. Fayette: near Nuttallburg (Nuttall). Preston: near Aurora ( $\mathrm{Mr}$. \& Mrs. Stecle).

\section{PHALARIS, L.}

P. ARundinacea L. Reed Canary-grass. tion.

Wet places. Wood. in a spring rill near Kanawha Sta-

P. Canariensis Linn.

Established at Morgantown, Monongalia County (Shcldon, 3598).

$$
\text { ARISTIDA, L. }
$$

A. Dichотома, Michx. Poverty Grass.

Fayette: near Nuttallburg (Nuttall). Preston: near Aurora $(M r$. \& $M r s$. Steele).

A. Oligantha Michx. Triple-awned Grass.

Dry banks. Kanawha: near Charleston (Barnes).

A. Lanosa Muhl. (A. lanata Poir.)

Fayette: near Nuttallburg (Nuttall).

A. GRACILIS Ell.

Monongalia: dry soils at Morgantown (Sheldon).

\section{ANTHOXANTHUM Linn.}

A. ODORATUM Linn.

Established rather extensively about Morgantown, Monongalia County (Sheldon).

$$
\text { MILIUM Linn. }
$$

M. EFFusum Linn.

Pocahontas: in Cranberry Glades (Sheldon, 3863).

MUHLENBERGIA, Schreber.

M. SOBOLIFERA (Muhl.) Trin.

Fayette: near Nuttallburg (Nuttall). 
M. Mexicana (L.) Trin.

Fayette: near Nuttallburg (Nuttall). Monongalia: on campus, Morgantown (Millspaugh). Preston: near Aurora (Mr. \& Mrs. Steele). Randolph: banks of Dry Fork near Harman (Greenman, 46).

M. sylvatica (Torr.), Torr. \& Gray.

Fayette: near Nuttallburg (Nuttall).

M. DIfFUSA, Schreb. Nimble Will.

Dry ground. Monongalia: along Decker's Creek. Fayete: Nuttallburg (Nuttall). Preston: near Aurora (Mr. \& Mrs. Steele). Randolph: near Elkins (Greenman, 80).

BRACHYELYTRUM, Beauv.

B. ERECTUM (Schreb.) Beauv.

(B. aristatum, Beauv.)

Rocky woods. Webster: near Welch Glade. Tucker: near the Falls of Blackwater. Fayette: near Nuttallburg (Nuttall).

Var. glabratum, Vasey, in Millsp. Prel. Cat. F1. W. Va., 469.

A new variety discovered by Mr. L. W. Nuttall. It agrees with the species except that it is perfectly smooth, and has an awn-pointed second glume which is about onehalf the length of the flowering glume. Its most striking peculiarity is that it has invariably two culms from each rootstock.

High, rocky woods. Fayette: near Nuttalburg, rare (Nuttall).

\section{PHLEUM, L.}

P. pratense, L. Timothy.

A common escape from cultivation throughout the State. Found even in the forests of the highest Alleghanies.

S. NEGLECTus Nash.

\section{SPOROBOLUS R. Br.}

In dry places. Upshur: near French Creek (Sheldon. 2I73).

S. vaginAEFLoRus (Torr.) Wood.

In dry soils. Preston: near Aurora (Mr. \& Mrs. Steele). Monongalia: near Morgantown (C.F. Sheldon).

CINNA, I.

C. Latifolia (Trev.) Griseb.

(C. pendula Trin.).

Pocahontas: valley of the East Fork of the Greenbrier River (Greenman, 88).

C. ARundinacea L. Wood Reed-grass.

Wet places. Randolph: along Tygart's Valley River near Huttonsville. Fayette: near Nuttallburg (Nuttall). 


\section{AGROSTIS, L.}

A. INTERmedia Scribn.

In dry soils, Preston: near Aurora (Mr. \& Mrs. Steele).

A. Altissima (Walt.) Tuckerman.

Sandy moist places, Preston: near Aurora (Mr. \& Mrs. Steele).

A. ALBA L. White Bent-grass. (incl.var. vulgaris (With. Thurb.)

Meadows and fields. A frequent escape in the western counties. Fayette: near Nuttallburg (Nuttall).

A. CANINA L.

On shaded banks of the Guyandotte River below Baileysville, Wyoming County, altitude, I,I00 feet, August I3, I900 (Morris, I I97).

Forma, ARIstata. Millsp. Prel. Cat. Fl. W. Va., 470.

Damp meadowlands. Monongalia: along Falling Run (Millspaugh, 244). Wyoming: near Baileysville (Morris, II97).

A. Perennans (Walt.), Tuckerm. Thin Grass.

Damp, shady places. Randolph: along Cheat River in clearings. Monongalia: near Little Falls. Fayette: near Nuttallburg (Nuttall). Preston: near Aurora (Mr. \& Mrs. Steele).

A. hyemalis (Walt.), B. S. P. Hair Grass. A. Scabra, Willd.

Moist fields. Preston: near Terra Alta. Fayette: near Nuttallburg (Nuttall).

CALAMAGROSTIS Adans.

C. CINNoides (Muhl.) Scribn.

Fayette: near Nuttallburg (Nuttall).

HOLCUS, L.

H. Lanatus L. Velvet Grass. "Old White Top." "Feather Grass."

Frequent in damp meadows. Monongalia: along the $\mathrm{F}$. M. \& P. R. R. Upshur: near Laurentz. Randolph: along Tygart's Valley River. Grant: near Bayard. Nicholas: in Collett's Glade. Fayette: near Hawk's Nest, and Kanawha Falls (James) ; Nuttallburg (Nuttall). Randolph: at Pickens (H. H. Smith, I390).

ARRHENA THERUM, Beauv.

A. Elatius (L.) Beauv. Oat Grass. (A. averaceum, Beauv.)

Fields and yards. Monongalia, abundant and becoming a nuisance.

The lower campus (half orchard) that yielded a good crop of Orchard-grass last season, was almost completely 
this species this year. Our analysis of this grass shows a nutritive ratio of only I :8.13. Fayette: near Nuttallburg (Nuttall).

\section{AVENA Linn.}

A. FATUA Linn.

Established at Morgantown, Monongalia County (Sheldon, 4309).

DANTHONIA, DC.

D. spicata (L.) Beauv. Wild Oat-grass.

Dry soil. Monongalia: near Beechwoods and Little Falls. Randolph: on a dry boulder, summit of Point Mountain, alt. 3,750 feet. Fayette: near Nuttallburg (Nuttall).

D. Compressa, Austin.

Dry banks. Tucker: near the Falls of Blackwater. Fayette: near Nuttallburg (Nuttall). Randolph: near Pickens (H. H. Smith, I355).

SPARTINA, Schreb.

S. Cynosuroides (L.) Willd. Fresh-water Cord-grass.

Ohio: on Bogg's Island (Mertz \& Guttenberg). Barbour: near Tygart Junction (Greenman, 43): Fayette: near Nuttallburg (Nuttall).

ELEUSINE Gærtn.

E. IndicA (L.), Gærtn. Dog's Tail or Wire Grass.

Yards and streets. Kanawha: near Cannellton. Monongalia: near Morgantown. Mason: near Point Pleasant. Berkeley: near Martinsburg. Fayette: near Nuttallburg (Nuttall).

DACTYLOCTENIUM Willd.

D. Aegyptium (L.) Willd. Crab-grass. Yard-grass.

Yards and lawns. Monongalia; on the campus.

TRICUSPIS Beauv.

T. SEslerioIdes (Michx.) Torrey. Tall Red Top. Triodia cuprea J. F. Jacq.

Dry or sandy fields. Monongalia near Little Falls. Wood: near Selden. Fayette: near Nuttallburg (Nuttall).

PHRAGMITES Trin.

P. Phragmites (L.) Karst.

In wet places. Kanawha: near Charleston (Holton). 
ERAGROSTIS, Beauv.

E. HYPNOIDES (Lam.), B. S. P.

E. reptans, Nees.

Shores of rivers. Summers: along New River near Hinton. Kanawha: at the Salinas (Holton). Wood: along the Ohio River near Parkersburg. Mason: near Point Pleasant. Ohio: near Wheeling (Mertz \& Guttenberg).

E. Eragrostis (L.) Karst. E. minor Host.

Waste places. Mason: near Point Pleasant (Millspaugh).

E. MAJOR, Host.

Fayette: near Nuttallburg (Nuttall).

E. PILOSA (L.), Beauv.

Jefferson: near Harper's Ferry (Mertz \& Guttenberg).

Fayette: near Nuttallburg (Nuttall); near Hawks Nest (Holton). Preston: near Aurora (Mr. \& Mrs. Steele).

E. Purshir, Schrader.

Sterile or sandy soils. Monongalia; near Little Falls, and on the campus.

E. CAPILlaris (L.), Nees.

Fayette: near Nuttallburg (Nuttall). Preston: near Aurora $(M r$. \& Mrs. Steele).

E. Frankil, steud.

Shores of rivers. Summers: along New River near Hinton (Millspaugh). Randolph: near Huttonsville (Greenman, 83).

E. PeCtinacea (Michx.) Steud.

Monongalia: dry soils near Morgantown (Sheldon).

EATONIA Raf.

E. obtusata (Michx.), Gray.

Rich woods. Fayette: near Nuttallburg (Nuttall.)

E. Pennsylvanica (DC.), Gray.

Moist opens. Monongalia: near Beechwoods and on the campus.

E. NitidA (Spreng.) Nash.

Dry woods. Monongalia : near Tibbs Run (Sheldon, 625).

\section{MELICA L.}

M. MUTICA, Walt.

Rich soil. Fayette: near Nuttallburg (Nuttall).

KORYCARPUS, Zea (I806).

(Diarrhena, Beauv. I8r2.)

K. Diandrus (Michx.) Kuntze. (K. Americana Kuntze.) Shaded tiver banks. Fayette: near Nuttallburg (Nuttall). 


\section{UNIOLA L.}

U. latifolia, Michx.

Shady places: Fayette: near Nuttallburg (Nuttall).

\section{DACTYLIS L.}

D. glomerata L. Orchard Grass.

Fields and meadows. Common throughout, especially in shady places.

\section{CYNOSURUS Linn.}

C. CRistatus Linn.

Established at Morgantown, Monongalia County (Sheldon, I6I7).

\section{POA L.}

P. ANNUA L. Low Spear-grass.

Ohio: near Wheeling (Mertz \& Guttenberg). Fayette near Nuttallburg (Nuttall).

P. COMPRESSA L. Wire Grass.

Sterile soil in crevices of rocks. Plentiful throughout the State.

Forma DePauperata, Millsp. Prel. Cat. F1. W. Va., 472.

On rocks. Monongalia: along Falling Run, especially at the cascade.

P. PRATENSis L. Blue Grass.

Dry soils and meadows. Common throughout the State, even in the higher Alleghanies.

P. trivialis L. Roughish Meadow-grass.

Moist meadows. Monongalia: along the Monongahela at Little Falls.

P. Sylvestris Gray.

Ohio: near Wheeling (Mertz \& Guttenberg).

P. ALsodes Gray.

Hillside woods. Monongalia : along Day Creek near Little Falls. Fayette: near Nuttallburg (Nuttail).

P. autumnalis Muhl.

P. flexuosa Muhl.

Tyler: near Long Reach.

P. BREVIFOLIA Muhl.

Ohio: Woods Run near Wheeling (Mertz \& Guttenberg).

Fayette: near Nuttallburg (Nuttall).

\section{PANICULARIA Fabr. ( 1763 )}

(Glyceria, R. Br. I8Io.)

P. Canadensis (Michx.) Kuntze.

Woods. Fayette: near Nuttallburg (Nuttall). 
P. Torreyana (Spreng.) Merr. (P. elongata Kuntze).

Wet woods. Webster: along the ridge of Buffalo Bull

Mountain. Fayette: near Nuttallburg (Nuttall).

P. nervata (Willd.) Kuntze. Foul Meadow-grass.

Moist meadows. Common throughout.

Forma Major. Millsp. Prel. Cat. F1. W. Va., 473.

Monongalia: sand bars in Monongahela River near Little Falls.

P. melicaria (Michx.) Hitchcock.

Fields, Randolph: near Pickens (H. H. Smith, I374).

\section{FESTUCA L.}

F. ovina Linn.

Waste grounds. Monongalia: near Morgantown (Sheldon, I 588).

F. OCTOFLORA Walt. (F. tenella, Willd.)

Dry open woods. Wirt: hills above Burning Springs.

F. elation L. Meadow Fescué.

(F. pratensis Huds.)

Fields. Monongalia: near Beechwoods. Fayette: near Kanawha River (James); near Nuttallburg (Nuttall). Meadows. Wood: near Kanawha Station, and elsewhere frequent.

F. NUTANS Willd.

Rocky woods. Randolph: on dry boulder, summit of Rich Mountain. Webster: roadsides near Long Glade. Fayette: near Nuttallburg (Nuttall).

\section{BROMUS L.}

B. HORDEACEus L. Soft Chess.

(B. mollis L.)

Wheat fields and waste grounds. Monongalia: on the campus.

B. Secalinus L. Cheat or Chess.

Wheat fields and waste places. A too common nuisance.

B. Racemosus L. Upright Chess.

Fields common throughout the State.

B. INERMIS Leyss.

Established at Morgantown, Monongalia County (Sheldon, I587).

B. CILIATUS L.

River banks. Wood: along the Little Kanawha River near Kanawha Station. Monongalia: along the Monongahela near Little Falls. Randolph: near Huttonsville (Greenman, 85).

$$
\text { AGROPYRON J. Gaertn. }
$$

A. Repens (L.) Beauv.

Established at Morgantown, Monongalia County (Sheldon, 4I33). 


\section{LOLIUM L.}

L. Italicum A. Br.

Common on lawns. Marion: at Fairmont (Boutlou). Upshur: near French Creek (F. E. Brooks).

L. Perenne L. Darnel. Rye Grass. English Blue Grass.

Lawns, introduced with "Lawn Grass Seed." Monongalia: at Morgantown. Fayette: near Nuttallburg (Nuttall).

\section{ELYMUS L.}

E. Virginicus L. Virginia Wild-rye.

River banks. Ohio : near Wheeling (Mertz \& Guttenberg). Mason: near Point Pleasant. Fayette: near Nuttallburg (Nuttall).

E. Canadensis, glaucifolius (Muh1.) Gray.

Dry banks and roadsides. Kanawha: along 8-Mile Creek. Mason: near Point Pleasant. Fayette: near Nuttallburg (Nuttall).

E. striatus Willd.

Rocky banks. Monongalia: along the Monongahela River below Morgantown.

Var. villosus Gray.

Monongalia: banks of the Monongahela below Morgantown.

E. hirsutiglumis Scribn. \& Sm.

River banks. Monongalia: near Morgantown (Sheldon).

E. intermedius Scribn. \& Smith.

Tucker: banks of the Blackwater River near Hendricks; and Pocahontas: East Fork of the Greenbrier River near Travelers Repose (Greenman, 56, 89).

HYSTRIX Moench. (1794).

(Asprella, Willd. I8og.)

H. Hystrix (L.) Millsp. Bottle-rush Grass.

Moist woodland banks. Scattering in Wood, Wirt, Calhoun, Gilmer, Lewis, Upshur, Monongalia, Randolph, Webster and Nicholas counties. Fayette: along the Gauley River: near Gauley Mountains; near Nuttallburg; near Kanawha Falls (James). Summers : near Hinton. Mason : near Point Pleasant. Harrison: near Lumberport. 
C. Rivularis Kunth.

\section{CYPERUS L.}

Tucker: bed of Shavers Fork near Parsons (Greenman, I30).

C. ovularis (Michx.) Torrey.

Dry fields. Mason: near Point Pleasant (Sheldon, 1943).

C. flavescens L. Galingale.

Low grounds. Monongalia and Marion: along the Monongahela River. Mason: near Point Pleasant. Fayette: near Nuttallburg (Nuttall). Preston: near Aurora (Mr. \& Mrs. Steele). Tucker: bed of Shavers Fork near Parsons and Railroad ditch near Hendricks (Greenman, 123, 124).

C. DIANDRUS Torr.

Fayette: near Nuttallburg (Nuttall). Preston: near Aurora ( $M r$. \& Mrs. Steele).

C. esculentus L. Edible Galingale.

Preston: near Rowlesburg (Mertz \& Guttenberg). Preston: near Aurora (Mr. \& Mrs. Steele).

C. StRigosus L. Lank Galingale.

Low grounds. Jackson: near Sandyville. Monongalia: near Morgantown. Fayette: near Nuttallburg (Nuttall). Preston: near Aurora (Mr. \& Mrs. Steele). Tucker: bed of Shavers Fork near Parsons (Greenman, 125).

C. REFRACTUS Engelm.

Fayette: near Hawk's Nest (T. C. Porter).

C. Retrofractus (L.) Torr.

Along the Guyandot River below Baileysville, Wyoming County, altitude I, Ioo feet, August I8, I900 (Morris, I236a).

C. Lancastriensis Porter.

Summers: shores of New River, near Hinton.

C. Filiculmis Vahl.

Along the Guyandot River below Baileysville, Wyoming County, altitude 1, I00 feet (Morris, 1267). Mason: near Point Pleasant (Sheldon, 1940).

K. pumila Michx.

KYLLINGA Rott.

Fayette: near Nuttallburg (Nuttall); opposite Gauley Junction (Eggleston, 5528).

DULICHIUM L. C. Richard.

D. Arundinaceum (L.) Britton. D. spathaceum Pers. Along streams. Randolph: Tygart's Valley River near Huttonsville (Millspaugh, Greenman, 92). Fayette: near Nuttallburg (Nuttall). 


\section{ELEOCHARIS R. Br.}

E. tuberculosa (Michx.) R. \& S.

Sands of New River. Fayette: near Nuttallburg (Nuttall).

E. OVATA (Roth.) R. \& S.

(E. obtusa Schult.)

Wet places. Fayette: along turnpike opposite Gauley Junction (Eggleston, 5527-6). Randolph: near Pickens ( $H$. H. Smith, 1362). Muddy places. Upshur: near Buckhannon. Randolph: Tygart's Valley River. Fayette: near Kanawha Falls (James); near Nuttallburg (Nuttall). Preston: near Aurora (Mr. \& Mrs. Steel). Monongalia: near Morgantown (Sheldon, 675). Randolph: Tygarts Valley River, near Elkins and Huttonsville; and Tucker: near Parsons (Greenman, 104, 105, 106, I07, 108).

E. PALUSTRIS (L.) R. \& S.

Ohio: near Wheeling (Mertz \& Guttenberg). Fayette: near Nuttallburg (Nuttall) :

Var. gLAUCESCENS (Willd.) Gray.

Jefferson: near Harper's Ferry (Mertz \& Guttenberg).

Preston: near Aurora (Mr. \& Mrs. Steele).

E. TENuis (Willd.) Schult. "Kill Cow." "Poverty Grass."

Damp places, abundant everywhere.

Note.-The above names are applied along Tygart's Valley River, where in places this species grows so abundant as to take whole fields, and as cattle will not thrive upon it, these names suggested themselves.

E. ACICUl.ARIS (L.) R. \& S.

Jefferson: near Harper's Ferry (Mertz \& Guttenberg).

E. TRICOSTATA Torrey.

In wet places. Monongalia: along Tibbs Run (Sheldon, 595).

\section{FIMBRISTYLIS Vahl.}

F. Autumnalis (L.) Roem. \& Schult.

Fayette: near Nuttallburg (Nuttall).

\section{SCIRPUS, L.}

S. Americanus Pers.

Jefferson: near Harper's Ferry (Mertz \& Guttenberg).

S. validus Vahl.

(S. lacustris Am. auth.).

Tucker: bank of Shavers Fork, near Parsons (Greenman, I2I). Glady places. Fayette: near Nuttallburg (Nuttall)? S. DEBILIS Pursh.

Wet places. Tucker: bed of Shavers Fork, near Parsons (Greenman, 69). 
S. SyLVATICUS L.

Glady places. Fayette: near Nuttallburg (Nuttall).

S. Atrovirens, Muhl.

Boggy places. Monongalia: along the Monongahela River. Fayette: near Nuttallburg (Nuttall). Randolph: near Pickens (H. H. Smith, I394).

S. POLYPHYLLUS Vahl.

Randolph: along Tygart's Valley River; near Pickens (H. II. Smith, I393); near Harman (Greenman, II7). Preston: near Aurora (Mr. \& Mrs. Steele). Fayette: near Nuttallburg (Millspaugh).

S. Lineatus Michx. Cotton Grass.

Low grounds. Monongalia: along Falling Run, above the campus.

S. CYPERINUS (L) Kunth. Wool Grass.

Wet meadow lands. Monongalia, frequent. Upshur: near Buckhannon. Webster: in Long Glade. Kanawha: up 8-Mile Creek. Tucker : bank of Shavers Fork, near Parsons; and Randolph: top of main ridge of the Alleghany Mountains opposite Spruce Knob (Greenman, I18, I20).

S. ERIOPHORUM Michx.

Low, wet places. Barbour: near Tygart Junction (Greenman, 326).

E. VIRGINICUM L.

\section{ERIOPHORUM L.}

Damp places. Fayette: near Nuttallburg. Preston: near Cranberry Summit (Mertz \& Guttenberg); near Aurora (Mr. \& Mrs. Steele).

\section{RYNCHOSPORA, Vah1.}

R. GLomerata (L.) Vah1. Beak-rush.

Low grounds. Webster: Upper Glade. Monongalia: near Morgantown. Fayette: near Nuttallburg (Nuttall). Preston: near Aurora (Mr. \& Mrs. Steele). Pocahontas: near Travelers Repose (Greenman, I22). Tucker: banks of the Blackwater River near Hendricks (Greenman, I27).

C. Folliculata L.

\section{CAREX L.}

Margins of streams. Tucker: near Falls of Blackwater.

C. INTUMESCENS Rudge.

Wet places. Fayette: near Nuttallburg. Tucker: near Falls of Blackwater. Randolph: in Blister Swamp near Gandy Creek (Greenman, 58). Preston: near Terra Alta. C. Asa-Grayi Bailey. Gray's Sedge. (C. Grayi Carey).

Meadows and copses. UTpshur: near Beech Fork. 
C. zupulina, Muhl. Hop Sedge.

Wet places. Upshur: near Laurentz (Millspaugh). Randolph: near Huttonsville (Greenman, 60).

C. utriculata Boott.

In a meadow at Bargers Spring, Summers County, altitude I,500 feet, July I3, I900 (Morris, 995).

C. LuRIda Wahl. Pale Sedge. (C. tentacula, Muhl.)

Swampy spots. Monongalia: along Falling Run. Wood: near Lockhart's Run. Fayette: near Nuttallburg (Nuttall). Randolph: Dry Fork River, near Harman (Greenman, 6r). Randolph: near Pickens (H.H. Smith, I352).

C. Baileyi Britton. Slender, Pale Sedge.

On mossy boulders. Webster: along Buffalo Bull Mountain, alt. 2,575 feet. Tucker: near Falls of Blackwater.

C. Frankir Kunth.

(C. stenolepis Torr.)

Damp meadows. Monongalia: along Falling Run. Fayette: near Nuttallburg (Nuttall).

C. sQuarRosa L.

River banks. Monongalia: mouth of Falling Run. Fayette: near Nuttallburg (Nuttall).

C. TYPHNOIDEs Schwein.

Banks of the Ohio river near Point Pleasant, Mason Co. (A. Lee Post $\mathrm{I} 725)$. Monongalia : near Morgantown (Sheldon 3582).

C. Shortiana Dewey.

Ohio: near Wheeling (Mértz, 3320).

C. STRICTA Lam.

Sphagnum Swamp. Fayette: near Nuttallburg (Nuttall). C. TORTA Boott.

Cold, damp places. Fayette: near Nuttallburg (Nuttall). C. PRASINA Wahl.

Fayette: bed of Keeney's Creek, near Nuttallburg (Nuttall).

C. CRINITA Lam.

Damp swales. General throughout the State even in the higher mountains.

C. CRInita x C. PRasina ? Bailey in Millsp. Prel. Cat. Fl. W. Va. 460.

Fayette: along a run in Sugar Camp Hollow (Nuttall).

C. virescens Muhl.

Banks and copses. Wood: near Lockhart's Run.

C. costellata Britton. (C. virescens costata Dew.)

Banks. Fayette: near Nuttallburg (Nuttall). Preston: near Aurora (Mr. \& Mrs. Steele).

C. TRICEPS Michx.

Dry pastures. Wood: near Kanawha Station. Fayette: woodland border of swamp near Nuttallburg (Nuttall). 
C. TEnUis Rudge.

(C. debilis Rudgei Bailey.)

On mossy boulders. Randolph : summit of Rich Mountain, alt. 2,850 ft.; undrained meadows of Tygart's Valley River; Point Mountain, on perfectly dry rock, alt. 3,650 ft.

A very common sedge in the mountains on mossy boulders and along runs. Beautiful growths occur all along the Blackwater Fork of Cheat. Fayette: near Nuttallburg (Nuttall.)

C. oblita Stend.

(C. venusta minor Boeck.)

Fayette: near Masterton's Swamp, Nuttallburg (Nuttall.)

C. OLIGOCARPA Schk.

Rich hillsides; Ohio: near Wheeling (Mertz, 3257).

C. Hitchicockiana Dewey.

Ohio: near Wheeling (Mertz).

C. GRACIllima Schw.

Low grounds. Tucker: near Falls of Blackwater. Fayette: near Nuttallburg (Nuttall).

C. Aмpнiвola Stend.

(C. grisea angustifolia Boott).

Fayette: on banks near Nuttallburg (Nuttall).

C. glaudodea Tuck.

Meadows; Fayette: near Nuttallburg (Nuttall).

C. GRANUlaris Muhl.

Monongalia: in a damp meadow near Morgantown (Sheldon 3588).

C. LAXIFLORA Lam.

Ohio: on Thomas Hill near Wheeling. Greenbrier: near White Sulphur Springs (Heller, I3I4).

Var. LATIFOLIA Boott.

Deep woods. Monongalia: banks of Day Creek. Wirt: banks of Straight Creek. Fayette: near Nuttallburg (Nuttall).

Var. Patulifolia (Dewey) Carey.

Fayette: shady bank, near Nuttallburg (Nuttall).

C. Digitalis, Willd.

In deep woods. Grant: near Bayard. Fayette: near Nuttallburg (Nuttall).

C. Laxiculmis Schw.

Glady places. Fayette: near Nuttallburg (Nuttall).

C. platyphylla Carey.

Fayette: near Nuttallburg (Nuttall).

C. rlantaginea Lam.

Rich wood. Fayette: near Nuttallburg (Nuttall).

C. varia Muhl.

Fayette: near Nuttallburg ( Nuttall).

C. Pennsylvanica Lam.

Ohio: near Wheeling (Mertz \& Guttenberg). Greenbrier: near White Sulphur Springs (Hellcr, 829). 
C. fedicellata (Dewey) Britton.

(C. communis Bailey).

Fayette: dry soil under cliffs, near Nuttallburg (Nuttall).

C. Jamesir Schwein.

Fayette: open woods near Nuttallburg (Nuttall).

C. POLYTRICHOIDES Willd. (C. leptalea Wahl.)

Fayette: sphagnum swamp near Nuttallburg (Nuttall).

C. Fraseri And. Frazer's Sedge.

The following remarks of Prof. T. C. Porter render it evident that this rare and odd sedge came originally from Randolph or Barbour county, each of which lies between the headwaters of the two Kanawhas:

"Muhlenberg, in his Descriptio uberior Graminum, etc., p. 265, under C. lagopus ?, which is C. Fraseri, Andrews, adds these words, 'Habitat in Tyger-Valley, Pennsylvania, eunde siccam habeo et vivam.' Kin, the German gardner who collected in Pennsylvania, brought it home, and his label reads thus: 'Deigher Walli in der Wilternus.' Dr. Gray has shrewdly conjectured that by 'Deigher Walli,' or Tygert Valley, is meant Tygart's Vallev, which lies further south in Virginia." In a footnote, Prof. Porter adds "a box containing the Carices of Muhlenberg has just been discovered ( 1877 ) in the herbarium of the Academy, Philadelphia, and the label attached to the specimens of Kin's collection places Tyger Valley 'prope amnem Kenahway.'"

As the two Kanawhas lie entirely within the State of West Virginia, there seems to be little doubt as to the propriety of including this species in this Flora.

Since writing the above Mr. Nuttall has found a plentiful station for this species near Nuttallburg in the Great Kanawha region.

C. stipata Muhl.

Ohio: near Wheeling (Mertz \& Guttenberg). Fayette: near Nuttallburg (Nuttall). Mercer: near Bluefield.

C. vUlpinoIdeA Michx.

Monongalia: along Falling Run. Wirt: near Burning Spring. Fayette: near Nuttallburg (Nuttall). Randolph: near Pickens (H. H. Smith I357).

C. RoseA Schk.

Fayette: R. R. bank near Nuttallburg (Nuttall).

Var. Radiata Dewey.

Open places. Monongalia: near Round Bottom. Wirt: along Straight Creek. Randolph: on Point Mountain, alt. 3,450 ft.; also on a dry rock, alt. 3,650 ft. (Millspaugh); western slope of the main ridge of the Alleghany Mountains above Horton (Greenman, I I6). Fayette: cliffs, rocks and banks, near Nuttallburg ( Nuttall). 
C. Retroflexa Muhl.

Fayette: Swampy place near Nuttallburg (Nuttall).

C. SPARganioIdes Muhl.

Fayette: wet open banks near Nuttallburg (Nuttall).

C. Murlenbergit Schk., var.-Bailey in Millsp. Prel. Cab. Fl.

An intermediate between the type and variety enervis, fidc

Bailey. Randolph: on an undrained meadow along Tygart's Valley River, near Cricard.

Var. Xalapensis (Kth.) Britton.

Opens. Lewis: along Leading Creek.

C. CEPHALophora Muhl.

Fayette: banks near Nuttallburg (Nuttall).

C. Brunescens (Pers.) Poir.

Pendleton: on Spruce Knob, alt. 4,860 ft. (A. B. Brooks 3307).

C. Brunnescens gracilior Britton. (C. canescens vulgaris Bailey).

On a dry conglomerate rock. Randolph: summit of Rich Mountain, alt. 2,850 ft.

C. TRIBULOIDES Wahl.

In a springy rill. Wood: near Kanawha Station. Var. TURBAta Bailey.

Fayette: low copse near Nuttallburg (Nuttall).

C. SCOPARIA Schk.

Open swales. Monongalia: along Falling Run. Fayette: banks of river and in sphagnum bog near Nuttallburg ( Nuttall) ; dry glade, Alderson Farm (Nuttall).

C. STRAMINEA Willd.

Dry soils. Fayette: near Nuttallburg (Nuttall).

C. SCABRATa Schwein.

Randolph: in a mountain ravine near Whitmar (Greenman, 59).

C. Bicknellii Britton.

Monongalia: in dry soil near Tibb's Run (Sheldon 593).

A R A C E AE.

ARISAEMA Mart.

A. TRIPHyllum (L.), Torr. Jack-in-the-Pulpit.

Rich, damp woods. Common throughout the State.

A. Dracontium (L.) Schott. Green Dragon.

Low grounds. Gilmer: near Glenville (Mapel).

SPATHYEMA Raf. (1808).

(Symplocarpus, Salisb. I8r2.)

S. foetida (L.), Raf. Skunk Cabbage.

Boggy meadows. Preston: near Terra Alta, Monongalia: on the Dille farm. 
Reported from: Brooke: near Wellsburg and Fowlers. Berkeley: near Martinsburg and Hedgesville. Barbour: near Belington. Cabell: near Milton. Grant: near Medley, Greenland, Mount Storm, Maysville and Petersburg. Green brier: near Trout Valley and Fort Spring. Hampshire: near Slanesville and Capon Bridge. Harrison: near Lost Creek, Bridgeport and Good Hope. Hardy: near Wardensville and Old Fields. Hancock: near Fairview and New Cumberland. Jefferson: near Kabletown and Summit Point. Jackson: near Odaville. Lewis: near Walkersville. Mercer: near New Bramwell and Princeton. Mason: near Maggie. Mineral: near Blaine. Marion: near Gray's Flats. Marshall; near St. Joseph and Glen Easton. Ohio: near West Liberty. Pocahontas : near Lobelia. Pleasants : near Schultz. Preston: near Terra Alta, Masontown, Eglon and Amblersburg. Randolph : near Lee Bell, Kerens and Cricard. Roane: near Clio. Summers: near Talcott and Clayton. Taylor: near Knottsville. Upshur: near Kanawha Haad and Hem. lock. Wayne: near Adkin's Mills. Wetzel: near Littleton, Pine Grove and Blake. Wirt: near Burning Springs, Evelyn and Reedy Ripple. Wood: near Rockport. Webster: near Replete.

\section{ACORUS.L.}

A. Calamus L. Calamus. Sweet-flag.

Swampy places. Lewis: along Stone Coal Creek. Randolph: near Valley Bend, Cricard, and on Point Mountain, alt. 3,050 ft. Gilmer: near Glenville (Mapel). Berkeley: near Martinsburg.

\section{ORONTIUM Linn.}

O. aquaticum Linn. Golden Club.

In watery bogs and slow streams. Grant: in Abram Creek (F. E. Brooks).

LEM N A C E AE.

\section{LEMNA L.}

L. MINOR L. Duck-weed.

Stagnant waters. Putnam: near Buffalo.

\section{CO M M E L I N A C E AE.}

TRADESCANTIA, L.

T. Virginiana L. Spiderwort.

Rich grounds. Kanawha: near Charleston (Barnes). Gilmer: near Glenville ( $\mathrm{Mapel}$ ). Fayette: near Nuttallburg (Nuttall). Wirt, along Straight Creek and near Burning Springs. Hardy: near Moorefield. 
T. PILOSA, Lehm. Hairy Spiderwort.

On boulders. Wirt: abundant beyond Burning Springs, and along Straight Creek.

COMMELINA L.

C. Virginica L. Day Flower.

Damp opens. Fayette: near Nuttallburg, rare (Nuttall). Hardy: near Moorefield (A. B. Brooks).

\section{J U N C A C E AE.}

J. Effusus L. Soft Rush.

\section{JUNCUS L.}

Marshy ground. Kanawha: near Charleston (James). Fayette: near Nuttallburg (Nuttall). Common throughout. Randolph: in Blister Swamp near Gandy Creek (Greenman, II2).

J. SETACEUS Rostk.

Fayette: near Loup reek (?) (James).

J. MARGINATUS Rostk.

Moist sandy soils. Kanawha: near Charleston (James). Common throughout. Fayette: near Nuttallburg (Nuttall). Preston: near Aurora (Mr. \& Mrs. Steele).

J. TENUIS Willd. "Poverty Grass."

Roadsides and ditches. Abundant throughout the State.

J. TENUIS ANTHELATUS Wiegand.

In reddish, clayey soil. Randolph: near Glady (Greenman, IIO).

J. acuminatus Michx.

Damp places. Monongalia and Marion: on the Monongahela River. Webster : in Welch and Long Glades. Fayette: near Nuttallburg (Millspaugh). Preston: near Aurora (Mr. \& Mrs. Steele).

J. Nodosus L.

Fayette: near Nuttallburg (Nuttall).

J. Canadensis J. Gay.

Monongalia: near Little Falls. Fayette: near Nuttallburg (Nuttall). Preston: near Aurora (Mr. \& Mrs. Steele).

J. BREvicaudatus (Engelm.) Fernald.

Randolph : in a cold spruce swamp near "The Sink", Gandy Creek; also at Glady; and Pendleton: on the main ridge of the Alleghany Mountains opposite Spruce Knob (Greenman. II 5 , II 3 ).

J. Canadensis subcaudatus Engelm.

Tucker: among rocks along the Blackwater River near Hendricks, and along Shavers Fork near Parsons (Greenman, 128, 129). 


\section{JUNCOIDES Adans.}

J. SALtUensis Heller. (Luzula saltuensis Fernald, L. vernalis Man. not DC. Juncoides pilosum Coville not Kuntze.

Ohio: on Thomas's Hill near Wheeling (Mertz \& Guttenberg). Fayette: near Nuttallburg (Nuttall). Upshur: near French Creek (F. E. Brooks).

J. Campestre (L.) Kuntze.

(Luzula campestris DC.)

Ohio: near Benwood (Mertz \& Guttenberg). Tyler: near Long Reach Fayette: near Nuttallburg (Nuttall). Monongalia: near Morgantown.

\section{ELA N T H A C E AE. \\ CHAMAELIRIUM Wiild.}

C. Luteum (L.), Gray. Blazing Star. Devil's-bit.

Glades. Preston: near Terra Alta and Kingwood. Webster: in Welsh, Long and Collett's Glades. Gilmer: near Glenville (Mapel). Preston: near Aurora ( $\mathrm{Mr}$. \& Mrs. Steele). Fayette: near Nuttallburg (Nuttall).

CHROSPERMA Raf. (1825)

(Amianthium, Gray i837)

C. muscaetoxicum (Walt.) Kuntze. Fly Foison.

Low rich grounds. Monongalia: Ice's Ferry. Preston: near Terra Alta.

\section{MELANTHIUM. L.}

M. Virginicum L. Bunch-flower.

Glades. Preston : near Terra Alta and in Morgan's Glade. Monongalia: glades near Booth's Creek. Webster: Second Glade. Nicholas: Collett's Glade.

M. PARViflorum (Michx.), Watson.

Greenbrier: near White Sulphur Springs (Mertz \& Guttenberg).

VERATRUM L.

V. vIRIDE, Ait. American White Hellebore.

Rich wet spots in deep mountain woods. Randolph: on Point Mountain. Webster: Buffalo Bull Mountain. Grant and Tucker: common along streams.

UVULARIA L.

U. perfoliata L. Bellwort.

Rich woods. Marion and Monongalia: along the Monongahela River. Gilmer: near Glenville (Mapel, Prof. Brown). Fayette: near Nuttallburg (Nuttall). Greenbrier: near White Sulphur Springs (Eggleston, 434I). 
U. SESSILIFOLIA L.

Oakesia sessilifolia (L.), Wats.

Low rich woods. Gilmer: near Glenville (Mapel). Fayette: near Nuttallburg (Nuttall). Mercer: near Beaver Spr. Greenbrier: near White Sulphur Springs (Eggleston, 4361 ).

U. GRANDIFLORA J. E. Smith.

On a north slope in rich woods along Horsepen Creek be tween McDowell County, West Virginia, and Tazewell County, Virginia, alt. I,900 ft., July 31, I900 (Morris, I Ioo). U. PUBERULA Michx.

In mountain woods. Greenbrier: near White Sulphur Springs (Eggleston, 4358).

\section{I L I A C E AE.}

\section{HEMEROCALLIS L.}

H. Fulva L. Day-lily. "Eve's Thread".

Roadsides and fields. A frequent escape from cultivation Monongalia: near Morgantown and Cassville. Hampshire. near Bloomery, where "it has become widely scattered by ploughing."

\section{ALLIUM L.}

A. vineale L. Field Garlic.

Cultivated fields. Jefferson: a vile and abundant weed in wheat fields. Monongalia: along the Monongahela below Morgantown.

Reported as a weed from: Berkeley: near Oakton, Martinsburg and Hedgesville. Barbour: near Belington. Braxton: near Lloydsville. Cabell: near Union Ridge. Dodd. ridge: near Smithton. Grant: near Medley. Greenbrier: near Trout Valley and White Sulphur Springs. Hampshire: near Slanesville, Three Churches, Capon Bridge, Bloomery, Dillon's Run, Higginsville and Springfield. Harrison: near Good Hope and Bridgeport. Hardy: near Wardensville and Moorefield. Jefferson: near Summit Point, Mohler's, Leetown, Charlestown, Ripon, Middleway and Kabletown. Jackson: near Sandy. Kanawha: near Blandon. Lincoln: near Hamlin. Mercer: near Princeton, and Concord Church. Marion: near Barracksville and Gray's Flat. Monongalia: near Morgantown. Ohio: near West Liberty. Preston: near Terra Alta, Reedsville, Amblersburg and Independence. Ritchie: near Ritchie C. H. Roane: near Walnut Grove. Summers: Talcott. Taylor: near Knottsville, Thornton and Grafton. Upshur : near Kanawha Head. Wayne: near Adkin's Mills. Wetzel: near Endicott and Blake. Wirt: near Reedy Ripple. Wood: near Waverly, Blennerhassett and Rockport. 
A. TRICoccum, Ait. Wild Leek. "Ramps."

Rich Mountain woods. Grant: near Bayard, abundant. Tucker: abundant along Blackwater Fork of Cheat. Greenbrier: near White Sulphur Springs. Randolph: near Pickens $(H . H$. Smith); near the mountain top above Horton (Greenman, 98). Ohio: near Wheeling; and Upshur: near Helvetia (Mertz \& Guttenberg).

A. CERnuUm Roth. Wild Onion.

Greenbrier: near White Sulphur Springs. Fayette: near Nuttallburg (Nuttall). Ohio: near Wheeling (Mertz \& Guttenberg). Summers: near Hinton. Monongalia: near Camp Eden, and banks of Decker's Creek.

A. Canadense L. Wild Garlic.

Ohio: near Wheeling (Mertz \& Guttenberg). Fayette near Loup Creek (James).

\section{LILIUM L.}

L. Philadelphicum L. Wild Red Lily. "Glade Lily."

Dry or damp grounds. Monongalia: in Clinton District (M. H. Brown) near Stewartstown, and Ice's Ferry. Marion . along the F., M. \& P. R. R. Gilmer: near Glenville ( $\mathrm{Ma}$ pel). Webster: in the glades, where it is called "Glade Lily" (Mapel). Hardy: near Moorefield (G.). Fayette: near Nuttallburg (Nuttall).

L. SUPERBUm L. Turk's-cap Lily.

Rich, low grounds. Randolph: summit Point Mountain, alt. 3,700 ft. Monongalia: near Morgantown and Stewartstown. Hardy : near Moorefield (Gamble). Upshur: near Newlin (H. H. Smith, I792). Pocahontas: at Travelers Repose (Greenman, 7I).

L. Canadense L. Wild Yellow Lily.

Moist meadows. Calhoun: along Laurel Run. Gilmer near Glenville (Mapel). Preston: near Aurora (Mr. \& Mrs. Steele). Randolph: summit Point Mountain, alt. 2,125 ft. Monongalia: near Ice's Ferry.

L. TIGRINUM. Ker. Tiger Lily.

Established from gardens. Jefferson, Berkeley, Morgan, Hampshire and Mineral : along the B. \& O. R. R. Calhoun: near Brookville. Monongalia: near Morgantown.

\section{ERYTHRONIUM L.}

E. Americanum Ker. Yellow Adder's Tongue. Dog's Tooth Violet.

Rich open woods along streams. Monongalia and Marion. along the. Monongahela River. Gilmer: near Glenville (Mapel; Brown). Fayette: near Nuttallburg (Nuttall) 
Frequent or common throughout the State. Hardy: near Moorefield (Gamble).

E. ALbIDUM Nutt.

Rich soil, Kanawha region, along Coal and Len's Creeks, Ohio Co., near Mt. de Chantal (Boutlou).

\section{QUAMASIA Raf.}

Q. hyacinthina (Raf.) Britton. (Camassia Fraseri Torr.)

Rich ground. Ohio: near Wheeling (Mertz \& Guttenberg). Gilmer: near Glenville ( $\mathrm{Mapel}$ ).

\section{ORNITHOGALUM L.}

O. umbellatum L. Star of Bethlehem.

Escaped from gardens. Monongalia: abundant and persistent on the line of the F., M. \& P. R. R. from Morgantown to Coburn's Creek. Gilmer: near Glenville ( $M a p e l$ ).

O. NuTANS L.

In a ravine above the glass factory north of Morgantown, Monongalia County (Boutlou).

\section{MUSCARI Mill.}

M. Botryoides (L.), Mill. Grape Hyacinth.

Escaped from gardens. Monongalia: near Uffington. Gilmer: near Glenville ( $M a p e l)$.

M. RACEMosum (L.) Mill.

Widely escaped and established at the junction of Aarons Run and Deckers Creek, Monongalia County, near Morgantown (Moffatt).

\section{ALETRIS L.}

A. FARINosa L. Star Grass. Colic Root.

Sandy moist soils. Webster: in Upper Glade. Fayette: near Nuttallburg (Nuttall).

\section{O N V A L L A R I A C AE.}

\section{ASPARAGUS L.}

A. officinalis. L. Asparagus.

Woods and opens; a frequent escape. Jefferson: near Shenandoah Junction, plentiful. Monongalia : plentiful along Cheat River, near Ice's Ferry, and at Stewartstown. Mason: near Point Pleasant. Berkeley: near Martinspurg. Hardy: near Moorefield.

\section{CLINTONIA Raf.}

C. Borealis (Ait.), Raf. Clinton's Lily.

Cold damp weeds. Grant: near Bayard. Preston: in Laurel hills. Tucker: near Davis. 
C. umbellulata (Michx.) Torr.

Rich woods. Randolph : on Rich Mountains. Grant: near Bayard. Tucker: in Land of Canaan. Gilmer: near Glenville (Brown, Mapel). Monongalia: near Morgantown. Kanawha: near Coalburg (James). Fayette: near Nuttallburg (Nuttall). Hardy: near Moorefield (Gamble). Barbour: near Tygart Junction (Greenman, 94). McDowell: near Elkhorn. Mercer: Bluestone Jc.

VAGNERA Adans.

V. RACEmosa (L.) Morong. (Unifolium racemosum (L.) Britton). Rich woods. Common throughout the State.

\section{UNIFOLUM Adans ( 1763 ).}

U. Canadense (Desf.), Greene. (Smilacina bifolia Canadensis A. Gray).

Low, rich woods. Common throughout the northern and eastern counties. Fayette: near Nuttallburg (Nuttall).

\section{DISPORUM Salisb.}

D. Lanuginosum (Michx.) Nichols. (Prosartes lanuginosa, Don.)

Rich woods. Monongalia: near Morgantown. Preston: near Terra Alta. Mineral: near Keyser (Workman). Fayette: near Nuttallburg (Nuttall). Grant: near Bayard. Mercer: Bluestone Jc. Greenbrier: near White Sulphur Springs (Eggleston, 4343). Preston: near Aurora (Mr. \& Mrs. Steele).

\section{STREPTOPUS Michx.}

S. ROSEUS Michx. Twisted Stalk.

Cold, damp woods. Upshur: along Sand Run. Preston : near Terra Alta. Tucker: near Blackwater Fails. Grant: near Bayard. Randolph : western slopes of Cheat Mountains.

\section{SALOMONIA Heist.}

(Polygonatum Adans.)

S. BIFLORA (Walt.) Britton.

Rich woods and wooded banks. Frequent generally

S. сомmutata (R. \& S.) Britton. (P. giganteum, Dietr.)

Edges of meadows. Nicholas: near Beaver Mills. Gilmer: near Glenville ( $\mathrm{Mapel}$ ). Monongalia: the Flats near Morgantown. Fayette: near Nuttallburg (Nuttall). 
CONVALLARIA Linn.

C. maJALis Linn. Lily-of-the-Valley.

High mountain woods. Pocahontas: near Winterburn (A. B. Brooks).

\section{MEDEOLA L.}

M. Virginiana L. Indian Cucumber.

Rich, damp woods. Upshur: along Sand Run. Grant and Tucker: in the mountains woods, plentiful. Gilmer: near Glenville ( $\mathrm{Mapel}$ ). Monongalia: near Morgantown. Kanawha: near Coalburg (James). Fayette: near Nuttallburg (Nuttall)., and elsewhere frequent. McDowell: near Elkhorn.

\section{TRILLIUM L.}

T. sessile L. Sessile-flowered Purple Trillium.

Moist woods. Monongalia and Marion: abundant along the Monongahela River. Gilmer: near Glenville (Brown).

T. erectum L. Purple Trillium.

Rich woods. Throughout the northern and eastern portions of the State. McDowell: near Elkhorn.

Var. AlBum Pursh.

Monongalia: near Morgantown. Gilmer: near Glenville (Mapel, Brown). Taylor: near Valley Falls (N.S. Hayes). Var. Declinatum Millsp. Prel. Cat. Fl. W. Va., 453 (I902).

Gilmer: near Glenville ( $\mathrm{Mapcl}$ ). Mason: near Point Pleasant. Jackson: near Ravenswood.

(Note:-This form appears to be sufficiently difierent from $T$. erectum, and $T$. cernum, to retain it; otherwise $I$ could not list the specimens gathered.)

T. grandiflorum (Michx.)、Salisb. White Trillium.

Rich woods. Abundant throughout the northern counties. Gilmer: near Glenville (Mapel, Prof. Brown). Fayette: near Nuttallburg (Nuttall). McDowell: near Elkhorn. Greenbrier: near White Sulphur Springs (Heller, 840).

T. CERNUUm L. Wake Robin.

Moist woods. Gilmer: near Glenville ( $\mathrm{Mapel}$ ). Fayette: near Nuttallburg (Nuttall).

T. Nivale Riddell. Dwarf White Trillium.

In moss of wet rocks. Monongalia: along Quarry Run. Grant: along Buffalo Creek. Randolph: along runs feeding Shaver's Fork of Cheat. Tucker: along Beaver Creek, and Blackwater Fork of Cheat.

T. undulatum Willd. (T. erythrocarpum Michx.)

Cold deep ravines along runs. Grant: near Bayari. Randolph: Cheat River (Millspaugh); near Whitmar (Greenman, 73). Tucker: along Black Water Fork. Fayette: near Nuttallburg, alt. 2,000 ft., rare (Nuttall). Mc- 
Dowell: near Elkhorn. Taylor: near Valley Falls (N. S. Hayes). Mercer: Bluestone Jc. Preston: near Aurora (Mr. \& Mrs. Steele).

\section{S M I L A CEAE.}

\section{SMILAX L.}

S. herbacea L. Carrion Flower.

Thickets. Monongalia: plentiful at the Flats. Fayette: near Nuttallburg, rare (Nuttall).

S. RotUndifolia L. Greenbrier.

Thickets, too common throughout the State.

S. GLAUCA Walt.

Dry thickets. Kanawha: near Charleston (Barnes). Fayette: near Nuttallburg (Nuttall). Monongalia: along Cheat River near Ice's Ferry.

S. tAMnifolia Michx.

Dry soils. Preston: near Aurora (Mr. \& Mrs. Steele).

S. Pseudo-China L.

Dry soils. Monongalia: plentiful on the Flats; near Morgantown.

S. HispidA Muhl. Bristly Sarsaparilla.

Rich soil. Marion: near Fairmont. Preston: near Aurora (Mr. \& Mrs. Steele).

\section{A M A.R Y L L D A C EA E.}

\section{HYPOXIS L.}

H. hirsuta (L.) Coville. Star Grass. Yellow-eyed Grass. (it. erecta L.).

Open places. Fayette: near Nuttallburg, common (Nuttall). Lewis: up Stone Coal Creek. Monongalia: along Cheat River near Camp Eden, plentiful. Hardy: near Moorefield (Gamble). Mercer: near Bluefield.

D I O S C O R E A CE AE.

\section{DIOSCOREA L.}

D. GLauca Muhl. Wild Yam-root. Colic-root. (D. villosa Flora).

Thickets and rich woods. Randolph: on Point Mountain, alt. 3,450 ft. Gilmer: near Glenville (Mapel). Fayette: near Nuttallburg, pistillate plants rare (Nuttall). Grant and Tucker: plentiful in the mountain woods. Monongalia: plentiful. Greenbrier: near White Sulphur Springs. Summers: near Hinton. Hardy: near Moorefield. McDowell: near Elkhorn. Mercer: Bluestone Junction. Preston: near 
Aurora (Mr. \& Mrs. Steele); near Albright (Bartlett). Barbour: (Greenman). Monongalia: (Jennings). Upshur: near Buckhannon (Pollock). Randolph: near Pickens ( $H$. H. Smith, I846).

I R I D A C E A E.

\section{IRIS L.}

I. verSiCOLOR L. Blue Flag.

Ditches and wet lands. Preston: near Terra Alta and Reedsville.

I. VERNA L. Dwarf Iris.

Hardy: near Moorefield (Gamble).

I. CRISTATA Ait. Dwarf Crested Iris.

Rich woods. Monongalia: near Morgantown, plentiful. Gilmer: near Glenville (Mapel; Brown). Fayette: near Nuttallburg (Nuttall). Marion: along the Monogahela River, plentiful.

\section{SISYRINCHIUM L.}

S. Angustifolium Mill. Blue-eyed Grass. S. Bermudianum Gray, not. L.

Moist grassy places. Fayette: near Kanawha Falls (James) ; near Nuttallburg (Nuttall). Gilmer: near Glenville (Mapel). Monongalia: near Morgantown, and elsewhere common.

S. GRAMINOIDES Bicknell.

(S. anceps S. Wats.)

Grassy places. Fayette: meadows near Nuttallburg (Nuttail). Monongalia: near Morgantown (Sheldon, I582).

S. muCronatum Michx.

Meadows. Preston: near Cranesville (Sheldon, I 509).

O R C H I D A C E AE.

\section{CYPRIPEDIUM L.}

C. Parviflorum Salisb. Small Lady's Slipper.

Rich woods. Monongalia: near Morgantown. Marion: near Opekiska and Fairmont. Gilmer: near Glenville ( $M a$ pel; Brown). Mineral: near Keyser (Workman). Hardy: near Moorefield (Gamble). Lewis: (Hinzman).

C. hirsutum Mill. Large Lady's Slipper. (C. pubescens Willd.)

Low woods. Same stations as the last. Fayette; near Nuttallburg (Nuttall). Slope of Kate's Mountain, alt. 3,300 ft. (Small; Eggleston, 4359; Heller, 821).

C. ACAUle Ait. Moccasin Flower.

Dry or moist woods. Monongalia: near Cheat View, plentiful. Preston: near Reedsville. Gilmer: near Glen- 
ville (Brown). Fayette: near Nuttallburg, alt. 2,000 ft., in laurel thickets. Marion: along the F. M. \& P. R. R. Mineral: near Keyser (Workman) Kanawha: near Coalburg (James). Hardy: near Moorefield (Gamble). Webster: near Tater Knob (H.H. Smith, I49I). Upshur: near Buckhannon (Pollock).

GALEORCHIS Rydb.

G. Spectabilis (L.) Rydb. Showy Orchid. (Orchis L.)

Rich woods. Monongalia: near Morgantown. Gilmer: near Glenville (Mapel; Brown). Fayette: near Nuttallburg, rare (Nuttall). McDowell: near Elkhorn.

\section{LYSIAS Salisb.}

L. orbiculata (Pursh) Rydb. (Habenaria orbiculata Torr.).

Rich woods. Randolpli: near summit of Rich Mountain (Millspaugh). Pendleton: in shade of spruce trees near the summit of Spruce Knob (Brooks). Preston: near Aurora (Mr. \& Mrs. Steele).

\section{PERULARIA Lindl.}

P. Flava (L.) Rydb.

(Hebenaria flava Gray).

Wet situations. Webster: in long Glade (Millspaugh).

\section{GYMNADENIOPSIS Rydb.}

G. Clavellata (Michx.) Rydb. (Hebenaria tridentata Hook.). Wet places. Webster : in Long Glade and Welsh Glade (Millspaugh). Fayette: near Nuttallburg (Nuttall).

\section{BLEPHARIGLOTTIS Raf.}

B. CILIARIS (L.) Rydb. (Habenaria ciliaris R. Br.).

Wet sandy bogs. Webster: Welch and Long Glades. Preston: near Terra Alta (Milispaugh). Fayette: near Nuttalburg, rare (Nuttall). Hardy: near Moorefield (Gamble).

B. LACERA (Michx.) Rydb. (Habenaria lacera R. Br.).

Bogs and moist thickets. Wood: near Lockharts Run (Millspaugh). Preston: near Cranberry Summit (Mertz \& Guttenberg). Fayette: near Nuttallburg (Nuttall). Upshur: near French Creek (Brooks).

B. psycodes (L.) Rydb. (Habenaria psycodes A. Gray).

Wet places. Randolph: on Rich Mountain; Grant: near Bayard; Preston: near Terra Alta; Wayne: near Central City (Millspaugh); Pocahontas: (Pollock).

B. ieramoena (A. Gray) Rydb. (Habenaria peramoena Gray).

Moist meadow. Upshur: near French Creek (Brooks). Preston: near Reedsville (Shcldon, 1896). 
ISOTRIA Rafin.

I. verticillata (Willd.) Raf. (Pogonia verticillata Nutt.). Woodlands. Upshur: near French Creek, very abundant, growing with Kalmia and Gaultheria; Webster: along the Gauley River (F. E. Brooks).

\section{POGONIA Juss.}

P. ophioglossoides (L.), Ker.

Boggy places. Preston: near Cranberry Summit (Mertz \& Guttenberg). Fayette: near Nuttallburg (Nuttall). Upshur: near French Creek (Brooks).

GYROSTACHYS. Pers. ( 1807 ).

(Spiranthes, Rich. I8I8).

G. CERnUA (L.). Kuntze. Ladies Tresses.

Wet meadows. Fayette: near Nuttallburg (Nuttall). Monongalia; frequent throughout. Preston: near Aurora (Mr. \& Mrs. Steele).

G. GRACILIS (Bigel.) Kuntze. Twisted Stalk.

Sandy woods and fields. Fayette: near Nutallburg, in hard, grassy ground (Nuttall). Monongalia: near Morgantown. Frequent throughout the State.

PERAMIUM Salisb. (I8I2).

(Goodyera, R. Br. I8I3.)

P. REPENS (L.) Salisb.

In deep evergreen forests. Grant: under Black Spruce near Bayard. Fayette: in deep Laurel thickets, rare, near Nuttallburg (Nuttall). McDowell: near Elkhorn. Preston: near Aurora ( $M r$. \& Mrs. Stcele).

P. Pubescens (Willd.) MacM. Rattlesnake Plantain.

Rich woods. Monongalia: along Decker's Creek and Tibb's Run. Fayette: near Nuttallburg, rare (Nuttall) Preston: near Aurora (Mr. \& Mrs. Steele). Nicholas: near Beaver Mills. McDowell: near Welch. Webster: $(H . H$. Smith, I860).

P. opHioides (Fernald) Rydb.

Cold mossy woodlands. Pocahontas: Cranberry Glades (Brooks, 3859).

P. Menziesii (Lindl.) Morong.

In deep rich woods, Webster: near Tater Knob $(H . H$. Smith, 1860).

T. Unifolia (Muhl.) B. S. P. TIPULARIA Nutt.

Upshur: common at French Creek (F.E. \& A.B.Brooks) 
TRIPHORA Nutt.

T. trianthophora (Sw.) Rydb.

Upshur: near French Creek (Brooks).

(Pogonia Sw.).

ACHROANTHES Raf. (1808).

(Microstylis, Nutt. 1818).

A. Unifolia (Michx.) Raf.

Rich woodlands. Greenbrier : near White Sulphur Springs (Mertz \& Guttenberg). Fayette: near Nuttallburg, rare (Nuttall).

LEPTORCHIS Thou. (I808).

(Liparis, Rich. 1818).

L. Liliifolia (L.) Kuntze. Tway-blade.

Rich woods. Monongalia: the Flats near Morgantown. Gilmer: near Glenville (Mapel, Brown). Fayette: near Nuttallburg (Nuttall).

CORALLORHIZA R. Br.

C. Corallorhiza (L.) Karst. Coral root. (C. innata R. Br.)

Deep, rich woods. Grant: near Bayard. Fayette: near Nuttallburg, alt. 2,000 ft. (Nuttall). Greenbrier: near White Sulphur Springs.

C. ODONTORHIZA (Willd.), Nutt.

Rich woods. Gilmer: near Glenville (Mapel). Fayette: near Nuttallburg, alt. 2,000 feet (Nuttall). Preston: near Aurora (Mr. \& Mrs. Steele).

C. multiflora Nutt.

Woodlands. Fayette: near Nuttallburg, alt. 2,000 feet (Nuttall). Preston: near Aurora (Mr. \& Mrs. Steele).

\section{APLECTRUM Nutt.}

A. hyemale (Muhl.) Torrey. (A. spicatum B. S. P.).

Rich woods. Monongalia: near Morgantown. Gilmer: near Glenville (Mapel). Fayette: near Nuttallburg (Nuttall).

LIMODORUM L. (1753).

(Calopgon, R. Br. 1813).

L. TUBEROSUM L.

C. pulchellus, R. Br.

Hardy: near Moorefield (Gamble). 


\section{SAURURUS L.}

S. CERnuUs L. Lizard's Tail.

Streams. Jefferson: near Harper's Ferry (Mertz \& Guttenberg). Brooke: near Wellsburg. Fayette: near Nuttallburg (Nuttall). Wayne: in a glade (Hopkins). Wood: near Parkersburg (Pollock).

\section{J U G L A N D A C E A E. \\ JUGLANS L.}

J. Cinerea L. Butternut. White Walnut.

Common throughout the State even in the valleys of the highest altitudes. Randolph: along Tygart's Valley River near Huttonsville (Greenman, 197).

J. NIGRA L. Black Walnut.

Rich soils, even in the higher Alleghanies. A very common and valuable timber tree throughout the State.

\section{HICORIA Raf.}

H. ovata (Mill.) Britton. Shag or Shellbark Hickory (Carya alba, Nutt.).

Low grounds, frequent throughout the State.

H. raciniosa (Michx.) Sarg. King Nut (Carya sutcata, Nutt.).

Rich soil. Monongalia : near Ice's Ferry.

H. Alba (L.) Britton. White Heart Hickory. (Carya tomentosa, Nutt.)

Woods, frequent throughout the State.

H. Glabra (Mill.) Britton. Pig Nut. (Carya porcina, Nutt.)

Dry soils, frequent throughout the State.

H. microcarpa (Nutt.) Britton. (Carya microcarpa, Nutt.)

Woodlands. Fayette: near Nuttallburg (Nuttall).

H. CORDIFORMIS (Wang.) Britton, Bitternut. (Carya amaraNutt.)

Low Woods. Greenbrier: near Fort Spring and Ronceverte. Fayette: near Nuttallburg (Nuttall). Webster : near Pugh (H. H. Smith, 1682).

\section{S A L I C A C E A E.}

\section{POPULUS L.}

P. Alba, L. White Poplar. Abele.

A frequent escape from cultivation. In many places in the State, where the tree is planted for ornament, this species spreads widely from the root, thus often becoming a pest in lawns and along streets. 
P. tremuloides, Michx. Aspen. Trembling Poplar.

Wooded hillsides. Wirt: along Little Kanawha River. Calhoun: near Grantsville. Gilmer: near Glenville. Monongalia: near Marion; along the Monongahela River. Summers: near Riffe. Mason: near Point Pleasant.

P. GRandidentata Michx. Large-toothed Aspen.

Preston: near Cranberry Summit. Ohio: near Wheeling (Mertz \& Guttenberg). Webster: on Tater Knob (H. H. Smith, I485).

P. Candicans Ait. Balm of Gilead.

Plentiful at Montana, along the Monongahela River in Marion Co. Monongalia: the Flats near Morgantown. Gilmer: near Glenville (Mapel). Fayette: near Nuttallburg, very likely introduced (Nuttall). Webster: Hacker Valley (H. H. Smith, ) 1602).

P. Deltoides Marsh. Cottonwood.

(P. monilifera Ait.).

Ohio: near Bogg's Run (Mertz \& Guttenberg). Mason: near Point Pleasant.

\section{SALIX L.}

S. NIGRA Marsh. Black Willow.

Along streams, frequent or common. The principal willow of the State.

Var. Falcata (Pursh.) Torr. Scythe-leaved Black Willow.

Along springy runs. Wirt: along Straight Creek. Lewis: along Leading Creek. Fayette: near Nuttallburg (Nuttall).

S. AMY DALOIDES And.

Fayette: near Nuttallburg (Nuttall).

S. Alba, var. vitellina (L.) Koch. White Willow.

Scattered, from protective or ornamental planting, along streams in many parts of the State.

S. Babylonica L. Weeping Willow.

A frequent escape as in the last species. Monongalia: near Morgantown. Jefferson: near Flowing Spring and Milltown.

S. DISCOLOR Muhl. Shining Willow.

Ohio: on Bogg's Islands (Mertz \& Guttenberg.)

S. Humilis Marsh. Prairie Willow.

Glady regions. Webster: near Upper Glade. Preston: near Terra Alta.

S. SERICEA Marsh. Silky Willow.

Along streams. Randolph: along Tygart's Valley River. Greenbrier: near White Sulphur Springs. Ohio: near Wheeling (Mertz, 264I).

S. CORDAta Muhl. Heart-leaved Willow.

Along streams, frequent. Lewis: along Leading Creek. Wood: near Parkersburg. Mason: niear Point Pleasant. 
COMPTONIA Banks.

C. PEREgrina (L.) Coulter.

(Myrica asplenifolia L.).

Dry soil, especially on hillsides. Hampshire: along Cacapon Creek (Brooks).

\section{B E T ULA CEA E.}

\section{CARPINUS L.}

C. Caroliniana, Walt. Blue or Water Beech.

Damp thickets and river banks. Wirt: near Elizabeth. Monongalia: near Morgantown, plentiful. Gilmer: near Glenville (Mapel). Upshur: near Buckhannon (Pollock). Fayette: near Nuttallburg (Nuttall). Summers: near Hinton. Marion: near Worthington. Jefferson: near Harper's Ferry (Mertz \& Guttenberg). Webster: Hacker Valley (H. H. Smith, I53 I).

\section{OSTRYA Scop.}

O. Virginiana (Mill.) Willd. Lever Wood. Iron Wood.

Rich woods and along streams. Wirt: near Elizabeth. Randolph: on Point Mountain; at first Top of Cheat there is a forest of this wood where trees are found, in numbers, from I-3 feet in diameter. Webster: on Buffalo Bull Mountain. Greenbrier: near White Sulphur Springs. Summers: near Hinton. Marion: near Worthington. Fayette: near Nuttallburg (Nuttall).

\section{CORYLUS L.}

C. Americana, Walt. Hazlenut.

Thickets, frequent throughout the State.

C. rostrata Ait. Beaked Hazlenut.

Mountainous regions. Upshur: summit on Staunton Pike Randolph: near Fords.

\section{BETULA L.}

B. Lenta L. Sweet Birch. Black Birch.

Rich Woods. Common throughout the State. Grows very large in the mountains. One specimen near Cheat Bridge measure $7 \mathrm{ft} .9$ in. in diameter. Preston: near Aurora (Mr. \& Mrs. Steele).

B. Lenta X Lutea. Millsp. Field Mus. Bot. I : 20I (I896). A number of specimens of this hybrid birch were found in 1892 at Pickens, Randolph Co. In all, the bark forms of each species were intermixed. All the specimens were small saplings about 2 in. in diameter (Millspaugh). 
B. LuteA Michx.f. Yellow Birch.

Higher mountain woods. Grant: near Bayard. Tucker: near Hulings. Braxton: near Sutton. Randolph: near Pickens. Fayette: near Nuttallburg (Nuttall).

B. Populifolia Marsh. White Birch.

Poor soils. Gilmer: near Glenville (Mapel). Randolph: near Winchester.

B. NigRA L. River Birch. Red Birch.

Along streams. Calhoun: along Little Kanawha River. Gilmer: near Glenville (Mapel). Greenbrier: near Fort Spring. Summers: near Greenbrier Stockyards; near Hinton. Kanawha: near Handley. Fayette: near Nuttallburg (Nuttall); and common along streams throughout the central and southern counties. A tree I4 feet $2 \frac{1}{2}$ inches in circumference was measured near Bargers Spring, Summers County (Morris).

\section{ALNUS Gaertn.}

A. Alnobetula (Ehrh.) K. Koch. Mountain Alder. (A. viridis DC.)

Along mountain streams, rare. Greenbrier: Columbia Sulphur Springs. Fayette: near Nuttallburg (Nuttall). Pocahontas: at Traveler's Repose. Randolph: along Cheat River.

A. Rugosa (DuRoi) K. Koch. Smooth Alder. (A. serrulata Willd.)

Low grounds and along rivers. Common, especially in the glade regions.

A. Incana (L.) Willd. Hoary Alder.

In wet places. Pocahontas: Cranberry Glades? (Brooks)

\section{F A G A C E A E.}

\section{FAGUS L.}

F. Americana Sweet. Beech.

General throughout the State.

CASTANEA Adans.

C. Dentata (Marsh.) Borkh. Chestnut. (C. vesca Americana Michx.).

Rocky hillside woods throughout the State.

C. pumila (L.) Mill. Chinquapin.

Dry hills. Fayette: near Nuttallburg, alt. 2,000 feet, frequent (Nuttall). Wayne: near Ceredo and Compton's Creek. Mercer: Beaver Spr., and Ingleside. A remarkably spreading and symmetrical individual was observed in a pasture at Bargers Spring, Summers County (Morris). 
Q. ALBA L. White Oak.

\section{QUERCUS L.}

All soils, plentiful throughout the State.

Q. Minor (Marsh.) Sarg. Post Oak. Iron Oak. (Q. stellata, Wang.)

Dry sterile soils, common.

Q. macrocarpa Michx. Burr Oak. Mossy-cup Oak.

Rich soils. Tyler: near Long Reach.

Q. Prinus L. Chestnut Oak.

Rocky woods. Frequent throughout the State.

Q. Acuminata (Michx.) Houda. Yellow Oak. (Q. Muhlenbergii Engelm.)

Rich, wooded valleys, especially in the mountains. Fayette: near Nuttallburg, rare (Nuttall).

Q. PAgodaefolia (E11.) Ashe.

Rich bottom lands. Kanawha: a few trees found on the north side of the river at Charleston (Brooks).

Q. BICOLOR Willd.

(Q. platanoides Sudw.).

Moist soils and borders of streams. Grant: along Lunice Creek; Hardy: along the South Branch, near Moorefield; Pocahontas: near Marlinton; Randolph: along the Tygarts Valley near Huttonsville; Upshur: rare near Lorentz (Brooks).

Q. RUBRA L. Red Oak.

Common throughout the State, in both rich and poor soils.

Q. Coccinea Wang.

Woodlands. Fayette: near Nuttallburg, apparently a second growth (Nuttall).

Q. velutina Lam. Black Oak. (Q. tinctoria Bartr.).

Dry woodlands. In large tracts in the Alleghanies of Mineral, Grant and Tucker counties. Gilmer: near Glenville (Mapel). Fayette: near Nuttallburg (Nuttall). Monongalia: near Ice's Ferry. Summers: near Hinton.

Q. PAlustris DuRoi.

Along streams. Monongalia: near Stumptown. Fayette: near Nuttallburg (Nuttall).

Q. Digitata (Marsh.) Sudw. Spanish Oak. (Q.falcata, Michx.)

Dry sandy soils throughout the western counties.

Q. NIGRA L. Black Jack Oak.

Common in dry or heavy clay soils throughout the center of the State. Hardy: near Moorefield.

Q. NAna (Marsh.) Sarg. Holly-leaved Oak. (Q. ilicifolia Wang.)

Sandy soils. Hampshire: near Romney. Hardy: near Moorefield.

Q. IMBRICARIA Michx.' Laurel Oak.

Rich woods. Monongalia: near Morgantown and Laurel Point. Hardy: near Moorefield. 


\section{U L M A C E A E.}

U. FUlva Michx. Slippery Elm.

\section{ULMUS L.}

Rich soils. Monongalia : near Morgantown, Laurel Point and Stumptown. Gilmer: near Glenville ( $\mathrm{Mapel}$ ). Fayette: near Nuttallburg (Nuttall). Mason: near Point Pleasant. Summers: along Greenbrier River. Webster: at Tate1 Knob (H. H. Smith, I492).

U. Americana L. White Elm.

Along rivers. Frequent throughout the State.

U. RACEMOSA Thomas. Corky Elm.

Near streams. Monroe: near Alderson. Summers: along Greenbrier River. Randolph: near Pickens (H. H. Smith, I704).

\section{CELTIS L.}

C. occidentalis L. Hackberry.

Woods and river banks. Jefferson: near Shenandoah Junction. Monongalia: near Morgantown. Jackson: near Ripley. Fayette: near Nuttallburg (Nuttall).

C. PUMila Pursh.

Among the rocks on the banks of both rivers at Harper's Ferry. Often fruiting copiously, on every twig, on trees but 2 or 3 feet high (Greene).

\section{OR A C E A E.}

\section{MORUS L.}

-M. RUBRA L. Black Mulberry.

Rich woods. Wood, Wirt, Calhoun and Gilmer (Mapel). Along the Little Kanawha River. Jefferson: frequent throughout. Greenbrier: near White Sulphur Springs. Fayette: near Nuttallburg, no large trees noted (Nuttall) M. Alba L. White Mulberry.

A frequent escape. Monongalia: near Morgantown. Jefferson: near Millville and Charlestown (Millspaugh); frequent about Harper's Ferry (Greene).

F. CARICA L. Fig.

\section{FICUS Linn.}

Fayette: on a ledge near the railroad from Gauley Bridge toward Gauley Junction (Eggleston, 5540).

\section{TOXYLON Rat ( 1817 ).}

(Maclura Nutt. I8I8).

T. POMIFERUM Raf. (Maclura aurantiaca Nutt.)

Banks of the Ohio River, Mason Co., near Point Pleasant. (Millspaugh). 


\section{BROUSSONETIA L'Herit.}

B. Papyrifera (L.) Vent. Paper Mulberry. "Cut Paper."

Escaped from cultivation. Jefferson: near Flowing Spring Mill and Milltown. Kanawha: near Montgomery Berkeley: near Martinsburg.

\section{HUMULUS L.}

H. Lupulus L. Hops.

Alluvial banks near streams. Very doubtfully native Randolph: near Cricard. Marshall : near Moundsville. Marion: near Clements, and Catawba. Mineral: opposite Cumberland, Md.

\section{CANNABIS L.}

C. sativa L. Hemp.

Fields and waste places. Escaped from cultivation. Jackson: frequent.

\section{U R T I C A C E A E.}

\section{URTICA L.}

U. Gracilis, Ait. Nettle.

Moist shady places. Common.

U. URENS L. Stinging Nettle.

Adventive. Hancock: near Holliday's Cove. Rare.

URTICASTRUM Fabr.

U. divaricatum (L.) Kuntze. (Laportea Canadensis Gaud.).

Moist rich woods. Fayette: near Nuttallburg (Nuttall); near Kanawha Falls (James). Preston: near Aurora ( $M r$. \& Mrs. Steele). Frequent throughout the State.

\section{ADICEA Raf. (I8I5). \\ (Pilea, Lindl. I82I).}

A. pumila (L.) Raf. Clear Weed. Rich Weed. ( $P$. pumila (L.) Gray).

Cool, moist, shady places. Common throughout the State.

BOEHMERIA Jacq.

B. CYLINDRicA (L.) Willd.

Damp places. Fayette: near Nuttallburg (Nuttall). Monongalia: near Uffington.

B. CYLINDRICA SCABRA Porter.

Barbour: in moist places near Tygart Junction (Greenman 340 ). 


\section{PARIETARIA Linn.}

P. Pennsylvanica Muhl.

Pocahontas: on a rocky hillside near Traveler's Repose (Greenman 342).

L O R A N T H A C A E.

PHORADENDRON Nutt.

P. Flavescens (Pursh.) Nutt. American Mistletoe.

Parasitic on Sugar-Maple and Black Locust, along the Great Kanawha River in Fayette County. On Black Walnut and Elm in Kanawha County. On Elms in Mason County and in Wood near Parkersburg. On Elms and Hickories, along the Ohio and Great Kanawha rivers, in Cabell County.

\section{S A N T A L A C E A E.}

PYRULARIA Michx.

P. PUBERA Michx.

Rich woods. McDowell: near Elkhorn. Nicholas Co., along Peter Creek (Boutlou). Upshur: near Buckhannon (Pollock).

\section{A R I S T OL O C H I A C E A E.}

\section{ASARUM L.}

A. Canadense L. Wild Ginger. "Colic Root."

Rich woods. Wirt: near Burning Springs. Gilmer : near Glenville (Mapel; Brown). Jefferson: 'near Flowing Spring Mill. Fayette: near Nuttallburg (Nuttall). Monongalia: near Uffington and Morgantown. McDowell: near Elkhorn. Mercer: Bluestone Jc., and common throughout the State.

\section{HEXASTYLIS Raf.}

H. Virginica (L.) Small.

(Asarum Virginicum L.).

Rich soil. Grant: near Bayard. Tucker: along Blacl: Water. Gilmer: near Glenville (Mapel, Brown). Greenbrier: near White Sulphur Springs. McDowell: near Elkhorn. Mercer: Bluestone Jc. and Bluefield. Upshur: near Buckhannon (Pollock). Fayette: at Hawks Nest (Holton).

H. Shuttlewortiin (J. Britten) Small. (Asarum J. Britten). In oak and beech woods near Bargers Spring, Summers County, altitude I,550 feet, July I3, I900 (Morris, 980). 


\section{ARISTOLOCHIA L.}

A. Serpentaria L. Virginia Snakeroot.

Rich woods. Wirt: near Burning Springs. Randolph: near Ford's and on Point Mountain. Gilmer: near Glenville (Broum). Grant: near Bayard. Tucker: near Davis. Mineral: near Keyser (Wortman). Fayette: near Nuttallburg (Nuttall). Wetzel: near Burton (Mertz, 2328).

A. macrophylla Lam. Dutchman's Pipe. (A. Sipho L' Her.). Rich woods, frequent throughout the State. Abandant in the following localities. Randolph: on Point Mountain (Millspaugh). Webster: Hacker Valley (H. H. Smith, I705). Preston: near Aurora (Mr. \& Mrs. Steele). Upshur: near Buckhannon (Pollock). Pocahontas: valley of the East Fork of Greenbrier River (Greenman, 204). Grant: near Bayard. Gilmer : near Glenville (Mapel, Brown). Fayette: near Nuttallburg (Nuttall). Mercer: Ingleside. Wirt: near Burning Springs (Mertz, 2329).

\section{POL Y GO N A CEA E.}

\section{ERIOGONUM Michx.}

E. Alleni, Watson, in A. Gray, Man. Ed. 6, 734 (I890).

Perennial, white tomentose throughout, the tall scape-like stem repeatedly dichotomous above; radical leaves lanceolate, long-petiolate, the upper in whorls of 4 or 5 , ovate to ovateoblong, very shortly petiolate, much reduced above; involucres mostly sessile; flowers glabrous, yellow, the segments elliptical. Near White Sulphur Springs, Greenbrier Co. (Allen, Britton, Small, Mackenzie).

\section{RUMEX L.}

R. Brittanica L. Water Dock.

Wet places. Ohio: on Bogg's Island (Meriz \& Guttenberg). Berkeley, near Martinsburg.

R. CRISPUS L. Curled Dock.

Waste places and cultivated fields. Frequent.

R. oвtusifolius L. Bitter Dock.

Waste grounds and cultivated fields, common.

R. CRispus x obtusifolius. Millsp. Prel. Cat. F1. W. Va., 433.

Waste places. Monongalia: streets of Morgantown.

R. sanguineus L. Bloody Dock. burg.

Damp places in waste grounds. Berkeley: near Martins-

R. CONGLOMERATUS Murray.

Shady places. Fayette: near Nuttallburg (Nuttall). 
R. acetosella L. Horse Sorrel.

Abundant everywhere; even along paths in the dense spruce forests of the higher mountains.

\section{FAGOPYRUM Gærtn.}

F. Fagopyrum (L.) Karst. Buckwheat. (F. esculentum Moench.) Waste grounds and cultivated fields. A frequent escape

\section{POLYGONUM' L.}

P. orientale L. Prince's Feather.

Escaped to waste places. Lewis: near Weston. Monongalia: near Morgantown. Fayette: near Nuttallburg, rare (Nuttall). Mineral: near Piedmont.

P. Pennsylvanicum L.

Low grounds. Common throughout the State.

P. Persicaria L. Lady's Thumb.

Waste grounds. Lewis: near Weston. Gilmer: vear Gilenville (Mapel). Fayette: near Nuttallburg (Nuttall). Mason: near Point Pleasant. Wood: near Parkersburg. Hardy: near Moorefield (Gamble). Randolph: near Pickens (H. H. Smith, I440).

Forma Albiflora. Millsp. Prel. Cat. F1. W. Va., 432.

A pure white-flowered form abundant near Point Pleasant.

P. Hydropiper L. Smartweed. Water Pepper.

Wet grounds. Monongalia: near Morgantown. Gilmer: near Glenville (Mapel). Fayette: near Nuttallburg. (Nuttall). Ohio: near Wheeling (Mertz \& Guttenberg.)

P. punctatum Ell. $\quad(P$. acre N. B. K.)

Wet places. Common throughout the State.

P. HYDROPIPEROIDES Michx. Mild Water-pepper.

Swampy places. Common throughout the State.

P. Virginianum L.

Thickets and in rich soils. Common throughout the State

P. Aviculare L. Door-weed. "Goose-grass."

About dwellings and roadsides. Common throughout the State.

P. ERECTUM L.

With the last, especially in streets.

P. TENUE Michx.

Dry pastures. Wood: near Kanawha Station. Monongalia: near Morgantown. Taylor: near Grafton, and common throughout the State.

P. CiLINODE Michx. 346).

Pendleton; in rocky places on Spruce Knob (Greenman 
P. sigittatum L. Tear-thumb.

Wet places. Common throughout the State.

P. ARIFOLIUM L.

Low grounds. Berkeley: near Martinsburg. Monongalia: near Morgantown, and elsewhere frequent.

P. Convolvulus L. Black Bindweed.

Gardens and waste places. Fayette: near Nuttallburg (Nuttall). Mason: near Point Pleasant.

P. CRistatum Engelm. \& Gray.

Along the Guyandot River below Baileysville, Wyoming County, altitude I, IOO-I,250 feet, August I9, I900 (Morris, 1255). Preston: near Aurora (Mr. \& Mrs. Steele).

P. scandens L. Climbing False Buckwheat.

Low grounds along streams, common.

\section{CHE N O POD I A C A E.}

\section{CHENOPODIUM, L.}

C. Album L. Lamb's Quarters. Pigweed.

Roadsides and waste places, common throughout the State. Var. viride (L.) Moq.

Dry sandy hillsides. Fayette: near Nuttallburg, alt. 2,000 feet, uncommon (Nuttall).

C. HYBRIDUM L.

Dry sandy hillsides. Fayette: near Nuttallburg, apparently indigenous (Nuttall). Jefferson: near Shepherdstown.

C. Murale L.

Plentiful along the cool northward bases of walls in the town of Harper's Ferry, Jefferson County (Greene).

C. URBICUM L.

Waste places, frequent.

C. Glaucum L.

Waste places. Monongalia: near Ice's Ferry.

C. Botrys L. Jerusalem Oak.

Ohio: near Wheeling. Jefferson: Shepherdstown.

C. Ambrosioides L. Mexican Tea.

Waste places. Common. Kanawha: along Great Kan. awha River. Taylor: near Grafton. Wood: near Parkersburg.

C. Anthelminticum L. Wormseed.

Plentiful along the Great Kanawha River. In Kanawha, Putnam and Mason counties. Fayette: near Nuttallburg. (Nuttall). Jackson: along C. \& P. pike. 


\section{SALSOLA Linn.}

S. Tragus Linn. Russian Thistle.

Monongalia: a few plants of this species appeared in an alfalfa field, in 1904, near Morgantown (Sheldon, 685).

\section{A M A R A N THA CEA E.}

\section{AMARANTHUS, L.}

A. HYBRIDUs L. Red Amaranth

(A. hypochondriacus L.).

Waste places. Ohio: on Bogg's Island (Mertz \& Guttenberg). Monongalia: near Morgantown. Fayette: nea1 Nuttallburg, common (Nuttall). Hardy: near Moorefield (Gamble).

A. paniculatus L.

Waste places. Monongalia: near Morgantown. Hardy: near Moorefield (Gamble).

A. Retroflexus L. Pigweed.

Ohio: on Bogg's Island (Mertz \& Guttenberg). Monongalia: near Morgantown.

A. GRAECIZANS L. Tumble-weed.

Waste places. Monongalia: near Morgantown.

A. spinosus L. Thorny Amaranth.

Waste grounds. Kanawha: near Charleston. Putnam: near Buffalo. Mason: near Point Pleasant. Wood: near Parkersburg, abundant. Jefferson: near Shepherdstown.

\section{P H Y T O L A C C A C E A E.}

\section{PHYTOLACCA, L.}

P. DECANDra L. Poke. Scoke. Garget.

All situations, even on the higher mountains. Common throughout the State.

\section{A I Z O A E A E.}

\section{MOLLUGO, L.}

M. verticillata L. Carpet-weed.

Waste and cultivated grounds. Monongalia: hills about Morgantown. Marion: near Fairmont. Fayette: near Nuttallburg, sandy banks of New River (Nuttall).

\section{POR T ULA CE AE. PORTULACA, L.}

P. oleracea L. Purslane. "Pussley."

A weed in cultivated grounds and gardens. Common throughout the State. 


\section{CLAYTONIA, L.}

C. Virginica L. Spring Beauty.

Common throughout the northern parts of the State, in rich open woods and along spring runs. Gilmer: near Glenville (Mapel; Brown). Hardy: near Moorefield (Gamble).

C. Caroliniana Michx.

Frequent with the former species. Marion, Preston, Wood, Wirt, Calhoun, Gilmer, Lewis, Upshur and Randolph counties. Fayette: near Nuttallburg (Nuttall). Ohio: near Wheeling (Mertz, 355).

\section{AGROSTEMMA L.}

A. Gituagic L. Corn Cockle.

(Lychnis Githago Scop).

A frequent weed in wheat fields throughout the State. Fayette: near Nuttallburg, in shady places (Nuttall).

\section{SILENE L.}

S. stellata (L.), Ait. Starry Campion.

Wooded banks, frequent. Wood, Wirt, Calhoun, Gilmer, Lewis, and Upshur, common. Preston: near Terra Alta; near Aurora ( $M r$. and Mrs. Steele). Summers: near Hinton. Greenbrier: near White Sulphur Springs. Fayette: near Nuttallburg (Nuttall). Hardy: near Moorefield (Gamble).

S. Virginica L. Fire Pink. Catchfly.

Open woods. Kanawha: near Charleston (Barnes).

Fayette: near Nuttallburg, very common (Nuttall). Mercer: near Bluefield. Monongalia: permian formations at Cassville, growing on a low roadside bank, fully exposed to the sun, but well supplied with root moisture (Morris).

S. Alba Muhl.

(S. nivea Otth.).

Wooded banks. Fayette: near Nuttallburg (Nuttall).

S. Caroliniana Walt. Wild Pink. (S. Pennsylvanica, Michx.)

Open woods. Monongalia: near Morgantown. Mineral: near Keyser (Workman). Gilmer: near Glenville (Mapel). Tampshire: Doe's Gully. Greenbrier Co., on Kate's Mountaiı, alt. 3,300 ft. (Small; Eggleston, 4355).

S. Antirrhina L. Sleepy Catchfly.

Dry places. Fayette: near Nuttallburg. (Nuttall). Preston: (Pollock).

\section{SAPONARIA L.}

S. officinalis L. Soap-wort. Bouncing Bet.

Becoming a very common weed along roadsides through- 
out the more settled portions of the State. In especially large areas along the B. \& O. R. R. and Shenandoah Valley R. R., in Jefferson Co. Calhoun: Grantsville. Gilmer: Glenville (Mapel). Webster: near Pugh (H. H. Smith. I668). Berkeley: Martinsburg. Summers: near Hinton. Fayette: near Nuttallburg (Nuttall). Hardy: near Moorefield (Gamble). Monongalia : near Lock 9.

V. VACCARIA (L.) Britton.

\section{VACCARIA Medic.}

Established in waste ground at Seebert, Pocahontas County (A. B. Brooks). Monongalia: near Morgantown (Sheldon, 3594).

\section{DIANTHUS L.}

D. Armeria L. Deptford Pink.

Fields, roadsides, and river banks. Marion: near Catawba (K. D. Walker). Gilmer: near Glenville (Mapel). Fayette: near Nuttallburg, rare (Nuttall); near Kanawha Fall: (James). Summers: near Hinton. Jefferson: at Harper's Ficrry (Mertz \& Guttenberg).

\section{ALSINE L. (I753 ante). \\ (Stellaria L. I753 post.)}

A. media L. Chickweed.

Damp places, common everywhere. Fayette : near Nuttallburg, blooms all winter (Nuttall).

A. Tennesseensis (Mohr) Small.

Woods and thickets. Ohio: near Wheeling (Mertz).

A. PUbera (Michx.) Britton. Great Chickweed.

Shady places, common. Monongalia, Marion and Preston counties. Gilmer: near Glenville ( $\mathrm{Mapcl}$ ). Fayette: near Nuttallburg (Nuttall). Mercer: near Bluefield (Millspaugh). Upshur: near Buckhannon (Pollock).

A. LONGifolia (Muhl.) Britton. Long-leaved Stitchwort.

Damp soils. Gilmer: near Glenville (Mapel).

\section{CERASTIUM L.}

C. vulgatum L. Large Mouse-ear Chickweed.

Waste ground and fields. Ohio: Cowan's Hill, near Wheeling (Mertz \& Guttenberg). Gilmer: near Glenville (Mapel). Wood: near Waverly and elsewhere, becoming frequent. Fayette: near Nuttallburg (Nuttall). Randolph: near Pickens (H. H. Smith, I386). 
C. viscosum L. Mouse-ear Chickweed.

Fields. Ohio: Cowan's Hill, near Wheeling (Merts \& (juttenberg). Fayette: near Nuttallburg (Nuttall). Monongalia: near Morgantown.

C. ARvense L. Field Chickweed.

Dry places. Frequent throughout the State.

C. Longepedunculatum Muhl. Star Chickweed. (C. nutans

Raf.). Woods: near streamlets; common in Monongalia and Marion counties.

SAGINA Linn.

S. PROCUMBENS Linn.

In moist places. Monongalia: near Morgantown (Sheldon, 429I).

ARENARIA L.

A. SERPYLlifolia L. Thyme-leaved Sandwort.

Sandy banks. Kanawha: near Charleston (Barnes). Hardy: near Moorefield (Gamble).

\section{SPERGULA L.}

S. Arvensis L. Field Spurry.

Fields. Preston: near Cranberry Summit (Mertz \& Guttenberg); near Terra Alta (Millspaugh); near Aurora (Mr. \& Mrs. Steele). Fayette: near Nuttallburg (Nuttall).

PARONYCHIA Adans.

P. пгснотома (L.) Nutt. Whitlow-wort.

Rocky places. Jefferson: near Harper's Ferry (Gray).

ANYCHIA Michx.

A. Candensis (L.), B. S. P.

(A. capillacea, D. C.)

Dry Woods. Fayette: near Nuttallburg, common (Nuttall).

A. POLygonoides Raf.

(Anychia dichotoma, Michx.).

This species first appeared in this locality in I895, at the bottom of a newly excavated railroad cut. Had the seeds been buried and dormant? (Nuttall, 1837). Morris says: "I should say, no. This species was common with and nearly as abundant as the next throughout the above mentioned counties. It is probable that the newly excavated cut proved, perhaps unusually suitable for the germination of scattering seeds."

A. DIVARICATA Raf.

Wyoming: near Baileysville (Morris I25 pt.). 
CASTALIA Salisb.

C. cdorata (Dryand), Woodv. \& Wood. White Water Lily. Nymphaea odorata, Ait.

In slack waters. Preston: near Pennsylvania line, rare.

\section{NYMPHAEA L.}

N. advena Soland. Yellow Pond Lily.

In slack waters. Preston: near Terra Alta. Wood: Wirt: Calhoun and Gilmer: along the Little Kanawha River. Upshur: near Lorentz. Morgan: along the Little Cacapon. Putnam: near Buffalo. Hardy: near Moorefield. Pocahontas: Marlinton (Kellerman).

\section{A G N OLI A C E A E.}

\section{MAGNOLIA L.}

M. Acuminta L. Cucumber Tree, 'Yellow Lin."

Rich woods. Monongalia : along the Monongahela River, especially near Little Falls, Opekiska, and Montana. Wirt: along Little Kanawha River. Randolph: on Point Mountain, alt. 2335-3700 ft. Webster: along Buffalo Bull Mounaains. Nicholas: at Beaver Mills, and Collett's Glade. Jackson: near Sandyville. Mineral: near Keyser. Preston: along B. \& O. R. R. Randolph: on Cheat Mountain, alt. $2800 \mathrm{ft}$. Gilmer: near Glenville ( $\mathrm{Mapel}$ ). Monroe: near Alderson. Summers: near Greenbrier Stock Yards; near Hinton. Kanawha: near Handley. Harrison: near Shinnston and Lumberport. Fayette: near Nuttallburg (Nuttall). Preston: near Aurora (Mr. \& Mrs. Steele).

M. TRIPETALA L. Umbrella tree. (M. Umbrella, Lam.).

Rich woods near streams. Wirt: near Burning Springs. Randolph: on Point Mountain, alt. $2800 \mathrm{ft}$. (Millspangh); near Pickens (H. H. Smith, 1944). Kanawha : near Charleston (Barnes; James). Nicholas: near Beaver Mills, alt. $2125 \mathrm{ft}$. Gilmer: near Glenville (Mapel). Monongalia : Little Falls. Summers: near Hinton. Harrison: near Shinnston and Lumberport. Fayette: near Nuttallburg, rare (Nuttall). Hardy: near Moorefield. McDowell: near Elkhorn. Mercer: Bluestone Junction and Ingleside.

"This species forms a very conspicuous part of the forests along Dry Fork and Crane Creek in McDowell County, and along the Guyandot River in Wyoming county. A great many young trees are now filling the places made vacant by 
the cutting of a few selected trees of other species. It is noticeable that until these trees reach the age of flowering and thereafter there is none of the characteristic umbrellalike clustering of the leaves on the axis of the season but they are strongly alternate and distant. This character confuses the species with Magnolia acuminata in the young large-leaved stage, unless the smoothness or pubescence of the leaf-buds be noted" (Morris). In Webster county, at Hacker Valley, this species is replacing "yeliow poplar" where that timber has been removed (H.H. Smith).

M. Fraseri Walt. Ear-leaved Magnolia.

Leaves oblong-obovate or spatulate, auriculate at the base, glabrous, 8 to 20 in. long; petals obovate-spatulate, with narrow claws, 4 in. long. Flower more graceful and cone of fruit smaller than in the preceding species.

Type locality doubtless in the Alleghanies of West Virginia, where it is abundant and in typical form.

Pocahontas: valley of the East Fork of Greenbrier River (Greenman, 192). Deep rich mountain woods. Randolph: on Point Mountain, alt. $3700 \mathrm{ft}$. (Millspaugh); near Pickens (H. H. Smith, 1945). Webster: on Buffalo Bull Mountain, alt. $3400 \mathrm{ft}$. Nicholas: near Beaver Mills, alt. 2125 ft. Fayette: near Nuttallburg (Nuttall). Summers : near Hinton. McDowell : near Elkhorn. Mercer: Bluestone Jc.

\section{LIRIODENDRON L.}

L. Tulipifera L. Tulip Tree, "White, Yellow, or Hickory Poplar."

Common in rich woods throughout the State. Magnificent trees in Randolph, Pocahontas, Greenbrier, Webster, Nicholas, and Preston counties. Summers: near Hinton. Fayette: near Kanawha Falls; near Nuttallburg, common (Nuttall). Hardy: near Moorefield (Gamble). Mercer: near Ingleside.

\section{A NONACEAE.}

\section{ASIMINA Adans.}

\section{A. triloba (L.), Dunal. Papaw.}

Rich soil near streams, common. Wood: near Kanawha Station (Millspaugh) ; near the mouth of the Little Kanawha (Michaux). Wirt: along Straight Creek Webster: Buffalo Bull Mountains, alt. 2 IOO ft. Nicholas: along Pete: Creek. Fayette: along Gauley River. Kanawha, Putnam and Mason: along the Great Kanawha. Gilmer: near Glen- 
ville $(\mathrm{Mapel})$. Greenbrier: near Ronceverte. Summers: near Hinton Marion: near Worthington, Fairmont and Montana. Monongalia: general. Fayette: near Nuttallburg, common (Nuttall). Morgan: near Cacapon. Hardy: nea: Moorefield. Mercer: near Ingleside. Randolph: near Pickens (H. H. Smith, 1475).

\section{R A N UN C UI, A CEAE.}

HYDRASTIS, Ellis.

H. Canadensis L. Yellow Root, Yellow Puccoon, Golden Seal.

Deep rich woods. Jackson: near Ripley. Wirt: near Burning Springs. Grant: near Bayard. Upshur: summit on Staunton pike. Lewis: along Leading Creek. Calhoun: along Laurel Run. Gilmer : near Glenville (Mapel; Brown). Monongalia: along Cheat River.

\section{CALTHA L.}

C. palustris, L. Marsh Marigold.

Two stations only known to me; Grant: in a spring run in deep woods near Bayard, about fifty plants within an area of one hundred feet. Preston: near Terra Alta.

\section{HELLEBORUS L.}

H. viridis L. Green Helleborc.

Hardy: near Moorefield (Gamble). It was from specimens sent to Dr. Gray from this station by Dr. Gamble, that the location "W. Va." was credited in the Manual.

\section{COPTIS Salisb.}

C. TRIFoliA (L.), Salisb. Gold Thread. (Isopyrum trifolium, Britton).

Deep, rich mountain woods. Preston : near Mill Run and Cranesville.

\section{XANTHORRHIZA L' Her.}

X. ApiIfolia L' Her. Shrub Yellow Root.

Rich rocky mountain woods. Nicholas: on Mumble-thePeg Creek, plentiful. Fayette: near Nuttallburg, common (Nuttall). Upshur:,(Pollock). Barbour: on river bank near Tygart Junction (Greenman, 184).

\section{ACTAEA L.}

A. RUBRA (Ait.), Willd. Red Baneberry. (A. spicata rubra Ait.). Rich woods, infrequent. Randolph: on Point Mountain. Grant: near Bayard. Tucker: near Davis. 
A. ALBA (L.) Mill., White Baneberry.

Rich woods. Randolph: on Point Mountain. Tucker: near Davis. Grant: near Bayard. Fayette: near Nuttallburg (Nuttall). Preston: near Aurora (Mr. \& Mrs. Steele).

\section{CIMICIFUGA L.}

C. Ámericana Michx. American Bug-bane.

Plentiful throughout the mountain regions of Mineral, Hampshire, Grant and Tucker counties. Hardy : near Moorefield; Randolph, Pendleton, Webster, Pocahontas and Greenbrier counties. Fayette: near Nuttallburg (Nuttall). McDowell: near Elkhorn. Preston: near Aurora (Mr. \& Mrs. Steele).

C. Racemosa (L.), Nutt. Rattle-weed, Black Cohosh.

Rich opens and clearings. Wood: near Leachtown. Lewis: along Stone Coal Creek. Randolph: near Valley Bend; Point Mountain, alt. 3300 ft. Webster: Buffalo Bull Mountains. Gilmer: near Glenville (Mapel). Preston: near Aurora $(M r$. \& Mrs. Steele). Fayette: near Nuttallburg (Nuttall) and frequent throughout the State.

\section{AQUILEGIA L.}

A. Canadensis L. Wild Columbine.

Damp, rocky places. Mineral: along Knobby mountains. Monongalia: along Cheat River. Calhoun: along Little Kanawha River. Gilmer: near Glenville ( Mapel; Brozen). Fayette: near Nuttallburg (Nuttall). Hampshire: near Does Gully. Hardy: near Moorefield.

A small form 6 to 8 inches high, with small leaves and flowers. Mercer: near Beaver Spr.

\section{DELIPHINIUM L.}

D. TRICORnE Michx. Dwarf Larkspur.

Dry woods. Monongalia: along Cheat and Monongahela Rivers. Marion: along the Monongahela. Gilmer: near Glenville (Mapel; Brown). Frequent throughout the northern part of the State. Fayette: near Nuttallburg (Nuttall). Lewis: (Pollock).

Forma Albiflora Millsp. Prel. Cat. F1. W. Va., 322.

Monongalia: prevalent near Stumptown. The flowers are pure white with no tinge of blue.

D. AJAcis Linn. Larkspur.

Escaped and thoroughly established near Wheelers, Webster County (H. H. Smith. 1652). Monongalia: near Morgantown (Shunk). 
D. Consolida L. Field Larkspur.

Frequent in old fields and along roadsides. Lewis : along Stone Coal Creek. Monongalia : near Morgantown. Upshur: near Buckhannon. Randolph: near Beverly. Kanawha: near Kanawha Falls (James).

\section{ACONITUM L.}

A. uncinatum L. Wild Monk's Hood.

Deep, rich woods along streams. Randolph: along Cheat River near Cheat Bridge. Monongalia: near Camp Eden. Fayette: along New River near Nuttallburg (Nuttall).

A. Reclinatum A. Gray.

Woodlands. Randolph: on Cheat Mountain (Gray). Pendleton: in a damp depression on Spruce Knob; and Pocahontas: on the Greenbrier River, "in neither place did the plants appear to have the least tendency to trail: they were strictly erect ( $A$. B. Brooks).

\section{ANEMONE L.}

A. CYLINDRICA Gray. Long-fruited. Anemone.

Dry Woodlands. Wood: near Leachtown. Jackson: near Sandyville. Rare.

A. Virginiana L. Thimble-weed.

Open woods and meadows. Upshur: near Buckhannon (Millspaugh, Pollock). Monongalia: along the Monongahela River. Webster: near Hacker Valley (H. H. Smith, 1649). Randolph: Rich Mountains, alt. I825 ft. Fayette: near Nuttallburg, where it grows as tall as $4 \mathrm{ft}$. (Nuttall). Frequent throughout the State.

A. Canadensis L. Pennsylvania Anemone. (A. Pennsylvanica, L.).

Rich woods, rare. Calhoun: along Laurel Run. Gilmer: near Glenville (Mapel).

A. Quinguefolia L. Wind-flower. Wood Anemone. A. nemorsa, L.).

Margins of rich woods and opens. Calhoun: along Laurel Run. Gilmer: near Glenville (Mapel). Fayette: near Nuttallburg (Nuttall). And general throughout the State.

A. TRIFOLIA L.

Rich woods. Mercer: near Ingleside. McDowell: near Elkhorn. Monongalia: near Camp Eden. Greenbrier: in a dry ravine at White Sulphur Springs, alt. about 2,300 ft. (Heller).

The altitude of the first two stations in the southern part of the State is from 2,200-2,350 ft.; these localities are along 
the same range of mountains as the original station of Canby in Virginia. The Monongalia station in the extreme northern part of the State has an altitude of about $850 \mathrm{ft}$. and is near Knipes' Pennsylvania station. Though I have not as yet found the species at any point through the State that would connect these distant localities, yet I fully believe that many will be found in the future.

\section{HEPATICA Scop.}

H. Hepatica (L.)., Karst. Hepatica. Liver-leaf.

Rocky or rich woods. Gilmer: near Glenville (Mapel). Greenbrier: near White Sulphur Springs. Hardy: near Moorefield (Gamble). Mercer: near Bluefield. Monongalia: along Decker's Creek. Fayette: near Nuttallburg, where it often grows in clefts in rocks (Nuttall). And frequent throughout the northern portions of the State.

H. ACUta (Pursh.) Britt.

Rich woods. Gilmer: near Glenville (Mapel; Brown). Greenbrier: near White Sulphur Springs. Monongalia: near Cheat View. McDowell : near Elkhorn. Mercer: Bluestone Jc. Throughout the State, especially eastward in the mountains. More frequent than the preceding.

\section{SYNDESMON Hoffm'g.}

S. thalictroides (L.) Hoffm'g. Rue Anemone. (Thalictrum anemonoides, Michx.).

Woods and hillsides. Gilmer: near Glenville (Mapel). Fayette: near Nuttallburg (Nuttall). Monongalia: near Uffington and Morgantown. Hardy: near Moorefield (Gamble). Mercer: near Beaver Spr. Frequent throughout the State.

Forma rosea Millsp. Prel. Cat. F1. W. Va., 3 I9.

A beautiful rose colored clump of the species has persisted for two years in Monongalia at Little Falls near the Cascade.

\section{CLEMATIS L.}

C. Virginiana L. Virgin's Bower.

River banks, fence rows, etc., Monongalia: Marion: Preston: Wood. Webster: Long Glade (Millspaugh); near Hacker Valley (H. H. Smith, I648). Gilmer: Glenville (Mapel). Greenbrier: near White Sulphur Springs. Summers: near Hinton. Kanawha: near Charleston. Fayette: near Nuttallburg (Nuttall). Preston: near Aurora $(M r$. $\mathcal{E}$ Mrs. Stcele). Tucker: in a thicket near Parsons (Greenman, I90). 
C. VIORnA L. Leather Flower.

Thickets in rich soil. Monongalia : Little Falls. Marion: Valley Falls (K. D. Walker). Fayette: near Nuttallburg (Nuttall). Summers: near Hinton.

C. ovata Pursh.

Greenbrier Co.: in rocky soil high up on Kate's Mountain, near White Sulphur Springs (N. L. Britton; Mackenzie; Small; Heller 842).

\section{ATRAGENE L.}

A. Americana Sims.

(Clematis verticillaris DC.). Rocky woods. Monongalia: near Cheat View.

TRAUTVETTERIA F. \& M.

T. Carolinensis (Walt.) Vail. False Bug-bane. (T. palmata F. \& M.).

Plentiful along the Blackwater Fork of Cheat, about one mile below Davis in Tucker county. Fayette: near Hawk's Nest; and Loup Creek (James) ; near Nuttallburg, common (Nuttall) ; near Quinnimont (Pollard \& Maxon 26). Preston: (Pollock).

\section{RANUNCULUS L.}

R. obtusiusculus Raf. Water Plantain Spear Wort ( $R$. ambigens Wats.).

One station only; Upshur: in a marshy spot near Lorentz. (Millspaugh; Pollock).

R. ABortivus L. Small-flowered Crowfoot.

Damp, shady places, frequent throughout the State.

R. SCEleratus L. Cursed Crowfoot.

Moist places, common throughout the State.

R. MICRANTHUS Nutt.

In rich woods. Monongalia: near Seneca (Sheldon $4270)$.

R. Alleghaniensis Britton.

Mountain woods. Pocahontas: near Spruce $\mathrm{Knob}(A . B$. Brooks).

R. Hispidus Michx.

Dry woodlands and thickets. Greenbrier: near White Sulphur Springs (Eggleston, 4360).

R. RECURvatus Poir. Hooked Crow foot.

Rich, open woods. Abundant along the Monongahela River in Upshur, Barbour, Taylor, Marion, and Monongalia counties. Fayette: near Nuttallburg, not common (Nuttall). Hampshire: near Romney (Millspaugh, I568). Mercer: near Simmons. 
R. FASCiCUlaris Muhl. Early Crowfoot.

Dry or moist grassy hillsides. Monongalia : near Morgantown. Mineral: near Keyser (Workman). Gilmer: near Glenville (Mapel). Hardy: near Moorefield (Gamble).

R. septentrionalis Poir. Buttercup.

Moist, shady places. Fayette: near Nuttallburg (Nuttall), and general throughout the State.

R. Pennsylvanicus L. f. Pennsylvania Buttercup.

Damp woods. Monongalia and Marion: along the Monongahela River.

R. Repens L. Creeping Crowfoot.

Low grounds. Mineral: Banks of the Potomac near Keyser. Jefferson: near Shenandale Springs.

R. Bulbosus Linn.

Fields and roadsides. Monongalia : near Morgantown ( $A$. B. Brooks).

R. ACRIS L. Tall Buttercups.

Waste places infrequent. Wood: near Parkersburg, Jefferson: near Shenandoah Junction. Randolph: in clearings on Cheat Mountain, near Cheat Bridge, alt. 3,350 ft.; near Pickens (H. H. Smith, 1388). Preston: near Terra Alta Workman). Gilmer: near Glenville (Mapel).

\section{THALICTRUM L.}

T. DIOICUM L.

Rocky woods: frequent throughout the State. Especially noticeable in the Alleghanies.

T. polygamum Muhl. Common Meadow Rue.

Damp meadows and near streams and ditches. Randolph: rich growths in the bottoms along Tygart's Valley River Fayette: near Kanawha Falls (James); near Nuttallburg, alt. 2,000 ft. (Nuttall). Gilmer: near Glenville. Frequent throughout the State.

T. purpurascens L. Purplish Meadow Rue.

Dry, open woods and rocky hillsides. Wirt: near Elizabeth; near Burning Springs. Webster: near Upper Glade. Randolph: along Tygart's Valley River, alt. 2,I00 ft. Fayette: near Nuttallburg (Nuttall).

T. CLAVATUM DC.

Fayette: near Nuttallburg, in sandy clefts of rocks around waterfall, alt. 2,000 ft., one station (Nuttall). 
BERBERIS L.

B. Canadensis Mill. Barberry.

Fields and roadsides. Mercer : near Beaver Spring, where it partakes of the nature of a weed (Millspaugh). Summers: near Hinton (Eggleston, 5579). Monroe: along Indian Creek (F.E. Brooks).

B. VULGARIS L.

Thickets and roadsides. Monongalia: a wide escape near Laurel Point. Mercer: a wide escape near Ingleside.

CAULOPHYLLUM Michx.

C. thalictroides (L.) Michx. Blue Cohosh.

Deep, rich woods. Randolph: Rich. Mountains; Point Mountain, alt. 3,300 ft. Monongalia: along the Monongahela River. Gilmer: near Glenville (Mapel). Fayette: near Nuttallburg (Nuttall). Mercer: near Bluefield. Frequent throughout the State.

JEFFERSONIA Barton.

J. Diphylla (L.) Pers. Twin-leat.

Rich woods. Monongalia: near Morgantown. Marion: near Glover's Gap. Wetzel: near Littleton. Marshall: near Glen Easton. Gilmer: near Glenville (Mapel; Brown). Cabell: near Huntington (Selby). Lewis: near Lorentz Pollock).

\section{PODOPHYLLUM L.}

P. peltatum L. May Apple. Mandrake.

Frequent throughout the State, in some rich spots very abundant. Randolph: Rich Mountains, alt. I,6Io-2,I25 ft.; Point Mountain, alt. 3,300 ft. Fayette: near Nuttallburg (Nuttall). Hardy: near Moorefield (Gamble). Mercer: Bluestone Jc.

MEN IS PER M A C E A E.

\section{MENISPERMUM L.}

M. Canadense L. Moonseed.

Thickets. Wirt: along Little Kanawha River. Marion and Monongalia: along the Monongahela. Greenbrier: near White Sulphur Springs. Fayette: near Nuttallburg, pistillate plants, rare (Nuttall). Randolph: along Tygarts Valley River near Huttonsville (Greenman, I75). Hardy: near 
Moorefield. Mercer: near Princeton; and frequent along streams throughout the State.

\title{
C A L Y C A N T H A CEA E.
}

\author{
BUTNERIA Duham (I755). \\ (Calycanthus, L. I759).
}

B. FLORIDA (L.) Kearney. Allspice. Sweet-scented Shrub.

Rich woods. Randolph: near Fords, on Staunton Pike, thence frequent over Rich Mountains. Webster and Nicholas: along Buffalo Bull Ridge. Fayette: along the Gauley River near Gauley Mountains. Summers : near Hinton.

B. FERTILIS (Walt.) Kearney (B. laerigatus in Flora).

Rich woods. McDowell : back of $\mathrm{R}$. R. water tank neai Welsh.

SASSAFRAS, Nees \& Eberm.

S. Sassafras (L.) Karst. Sassafras. (Sassafras officinale Nees.). Thickets and opens. Abundant throughout the State.

BENZOIN Fabric.

B. Benzoin (L.) Coulter. Wild Allspice. Spice-bush. (Lin. dera Benzoin Blume).

Low woods. Common throughout the State.

P A P A VER A CEA E.

\section{SANGUINARIA L.}

ङ. Canadensis L. Blood-root "Coon-root."

Rich woods, frequent. Randolph: Point Mountain. Monongalia : near Morgantown. Jefferson: near Flowing Spring, and Shenandale Spring. Gilmer: near Glenville (Mapel). Fayette: near Nuttallburg (Nuttall). Hardy: near Moorefield (Gamble). Greenbrier: near White Sulphur Springs (Eggleston, 4340). Throughout the State.

\section{STYLOPHORUM Nutt.}

S. Dyphllum (Michx.) Nutt. Celandine Poppy.

Old fields. Ohio: near Wheeling (Mertz \& Guttenberg). Fayette: near Nuttallburg (Nuttall). 


\section{CHELIDONIUM L.}

C. majus L. Celandine.

Waste grounds, near dwellings. Monongalia: near Easton; near Morgantown. Jefferson: near Charlestown, abundant. Lewis: near Weston. Hardy: near Moorefield (Gamble). Hampshire: near Romney.

\section{PAPAVER L.}

P. Dubium L. Smooth-fruited Corn-poppy.

Cultivated grounds, and waste fields. Jefferson: near Shenandoah Jc.; near Charlestown, abundant. Berkeley: near Hedgesville, a weed.

\section{ADLUMIA Raf.}

A. Fungosa (Ait.) Greene.

Monongalia: climbing over rocks, Tịbb's Run (Millspaugh). Pendleton: top of Alleghany Mountains above Horton (Greenman, 195).

G. Glaucium (L.) Karst.

\section{GLAUCIUM Juss.}

Jefferson: near Charlestown, Shenandoah Junction, Spring Mill, and Milltown.

BICUCULLA Adans.

B. Cucullaria (L.) Millsp. Prel. Cat. F1. W. Va., 327: Dutchman's Breeches.

Rich woods. Monongalia : near Morgantown; along Cheat River. Gilmer: near Glenville (Mapel). Hardy: near Moorefield (Gamble). Fayette: near Nuttallburg (Nuttall). Lewis: (Pollock).

B. Canadensis (Goldie) Millsp. ibid. Squirrel Corn.

Rich woods. Monongalia and Marion: along the Monongahela River. Preston: along Cheat River. Fayette: near Nuttallburg (Nuttall).

B. eximia (Ker.) Millsp. ibid.

Pocahontas: summit of Spruce Knob, alt. 4,80o ft. (Hopkins).

CAPNOIDES Adans ( 1763$)$.

(Neckeria, Scop. I777.)

C. sempervirens (L.) Borck. Pale Corydalis. (N. glauca (L.) Millsp.).

On rocks. Randolph: on Lone Sugar Knob. Gilmer: near Glenville $(\mathrm{Mapel})$. Fayette: near Nuttallburg (Nutt- 
all). Preston: near Aurora (Mr. \& Mrs. Steele). Pocahontas: (Pollock).

C. flavulum (Raf.) Kuntze. Yellow Corydalis. N. Aavula (Raf.) Millsp.

Rich soils. Common throughout the northern counties. Fayette: near Nuttallburg (Nuttall). Hardy: near Moorefield.

C. Aureum (Willd.) Kuntze. Golden Corydalis. (N. aurea, (Willd.) Millsp.).

Along streams. Hardy: near Moorefield (Gamble). Ohio: near Wheeling (Mertz, I25).

\section{FUMARIA L.}

F. cFificinalis L. Fumitory.

Waste places. Hardy: near Moorefield (Gambie).

B R A S S I C A C E A E.

(Cruciferae.)

\section{LEPIDIUM L.}

L. Virginicum L. Wild Peppergrass.

Fields and roadsides, common throughout the State.

L. Campestre (L.), R. Br. English Peppergrass, "Glenn-weed," "Glenn-pepper," "Crowd-weed."

An exceedingly abundant weed in Jefferson and Berkeley counties, where it is known as "Glenn-weed," it being first noticed in the fields of Colonel Glenn; who tells me that the weed was quite plentiful, however, in these fields before he purchased them, having been brought there in clover seed bought in Hagerstown, Md., and sown by the previous owner of the farm. The weed is now the worst pest in the large wheat fields of those counties.

\section{SISYMBRIUM L.}

S. officinale (L.), Scop. Hedge Mustard.

Roadsides and ditches, too common throughout the State. S. ALtissimum Linn:

Established in waste grounds at Morgantown, Monongalia County (Sheldon, 3599).

\section{BRASSICA L.}

B. NigrA (L.) Koch. Black Mustard.

Fields and waste places. Fayette: near Nuttallburg, much eaten as "greens" in spring (Nuttall). A common weed throughout the State. 
B. ARvensis (L.), B. S. P. Charlock, "Crowd-weed," "Krautweed." (B. Sinapistrum Boiss.).

A miserable weed in wheat fields in Jefferson and Berkelev counties. Less abundant elsewhere throughout the State.

R. SAtivus I. Radish.

\section{RAPHANUS L.}

Frequently perșistent in waste grounds and cultivated fields, in many parts of the State.

BARBAREA R. Br.

B. Barbarea (L.) MacM. Yellow Rocket. (B. vulgaris R. Br.). Becoming a weed in many places in Jefferson: Berkeley: Morgan: Hardy: near Moorefield (Gamble); and other counties.

B. PRAecox (Smith), R. Br. Scurvy Grass.

Running wild near Charlestown in Jefferson; and Lewis: neảr Weston (Millspangh). Ohio: near Wheeling (Richardson).

\section{IODANTHUS T. \& G.}

I. Pinnatifidus (Michx.) Steudel.

Banks of the Ohio River. Ohio: near Elm Grove (Curtis).

RORIPA Scop. I760).

(Nasturtium, R. Br. I8I2.)

R. Nasturtium (L.), Rusby. Water Cress. (Nasturtium officinale, $\mathrm{R}$. Br.).

Cold spring runs, frequent. Jefferson: near Shenandale Springs; near Flowing Spring: Lewis: on Leading Creek. Wirt: near Elizabeth.

R. sylvestris (L.), Bessey. Yellow Wood-cress. (Nasturtium sylvestre, R. Br.).

Moist places in open woods, frequent. Monongalia: near Morgantown. Preston: Coldi Spring. Marion: near Montana. Jefferson : near Shenandale Springs.

R. oвtusa (Nutt.) Britton. (Nasturtium obtusum Nutt.).

River banks. Mason: near Point Pleasant.

R. palustris (L.) Bessey. Marsh Cress. (Nasturtium palustre, DC.).

Marshy places and glades, infrequent. Webster: near Welch Glade. Kanawha: near Charleston. Preston: near Kingwood. Fayette: near Nuttallburg, rare (Nuttall). Mason: near Point Pleasant, banks of the Ohio. Wood: near Parkersburg. 
R. HISPIDA (Desv.) Britton. (N. palustre var. hispiaum, Gray). Preston: near Kingwood.

R. Armoracia (L.) Hitch. Horseradish. (Nasturtium Armoracia Fries.).

Escaped from cultivation in many waste places and fields. Marshall: frequent in several places where it is complained of as a weed difficult to eradicate. Jefferson: near Shenandoah Junction.

\section{CARDAMINE L.}

C. PARVIFLORA Linn.

On rocks. Monongalia : near Morgantown (A.B. Brooks); near Uffington (Sheldon, I366).

C. HIRSuTA L. Small Bitter Cress.

Wet places. Monongalia: Monongahela River below Morgantown; above Little Falls. Marion: near Catawba and elsewhere. Fayette: near Nuttallburg (Nuttall). Randolph: near Pickens (H. H. Smith, I406).

C. Bulbosa (Schreb.) B.S.P. Spring Cress. (C. rhomboidea, DC.).

Wet meadows and springy places. Preston: Cold Spring. Monongalia: road to Cheat River beyond Easton. Wood: Kanawha Station. Fayette: near Nuttallburg, common (Nuttall). Mercer: near Bluefield.

C. pUrpurea(Torr.) Britt. (C. rhomboidea, var. purpurea, Torr.).

Damp places. Monongalia: near Morgantown (Millspaugh). Ohio: in a spring marsh near Wheeling (Mertz, I44b). Upshur (Pollock).

C. ROTUNDIFolia Michx. Mountain Water Cress.

Cool Springs. Preston: Cold Spring. Jefferson: Flowing Spring. Wirt: near Burning Springs. Calhoun: Laurel Run. Monongalia: near Morgantown. Ohio: near Wheeling (Merti, I45).

C. PRATENSIS Linn.

Wet meadows. Ohio: near Wheeling (Richardson).

C. Pennsylvanica Muhl.

Wet places. Randolph: borders of Gandy Creek (Green$\operatorname{man} 374,373$ ) and vicinity of Horton.

\section{DENTARIA L.}

D. Diphylla Michx. Pepper Root.

Common in deep, cool ravines and in the mountains. Monongalia: Wirt: Wood: Calhoun: Lewis: Upshur: near Buckhannon (Pollock) Jefferson : Grant: and Tucker counties. Gilmer: near Glenville (Mapel; Brown). Fayette: Hawk's Nest (Porter); near Nuttallburg (Nuttall). 
D. HeTEROPHYLla Nutt.

Rocky, moist places. Monongalia :near Little Falls; opposite Uffington. Fayette: near Nuttallburg (Nuttall).

D. Laciniata Muhl.

Moist woods, frequent. Monongalia: opposite Beechwoods and Uffington; near Little Falls. Preston: Cold Spring and elsewhere. Gilmer: near Glenville (Mapel). Fayette: near Nuttallburg (Nuttall). Hardy: near Moorefield (Gamble).

var. INTEGRIFolia (Schulz) Fernald.

Monongalia: near Morgantown (Grose $357 \mathrm{I}$ visa). var. MULTIFIDA Muhl.

Rich woods. Monongalia: near Little Falls.

\section{BURSA Weber.}

B. Bursa-pastoris (L.) Britton. Shepherd's Purse. (Capsella Bursa-pastoris Medic).

Fields and roadsides, common throughout the State.

\section{CAMELINA Crantz.}

C. sativa (L.) Crantz. False Flax.

Fields and waste grounds. Mineral : near Keyser (Wortman). Jefferson: near Charlestown; near Shenandoah Jc.

C. microcaripa Andrz.

Waste ground. Jefferson: near Shenandoah Jc. (Mills. paugh).

DRABA L.

D. Ramosissima Desv. Whitlow Grass.

On wet cliffs. Jefferson: cliffs along Shenandoah River between Millville and Harper's Ferry. Tucker: Cliffs near Falls of Blackwater. Hardy: near Moorefield.

D. VERna L. Shad Flower.

Sandy wastes and roadsides. Monongalia : banks of Falling Run; banks of Monongahela below Morgantown, and neai Little Falls. Marion: near Opekiska. Fayette: near Nuttallburg (Nuttall). Greenbrier Co.: on Kate's Mountain, alt. $3,300 \mathrm{ft}$. (Small).

\section{STENOPHRAGMA Celak.}

S. Thaliana (Linn.) Celak. (Sisymbrium Thalianum Gay). Waste grounds. Fayette: near Nuttallburg (Nuttall). 
A. Patens Sulliv.

\section{ARABIS L.}

Moist rocky places in woods. Monongalia: near Monongahela River at Uffington. Preston: Cold Spring.

A. Laevigata (Muhl.), Poir.

Rocky places, frequent. Monongalia: near Morgantown, and little Falls. Marion: near Catawba. Fayette: near Nuttallburg, common (Nuttall). Lewis: (Pollock).

A. Canadensis L. Sickle-pod.

Woods, and along cool runs. Monongalia: near Granville, near Morgantown and Uffington.

A. LyRATA L.

Rocky or sandy places. Monongalia: banks near mouth of Cheat River. Mercer: near Ingleside.

\section{ERYSIMUM L.}

E. Cheiranthoides L. Worm-seed Mustard.

Roadsides and railroad embankments. Monongalia: near Morgantown. Jefferson: near Shenandoah Junction. Mineral: near Piedmont.

\section{HESPERIS L.}

H. matronalis L. Dame's violet.

Escaped to waste places. Monongalia: cinders of railroad banks near Morgantown.

$$
\text { C A P P A R I D A C E E. }
$$

\section{CLEOME L.}

C. spinosa L. Spider Flower.

C. pungens Willd.

Escaped from farther south, at Barboursville near the Guyandot River, Cabell county (James). Webster: Hacker Valley (H. H. Smith, I737).

D R O SER A CEA E.

DROSERA L.

D. Rotundifolia L. Sundew.

Glades. Preston: Cranberry Summit: Morgan's Glade; and Terra Alta (Millspaugh); near Aurora (Mr. \& Mrs Steele).

$$
\text { PODOSTE MON A CEAE. }
$$

PODOSTEMON Michx.

P. Ceratophyllum Michx.

"Three well marked stages, (a) an entirely sessile growth 
on new surfaces, (b) matted growth of previous seasons on old surfaces, with stems an inch or two high, (c) very old masses with stems from five to eight inches high or as long where the current prevented an erect habit; in the Guyandot River below Baileysville, Wyoming county, alt. I,IOO ft., August 15, 1900. (Morris, 1210).

\section{R A S S ULA CE AE.}

\section{SEDUM L.}

S. PUlChellum Michx.

Rocky places. Jefferson: near Harper's Ferry (Gray).

S. Nevir Gray.

Dry, rocky places. Greenbrier: near White Sulphur Springs (Millspaugh, Eggleston 4367).

S. ternatum Michx. Stone-crop. State.

On rocks in deep woods and opens. Throughout the

S. TELEPHioIdes Michx.

Drier situations. Throughout the northern counties. Jefferson: Harper's Ferry (Gray). Hardy: near Moorefield (Gamble). Hampshire: near Romney.

S. Telephium, L. Live-for-ever.

Along railroad banks. Jefferson, Morgan and Berkeley counties.

\section{PEN T H O R C E AE.}

\section{PENTHORUM L.}

P. SEDOIDES L. Ditch Stone-crop.

"Open wet places, and ditches. Throughout the State. Very luxuriant specimens three feet and more high were noted in the delta of a spring, under limestone cliffs, below Baileysville, Wyoming county" (Morris).

\section{P A R N A S S I A C E AE.}

\section{PARNASSIA Linn.}

P. GRANDIFLORA DC.

Wet banks. Fayette: near Kanawha Falls (Selby).

P. ASARIFOliA Vent.

Wet places. Upshur: near French Creek (A. B. Brooks). 


\section{S A X IF R A G A C E AE.}

ASTILBE Hamilton.

A. Biternata (Vent.) Britton. Goats-beard. (A. decandra Don.). Rich woods. Mercer: near Wills.

\section{SAXIFRAGA L.}

S. Virginiensis Michx. Early Saxifrage.

Exposed moist rocks and opens. General throughout the State.

S. Pennsylvanica L. Swamp Saxifrage.

Glades. Preston: Morgan's and Terra Alta Glades.

S. micranthidifolia (Haw.) B. S. P. Lettuce Saxifrage. (S. crosa, Pursh.).

Spring-rills in deep woods. Monongalia: near Camp Eden. Grant: near Bayard. Tucker: along the Blackwater.

\section{THEROFON Raf.}

T. Aconitifolium (Nutt.) Millsp. Prel. Cat. Fi. W: Va., 36r. (Boykinia aconitifolia, Nutt.).

Creek beds. Fayette: near Nuttallburg; plentiful along the beds of creeks at an alt. of I,800 ft., does not extend down these beds as far as the shores of New River (alt. I, IOO ft.). Often 3-celled, sometimes the flowers 6-parted, few 7-parted (Nuttall).

\section{TIARELLA L.}

T. CORDifolia L. False Mitre-wort.

Rich, moist woods. Common throughout the Alleghanies and the foot-hills. Monongalia: near Morgantown. Gi1mer: near Glenville (Mapel). Fayette: near Nuttallburg (Nuttall). Greenbrier: near White Sulphur Springs. McDowell: near Elkhorn. Mercer: near Bluestone Jc. (Millspaugh). Upshur: near Buckhannon (Pollock).

\section{MITELLA L.}

M. Diphylla L. Mitre-wort. Bishop's Cap.

Rich, shady woods. Common throughout the State.

\section{CHRYSOSPLENIUM Linn.}

C. Americanum Schwein.

In damp, shady situations. Monongalia: near Richard (Sheldon, 4279). 


\section{HEUCHERA L.}

H. villosa Michx.

Rocky places. Fayette: Kanawha Falls (James); Loup Creek (James). Nuttallburg (Nuttall); above Gauley Bridge and at Gauley Junction (Eggleston, 5534, 554I); near Hawks Nest (Holton); Kanawha: near Coalburg (James).

H. Americana L. Alum-root.

Rich, damp woods. Monongalia : near Morgantown, and frequent elsewhere. Gilmer: near Glenville (Mapel). Fayette: near Nuttallburg (Nuttall). Greenbrier: near White Sulphur Springs. Grant: near Bayard. Tucker: near Davis. Hampshire: near Romney. Ohio: near Wheeling (Mertz, 859). Upshur: near Buckhannon (Pollock).

H. PARVIFLORA Bartl.

(H. Rugelii, Shuttlw.). all).

Shaded cliffs. Fayette: near Nuttallburg, common (Nutt-

H. PUBEscens Pursh.

Rich, deep woods. Randolph: near Durbin (Greenman $376)$.

HYDRANGEA L.

H. ARBORESCENS L. Wild Hydrangea.

Rich opens. Monongalia: near Morgantown. Marion: Opekiska. Wood: near Kanawha Station. Wirt: near Burning Springs. Gilmer: near Glenville ( $\mathrm{Mapel}$ ). Lewis : along Stone Coal Creek. Throughout the above range the flowers were all fertile. Fayette: near Hawk's Nest (James) ; near Kanawha Falls (James); near Nuttallburg (Nuttall). Randolph: at Pickens (H. H. Smith, I39I). Preston: near Aurora (Mr. \& Mrs. Steele).

A form with grass-green marginal radiant flowers, in a deep ravine in Fayette: near Nuttallburg.

Var. Kanawhana Millsp. Prel. Cat. Fl. W. Va., 363.

Low straggling bush, leaves small, paler beneath, acuminate, somewhat cordate at the base; cymes very open and branching, marginal radiant flowers many, I in. broad, fertile flowers nearly glabrous, smaller than in the species. Along the Little Kanawha River from Kanawha Station to Glenville.

\section{G R O S S ULA R I A CE AE.}

RIBES I.

R. Cynosbati L. Prickly Gooseberry.

Deep rocky woods. Randolph: along Cheat River, alt. 
3,360 ft. ; Point Mountain, alt. 3,700 ft.; Rich Mountain, alt. 2,700. Grant: near Bayard. Preston: near Terra Alta (Millspangh); near Aurora (Mr. \& Mrs. Steele). Upshur: near Buckhannon (Pollock). Pendleton: branch of Two Spring Creek above Horton (Greenman, I39) ; and frequent throughout the northern and eastern counties. Hardy: near Moorefield (Millspaugh). Nicholas: at Richwood ( $H$. $H$. Smith, I743).

R. Rotundifolium Michx. Smooth Gooseberry.

Rich, cool, mountain woods, with the last, frequent (Millspaugh). Pendleton: on Spruce Knob; and Pocahontas: at Durbin (Greenman I4O, I4I).

R. PRostratum L'Her.

Pocahontas: summit of Spruce Knob, alt. 4,8c0 ft. (Millspaugh). Pendleton: west slope of Spruce Knob (Greenman, I42) (Hopkins).

R. FLORIDUM L'Her. Wild Black Currant.

Rich woods. Randolph: near Beverly. Grant: near Bayard. Preston: near Terra Alta. Fayette: near Nuttallburg (Nuttall). Ohio: near Wheeling (Mertz, 829).

H A M A M ELID A CE AE.

\section{HAMAMELIS L.}

H. Virginiana L. Witch-hazel.

Common in damp woods throughout the State.

\section{LIQUIDAMBAR L.}

L. Styraciflua L. Sweet-gum. Alligator-wood.

Rich woods. The distribution of this species in the State according to my notes of travel is, from east to west, as follows: Beginning near the source of Peter Creek, in Nicholas county, it follows that stream to its junction with the Gauley River, down this to its confluence with the New River to form the Great Kanawha, which latter it follows to the mouth of Elk River, whence it bears northward up Eight Mile Creek to the Pocotaligo and its Middle Fork, across to Mill Creek in Jackson, which it follows to the Ohio.

It is also noted in Gilmer: near Glenville ( $\mathrm{Mapel}$ ). Fay ette: near Kanawha Falls (James) ; near Nuttallburg (Nuttall). Cabell: near Huntington (Selby). I have also met with it in Summers: near Hinton; and along the Greenbrier River in that county. 


\section{P L, A T.A N A C E AE.}

\section{PLATANUS L.}

P. occidentalis L. Sycamore. Buttonwood.

All soils. Wood: at the mouth of the Little Kanawha (Michaux). Common throughout the State.

R O S A C E AE.

OPULASTER Medic.

(Physocarpa Raf. I836.)

O. opulifolius (L.) Kuntze. Nine-bark (Physocarpa opulifolia Raf.).

Along streams, common. Monongalia: along the Monongahela River. Webster: Upper Glade. Fayette: near Nuttallburg (Nuttall). Hardy: near Moorefield. Upshur: near Buckhannon (Pollock).

\section{SPIRAEA L.}

S. corymbosa Raf. Birch-leaved Meadow-sweet. (S. betulifolia Pall.).

Rich mountain woods. Webster: near Upper Glade. Hardy: near Moorefield (Gamble).

S. Virginiana Britt. West Virginia Meadow-sweet.

Discovered I8go along the Monongahela River near Morgantown. The following description is taken from Prof Britton's account of the plant in "Bull. Torrey Club," Dec. I890.

"A glabrous shrub, the branches forming long wands, erect or reclining, I-4 ft. long. Leaves oblong or slightly oblanceolate, thin, obtuse or short-pointed at the apex, rounded or cuneate at the base, $I^{1} \frac{1}{2}$ to 2 in. long, 5-8 lines wide, green above, pale beneath, entire or with a few low serration, in the upper half; petioles 2 lines long; pedicels and peduncles pale and glaucous; flowers about 2 -lines broad, in terminal compound corymbs I-3 in. across; calyx teeth 5 , triangular, blunt, about the length of the short-campanulate tube, distinctly glaucous; petals 5 , white, ovate-orbicular, obtuse; stamens $15-20$, persistent; styles $5-6$; follicles in the specimens examined, 5-6, apparently sterile, included in the persistent calyx."

"On damp rocks along the Monongahela River, Morgantown, West Virginia, collected by Dr. C. F. Millspaugh in flower, June 20th, I890, and in apparently imperfect fruit 
late in September. Coilected also by Mr. G. R. Vasey in the mountains of North Carolina, I878."

"Spiraea betulaefolia, Pall. and $S$. corymbosa, Raf., have much longer follicles exserted beyond the calyx, broader. thicker, and dentate leaves, and are different in habit. Rafinesque published a number of species in his New Flora, but none of them can apply to this one."

- Upshur: near Buckhannon (Pollock).

S. SAlicifolia Linn. Queen of the Prairie.

Meadows. Monongalia: near Morgantown. Preston: near Terra Alta (Millspaugh); near Aurora (Mr. \& Mrs. Steele). Fayette: near Nuttallburg (Nuttall). Forming a hedge along a woodland swamp between Harvey and Trap Hill, Raleigh county (Morris). Upshur: near Buckhannon (Pollock). Randolph: at Elkins (Greenman, I89).

S. tomentosa L. Hardhack. Steeple-bush.

Low grounds. Webster: Welch Glade. Wood: near Lockhart's Run. Fayette: near Nuttallburg (Nuttall). Randolph: in a pasture at Elkins (Greenman, I88).

\section{ARUNCUS Adans.}

A. Aruncus L. Goat's Beard.

(Spiraea Aruncus L.).

Rich ground and along streams. Monongalia: near Morgantown, Uffington, Little Falls, Day Creek and Granville along the Monongahela. Randolph: along Middle Fork, on Rich Mountains, alt. 2,125 ft. Gilmer: near Glenville ( $\mathrm{Ma}$ pel).

\section{PORTERANTHUS Britton (I894).}

(Gillenia, Moench. I802 not Adans I763.)

P. tRifoliatus (L.) Britton. Bowman's Root. Indian Physic. (Gillenia trifoliata Moen.).

Rich woods, frequent. Webster: Welch and Long Glades. Monongalia: along the Monongahela River from Little Falls to Beech Woods. Mineral : near Keyser (Wortman). Summers: near Hinton. Hardy: near Moorefield (Gamble). Mercer : near Ingleside. Greenbrier Co., on Kate's Mountain, alt. 3,300 ft. (Small; Eggleston, 4342).

P. Stipulatus (Muhl.), Britton. American Ipecac. (Gillenia stipulacea Nutt.).

Borders of woods. Wood: between Kanawha Station and Lockhart's Run.

\section{RUBUS L.}

R. Americanus (Pers.) Britton. (R. triflorous Rich.). Open places. Preston: at Craneville Glade (Rumsey). 
R. odoratus L. Purple-flowering Raspberry. 'Thimble-berry.

Damp rocky places. Monongalia : near Little Falls. Grant: near Bayard. Randolph: near Crickard P. O. Fayette : nea: Kanawha Falls (James); near Nuttallburg (Nuttall). Preston: near Aurora (Mr. \& Mrs. Steele). Summers: near Hinton. Hardy: near Moorefield (Gamble). Upshur: near Buckhannon (Pollock).

R. Columbianus (Millsp.) Rydb.

Leaves ample, 5-7-incised, divisions oblong-lanceolate long and taper pointed, sharply and mostly double serrate. Inflorescence smaller, more compact and the fruit smaller than in the previous species and of a more decided musky taste. Monongalia: coo1 woods, Tibbs Run.

R. strigosus Michx. Wild Red Raspberry.

Thickets Fayette: near Nuttallburg, not plestiful (Nuttall). Pocahontas: Spruce Mountain (Hopizins).

R. occidentalis L. Black Raspberry.

Frequent throughout the State.

R. Nigrobaccus Bailey. High Blackberry. ( $R$. villosus of Flora.)

Common everywhere in the State.

R. ARGUTUs Link. fide Rydberg.

Roadsides, Randolph: near Pickens (H. H. Smith, I384). R. Baileyanus Britton. (R. villosus humifusus T. \& G.).

Woods and river banks. Fayette: near Nuttallburg ( $N u t t-$ all).

Var. FRONDOSUs Bigel.

Fayette: near Nuttallburg (Nuttall). Preston : near Tunnellton

R. Millspaughii Britt.

This species was described in "The Bulletin of the Torry Club" for I891, page 366 , as follows :

"Ascending, wand-like, entirely unarmed or with a very few weak prickles above, glabrous throughout or the younger shoots scurfy pubescent. Stems one and one-half to four meters long; leaves long petioled, pedately 5 -foliate or some of those one the twigs 3 -foliate; leaflets thin, oval, glabrous on both sides, long-acuminate at the apex, mostly rounded at the base, I2-I $5 \mathrm{~cm}$. long, about $5 \mathrm{~cm}$. wide, sharply, but not deeply serrate; stock of the terminal leaflet 7-10 cm. iong; inflorescence loosely racemose; bracts linear-lanceolate; acuminate ; fruit black, about io mm. long."

"Nearest to R. villosus, but evidently a distinct species. Curiously enough there is a leaf of this species glued down on the sheet of R. Canadensis, L in herb Linn., and it appears to have been included in his description of that species -the specimens furnished by Kalm." 
Near the summit of Point Mountain in Randolph county at an altitude of 3,500 $\mathrm{ft}$., also along the Gandy in great profusion. Pendleton and Pocahontas: on Little Rich Mountains abundant. The mountaineers claim that it is upon this species that the bears grow fat for their period of hibernation, the fruit being late to ripen and very nutritious.

Mr. John K. Small remarks in his Studies of Botany of the Southeastern United States, Bull Torr. Club, 2I :I9 (1894) in reporting this species: "Grows on the walls of the canon at Tallulah Falls, Ga. Collected in flower in April, 1893 ; alt. I,600 ft. This most likely locates the southern limit of the geographical range of $R$. Millspaughii. Being a typical high mountain species, it is not to be expected much below 1,600 feet, and just south of the above-mentioned locality the Blue Ridge 'runs out' into the plains. Further south the land decreases gradually in altitude until the gulf is reached. According to my observations the above plant thrives most vigorously at altitudes ranging from 4,500 to $6,000 \mathrm{ft}$. It grows well at lower elevations, but never in such a prolific manner." Mr. Small and Anna M. Vail also give in their "Report of the Botanical Explorations of Southwestern Virginia, I892," the following additional localities for this species: "Above Fox Creek, on Pine Mountain in deep woods, ait. 3,000 ft. Summit and slopes of Mt. Rodgers, alt. 4,000 to 5,7I9 ft. Summit of White Top Mountain, alt. $5,678 \mathrm{ft}$. Slopes and summit of White Rock Mountain, alt. 3,000 to 4,400 ft."

The species was also collected by Mr. T. H. Kearney, Jr., at the summit of 'Thunderhead (about 6,500 ft.), and on Chilhowee Mountain (at about I,500 ft.), in Blount Co., Tennessee. Reports of the collection of the species in the mountains of North Carolina, New Hampshire, and norther! Michigan have been made.

The species is reported as common in thickets at Wilton and Southport, Maine (Fernald), and at Deering, Maine (Blankinship).

R. Canadensis L. Dewberry.

Abundant on dry hillsides throughout the State.

iR. Allegheniensis Porter. Leucretia Dewberry. (R. Canadensis roribaccus Bailey).

Dry hillsides. Randolph: near Beverly.

This new variety of the species is described by Prof. Bailey in the American Garden, November, I890, as follows: "Plant larger and stronger; leaflets broad below, usually triangular-ovate, doubly serrate with small teeth, and more or less notched and jagged; peduncles longer, st:aighter and 
stouter, habituously more numerous and more conspicuously overtopping the leaves; flowers very large (sometimes two inches across) ; sepals uniformly larger, some of them much prolonged and leaf-like and conspicuously lobed (sometimes becoming an inch long and wide) ; fruit much larger."

R. HISPIDUs L. Running Swamp-Blackberry.

Glade regions of Preston, Randolph and Webster counties. Fayette: near Nuttallburg (Nuttall).

R. TRIVIALis Michx. Low Bush-blackberry.

Sandy soil. Kanawha: near Charleston (James).

\section{DALIBARDA L.}

D. REPENS L.

Deep mountain woods. Grant: near Bayard. Tucker: along Blackwater Fork of Cheat (Millspaugh). Preston: near Aurora ( $M r$. \& Mrs. Stecle).

\section{DRYMOCALLIS Fourr.}

D. ARguta (Pursh.) Rydb.

(Potentilla arguta Pursh.).

Dry situations. Upshur: near French Creek ( $A$. $B$. Erooks).

\section{FRAGARIA L.}

F. Virginiana Duches. Wild Strawberry.

Moist woodlands and fields, common. Monongalia: near Morgantown (Brozen). Gilmer: near Glenville (Mapel) Fayette: near Nuttallburg (Nuttall). Hardy: near Moorefield (Gamble).

F. vesca L. Northern Wild Strawberry.

Shady woods, less common than the last. Kanawha: nea1 Charleston (James). Fayette: near Nuttallburg (Nuttall).

\section{DUCHESNEA J. E. Smith.}

D. Indica (Andr.) Focke. Indian Strawberry. (Fragaria Indica Andr.).

Escaped to waste places. Monongalia: near Morgantown. Kanawha: near Charleston (Barnes, Coulter, C. L. Brooks).

\section{ULMARIA Hill.}

U. RUBRA Hill.

(Spiraea lobata Gronov.).

Escaped and established near Pickens, Randolph County (H. H. Smith, I 542).

U. Ulmaria (L.) Barnhart. (Spiraea Ulmaria Linn.).

Escaped and established near Pickens, Randolph County (H. H. Smith, I 543). 


\section{POTENTILLA L.}

P. Monspeliensis L.

(P. Norvegica L.).

Fields and wet places. Wood: near Kanawha Station. Monongalia : near Morgantown. Grant: near Bayard. Fay. ette: near Nuttallburg, rare (Nuttall). Tucker: near Davis, Randolph: near Pickens (H. H. Smith, 1365).

P. Canadensis L. Cinquefoil.

Dry fields, an abundant weed throughout the State. Var. Simplex (Michx.) T. \& G.

Dry fields. Ohio: near Wheeling (Mertz \& Guttenberg).

P. SUlPHUREA Lam.

(P. recta Am. Auth.).

Found growing in abundance in a meadow near South Fairmont, Marion County (Boutlou).

\section{WALDSTEINIA Willd.}

W. Fragarioides (Michx.) Tratt. Barren Strawberry.

Wooded hillsides. Grant: near Bayard. Greenbrier : near White Sulphur Springs (Heller, 830).

\section{GEUM L.}

G. RIVALE Linn.

In swampy places. Preston: in "Pine Swamp" near Cranesville (A. B. Brooks).

G. Canadense Jacq.

(G. allum Gmel.).

Woods and thickets. Calhoun, Wood, Grant, Mineral, and Monongalia Counties. Fayette: near Kanawha Falls (James) ; near Nuttallburg (Nuttall). Summers : near Hinton (Millspaugh). Webster: on Tater Knob (H. H. Smith, I477).

G. Virginianum L.

Borders of woods and low grounds. Wood, Wirt, Calhoun, Gilmer, Marion, Lewis, Monongalia, and Jefferson Counties. Fayette: near Nuttallburg (Nuttall)

G. FLAVUM (Porter) Bicknell.

Along Madam Creek opposite Hinton, Summers County, alt. I,500 ft., July 9, I900 (Morris, 965) ; along the Guyandot River below Baileysville, Wyoming County, alt. I,250 ft., August I 5, Igoo (Morris, I218).

G. VERnum (Raf.) T. \& G.

Moist places. Monongalia: near Morgantown, Little Falls. Marion: near Opekiska.

\section{AGRIMONIA L.}

A. Hirsuta (Muhl.) Bicknell.

(A. Eupatoria of Flora). Borders of woods, frequent throughout the State. In a 
thicket about a spring near Kegley, Mercer County, alt. 2, I00 ft., July 21, I900 (Morris, I042). Preston: near Aurora (Mr. \& Mrs. Steele).

A. PARviflora Soland.

Woods and glades. Randolph: on Lone Sugar Knob. Preston: near Terra Alta. Webster : Long Glade. Nicholas : Collett's Glade. Tucker : near Davis. Fayette : near Nuttallburg, alt. 2,000 ft. (Nuttall). Summers: near Hinton. Monroe: near Alderson.

A. Brittoniana Bicknell.

Dry, open field. Webster: summit of Lone Tree Knob (Millspaugh, 450).

A. GRYPOSEPALA Wallr.

Dry situations: Randolph: at Ford's (Millspaugh, 425). A. mollis (T. \& G.) Britton.

Dry woods and thickets. Preston: near Aurora (Mr. \& Mrs. Stecle).

A. Striata Michx.

Dry woods. Randolph : summit of Rich Mountain (Millspaugh, 450). Pocahontas: valley of the East Fork of the Greenbrier River (Greenman, I37).

\section{SANGUISORBA L.}

S. Canadensis L. Burnet. (Poterium Canadense Gray). Rich, moist woods. Randolph: along Cheat River. Tucker: along Blackwater Fork of Cheat. Monongalia: Cheat River near Camp Eden. Preston: Terra Alta (Millspaugh); at Read (Greenman, I7I).

\section{ROSA L.}

R. SETIGera Michx.

Escaped and established commonly about Fairmont, Marion County (Boutlou).

R. Carolina L Carolina Rose.

Damp places. Wood: near Kanawha Station, profuse. Upshur: near Buckhannon. Webster: Long Glade. Randolph; meadows along Tygart's Valley River. Fayette: near Nuttallburg (Nuttall).

R. Humilis Marsh. Dwarf Wild Rose.

Dry soils. Wood: near Kanawha Station. Monongalia: near Uffington and Beechwoods. Gilmer: near Glenville (Mapel). Fayette: near Nuttallburg (Nuttall). Summers: near Hinton.

R. LUCIDA (Ehrh.) Larger Wild Rose.

Shaded hillsides. Kanawha: near Charleston (James, 
Barnes). Monongalia: plentiful along Cheat River above Camp Eden. Fayette: Kanawha Falls (James).

R. RUGosA Thunb.

Established at Morgantown, Monongalia County (Sheldon).

R. RUbiginosa L. Sweet-brier. Eglantine.

Frequent along roadsides and thickets. Nicholas: along Gauley River. Randolph: Staunton Pike on Cheat Mountains. Summers: near Hinton. Monongalia: near Ice's Ferry.

R. CANINA L.

Rocky Banks. Fayette: near Nuttallburg (Nuttall).

POM A C E AE.

\section{SORBUS L.}

S. Americana Marsh. Mountain Ash. (Pyrus Americana DC.). Damp mountain woods. Randolph: near Cheat Bridge. Grant: near Bayard. Tucker: near Davis; and along the Blackwater. Preston: near Aurora (Mr. \& Mrs. Steele). Pendleton: on Spruce Knob (Greenman, I48).

\section{MALUS Hill.}

M. Coronaria (L.) Mill. Wild Crabapple. (Pyrus coronaria L.).

Opens and damp places. Monongalia: frequent about Morgantown. Marion: along the Monongalia River. Gilmer: near Glenville (Mapel). Upshur: near Buckhannon (Pollock). Pocahontas: near Travelers Repose (Greenman, I70). Fayette: near Nuttallburg (Nuttall). Mercer: near Beaver Spr. Greenbrier: near White Sulphur Springs (Eggleston, 4324, 4369).

M. angustifolia (Ait.) Michx. Narrow-leaved Crab. (Pyrus angustifolia Ait.).

Glady regions of Preston, Webster and Nicholas Counties (Millspangh). Pocahontas: near Durbin (Greenman, I43).

M. GLAUCESCENS Rehder.

Greenbrier : near White Sulphur Springs (Eggleston 4323, 4363). Summers: near Hinton (Eggleston, 5571).

ARONIA Medic.

A. Arbutifolia (L.) Medic. Choke Berry. (Pyrus arbuti. folia L.).

Damp places. Webster: Upper Glade. Preston: near Terra Alta. Nicholas: Collett's Glade. Fayette: near Nutt- 
allburg, alt. 2,000 ft. (Nuttall). Upshur: near Buckhannon (Pollock).

A. NIGRA (Willd.) Britton. (P.arbutifolia melanocarpa Michx.)

Preston: Reedsville Glade; Morgans' Glade. Webster: Upper and Welch Glades.

AMELANCHIER Medic.

A. Canadensis (L.) Medic. Shad Bush. June Berry.

Common generally, even in the higher mountains.

A. Botryapium (L.f.) D.C. (A. canadensis oblongifolia T. \& G.) Open woodlands. Preston: near Reedsville (Rumsey).

C. Amara Ashe.

\section{CRATAEGUS L.}

Summers: near Hinton (Eggleston, 5578, 5592).

C. Roanensis Ashe.

Summers: near Hinton (Eggleston, 557.2, 5588). Hampshire : near Romney (Brooks).

C. Margaretta Ashe.

(C. Brownii Britton).

Greenbrier: near White Sulphur Springs (Eggleston, $4317-21,4326,4329,4362,4368)$; on Kate's Mountain (Small). Fayette: near Thurmond (Eggleston, 5555-6, 5559, 5561). Summers: near Hinton (Eggleston, with red fruit 5582 , with yellow $5583 ; 5589$ ).

C. StRAMinea Beadle.

Greenbrier: near White Sulphur Springs (Eggleston 4322, 4330, 4370). Summers : near Hinton (Eggleston 5563, 5570).

C. PRuinosa (Wendl.) K. Koch.

Greenbrier : near White Sulphur Springs (Eggleston 4327, 4372). Summers: near Hinton (Eggleston, 5586).

C. succulenta Schrad.

(C. macrantha Lodd.)

Greenbrier: near White Sulphur Springs (Eggleston 4328, 437I). Summers: near Hinton (Eggleston, 5593).

C. macrosperma Ashe. forma.

Greenbrier: about the base of Kate's Mountain near White Sulphur Springs (Eggleston, 4332). Monongalia: near Morgantown (Brooks).

C. Pausiaca Ashe.

Upshur: near Buckhannon (Brooks).

C. FoetinA Ashe.

Grant: along Abram Creek (Brooks).

C. Holmesiana Ashe.

Monongalia : near Morgantown (Brooks).

C. spathulata Michx.

Rocky woods. Mercer: near Beaver Spring, and Wills. McDowell: near Elkhorn. 
C. CORdata (Mill.) Ait.

Rocky mountain woods. Mercer: near Beaver Spring, Ada, and Ingleside.

C. Oxyacantha L.

River banks. Fayette: near Nuttallburg (Nuttall).

C. Apiffolia (Marsh.) Michx.

Rocky woods. Mercer: near Ingleside and at Beaver Spring.

C. Coccinea L. Scarlet Thorn.

Thickets. Calhoun: Lower Leading Creek. Monongalia: near Ice's Ferry. Marion: near Opekiska. Gilmer: near Glenville (Mapel). Fayette: near Nuttallburg (Nuttall). Upshur: near Buckhannon. Summers: near Hinton. Hardy: near Moorefield (Gamble). Mercer: near Ingleside.

C. mollis (T. \& G.) Scheele. (C. coccinea, var. mollıs, T. \& G.)

Mercer: near Beaver Spring and Ingleside.

C. tomentosa L. Black Thorn.

Uplands. Monongalia: near Stewartstown and Uffington; road to Dorsey's Knob. Randolph: near Cheat Bridge. Tucker : along the Blackwater. Wirt: near Elizabeth. Wood : near Lockhart's Run. Summers : near Hinton. McDowell : near Elkhorn. Grant: near Abram Creek (Brooks).

C. punctata Jacq.

Borders and open woods. Monongalia: near Ice's Ferry; Cheat River above Camp Eden. Greenbrier: near White Sulphur Springs (Eggleston 4325, Mllispaugh). Fayette: near Nuttallburg (Nuttall).

C. Crus-galli L. Cockspur Thorn.

Thickets. Monongalia: frequent. Marion: near Hough. town. Preston: near Reedsville and Terra Alta. Mineral: near Keyser. Jefferson: near Shenandoah Junction. Upshur: near Buckhannon. Fayette: near Nuttal!burg (Nuttall); near Gauley Bridge (Eggleston, 5538). Greenbrier: near White Sulphur Springs. Summers: near Hinton Hardy: near Moorefield (Gamble). Mercer: near Beaver Springs and Ingleside.

C. Flava Ait. Summer Haw.

Shady river banks. Fayette: banks of New River near Nuttallburg (Nuttall). Mercer: near Ingleside; along stream opposite Wills. McDowell: along Tug Fork, near Welch.

C. UNIFLORA Muench.

(C. parviflora Ait).

Sandy woods. Mercer: along streamlet near Ingleside (Millspaugh). Summers: near Hinton (Eggleston: with red fruits 5573, with yellow fruits 5574). 
C. rotundifolia (Ehrh.) Bork.

Greenbrier Co.: on Kate's Mountain, alt. 3,300 ft. (Small).

\section{R U P A C E AE.}

\section{PRUNUS L.}

P. Americana Marsh. Wild Yellow or Red Plum.

River banks and woodlands. Hardy: near Moorefield. Monongalia: near Morgantown. Marion: near Opekiska. Tyler: near Long Reach (Col. Johnson). Wood: near Lockhart's Run. Gilmer: near Glenville (Mapel). Randolph: near Pickens. Fayette: near Nuttallburg (Nuttall); above Gauley Bridge (Eggleston, 5530).

P. ANGUSTIFolia Michx.

(P. chicasa Michx.).

Thickets. Monongalia: Permian formations near Cassville. Tyler: near Long Reach.

P. Pennsylvanica, L. f. Wild Red Cherry.

Rocky woods. Very common throughout the eastern portion of the State.

P. Virginiana L Choke Cherry.

Moist, shady places, common.

P. SERotina Ehrh. Wild Black Cherry.

Common throughout the State, where it often forms extensive and very valuable forests. This is especially true of the tree in the central eastern section.

A. Persica L. Peach.

\section{AMYGDALUS Linn.}

Several trees were growing in the woods along Dry Fork above Peeryville, McDowell County, alt. I,300 ft., August 4, Ig00 (Morris, I I30).

\section{A E S A L P I N A E A E.}

\section{CERCIS L.}

C. Canadensis L. Red-bud. Judas' Tree.

Especially common on hillsides along the Great Kanawha River in Putnam and Mason Counties. Monongalia: along Cheat River; and the Monongahela. Wirt : along Little Kanawha River. Gilmer: near Glenville (Mapel). Upshur: near Buckhannon (Pollock). Fayette: near Nuttallburg (Nuttall). Summers: near Hinton.

CASSIA L.

C. Marilandica L. Wild Senna.

Sandy alluvium. Fayette: near Nuttallburg (Nuttall). 
near Gauley Bridge. Gilmer: near Glenville (Prof. Brown). Kanawha: near Pocotaligo. Jackson: near Sandyville. Jefferson: near Harpers Ferry. Mason: near Point Pleasant. Harrison : near Shinnston. Summers : near Hinton. Monongalia: near Morgantown. Hardy: near Moorefield (Gam. ble).

C. Medsgeri Shafer.

Dry gravelly situations. Ohio: near Wheeling (Guttenberg).

C. Chamaechrista L. Partridge Pea.

Sandy fields. Monongalia: near the mouth of Cheat River. Marion: near Opekiska. Fayette: near Nuttallburg (Nuttall)

C. Nictitans L. Wild Sensitive Plant.

Sandy places. Monongalia : along the Monongahela River. Marion: near Clements. Fayette: near Nuttallburg (Nutiall); near Hawks Nest (Holton). Barbour: near Tygart Junction (Greenman, I8I). Mason: near Point Pleasant. Summers: near Hinton. Hardy: near Moorefield (Gamble).

C. nictitans commixta (Poll. \& Max.) comb. nov.

Chamaecrista nictitans commixta Pollard \& Maxon, Proc. Biol. Soc. Wash., I4:163 (I90I).

Plant of low stature, very densely and divaricately branching, the stems finely pubescent or puberulent: leaves resembling those of nictitans but often with more numerous leaflets; petiolar gland cupulate or truncate, usually nearly sessile; flowers and fruits as in C. nictitans.

Collected by Charles L. Pollard and William R. Maxon in alluvial soil along New River at Quinnimont, Fayette County, August 2I, I899. Their No. 3I, the type, is in the National Herbarium as No. 357,069 .

\section{GLEDITSIA L.}

G. triacanthos L. Honey or Black Locust.

Rich woods. Monongalia: near Morgantown. Wirt: on Nigh-Cut Hill. Randolph: Rich Mts.; Point Mt., alt. 233,700 ft. Gilmer: near Glenville. Hardy: near Moorefield.

\section{GYMNOCLADUS Lam.}

G. DioicA (L.) Koch. Kentucky Coffee-tree. (G. Canadensis, Lam.).

Rich woods, infrequent. Randolph: Point Mountain, beyond Valley Bend. Webster: Buffalo Bull Mountain, along ridge. 


\section{P A P I L I N A CE AE.}

\section{BAPTISIA Vent.}

B. tinctoria (L.) R. Br. Wild Indigo. "Shoo Fly."

Sandy opens. Randolph: along Middle Fork; along Tygart's Valley River; Point and Rich Mountains. Webster: beyond Addison. Nicholas : between long and Collett's Glades. Gilmer: near. Glenville (Mapel). Kanawha: near Coalburg (James). Fayette: near Nuttallburg (Nuttall). Upshur: near Buckhannon (Pollock). Preston: near Terra Alta.

B. villosa (Walt.) Nutt.

Rocky woods. Mercer: near Ingleside.

B. australis (L.) R. Br. Blue False Indigo.

River shores. Ohio: along the Ohio near Wheeling (Mertz \& Guttenberg). Along New River. Fayette: near Nuttallburg, common (Nuttall). Summers: near Hinton, abundant. Mercer: Beaver Springs.

\section{LUPINUS L.}

L. PERENNIS L. Lupine.

Sandy soils. Monongalia : near the mouth of Cheat River.

\section{ULEX Linn.}

U. Europaeus L. Gorse.

Largely escaped and fully naturalized on a cleared hillside, with no evidence of a previous habitation. Randolph: near Pickens, where it is called "Thistle" (H. H. Smith, 2022).

\section{CYTISUS Linn.}

C. scoparius (L.) Link. Broom.

An extensive naturalization of this species occurs with the Ulex near Pickens, Randolph County, where it is used for house brooms and called, locally, "Ginster" (H. H. Smith, I928).

\section{MEDICAGO L.}

M. sativa L. Lucerne.

Dry places. Monongalia: in cinders of railroad near Morgantown, where it has persisted for several years.

M. Lupulina L. Black Medic.

Dry places. Marion: near Catawba (Walker). Monon. galia: near Uffington. 


\section{MELILOTUS Juss.}

M. Officinalis (L.) Lam. Yellow Melilot.

Ohio: near Wheeling (Mertz \& Guttenberg).

M. Alba Desv. White Melilot. Sweet Clover. Bokhara Clover.

Roadsides and ditches. Jackson: near Sandyville. Wood: near Parkersburg. Monongalia: near Morgantown. Berkeley: near Martinsburg. Jefferson: near Summit Point, and Shenandoah Junction Mason: near Pt. Pleasant. Mineral: near Keyser. Hardy: near Moorefield.

\section{TRIFOLIUM L.}

T. ARIEnse L. Rabbit-foot Clover.

Established in many places along roadsides and in old fields. Kanawha : near Pocotaligo. Jackson: along C. \& P. Pike. Mineral: near Keyser (W'orkman). Cabell: near Barboursville (James). Jefferson: near Charlestown. Hampshire : near Romney.

T. pratense L. Red Clover.

A common escape to fields; roadsides, and open woods; even in the higher Alleghenies. With pure white flowers. Randolph: near Pickens (H. H. Smith, I367).

T. Virginicum Small, Mem. Torr. Club, 4:I I2 (i894) Tab. 75.

Perennial from a large and long root, diffusely branched from the summit of the root; branches 2 to $4 \mathrm{~cm}$. long, strictly prostrate, pubescent; leaves 3 -foliate, petiole 4 to $8 \mathrm{~cm}$. long; leaflets linear, linear-lanceolate or oblanceolate, $I$ to 4 $\mathrm{cm}$. long, acute or cuspidate, serrate-dentate, glabrous above, more or less silky. beneath, conspicuously veined; sepals ovate, conspicuous; inflorescence in terminal, globose heads, about $2.5 \mathrm{~cm}$. in diameter; flowers whitish, more or less crowded on slender pedicels, .2 to $.4 \mathrm{~cm}$., standard emarginatemucronate, striate; calyx clothed with long silky hairs, the teeth subulate, nearly half the length of the corolla. Pods and seeds not seen.

Growing on the rocky slopes of Kate's Mountain, Greenbrier County, in company with Clematis ovata (Small; Mackenzie; Heller, 843).

This species is most closely related to $T$. stoloniferum by its flower, but in all other respects it differs from that and all the other eastern American species.

T. REPENS L. White Clover.

Fields, open woods, and waste places; common throughout the State. 
T. HYBRIDUm L. Alsike Clover.

Becoming frequent in fields and meadows. Monongalia: on the University Campus.

T. STOLONIFERUM Muhl.

In dry woodlands. Monongalia: near Marilla (Sheldon, I640).

T. AUREUM Poll.

(T. agrarium Linn.).

Sandy hills and roadsides. Upshur: near Buckhannon: summit on Staunton Pike. Randolph: Cheat Mountain Battlefield. Cabell : near Huntington (James). Hampshire : near Romney. Preston: near Aurora (Mr. \& Mrs. Stecle). T. PROCUMBENS L.. Low Yellow Clover.

Sandy fields, and roadsides. Kanawha: near Charleston (Barnes). Jackson: plentiful in fields and along roads. Fayette: near Nuttallburg (Nuttall).

\section{CRACCA L. (I753).}

(Tephrosia Pers. I807).

C. Virginiana L. Goats Rue.

(T. Virginiana Pers.).

Dry sandy soils. Monongalia: near Morgantown (Millspaugh). Gilmer: near Glenville (Mapel). Webster Co. on an "island" in Upper Glade. Kanawha: near the Salinas (Holton).

\section{ROBINIA L.}

R. Pseud-Acacia L. Yellow Locust.

Common throughout the State, even in the higher mountains.

Dr. Asa Gray, in his account of a "Botanical Excursion to the mountains of North Carolina," says: "On the rocky banks of the Potomac below Harper's Ferry, we saw for the first time the common Locust tree (Robinia Pseudacacia) decidedly indigenous. It probably extends to the southern confines of Pennsylvania; and from this point south, it is everywhere abundant, but we did not meet with it east of the Blue Ridge." The Blue Ridge forms our eastern boundary line between Jefferson county and the State of Virginia. Our State is therefore the eastern extension of this species, though it extends farther north into Pennsylvania.

R. HISPIDA L. Bristly or Rose Acacia.

Rich soils. Monongalia: near Morgantown; near Cheat River. Preston: in Laurel Hills. Summers: near Hinton R. viscosA Vent.

Highlands. Ohio: near Wheeling (Mertz, 576). 


\section{ASTRAGALUS L.}

A. Carolinianus L.

(A. Canadensis L.).

River banks. Monongalia: near Camp Eden. Preston: along Cheat River. Webster: Long Glade. Fayette: near Hawk's Nest (James); near Nuttallburg (Nuttall). Summers: near Hinton.

Specimens found by Mr. Nuttall in his locality resemble so completely Linnaeus' description of A. Carolinianuswhich, however, is not sufficiently different from his A. Canadensis published later, to consider these as two species-the former must, therefore, take precedence and stand for the species.

A. Distortus T. \& G.

Since the publication of the Preliminary Catalogue, Dr. Gamble has succeeded in securing fruits of this species, reported generically only in that work. This is the farthest eastern extension of the species, which ranges, according to the manual, from Illinois to Iowa, Missouri, Kansas and Texas.

Hardy: near Moorefield (Hopkins; Gamble)

STYLOSANTHES Swartz.

S. biflora (L.), B. S. P. Pencil Flower. (S. elatior, Sw.).

Dry, open woods. Wirt: near Burning Springs. Upshur: summit on Staunton Pike. Summers: near Hinton. Fayette: rocky banks of $\mathrm{Ne}_{w}$ River near Nuttallburg, (Nuttall). Monongalia: near Camp Eden.

S. RIPARIA Kearney. (S. procumbens, Siv.). Shores of New River. Summers: near Hinton.

First report of this species north of Tennessee.

MEIBOMIA Heist.

(Desmodium Desv.)

M. NUdiflora (L.). Kuntze. Tick Tree-foil.

Rich woods, common. Wood: near Leachtown. Randolph : on Point Mountain. Webster: Buffalo Bull Mountain. Gilmer: near Glenville (Brown). Fayette: near Nuttallburg (Nuttall). Summers: near Hinton. Preston: near Aurora Mr. \& Mrs. Steele).

M. PAUCiflora (Nutt.) Kuntze.

In woods along Laurel Branch east of Oceana, Wyoming county, alt. 2,000 feet, August 22, I900 (Morris, I29I).

M. GRANDIFLORA (Walt.). Kuntze. D. acumintum, D. C.

Rich woods. Monongalia: Marion: Preston: Wetzel: Mineral: Jefferson: Berkeley and Calhoun counties. Fayette: 
near Kanawha Falls (James); near Nuttallburg (Nuttall). Summers: near Hinton. Kanawha: near Charleston; and frequent throughout the State. Randolph: near Pickens ( $H$. H. Smith, I549).

M. michauxII Vail. "Hive Vine." (D. rotundifolium, DC.).

Dry, rocky woods. Monongalia: near Morgantown. Lewis: along Learling Creek. Upshur: near Lawrence. Fayette: near Nuttallburg, alt. I,500 ft. (Nuttall).

M. ochroleuca (M. A. Curtiss) Kuntze. ville.

Mineral: along Knobby Mountains. Jefferson: near Mill-

M. Canescens (L.) Kuntze.

Open woods and clearings. Wood: near Lockhart's Run. Monongalia : campus, Morgantown. Summers: near Riffe and Wolf Creek. An abundant weed. Fayette: near Nut. tallburg (Nuttall). Monroe: near Alderson; the worst weed in some fields. Mason: near Point Pleasant.

M. BRACTEOSA (Michx.) Kuntze. (D. cuspidatum Hook).

Thickets. Monongalia: along Decker's Creek; near the mouth of Cheat River. Marion: near Little Falls. Wood: near Kanawha Station. Fayette: near Nuttallburg (Nuttall). Summers : near Hinton.

M. Dillenir (Darl.) Kuntze.

Open woodlands. Monongalia, Wood, Marion, 'Wirt, Upshur, Lewis, Jefferson, Berkeley, Grant and Preston counties. Monroe: near Alderson. Wyoming: along the Guyandot River near Baileysville, a very troublesome weed in fields (Morris, I254).

M. paniculata (L.) Kuntze.

Copses. Wood, Wirt, Calhoun and Gilmer, along the Little Kanawha River. Monongalia and Marion: along the Monongalia River. Fayette: near Nuttallburg (Nuttall). Summers : near Hinton.

M. Canadensis (L.) Kuntze.

Dry, but rich woodlands. Monongalia: at The Flats. Marion: near Opekiska. Wood: near Kanawha Station. Mason: at Point Pleasant.

M. Laevigata (Nutt.) Kuntze.

In dry woods. Preston: near Aurora (Mr. \& Mrs. Steele). M. RIGIDA (Ell.) Kuntze.

Dry hillsides. Monongalia: near Morgantown: on Dorsey's Knob. Mineral : along Knobby Mts. Marion: opposite Montana. Ohio: near Wheeling (Mertz, 628).

M. oвtusa (Muhl.) Vail. (D. ciliare DC.).

Dry hillsides. Monongalia: Cheat View, Little Falls, Beechwoods. Marion: near Houghtown. Lewis: near Weston. Upshur: near Buckhannon. 
M. Marilandica (L.) Kuntze.

Copses. Grant: near Bayard. Mineral: near Keyser. Berkeley: North Mountain. Morgan: near Hancock. Jefferson: Shenandoah Jc. Summers: near Hinton.

M. ARENICOLA Vail.

(D. lineatum DC.).

Dry soils. Jefferson: near Shenandale Springs. Gilmer: near Glenville ( $\mathrm{Mapel}$ ).

\section{LESPEDEZA Michx.}

L. Repens (L.) Bart. Bush Clover.

Dry, sandy soils. Monongalia: hills near The Flats; banks of the Monongalia below Dille's. Wood: near Kanawha Station. Kanawha: Charleston (James). Summers: near Hinton. Ohio: near Wheeling (Mertz 635). Jefferson: near Harper's Ferry (Mertz).

L. violacea (L.) Pers.

Dry copses. Monongalia: near Morgantown. Wood: near Parkersburg. Wirt: near Burning Springs. Fayette: near Nuttallburg (Nuttall). Summers: near Hinton.

L. Virginica (L.) Britt.

River shores. Summers: near Hinton, along New River.

L. Stuvei Nutt.

Mountain sides. Fayette: near Nuttallburg (Nuttall).

L. FRUTESCENs (L.) Britton. (L. Stuvei intermedia et reticulata Wats.).

River banks. Fayette: near Nuttallburg, along New River, plentiful ( Nuttall).

L. HiRTA (L.) Ell. (L. polystachya Michx.).

Dry opens. Fayette: near Nuttallburg, alt. 2,000 ft. (Nuttall). Preston: near Aurora $(M r . \mathcal{E} M r s$. Steele $)$.

L. Capitata Michx.

Dry sandy soil. Ohio: River banks, frequent. Menongalia: near Morgantown. Marion: near Catawba, and Houghtown.

L. StRiATA ('Thunb). Hook \& Arn. Japanese Clover.

Dry, red soils. Spreading profusely along the C. \& O. R. R. in Kanawha, Putnam and Cabell counties.

\section{VICIA L.}

V. Carolinana Walt. Carolina Vetch or Tare.

River banks and edges of glades. Webster: Welsh Glade; island in Long Glade. Wood: shores of Little Kanawha River. Gilmer: near Glenville (Mapel). Fayette: near Nuttallburg (Nuttall). Monongalia: near Cassville. Greenbrier Co., Kate's Mountain, White Sulphur Springs, alt. 3,000 ft. (Small; Eggleston 4349). 
L. venosus Muhl.

\section{LATHYRUS L.}

Shady banks. Mineral: banks of the Potomac near Keyser. Fayette: near Nuttallburg, a form IO-I 5 flowered, with winged stem and stipules 3-I 5 lines long (Nuttall) Greenbrier Co., Kate's Mountain, alt. 3,300 ft. (Small; Eggleston 4357; Heller 828).

L. LATIFOLIUS Linn.

Established on the University campus at Morgantown, Monongalia Co. (Sheldon 3597, July I909.)

\section{FALCATA Gmel.}

F. comosa (L.) Kuntze. (Amphicarpa monoica El1.). Rich, damp thickets. Monongalia and Marion: along the Monongahela River. Fayette: near Nuttallburg (Nuttall). Preston: near Aurora (Mr. \& Mrs. Steele).

\section{APIOS Moench.}

A. Apios (L.) MacM. Ground Nut. (A. tuberosa Moen.). Low grounds along streams. Frequent throughout the State. Fayette: near Nuttallburg, not common (Nuttall).

$$
\text { GALACTIA P. Br. }
$$

G. Regularis (L.) B. S. P.

Fayette: near Quinnimont on bottom lands of New River (Pollard \& Maxon 29).

\section{PHASEOLUS L.}

P. polystachyus (L.) B. S. P. Wild Kidney Bean. (P. perennis Walt.).

Copses. Wood: near Kanawha Station. Monongalia: near Morgantown. Mason: near Point Pleasant.

\section{STROPHOSTYLES E11.}

S. Helvola (L.) Britton.

(Phaseolus helvolus L.).

Sandy river banks. Mason : banks of the Ohio near Point Pleasant. Fayette: near Nuttallburg, with the inner surface of the petals pink (Nuttall); Quinnimont (Pollard \& Maxon 32 ).

$$
\begin{gathered}
\text { G E R I A N I A C AE. } \\
\text { GERANIUM L. }
\end{gathered}
$$

G. maculatum L. Wild Geranium.

Open woods and clearings, frequent throughout the State. A small form with leaves round in outline and from I-2 ir. in diameter at Bluefield, Mercer county. 
G. Robertianum L. Herb Robert.

Rocks of cool, shaded ravines, rare. Marion: near Fairmont. Gilmer : near Glenville ( $\mathrm{Mapel}$ ).

G. Carolinianum L. Cranesbill.

Fields, meadows and waste places. Mercer: near Ingleside. Fayette: near Nuttallburg (Nuttall). Kanawha: near Charleston (Barnes). Monogalia: on the University Campus; and frequent throughout the State.

\section{OX A L I D A CE AE. \\ OXALIS L.}

O. Acetosella L. Wood Sorrel.

Deep, rich, mountain woods. Randolph: on Point Mountain; Cheat Mountain near Cheat Bridge, where this species grows in such profusion as to actually carpet the Spruce forests. Grant: near Bayard. Tucker: near Davis; and Land of Canaan. Gilmer: near Glenville (Mapel). Fayette : near Nuttallburg (Nuttall).

O. VIOLACEA L.

Rich, cool woods. Randolph: on Point Mountain. Monongalia: up Falling Run; at Uffington and Little Falls. Marion: near Beechwoods; Opekiska and Catawba. Gilmer: near Glenville ( $\mathrm{Mapel}$ ). Fayette: near Nuttallburg, rare (Nuttall). Lewis: near Lorentz (Pollock). Hardy: near Moorefield (Gamble). Mercer: near Beaver Spring.

O. stricta L. Sheep Sorrel.

Fields, cultivated grounds and roadsides. Common throughout the State.

O. RECURVA Ell. all).

Open places. Fayette: near Nuttallburg, common (Nutt-

O. crimosa Small.

In woods and opens. Preston: near Aurora (Mr. \& Mrs. Steele).

O. Filipes Small.

Monongalia: common about Morgantown (Sheldon).

O. GRANDIS Small.

Monongalia: along the railroad south of Morgantown (Sheldon I392). 
L I N A CE AE.

\section{LINUM L.}

L. Virginianum L. Wild Flax.

Open woods, borders and roadsides. Wood, Wirt, Calhoun, Gilmer, Lewis and Upshur. Randolph: along Tygart's Valley River. Webster and Nicholas; near Richwood (H. H. Smith I740). Kanawha: near Peabody; near Coalburgh (James). Jackson, Monongalia, and Preston. Fayette: near Gauley Bridge; near Kanawha Falls (James); near Nuttallburg (Nuttall). Kanawha: near Charleston (James).

L. STRIATUM Walt.

Damp places. Webster: in Upper and Long Glades Preston: in glades near Terra Alta and Reedsville (Millspaugh); near Aurora (Mr. \& Mrs. Steele). Fayette: near Nuttallburg, in sphagnous bog (Nuttall). Monongalia: at Camp Eden.

L. usitatissimum L. Flax.

An uncommon adventive. Fayette: along the C. \& O. R. R., near Nuttallburg (Nuttall).

\section{R U T A C E AE.}

\section{XANTHOXYLUM L.}

X. Americanum Mill. Prickly Ash. Toothache-tree.

Rocky woods, becoming rare. Jefferson: near Flowing Spring. Monongalia : Decker's Creek. Taylor: along Cheat River.

R. GRAVEOLEns L. Rue.

$$
\text { RUTA L. }
$$

Escaped from gardens. Randolph: on Point Mountain along the road about half way to the summit.

\section{PTELEA L.}

P. trifoliata L. Wafer Ash. Hop-tree.

River banks. Jefferson: near Harper's Ferry (Mertz \& Guttenberg). Hancock: along Oak Run. Brooke: on Short Creek. Summers: on banks of New River, near Hamilton, common.. 


\section{AILANTHUS Desf.}

A. glandulosa Desf. Tree of Heaven.

Naturalized from China. The seeding-in of this cultivated species is so profuse in the following localities as to render it a great nuisance. Monongalia: near Morgantown. Gilmer: De Kalb P. O. Lewis: near Weston. Kanawha: at Pocotaligo. Jackson : near Sandyville. Marion: Fairmont. Gilmer: near Glenville (Mapel). Jefferson: near Harper's Ferry, and Charlestown.

\section{POL Y G A L A C E AE.}

POLYGALA L.

P. VIRIDESCENS L. Red Milkwort.

(P. sanguinea L.).

Sandy fields. Wood: near Kanawha Station. Webster: Upper Glade. Preston: near Terra Alta. Fayette: near Nuttallburg, alt. 2,400 ft. (Nuttall). Preston: near Aurora (Mr. \& Mrs. Steele).

Forma. ALBIFLORA Wheelock.

In the glades of Webster and Preston counties.

P. Mariana Mill.

Damp places. Preston: near Terra Alta.

P. fastigiata Nutt.

P. Curtissii Gray.

Glady spots. Fayette: near Nuttallburg (Nuttall).

P. CRuciata L. Cross Milkwort.

Margins of Glades. Preston: near Reedsville. Webster: Upper Glade.

P. verticillata L. Whorled Milkwort.

Dry places. Wirt: near Burning Springs. Lewis: near Leading Creek. Upshur: near Buckhannon. Summers: near Hinton.

P. Ambigua Nutt.

Dry soils. Wood : near Lockhart's Run. Wirt : near Elizabeth. Calhoun : on Nigh-Cut Hill. Monongalia: hills below Morgantown. Fayette: near Nuttallburg (Nuttall). Preston: Aurora ( $M r$. \& Mrs. Steele).

P. Nuttallin T. \& G.

Mountain woods. Fayette: near Nuttallburg, alt. 2,000 ft. (Nuttall).

P. Senega L. Seneca Snake-root.

Rocky soils and rich bottoms. Mineral: near Keyser 
(Workman). Webster: in Welsh, Long and Collett's glades. Preston: Reedsville glade. (Long glade is said to be white with this species when it is in bloom.)

P. PAUCifolia Willd. Fringed Polygala.

Rocky woods. Mineral: near Keyser along the Knobby Mountains (Workman). Hardy: near Moorefieid (Gamble.) Greenbrier Co., Kate's Mountain, alt. 3,300 ft. (Small).

CHAMAESYCE S. F. Gray.

C. glystoperma pubescens (Engelm.). (Euphorbia glyptosperma pubescens Englm.).

Sandy soil. Mason: banks of the Ohio river, near Point Pleasant. The only station so far known in the State.

C. maculata (L.) Small. Spotted Spurge. (Euphorbia maculata L.).

Arid soils. Common even in the higher Alleghanies. Mineral Co., opposite Cumberland, Md. (Small). Tucker: near Parsons (Greenman).

C. Preslir (Guss.) Arthur.

(Euphorbia Preslii Guss.).

Dry soils and pastures. Common throughout the State, even in the higher Alleghanies. Tucker: near Parsons and Hendricks (Greenman).

\section{DICHROPHYLLUM. K1. \& Garcke.}

D. marginatum (Pursh.) Kl. \& Garcke. (Euphorbia marginata Pursh.).

An escape from cultivation. Taylor: permanently established near Mannington ( Mapel). Monongalia: the Flats near Morgantown.

\section{TITHYMALOPSIS K1. \& Garcke.}

T. CORollata (L.) Small. Flowering Spurge. (Euphorbia corollata L.).

Dry soils. Wirt: near Elizabeth. Lewis: along Leading Creek. Upshur: near Laurentz. Randolph: on Lone Sugar Knob, alt. 2,80o ft. Webster: Long Glade. Nicholas: Mumble-the-peg Creek. Kanawha: along 8-Mile Creek. near Pocataligo. Jackson: Fisher's Point. Wood: on Limestone Ridge. Gilmer: near Glenville (Mapel). Fayette: near Nuttallburg (Nuttall). Grant: near Bayard. Monongalia : plentiful along Cheat River, near Camp Eden. Greenbrier: near White Sulphur Springs. Monroe: near Alderson. 
Summers : near Hinton. Mason: near Point Pleasant. Barbour: near Tygart Junction; and Tucker near Parsons (Grecnman).

\section{POINSETTIA R. Grah.}

P. Dentata (Michx.). Small.

(Euphorbia dentata Michx.). Rich soil. Ohio: near Wheeling.

\section{TITHYMALUS Adans.}

T. Darlingtonir(Gray) Small. (Euphorbia Darlingtonii A.Gray). Damp woods. Pocahontas: along the mountains (Hopkins). Randolph: Blister Swamp near Gandy Creek (Greenman).

T. obtusatus (Pursh.) Small. (Euphorbia obtusata Pursh.). Rich soil. Ohio: near Wheeling (Mertz \& Guttenberg).

T. Cyparissias (L.) Hill. "Grave-yard-weed." (Euphorbia Cyparissias L.):

A frequent escape from cemeteries. Monongalia: near Morgantown. Cabell: near Huntington.

T. сомmutatus (Englm.). K1. \& Garcke. (Euphorbia commutata Englm.).

Woodlands. Jefferson: near Harper's Ferry (Mertz \& Guttenberg); near Shepherdstown. Mineral: near Keyser (Workman) along Knobby Mountains. Sunimers: near Hinton, abundant. Hardy: near Moorefield (Gamble). Hampshire : near Romney.

T. Lathyrus (L.) Hill. "Mole-weed." (Euphorbia Lathyrus L.).

Escaped from gardens, where it is cultivated with the idea of keeping out moles. Randolph: roadside up Point Mountain.

\section{ACALYPHA L.}

A. VirginicA L.

Fields and waste places. Common throughout the State. forma intermedia Millsp. Prel. Cat. Fl. W. Va., 436.

A form apparently uniting A. Virginica, L. with A. Caroliniana, E11. especially in the matter of leaves and bracts, is found near Nuttallburg (Nuttall), and Hawk's Nest; as well as along New River opposite Hinton.

\section{PHYLLANTHUS Linn.}

P. Carolinensis Walt.

Dry situations. Mason: near Point Pleasant (Sheldon I940). 
CALLITRICHE Linn.

C. heterophylla Pursh.

Fayette: near Nuttallburg ( Nuttall).

\section{EMPETRACEAE.}

[Corema Conradi, Torr., mentioned in Botanical Gazette, Vol. 2, P. I36, as occurring near Hawk's Nest, Fayette Co., is proven by Prof. James to be another plant, it should therefore not yet be credited to this State.]

\section{B U X A C E AE.}

\section{PACHYSANDRA Michx.}

P. PROCumbens Michx.

Credited to West Virginia in Britton \& Brown, Illustrated Flora, vol. 2, p. 384. I have not seen the specimen authenticating this reference.

\section{I M N A T H A C E AE.}

\section{FLOERKEA Willd.}

F. PROSERPINACOIDES Willd. False Mermaid.

Wet places. Ohio: near Wheeling (Mertz \& Guttenberg), Preston: glades near Terra Alta. Lewis: near Lorentz (Pollock).

\section{A N A C A R D I A C EAE.}

\section{RHUS L.}

R. Hirta (L.) Sudw. Staghorn Sumach. (R. typhina L.).

Dry hillsides. Gilmer: DeKalb Postoffice; near Glenville ( $\mathrm{Mapel}$ ). Monongalia : near Stewartstown. Summers: near Hinton. Fayette: near Nuttallburg (Nuttall). Randolph: near Pickens ( $H$. H. Smith I376). Preston: near Aurora (Mr. \& Mrs. Steele).

During the season of 1890 to I89r hundreds of plants of this species were noted in Monongalia county to have the inflorescence reverted to leaves, making a strikingly beauti- 
ful proliferation when young. This effect, according to Dr. Britton, formed the type of the Linnæan species, Datisca hirta (L. Sp. P1. I037) collected by Kalm near Philadelphia, $\mathrm{Pa}$.

R. glabra L. Smooth Sumach.

Frequent or very common in all parts of the State, in rocky or barren soils. Randolph: Point Mountain, alt. 2,200 ft.; Cheat Mountains, ait. 2,700-3,600 ft. Cabell : near Barboursville (James). Summers: near Hinton. Fayette: near Nuttallburg, not common (Nuttall). Hardy: near Moorefield (Gamble).

R. Copallina L. Dwarf Sumach.

Dry fields and rocky places. Wood: near Lockhart's Run, plentiful. Webster: Buffalo Bull Mountains, alt. 2,575 ft. Randolph: Cheat Mountains, alt. 3,200 ft.; near Hodam ( $H$. H. Smith I527). Preston: near Aurora (Mr. \& Mrs. Steele). Monongalia: near Ice's Ferry. Fayette: near Nuttallburg, alt. 2,000 ft., common (Nuttall); and frequent throughout the State. Mercer: Beaver Springs and Ingleside.

R. vernix L. Poison Sumach. Poison Elder. ( $R$. venenata DC.)

Swampy places. Randolph: Stalnaker Run. Preston: near Terra Alta, infrequent.

R. Radicans L. Poison Vine. Poison Ivy. Including (R. toxicodendron L.).

Thickets and low grounds, very common throughout the State. Monongalia: abundant everywhere in the neighborhood of streams. Webster: Buffalo Bull Mountains, alt. 2,I00 ft. (Millspaugh); Hacker Valley (H. H. Smith I532). Kanawha: Pocotaligo. Gilmer: near Glenville (Mapel). Fayette: near Nuttallburg (Nuttall). Summers: near Hinton. Mason: near Point Pleasant. Mercer: Princeton, near Ingleside and Wills..

R. aromatica Ait. Fragrant Sumach. (R. canadensis Marsh.).

Dry or stony soils. Brooke: roadside between Wellsburg and Bethany College (Mertz \& Guttenberg). Hardy: near Moorefield (Gamble).

\section{COTINUS Adans.}

C. COTINoIdes (Nutt.) Britton. (Rhus cotinoides Nutt.). Established near Morgantown, Monongalia Co. (Sheldon.) 


\section{A Q U I F O L I C E AE.}

\section{ILEX L.}

I. opACA Ait. American Holly.

Moist woodlands. Marion: near Nuzums. Randolph: near Rich Mountains and Laurel Hills. Kanawha: near Charleston (Barnes). Fayette: near Hawk's Nest, large trees 8-I2 inches in diameter (Porter; Holton) ; Nuttallburg, in moist woods common (Nuttall). McDowell: near Elkhorn. Upshur: near Newlin (H. H. Smith I769); near Buckhannon (Pollock).

I. MONTICOLA Gray.

Damp woods. Randolph: near summit of Point Mountain; Rich Mountain, near Lone Sugar Knob; Cheat Mountain, near "The Battle Field." Kanawha: near Charleston (James). Upshur: near Newlin (H.H. Smith I762); near Buckhannon (Pollock). Preston: near Aurora (Mr. \& Mrs. Steele). Randolph: dry banks of Tygarts Valley River near Huttonsville (Greenman I37).

I. MOLLIS Gray.

Deep woods. Fayette: near Nuttallburg (Nuttall).

I. verticillata (L.) Gray. Black Alder. Winterberry.

Frequent in swampy places, throughout the central and northern counties. Fayette: near Nuttallburg (Nuttall); near Thurmond (Eggleston 5562). Preston: near Aurora (Mr. \& Mrs. Steele). Pocahontas: near Durbin (Greenman I36). Upshur: uear Buckhannon (Pollock).

\section{ILICIOIDES Dumort.}

I. mucronata (L.) Britton. (Vaccinium L., Nemopanthes fascicularis Raf.)

Pendleton: summit of Spruce Knob; Preston: at Cranesville; Pocahontas : head of Greenbrier River (Brooks). Randolph: in Blister Swamp near Gandy Creek (Greenman I54).

\section{CELA S T R A CE AE.}

\section{EUONYMUS L.}

E. atropurpureus Jacq. Burning Bush.

Margins of woods and thickets. Jackson: near Sandyville. Gilmer: near Glenville (Mapel). Randolph: banks of Tygarts River; near Huttonsville (Greenman I74). Fayette: near Nuttallburg, rare (Nuttall). 
E. Americanus L. Strawberry Bush.

Rocky, wooded river banks. Fayette: along the Great Kanawha River, below Gauley Bridge ; near Nutallburg, common (Nuttall). Kanawha: near the Salinas (Holton).

E. obovatus Nutt. (E. Americanus var. obovatus T. \& Gray).

Of this species Rev. A. Boutlou says: "In Marshall Co., at Board Tree, Cameron, Belton, etc., this is the most common form; while the trailing rooting form I have met almost everywhere I have been in the State.'

\section{PACHYSTIMA Raf.}

P. Canbyi A. Gray.

Credited to West Virginia in Britton \& Brown, Illustrated Flora, vol. 2, p. 395. I have not seen the specimen authenticating this reference.

\section{CALASTRUS L.}

C. scandens L. Climbing Bitter-sweet. Wax-work.

Thickets, fence rows and along streams, frequent. Wood: near Limestone Ridge. Monongalia and Marion : along the Monongahela River. Fayette: near Nuttallburg (Nuttall).

\section{S T A P H Y LE A C E AE.}

\section{STAPHYLEA L.}

S. TRIFOLIA L. Bladder-nut.

Rocky woods, thickets and opens. Wirt: near Elizabeth Monongalia: near Morgantown and Stumptown. Gilmer: near Glenville. Fayette: near Nuttallburg (Nuttall). Upshur: near Buckhannon (Pollock). Jefferson: at Harpers Ferry (Greene).

\section{A C E R A C E AE.}

\section{ACER L.}

A. Pennsylvanicum L. Striped Maple.

Rich, cool woods. Randolph: on Point Mountain; Staunton Pike on Cheat Mountain. Webster: on Buffalo Bull Mountain. Grant: near Bayard. Tucker: on Blackwater Fork of Cheat. Fayette: near Nuttallburg (Nuttall) and elsewhere in the mountains. McDowell: near Elkhorn. Preston: near Aurora (Mr. \& Mrs. Steele).

Although in most situations in the State this species is a 
mere shrub, yet near Welsh in Grant Co. it assumes the proportions of a beautiful tree 20 to $40 \mathrm{ft}$. high with a strict columnar trunk 5 to 8 inches in diameter (Millspangh). Greenbrier Co., on Kate's Mountain, near White Sulphur Springs, alt. 3,300 ft. (Small; Heller 827).

A. SPICATUM Lam. Mountain Maple.

Same localities as previous species, except Fayette, but more plentiful where found. Greenbrier: near White Sulphur Springs. McDowell: near Elkhorn. Preston: near Aurora (Mr. \& Mrs. Steele). Pocahontas: (Pollock).

A. Saccharum Marsh. Sugar Maple. (A. saccharinum, Wang., not L.).

Plentiful throughout the State, especially, however, in the central counties. Randolph: Summit Point Mountain, alt. 3,700 ft. Webster: Buffalo Bull Range, alt. 27-3,600 ft. In Randolph Co., near Pickens, the sugar maple grows to a diameter of 2 to $4 \mathrm{ft}$., and a height of 50 to $70 \mathrm{ft}$. (Millspaugh).

A. NIGRUM Michx. Black Sugar Maple.

With the preceding, and almost as plentiful.

A. saccharinum L. White or Silver Maple. (A. dasycarpum, Ehrh.).

Banks of Little Kanawha: Gauley River: Great Kanawha, and Ohio. Fayette: near Nuttallburg (Nuttall); and elsewhere frequent. Large trees 2 to $4 \mathrm{ft}$. in diameter in Randolph Co., near Pickens. At one station in Webster Co., on Buffalo Bull Mountain, nearly every tree of this species is of the Bird's-eye type (Millspaugh).

A. Rubrum L. Red or Swamp Maple.

Common throughout the State, where it grows upon the hills and in the mountains, as well as in low places. Wood: near the mouth of the Little Kanawha (Michaux).

A. Negundo L. Box Elder, Ash-leaved Maple.

Common near rivers throughout the northern and middle counties. Lewis: along Leading Creek (Millspaugh, Pollock). Gilmer: near Glenville ( $\mathrm{Mapel}$ ). Summers: near Hinton. Berkeley: near Martinsburg. Hardy: near Moorefield (Gamble). Fayette: near Nuttallburg (Nuttall). Wood: near the mouth of the Little Kanawha (Michaux)

$$
\text { S A P I N D A C E ȦE, }
$$

\section{AESCULUS L.}

Ae. glabra Willd. Ohio or Fetid Buckeye.

Low lands near streams. Wirt: along Straight Creek. Gilmer: near Glenville (Brozen). Along the Ohio River, common. Monongalia : near Uffington. 
AE. OCTANDra Marsh. Sweet Buckeye.

(Ae. Alava, Ait.).

Rich mountain woods. Webster : Buffalo Bull Mountains, alt. 2, I00 ft. Gilmer: near Glenville (Mapel). Lewis : near Weston (H. H. Smith I75I). Wood: near the mouth of the Little Kanawha (Michaux). Mercer : near Ingleside (Millspaugh 1468). Summers: near Hinton. Kanawha: near Charleston and Handley. Marion: near Worthington. An immense tree of this species, measuring twenty feet in circumference at the ground, twelve feet at the height of one's shoulder, and nearly if not quite one hundred feet high, stood by the bank of Dry Fork above Peeryville, McDowell County (Morris). Randolph: along Tygarts Valley River above Huttonsville (Greemman 196).

Var. HybRIDA (DC) Sarg.

Woodlands. Fayette: near Nuttallburg (Nuttall). Ae. Pavia L.

Rich lands along streams. McDowell: along Tug Fork near Elkhorn.

\section{B A L S A M I N A C E AE.}

\section{IMPATIENS L.}

I. Aurea, Muhl. Pale Touch-me-not.

(I. pallida, Nutt.).

Rich soils near streamlets. Gilmer: near Glenville ( $M a-$ pel). Kanawha: near Charleston. Fayette: near Nuttallburg (Nuttall); and common throughout the State. Also common in the deep, primitive forests along spring runs. Randolph: on Rich and Cheat Mountains. Grant: near Bayard. Tucker: along the Blackwater Fork of Cheat (Millspaugh); vicinity of Parsons (Greenman I5I). Hardy: near Moorefield (Gamble). Preston: near Aurora ( $M r$. \& Mrs. Stecle).

I. BIFLORA Walt. Spotted Touch-me-not.

(I. fulva Nutt.)

Shady, moist places, more common than the preceding species and generally seeking lower altitudes (Millspaugh) Tucker: near Hendricks (Greenman I50).

\section{R H A M N A C E AE.}

\section{RHAMNUS L.}

R. Lanceolata Pursh.

In moist soil. Hampshire: at Little Cacapon, rare (Brooks).

R. ALNIFOLIA L'Her.

In swampy places. Head of East Fork Greenbrier River (Brooks). 
R. Caroliniana Walt.

McDowell: along Tug Fork of the Big Sandy River near Elkhorn; at Welch, along the same stream.

CEANOTHUS L.

C. Americanus L. New Jersey Tea.

Dry open woods. Upshur: summit on Staunton Pike (Millspaugh, Pollock). Jackson: Sandyville. Gilmer: Glenville (Mapel). Fayette: near Nuttallburg (Nuttall). Hady: near Moorefield.

\section{I T A C E AE,}

\section{VITIS L.}

V. Labrusca L. Northern Fox-grape.

Damp, rich thickets. Randolph: near Valley Head. Gilmer: near Glenville ( $\mathrm{Mapel}$ ). Summers: near Hinton.

An immense specimen of this grape was found in Randolph Co., near Pickens; its trunk measured 22 inches in diameter and its two main branches 8 inches each (Millspaugh).

V. AEstivalis Michx. Summer Grape.

Thickets. Wirt: along Straight Creek. Randolph: on Point Mountain. Fayette: near Nuttallburg, common (Nuttall). Summers: near Hinton.

V. CORdifolia Michx. Frost Grape.

Thickets and banks of streams. Wirt: along Straight Creek. Randolph: at Valley Head; along Point Mountain Road. Fayette: near Nuttallburg, common. (Nuttall). Summers : near Hinton. Kanawha: near Charleston.

V. vULPINA L.

Banks of streams. Randolph: near Valley Head; Point Mountain Road. Summers : near Hinton. Jefferson: Shenandoah Jc.

V. BICOLOR LeConte. Winter Grape.

Rich woods, Webster: Hacker Valley (H. H. Smith I 592 ).

V. RUPESTRIS Scheele, Sand Grape. Sugar Grape.

Rocky banks of New River in Fayette near Nutallburg, plentiful (Nuttall).

V. ROtundifolia Michx. Muscadine. Southern Fox Grape.

Rich river banks. Randolph: near Valley Head. Fayette : near Nuttallburg, rare, on mountain side climbing over trees (Nuttall). Summers : near Hinton. 
AMPELOPSIS Michx.

(Cissus Pers.).

A. CORDATA Michx.

(Vitis indivisa Willd.).

River banks. Ohio: near Wheeling (Mertz \& Guttenberg). Summers: near Hinton.

A. TRICUSPIDATA Sieb. \& Zucc.

Established at Morgantown, Monongalia County (Sheldon).

\section{PAR̄THENOCISSUS Planch.}

P. Quinguefolia (L). Planch. Virginia Creeper. American Ivy. (Ampelopsis quinquefolia Michx.).

Woods and thickets. Gilmer: near Glenville ( $\mathrm{Mapel}$ ). Fayette: near Nuttallburg (Nuttall). Webster: at Hacker Valley (H. H. Smith I 59I). Randolph: along Tygarts Valley River near Huttonsville (Greemman I63); and common throughout the State.

\section{T I L I A C E AE.}

\section{TILIA L.}

T. Americana L. Linden. Basswood.

Rich woods. Gilmer: at DeKalb P. O. Randolph: on Point Mountain. Grant: near Bayard. Monongalia: near Morgantown, Uffington and Little Falls. Mason : near Point Pleasant. McDowell : near Elkhorn. Large growths of this species found at Pickens, Randolph Co., with trunk diameters of 26 to 38 inches, and height 60 to roo feet (Mills. paugh). Fayette: near Nuttallburg (Nuttall).

T. Heterophylla Vent. White Basswood. Lin."

Deep woods. Jefferson: near Charlestown, near Flowing Spring Mill. One specimen cut at Nuttallburg, Fayette County, had a diameter of 26 inches (Millspaugh; Nuttall). Pocahontas: valley of the East Fork of Greenbrier River (Greenman I94).

T. Michauxim Nutt.

Deep woods, Randolph: near Pickens (H. H. Smith I288, I342). Upshur: near Buckhannon (Pollock).

\section{A L V A C E AE.}

\section{ALTHAEA L.}

A. Rosea Cav. Hollyhock.

Appears annually along the B. \& O. R. R. tracks in Berkeley: near North Mountain, apparently a thorough establishment. 


\section{MALVA L.}

M. Rotundifiolia L. Common Mallow.

Cultivated grounds and waysides, a common weed.

M. moschata L. Muck Mallow.

Roadsides and meadows escaped from cultivation. Lewis: along Stone Coal Creek. Upshur: near Lorentz. Monongalia: near Morgantown. Randolph: near Pickens $(H . H$. Smith I540, I54I). Kanawha: at the Salinas (Holton).

S. SPINOSA L.

\section{SIDA L.}

Waste grounds and fields. Monongalia: near Morgantown, common. Fayette: near Nuttallburg (Nuttall); near Guyandot (Holton). Jefferson: near Shepherdstown. Mason: near Point Pleasant.

S. HERMAPHRODITA (L.) Rusby

(S. Napaea Cav.).

Rocky banks along the Great Kanawha River. Kanawha : opposite Cannelton. Fayette: Quinnimont; near Nuttallburg, frequent and always with 8 carpels (Nuttall). Mason: near Point Pleasant.

\section{ABUTILON Gaertn.}

A. Abutilon (L.) Rusby. Indian Mallow. American Jute. (A. avicennae Gaertn.).

A too common weed in waste and cultivated soils. Monongalia, Wood, Wirt, Calhoun; Mason: near Point Pleasant; near Brighton. Fayette: near Nuttallburg (Nuttall). Berkeley: near Martinsburg, a very bad weed.

\section{HIBISCUS L.}

H. Moscheutos L. Swamp Rose Mallow.

Brackish, marshy places, and ditches near salt works. Mason: near Point Pleasant. Kanawha: near Charleston. Fayette: near Nuttallburg (Nuttall). The pink form, Jackson: near Sandyville. Hardy: near Moorefield.

H. Trionum L. Bladder Ketmia.

Cultivated grounds. Monongalia: a weed in the Station experimental plats.

\section{H Y P E R I C A C E AE.}

ASCYRUM L.

A. hypericoides L. St. Andrew's Cross. (A. Crux-Andreae L.). Dry sandy places. Upshur: summit of Staunton Pike. Randolph: Rich Mountains; along Tygart's Valley River. Fayette: near Gauley Bridge: near Nuttallburg (Nuttall). Cabell: near Barboursville (James). 
HYPERICUM L.

H. PROLIFICUM L.

Glade regions. Webster: Upper Glade. Preston: nea1 Reedsville. Gilmer: near Glenville $(\mathrm{Mapel})$. Fayette: near Kanawha Falls (James); near Nuttallburg (Nuttall).

H. Densiflorum Pursh. Shrubby St. John's Wort.

Glade regions and moist meadows. Wood: near Lockhart's Run. Webster: in the glades. Preston: Terra Alta, and Reedsville glades. Fayette: near Nuttallburg (Nuttall). Preston: near Aurora (Mr. \& Mrs. Steele). Pocahontas: near Durbin (Greenman 316).

H. virgatum acutifolium Coulter.

Fayette: near Hawk's Nest (Porter); near Nuttallburg (Nuttall).

H. PERforatum L. St. John's Wort. "St. John."

Fields and roadsides. Randolph: along Tygart's Valley River. Monongalia, Marion, Preston, Grant: near Bayard. Fayette: near Nuttallburg (Nuttall). Not yet very plentiful in the State.

H. maculatum Walt. Spotted St. John's Wort.

Glade regions and wet places. Wood: near Kanawha Station. Wirt: near Elizabeth. Preston: near Reedsville and Terra Alta (Millspangh); near Aurora (Mr. \& Mrs. Stecle). Webster: Upper, Long, and Welsh Glades. Fayette: near Hawk's Nest and Kanawha Falls (James); near Nuttallburg (Nuttall).

H. mutilum L.

Ditches and low grounds, common throughout the State

H. Canadense L. Canadian St. John's Wort.

Glade regions of Preston and Webster Counties (Millspaugh). Preston: near Aurora (Mr. \& Mrs. Steele). Fayette; near Nuttallburg, in spahgnum bogs (Nuttall).

H. ADPRESSUM Barton.

Moist grounds. Greenbrier: near White Sulphur Springs.

H. punctatum Lam.

Nicholas: near Richwood; and Webster: at Hacker Valley (H. H. Smith I747, I560).

H. ELLIPTICUM Hook.

In spagnum glades. Preston: near Terra Alta.

H. GYMNANTHum Engelm. \& Gray.

Low moist places. Pocahontas: valley of the East Fork of Greenbrier River (Greenman 4I8). 


\section{SAROTHRA L.}

S. Gentianordes L. Orange Grass. (Hypericum Sarothra Michx.).

Dry fields. Monongalia: near The Flats. Wood: near Kanawha Station. Fayette: near Kanawha Falls (James).

\section{TRIADENUM Raf.}

T. Virginicum (L.) Raf. (Hypericum Virginicum L.). Swampy glades. Fayette: near Nuttallburg (Nuttall).

\section{I S T A C E AE.}

\section{HELIANTHEMUM Pers.}

H. majus (L.) B. S. P. Frost-weed.

Dry soils. Preston: near Terra Alta.

\section{LECHEA L.}

L. MINOR L. Pin-weed.

Dry places. Summers : near Hinton. Fayette; near Nuttallburg, alt. I,600 ft. (Nuttall).

L. LegGetTiI Britt. \& Hollick.

L. minor Lam.

Dry sandy places. Fayette: near Nuttallburg, plentiful at an alt. of 2,000 ft. (Nuttall).

\section{I O L C E AE.}

\section{VIOLA L.}

V. PEDAta L. Bird's-foot Violet.

Sandy soils. Monongalia: at The Flats. Mineral: along the Potomac near Keyser (Workman). Randolph: on Poini Mountain. Greenbrier Co., on Kate's Mountain, alt. 3,300 ft., near White Sulphur Springs (Small; Eggleston 4350, 4354; Heller 807). Mercer Co., near Bluefield. MacDowell Co., at Fletcher's (Millspaugh).

Var. BICOLOR Pursh. Pansy Violet.

Hardy: near Moorefield (Gamble). Hampshire: near Romney (Hopkins). Greenbrier Co., on Kate's Mountain, alt. 3,300 ft. (Small).

V. PALMATA L.

Near runs in moist ground. Monongalia : near The Flats, Morgantown. Gilmer: near Glenville (Mapel). Fayette: near Nuttallburg (Nuttall). Ohio: near Wheeling (Richardson).

V. cucullata Ait. Common Blue Violet.

Low grounds; common throughout the State. Upshur: near Buckhannon (Pollock). 
V. AFFinis LeConte.

About a spring near Kegley, Mercer County, alt. 2,090 ft., July 21, 1900 (Morris 1046).

V. Papilionacea Pursh.

On Great Bend, Tunnel Mountain, Summers County, alt. I,700 ft., July I4, I900 (Morris 1023); along Horsepe^1 Creek, McDowell County, alt. I,900 ft., July 30, 1900 (Mor. ris 1104$)$.

V. SagitTata Ait. Arrow Leaved Violet.

Dry or moist, sandy places. Monongalia: near Morgantown, Uffington and Little Falls. Preston: near Cold Spring. Gilmer: near Glenville ( $\mathrm{Mapel}$ ). Fayette: near Nuttallburg (Nuttal).

V. BLANDA Willd. Sweet White Violet.

Damp woods. Monongalia: near Morganton. Gilmer: near Glenville (Mapel). Upshur: (Pollock). Fayette: near Nuttallburg (Nuttall); and frequent throughout the State. McDowell: near Elkhorn.

V. ALSOPHILA Greene.

( $V$. amoeria LeConte).

McDowell: along Horsepen Creek, alt. I,850 ft. (Morris I IOI, I IOO).

V. PRIMULAEFOLIA L.

Damp soils. Fayette: near Nuttallburg (Nuttall). McDowell : near Elkhorn.

V. SORORIA Willd.

In woodlands. Monongalia : near Morgantown (Sheldon).

V. HIRSUTULA Brainerd. ( $V$. villosa Auth.).

Dry situations. Monongąlia: near Dellslow; and Fayettc: near Ronceverte (Sheldon 207, InO5).

V. Fimbriatula J. E. Smith.

Sandy soil near Bretz and other locations in Preston County; also in Monongalia County (Sheldon 4i Io).

V. Lanceolata L. Lance-leaved Violet.

Boggy places. Monongalia: up Falling Run, above Morgantown, the only station so far known to me.

V. ROTUNDIFolia Michx. Round-leaved Violet.

Cold woods. Randolph: Rich Mountains, alt. 2, I Io ft. Gilmer: near Glenville ( $\mathrm{Mapel}$ ). Fayette: near Nuttallburg (Nuttall). Mercer: near Ingleside and Wills.

V. PUbescens Ait. Yellow Violet.

Rich woods. Mineral: neat Keyser (Workman). Randolph: Rich Mountains, alt. 2,I25 ft. Grant: near Bayard. Tucker: along Blackwater Fork of Cheat. Gilmer: near Glenville (Mapel). Fayette: near Nuttallburg (Nutall). Monongalia: near Cassville. Upshur: near Buckhannon (Pollock). 
V. Scabriuscula (T. \& G.) Schwein.

- Rich woods. Monongalia: near Morgantown, common.

Fayette: near Nuttallburg (Nuttall).

V. hastata Michx.

Woodlands. Fayette: near Nuttallburg (Nuttall). Upshur: near Buckhannon (Pollock). Mercer: oak woods, near Bluefield. McDowell : near Elkhorn.

V. TRILOBA Schwein.

Dry woodlands. Upshur: near Buckhannon (Pollock). Monongalia: near Morgantown (Sheldon).

V. CONSPERSA Reichb. (V. Muhlenbergii, Labradorica Auth.). Low shaded places, Upshur: near Buckhannon (Follock).

V. Canadensis L. Canada Violet.

Rich woods. Monongalia: magnificent specimens in great profusion along the woods bordering the F. M. \& P. R. R., between Beechwoods and Little Falls; near Uffington. Marion: near Opekiska and Catawba. Gilmer: near Glenville (Mapel). Fayette: near Nuttallburg (Nuttall). McDowell: near Elkhorn. Upshur: near Buckhannon (Pollock).

V. striata Ait. Pale Violet.

Along runs. Monongalia: the most common species. Fayette: near Nuttallburg ( Nuttall). Hardy : near Moorefield (Gamble). McDowell: near Elkhorn. Upshur: near Buckhannon (Pollock).

V. LABRAdORICA Schrank. Viola canina var. Muhlenbergii, Traut.

Fayette County, at foot of cliff at Nuttallburg (Nuttall).

V. Rostrata Pers. Long-spurred Violet.

Hillside. Fayette: near Nuttallburg (Nuttall). Greenbrier: near White Sulphur Springs (Eggleston 4366). Upshur: near Buckhannon (Pollock).

V. Rafinesouei Greene. Field Pansy. ( $V$. tenella Muhl. not Poir.).

Greenbrier: near White Sulphur Springs (Eggleston 4365). Monongalia : near Morgantown (Sheldon 200).

Fields and rocky opens. Monongalia, common. Mineral: near Keyser (Workman). Fayette: near Nuttallburg (Nuttall).

V. AFFinis X CUCUllata Brainerd.

Tucker: near Parsons in "The Sluice" and along the bottom of the creek. (Greenman 301, 303, 304, 305, 306).

"These five sheets, Nos. 301, 303-6, collected at the same time and apparently at stations not far apart, are I believe $V$. affinis $x$ cuculata and its offspring; see Rhod. 8:49. March, I806. The hybrid has since been found in several other stations.

The long auricles are found characteristic of $V$. cucul- 
lata; but when pure it never has purplish or purple-dotted capsules. The green-capsuled plants seem to be seedlings with the recessive character in color of capsule. The black seeds are nearly those of $V$. cucullata, black being dominant over buff-the color of the seeds of $V$. affinis. . (In my cultures of this hybrid the recessive buff re-appears in some of the offspring. See Science $25: 940-944$. June 14, 1907.)

In No. 305 (but not in 306 ) the short auricles of $V$. affinis appear with the green capsules of $V$. cucullata."-Ezra Brainerd.

\section{CUBELIUM Raf.}

C. CONCOLOR (Forst.) Raf. Green Violet. (Solea concolor Ging.).

Rich woods. Wirt: near Burning Springs. Calhoun: along Laurel Run. Gilmer: near Glenville (Mapel).

\section{P A S S I F L O R A C E AE.}

\section{PASSIFLORA L.}

P. LuteA L. Yellow Passion-flower.

Hillsides. Fayette: near Nuttallburg (Nuttall). Preston: near Aurora (Mr. \& Mrs. Steele).

P. InCARnata L. Passion-flower.

Dry soil. Gilmer : near Glenville (Mapel).

\section{A C T A C E AE.}

OPUNTIA Mill.

O. Opuntia (L.) Coulter. Prickly Pear. (O. vulgaris Mill.).

Open fields and among scrub pines in the Devonian formations of Hardy: near Moorefield, where it is a prevalent weed in many places.

\section{TH Y M E E A C E $\mathrm{E}$.}

\section{DIRCA L.}

D. PALUSTRIS L. Leatherwood.

Damp woods. Jackson: near Ripley. Wirt: near Eliza* beth. Calhoun: near White Pine and Brookville. Greenbrier: White Sulphur Springs. Fayette: near Nuttallburg (Nuttall).

\section{Y T H R A C E AE.}

ROTALA Linn.

R. RAMOSIOR (L.) Koehne.

Wet places. Kanawha: at the Salinas (Holton). 
PARSONSIA P. Br. (I756).

(Cuphea P. Br. I756).

P. Petiolata (L.) Rusby. "Tar-weed." (C. viscosissima Jacq.) Dry soils, and fields. Monongalia, Marion, Wood, Wirt, Calhoun, Gilmer, Lewis, Upshur and Randolph. 'Fayette: near Gauley Bridge; near Nuttallburg (Nuttall); near Hawk's Nest (James). Greenbrier: near White Sulphur Springs. Summers: near Hinton. Monroe: near Alderson. Berkeley: near Martinsburg, and elsewhere. Preston: near Aurora ( $M r$. \& Mrs. Steelc). Kanawha: along the Kanawha River near Cabin Shoals (Holton).

\section{EL A S T O M A C E AE.}

\section{RHEXIA L.}

R. Virginica L. Meadow Beauty.

Moist, sandy meadows, and river shores. Monongalia: near Camp Eden; Little Falls. Wood: near Lockhart's Run. Wirt: near Burning Springs. Upshur: near Lorentz. Randolph: along Tygart's Valley River. Berkeley: near Martinsburg. Putnam: near Buffalo. Webster: Hacker Valley (H. H. Smith I533). Kanawha: at the Salinas (Holton).

O N A GR A C E AE.

ISNARDIA L.

I. PALUSTRIS L.

Sandy soil. Fayette: in a Sand bar in New River near Nuttallburg (Nuttall). Kanawha: near the Salinas (Holton).

\section{LUDWIGIA L.}

L. alternifolia L. Seed-box.

Wet banks. Wood: near Lockhart's Run. Monongalia : along Cheat River near Camp Eden. Fayette: near Kanawha Falls (James); near Nuttallburg (Nuttall). Kanawha: near the Salinas (Holton).

L. LINEARFOLIA Britt.

With or near the species. Wood: near Lockhart's Run. Fayette: near Nuttallburg (Nuttall).

Described by Prof. Britton in "Bull. Torrey Club," Dec., I8go, as follows : 
"Two or three feet high, divergently branched, the branches ascending. Leaves linear, elongated, 2-4-in. long, $I^{1} / 2-4$-lines wide, acute; flowers solitary in the axils of the upper leaves or bracts, yellow; sepals ovate-lanceolate acute, narrower than those of $\mathrm{L}$. alternifolia; branches and both sides of the leaves somewhat pubesent. Petals apparently remaining on the plant longer than those of L. alternifolia, which, as Dr. Millspaugh observes, commonly fall away when the plant is shocked."

"Appearing very distinct from typical L. alternifolia, but presumably but a variety of it. From the description it may be the Rhexia linearifolia, Poir, in Lam. Encycl. vi. 2, said to come from Carolina."

\section{CHAMAENERION Adans.}

C. angustifolium (L.) Scop. Fire-weed. (Epilobium spicatum Lam.).

In new clearings. Mineral: Grant: and Tucker: along the W. Va. Cent. R. R. Randolph : summit of Point Mountain, alt. 3,700 ft. Cheat Mountain, alt. 27-3,600 ft.; 11ear Pickens (H. H. Smith I $396 ;$ I454). Preston: near Aurora (Mr. \& Mrs. Stecle). Pendleton: on Spruce Knob (Greenman 173). Hardy: near Moorefield (Gamble).

\section{EPILOBIUM L.}

E. Coloratum Muhl. Willow-herb.

Ditches, and wet rocks. Greenbrier: near White Sulphur Springs. Fayette: near Nuttallburg (Nuttall). Preston: near Aurora $(M r$. \& Mrs. Steele). Mason: near Pt. Pleasant; and frequent throughout the State.

E. LINEARE Muhl.

In swamps, Preston: near Aurora (Mr. \& Mrs. Steele). Pocahontas: in Cranberry Glades (Sheldon 3848).

E. ADENOCAULON Haussk.

Wet places. Randolph: in Blister Swamp near Gandy Creek (Greenman 427).

\section{ONAGRA Adans.}

O. BIennis (L.) Scop. Evening Primrose. (Oenothera biennis L). Frequent or common, throughout the State.

Var. GRANDIFLORA (Ait.) Lindl.

Frequent. Randolph: Cricard, P. O.; Point Mountain. Wood: near Kanawha Station. Preston: near Tunnellton; near Terra Alta (Millspaugh); near Aurora (Mr. \& Mrs. 
Stecle). Webster: at Hacker Valley (H. H. Smith I57I). Greenbrier: near White Sulphur Springs. Hardy: nea: White Sulphur Springs. Hardy: near Moorefield (Gamble).

O. ARgillicola. Mackenzie, Torreya 4:56. 1904).

Biennial, with numerous stems ascending from the same root, $5^{-\mathrm{I}} 5 \mathrm{dm}$. hgh. Stems puberulent, but otherwise without pubescence; leaves of the stemless plant of the first year rosulate, $6-\mathrm{I} 5 \mathrm{~cm}$. long, the blades oblanceolate, $\mathrm{I} 5 \mathrm{~mm}$. or less wide, sinuate, acute, puberulent on both sides, the mid-nerve strongly developed, tapering at the base to a long, rather narrowly winged petiole; cauline leaves of the flowering plants of the second year, with narrowly linear-lanceolate blades, the well-developed ones $6-8 \mathrm{~cm}$. long, $7 \mathrm{~mm}$. or less wide, remotely sinuate-dentate, acute, glabrous or slightly puberulent, tapering to a petiole-like base and often strongly decurrent on the stem, forming well-developed ridges; calyxtube $3-4 \mathrm{~cm}$. long and longer than the sepals, perfectly glabrous, as also are the sepals, the tips of the latter free, spreading, often $3-4 \mathrm{~cm}$. long; petals bright yellow, obcordate, crenulate, $3-4 \mathrm{~cm}$. long, so that the open flower is often $6-8$ $\mathrm{cm}$. across ; capsules perfectly glabrous, $2-3 \mathrm{~cm}$. long, sessile, gradually tapering upward from the broad base and often strongly curved, somewhat quadrangular, strongly ribbed; seeds angled, I-I. $5 \mathrm{~mm}$. long.

Open sunny clay banks and along rivers, in loose, rocky soil. Greenbrier: near White Sulphur Springs, August 27, 1903 (Mackenzie 373).

KNEIFFIA Spach.

K. Pumila (L.) Spach.

(Oenothera pumila L.).

Dry fields, frequent throughout the State, especially in the northern section. Hardy: near Moorefield (Gamble). Randolph: near Pickens ( H. H. Smith I387).

K. FRUTicosA (L.) Raim. Sun-drops. "Wild Beet." (Oenothera fruticosa L.).

Common in most soils, and in cultivated fields as a weed. Summers: near Talcott and Lowell. Marion: near Worthington. Gilmer: near Glenville (Mapel). Fayette: near Nuttallburg (Nuttall).

K. Fruticosa Differta (Millsp. Prel. Cat. Fl. W. Va., 366(i892).

Damp meadows, Wood County, near Lockhart's Run, the most common form.

Stems I to $2 \mathrm{ft}$. high, nearly smooth, branching diffusely from every axil. Flowers profuse, large. Lower leaves ovate. Capsules narrowly winged, very short; apical inflorescence strongly cymose. 
K. LINEARIS (Michx.) Spach.

(Oenothera linearis Michx.).

Damp places. Wood: near Kanawha Station. Wirt: near Elizabeth. Calhoun: near Grantsville. Gilmer: near Glenville (Mapel). Upshur: near Buckhannon.

K. Longipedicellata Small, Bull. Torr. Club, 23 :I78. (I896).

Annual, slender, bright green, more or less puberulent. Stem erect or assurgent, 4-7dm. tall, red, slender, simple or sparingly branched above; basal leaves spatulate or obovatespatulate, 4-cm. long; stem leaves few, linear-lanceolate, $3-9 \mathrm{~cm}$. long, obtuse or acuitish, entire, often somewhat undulate, narrowed into a short petiole; flowers yellow, subtended by leaf-like bracts in terminal racemes which are sometimes corymbosely arranged; caylx hirsute, its tube slender, I-I.5 $\mathrm{cm}$. long, its segments linear, longer than the tube, the tips free in the bud; petals obovate, $2 \mathrm{~cm}$. long, many-nerved, emarginate, stamens less than one-half as long as the petals; styles slender, two-thirds as long as the petals; capsule narrowly obovoid, $I \mathrm{~cm}$. long, its angles winged, its faces ridged, on pedicels longer than the body; seeds irregularly oblong, $.8 \mathrm{~mm}$. long, brown, minutely papillose.

Between K. subglobosa and K. linearis. It differs from the former in its sparsely leafy, usually nearly simple stem, the hirsute calyx, the larger flowers and the narrowly obovoid capsules. It can easily be separated from $\mathrm{K}$. linearis by its pedicel, which exceeds the body of the capsule.

Moist places. Webster: at Long Glade (Millspaugh 579, July I890).

G. BIENNIS, L.

\section{GAURA L.}

Dry banks. Webster: near Taylor. Greenbrier: near White Sulphur Springs. Fayette: near Nuttallburg (Nuttall). Harrison: near Lumberport. Berkeley: near Martinsburg. Hardy: near Moorefield (Gamble).

\section{CIRCAEA L.}

C. Lutetiana L. Enchanter's Nightshade.

Low grounds, and wet woods. Lewis: up Stone Coal Creek. Gilmer: near Glenville (Brown). Fayette: near Nuttallburg (Nuttall). Webster: on Tater Knob ( $H . H$. Smith 1478). Preston: near Aurora (Mr. \& Mrs. Steele). C. ALPINA L.

Deep, rich woods. Randolph, Grant, Tucker and Pendleton: prevalent in the Alleghanies. Gilmer: near Glenville ( Mapel). Monongalia: along Cheat River, above Camp Eden. Preston: near Aurora (Mr. \& Mrs. Steele). 


\section{ARALIA L.}

A. spinosa L. Angelica Tree. Hercules' Club

Rich Mountain woods. Webster: Buffalo Bull Mt. alt. 2,595 ft., plentiful (Millspaugh); Hacker Valley (H. $H$. Smith I 584). Preston: near Rowlesburg. Summers: along the Greenbrier River; near Hinton. Fayette: near Nuttallburg (Nuttall); at Gauley Bridge, abundant. Randolph: near Whitman (Greenman 160). Monongalia: near Morgantown.

A. Racemosa L. Spikenard.

Deep, cold woods, frequent in the Alleghanies. Randolph: Cheat Mountains, alt. 3,350 ft.; Point Mountain, alt. 3,560 $\mathrm{ft}$. Hampshire: Ice Mountain. Tucker: near Falls of Blackwater. Gilmer: near Glenville (Mapel). Greenbrier: near White Sulphur Springs. Fayette: near Nuttallburg (Nuttall). Summers: near Hinton. Preston: near Aurora (Mr. \& Mrs. Steele).

A. Nudicaulis L. Wild Sarsaparilla.

Rich woods, frequent. Monongalia: the Flats, Rich Woods, and along the Monongahela. Marion: near Opekiska. Randolph: on Point Mountain. Grant: rear Bayard.

A. Hispida Vent.

Rocky soils. Tucker: near Davis, along Blackwater Fork of Cheat.

\section{PANAX L.}

P. Quinguefolium L. Ginseng. "Sang."

Rich, deep woods. Wirt: near Burning Springs. Jackson: near Ripley. Gilmer: near Glenville (Mapel). Grant: near Bayard. Randolph: Rich, Cheat and Point Mountains. (One store at Crickard P. O. buys from this neighborhood $\$ \mathrm{I}, 500$ worth annually of the mountaineers.) Webster: Buffalo Bull range. Fayette: near Nuttallburg (Nuttall). Summers: near Hinton. Preston: near Aurora ( $M r$. \& Mrs. Steele).

P. TRIFOLIUM L.

(Aralia trifolia Decn.).

Moist woods. Upshur: near Buckhannon (Pollock), and near Newlon ( $A$. B. Brooks). 


\section{A P I A C E AE. \\ (Umbelliferae.)}

\section{DAUCUS L.}

D. Carota L. Wild Carrot. "Devil's Plague."

Fields, meadows, and roadsides. Lewis, Randolph, Monongalia, Marion, Berkeley, Morgan, Mineral, Preston, Grant, and Kanawha counties. Gilmer: near Glenville (Mapel). Fayette: near Nuttallburg. Jefferson: near Shenandoah Junction; near Charlestown; and Summit Point. Greenbrier: near Caldwell and White Sulphur Springs. Summers: near Greenbrier Stock Yards, and Hinton. Mason: near Point Pleasant. Mercer: near Ingleside; and reported from every county in the State.

Forma RoseA. Millsp. Prel. Cat. Fl. W. Va., 369.

With rose colored flowers, a frequent form in Monongalia: near Morgantown; and along the Kingwood Pike.

\section{ANGELICA L.}

A. Curtisir Buckley.

Sandy river banks. Monongalia: near Camp Eden. Preston: near Reedsville. Grant: near Bayard. Greenbrier: near White Sulphur Springs.

A. villosa (Walt.) B.S.P. Hairy Angelica. (A. hirsuta, Muhl.)

Frequent in dry woods and glady meadows. Webster: Long and Welsh Glades. Jackson : near Sandyville. Tucker : near Davis. Fayette: near Nuttallburg (Nuttall). Monongalia: near Camp Eden. Preston: near Terra Alta. Randolph: on Point Mountain, alt. 2,540 ft. Hardy: near Moorefield (Gamble). Preston: near Aurora (Mr. \& Mrs. Steele).

A. Atropurpurea L. Purplish Angelica. (Archangelica atropurpurea, Hoffm.).

Low grounds and river banks. Grant: near Bayard. Preston: near Terra Alta. Tucker: near Davis. Fayette: near Kanawha falls (James).

OXYPOLIS Raf. (1825).

(Tiedemannia DC. 1829.)

O. RIGIDIOR (L.) C. \& R.

(O. rigidus $\mathrm{Raf}$.

Swampy spots. Randolph: along Shaver's Fork of Cheat. Fayette: near Nuttallburg (Nuttall). Preston: near Terra Alta (Millspangh); near Aurora (Mr. \& Mrs. Steele). Pocahontas: in Cranberry Glades (Sheldon 3821). 


\section{PSEUDOTAENIDIA Mackenzie.}

Glabrous and glaucous erect perennials from stout horizontal to perpendicular rootstocks. Leaves ternately decompound, the leaflets entire. Umbels borne on terminai and lateral peduncles, compound, the rays very unequal in length. Involucre and involucels none or rarely of one or two bractlets. Corolla not seen.* Calyx-teeth short, but evident. Fruit thick, strongly flattened dorsally, oval or obovate, glabrous. Dorsal and intermediate ribs of carpel filiform and very much narrower than the intervals, coming together at base and apex to form short prominent ridges; lateral ribs thick, broadly winged and contiguous to those of the other carpel so as to form a broad one-edged margin around the fruit, nerved dorsally at the inner margin and also near the outer margin. Oil-tubes solitary in the intervals or often two in the intervals nearest the lateral ribs; two entirely developed and two partially developed on the commissural side. Top of fruit thickened by the converging ribs, but stylopodium absent or much depressed. Seed-face plane, the back rounded.

P. MONTANa Mackenzie, Torreya 3:I59 (I903).

Plant 4-8 dm. high, entirely glabrous; stems striate; leaves several, the blades two or three times ternately compound; the segments entire, ovatc or oblong-lar.ceolate, oval or ublanceolate, glaucous and strongly veined beneath, sessile or stalked, I0-30 $\mathrm{mm}$. long, 6-20 mm. wide, mucronate, often inequilateral at base; petioles dilated at base, striate and clasping the sten ; perluncles $6-20 \mathrm{~cm}$. long; rays of umbels 8-I2, I-5 cm. long; rays of umbellets usually slightly more numerous, 3-7 $\mathrm{mm}$. long; fruit 5-6 $\mathrm{mm}$. long, $4 \mathrm{~mm}$. wide, the lateral ribs I $\mathrm{mm}$. wide.

The plant exactly resembles Taenidia integerrima (L.) Drude in everything except the fruit.

In dry open woods, in a clayey soil intermixed with loose rocks. Greenbrier: side of Kate's Mountain, August 29, 1903 (Mackenzie).

\section{SIUM Linn.}

S. cicutaefolium Gmel.

In swampy places. Randolph: on Tygart Valley River near Huttonville (Greenman 434).

*Almost certainly yellow. 
HARPERELLA Rose.

H. vivipara Rose.

Wet bottomlands. Jefferson: near Harper's Ferry(Aiken).

\section{HERACLEUM L.}

H. Lanatum Michx. Cow Parsnip.

Wet grounds. Lewis: along Leading Creek. Upshur: near Lorenz. Randolph: along Tygart's Valley River. Webster: Welch Glade.

IMPERATORIA Linn.

I. Ostruthium Linn.

Established in field and along a road at McClure, Webster County (H. H. Smith I558).

\section{PASTINACA L.}

P. sativa L. Wild Parsnip.

Waste grounds and cultivated fields. Randolph: Cheat Mountains, alt. 3,350 ft. Jefferson: near Shenandoah Jc. Greenbrier: near White Sulphur Springs. Mineral: opposite Cumberland. Morgan: near Hancock. Wood: near Kanawha Station. Mason: near Point Pleasant. Hardy: near Moorefield (Gamble).

\section{THASPIUM Nuttall.}

T. tRifoliatum (L.) Britton.

Mountain woodlands. Hardy: near Moorefield (Gamble).

T. trifoliatum aureum (Nutt.) Britton. Meadow Parsnip.

Thickets and meadows. Frequent throughout the State.

T. BARBINODE (Michx.) Nutt.

Greenbrier: near White Sulphur Springs (Eggleston 4347).

Rich woods. Mercer: plentiful near Bluestone Jc. Taylor: near Grafton (Pollock).

\section{LIGUSTICUM L.}

L. Canadense (L.) Britton.

Rich soil. Grant: near Bayard, plentiful along the Blackwater Fork of Cheat River. 


\section{ERYNGIUM L.}

E. aquaticum L. Rattle-snake Master. (E. yuccaefolium Michx.).

Swampy places. Webster: at Welch Glade.

\section{SANICULA L.}

S. Marilandica L. Black Snake-root.

Rich woods. Monongalia: near Morgantown. Preston: near Terra Alta (Millspangh); near Cranberry Summit (Mertz 995) ; near Aurora (Mr. \& Mrs. Steele). Fayette: near Nuttallburg (Nuttall).

S. Canadensis L.

Rich soil. Monongalia: near Little Falls. Marion: near Opekiska. Fayette: near Nuttallburg (Nuttall).

S. TRIFOliATA Bicknell.

Along Madam Creek opposite Hinton, Summers County. alt. I,500 ft., July 9, I900 (Morris 96I). Monongalia : near Marilla (Sheldon I632).

S. GREGARIA Bicknell.

Woods and thickets. Summers: near Hinton (Eggleston $5567)$.

\section{TAENIDIA Drude.}

T. integerrima (L.) Drude.

(Pimpinella integerrima L.).

Rocky hillsides. Lewis: along Stone Coal Creek, and Randolph: on Point Mountain (Millspaugh). Preston: near Aurora ( $M r$. \& Mrs. Steele). Upshur: near Buckhannon (Pollock). Monongalia: near Seneca (Sheldon 3574).

\section{CHAEROPHYLLUM L.}

C. Procumbens (L.) Crantz.

Ohio: Elm Grove, near Wheeling (Mertz \& Guttenberg).

WASHINGTONIA Raf. (I8I8). (Osmorrhiza Raf. I8I8).

W. Claytoni (Michx.) Britton.

(O. brevistylis DC.). Rich woods. Wirt: above Elizabeth. Gilmer: near Glenville (Mapel; Broien). Monongalia: opposite Roundbottoms. Grant: near Bayard. Fayette: near Nuttallburg (Nuttall). Ohio: near Wheeling (Mertz 1042). Upshur: near Buckhannon (Pollock). 
W. LONGistyLIS (Torr.) Britton.

Rich woods. Monongalia: near Morgantown. Marion: near Fairmont. Tucker: near Davis. Wirt: above Elizabeth.

\section{ZIZIA Koch.}

Z. CORDATA (Walt.) DC.

River banks. Fayette: near Nuttallburg (Nuttall). Greenbrier: near White Sulphur Springs (Eggleston 4345).

Z. Aurea (L.) Koch.

Damp places. Monongalia: The Flats, and along the Monongahela. Marion: near Opekiska. Fayette: near Nuttallburg ( Nuttall).

Z. BebbiI (C. \& R.) Britton. (Z. aurea, var. Bebbii C. \& R.). Woodlands. Fayette: near Nuttallburg (Nuttall).

Of specimens gathered in Mason, near Pt. Pleasant, Prof. Coulter says: "Flowers too white, and altogether it does not quite fit, and is indeterminable on account of the immature fruit." Greenbrier Co., on Kate's Mountain, alt. 3,300 ft. (Small; Heller 84I).

\section{CICUTA L.}

C. maculata L. Spotted Cow-bane. Beaver Poison.

Swampy spots, and wet meadows. Randolph: along Tygart's Valley River plentiful. Fayette : along Loup Creek (James) ; near Nuttallburg (Nuttall). Putnam: near Scott's Depot (James). Gilmer: Glenville (Brown); near DeKalb P. O. Morgan: near Cacapon. Monongalia: near Ice's Ferry. Mason: near Point Pleasant.

C. BULbifERA L.

Wet places. Mason: near Pt. Pleasant.

DERINGA Adans. ( 1763 ). (Cryptotaenia, DC. I829).

D. Canadensis (L.) Kuntze. Honewort. (Cryptotaenia Canadensis (L.) DC.).

Shady rocks. Monongalia: Roundbottoms and Little Falls Marion: near Opekiska. Fayette: near Nuttallburg (Nuttall). Tucker: rich woods near Parsons (Greenman I45).

\section{HYDROCOTYLE L.}

H. Americana L. Water Pennywort.

Along streams. Jefferson: near Flowing Spring. Randolph: above Cricard P. O. Grant: near Bayard. Green- 
brier: near White Sulphur Springs. Preston: at Cranberry Summit (Mertz 990); near Aurora (Mr. \& Mrs. Steele).

\section{ERIGENIA Nutt.}

E. Bulbosa (Michx). Nutt. Harbinger of Spring.

Rich open woods. Monongalia: opposite Granville, plentiful. Fayette: near Nuttallburg (Nuttall).

\section{COR N A CE AE.}

\section{CORNUS L.}

C. FIORIDA L. Flowering Dogwood.

Dry woods. Monongalia throughout, some quite large trees near Morgantown. Wood, Wirt and Calhoun counties. Gilmer: near Glenville (Brozen; Mapel). Lewis and Upshur (Millspaugh; Pollock). Randolph: on Cheat Mountains, alt. 3,600 ft. Marion: Webster: Hacker Valley ( $H$. H. Smtih I 588). Fayette: near Nuttallburg (Nuttall). Hardy: near Moorefield (Gamble). Mercer: near Bluefield. Greenbrier Co., on Kate's Mountain, alt. 3,300 ft. (Small).

C. Circinata L'Her. Round-leaved Dogwood.

Damp, cool woods. Rare. Upshur: near Lorentz.

C. Амомum Mill. Kinnikinnik. (C. sericea L.).

Wet places. Grant: near Bayard. Randolph: along Tygart's Valley River. Nicholas: along Peter Creek. Fayette: near Nuttallburg (Nuttall). Upshur: near Buckhannon (Pollock).

C. StOlONifera Michx. Red Osier.

Moist places. Ohio: near Wheeling (Mertz 1056).

C. Candidissima Marsh. Panicled Cornel. (C. paniculata L'Her.)

Thickets and river banks. Monongalia : near Morgantown. Marion: Montana; along Beaver Creek. Randolph: Cheat River, alt. 2,700 ft. Summers: near Hinton. Hampshire: near Romney.

C. ALTERNIFOLIA L. f.

Hillside copses. Monongalia, Marion, Preston, Wood and Calhoun counties. Gilmer: near Glenville ( $\mathrm{Mapel}$ ). Preston: near Aurora (Mr. \& Mrs. Steele). Lewis: along Leading Creek. Upshur: near Lawrence (Millspaugh); near Buckhannon (Pollock); near Newlin (H. H. Smith I784). Fayette: near Nuttallburg (Nuttall). Greenbrier: near White Sulphur Springs. Summers: near Hinton.

C. Canadensis L. Dwarf Cornel.

Deep woods. Hampshire: on Ice Mountain (Rumsey). Pendleton: summit of Spruce Knob (A.B. Brooks). 


\section{NYSSA L.}

N. sylvatica Marsh. Black Gum.

Various situations throughout the State. Wood: near Leachtown. Wirt: along Straight Creek. Calhoun: near Brookville. Gilmer: near Glenville ( $\mathrm{Mapel}$ ). Monongalia : near Morgantown. Randolph: on Point Mountain. Webster: on Buffalo Bull Mountains. Fayette: near Nuttallburg (Nuttall). Summers : near Hinton; and along the Greenbrier River. Kanawha: near Handley. Mercer: near Ingleside. Immense growths of this species at Pickens, Randolph Co., with trunks 3 to $4 \mathrm{ft}$. in diameter (Millspaugh).

CLETHR A CE AE.

\section{CLETHRA L.}

C. Acuminata Michx. White Alder.

Wooded banks. Fayette: along the Gauley River at the base of the Gauley Mountains : near Nuttallburg, uncommon (Nuttall).

P Y R OL A CE AE.

\section{PYROLA L.}

P. elliptica Nutt. Shin-leaf.

Rich woods. Kanawha: near Charleston (James). Preston : near Terra Alta.

P. RotUNDIFolia L. Shin-leaf.

Sandy woodlands, frequent. Upshur : summit on Staunton Pike. Gilmer: near Glenville ( $\mathrm{Mapel}$ ). Fayette: near Nuttallburg (Nuttall). Grant: near Bayard. Hardy: near Moorefield (Gamble).

\section{MONESES Salisb.}

M. uniflora (L.) A. Gray. One-flowered Pyrola. (M. grandiflora S. F. Gray).

Deep, cold woods. Gilmer: near Glenville (Mapel). Preston: along Laurel Hills. Monongalia : near Cheat View, and along Quarry Run.

CHIMAPHILA Pursh.

C. umbellata (L.) Nutt. Prince's Pine.

Dry woods, rare compared with the next. Monongalia: along Decker's Creek; and on Laurel Hills in pine thickets. 
C. maculata (L.) Pursh. "Pipsisseway."

Rich woods, frequent throughout the northern, eastern, and central counties. Gilmer: near Glenville (Mapel; Brown). Kanawha: near Charleston (James). Fayette: near Hawk's Nest, and Kanawha Falls; near Nuttallsburg (Nuttall). Hardy; near Moorefield (Gamble).

\section{MONOTROPA CEAE.}

MONOTROPSIS Schwein.

M. odoRAta E11.

Upshur: growing at the edge of a sandy bottom along Little Bush Run of French Creek (Brooks).

\section{MONOTROPA L.}

M. UnIfloRA L. Indian-pipe. Corpse-plant.

Deep, rich woods. Wirt: near Elizabeth. Kanawha: near Charleston (James). Gilmer: near Glenville (Mapel). Fayette: near Nuttallburg (Nuttall). Preston: near Terra Alta (Millspaugh); near Aurora (Mr. \& Mrs. Steele. Webster : deep woods on Tater Knob (H. H. Smith I495). Grant: near Bayard. Hardy: near Moorefield (Gamble).

\section{HYPOPITYS Hill.}

H. Hypopitys (L.) Small. Pine Sap. (Monotropa Hypopitys L.).

Deep, rich woods. Wirt: near Elizabeth. Fayette: near Nuttallburg (Nuttall). Gilmer: near Glenville (Mapel, Brown). Kanawha: near Charleston (James). Upshur: summit on Staunton Pike. Preston: near Aurora ( $M r$. \& Mrs. Steele).

\section{E R I C A C E AE.}

\section{AZALEA L.}

A. Arborescens Pursh. Smooth Azalea.

Glades and along mountain streams. Fayette: near Nuttallburg (Nuttall). Webster: Upper and Welch Glades.

A. Canescens Michx. Hoary Azalea.

Hampshire: Mutton Run, near Cacapon Springs; Dillon's Run, near Cacapon River.

Specimens in full leaf were noted at these points that differ widely from $\mathrm{R}$. nudiflorum and $\mathrm{R}$. calendulaceum, and 
seem, so far at least, to be this species. Greenbrier Co., Kate's Mountain, alt. 3,300 ft. (Small).

A. viscosa L. Sticky Azalea.

Glades and cool ravines. Preston: Kingwood glades. Kanawha: near Charleston (Barnes). Fayette: near Nuttallburg (Nuttall) ; near Hawk's Nest (James). Webster: near Long Glade. Upshur: near Buckhannon (Pollock). Var. GLAUCA Michx. "Cinnamon Honeysuckle."

Rocky streams in the higher mountains. Tucker: along the Blackwater Fork of Cheat.

Var. NitidA (Pursh.) Britton.

Glades. Webster : in Long and Upper Glades.

A. Nudiflora L. "Wild Honeysuckle." Pinxter Flower.

Rocky places along streams. Common throughout the northern, central, and eastern portions of the State. Mercer: near Princeton 6-8 ft. high.

A. Lutea L. Flaming Azalea.

Mountain woods. Monongalia: Cheat View. Mineral: near Keyser (Workman). Gilmer: near Glenville (Mapel). Fayette: near Nuttallburg, alt. I,800 ft., flowers with a delicate fragrance quite distinct from that of other Azaleas (Nuttall). Summers: near Hinton. Preston: along the Laurel Hills. McDowell: near Elkhorn. Mercer: near Princeton and Bluefield. Upshur: near Buckhannon (Pol. lock).

\section{RHODODENDRON L.}

R. maximum L. Great Laurel.

Deep rich woods, forming the most dense and tangled thickets in the mountains. Western limit on the Great $\mathrm{Ka}$ nawha River near Charleston, Kanawha County. Common throughout the eastern and northern portions of the State

R. Catawbiense Michx. Lilac-colored Laurel.

Deep, rich mountain woods, rare. Pendleton: near Cherry Grove. Fayette: near Nuttallburg, where it prefers the face of cliffs (Nuttall). Greenbrier: Top of Alleghanies. Summers: near Hinton.

\section{MENZIESIA Smith.}

M. pilosa (Michx.) Pers.

(M. globularis Salisb.).

Pocahontas: summit of Spruce Knob, alt. 4,800 ft. (Hopkins). 
K. Latifolia L. Mountain Laurel. Calico-bush. Spoon-wood.

Dry or moist hillsides and thickets; forming impenetrable masses in the mountains. Calhoun: Laurel Run. Upshur: Sand Run (Millspaugh, Pollock). Webster: Buffalo Bull Mountains. Kanawha: near Charleston (Barnes). Nicholas: near Beaver Mills. Monongalia: near Ice's Ferry and Cheat View. Preston: Laurel Hills, thence southward throughout the eastern counties. Fayette: near Nuttallburg (Nuttall). Jefferson: near Harper's Ferry (Mertz \& Guttenberg).

K. Angustifolia L. Sheep-laurel. Lamb-kill.

Hillsides. Calhoun: Laurel Run. Upshur: Sand Run. Nicholas : near Beaver Mills. Randolph: near Cheat Bridge. Hardy: near Moorefield.

\section{PIERIS D.Don.}

P. Mariana (L.) Bth. \& Hook. Stagger Bush. (Andromeda Mariana L.).

Low grounds. Webster: Long Glade. Summers: near Hinton. Pocahontas: near Greenbank (Swank).

P. FloRibunda (Pursh.) Bth. \& Hook. (Andromeda floribunda Pursh.).

Mountain woodlands. Greenbrier: in the mountains (Rumsey).

\section{XOLISMA Raf.}

X. Ligustrina (L.) Britton. "Seedy Buckberry."

Wet grounds. Preston: Morgan's Glade and Terra Alta. Upshur: near Buckhannon. Webster: Upper, Long and Welch Glades. Nicholas: Collett's Glade. Fayette: near Nuttallburg (Nuttail).

Var. PUBESCENs (Gray). (Andromeda ligustrina pubescens A. Gray).

Swampy place. Fayette: near Nuttallburg, alt. 2,000 ft.: a variation with a six-lobed corolla and six-celled ovary (Nuttall).

\section{OXYDENDRUM DC.}

O. Arboreum (L.) DC. Sour Gum.

Rich woods. Wood: near Leachtown. Randolph: near Valley Bend. Gilmer: near Glenville ( $\mathrm{Mapel}$ ). Kanawha: near Charleston (James). Fayette: near Nuttallburg (Nutt- 
all). Summers: along Greenbrier River and rear Hinton. Marion: near Shinnston and Clements. Monongalia: near Beechwoods. Mercer: Beaver Spr. and Ingleside. Webster near Tater Knob (H. H. Smith 1498).

\section{EPIGAEA L.}

E. REPENS L. Trailing Arbutus.

In moss of shady woods. Monongalia, and Preston : along the Laurel Hills. Gilmer: near Glenville ( $\mathrm{Mapel}$ ). Mineral: near Keyser (Workman). Kanawha: near Charleston (James). Fayette: near Nuttallburg (Nuttall). Hardy: near Moorefield (Gamble). Mercer: near Bluefield.

\section{GAULTHERIA L.}

G. Procumbens L. Wintergreen. Tea-berry. Mountain Tea.

Cool rich woods. Throughout the mountainous regions of the State.

\section{A C C I N I A C E AE.}

\section{GAYLUSSACIA H. B. K.}

G. Dumosa (Andr.) T. \& G. Dwarf Huckleberry.

Damp, sandy soils. Kanawha: near Charleston (James). Hardy: near Moorefield.

G. FRoNdosa (L.) T. \& G. Dangleberry.

Low copses. Fayette: near Hawk's Nest (James). Webster : Upper Glade.

G. Resinosa (Ait.) T. \& G. Huckleberry.

Wirt: near Burning Springs. Monongalia: near Laure! Hills. Upshur: near Buckhannon (Pollock). Marion: near Forksburg. Fayette: near Nuttailburg (Nuttall). Frequent throughout the State.

\section{POLYCODIUM Raf.}

P. stamineum (L.) Greene.

Open woods. Wirt: near Burning Springs. Mineral. along Knobby Mts. (Workman). Gilmer: near Glenville (Mapel). Fayette: near Nuttallburg (Nuttall). Grant: near Bayard. Tucker: near Davis. Hardy: near Moorefield (Gamble). Mercer: near Pluefield (Millspaugh). Webster : Hacker Valley (H. H. Smith I589). Upshur: near Buckhannon (Pollock). 


\section{VACCINIUM L.}

V. NigRUM (Wood) Britton.

Dry rocky soil. Pendleton: on Spruce Mountain. Monongalia : near Morgantown (Brooks).

V. Pennsylvanicum Lam. Dwarf Blueberry.

Dry hills. Gilmer: near Glenville ( $\mathrm{Mapel})$. Brooke : near Wellsburg (Mertz \& Guttenberg).

V. vacillans Kalm. Low Blueberry.

Opens. Brooke: near Wellsburg (Mertz \& Guttenberg). Fayette: near Nuttallburg, alt. 2,00o ft. (Nuttall). Upshur: near Buckhannon (Pollock). Webster: on the highest rock: at the summit of Tater Knob (H. H. Smith I497).

V. corymbosum L. Swamp Blueberry. "Seedy Deerberry."

Swampy thickets. Preston: Kingwood Glades; Terra Alta

Glades. Webster: Welch, Upper and Long Glades.

V. PALlidum Ait.

Glady regions. Webster: in Upper Glade. Upshur: near Buckhannon (Pollock).

V. Canadense Richards.

In wet places. Preston: in a swamp near Cranesville ( $A$. B. Brooks; Rumsey; Sheldon I497).

\section{OXYCOCCUS Hill.}

O. macrocarpus (Ait.) Pers. Cranberry. (Vaccinium macrocarpon Ait.).

Glades. Webster: Welch, Long and Upper Glades. (This station will be lost in a few years, as drainage is being practised here to reclaim the land). Preston: Glade Farms, Morgan's Glade, Cranberry, Reedsville and Terra Alta.

O. Oxycoccus (L.) Mac.M.

In cold bogs. Pocainontas: Cranberry Glades; abundant in the open glades; Round and Flag Glades (Sheldon) Tucker: in open glades (Brooks). Preston: near Cranesville (Sheldon I458).

O. erythrocarpus (Michx.) Pers.

(Vaccinium Michx.)

Pocahontas: summit Spruce Knob, alt. 4,800 ft.

\section{CHIOGENES Salisb.}

C. Hispidula (L.), T. \& G. Creeping Snowberry.

Tucker: on rocks in the mist of Blackwater Fall. 
D I A PE N S I A C E AE.

G. APHYLLA L.

$$
\text { GALAX L. }
$$

Woodlands. Fayette: near Nuttallburg (Nuttall). Monongalia: Dille Farm near Morgantown.

$$
\text { P R I M U L A C AE. }
$$

\section{SAMOLUS L.}

S. FLORIBUndus H. B. K.

Sandy places. Summers: shores of New River near Hinton (Millspaugh, Eggleston 5518).

\section{LYSIMACHIA L.}

L. QUADRIFOLIA L. Loosestrife.

Moist soils. In all sections of the State visited.

L. TERRESTRIS (L.), B. S. P. - (L. stricta Ait.).

Wet places. Fayette : near Nuttallburg, in railroad ditches, infrequent (Nuttall). Ohio: on Bogg's Island (Mertz \& Guttenberg). Wood: near Kanawha Station.

L. Nummularia L. Money-wort.

Escaped from cultivation. Wood: near Lockhart's Run, profuse.

STEIRONEMA Raf.

S. ciliatum (L.) Raf.

Low grounds and ditches. Randolph: on Rich Mountain, alt. I,6IO-2,125 ft.; on Point Mountain (Millspaugh); Dry Fork River near Harman (Greenman 237). Grant: near Bayard. Gilmer: near Glenville (Brown). Fayette: nea: Nuttallburg (Nuttall); near Hawk's Nest. Tucker: near Davis.

S. LANCEOLATUm (Walt.) Gray.

Low grounds. Wood: near Kanawha Station. Lewis : along Stone Coal Creek. Randolph: along Tygart's Valley River. Monongalia: Camp Eden.

Var. ANGUSTIFOLIUm (Lam.) Gray.

Low grounds. Monongalia: Sandy banks of Cheat River above Camp Eden.

NAUMBERGIA Moench.

N. thyrsiflora (L.) Duby. Pyramidal Loosestrife. (Lysimachia L.). 
Wet meadows near the mountains. Upshur: near Buckhannon. Randolph: along Tygart's Valley River. Morgan : near Hancock.

\section{TRIENTALIS L.}

A. Americana (Pers.) Pursh. Star Flower.

Damp cool woods. Mineral : near Piedmont. Grant: near Bayard. Tucker: along Blackwater Fork of Cheat. Preston: near Terra Alta. Monongalia: near Laurel Point.

\section{ANAGALLIS L.}

A. ARvensis L. Poor Man's Weather-glass.

Waste places. Jefferson: near Bolivar Heights (Mertz $\mathcal{E}$ Guttenberg); in crevices of old walls at Harpers Ferry (Greene). Wayne: near Fort Gay (Hopkins).

\section{DODECATHEON L.}

D. Meadia L. Shooting -Star.

Rich woods. Mineral : near Keyser (Workman). Hardy: near Moorefield (Gamble).

E B E N A C E AE.

DIOSPYROS L.

D. Virginiana L. Persimmon. Date Plum.

Thickets and opens. Wood: throughout. Fayette: near Nuttallburg (Nuttall). Gilmer: near Glenville (Mapel). Monongalia: near Morgantown. Wirt: along Little Kanawha River. Jackson: near Ripley. Lewis: along Leading Creek.

S T YR A C A CEAE.

MOHRODENDRON Britton.

M. Carolinum (L.) Britton. "Shittimwood." (Halesia tet raptera $\mathrm{L}$.).

Banks of streams. Fayette: near Nuttallburg (Nuttall). below Gauley Bridge. Summers : near Hinton, abundant.

$$
\text { O L E A C E AE. }
$$

\section{FRAXINUS L.}

F. Americana L. White Ash.

Rich woods. Frequent throughout the State. Fine speci- 
mens of very large growth in Randolph Co., especially on Channel ridge where the species grows very tall, and at Pickens where specimens were measured from 3 to $6 \mathrm{ft}$. in diameter.

F. Pennsylvanica Marsh. Red or Black Ash. (F. pubescens Lam.).

Low grounds. Randolph: along Tygart's Valley River. Upshur: near Lawrence. Fayette: near Nuttallburg (Nuttall). Wood: along the Little Kanawha River. Mason: neat Point Pleasant.

F. Lanceolata Borck. Green Ash. (F. viridis Michx.). Along streams. Summers: near Hinton. Frequent throughout the State.

F. NIGRA Marsh. Black Ash.

(F. sambucifolia Lam.).

Wet woods. Wirt: along Straight Creek. Fayette: nea1 Nuttallburg, rare (Nuttall). Randolph: on Point Mountain. Webster: Buffalo Bull Mountain. Monongalia: near Ice's Ferry. Summers : Hinton.

\section{CHIONANTHUS L.}

C. Virginica L. Fringe Tree.

River banks. Jackson: near Sandy and Ripley. Fayette: near Nuttallburg, plentiful (Nuttall); along Gauley River near Gauley Mountains (Millspaugh); near Thurmond (Eggleston 5560) ; near Hawks Nest (Holton) in fruit. Summers: near Hinton. Monongalia: along Tibb's Run.

\section{LIGUSTRUM L.}

L. vUlgare L. Privet.

Escaped from cultivation to waste places. Kanawha: near Charleston (Barnes). Randolph: near Pickens ( $H . H$. Smith 2024).

LO GA N I A C E AE.

CYNOCTONUM J. G. Gmel.

(Mitreola R. Br. I8Io).

C. Mitreola (L.) Britton.

(M. petiolata T. \& G.)

Damp soil. Fayette: near Nuttallburg; rare (Nuttall). 
SABBATIA Adans.

S. Angularis (L.) Pursh. Centaury.

Rich soil. Jackson and Wood counties, frequent. Gilmer: near Glenville (Mapel). Fayette: near Nuttallburg (Nuttall). Monongalia: near Easton. Summers: near Hinton. Harrison: near Lumberport. Marion: near Clements.

S. lanceolata (Walt.) T. \& G.

Wet places. Marion: near Fairmont (Boutlou).

\section{GENTIANA L.}

G. QUinguefolia L. Four-leaved Gentian. (G. quinqueflora, Lam.).

Opens. Doddridge: near Long Run. Hardy: near Moorefield (Gamble). Preston: near Aurora (Mr. \& Mrs. Steele).

G. Andrewsir Griseb. Andrew's Gentian.

Moist woods. Fayette: near Nuttallburg, alt. 2,000 ft (Nuttall). Monongalia: near Cheat View. Preston: near Reedsville. Webster: (H. H. Smith).

G. Saponaria L. Soapwort. Gentian.

Moist woods. Fayette: near Kanawha Falls (Selby).

Preston: near Aurora (Mr. \& Mrs. Steele).

G. LINEARIS Froel.

Boggy places. Preston: near Terra Alta and Morgan's Glade.

G. Flavida A. Gray. Yellow Gentian.

In moist soils, Preston: near Aurora (Mr. \& Mrs. Steele).

\section{OBOLARIA L.}

O. Virginica L. Pennywort.

Moist woods. Gilmer: near Glenville ( $M a p e l)$. Upshur: near Buckhannon (Pollock). Fayette: near Nuttallburg (Nuttall). McDowell: near Elkhorn.

BARTONIA Muhl.

B. Virginica (L.) B. S. P. Yellow Bartonia.

In moist situations. Preston: near Aurora (Mr. \& Mrs. Steele). 
M E N Y A N T H A CEAE.

\section{MENYANTHES Linn.}

M. TRIFoliata Linn. Buckbean.

In cool bogs, Preston: near Cranesville (Sheldon). Pocahontas: Cranberry Glades (Brooks).

\section{A P O C N A C E AE.}

\section{APOCYNUM L.}

A. Androsaemifolium L. Spreading Dog's-bane.

Meadows, fields, and borders of thickets. Randolph: near Cricard P. O.; near Valley Head. Greenbrier: near White Sulphur Springs. Mason: near Point Pleasant, Brighton and Buffalo.

A. CANNABinum L. "Rheumatism weed." Indian Hemp. "Wild Cotton."

Moist grounds, fields, and banks of streams. Frequent or common throughout the State. Tucker: banks of the Blackwater River near Hendricks (Greenman 274). Jefferson: dry uplands near Harpers Ferry (Greene).

A. ALBUM Greene.

Jefferson: alluvial banks of both rivers at Harpers Ferry (Grecne).

A. pubescens R. Br.

Hardy: near Moorefield (Gamble).

\section{A S C L E P I A D A CEAE.}

\section{ASCLEPIAS L.}

A. tuberosa L. Pleurisy-root.

Fields and meadows. Wood: near Leachtown. Monongalia: near Morgantown and at Camp Eden. Lewis: along: Leading and Stone Coal Creeks. Webster: near Long Glade. Fayette: near Nuttallburg (Nuttall).; near Kanawha Falls (James); near Gauley Bridge (Millspaugh); near Hawk's Nest (Holton). Jackson: near Fisher's Point. Gilmer: near Glenville (Mapel; Brown). Doddridge: near Smithton. Jefferson: near Shenandoah Jc. Berkeley: near Martinsburg. Hardy: near Moorefield (Gamble). Upshur: near Buckhannon (Pollock).

A. RUBRA L.

Damp woods. McDowell : near Elkhorn. 
A. purpurascens L. Purple Milk-weed.

Damp grounds. Fayette: near Nuttallburg (Nuttall); near Quinnimont. Hardy: near Moorefield (Gamble).

A. VARIEGATA L.

Dry woods. Wirt: near Elizabeth. Upshur: near School House Summit. Hardy: near Moorefield (Gamble). Fayette: near Nuttallburg (Nuttall). Kanawha: near Charleston (Holton).

A. Incarnata L. Swamp Milk-weed.

Wet places. Wirt: near Burning Springs. Gilmer : along Tanner's Fork. Randolph: along Tygart's Valley River, alt. I,963-2,200 ft. Fayette: near Nuttallburg (Nuttall). Monongalia: near Stewartown. Summers: near Hinton. Kanawha: near Charleston. Marion: near Worthington Upshur: near Buckhannon (Pollock).

A. PULCHRA Ehrh.

Hardy: near Moorefield (Gamble).

A. Syriaca L. Milk-weed.

(A. Cornuti, Dec.).

Fields and roadsides. Common throughout the State, even in the wildest portions. Preston : near Aurora ( $M r . \&$ Mrs. Steele).

A. exaltata (L.) Muhl. Poke Milk-weed. (A. phytolaccoides, Pursh.).

Moist copses. Randolph: near Valley Bend; on Point Mountain, alt. I,963-3,300 ft. Preston: near Terra Alta. Grant: near Bayard. Tucker: near Davis. Fayette: near Nuttallburg (Nuttall). Preston: near Aurora (Mr. \& Mrs. Steele).

A. amplexicaulis J. E. Smith.

(A. obtusifolia Mx.)

Dry fields, Mason: near Point Pleasant (Sheldon).

A. QUADRIFolia Jacq. Four-leaved Milk-weed.

Open woodlands. Mineral: near Keyser (Workman). Kanawha: near Charleston (Barnes). Gilmer: near Glenville (Mapel). Fayette: near Nuttallburg (Nuttall). Monongalia: near Mapel Run (Brown). Summers: near Hinton. Hardy: near Moorefield (Gamble).

\section{ACERATES Ell.}

A. viridiflora (Raf.) Eaton. Green-flowered Milk-weed.

Berkeley: near Martinsburg (Mertz \& Guttenberg). Mineral: near Keyser. Jefferson : near Charlestown. Jackson: along Limestone Ridge. 
GONOLOBUS Michx.

G. LAEvis Michx.

Climbing over weeds and fences. Mason: near Point Pleasant. Putnam: near Buffalo. An abundant weed about Charleston, Kanawha County (Boutlou); in the Salinas (Holton).

\section{CON VOL V U L A C E AE.}

QUAMOCLIT Moench.

Q. coccinea (L.) Moench. Scarlet Morning-glory. (Ipomoea L.)

Waste grounds. Monongalia: near Morgantown. Máson: near Point Pleasant.

\section{IPOMOEA L.}

I. HEDERACEA Jacq. Ivy-leaved Morning-glory.

Waste places. Mason: sandy banks of the Ohio near Point Pleasant. Fayette: near Nuttallburg, banks of New River (Nuttall). Berkeley: near Martinsburg. Hardy: near Moorefield (Gamble). Kanawha: at the Salinas (Holton).

I. PURPUREA (L.) Roth. Morning-glory.

Fields, cultivated grounds and waste places. Mineral: near Keyser (Workman). Fayette: near Nuttallburg (Nuttall). Wood: near Lockhart's Run. Monongalia: near Morgantown. Jackson: near Sandyville. Mason and Putnam: an abundant weed in corn fields. Mineral Co., opposite Cumberland, Md. (Small).

I. Pandurata (L.) Meyer. "Wild Sweet Potato."

Fields, roadsides, and waste places. Monongalia: near Stewartown, Morgantown and Glenville. Marion: near Houghtown, Opekiska, and Fairmont. Wood: near Kanawha Station and Lockhart's Run. Gilmer: near Glenville (Mapel). Fayette: near Nuttallburg (Nuttall). Greenbrier: near White Sulphur Springs. Monroe: near Alderson. Mason: near Point Pleasant. Summers : near Hinton. Hardy: near Moorefield (Gamble).

Reported as a troublesome weed, from the following localities: Marion: near Canton, Farmington, Barracksville, Eldora, and Worthington. Taylor: near Grafton. Harrison: near Clarksburg, Bridgeport, Good Hope, Mt. Clair, and Wallace. Hampshire: near Slanesville, Concord, Three Churchs, Bloomery, Dillon's Run, and Springfield. Jefferson: near Summit Point, Shenandoah Jc., Middleway, and 
Kabletown. Jackson: near Douglass, Lone Cedar, Grass Lick, Garfield, Wilding, Odaville, Belgrove, and Kentuck. Ohio: near Alum Grove. Hancock: near New Cumberland. Lincoln: near Hamlin. Wood: near Waverly, Belleville, Tyner, Jerry's Run, Fountain Spring, Blennerhassett, Murphy's Mills, Deer Walk, and Rockport. Lewis : near Vadis, and Aberdeen. Wirt: near Burning Springs, Morris, Elizabeth, and Reedy Ripple. Summers: near Forest Hill, Talcott, Clayton, and Indian Mills. Preston: near Masontown, and Reedsville. Wetzel: near Endicott, Pine Grove, New Martinsville, and Blake. Mineral: near Patterson's Depot, and Piedmont. Berkeley: near Oakton, Martinsburg, and Gerardstown. Webster : near Replete. Ritchie: near Ritchie C. H., and Highland. Mercer: near Princeton, Concord Church, Bramwell and New Hope. Cabell: near Union Ridge and Milton. Kanawha : near Pocotaligo, Blandon and Gazil. Monroe: near Cashmere, and Johnson's X Roads. Wayne: near Adkin's Mills, and Stone Coal. Randolph: near Florence. Doddridge: near Smithton, Center Point, and Leopold. Fayette: near Fayetteville. Braxton: near Bulltown, Lloydsville, Frametown, and Newville. Tyler: near Wick, and Long Reach. Roane: near Newton, Looneyville, Clio, Reedy, Walnut Grove and Peneil. Upshur: near Evergreen, Kanawha Head, and Overhill. Barbour: near Pepper. Marshall: near Meighen, and Welcome. Grant: near Medley, and Greenland. Raleigh: near Egeira, and Raleigh C. H. Greenbrier: near Traut Valley, and White Sulphur Springs. McDowell: near Squire Jim. Mason: near Maggie. Taylor: near Thornton, and Meadland. Brooke: near Wellsburg, and Fowler's. Pleasants: near Schultz. Putnam: near Carpenter's. Hardy: near Old Fields. Clay: near Valley Fork.

I. LACUNOSA L.

Fayette: sandy banks of New River near Nuttallburg (Nuttall). Kanawha: at the Salinas (Holton). Jefferson: near Harpers Ferry (Merriam). Summers: near Hinton.

\section{CONVOLVULUS L.}

C. spithamaeus L.

Rocky soil. Mineral: near Keyser (Workman).

C. Sepium L. Hedge Bindweed.

Alluvial soils. Monongalia: near Little Falls; and along

Decker's Creek. Mason: near Point Pleasant.

C. REPENS L.

Rocky river banks. Fayette: banks of New River near Nuttallburg (Nuttall). Monongalia: below Morgantown. 
CU S C U T A C E AE.

\section{CUSCUTA L.}

C. Epithymum Murr. Clover Dodder.

(C. trifolii Bab.).

Parasitic on Clover. Greenbrier: near White Sulphur Springs.

C. ARVENSIS Beyrich.

On Ambrosia artemisiaefolia in very dry grounds below

Baileysville, Wyoming County, alt. I,I50 ft., August I3I9, I900 (Morris, I203a).

C. Gronovir Willd. Dodder.

Parasitic on grasses, sedges, and low weeds. Preston: near Aurora (Mr. \& Mrs. Steele). Kanawha: opposite Charleston (Holton). Frequent in wet places throughout the State.

C. PARADOXA Raf.

(C. glomerata Choisy.)

Parasitic on Compositae. Monongalia: near Little Falls.

POLEM ON I A E AE.

PHLOX L.

P. PANiculata Linn.

Fayette: near Nuttallburg (Nuttall).

P. paniculata, Var. acuminata (Pursh.) Chapm.

Monroe: banks of Greenbrier River (Nuttall). Preston: near Aurora ( $M r$. \& Mrs. Steele). Tucker: rich woods near Parsons (Greenman 273).

P. maculata L. Wild Sweet William.

Grassy woodlands along streams. Wirt: above Burning Springs, plentiful. Upshur: near Lorentz. Randolph: along Tygart's Valley River from Beverly to Valley Head. Hardy: near Moorefield (Gamble).

P. AMOENA Sims.

Dry open woods. Fayette: near Nuttallburg, frequent (Nuttall).

P. REPTANS Michx.

Deep damp woods. Gilmer: near Glenville (Mapel). Grant: near Bayard. Tucker: near Davis. Fayette: nea: Nuttallburg (Nuttall). Mercer: near Bluefield. Upshur : near Buckhannon (Pollock).

P. DIVARICATA L.

M. \& G.

Rocky woods. Monongalia : near Morgantown and Stumptown. Gilmer: near Glenville (Mapel, Brozen). Hardy: near Moorefield (Gamble). Fayette: near Nuttallburg (Nuttall). Mercer: near Bluefield. Lewis: (Pollock). 
P. subulata L. Moss Pink.

Dry rocky places. Mineral: near Keyser (Workman). Fayette: near Hawk's Nest (James). Monongalia and Marion: near the F. M. \& P. R. R. Hardy: near Moorefield (Gamble; F. E. Brooks). Mercer: near Bluefield. - Greenbrier Co., dry, stony ledges on Kate's Mountain, alt. 3,300 ft. (Small; Heller 812).

P. Britronir Small, Bull. Torr. Club, 27 :279. (1900).

Perennial, deep green: Stems copiously branched; branches matted, forming wide tufts, glandular-pilose: leaves numerous, small ones often clustered in the axils of the larger; blades subulate or narrowly linear-subulate, 5-10 $\mathrm{mm}$. long, ciliate, especially near the base: calices $5-6 \mathrm{~mm}$. long, glandular-pubescent like the branches; segments subulate, about as long as the tube: corolla white: tube curved, about $\mathrm{Icm}$. long; limb I2-I $3 \mathrm{~mm}$. wide; segments cuneate, with two pale magenta spots near the base, cleft by a Vshaped sinus about $3 \mathrm{~mm}$. deep, usually with a minute tooth in each sinus, tips acute or acutish.

A relative of $\mathrm{P}$. subulata but more delicate in all its parts. (Note the contrasting characters as tabulated in the origina! description of the species).

The specimens upon which the species is based were collected by Dr. N. L. Britton, at White Sulphur Springs, Greenbrier County, May I898.

\section{POLEMONIUM L.}

P. REPTANS L. Greek Valerian.

Damp woods. Monongalia: in Brand's Woods near Easton, where it is used by the people of that neighborhood as a stomachic and tonic. Rich woods, near Morgantown. Gilmer : near Glenville (Mapel).

P. Van Bruntiae Britton. Jacob's Ladder. (P. coeruleum A. Gray.

Moist opens. Preston : near Cranberry Summit (Mertz \& Guttenberg).

H Y D R O P H Y L L A CE AE.

\section{HYDROPHYLLUM L.}

H. macrophyllum Nutt. Small-leaved Water-leaf

Rich woods. Gilmer: near Glenville (Mapel). Wetzel: near Burton (Mertz \& Guttenberg). 
H. Virginicum L. Water-leaf.

Rich woods. Monongalia and Marion: along the Monongahela River. Ohio: near Wheeling (Mertz \& Guttenberg). Hampshire: near Romney.

H. Canadense L. Canadian Water-leaf.

Damp rich woods. Monongalia and Marion: along the Monongahela River. Fayette: near Kanawha Falls (James). Ohio: near Wheeling (Mertz \& Guttenberg).

H. appendiculatum Michx.

Ohio: Thomas' Hill, near Wheeling (Mertz \& Guttenberg). Mineral: near Keyser (Workman). Grant: near Bayard. Tucker: near the Falls of Blackwater.

\section{PHACELIA Juss.}

P. Bipinnatifida Michx.

Rich soil. Fayette: near Nuttallburg, on or among damp mossy rocks (Nuttall).

P. Purshin Buckley.

Moist wooded banks. Monongalia: banks of Decker's Creek. Mineral: near Keyser-(Workman). Gilmer: near Glenville (Mapel).

P. DUbia (L.) Small.

(P. parviflora Pursh.).

Shaded banks. Fayette: near Nuttallburg (Nuttall).

B O R A G I N A C E AE.

HELIOTROPIUM Linn.

H. INDICUM Linn.

Established on waste grounds near Harpers Ferry, Jefferson County (Merriam).

\section{CYNOGLOSSUM L.}

C. officinale L. Hound's Tongue. "Dog-burr."

Waste places and roadsides. Jefferson: near Shenandoah Jc. Gilmer: near Glenville (Brown). Hardy: near Moorefield (Gamble). Mercer: generally frequent.

C. Virginicum L. Wild Comfrey.

Wood openings. Calhoun: near White Pine. Grant: near Bayard. Preston: near Terra Alta. Monongalia: near Morgantown. Gilmer: near Glenville ( $\mathrm{Mapel}$ ). Summers: near Hinton. 
LAPPULA Moench (I794).

(Echinospermum Sw. I8I8.)

L. Virginiana (L.) Greene. Beggar's-lice.

Borders and thickets. Frequent throughout the State.

\section{MERTENSIA Roth.}

M. VirginicA (L.) DC. Virginian Cowslip. Lung-wort. Bluebells.

Rich woods. Monongalia and Marion; along the Monongahela River. Lewis: along Stone Coal Creek: Wirt: near Elizabeth. Upshur: near Laurentz (Millspangh, Pollock). Gilmer: near Glenville (Mapel, Brown). Hardy: near Moorefield (Gamble).

\section{ONOSMODIUM Michx.}

O. Carolinianum (Lam.) DC.

Summers: banks of New River near Hinton.

\section{MYOSOTIS L.}

M. PALUSTRIS (L.) Lam. Forget-me-not.

Damp places. Jefferson: near Harper's Ferry (Mertz \& Guttenberg).

M. LAXA Lehm.

About a spring near Kegley, Mercer County, alt. 2,09o ft., July 21, I900 (Morris IO4I).

M. ARvensis (L.) Lam. Scorpion grass.

Grassy places. Randolph: near Helvetia (Guttenberg).

\section{SYMPHYTUM L.}

S. officinale L. Comfrey.

Waste places. Gilmer: near Glenville ( $\mathrm{Mapel}$ ). Mercer: near Ingleside.

\section{LITHOSPERMUM L.}

L. ARvense L. Corn Cromwell.

Fields. Ohio: near Wheeling (Mertz \& Guttenberg). Fayette: near Nuttallburg (Nuttall).

L. Latifolium Michx. Stone-seed.

Ohio: Cowan's Hill near Wheeling (Mertz \& Guttenberg). Fayette: near Nuttallburg; rare, not found in r89I (Nuttall). Monongalia: near Little Falls (Walker). 
L. Canescens (Michx.) Lehm. Puccoon.

Open woods. Mineral : on Knobby Mountain (Workman). Hardy: near Old Fields (Hopkins); and Moorefield (Gamble). Hampshire: near Romney (Millspaugh).

\section{ECHIUM L.}

E. vulgare L. "Blue Weed." "Blue Devils." "Blue Thistle." "Blue Stem."

Fields and waste ground. Jefferson: abundant especially near Charlestown, where there are many fields absolutely blue with the plant. Dr. Gray says of his trip through this country: "From the moment we entered the valley, we observed such immense quantities of Echium vulgare, that we were no longer surprised at the doubt expressed by Pursh whether it were really an introduced plant;" near Shenandoah Junction; Shepherdstown and Harper's Ferry. Randolph: along Tygart's Valley River; near Huttonsville, and up Riffles Creek. Berkeley: near Martinsburg, plentiful. Morgan: near Hancock, Cacapon and Orleans X Roads. Mineral: near Keyser, and Piedmont. Fayette: near Kanawha Falls (James); near Nuttallburg (Nuttall). Kanawha: opposite Coalburg. Summers : near Hinton. Jefferson : near Summit Point. Greenbrier: near White Sulphur Springs. Hardy: near Moorefield.

Also reported from: Jefferson : near Ripon, Summit Point, Middleway and Kabletown. Berkeley: near Oakton and Hedgesville. Morgan: near Rock Gap. Hampshire: near Slanesville, Concord, Romney, Three Churches, Dillon's Run, Higginsville, Sedan, Purgitsville and Springfield. Mineral: near Blaine. Hardy: near Moorefield, Wardensville and Old Fields. Grant: near Medley, Greenland and Petersburg. Tucker: near Hendricks and St. Georges. Pendleton : near Franklin and Upper Tract. Pocahontas: near Lobelia. Greenbrier: near Williamsburg and Fort Spring. Summers • near Talcott. Mercer: near Princton and New Hope. McDowell: near Squire Jim. Wood: near Waverly. Lewis: near Vadis and Aberdeen. Barbour: near Old Field. Webster: near Replete. Wetzel: near Endicott. Doddridge: near Smithton. Marshall: near Lowdenville. Roane: near Clio and Walnut Grove. Kanawha : near Tornado. Taylor: near Thornton; and Clay: near Valley Fork. 


\section{VERBENA L.}

V. officinalis L. Vervain.

Waste grounds. Jefferson: plentiful at Harpers Ferry (Mertz \& Guttenberg; Greene).

V. URTicaefolia L. White Vervain.

Waste or open grounds. Common throughout the State.

V. hastata L. Blue Vervain.

Damp waste grounds and roadsides. Frequent throughout the State. In some regions rare.

V. Angustifolia Michx.

Roadsides and waste places. Throughout Jefferson County. Hardy : near Moorefield (Gamble).

\section{LIPPIA L.}

L. Lanceolata Michx. Fog Fruit.

Waste grounds. Ohio: near West Wheeling (Mertz \& Guttenberg). Mason: banks of the Ohio River near Point Pleasant.

\section{A B I A T AE.}

ISANTHUS Michx.

I. BRachiatus (L.) B. S. P. False Pennyroyal. (I. coeruleus, Michx.).

Mineral: opposite Cumberland, Md. (Mertz \& Guttenberg).

\section{TEUCRIUM L.}

T. Canadense L. Germander. Wood Sage.

Low grounds. Wood: ditches near Kanawha Station. Jackson: near Sandyville. Gilmer: near Glenville (Mapel). Cabell: near Barboursville (James). Fayette: near Nuttallburg, profile of expanded flower resembles a deer's head (Nuttall). Monongalia: near Ice's Ferry. Hardy: near Moorefield (Gamble).

\section{TRICHOSTEMA L.}

T. діснотомum L. Bastard Pennyroyal.

Dry fields. Mason: near Brighton. Hardy: near Moorefield (Gamble). 


\section{SCUTELLARIA L.}

S. LAteriflora L. Mad-dog Skull-cap.

Wet shady places. Frequent throughout the State.

S. CORDIFOLIA Muhl. var. MINOR (Chapm.). (S. versicolor minor Chapm.).

Rich soil. Fayette: near Nuttallburg (Nuttall). On .visiting Mr. Nuttall's station for this species, a moss covered boulder, I was impressed with the great beauty of this little skull-cap, which, in its mossy bed, resembled a bright blue bit of color upon a Fairy's palette.

S. SAXATILIs Riddell.

Moist shady banks. Fayette: near Nuttallburg (Nuttall); along the north bank of the Great Kanawha River near Kanawha Falls. Jefferson: under cliffs along the Shenandoah River at Harpers Ferry (Greene).

S. SERRATA Andrews.

Woodlands: Kanawha: near Charleston (Barnes). Putnam: near Buffalo. Fayette: near Nuttallburg (Nuttall). S. INCANA Muhl.

(S. canescens Nutt.).

Ditches and moist places. Wirt: near Elizabeth. Kanawha: up 8 Mile Creek (Millspaugh). Upshur: near Buckhannon (Pollock).

S. PILOSA Michx.

Dry mountain sides. Fayette: near Nuttallburg (Nuttall). Var. HiRsuta (Short) Gray.

With the preceding. Fayette: near Nuttallburg (Nuttall). S. INTEGRIFOLIA L.

Low grounds. Wood: near Kanawha Station, abundant. S. PARvula Michx.

Sandy banks. Wood: near Parkersburg (Mertz \& Guttenberg). Mason: near Point Pleasant.

S. GALERICULATA L.

Wet shady places. Kanawha: near Charleston (Barnes); near Pocataligo. Jackson: near Fisher's Point. Gilmer . near Fisher's Point; near Glenville (Browm).

Waste grounds, escaped from.gardens. Randolph: near Forma Albiflora Millsp. Prel. Cat. Fl. W. Va., $42 \delta$.

Kanawha: near Charleston (Barnes).

S. NERvosA Pursh.

Moist thickets. Monongalia: on The Flats near Morgantown (Millspaugh). Ohio: near Wheeling (Mertz 2062). 


\section{MARRUBIUM L.}

M. vUlgare L. Horehound.

Waste grounds, escaped from gardens. Randolph: near Ford's. Jefferson: near Shepherdstown, plentiful.

AGASTACHE Clayt. ( 1762 ).

(Lophanthus. Benth, I834.)

A. Nepetoides (L.) Kuntze. Giant Hyssop.

Ohio: near Wheeling (Mertz \& Guttenberg).

A. scrophulariaefolia (Willd.) Kuntze.

Woods and thickets. Preston: near Aurora (Mr. \& Mrs. Steele).

\section{MEEHANIA Britton.}

M. CORDATA (Nutt.) Britton

Moist, shady ravines. Kanawha: near Charleston (Barnes). Fayette: near Kanawha Falls (James). Gilmer: near Glenville (Mapel; Brown). Randolph: summit of Point Mountain, alt. 3,700 ft. Monongalia: near Round Bottoms; opposite Little Falls. Fayette: near Nuttallburg (Nuttall). Upshur: near Buckhannon (Pollock). Taylor: near Grafton (Canby). Hancock: near Fairview (Hill).

\section{NEPETA L.}

N. Cataria L. Catnip.

Roadsides and waste places. Common throughout the State.

Found at various points in the higher Alleghanies, remote from dwellings.

\section{GLECOMA L.}

G. HEDERACDA L. Ground Ivy. Gill-over-the-ground. (Nepeta Bth.).

Abundant throughout the settled portions of the State.

\section{PRUNELI.A L.}

P. vUlgaris L. Heal-all.

All situations, common throughout the State.

Forma ALBIFLoRA (Boggenhard) Britt.

Jackson: on Limestone Ridge. 
SYNANDRA Nutt.

S. Hispidula (Michx.) Britton.

Wet places. Ohio: near Wheeling (Mertz).

PHYSOSTEGIA Benth.

P. Virginiana (L.) Benth. False Dragon-head.

Wet places. Fayette: near Nuttallburg (Nuttall); near Kanawha Falls (James).

\section{GALEOPSIS L.}

G. Tetrahit L. Hemp Nettle.

Waste places. Preston: near Terra Alta; near Cranberry Summit (Mertz \& Guttenberg); near Aurora (Mr. \& Mrs. Steele).

\section{LEONURUS I.}

L. CARdiaca L. Motherwort.

Waste places near dwellings. Monongalia: near Ice's Ferry. Hardy: near Moorefield. Mercer: near Princeton. Jefferson: near Shenandoah Jc.

\section{LAMIUM L.}

L. Amplexicaule L. Dead Nettle.

Escaped from Gardens. Fayette: near Nuttallburg (Nuttall). Monongalia: plentiful on the College Campus.

L. maculatum L.

Escaped and established from gardens. Preston: at Bruceton (Rumsey).

L. PURPUREUM Linn.

Established in waste grounds near Morgantown, Monongalia County (Sheldon 4267 ).

\section{STACHYS L.}

S. TENUIFOLIA Willd.

In a moist field, Monongalia: near Sabraton (Sheldon 4322).

S. palustris L. Hedge Nettle.

Wet grounds. Gilmer: near Glenville (Brown).

S. ASPERA Michx.

Damp places. Fayette: near Nuttallburg (Nuttall). 
Var. GLABRA Gray.

Damp places. Mason: banks of the Ohio River near Point Pleasant, common.

S. CORDATA Ridd.

Rocky thickets. Wirt: near Elizabeth.

\section{SALVIA L.}

M. Lyrata L. Sage.

Meadows. Monongalia: near Morgantown. Fayette: near Nuttallburg (Nuttall). Gilmer: near Glenville ( $M a$ pel). Mercer: near Ingleside.

MONARDA L.

M. didyma L. Bee-balm. Oswego-Tea.

Mòist places. Randolph : near Cheat Bridge, alt. 3,350 ft. : near Valley Head. Mineral : near Davis. Grant and Tucker on W. Va. Central R. R. Monroe: near Alderson. Hardy: near Moorefield (Gamble). Randolph: near Pickens (H. H. Smith 1409). Preston: near Aurora (Mr. \& Mrs. Steele).

M. Fistulosa L. Wild Bergamot.

Dry soils. Wirt: near Elizabeth. Gilmer: near DeKalb, abundant; near Glenville ( $\mathrm{Mapel}$ ). Randolph: summit of Point Mountain, alt. 3,700 ft. Monongalia : near Ice's Ferry. Fayette: near Nuttallburg (Nuttall); near Kanawha Falls (James). Kanawha: near Coalburg (James). Greenbrier: near White Sulphur Springs. Marion: near Fairmont. Hardy: near Moorefield (Gamble).

M. MEDIA Willd.

Moist grounds. Mineral: along Abraham's Creek. Summers: near Greenbrier Stockyards. Monroe: near Alderson.

M. SCABRA Beck.

Shady places. Fayette: near Nuttallburg (Nuttall).

M. Clinopodia L. Basil Balm.

Woods and thickets, Webster: near Tater Knob; and Nicholas: near Richwood ( $H$. H. Smith I4I4, I52I, I744). Preston: near Aurora (Mr. \& Mrs. Steele).

\section{BLEPHILIA Raf.}

B. hirsuta (Pursh.) Torrey.

Fields and fence rows. Randolph: summit of Rich Mountain, alt. 3,000 ft. Fayette: near Hawk's Nest (James). Preston: near Terra Alta. 
B. ciliata (L.) Raf.

On dry banks at Bargers Spring, Summers County, alt. I,500 ft., July I3, I900 (Morris, 999); thickets near Kegley, Mercer County, alt. 2,100 ft., July 27, I900 (Morris. 1067).

\section{HEDEOMA Pers.}

H. PUlegioides (L.) Pers. American Pennyroyal.

Dry fields and woods. Common throughout the State.

\section{MELISSA L.}

M. officinalis L. Balm.

Escaped from gardens. Kanawha: up 8-Mile Creek. Fayette: near Nuttallburg (Nuttall).

\section{CLINOPODIUM L. (I753).}

(Calamintha Moench. I794.)

C. Calamintha (L.) Kuntze. Basil.

Dry soils. Upshur: near Buckhannon. Fayette: near Nuttallburg, rare (Nuttall). Randolph: near Crickard P. O.

C. vUlgare Linn. Basil.

Roadside, Randolph: near Pickens (H. H. Smith I368, I476). Preston: near Aurora (Mr. \& Mrs. Steele).

KOELLIA Moen. ( I794).

(Pycnanthemum, Michx. 1803.)

K. Flexuosa (Walt.) MacM.

(P. linifolium Pursh.)

Dry grounds. Wood: near Kanawha Station and Lockhart's Run. Fayette: near Kanawha Falls (James); near Nuttallburg (Nuttall). Preston: near Terra Alta. Summers: near Hinton. Monroe: near Alderson. Upshur: near Buckhannon (Pollock).

K. verticillata (Michx.) Kuntze.

(P. Torreyi Bth.).

Dry soil. Fayette: near Nuttallburg (Nuttall). Summers: near Hinton.

K. CLinopodioides (T. \& G. ) Kuntze.

Dry soil. Nicholas: near Beaver Mills, alt. 2,125 ft.

K. Pycnanthemoides (Leavenw.) Kuntze. (P. Tullia Bth.).

Open woods and banks. Fayette: near Nuttallburg, common (Nuttall). 
K. Incana (L.) Kuntze. Mountain Mint.

Dry soils. Wirt: near Burning Springs and Elizabeth

Monongalia: near Morgantown. Fayette: near Nuttallburg (Nuttall).

K. montana (Michx.) Kuntze.

Rocky river banks. Fayette: near Nuttallburg, rare. Not found in I89I (Nuttall).

K. mutica (Michx.) Britton.

Sandy soils. Webster: Hacker Valley (H. H. Smith I 557).

K. Virginiana (L.) McM.

Dry roadsides and pasture lands. Randolph: near Elkins (Greenman 473).

\section{CUNILA L.}

C. origanoides (L.) Britton. Dittany. (C. Mariana L.).

Dry hillsides. Gilmer: near Glenville ( $\mathrm{Mapel}$ ). Fayette: near Nuttallburg (Nuttall). Wetzel: near Burton (Mertz \& Guttenberg).

\section{LYCOPUS L.}

L. UNIFLORUS Michx.

L. communis and membranaceus of Bicknell. Randolph: near Cheat Bridge (Sheldon 2609).

L. Virginicus L. Bugle Weed.

Low, wet grounds. Common throughout the State.

L. Americanus Muhl. (L. sinuatus Ell.).

Low, wet ground. Mason: near Point Pleasant.

\section{MENTHA L.}

M. spicata L. Spearmint.

( $M$. viridis L.)

Low grounds and damp places. Frequent, even at the. higher altitudes.

M. PIPERITA L. Peppermint.

A frequent escape along springy brooklets. Gilmer: along Tanner's Fork. Randolph: on Point Mountain, alt. 3,050 ft. Jackson: near Sandyville. Gilmer: near Glenville (Mapel). Fayette: near Nuttallburg, alt. 2,000 ft. (Nuttall). Summers: near Hinton. Preston: near Aurora ( $M r . \&$. Mrs. Steele).

M. ARVEnsis glabrata (Benth.) Fernald.

Moist places. Randolph: along Gandy Creek (Greenman: 469). 
M. SATiva L. Whorled Mint.

Monroe: banks of Greenbrier River (Nuttall).

M. Canadensis L. Wild Mint.

Wet places. Randolph: along the road up Point Mountain, alt. 2,325 ft. Fayette: near Nuttallburg, rare (Nuttall). Tucker: near Hendricks (Greenman 243). Summers : near Hinton.

\section{COLLINSONIA L.}

C. Canadensis L. Rich-weed. Stone-root.

Rich, damp woods. Randolph: along Staunton pike up Riffles Creek, alt. 2,700 ft. Gilmer : near Glenville (Mapel). Fayette: near Nuttallburg, some plants with elliptical leaves, acute at both ends $3 \frac{\mathrm{T}}{2}$ in. wide by ro inches long ( Nuttall). Monongalia: near Camp Eden. McDowell: near Elkhorn. Preston: near Aurora (Mr. \& Mrs. Steele). Tucker: rich woods near Parsons (Greenman 319, 322).

PERILLA Ard.

P. frutescens (L.) Britton.

Established in waste grounds, Jefferson: at Harpers Ferry (Greene).

P. frutescens Nankinensis (Lour) Britton.

Escaped and established in waste places. Monongalia: near Morgantown (Millspaugh). Monroe: at Sweet Springs (Steele).

P. ocy moides L. var. crispa Bth.

Waste ground. Monongalia: near Morgantown.

$$
\text { S O L A N A E AE. }
$$

\section{SOLANUM L.}

S. Dulcamara L. Bitter-sweet.

Damp places. Frequent throughout the State, but not so much so as the next.

S. Nigrum L. Common Nightshade.

Fields, roadsides, and cultivated grounds. Common throughout the State.

S. Carolinense L. Horse Nettle. "Radical Weed."

Becoming a detestable weed in fields and forests. Calhoun : along Leading Creek. Wood: near Kanawha Station. Wirt: near Elizabeth. Randolph: near Cricard P. O. Webster: on Buffalo Bull Mountains. Nicholas: near Beaver Mills. Gilmer: near Glenville (Mapel). Fayette: near 
Nuttallburg (Nuttall). Monongalia: near Ice's Ferry. Cabell: near Barboursville. Greenbrier: near White Sulphur Springs. Monroe: near Alderson. Summers: near Hinton. Kanawha: near Charleston. Mason: near Point Pleasant. Jefferson: near Flowing Springs, and Shepherdstown. Mercer: near Ingleside.

Reported as a troublesome weed from: Harrison: neat Clarksburg, Wilsonburg, Good Hope, Mt. Clair, and Wallace. Ohio: near Elm Grove and West Liberty. Wood: near Waverly, Belleville, Deer Walk and Kanawha Station. Hardy: near Moorefield and Wardensville. Grant: near Medley and Petersburg. Jefferson : near Moore's and Kabletown. Summers: near Forest Hill and Talcott. Wetzel: near Endicott, Pine Grove, New Martinsville and Blake. Mineral: near Patterson's Depot, and Blaine. Wirt: near Burning Springs, Morris, Evelyn, and Reedy Ripple. Jackson: near Grass Lick, and Odaville. Cabell: near Union Ridge, and Barboursville, Taylor: near Knottsville. Wayne: near Stone Coal, and Adkin's Mills. Doddridge: near Smithton, and Center Point. Marshall: near Knoxville, and Welcome. Braxton: near Bulltown, and Tate Creek. Berkeley: near Hedgesville. Mercer: near Bramwell, and New Hope. Roane: near Looneyville, Clio, Reedy, and Peneil. Pocahontas: near Lobelia. Kanawha: near Blandon. Greenbrier: near Trout Creek. McDowell: near Squire Jim. Mason: near Maggie. Brooke: near Wellsburg. Marion: near Mannington. Taylor: near Grafton. Upshur: near Kanawha, Head, Overhill, and Hemlock. Hampshire: near Higginsville and Springfield. Tyler: near Long Reach. Webster: near Welch Glade. Clay: near Valley Fork. Randolph : railroad tracks at Elkins (Greenman 268). This species and Verbesina occidentalis were the commonest weeds throughout the southeastern counties (Morris).

S. Rostratum Dunal.

Waste places. Wayne: near Bowen (Rumsey).

S. TUBEROSUm Linn.

Established at the edge of a wood, Randolph: near Pickens ( $H$. H. Smith I442).

\section{PHYSALIS L.}

P. Philadelphica Lam.

Rich opens. Fayette: near Nuttallburg (Nuttall).

P. angulata L. Ground Cherry.

Open rich grounds. Grant: near Bayard. Gilmer : near Glenville (Mapel). Wood: near Kanawha Station. 
P. PUBESCENS L.

Low grounds. Fayette: near Nuttallburg (Nuttall). Ohio: near Wheeling (Mertz \& Guttenberg). Jefferson: near Shepherdstown. Mason: near Point Pleasant.

P. Virginiana Mill.

- Light sandy soils. Monongalia: near Beechwoods Jefferson: near Shepherdstown. Jackson: near Ripley. Hardy: near Moorefield (Gamble).

P. VISCOSA L.

Low grounds. Ohio: near Wheeling (Mertz \& Guttenberg). Hardy: near Moòrefield (Gamble).

P. Lanceolata Michx. town.

P. HETEROphyLLA Nees.

Fayette: near Quinnimont on bottom lands of New River (Pollard \& Maxon 38). Preston: near Aurora (Mr. \& Mrs. Steele).

$$
\begin{aligned}
& \text { PHYSALODES Boehm. ( } 1760) . \\
& \text { (Nicandra, Adans, I763). }
\end{aligned}
$$

P. Physalodes (L.) Britton. Apple-of-Peru.

Waste grounds. Lewis: near Weston. Mineral: near Piedmont.

\section{LYCOPERSICON Mill.}

L. LyCopersicon (L.) Karst.

Established on the banks of Tygarts Valley River below Huttonsville (Greenman 2I4).

\section{LYCIUM L.}

L. vulgare (Ait. f.) Dun. Matrimony Vine.

A frequent and persistent escape. Berkeley : near Martinsburgh. Jefferson:- near Shepherdstown. Mason: banks of the Ohio near Point Pleasant.

\section{DATURA L.}

D. Stramonium L. "Jimson-weed." Jamestown-weed. Stinkweed.

Waste places. A common weed throughout the State.

D. tatula L. Purple Thorn-apple.

With the last. Common throughout the State. 
PETUNIA Juss.

P. violacea Lindl.

Escaped to waste grounds. Monongalia: near Morgantown, common, where it persists annually. Mason: near Point Pleasant. Jefferson: near Shepherdstown.

S C R O P H ULA R I A C E AE.

VERBASCUM L.

V. Thapsus L. Mullein.

Old fields and pastures. Common throughout the State. V. Blattaria L. Moth Mullein.

Fields and waste places. Wirt: along the Little Kanawha River. Gilmer: near Glenville (Mapel). Webster: on Tater Knob; and Randolph: near Pickens (H. H. Smith I505, I 547). Fayette: near Nuttallburg (Nuttall). Monongalia and Marion, common. Grant: near Bayard. Jefferson: near Charlestown. Berkeley: near North Mountain. Preston: near Aurora (Mr. \& Mrs. Steele).

V. Lychnitis L. Yellow Moth-mullein.

Fields and wastes. Kanawha: roadside, up 8 Mile Creek Mineral: opposite Cumberland, Md.

\section{LINARIA Hill.}

L. Linaria (L.) Karst. Toad Flax. "Devil's Flax." "Wild Flax." "Indian Hemp." "Impudent Lawyer."

Fields, roadsides and waste places. Monongalia: neat Stewartown. Jefferson: near Charlestown and Shenandoah Junction. Jackson: near Sandyville. Berkeley: near North Mountain. Mineral: near Piedmont and Keyser. Hardy: near Moorefield (Gamble). This species first appeared near near Nuttallburg in Fayette Co., this year-I895 (Nuttall).

Also reported as a weed from: Harrison: near Good Hope. Ohio : near West Liberty. Wood: near Jerry's Run, Fountain Spring, Blennerhassett, and Rockport. Jefferson: near Molers. Wetzel: near Endicott. Mineral: near Piedmont. Wirt: near Burning Spring, Morris and Reedy Ripple. Jackson: near Lone Cedar, Garfield, and Belgrove. Cabell: near Union Ridge. Taylor: near Thornton and Meadland. Wayne: near Stone Coal. Marshall: near St. Joseph. Berkeley: near Martinsburg. Greenbrier: near White Sulphur Springs. Mason: near Grimm's Landing and Maggie. Upshur: near Kanawha Head and Overhill. Tyler: near Long Reach. Webster: near Welch Glade. 
Preston: near Tunnelton and Terra Alta. Hancock: near New Cumberland and Fairview. Ritchie: near Ritchie C. H. Monroe: near Pickaway. Morgan: near Rock Gap. Tucker: near Texas. Raleigh : near Raleigh C. H.

CYMBALARIA Medic.

C. Cymbalaria (L.) Wettst.

Thoroughly established in Harpers Ferry, Jefferson County, and the neighborhood; where it hangs in heavy masses from wall crevices (Greene).

\section{SCROPHULARIA L.}

S. Marilandica L. Figwort.

Fields and waste places. Monongalia: near Morgantown. Fayette: near Nuttallburg (Nuttall). Greenbrier: near White Sulphur Springs. Kanawha: near Charleston (Millspaugh). Preston: near Aurora (Mr. \& Mrs. Steele).

S. LEPORELla Bicknell.

Woods and roadsides. Monongalia: quite a patch of this; species near Morgantown (Sheldon 4458).

\section{COLLINSIA Nutt.}

C. VERNA Nutt.

Moist soil. Gilmer: near Glerville (Mapel, Brown). Ohio: on Wheeling Hill (Mertz \& Guttenberg). Monongalia : near Cassville.

\section{CHELONE L.}

C. GLAbra L. Snake Head.

Wet places. Gilmer: near Glenville (Mapel). Fayette : near Nuttallburg, uncommon (Nuttall). Wirt: near Burning Spring. Preston: near Aurora (Mr. \& Mrs. Steele).

C. OBLIQUA L.

Wet places. Randolph: along Cheat River near Cheat Bridge. Monongalia: near Camp Eden.

\section{PENTSTEMON Soland.}

P. Hirsutus (L.) Willd. Beard-tongue. (P. pubescens Sol.). Dry or rocky places. Kanawha: near Charleston (Barnes). Gilmer: near Glenville ( $\mathrm{Mapel}$ ). Cabell: near Barboursville (James). Hardy: near Moorefield (Gamble). Hampshire: near Doe's Gully. 
P. CANEscens Britton.

Slopes of Kate's Mountain, alt. 3,300 ft., near White Sulphur Springs, Greenbrier Co. (Small; Eggleston 4353).

P. Digitalis (Sweet) Nutt.

Rich soil. Monongalia: along the Monongahela River, frequent. Wood: near Kanawha Station. Fayette: near Kanawha Falls (James). Hardy: near Moorefield. Hamp. shire: near Doe's Gully. Rich soil. Fayette: near Nuttall. burg, in open woods (Nuttall).

\section{MIMULUS L.}

M. RINGEns L. Monkey Flower.

Wet places. Upshur: along Stone Coal Creek. Fayette: near Kanawha Falls (James); near Nuttallburg (Nuttall). Randolph: along Tygarts Valley River. Frequent throughout the State.

M. ALAtus Soland. Winged Monkey Flower.

Wet places. Greenbrier: near White Sulphur Springs Putnam: near Buffalo.

\section{GRATIOLA L.}

G. Virginiana L. Gratiola.

Ditches. Common throughout the State.

G. SPHAEROCARPA Ell.

Damp places. Fayette: near Nuttallburg, on mossy banks in bed of creek (Nuttall).

\section{ILYSANTHES Raf.}

I. DubiA (L.) Barnhart. False Pimpernel. (I. gratioloides Bth.). Wet places. Fayette: near Kanawha Falls (James). Along Little Kanawha River (Mertz \& Guttenberg). Preston: near Aurora (Mr. \& Mrs. Steele). Kanawha: at the salinas (Holton).

\section{VERONICA L.}

V. Anagallis aquatica L. Water Speedwell.

Banks and ditches. Fayette: near Kanawha Falls (James).

V. Americana Schw. American Brooklime. "Wallink."

Brooks and ditches. Monongalia : the Flats near Morgantown. Randolph: on Point Mountain, alt. 3,050 ft. (where it is called "Wallink," and is used internally to bring out rashes) (Millspaugh); in Blister Swamp (Greenman 277). Webster: near Addison, alt. 2,000 ft. Mercer: near Beaver Springs. 
V. officinalis L. Speedwell. "Gypsy Weed."

Rich, deep woods and opens. Randolph: on Rich Mountains. Gilmer: near Glenville (Mapel). Kanawha: near Charleston (James). Fayette: near Nuttallburg (Nuttall). Tucker: dry mountain road near Parsons (Greenman 239). Monongalia: along Decker's Creek. Greenbrier: near White Sulphur Springs; and frequent throughout the State. Mercer: near Beaver Spr., and Bluefield.

V. SERPyllifolia L. Thyme-leaved Speedwell.

Roadsides, fields and lawns. Monongalia: near Morgantown. Gilmer: near Glenville ( $\mathrm{Mapel}$ ). Fayette: near Nuttallburg (Nuttall). Ohio: near Wheeling (Mertz \& Guttenberg). Mercer: near Bluefield.

V. PEREgrina L. Neck Weed. Purslane Speedwell.

Waste places. Monongalia: near Morgantown. Fayette: - near Nuttallburg (Nuttall). Ohio: Thomas Hill, near Wheeling (Mertz \& Guttenberg).

V. Arvensis L. Corn Speedwell.

Cultivated grounds. Gilmer: near Glenville (Mapel). Ohio: Thomas Hill, near Wheeling (Mertz \& Guttenberg). Fayette: near Nuttallburg (Nuttall).

\section{LEPTANDRA Nutt.}

L. Virginica (L.) Nutt. Culver's Physic.

(Veronica L.).

Rich woods and borders. Webster: Long Glade. Jackson: near Sandyville. Fayette: near Nuttallburg (Nuttall).

\section{DASYSTOMA Raf.}

D. Pedicularia (L.) Bth.

(Gerardia L.).

Hardy: near Moorefield (Gamble). Fayette: near Nuttallburg (Nuttall).

D. Virginica (L.) Britton. Oak-leaved Gerardia. (G. quercifolia Pursh.)

Open woods. Fayette: near Nuttallburg (Nuttall).

D. Flava (L.) Wood. False Foxglove. (Gerardia L.). Open woods. Wood: near Leachtown. Fayette: near Kanawha Falls and Hawk's Nest (James) ; near Nuttallburg (Nuttall). Gilmer: near Glenville ( $M a p e l)$. Hardy: near Moorefield (Gamble).

D. Laevigata Raf.

(Gerardia Raf.).

Oak woods. Fayette: near Nuttallburg, alt. 2,000 ft. (Nuttall). Preston: near Aurora (Mr. \& Mrs. Steele). 


\section{DIGITALIS Linn.}

D. PURPUREA Linn. Foxglove.

Thoroughly established in open woods, near Pickens, Randolph County (H. H. Smith 1236).

\section{GERARDIA L.}

G. tenuifolia Vahl. Slender Gerardia.

Dry soil. Mineral: near Keyser (Workman). Fayette: near Nuttallburg, alt. 2,000 ft. (Nuttall). Randolph: nea: Elkins.

G. auriculata Michx.

Fields. Monongalia: near Little Falls, with pure white flowers.

G. PAupercula (Gray) Britton.

Damp situations. Fayette: near Hawk's Nest (Holton). BUCHNERA L.

B. Americana L. Blue Hearts.

Moist, sandy ground. Putnam: near Buffalo.

CASTILLEJA Mutis.

C. coccinea (L.) Spreng. Painted Cup.

Sandy soils. Monongalia: along the Monongahela at Uffington, and below Morgantown. Randolph: near Valley Head. Preston: near Terra Alta. Hampshire: near Romney.

\section{PEDICULARIS L.}

P. Canadensis L. Louse-wort.

Copses, woods and banks. Preston: near Terra Alta. Fayette: near Nuttallburg (Nuttall). Gilmer: near Glenville (Mapel). Greenbrier: near White Sulphur Springs. Summers: near Hinton. McDowell : near Elkhorn. Upshur: near Buckhannon (Pollock).

\section{MELAMPYRUM L.}

M. Lineare Lam. Cow-wheat. (M. Americanum Michx.) Rich, open woods. Preston: near Terra Alta. 


\section{UTRICULARIA Linn.}

U. CORNUTA Michx. Bladderwort.

Bogs and borders of ponds. Pocahontas: Big Glade (Sheldon); Cranberry Glades (E. A. Brooks).

U. BIFLORA Lam.

Hardy: in a small bog near Moorefield (A. B. Brooks).

O R O B A N CH A CEAE.

LEPTAMNIUM Raf. (I8I8). (Epifagus Nutt. I8I8.)

L. Virginianum (L.) Raf. Beech-drops. Cancer-root.

Parasitic upon the roots of the beech. Wirt: near Elizabeth. Gilmer: near Glenvilie (Mapel). Fayette: near Nuttallburg (Nuttall). Monongalia: near Morgantown. Randolph: near Pickens (H. H. Smith). Preston: near Aurora (Mr. \& Mrs. Steele). Tucker: moist woods near Parsons (Greenman 261).

\section{CONOPHOLIS Wallr.}

C. Americana (L. f.) Wallr. Cancer Root.

Oak woods. Among fallen leaves. Ohio: near Wheeling (Mertz 1838). Monongalia: along Decker's Creek and near Little Falls. Gilmer: near Glenville (Mapel). Fayette: near Nuttallburg (Nuttall). McDowell: near Elkhorn. Mercer: near Bluefield. Summers: near Hinton (Eggleston $5569)$.

$$
\begin{aligned}
& \text { THALESIA Raf. I8I8). } \\
& \text { (Aphyllon A. Gray I848.) }
\end{aligned}
$$

T. Uniflora (L.) Britton. One-flowered Cancer-root.

Damp woodlands and opens. Monongalia : near Morgan. town. Wirt: near Elizabeth. Gilmer: near Glenville (Mapel). Fayette: near Nuttallburg (Nuttall).

B I G NON I A C E AE.

TECOMA Juss.

T. RadiCans (L.) DC. Trumpet-Creeper.

Moist soil. Monongalia: near Little Falls. Marion: near 
Fairmont. Fayette: near Nuttallburg (Nuttall). Webster: Hacker Valley (H. H. Smith I647). Summers: near Hinton. Mason: near Point Pleasant; and frequent throughout. the State.

\section{BIGNONIA L.}

B. CRUCIGERA Linn.

(B. capreolata Linn.)

Kanawha Co., rich soil between Cannelton and Brownstown, where it grows luxuriantly along the banks of the Great Kanawha River. Its evergreen foliage makes it a conspicuous feature of the winter landscape (Boutlou). Randolph: near Pickens.

\section{CATALPA Scop.}

C. Catalpa (L.) Karst. Indian Bean Tree. "Catawba." (C. bignonioides Walt.).

River banks. Marion: near Catawba, a place named after this tree, which would render it apparent that the tree was native here, which I hold to be true. Wood: near Leachtown. Gilmer: near DeKalb and along Leading Creek. Monongalia: near Ice's Ferry and Stewartown. Wirt: near Elizabeth. Upshur: along Big Sandy Run, alt. I,827 ft. Mason: near Point Pleasant. Webster: near Pugh $(H . H$. Smith I660).

C. SPECIOSA Warder.

Mason Co., banks of the Ohio River, near Point Pleasant.

$$
\text { A C A N T H A C E AE. }
$$

RUELLIA L.

R. CILIOSA Pursh.

Margins of woods. Wood: near Leachtown. Wirt: near Elizabeth. Calhoun: near Grantsville. Gilmer: near DeKalb. Lewis: along Stone Coal Creek. Upshur: near Laurentz. Nicholas: along the Gauley River. Kanawha: near Cannelton.

\section{DIANTHERA L.}

D. Americana L. Water Willow.

In streams. Ohio: near Wheeling (Michaux). Common throughout the State. 
PHRYMACEAE.

\section{PHRYMA L.}

P. Leptostachya L. Lop-seed.

Rich open woods. Greenbrier: near White Sulphur Springs. Fayette : near Kanawha Falls (James); near Nutt. allburg (Nuttall). Kanawha: near Charleston. Mason: near Point Pleasant. Webster: at Pugh (H. H. Smith 1653). Preston: near Aurora (Mr. \& Mrs. Steele); and frequent throughout the State.

\section{P L A N T A G I N A C E AE.}

\section{PLANTAGO L.}

P. MAJOR L. Plantain.

Waste ground. Ohio: near Wheeling (Mertz \& Guttenberg). Fayette: Nuttallburg (Nuttall). Monongalia: Morgantown.

P. Rugelir Decne. Common Plantain.

Common throughout the State, near dwellings.

P. Lanceolata L. "Buck Plantain." "Ripple." "Buck-horn Plantain."

Becoming a common weed throughout the State; very little as yet however in Jefferson, Berkeley and Morgan counties. Greenbrier: near White Sulphur Springs. Fayette: near Nuttallburg (Nuttall). Mercer: near Princeton.

P. VirginicA L. White Plantain.

Sandy soils. Fayette: near Nuttallburg (Nuttall). Monongalia: near Morgantown; and frequent throughout the State. Hardy: near Moorefield.

P. ARISTAta Michx.

Dry meadows near Bargers Spring, Summers County, alt. I,500 ft., July I3, Ig00 (Morris 983). Marion: near Farmington (Boutlou). Monongalia: found, in I912, near Morgantown (Sheldon 4459). Wirt: near Newark (Hoff).

\section{R U B I A C E AE.}

\section{HOUSTONIA L.}

H. COERULEA L. Bluets. Innocents.

Moist fields. Monongalia: Marion: Preston: Wood: Wirt: Calhoun: Lewis: and Upshur. Gilmer: near Glenville (Brown; Mapel). Kanawha (James). Mineral: Jef- 
ferson: Berkeley: and Morgan. Fayette: near Nuttallburg (Nuttall). Hardy: near Moorefield (Gamble).

Forma AlBiflora Millsp. Prel. Cat. Fl. W. Va., 375.

Grassy places, Permian formations. Monongalia: near Cassville.

H. SERPYLLIFOLIA Michx.

Rocky places. Tucker: rocks below the falls of Blackwater. Monongalia: on rocks in Tibb's Run. Fayette: near Nuttallburg (Nuttall).

H. PURPUREA L.

Wooded opens. Gilmer: near Glenville (Mapel). Fayette: near Nuttallburg (Nuttall); Kanawha Falls (James). Hampshire: near Romney. Greenbrier: near White Sulphur Springs. Summers: near Hinton. Monongalia: near Ice's Ferry (Millspaugh). Randolph: near Cheat Bridge (Sheldon 2634).

Var. CALYCOSA Gray.

Greenbrier: near White Sulphur Springs.

H. ciliolata Torr.

Monongalia: near Morgantown. Fayette: near Nuttallburg (Nuttall). Greenbrier: near White Sulphur Springs.

H. LONGIFOLIA Gaertn.

Dry soils, the most common form of the species. Wood: near Lockhart's Run. Wirt: near Burning Springs. Calhoun: near Grantsville. Gilmer: near DeKalb. Lewis: up Stone Coal Creek. Upshur: near Buckhannon. Randolph: near Cricard P. O. Cabell: near Barboursville (James). Fayette: near Nuttallburg (Nuttall). Mercer: near Bluefield. Jefferson: at Harpers Ferry (Greene).

H. TENUIFOLIA Nutt.

Greenbrier: near White Sulphur Springs (Millspaugh).

\section{CEPHALANTHUS L.}

C. occidentalis L. Button-bush.

Along streams. Monongalia : along the Monongahela and Cheat Rivers. Preston: general in the glades and along streams. Grant: near Bayard. Randolph: along Tygart's Valley River; near Cheat Bridge. Fayette: near Nuttallburg (Nuttall); near Kanawha Falls (James). Monroe: near Alderson. Summers: near Riffe and Hinton. Barbour: near Tygart Junction (Greenman 226).

\section{MITCHELLA L.}

M. REPEnS L. Partridge-berry.

Rich woods. under evergreens. Upshur: Sand Creek. 
Grant: near Bayard. Tucker: along Blackwater. Mineral: Knobby Mts. (Workman). Randolph: along Cheat River. Gilmer: near Glenville ( $\mathrm{Mapel}$ ). Fayette: near Nuttallburg (Nuttall). Kanawha: near Coalburg (James).

\section{DIODIA L.}

D. TERES Walt. Button-weed.

Sandy river banks. Ohio: along Bogg's Run, near Wheeling (Mertz \& Guttenberg). Preston: banks of Cheat River. Fayette: near Nuttallburg, rare (Nuttall).

\section{SHERARDIA Linn.}

S. ARVENSIS Linn.

A single plant found, in 1908, on the lawn of the Experiment Station at Morgantown (Sheldon 325I).

\section{GALIUM L.}

G. Aparine L. Goose-grass. Cleavers.

Shaded places. Frequent throughout the State.

G. PILOSUm Ait.

Dry copses. Fayette: near Nuttallburg (Nuttall) ; near Kanawha Falls (James). Kanawha: near Coalburg (James). Monongalia: near Little Falls and Uffington; near Camp Eden. Pocahontas: rocky hillsides near Travelers Repose (Greenman 310).

G. Claytoni Michx.

Swampy places. Randolph: near Pickens (H. H. Smith I404); near Read, and Pocahontas: near Durbin (Green-. man 307, 289).

G. Circaezans Michx. Wild Liquorice.

Rich woods. Wood: near Lockhart's Run. Monongalia: Rich woods near Morgantown; Ice's Ferry and Camp Eden. Fayette: near Nuttallburg (Nuttall). Upshur: near Buckhannon (Pollock).

G. LANCEOlatum Torr.

Dry woods. Monongalia: near Morgantown. Ohio: near Wheeling (Mertz \& Guttenberg). Fayette: near Nuttallburg (Nuttall).

G. TRIFLORUm Michx. Sweet-scented Bedstraw.

Rich woodlands. Lewis: along Leading Creek. Upshur: near Lorentz. Webster: along Buffalo Bull range. Monongalia: near Morgantown. Fayette: near Nuttallburg (Nuttall). Preston: near Aurora (Mr. \& Mrs. Steele). Tucker: rich woods near Parsons (Greenman 315). 
G. Latifolium Michx.

Fayette: near Nuttallburg, uncommon (Nuttall). Preston: near Rowlesburg (Mertz \& Guttenberg). Pocahontas: near Durbin (Greenman 312). Webster: in Long Glade (Millspaugh). Randolph: near Pickens (H.H. Smith I 552). G. TRIFIDUM L. Small Bedstraw.

Low, wet grounds. Monongalia, Lewis, Upshur, Gilmer, Calhoun, Wirt, Wood, and Webster: Long Glade. Fayette. near Nuttallburg (Nuttall). Preston: near Aurora (Mr. E. Mrs. Steele).

G. Concinnum Torr. \& Gray.

Low, wet grounds. Wood: near Kanawha Station. Wirt: near Elizabeth. Lewis: along Leading Creek. Randolph: near Valley Bend.

G. ASPrellum Michx. Rough Bedstraw.

Alluvial bottoms. Monongalia: along the Monongahela River. Greenbrier: near White Sulphur Springs (Mertz \& Guttenberg). Preston: near Aurora (Mr. \& Mrs. Steele). Randolph: along Gandy Creek (Greenman 308).

C A P R I F O I A C E AE.

\section{SAMBUCUS L.}

S. Canadensis L. Common Elder.

Rich soils; common in bottoms and along fences throughout the State; even in the pine and spruce forests of the higher mountains; alt. on Point Mountain 3,050 ft.

S. PubEns Michx. Red-berried Elder. (S. racemosus L.).

Deep, rich mountain woods, near rivulets. Abundant in Randolph, Grant and Tucker Counties. Fayette: near Nuttallburg ( Nuttall).

Forma ALBICOCCA Britton.

With the species rare. Randolph: on Point Mountain. Grant: near Bayard.

\section{VIBURNUM L.}

V. Alnifolium Marsh. Michx. Hobble-bush. ( $V$. lantanoides Michx.).

Cold, rich ravines. Randolph: near the summit of Point Mountain (Millspaıgh); near Pickens (H. H. Smith I537). Preston: near Aurora (Mr. \& Mrs. Steele). Grant: near Bayard. Tucker: along the Blackwater.

V. ACERIfolium L. Arrow Wood. Dockmackie.

Cool, rocky woods. Throughout the mountains of the eastern counties. Randolph: near Glady (Greenman 286). 
Preston and Monongalia: along Cheat River. Gilmer: near Glenville ( Mapel). Mineral: near Keyser (Workman). Fayette: near Nuttallburg (Nuttall). Grant: near Bayard. V. Dentatum L. Arrow-wood.

Wet places or damp thickets. Upshur: near the Summit on Staunton Pike. Fayette: near Nuttallburg, rare (Nuttall). V. NUDUM L.

Rich woods. Randolph: at Ford's, near the Middle Fork River. Webster: Upper Glade.

V. Lentago L. Sweet Viburnum. Sheep-berry.

Rich banks of streams. Randolph: on Point Mountain, alt. 3,66o ft. Upshur: near Buckhannon (Pollock).

V. Opulus Americanum (Mill.) Ait.

In woodlands and along streams. Tucker: Canaan Valley (Brooks).

V. CASSINOIDES L.

In wet soil. Webster: along the Gauley River. Pendleton: along Big Run. Monongalia: along Deckers Creek (Brooks); near Tibbs Run (Sheldon 623). Preston: near Aurora ( $M r$. \& Mrs. Steele); near Cranesville and Masontown (Sheldon I457, 4III). Upshur: near Buckhannon (Pollock).

V. PRUNIfolium L. Black Haw. Nanny-berry.

Copses and edges of woods. Wirt: near Burning Springs. Mineral: near Keyser (Workman). Gilmer: near Glenville (Mapel, Brown). Monongalia: near Morgantown (Grose). Fayette: near Nuttallburg (Nuttall). Upshur: near Buckhannon (Pollock). Summers: near Hinton.

\section{TRIOSTEUM L.}

T. Perfoliatum L. Tinker's Weed. Wild Coffee.

Rich borders, infrequent. Randolph: Cheat Mountains, alt. 4,600 ft. Gilmer: near Glenville ( $\mathrm{Mapel})$. Pocahontas: roadside near Travelers Repose (Greenman I52). Fayette: near Nuttallburg (Nuttall). Hardy: near Moorefield (Gamble). Mercer: near Princeton.

\section{LINNAEA Linn.}

L. Americana Forbes.

In cold woods. Randolph: at Big Sinks, in a sphagnum bog near Osceola, alt. 4,000 ft. (A. B. Brooks). 
SYMPHORICARPOS Juss.

S. Symphoricarpos (L.) MacM.

(S. orbiculata Moench.).

Dry places. Nicholas: near Peter Creek. Fayette: near Nuttallburg, alt. 2,000 ft. (Nuttall).

\section{LONICERA L.}

L. Dioica L. Smooth Honeysuckle.

Rocky soils. Monongalia: near Morgantown.

L. JAPONICA Thunb.

Escaped from cultivation. Mason: banks of the Ohio near Point Pleasant. Jefferson: near Shepherdstown. Taylor: near Grafton.

L. Canadensis Marsh.

In moist woods. Pendleton: on Spruce Knoi (Brooks).

L. SEMPERVIRENS Linn.

Monongalia: near Morgantown in a pasture east of Dorsey School House, probably an established escape (Sheldon 3589).

DIERVILLA Moench.

D. Diervilla (L.) MacM. Bush Honeysuckle. (D. trifida Moench.)

Thickets. Monongalia: near Morgantown, along Deckers Creek. Pendleton: on Spruce Mountain (Brooks).

\section{A L E R I A N A C E AE.}

\section{VALERIANA L.}

V. PAUCIFLORA Michx. Valerian.

Fields and open woods. Ohio: near Moundsville (Mertz $\mathcal{E}$ Guttenberg); Bethany (Richardson). Hancock: near Fairview (Hill).

\section{VALERIANELLA Poll.}

V. CHenopodifolia (Pursh) DC.?

Monongalia: in woods along Deckers Creek near Marilla (Sheldon 357). The specimen is too young for positive settlement of the species. 
D I P S A C A C E AE.

\section{DIPSACUS L.}

D. sylvestris Huds. Teasel. "Water Thistle." Huttonweed."

Roadsides and waste places. Wirt: near Burning Springs and Elizabeth. Marion: near Worthington; near Fairmont and Houghton in great quantity. Webster: Buffalo Bull Mountains, alt. 2, IOO ft. Fayette: near Crescent; near Nuttallburg (Nuttall). Kanawha: along the Kanawha and Pocataligo Rivers. Jackson: along Allen's Fork. Gilmer: near Glenville (Mapel). Jefferson: near Flowing Spring and Shenandoah Jc. Randolph: Cheat Mountains near Cheat Bridge, alt. 2,700 ft.; near Huttonsville. Greenbrier: near White Sulphur Springs, near Fort Spring. Monroe: near Alderson. Summers: near Hinton. Monongalia: along Deckers Creek. Harrison: near Lumberport. Mineral: opposite Cumberland. Berkeley: near Martinsburg. Hardy: near Moorefield. Mercer: near Ingleside, and Ada.

\section{CUCUR B I T A C E AE.}

\section{CUCURBITA L.}

C. OVIFERA L. Gourd.

Escaped to waste grounds. Monongalia: about Morgantown. Jefferson: near Shepherdstown.

\section{CITRULLUS L.}

C. Citrullus (L.) Karst. Watermelon. (C. vulgaris Schrad.). Escaped to waste grounds. Mason: banks of the Ohio near Point Pleasant.

\section{CUCUMIS L.}

C. Melo L. Musk Melon.

Escaped to waste grounds. Mason: banks of the Ohis near Point Pleasant. Monongalia : waste grounds, near Morgantown.

\section{MICRAMPELIS Raf.}

M. Lobata (Michx.) Greene.

(M. echinata Raf.).

Escaped from gardens, where it is frequently grown as a vine for fences and rock work ornamentation. 


\section{SICYOS L.}

S. Angulatus L. Star Cucumber.

Damp places. Monongalia: along Deckers Creek; near Easton. Fayette: near Nuttallburg (Nuttall). Preston: near Aurora (Mr. \& Mrs. Steele).

\section{A M P A N ULA CE AE.}

\section{CAMPANULA L.}

C. Rotundifolia L. Harebell.

Moist rocks. Mineral: along the Potomac, near Keyser (Workman). Gilmer: near Glenville' (Mapel). Tucker: along the Blackwater.

C. APARINOIDEs Pursh. Marsh Bellflower.

Wet meadows. Preston: near Terra Alta.

C. Americana L. Tall Bellflower.

Rich woods, or even on dry rocks. Monongalia: near Ice's Ferry. Wood, Wirt and Calhoun counties, general. Gilmer: near Glenville (Mapel, Brown). Lewis, and Upshur. Randolph: near Cheat Bridge, alt. 3,650 ft., with wands 4-6 ft. high. Webster: in the glade region. Fayette: near Nuttall, burg (Nuttall); along Loup Creek (James). Kanawha and Jackson : general. Greenbrier: near White Sulphur Springs. Summers: near Hinton. Marion: near Worthington, and near Fairmont. Preston: near Aurora (Mr. \& Mrs. Steele).

C. DIVARICATA Michx.

Dry banks. Summers: near Talcott. Greenbrier: near White Sulphur Springs. "Millspaugh \& Nuttall mention 'the rare Campanula divaricata $\mathrm{Mx}$.' If the southern counties are to be taken into consideration in rating the occurrence of species in the State, then this species can not be accounted 'rare' for the more rocky hillsides throughout are heavily blue-dotted in the Summer with its delicate panicles." (Morris).

\section{LOBELIA L.}

L. CARdinalis L. Cardinal Flower.

Low grounds, and low banks of streams. Nicholas: Collett's Glade. Gilmer: near Glenville (Mapel, Brown). Randolph: near Cricard P. O. Greenbrier: near White Sulphur Springs. Summers: near Talcott, and Hinton. Kanawha : near Kanawha City. Mason : near Brighton. Frequent throughout the State. Hardy: near Moorefield (Gamble). Preston: near Aurora (Mr. \& Mrs. Steele). 
L. Syphilitica L. Great Blue Lobelia.

Low, wet grounds. Randolph: near Elkins, and along the valley of Tygart's. Gilmer: near Glenville ( $\mathrm{Mapel}$ ). Fayette: near Nuttallburg. Greenbrier: near Winite Sulphur Springs. Monongalia: near Morgantown. Summers: near Hinton. Jefferson: near Shepherdstown. Hardy: near Moorefield (Gamble). Preston: near Aurora (Mr. \& Mrs. Steele).

forma ALBIFLORA Britton.

With the species. Randolph: near Huttonsville, frequent.

L. PUberula Michx.

Low grounds. Fayette: near Nuttallburg (Nuttall) Monongalia: near Morgantown (Millspaugh); near Burnt House (Sheldon 4250).

L. Amoena Michx. var. GLANdulifera Gray.

Swampy spots. Fayette: near Nuttallburg, alt. 2,000 ft., rare. (Nuttall).

L. Leptostachys A. DC.

Sandy soil. Wood: near Leachtown. Summers: near -Hinton.

L. SPICATA Lam.

Sandy hillsides. Monongalia : near Ice's Ferry, and above Camp Eden. Upshur: near Buckhannon (Millspangh, Pollock).

Var. PARVIFLORA Gray.

Wet places. Gilmer : near Glenville ( $\mathrm{Mapel}$ ).

L. INFLATA L. Indian Tobacco. Lobelia.

Dry soils. Common throughout the State.

Var. simplex (Raf.). Millsp. Prel. Cat. Fl. W. Va., 398.

Dry places. Randolph: near Cricard P. O. Characters of the species, but simple stemmed.

Having noted that this form perpetuated itself at one station in New York State, near Binghamton, for five years, I have decided that it is a true variety. Approaching the question from another point of view : I worked over a field near Morgantown this season, examining 783 small plants of L. inflata, many of which were not over four inches high, without finding a single simple-stemmed plant among them At the station above named, as well as that in New York, there was a goodly amount of the variety, with none of the species in the immediate neighborhood.

\section{SPECULARIA Heist.}

S. Perfoliata (L.) A. DC. Venus' Looking-glass.

Dry soils. Monongalia: near Morgantown. Upshur: near Buckhannon. Gilmer: near Glenville (Mapel). Fay. 
ette: near Nuttallburg (Nuttall). Hardy: near Moorefield (Gamble). Randolph: near Pickens (H. H. Smith 1363).

C I C H O R I A C E AE.

(Compositae.)

ADOPOGON Neck. (1790).

(Krigia, Schreb. I79I.)

A. Dandelion (L.) Kuntze. Dwarf Dandelion.

Kanawha: near Charleston (?) (James).

A. Virginicum (L.) Kuntze. (A. amplexicauiis Kuntze.)

Moist woods and opens. Monongalia : near Morgantown. Wood: near Lockhart's Run, becoming a bad weed (Hopkins).

\section{HYPOCHAERIS Linn.}

H. RADICATA Linn.

Established at Morgantown, Monongalia County (Sheldon).

\section{CICHORIUM L.}

C. Intybus L. Chicory.

Fields. Jefferson: two stations near Shepherdstown. Greenbrier: near White Sulphur Springs (Mertz \& Guttenberg).

\section{TRAGOPOGON L.}

T. PORRIFolius L. Salsify. Oyster-plant.

Waste grounds. Morgan: near No. I2 Water Tank.

\section{CHONDRILLA L.}

C. JUNCEA L. "Naked-weed." "Skeleton-weed."

Fields and roadsides. Hampshire: near Bloomery, where the name Naked-weed has been given it on account of the minuteness of the leaves. Jefferson: near Summit, where it is called Skeleton-weed, for the same reason; near Charlestown. Berkeley: near Martinsburg (Millspaugh); near Bunkerhill (Sheldon 1983).

\section{TARAXACUM Haller.}

T. Taraxacum (L.) Karst. Dandelion. ( $T$. officinale Web.). All situations. Frequent throughout the State. 
T. ERYThrospermum Andrz.

Greenbrier: near White Sulphur Springs (Eggleston 4335). Monongalia : common about Morgantown; and various places in Preston County (Sheldon).

\section{SONCHUS L.}

S. Oleraceus L. Sow-thistle.

Waste grounds. Ohio: near Wheeling (Mertz \& Guttenberg).

S. ASPER (L.) All. Spiny leaved Sow-thistle.

Roadsides and wastes. Monongalia: near Morgantown. Marion: near Fairmont. Hampshire: near Slanesville. Wetzel: near Littleton. Lewis : near Vadis. Cabell: near Union Ridge. Mercer: near Concord Church. Fayette: near Nuttallburg (Nuttall). Doddridge: near Smithton. Ohio: near Wheeling (Mertz 1632). Webster: at Tater Knob ( $H$. H. Smith I 524).

\section{LACTUCA L.}

L. Scariola L. Prickly Lettuce.

Fields. Monongalia: near Laurel Point, where it has become a troublesome weed.

L. Canadensis L. Wild Lettuce. Horse-weed. "Devil-weed." Meadows and fence-rows. Common throughout the State.

L. SAGitrifolia Ell. "Devil's Iron-weed." (L! integrifolia Gr. Man.)

Fields and roadsides. Monongalia: near Morgantown. Mason: near Point Pleasant. Fayette: near Nuttallburg (Nuttall). Webster: near Tater Knob (H. H. Smith I 525). Jackson: near Douglas. Preston: near Aurora (Mr. \& Mrs. Steele).

L. HIRSUTA Muhl.

Dry open mountain sides. Fayette: near Nuttallburg (Nuttall).

L. SPICATA (Lam.) Hitch (L. leucophaea A. Gray.)

Low woodlands. Fayette: near Nuttallburg (Nuttail).

Monroe: near Alderson. Ohio: near Wheeling (Mert: 1630). Preston: near Aurora (Mr. \& Mrs. Steele).

L. VILlosa Jacq. (Mulgedium acuminatum DC.)

Borders. Fayette: near Nuttallburg (Nuttall). Monroe: near Alderson. Preston: near Terra Alta.

L. Floridana (L.) Gaertn.

Open banks and borders of woods. Fayette: near Nuttallburg (Nuttall). 


\section{CREPIS Linn.}

C. CAPILlaris (L.) Wallr.

Established near Morgantown, Monongalia County, I808 (Sheldon 3317).

\section{HIERACIUM L.}

H. Canadense Michx. Hawkweed.

Dry woods. Webster: near Upper Glade.

H. Paniculatum L.

Moist grounds. Preston: near Cranberry Summit (Mert\% \& Guttenberg) ; near Aurora (Mr. \& Mrs. Steele). Fayette: near Nuttallburg (Nuttall).

H. venosum L. Rattlesnake-weed.

Openings, and edges of dry woods. Frequent throughout the State.

H. SCABRUM Michx.

Dry open woods. Fayette: near Nuttallburg (Nuttall). Monongalia: along Decker's Creek. Preston: near Terra Alta (Millspaugh); near Aurora (Mr. \& Mrs. Steele).

H. Alleghaniense Britton. Manual 2nd edit. 905. (I905).

Stem rough-hairy below, glabrous or nearly so above, about $6.5 \mathrm{dm}$. tall. Lower and middle stem-leares oblanceolate, I-I.5dm. long, $3 \mathrm{~cm}$. wide or more, obtuse, cuspidate, tapering into narrowly margined pubescent petioles, thin, pale and pubescent along the midvein beneath, green above, with a few very distant minute glandular teeth; upper leaves oblong to oblong-lanceolate, acute, narrowed to a sessile base; inflorescense paniculate, its branches filiform, glandular; heads rather numerous: involucre about 7 nim. high, its bracts linear-lanceolate, sparingly glandular, a iittle shorter than the brown pappus: young achenes truncate.

Preston: in the Alleghany Mountains near Aurora, August 29, I898 (Mr. \& Mrs. Steele).

H. Gronovit L.

Dry soils. Fayette: near Nuttallburg, alt. 2,000 ft. (Nuttall). Upshur: summit on Staunton Pike.

H. LONGI PILUM Torr.

Dry situations. Monongalia: Decker's Creek, near Morgantown. Fayette: near Kanawha Falls, and Hawk's Nest (James).

\section{NABALUS Cass.}

N. Altissimus (L.) Hook.

(Prenanthes L.)

Rich moist woods. Fayette: near Nuttallburg (Nuttall). Preston: near Aurora (Mr. \& Mrs. Steele). 
N. Albus (L.) Hook.

(Prenanthes L.)

Open woods. Hardy: near Moorefield (Gamble). Pres* ton: near Aurora (Mr. \& Mrs. Steele).

N. Serpentarius (Pursh). Hook. Gall-of-the-Earth. (Prenanthes Pursh.)

Sandy woods. Randolph: near Cheat Bridge, alt. 3,550 ft. Summers: near Hinton. Marion: near Catawba. Fayette: near Nuttallburg (Nuttall).

\section{A M B R O S I A C E AE.}

(Compositae.)

\section{AM̄BROSIA L.}

A. TRIFIDA L. Great Rag-weed.

Moist places. Common or abundant throughout the State. Var. integrifolia (Muhl.) T. \& G.

With the species, uncommon. Monongalia: near Morgantown. Wood: near Parkersburg. Fayette: near Nuttallburg (Nuttall). Berkeley: near Martinsburg.

A. artemisiaefolia L. Rag-weed.

Fields and roadsides. Abundant throughout the State.

A. Bidentata Michx.

Meadows. Hardy: near Moorefield (A. B. Brooks). This extends the distribution of this species eastward from Illinois.

\section{XANTHIUM L.}

X. Commune Britton.

Dry situations. Tucker: along the Blackwater River near Hendricks (Greenman 537).

X. spinosum L. Spiny Clotbur.

Waste lands along rivers. Kanawha: at Stockton's. Mineral: near Piedmont. Jefferson: near Shepherdstown. Wood: near Parkersburg. Berkeley: near Martinsburg. Mineral : opposite Cumberland, Md. (Small).

X. glabratum (DC) Britton. Clotbur. Cockle-bur. (X. Strumarium of Flora.)

Low waste grounds. Monongalia, Marion and Gilmer counties. Wood: near Parkersburg. Lewis: near Weston. Jefferson: near Shepherdstown.

X. Canadense Mill.

Low waste grounds. Common throughout the State. 
C A R D U A C E AE.

(Compositae.)

VERNONIA Schreb.

V. GIgAnteA (Walt.) Britton. Iron-weed. ( $V$. altissima Nutt).

Low grounds. A frequent weed throughout the northern, central and western portions of the State. Fayette: near Nuttallburg (Nuttall).

V. maxima Small.

Moist river bottom. Monongalia :near Morgantown (Millspaugh 677).

V. maxima pubescens Morris, Proc. Biol. Soc. Wash., i5:25. (I9OI).

In gross characters like the species. Reaching $10^{\circ}$ or over, more or less pubescent. Leaves thin, lanceolate, acuminate. the upper finely, the lower sharply doubly serrate, $3^{\prime}-12^{\prime}$ long, $1 / 2^{\prime}-2 \frac{1}{2} 2^{\prime}$ wide, finely pubescent below, somewhat so above; inflorenscence open, its branches rather erect, the peduncles bracteate for $2^{\prime \prime}-5$ " below the heads; the heads long-peduncled or the centre ones nearly sessile; the bracts greenish purple, acute to short-acuminate, ciliate, erect; corollas light to dark pink, not purple; otherwise as in the species.

Collected among plants of the species along Hound Creek, below Baileysville, Wyoming County, alt. I, 100-I,200 ft, August 20, I900 (Morris, 1274). Type specimen is deposited in the U. S. National Herbarium.

Through a misapprehension of the case the subspecies pubescens was referred (Proc. Biol. Soc. Wash. I3:I79, October. I900) to Vernonia gigantea of the Atlantic seaboard, which does not occur in the Alleghenies or westward. The species so common throughout the latter range is $V$. maxima Small (Bull. Torr. Bot. Club, $27: 280$, May, I900). Hence the name of the subspecies collected near Baileysville, West Virginia, is Vernonia maxima pubescens. (Morris).

V. Noveboracensis (L.) Willd. Iron weed.

In meadows and pastures, common throughout the State.

Var. LATIFolia Gray.

Meadows and fields. Mason : near Point Pleasant. Monon-galia: near Morgantown. Fayette: near Nuttallburg (Nuttall).

\section{ELEPHANTOPUS L.}

E. Carolinianus Willd.

Dry banks. Fayette: near Nuttallburg (Nuttall). 
E. romentosus L. "Tobacco Weed." "Devil's Grandmother." Fields. Harrison: near Quiet Dell. Upshur: near Lorentz.

\section{EUPATORIUM L.}

E. PURPureum L. Queen of the Meadow. "Quill-wort."

Low grounds. Common throughout the State. Cheat Mountains in Randolph at an altitude of 3,600 feet. Numerous specimens were measured which were over twelve feet high (Morris).

E. maculatum Linn.

Dry Fork River near Harman, Randolph Co. (Greenman 236).

E. maculatum amoenum (Pursh) Britton.

Rich woods along runs. Grant: Buffalo Creek near Bayard. Tucker: Beaver Creek near Davis.

E. HYSSOPIFOLIUM L.

Sterile soil. Jefferson: near Shepherdstown.

E. PUbescens. Muhl.

Dry hillsides. Fayette: near Nuttallburg (Nuttall). Jefferson: near Shepherdstown. Monongalia: near Morgantown and Camp Eden. Preston: near Aurora (Mr. and Mrs. Steele).

E. Altissimum L. Tall Boneset.

River banks. Monongalia: near Little Falls and Beechwoods.

E. TRIFOLIATUM Linn.

In moist soils, Preston: near Aurora (Mr. and Mrs. Steele).

E. Sessilifolium L. Upland Boneset.

River banks. Monongalia: near Beechwooc's. Fayette: near Nuttallburg, plentiful (Nuttall).

E. Perfoliatum L. Boneset. Thorough-wort.

Damp places. Common throughout the State.

E. Ageratoides L. White Snake-root.

Rich woods. Monongalia : along Decker's Creek and else-where plentiful. Randolph: Cheat Mountains near Cheat Bridge. Marion: near Worthington. Fayette: near Nuttallburg (Nuttall). Hardy: near Moorefield (Gamble). Preston: near Aurora (Mr. and Mrs. Steele). Tucker: rich woods near Parsons (Greeman 242).

E. AROMAticum L.

Rich soil. Fayette: near Nuttallburg. Hardy: near Morefield (Gamble). 
E. coelestinum L. Mist-flower.

Rich soils. Putnam. Jackson, Wood and Monongalia. A common weed. Randolph: along Tygart's Valley River. Barbour: near Tygart Junction (Greenman 263). Harrison along the "Monongah" R. R. Summers: near Hinton. Fayette: near Nuttallburg (Nuttall). Putnam: near Buffalo. Kanawha: near Charleston. Mason: near Point Pleasant. Marion: near Montana and Worthington. Jefferson: near Shepherdstown. Nicholas: near Richwood (H. H. Smith I749).

\section{KUHNIA Linn.}

K. eupatorioides L. False Boneset.

In dry soil. Greenbrier: near White Sulphur Springs (Britton). Berkeley: near Martinsburg (Sheldon 2663).

$$
\begin{aligned}
& \text { LACINIARIA Hill (I762). } \\
& \text { (Liatris, Schreb. I79I). }
\end{aligned}
$$

L. SPICATA (L.) Kuntze.

Among rocks, banks of New River (Selby). Fayette: near Nuttallburg, heads 5-flowered (Nuttall).

L. SCARIOSA SQUarrulosa (Michx.) Small.

Greenbrier Co.: White Sulphur Springs (Brown):

\section{CHRYSOPSIS Nutt.}

C. Mariana (L.) Nutt.

Dry, rocky roadside. Fayette: R. \& K. turnpike near Nuttallburg (Nuttall).

$$
\text { SOLIDAGO L. }
$$

S. FLExicaulis L.

(S. latifolia L.)

Moist, shaded banks. Monongalia: banks of the Monongahela and Cheat Rivers. Favette: near Nuttallburg (Nutall). Webster: (H. H. Smith I839). Open rich places. Fayette: along the railroad track near Gauley Junction (Eggleston 5543). Preston: near Aurora (Mr. \& Mrs. Stecle).

S. CAESIA L.

Rich woodlands. Frequent throughout the State.

S. Curtisin Torr. \& Gray.

Woodlands. Fayette: near Nuttallburg, common (Nuttall). 
S. BICOLOR L.

Dry fields and copses. Frequent throughout the State.

S. MONTICOLA Torr. \& Gray.

Woods and opens. Fayette: near Nuttallburg, alt. 2,000

ft. (Nuttall). Webster: (H. H. Smith I8I6). Preston:

near Aurora (Mr. \& Mrs. Steele).

S. PUberula Nutt.

Sunny opens. Fayette: near Nuttallburg (Nuttall).

S. Uliginosa Nutt.

Swampy places. Randolph: top of Allegheny Mountains near Horton (Greenman 493).

S. SPECIOSA Nutt.

Cliffs and banks. Fayette: near Nuttallburg (Nuttall).

S. odora Ait. Sweet Golden-rod.

Fayette: near Nuttallburg (Nuttall). Nicholas: near Richwood (H. H. Smith I746).

S. RUgosa Mill.

Borders of fields and copses. Along Cheat River. Randolph, Tucker, Preston and Monongalia counties. Fayette: near Nuttallburg (Nuttall). Shores of the Monongahela in Barbour, Taylor and Marion counties. Preston: near Aurora (Mr. \& Mrs. Steele).

S. ULMifolia Muhl.

River banks. Ohio: Thomas Hill near Wheeling (Mertz $\mathcal{E}$ Guttenberg). Brooke: (Mertz \& Guttenberg). Fayette: near Nuttallburg (Nuttall).

S. BootTiI Hook.

Dry open woods. Putnam: near Buffalo. Fayette: near Nuttallburg (Nuttall).

S. ARGUTA Ait.

River banks. Ohio: banks of the Ohio River near Wheeling (Mertz \& Guttenberg). Preston: near Aurora (Mr. \& Mrs. Steele).

S. NEGLECTA T. \& G.

Swampy places. Upshur: near Buckhannon (Pollock). Fayette: near Quinnimont (Pollard \& Maxon 33, 34). Preston: near Aurora (Mr. \& Mrs. Steele).

S. JUNCEA Ait. "Yellow Top."

Fields and waste places. Common throughout the northern, central and western counties. Fayette: near Nuttallburg (Nuttall); near Gauley Bridge and near Thurmond (Eggleston 5524, 5558). Preston: near Aurora ( $M r$. \& Mrs. Stecle). Berkeley: near Martinsburg. Mason: near Point Pleasant. Hardy: near Moorefield (Gamble).

Var. SCABRElla (T. \& G.) Gray.

With the species. Frequent. 
Var. RAmosa Porter \& Britton.

River banks. Monongalia : near Morgantown, below high

water mark along the Monongahela.

S. SERotina Ait.

Fayette: near Nuttallburg (Nuttall). Monongalia: near Morgantown (Millspaugh). Preston: near Aurora (Mr. \& Mrs. Steele).

Var. GIGANTEA (Ait.) Gray.

Thickets. Gilmer: near Glenville (Mapel). Preston: near

Rowlesburg.

S. RUPESTRIS Raf.

Rocky river banks. Fayette: along the Gauley at Gauley

Mountain; Kanawha Falls and Hawk's Nest (James).

S. Canadensis L.

Borders and waste fields. Common throughout the State.

S. NEMORALIS Ait.

Dry, sterile fields. Fayette: near Nuttallburg (Nuttall). Preston: near Aurora (Mr. \& Mrs. Steele). Common throughout the northern counties.

\section{EUTHAMIA Nutt.}

E. GRAminifolia (L.) Nutt.

(Solidago lanceolata L.)

River banks. Along Cheat River throughout its length Along the Monongahela in Marion, Taylor and Monongalia counties. Gilmer: along the Little Kanawha (Mapel). Mason: near Point Pleasant. Fayette: near Nuttallburg (Nuttall). Preston: near Aurora (Mr. \& Mrs. Steele).

E. Caroliniana (L.) Greene.

Sandy fields. Monongalia : near Morgantown.

\section{SERICOCARPUS Nees.}

S. Linifolius (L.) B. S. P.

On dry shaded banks along the road above Hinton, Summers County, alt. I,400 ft., July 7, I900 (Morris 950).

S. Asteroides (L.) B. S. P. White-topped Aster.

Dry grounds. Frequent or common throughout the State. Kanawha: near Charleston (James). Greenbrier: near White Sulphur Springs. Fayette: near Nuttallburg (Nuttall). Greenbrier Co., mountains about White Sulphur Springs, alt. 3,000 ft. (A. Brozen).

BRACHYCHAETA T. \& G.

B. sphacelata (Raf.) Britton. (B. cordata T. \& G.) Dry woods. Fayette: near Nuttallburg, plentiful (Nuttall). 


\section{BELLIS Linn.}

B. PERENNIS Linn.

Becoming rather common in the cemetery and in yards at Morgantown, Monongalia County, I907 (Sheldon 2623).

\section{ASTER L.}

A. Claytoni Burgess.

Along rocky banks east of Oceana, Wyoming County, alt. I,300 ft., August 22, I900 (Morris I294a).

A. MACROPHYLLUS L.

Open woods. Fayette: near Nuttallburg (Nuttall). Preston: near Aurora (Mr. \& Mrs. Steele). Kanawha: at the Kanawha salinas (Holton).

A. SAgittifolius Willd.

On dry banks between Piney, Raleigh County, and Jumping Branch, Summers County, alt. 2,200-3, I00 ft., August 24, I900 (Morris I338).

A. patens Ait.

Rocky river banks. Fayettte: near Nuttallburg (Nuttall); opposite Gauley Junction (Eggleston 5523). Summers: near Hinton.

A. PHLOGIFolius Muhl.

Open woods. Fayette: near Nuttallburg (Nuttall). Preston: near Aurora (Mr. \& Mrs. Steele).

A. Laevis L.

Rocky river banks. Monongalia: near Little Falls. Fayette: near Nuttallburg, plentiful (Nuttall); near Thurmond (Eggleston 5552).

A. undulatus L.

Dry woods. Mason: near Point Pleasant. Kanawha: near Charleston. Fayette: near Nuttallburg, common (Nuttall); near Gauley Bridge (Eggleston 5546). Summers: near Hinton (Eggleston 5580).

A. CORDIFOLIUS L.

Woodlands. Monongalia: near Morgantown and Little Falls. Fayette: near Kanawha Falls (James) ; near Nuttallburg (Nuttall); near Gauley Bridge (Eggleston 5542). Mason: near Point Pleasant. Preston: near Aurora (Mr. \& Mrs. Steele). Kanawha: at the Kanawha salinas (Holton).

A. Lowrieanus Porter.

Woodlands and opens. Monongalia: near Morgantown, abundant. Preston: near Aurora (Mr. \& Mrs. Steele).

A. Lowrieanus lancifolius Porter.

In woodlands, Preston: near Aurora (Mr. \& Mrs. Steele). 
A. Tradescanti $L$.

In fields and swampy places, Preston : near Aurora ( $M r$. \& Mrs. Steele).

A. PURPURATUs Nees.

(A. virgatus E11.)

Rocky river banks. Fayette: near Nuttallburg (Nuttall).

Preston: along Cheat River. Monongalia : near Camp Eden. A. Polyphyllus Willd.

(A. Faxoni Porter.)

Rocky or gravelly soils. Barbour: near Tygarts Junction (Greenman 503).

A. ERICOIDES L.

Dry open places. Fayette: near Nuttallburg (Nuttall). Mason: near Point Pleasant. Wood: near Parkersburg. Monongalia: near Morgantown.

Var. Depauperatus Porter. (var. pusillus A. Gray.)

Dry fields. Monongalia: plentiful about Morgantown. Var. PILosus (Wiild.) Porter.

Roadsides, etc. Fayette : near Nuttallburg, common (Nuttall). Preston: near Aurora (Mr. \& Mrs. Steele). Monongalia : near Morgantown. Marion: near Fairmont. Var. villosus T. \& G.

Dry open places. Pocahontas: near Travelers Repose (Greenman 502).

A. lateriflorus (L.) Britt. (A. miser Man. A. diffusus Ait.)

Dry or moist grounds: Monongalia: near Morgantown. Frequent throughout the northern counties. Hardy: near Moorefield (Gamble). Ohio: near Wheeling (Mertz I 254). Preston: near Aurora (Mr. \& Mrs. Steele).

A. HiRsuticaulis Lindl. "Nail-rod."

Fields and roadsides. Cabell : near Barboursville. Monongalia: near Morgantown; and common throughout the northern, central and western counties.

A. multiflorus Ait.

Hardy: near Moorefield (Gamble).

A. Dumosus L.

Hardy: near Moorefield (Gamble.)

A. DIVARICATUS L.

(A. corymbosus Ait.)

Fayette: in the gulf where the old highway goes over the mountain above Gauley Bridge (Eggleston 5533, 5550.). Webster: (H. H. Smith). Preston: near Aurora (Mr. \& Mrs. Steele). Monongalia: near Morgantown (Millspaugh).

A. Vimineus Lam.

Shaded roadsides and fields. Fayette: near Nuttallburg, alt. 2,000 ft., plentiful (Nuttall). Monongalia: near Morgantown. Mason: near Point Pleasant.

Var. Foliolosus (Aiton) A. Gray.

Monongalia : near Morgantown, Uffington and Little Falls, common. 
A. PANiculatus Lam.

(A. simplex Willd).

Low grounds. Fayette: near Nuttallburg (Nuttall). Mason: near Point Pleasant. Putnam: near Buffalo. Ohio: near Wheeling (Mertz I270).

A. SALICIFOLIUS Lam.

Near streams. Monongalia and Preston: banks of Cheat River (Millspaugh). Fayette: near Gauley Junction (Eggleston 5520) ; near Gauley Bridge, with white flowers (Eggleston 5549), with blue flowers (Egglestton 555I).

A. Novi-Belgi L.

Damp meadows. Monongalia: near Morgantown.

A. PRENANTHOIDEs Muhl.

Rich woods and borders of streams. Randolph: Cheat Bridge, alt. 3,360 ft. Monongalia: shore of Monongahela above Morgantown. Fayette: near Nuttallburg (Nuttall); near Gauley Bridge (Eggleston 5536). Preston : near Aurora $(M r$. \& Mrs. Steele).

A. Novae-Angliae L. New England Aster.

Fields and moist grounds. Marion: near Fairmont and Palatine (Boutlou).

A. PUNICEUS L.

Swampy places. Fayette: near Nuttallburg, uncommon (Nuttall). Hardy: near Morefield (Gamble). Preston: near Aurora (Mr. \& Mrs. Steele).

A. PUNiCEUs firmus (Nees) T. \& G.

Wet places. Randolph: near Whitmer and along Dry

Fork River near Harmon (Greenman 505, 504).

A. acuminatus Michx.

Cool, rich woods. Randolph: near Cheat Bridge. Fayette: near Kanawha Falls (James.)

A. Shortir Hook.

Edges of woodlands. Monongalia: near Morgantown (Sheldon 4582).

\section{ERIGERON L.}

E. Annuus (L.) Pers. Daisy Fleabane. Sweet Scabious.

A weed in meadows and fields. Common throughout the State.

E. RAmosus (Walt.) B.S.P. Daisy Fleabane. (E. strigosus Muhl.) Fields and waste places. Monongalia: the Flats and Uffington. Fayette: Nuttallburg (Nuttall).

E. PUlchellus Michx. Robin's Plantain. (E. bellidifolius Muhl.) Copses, common throughout the State.

E. Philadelphicus L. Common Fleabane.

Moist ground. Frequent throughout the northern counties. 


\section{LEPTILON Raf.}

L. Canadense (L.) Britton. Butter-weed. Horse-weed. Waste places. Common throughout the State.

\section{DOELLINGERIA Nees.}

D. umbellata (Mill.) Nees.

(Aster Mill.)

Moist thickets. Along Cheat River in Randolph, Tucker, Preston and Monongalia counties. Fayette: near Nuttallburg (Nuttall). Preston: near Aurora (Mr. \& Mrs. Stecle).

D. INFIRMA (Michx.) Greene.

(Aster Michx.)

Mountain woods. Randolph: Point Mountain, alt. 2,800 ft. Fayette: near Nuttallburg (Nuttall).

\section{IONACTIS Greene.}

I. LiNARIIFolius (L.) Greene.

(Aster L.)

Rocky places. Fayette: near Nuttallburg, along the banks of New River below high water mark, common (Nuttall); New River Falls (Eggleston 552I). Barbour: near Tygart Junction (Greenman 223).

\section{GIFOLA Cass.}

G. Germanica (L.) Dumort. (Gnaphalium Germanicum L.) Along the road north of Athens, Mercer County, alt. 2,500 ft., July I8, I900 (Morris I034).

\section{ANTENNARIA Gaertn.}

A. Plantaginifolia (L.) Richards. Everlasting.

Sterile hills. Frequent or common throughout the State.

A. Parlinir Fernald.

Greenbrier: near White. Sulphur Springs (Eggleston 4348). Monongalia: near Morgantown (Sheldon 299).

A. PROPINQUA Greene, Pittonia 4:83. (1899).

Near A. arnoglossa but more slender and only half as large, the stolons relatively more elongated and more copiously beset with black gland-tipped hairs, the bracts of the flowering branch often rather strongly ciliate with them: mature leaves one and a half to two inches long, with almost elliptic blade and short petiole, mucronately acute, bright green and nearly veinless above, white-tomentose and obviously triple-nerved beneath: flowering branches (in the male, which alone is known) three to six inches high, bearing 4 or 5 involucres, the terminal one sessile, the rest short-pedicel- 
late: bracts biserial, with large clear-white oblong-ovate obtuse tips: bristles of the pappus only slightly dilated and sharply serrulate at the tip.

Collected only by E. L. Greene, on an open hillside at Harper's Ferry, May I4, I898; only one large patch seen, and that male. Though obviously a near relative of $A$. Parlinii and arnoglossa, its small size, very differently shaped foliage and peculiarly narrowed and serrulated male pappus. bristles, compel one to regard it as wholly distinct. I hope that the female plant may be detected at no distant time. There is no A. arnoglossa in the Harper's Ferry region.

A. NEodioica Greene.

Dry situations. Monongalia: near Morgantown (Sheldon I624).

A. fallax Greene.

Monongalia : near Morgantown (Sheldon 349).

A. SOLITARIA Rydb.

Dry hillsides. Tucker: near Parsons (Greenman 240).

ANAPHALIS DC.

A. margaritacea (L.) Bth. \& Hook. Pearly Everlasting.

Dry hills and woods. Monongalia : along Decker's Creek. Marion: above Opekiska.

\section{GNAPHALIUM L.}

G. obtusifolium L. Everlasting. (G. polycephalum Michx.)

Old fields. Frequent or common throughout the northern and central counties. Fayette: near Nuttallburg (Nuttall).

- Hardy: near Moorefield (Gamble). Webster: on Taters Knob (H. H. Smith). Preston: near Aurora (Mr. \& Mrs. Steele).

G. Uliginosum L. Low Cud-weed.

Low grounds. Grant: near Davis. Gilmer: near Glenville (Mapel; Brown). Monongalia: near Morgantown. Randolph: top of Alleghany Mountains (Greenman 266). Mason: near Point Pleasant. Wood: near Parkersburg. Preston: near Aurora (Mr. \& Mrs. Steele).

G. Purpureum L. Purplish Cud-weed.

Sandy soil. Monongalia: near Beechwoods and Ice's Ferry. Fayette: near Nuttallburg (Nuttall).

G. DECURRENS Ives.

Open places, Preston: near Aurora (Mr. \& Mrs. Steele). Randolph: top of Alleghany Mountains (Greenman 266a). 
I. Helenium L. Elecampane.

Fields. Wirt: near Burning Springs. Upshur: near Lorentz. Nicholas: along Mumble-the-peg Creek. Fayette: near Nuttallburg (Nuttall). Greenbrier: near Ronceverte. Jefferson: near Shepherdstown. Hampshire: near Romney. Monongalia: near Stumptown. Webster: near Pugh $(H . H$. Smith I655). Preston: near Aurora (Mr. \& Mrs. Steele).

\section{POLYMNIA L.}

\section{P. Canadensis L. Leaf Cup.}

Moist shaded ravines. Fayette: near Kanawha Falls and Hawk's Nest (James; Porter) ; near Nuttallburg (Nuttall) ; near Gauley Junction (Eggleston 5544). Hardy: near Moorefield (Gamble). Wood: at the mouth of the Little Kanawha (Michaux).

Var. RADIATA Gray.

Rich rocky soil. Fayette: near Nuttallburg (Nuttall).

P. Uvedalia L.

Rich soil. Randolph : frequent along Tygarts Valley River. Fayette: near Nuttallburg (Nuttall). Ohio: near Wheeling (Mertz I380).

\section{SILPHIUM L.}

S. Asteriscus L.

Dry sandy soil. Wirt: beyond Burning Springs. Jackson: near Ripley.

S. TRIFOliatum L. Rosin-weed.

Dry hills and banks. Fayette: near Nuttallburg (Nuttall); at Hawks Nest (Holton). Greenbrier Co., near White Sulphur Springs, alt. 3,00o ft. (A. Brown).

S. PERfoliatum L. Cup Plant.

Along streams. Fayette: near Hawks Nest (James); near Nuttallburg (Nuttall).

\section{CHRYSOGONUM L.}

C. Virginianum L.

Dry soils. Hardy: near Moorefield (Gamble).

\section{PARTHENIUM L.}

P. INTEgRIfolium L. Sneeze-wort.

Dry soils. Fayette : near Nuttallburg, banks of New River 
below high water mark, plentiful (Nuttall). Greenbrier : near White Sulphur Springs (Mertz \& Guttenberg).

\section{HELIOPSIS Pers.}

H. scabra Dunal. Ox-eye.

Fields. Gilmer: near Glenville (Mapel).

H. helianthoides (L.) B. S. P. (H. lacvis Pers.)

Open places, Webster:'near Pugh (H. H. Smith 1654). Fayette: near Nuttallburg (Nuttall). Preston: near Aurora (Mr. \& Mrs. Steele): Tucker: near Parsons (Greenman 24I).

\section{ECLIPTA L.}

E. Alba (L.) Hassk. (Eclipta procumbens, and E. erecta, Michx.)

Wet river banks. Mason: banks of the Ohio near Point Pleasant. Ohio: near Wheeling (Mertz \& Guttenberg). Fayette: R. R. bank, Nuttallburg (Nuttall).

\section{RUDBECKIA L.}

R. laciniata L. Cone-flower.

Low grounds. Monongalia: Little Falls, Beechwoods, Uffington, and Morgantown. Fayette: near Nuttallburg (Nuttall).

Var. Humilis Gray. town.

Monongalia: banks of Monongahela River below Morgan-

R. FULGIDA Ait.

Fields and Meadows. Monroe: abundant near Alderson. Hardy: near Moorefield (Gamble).

R. TRILOba L. Brown-eyed Susan.

Dry fields. Gilmer: near Glenville $(\mathrm{Mapel})$. Greenbrier : near White Sulphur Springs.

R. Hirta L. "Nigger Head." "Yellow Daisy." Brown-eyed Susan.

Becoming too frequent in meadows. Randolph: Cricard P. O. (Millspaugh); near Pickens (H. H. Smith 1372). Preston: near Aurora (Mr. \& Mrs. Steele). Throughout the Ohio River counties. Fayette: along Loup Creek (James) ; near Nuttallburg (Nuttall). Wood: near Kanawha Station.

R. SPECIOSA Wender.

Dry soils. Ohio: near Wheeling (Mertz \& Guttenberg). 


\section{BRAUNERIA Neck (I790).}

(Echinacea, Moench. I794.)

B. purpurea (L.) Britton. Purple Cone-flower. (Echinacea L.)

Along the C. \& O. R. R. Fayette: near Nuttallburg; a rough, bristly form ( Nuttall); near Thurmond (Eggleston 5557). Adventive from the west.

\section{HELIANTHUS L.}

H. Laetiflorus Pers.

Dry opens. Fayette: near Nuttallburg.

H. occidentalis Riddell. Western Sunflower.

Banks of New River. Fayette: near Nuttallburg; infrequent (Nuttall) ; at Hawk's Nest (Holton).

Var. Dowellianus T. \& G.

Dry soils. Fayette: near Nuttallburg (Nuttall).

H. Tomentosus Michx.

Banks of New River. Fayette: near Nuttallburg (Nuttall).

H. GROSSE-SERRATUS Martens. Large-toothed Sunflower.

Dry fields. Upshur: near Buckhannon.

H. giganteus L. Giant Wild Sunflower.

Low grounds. Randolph: near Cheat Bridge. Fayette: near Nuttallburg, plentiful (Nuttall); at Hawks Nest (Holton). Preston: near Terra Alta (Millspaugh); near Aurora (Mr. \& Mrs. Steele).

H. Laevigatus Torr, \& Gray.

Thickets. Preston: near Terra Alta.

H. DORONICOIDES Lam.

Dry grounds. Ohio: on Bogg's Island (Mertz \& Guttenberg). Hardy: near Moorefield (Gamble).

H. Microcephalus T. \& G.

(H. parviflorus Bernh.)

Thickets. Summers: near Hinton. Greenbrier: near White Sulphur Springs. Fayette: near Nuttallburg (Nuttall); near Gauley Junction (Eggleston 5547). Preston: near Terra Alta (Millspaugh); near Aurora (Mr. \& Mrs. Steele). Ohio: near Wheeling (Mertz 1452).

H. Divaricatus L.

Thickets and dry places. Fayette: near Nuttallburg (Nuttall). Jackson: up 8 mile creek.

H. HIRSUTUs Raf.

Dry banks. Fayette: near Nuttallburg, rare (Nuttall). Mason: banks of the Ohio near Point Pleasant. Hardy: near Moorefield (Gamble). 
H. STRUMOSUS L.

River banks and low copses. Monongalia: along Decker's Creek (Millspaugh). Preston: near Aurora (Mr. \& Mrs. Steele).

H. TRACHELIIFOLIUS Mill.

Mountain woods. Fayette: near Nuttallburg, unconmon (Nuttall).

H. DECAPETALus L.

Rich open woods. Monongalia: near Little Falls and Uffington. Fayette: near Nuttallburg, the most commori species here; petals mostly 8 (Nuttall). Ohio: near Wheeling (Mertz I442).

\section{VERBESINA L.}

V. occidentalis (L.) Walt. Crownbeard.

Rich soil. Fayette: near Nuttallburg (Nuttall) ; and along the Great Kanawha River to its mouth. Jackson: up 8-Mile Creek. Wood: near Lockhart's Run. Monongalia: near Morgantown. Summers: near Hinton. Jefferson: near Shepherdstown. Berkeley: near Martinsburg. Greenbrier: near White Sulphur Springs (Eggleston 5514). Kanawha: near Charleston (Holton).

V. Alternifolia (L.) Britton. (Actinomeris alternifolia DC.)

Rich soil. Ohio: near Wheeling (Mertz \& Guttenberg). Preston: near Aurora ( $M r$. \& Mrs. Steele). Fayette: near Nuttallburg, common (Nuttall). Monongalia: near Morgantown. Kanawha: near Charleston (Millspaugh); near Kanawha Salinas (Holton).

\section{COREOPSIS L.}

C. Lanceolata villosa Michx.

Rich soil. Fayette: banks of New River near Nuttallburg (Nuttall).

C. pubescens E11.

Rich shady place. Fayette: near Nuttallburg (Nuttall).

C. AURiculata L.

Rich banks. Fayette: near Nuttallburg (Nuttall); near

Hawks Nest (Porter). Monroe: near Alderson.

C. MAJOR Walt.

(C. senifolia Michx.)

Shady woods. Greenbrier: near White Sulphur Springs (Mertz \& Guttenberg).

Var. stellata Torr. \& Gray.

(C. stellata Nutt.)

Fayette: banks of New River near Nuttallburg (Nuttall). 
C. TRIPTERIS L. Tall Coreopsis.

Rich ground. Jackson: plentiful along 8-Mile Creek and on Limestone Ridge. Fayette: near Nuttallburg (Nuttall); at Hawks Nest (Holton). Monongalia: near Little Falls.

\section{BIDENS L.}

B. CERNUA Linn.

Wet places. Randolph: along Dry Fork River near Harmon (Greenman 543).

B. Frondosa L. Begger's Ticks. Stick-tights. Pitchforks.

Damp, waste places. Common throughout the State.

B. MELANOCARPA Wiegand.

(B. frondosa Torr. not L.)

Damp, waste places. Monongalia: near Morgantown (Millspaugh 775).

B. Connata Muhl. Swamp Beggar's Tick.

Wet places. Frequent throughout the State. Kanawha:

a small form at the Kanawha salinas (Holton).

B. comosa (Gray) Wiegand.

Damp open places. Fayette: near Nuttallburg (Nuttall). Kanawha: a dwarf form of the species at the Kanawha salinas. (Holton). Monongalia: near Morgantown and frequent throughout the State.

B. Laevis (L) B. S. P.

(B. chrysanthemoides) Michx.

Wet places. Frequent throughout the State.

B. Bipinnata L. Spanish Needles.

Dry places. Abundant throughout the State. Tücker: bank of Blackwater River near Hendricks (Greenman 250). B. TRICHOSPERMA (Michx.) Britton.

Fields. Kanawha: near Charleston. Monongalia: near Morgantown.

GALINSOGA Ruiz \& Pav.

G. PARviflora Cav.

Waste grounds. Mason: near Point Pleasant. Wood: near Parkersburg.

MARSHALLIA Schreb.

M. GRANDIFlora Beadle \& Boynton.

Moist soils, Upshur: near Buckhannon (Pollock).

M. obovata platyphylla (Curtis) Bead. \& Boynt.

Barbour: near Tygart Junction (Greenman 2I7). 


\section{HELENIUM L.}

H. autumnale L. Sneeze-weed.

Alluvial river banks. Wirt: along the Little Kanawha River. Fayette: near Nuttallburg (Nuttall). Monongalia: near Morgantown. Randolph: near Cheat Bridge, alt. 3,660 ft. Summers: near Hinton (Millspaugh, Eggleston 5519). Hardy: near Moorefield (Gamble). Preston: near Aurora (Mr. \& Mrs. Steele).

\section{TAGETES Linn.}

T. PATULA L.

Escaped from cultivation and established along the railroad near Laurel Creek, Fayette County, near Quinnimont (Pollard \& Maxon 20).

\section{ACHILLEA L.}

A. Millefolium L. Yarrow. Milfoil.

Common throughout the State, even in the most inaccessible portions of the virgin forests in the Alleghanies, where it certainly appears native. Randolph: Point Mountain, alt. 3,300 ft. . Nicholas: Buffalo Range, alt. 2,875 ft.

\section{ANTHEMIS L.}

A. Cotula L. Dog's Fennel. May-weed.

Fields and waste grounds. Common throughout the State.

A. ARvensis L. Chamomile.

Waste places. Morgan: along the B. \& O. R. R. near No. I2 Water Tank.

\section{CHRYSANTHEMUM L.}

C. Leucanthemum L. Ox-Daisy. "Sheriff Pink."

Becoming too plentiful as a weed in fields, in the following counties: Monongalia, Marion, Hampshire: where it is often knówn as Sheriff Pink; Jackson, Preston, Kanawha: near Charleston (James); Cabell: near Barboursville (James). Grant, Lewis, Upshur, Randolph, Berkeley: near Martinsburg; Fayette: near Nuttallburg (Nuttall). Greenbrier: near Ronceverte, Caldwell, Fort Spring, and White Sulphur Springs. Hardy: near Moorefield (Gamble). Mercer: near Princeton and Ingleside. 


\section{MATRICARIA L.}

M. matricarioides (Less.) Porter. Wild Chamomile. (M. discoidea DC.)

Established on B. \& O. R. R. bank, Morgan: near No. I2 Water Tank.

\section{TANACETUM L.}

T. vUlgare L. Tansy.

Escaped to roadsides. Gilmer: near DeKalb. Lewis: near Weston. Grant: near Davis. Wood: near Parkersburg. Jefferson: near Shepherdstown. Monongalia: on Kingwood Pike.

\section{ARTEMISIA Linn.}

A. ANNUA L. Wormwood.

River banks and waste places. Wood: near Parkersburg (Millspaugh). Mason: near Point Pleasant, where it is said to have been introduced by an early French physician (Sheldon 924). Jefferson: at Harpers Ferry (Greene).

\section{ERECHTITES Raf.}

E. hieracifolia (L.) Raf. Fireweed.

Moist woods and banks, especially new fallows. Randolph: near Cheat Bridge, alt. 3,700 ft. Fayette: near Nuttallburg (Nuttall). Monongalia: near Uffington and Morgantown. Preston: near Aurora (Mr. \& Mrs. Steele).

\section{MESADENIA Raf.}

M. Renirormis (Muhl.) Raf. Great Indian Plantain. (Cacalia Muhl.)

Rich woods. Marion: along the F. M. \& P. R. R., especially near Opekiska. Summers : near Greenbrier Stock Yards. Monroe: near Alderson and Wolf Creek. Preston: near Terra Alta. Ohio: Bogg's Island, near Wheeling (Mertz \& Guttenberg).

M. Atriplicifolia (L.) Raf. Pale Indian Plantain.' (Cacalia L.).

Rich woodlands. Epshur: near Lorentz. Monongalia: banks of Cheat River, near Camp Eden. Ohio: near Wheeling (Mertz \& Guttenberg). Fayette: near Nuttallburg (Nuttall); near Thurmond (Eggleston 5553). Preston: near Aurora (Mr. \& Mrs. Steele). 
SYNOSMA Raf.

S. suaveolens (L.) Raf. Indian Plantain.

(Cacalia L.)

Rich banks. Monongalia and Marion: from Opekiska to Morgantown along the Monongahela River, frequent. Preston: near Terra Alta. Summers: near Hinton. Ohio: near Wheeling (Mertz \& Guttenberg).

\section{SENECIO L.}

S. ANTENNARIIFolius Britton.

On a loose slate hillside. Greenbrier: near White Sulphur Springs (Allen \& Britton, Mackenzie).

Perennial, tufted in mostly large clumps; stems slender, 2-4.5 dm. high, loosely white-woolly. Leaves nearly all basal, commonly numerous, oval to spatulate, angulateiy few-toothed or entire, mostly obtuse, narrowed into a petiole as long as the blade or longer, densely white-tomentose beneath, green and finally glabrous above, $2-4 \mathrm{~cm}$. long; stem-leaves small, spatulate, laciniate, or the upper narrowly linear and entire; heads. slender-peduncled, rather less than $25 \mathrm{~mm}$ broad; rays golden-yellow, showy; involucre $6 . \mathrm{mm}$ high. white-woolly; achenes glandular-pubescent.

S. vUlgaris L. Groundsel.

Roadsides, fence rows, streets, and waste places; adventive from Europe. Frequent.

S. AUREus L. Golden Rag-wort.

Damp places in open woods. Frequent throughout the State.

S. obovatus Muhl.

Damp places. Lewis: near Weston. Monongalia: near Morgantown (Millspaugh). Greenbrier: near White Sulphur Springs (Eggleston 4346). Ohio: near Wheeling (Mertz I567).

S. Balsamitae Muhl.

Rocky open woods. Fayette: near Nuttallburg (Nuttall). Monongalia: near Morgantown. Mercer: near Beaver Springs.

\section{ARCTIUM L.}

A. LAPPA L. Burdock.

Waste grounds, near dwellings. Abundant everywhere.

A. MINUs Schk.

Fayette: near Nuttallburg (Nuttall). 


\section{CENTAUREA Linn.}

C. NIGRA Linn.

Appeared in I9I I in the alfalfa field of Mr. L. A. Bond, at Lost Creek, Harrison County, and in 1912 in his oat field (Sheldon).

\section{CARDUUUS L.}

C. lanceolatus L. Common Thistle.

Fields, waste grounds, and roadsides. Common.

C. AĹtissimus L. Tall Thistle.

Fields and moist copses, frequent. Monongalia, Marion and Preston counties. Fayette: near Nuttallburg (Nuttall). Summers: near Greenbrier Stock Yards. Hardy: near Moorefield. Preston: near Aurora (Mr. and Mrs. Steele). Tucker: banks of the Blackwater River near Hendricks (Greenman 320).

C. DISCOLOR (Muhl.) Nutt.

Fields. Jefferson: near Charlestown; Summit Point and near Shepherdstown.

C. Virginianus (L.) Pers. Virginia Thistle.

Woods and opens. Summers: near Hinton. Preston: near Terra Alta. Frequent throughout the State.

C. Muticus (Michx.) Pers. Swamp Thistle.

Wet places. Randolph: near Cheat Bridge, alt. 3,700 ft. Upshur: near Lorentz. Kanawha: near Charleston. Preston: near Terra Alta; near Aurora ( $M r$. and Mr.s. Steele).

C. odoratus (Muhl.) Porter. Pasture Thistle. (Cirsium pumilum, Spr.)

Dry fields. Greenbrier: near White Sulphur Springs. Preston: near Terra Alta; near Cranberry Summit (Mertz \& Guttenberg) ; near Aurora (Mr. and Mrs. Steele).

C. Arvensis (L.) Robs. Canada Thistle.

Dry fields, becoming troublesome in many localities. Jefferson: plentiful near Charlestown, where it was doubtless brought in baled hay by the Federal troops during the war. Randolph: on the apex of Point Mountain, alt. 3,700 ft., in a field owned and cultivated two years ago by a Connecticut gentleman, who probably brought the seed there from the east. Greenbrier: near White Sulphur Springs. Jefferson: near Summit Point and Shenandoah Junction. Hancock: near Holliday's Cove. Brooke: at Wellsburg.

Reported also from Hampshire: near Slanesville and Capon Bridge. Brooke : near Wellsburg. Ohio: near Beech Glen School House. Summers: near Jumping Branch. Putnam: near Hurricane, Paradise and Confidence. Jeffer- 
son: near Summit Point, Middleway, Mohler's, Shenandoah Junction, Leetown and Charlestown. Lewis: near Camden Harrison: near Shinnston and Wallace. Mineral: near Patterson's Depot (since destroyed). Berkeley: near Martinsburg and Gerrardstown. Wirt: near Burning Springs. Wetzel: near Endicott. Jackson: near Sandy and Silverton. Kanawha: near Pocotaligo and Gazil. Mercer: near Concord Church. Wayne: near Stone Coal. Braxton: near Bulltown and Tate Creek. Tyler: in Mead dist. Roane: near Newton and Looneyville. Upshur: near Evergreen. Wood: near Murphy's Mills, Volcano, Parkersburg, and Rockport. Ritchie: near Berea. Fayette: near Mountain Cove. Marshall: near Meighen. Hardy: near Wardensville. Preston: near Independence, I889-9I. Monroe : neai Union. Greenbrier: near Trout Valley and Lewisburg. Grant: near Greenland. Hancock: near Holliday's Cove. Taylor: near Grafton. Cabell: near Milton. Clay: near Valley Fork. Doddridge: near Leopold.

The presence of this weed in the localities noted in the second paragraph, where not corroborated in the first, is open to doubt. 


\section{PART II.}

\section{The Fossil Flora of West Virginia}

\section{By DAVID WHITE}

The following list includes the species of fossil plants published by various geologists as having been found in the upper Paleozoic and Pleistocene formations of West Virginia. The plant-bearing beds represented belong to the Pocono, the basal formation of the Mississippian ("Lower Carboniferous") series; the Princeton conglomerate member, of Chester age, also in the Mississippian series; several formations in the Pottsville group, the basal division of the Pennsylvanian ("Upper Carboniferous") series; and from various members or beds in the Allegheny, Conemaugh and Dunkard formations which constitute the remainder of the Pennsylvanian and the Permian in the bituminous regions of the Appalachian trough. The Quaternary system is represented by the Carmichaels clay, an interglacial deposit of pre-Wisconsin age.

In West Virginia the fossil plants of some of the formations, like the Allegheny, for example, have received very little attention, and the lists for these formations are accordingly short, while in others, like the Quinnimont and Kanawha (both of Pottsville age), our paleobotanical knowledge is based preduminantly on material from this state. The floras of the Conemaugh have had but little study, and their differentiation from those of the Monongahela, on the one hand, or from those of the Allegheny on the other, is therefore at present very incomplete. 'The composition and characteristics of the plant life of the Monongahela also are but little understood, though it is known that the floras contain much that is present in, though not peculiar to, the Dunkard (basal Permian). The remains of a meager flora occurring near the top of the Greenbrier limestone in West Virginıa are wholly unstudied and unrecorded. Consequently they do not appear in the list. Further study will greatly enlarge a number of the lists from formations containing large and interesting floras that are as yet comparatively unstudied.

Many of the names quoted in the accompaning lists are based 
on preliminary examinations and are tentative, the collections having in a large number of cases not yet been fully studied.

The differentiation of the floras of the lower Pottsville which Doctor White has called the Pocahontas group; of the middle Pottsville, which he has termed the New River group; and of the upper Pottsville for which he has adopted the name, Beaver River group, is fairly well established. As initial, or invasion, stages the Raleigh sandstone and the Nuttall sandstone lentil of the Sewell formation are logically placed by the writer in the New River and Beaver River groups, respectively, of Dr. White. His Pocahontas and New River groups fall within the time covered by the "Millstone Grit", and the Lower Coal Measures of Great Britain, the Beaver River group being referable approximately to the Middle Coal Measures and the "transition" series of Great Britain, while the Allegheny goes, for the most part, together with the Conemaugh and Monongahela, into the Upper Coal Measures of Great Britain. The beds up to an horizon possibly as high as the base of the Kittanning group of the coals in the Allegheny formation are of Westphalian age, the higher Pennsylvanian beds being of Stephanian age as these periods are defined in Continental Europe.

The Dunkard formation, the division originally proposed by Doctor White in substitution for the "Upper Barren Measures" of the Pennsylvania State Reports, is here used for convenience and conformity with the West Virginia state nomenclature in place of Washington and Greene, the two formations into which the Dunkard has been divided.

The thanks of the writer are due to Doctor White and the other geologists of the State for the stratigraphic references of several of the fossil plant collections made from beds in the Allegheny and Conemaugh formations, the precise stage of these beds not having been determined prior to the detailed areal work now in progress by the State.

Among the species in the list there are many that have not been recorded from localities outside of West Virginia, though most are known to occur in beds of the same age in other parts of the Appalachian coalfields. In order, however, to avoid the description of new species which must necessarily be accompanied by illustrations, thus unduly increasing both the volume and the expense of publication, the list is confined to species already reported from West Virginia, or - as in the cases of Lewis Tunnel in Virginia, or Brown's Mills and Jollytown in Greene County, Pennsylvania-from localities near the state boundary.

The names inscribed in this list are based upon the identifications of a number of geologists and paleontologists and represent not merely the work of different men but also the work of different periods or stages in the growth of our knowledge of the 
paleobotany of the upper Paleozoic. The identifications, therefore, represent the diverse points of view and the interpretations of men varying as to their special lines of scientific work, as to their familiarity with fossil floras, and as to their periods of study. The result is the introduction of some incompatible identifications and some confusion in the lists. There are doubtiess cases in which the same species is listed under different names, while, in others, plants differing as to species, or even as to genera, are probably recorded under a single name. The results are the records as present of species not present in a given formation and the omission of others. The writer has attempted to eliminate the greater number of the paleobotanical and stratigraphic errors thus introduced. In numerous cases, however, for the sake of conservatism this has not been attempted. Obviously the errors cannot in all cases be eliminated without consultation of the original specimens, which appears to be for the most part impossible.

In the first list, which is systematic and approximately complete, the name of the species is accompanied by the citation of either the place of its original publication or the publication in which the generic combination was first introduced. Other references are to papers relating to the floras of West Virginia. The lists given in Volume II of the State Reports are not cited for the reason that these lists are quoted from a paper of earlie: date by the writer (Bulletin of the Geological Society of America, vol XI), which is cited. In passing mention should be made of the list of fossil plants of the State printed in Professor Millspaugh's "Preliminary Catalogue of the Flora of West Virgina" (see Bul. 24, W. Va. Agric. Exp. Sta., I892, pp. 519-527). The fossil plant list in this publication was contributed by America's great patron of paleobotany, R. D. Lacoe, of Pittston, $\mathrm{Pa}$. It is based in part on identifications made by Lesquereux in his last and enfeebled years, and in part on studies made by Mr. Lacoe who was, himself, a high authority on the Paleozoic floras. Lack of comparison of the specimens studied by Fontaine and White is responsible for certain variations in identification.

Full data descriptive of the formations of the Pennsylvanian and Permian in West Virgina, the position of the coals and their stratigraphic relations will be found in Volume II A of the State Reports, to which the reader is referred for all geologic information respecting the plant beds.

\section{SYSTEMATIC LIST OF SPECIES.}

Archaeopteris alleghanensis (Meek) Font. \& I. C. White. Bull. Phil. Soc. Wash.. Washington, vol. I, Appendix VIII, r874, p. xviii, pl. i, fig. 2 a. b. [Pocono sandstone.] 
ArchaEopteris Bockschiana (Goeppert) Lesquereux?

Second Geol. Surv. Pa., Rept. Prog. P. (Coal Fl.), vol. I, I880, p. 306 , pl. xlix, figs. I-4. [Pocono sandstone[.

ArchaEOPtERIS $c f$. STRICTA Andrews.

Rept. Geol. Surv. Ohio, vol. 2, pt. 2, I875, Palaeont, p. 4I8, pl. xlix, figs. 2, 2a. D. White. Bull. Geol. Soc. Am., vol. I I, I900, p. I62. [Pottsville group: Nuttall sandstone lentil of Sewell formation].

Árchaeỏpteris n. sp. D. White.

Bull. Geol. Soc. Am., vol. 6, I895, p. 3i8. [Nuttall sandstone lentil of Sewell formation].

Triphyllopteris Lescuriana (Meek) Fontaine.

Amer. Jour. Sci., 3rd ser., vol. I3, I877, p. I23. [Pocono sandstone].

Triphyllopteris virginiana Meek.

Bull. Phil. Soc. Washington, vol. I. Appendix VIII, I874, p. xviii. [Pocono sandstone].

This is probably the plant listed by Fontaine (Amer. Jour. Sci., 3rd ser., vol. $7, \mathrm{I} 874$, p. 578 ) as Cyclopteris valida.. It is one of the forms of a polymorphous type, all of which are characteristic of the lower Mississippian.

Anermites $c f$. tenuifolius (Goeppert) D. White.

Better known as Adiantites tenuifolius (Goppert) Stur. Abh. K.-K. Geol. Reichsanst, vol. 8, No. I (Culm-F1.), pt. I, I 875 , p. 65 , pl. xvi, figs. 2, 3. D. White: Bull. Geol. Soc. Am., vol. 6, I895, p. 315. [Pottsville group: Quinnimont formation].

Aneimites adiantoides (Lindley \& Hutton) Bailey.

Mem. Geol. Surv. Ireland, Expl. Sheet 137, Dublin, 1859, p. I2. Described by Lindley \& Hutton as Sphenopteris adiantoides Foss. F1. Great Britain, vol. 2, I834, p. 9I, pl. cxv. Fontaine: Amer. Jour. Sci., 3rd ser., vol. I I, 1876, p. 378. [Pottsville group; Clark? and Sewell formations].

ANeimites FERTILIS D. White.

Smithsonian Misc. Coll., vol. 47, pt. 3, I904, p. 323, pl. xlvii. The small fruits bearing the name Wardia fertilis are the seeds of Aneimites fertilis. [Pottsville group; Clark formation]

Aneimites (Adiantites) sp. D. White.

Bull. Geol. Soc. Am., vol. 6, I895, p. 313. [Princeton conglomerate (Mississippian)].

EREmopteris artemisiaefolia (Sternberg) Schimper.

Pal. Veg., vol. I, I869, p. 4I6, pl. xxx, fig. 4; Millspaugh, Bull. No. 24, W. Va. Agric. Exp. Sta., Morgantown, I892, p. 521. [Pottsville group, Kanawha formation]. 
EREMopteris $c f$. ELEGANS (Ettingshausen) Lesquereux.

Second Geol. Surv. Pa., Rept. Prog. P. (Coal Fl.), vol. I, I880, p. 294, pl. liii, figs. 7, 7a. Bull. Geol. Soc. Am., vol. 6 , I895, p. 3I5. [Pottsville group; Quinnimont and Sewell formations].

EREMOPTERIS $c f$. DECOM POSITA (Kidston) D. White.

Trans. Roy. Soc. Edinburgh, vol. 30, I882, p. 538, pl. xxxii, figs. I, Ia, 4, 5. D. White: Bull Geol. Soc. Am., vol. 6, I895, p. 3I3. [Princeton conglomerate (Mississippian)]. EREMOPTERIS MICROPHYLLA Lesq?

Second Geol. Surv. Pa., Rept. Prog. P. (Coal Fl.) vol. I, I880, p. 296, pl. liii, figs. 6, 7, 7a. D. White: Bull. Geol. Soc. Am., vol. 6, I895, p. 315. [Pottsville group; Quinnimont formation].

EREMOPTERIS CHEATHAMI Lesquereux.

Second Geol. Surv. Pa., Rept. Prog. P. (Ccal F1.), vol 3, I884, p. 770, pl. civ, figs. 2-4. [Pottsville group; Seweli formation].

Eremop'teris macilenta (Lindley \& Hutton) D. White?

Known in the literature as Sphenopteris macilenta Lindley \& Hutton: Foss F1. Great Britain, vol. 2, I835, p. 193, pl. cli. Fontaine: Amer. Jour. Sci., New Haven, 3rd ser., vol. I I, I876, p. 38I (identification subject to question). [Pottsville group; Clark and Sewell formations].

Eremopteris $c f$. Sauveuri (Crepin) D. White.

Bull. Geol. Soc. Am., vol. I I, I900, p. I63. [Pottsville group; Kanawha formation].

EREMOPTERIS $c f$. LINCOLNIANA D. White.

Twentieth Ann. Rept. U. S. Geol. Survey, r898-'99, pt. 2, I900, p. 869, pl. cxcii, figs. I, Ia. Bull. Geol. Soc. Am., vol. I I, I900, p. I6I. [Pottsville group; Kanawha formation].

EREMOPTERIS n. sp. D. White.

Bull. Geol. Soc. Am., vol. I I, I900, p. I6I. [Pottsville greup; Kanawha formation.]

EREMopteris SP. D. White.

Bull. Geol. Soc. Am., vol. 6, I895, p. 318. [Pottsville group; Nuttall sandstone lentil of Sewell formation].

Cheilanthites trifoliolatus (Artis) Goppert.

Nov. Act. Acad. Caes. Leop.-Car., vol. I7, Suppl. (Sys. Fil. Foss.), 1836, p. 245. Better known as Psendopecopteris trifoliolata. Second Geol. Surv. Pa., Rept. Prog. P. (Coal F1.), vol I, I880, p. 2I7, and Sphenopteris trifoliolata: Dict. Sci. Nat., vol. 57 (Prodrome), r828, p. 59 (50). D. White: Bull. Geol. Soc. Am., vol. I I, I900, pp. I6I, I63. [Pottsville group; Kanawha formation]. 
Cheilanthites obtusilobus (Brongniart) Goeppert

Nov. Act. Acad. Caes. Leop.-Car., vol. I7, Suppl.(Sys. Fil. Foss.), 1836 , p. 246. This is the Pseudopecopteris obtusiloba (Brongniart) of Lesquereux: Second Geol. Surv. Pa., Rept. Progr. P. (Coal Fl.), vol. 3, I884, p. 753. D. White: Bull. Geol. Soc. Am., vol. I I, I900, p. I7o. [Conemaugh formation].

The report of the species by Fontaine (Amei. Jour. Sci. (3), vol. 7,1874, p. 574) from the Sewell formation is undoubtedly erroneous. It is probable that the Sewell plant is close to, if not identical with, that described by Lesquereux (Coal Flora, vol. 3, I884, p. 770, pl. civ, figs. 2-4) as Eremopteris Cheathami.

Cheilanthites obtusilobus var. dilatata (Lesquereux).

The Pseudopecopteris obtusiloba dilatata (Lesquereux) Lacoe: Cat. Palaeoz. Plts. N. Amer., Pittston, I884, p. 9. D. White: Bull. Geol. Soc. Am., vol. I I, Igoo, p. I63. [Pottsville group; Kanawha formation].

Cheilanthites solidus D. White.

This is the Sphenopteris solida of Lesquereux: Second Geol. Surv. Pa., Rept. Progr. P, vol. 3, 1884, p. 759, pl. ci, fig. 3. D. White: Bull. Geol. Soc. Am., vol. I I, 1900, p. I70. [Pottsville proup; Kanawha formation]. [Also in Conemaugh formation].

Cheilanthites squamosus (Lesquereux) D. White.

Bull. Geol. Soc. Am., vol. I I, I900, p. I70. This species, first described by Lesquereux as Pecopteris squamosa, has generally been recorded in the American literature as Pseudopecopteris anceps. It is possibly indistinguishable from the European plant known as Pecopteris sphenopteroides. [Allegheny and Conemaugh formations].

Cheilanthites $c f$. Nummularius (Gutbier) D. White.

This is the Pseudopecopteris cf. nummularia (Gutbier) of Lesquereux: Second Geol. Surv. Pa., Rept. Progr. P, (Coal F1.), vol. 3, r884, p. 75I, pl. ciii, figs. I-3. D. White: Bull. Geol. Soc. Am., vol. I I, I900, p. I68. [Pottsville group; Kanawha formation\}.

Diploth mema pachyderma (Fontaine $\&$ I. C. White) D. White. The Odontopteris pachyderma of Fontaine and I. C. White: Second Geol. Surv. Pa., Rept. Prog. PP., i88o, p. 53, pl. x. figs. 5-Io. [Dunkard formation (Permian).].

Diploth mema $c f$. JacQuoti Zeiller.

Bull. Geol. Soc. Am., vol. I I, I900, p. I63. [Pottsville group : Kanawha formation.]

MARIOPTERIS POTTSVILleA D. White. Twentieth Ann. Rept. U.

S. Geol. Surv. 1898-'99, pt. 2, 1906.

This is the plant listed as Pseudopecopteris muricata 
(Schlotheim) Lesquereux "form." by D. White: Bull. Geol. Soc. Am., vol. 6, I895, p. 315. [Pottsville group: Quinnimont formation.]

This species is everywhere present in the upper part of the lower Pottsville.

Mariopteris pottsvillea var. The plant listed as Pseudopecopteris muricata form. D. White.

Bull. Geol. Soc. Am., vol. 6, I895, p. 317. [Pottsville group; Sewell formation.]

Mariopteris inflata. (Newberry) D. White.

Bull. Geol. Soc. Am., vol. I I, I900, p. I6I. [Pottsville group; Kanawha formation.]

MARIOPTERIS PYgMaEA D. White.

Twentieth Ann. Rept. U. S. Geol. Surv., I898-'99, pt. 2, I900, p. 876, pl. cxcii, figs. 2-6. D. White: Bull. Geol. Soc. Am., vol. II, I900, p. I62. [Pottsville group; Kanawha formation.]

Mariopteris Sillimanni (Brongniart) D. White.

Nineteenth Ann. Rept. U. S. Geol. Surv., I897-1898 [1899], p. 479. D. White, Bull Geol. Soc. Am., vol. I I, I900, p. I70. [Allegheny and Conemaugh formations.]

MARIOPTERIS MURICATA (Schlotheim) Zeiller.

Expl. Carte Geol. France, Paris, vol. 4, Atlas, 1878, pl. clxvii, fig. 5 ; text I879, p. 7I. D. White, Bull. Geol. Soc. Am., vol. II, I900, p. I6r. [Pottsville group; Kanawha formation, and Nuttall sandstone lentil of Sewell formation.] MARIOPTERIS ACUTA (Brongniart) Zeiller.

Bull. Soc. Geol. France (3), vol. 7, 1879, p. 98. Recorded as Pseudopecopteris acuta (Brongniart) by Lesquereux: Second Geol. Surv. Pa., Rept. Progr. P. (Coal. Fl.), vol. I, I88o, p. 21 5, pl. xxxvii, fig. 6. D. White, Bull. Geol. Soc. Am., vol. 6, I895, p. 318. [Pottsville group; Nuttall sandstone lentil of Sewell formation, and the Kanawha formation.]

Mariopteris Andraena (v. Roehl.) D. White.

Bull. Geol. Soc. Am., vol. I I, I900, p. I63 [Pottsville group; Kanawha formation].

MARIOPTERIS LATIFOLIA (Brongniart) Zeiller?

Mem. Soc. Geol. Nord, Lille, vol. I, No. 3, I882, p. 5. This is the Pseudopecopteris latifolia (Brong.) Lesq. form.. D. White: Bull. Geol. Soc. Am., vol. 6, I895, p. 3I5. [Pottsville group; Quinnimont formation and Kanawha formation.]

Mariopteris $c f$. Dimorpha (Lesquereux) D. White. This is the Pseudopecopteris dimorpha Lesquereux: Second Geol. Surv. Pa., Rept. Progr. P (Coal Fl.), vol. I, I880, p. 20I, pl. 
xxxv, figs. I-6. D. White: Bull. Geol. Soc. Am., vol. 6 , I895, p. 3 I5. [Pottsville group; Quinnimont formation.] MARIOPTERIS SPHENOPTEROIDES (Lesquereux) Zeiller. n. var.

F1. foss. Bass. Houill. Valenciennes, Paris, Atlas, I886, pl. xix, figs. 3,4 ; text I888, p. I7 I. D. White, Bull. Geol. Soc. Am., vol. II, I900, p. I63. [Pottsville group; Kanawha formation.] [Also in Allegheny formation.]

MARIOPTERIS NERVOSA (Brongniart) Zeiller.

Expl. Carte Geol. France, Paris, vol. 4, Atlas, 1878, pl. clxvii, figs. I-4; text I879, p. 69. D. White, Bull. Geol. Soc. Am., vol. II, I900, p. I6I. [Pottsville group; Kanawha formation.] [Also in the Allegheny and Conemaugh formations.]

Mariopteris Newberryi (Lesquereux) D. White.

Bull. Geol. Soc. Am., vol. I I, I900, p. I70. [Allegheny formation.]

MARIOPTERIS? SPINULOSA (Lesquereux) D. W.

This is the Pseudopecopteris spinulosa of Lesquereux: Second Geol. Surv. Pa., Rept. Progr. P, I880, p. I95, pl. lvi, fig. I. Millspaugh: Bull. No. 24, W. Va. Agric. Exp. Sta., Morgantown, 1892 , p. 525 . [Conemaugh formation.]

Sphenopteris (Crossotheca) ophioglossoides (Lesquereux) D. White.

Bull. Geol. Soc. Am., vol. 8, 1806 [1897], p. 300 [Sorocladus]. D. White: Bull. Geol. Soc. Am., vol. I I, I900, p. I7o. [Allegheny and Conemaugh formations.]

SPHENOPTERIS SPINOSA Goppert.

Gatt. Foss. Pflanzen, pts. 3 \& 4, 1842, p. 104, pl. xii. D. White: Bull. Geol. Soc. Am., vol. I I, I900, p. I6I. [Pottsville group; Kanawha formation.]

SphenOPTERIS PATENTISSIMA (Ettingshausen) Schimper.

Pal. Veg., vol. I, I869, p. 407. D. White: Bull. Geol. Soc. Am., vol. 6, I895, p. 3I5. [Pottsville group; Quinnimont formation.]

Sphenopteris $c f$. Royi Lesquereux, Second Geol. Surv. Pa., Rept. Prog. P. (Coal Fl.), vol. 3, I884, p. 768, pl. civ, figs. 7-IO. [Pottsville group; Sewell formation.]

SPHENOPTERIS FURCATA Brongniart.

Dict. Sci. Nat., vol. 57 (Prodrome), 1828, p. 59 (50). D. White: Bull. Geol. Scc. Am., vol. I I, I900, p. I6I. [Pottsville group; Kanawha formation.]

SPHENOPTERIS ELEGANS (Brongniart) Sternberg.

Flora d. Vorwelt (Tentamen), I825, p. xv, pl. xxiii, fig. 2. Millspaugh, Bull. No. 24, W. Va. Agric. Exp. Sta., Morgantown, I892, p. 527. [Pottsville group; Kanawha formation.] 
Sphenopteris Distans Sternberg.

Flora d. Vorwelt (Tentamen), 1825, p. xvi. D. White: Bull. Geol. Soc. Anı., vol. 6, I895, p. 3I3. [Princeton conglomerate (Mississippian).]

SPHENOPTERIS DIVARICATA of Lesquereux.

Second Geol. Surv. Pa., Rept. Prog. P (Coal Fl.), vol. 3, I884, p. 767, pl. civ, fig. 6. D. White: Bull. Geol. Soc. Am., vol. 6, I895, p. 315. [Pottsville group; Quinnimont formation.]

A plant doubtfully referred to the same species occurs in the Nuttall sandstone lentil of the Sewell formation.

Sphenopteris $c f$. Goepperti (Ettingshausen) Schimper.

Pal. Veg., vol. I, I869, p. 4I2. D. White: Bull. Geol. Soc. Am., vol. 6, I895, p. 315. [Pottsville group; Quinnimont formation.]

Sphenopteris Hoeninghausir Brongniart.

Dict. Sci. Nat., vol. 57 (Prodrome), I828, p. 60 (5I). D. White: Smithsonian Misc. Coll. (Quarterly Issue), vol. 47, pt. 3, I 905 , p. 383, pl. liii, fig. 2. [Pottsville group; Clark, Quinnimont and Sewell formations.]

Sphenopteris RARINERVIS Fontaine.

Am. Jour. Sci. (3), vol. I I, I876, p. 38r. [Pottsville group; Clark formation.]

SPHENOPTERIS $c f$. LINEARIS Sternberg.

Flora d. Vorwelt (Tentamen), I825, p. xv, pl. xlii, fig. 4. D. White: Bull. Geol. Soc. Am., vol. I I, I900, p. I62. [Pottsville group; Kanawha formation.]

Sphenopteris subgeniculata (Stur) Rothpletz.

Botanisches Centralblatt, Cassel, vol. I-2, I880, III, GratisBeilage, No. I, p. I4, pl. iii, fig. I7. D. White: Bull. Geol. Soc. Am., vol. 6, I895, p. 313. [Princeton conglomerate (Mississippian).].

Sphenopteris $c f$. Geniculata Germar \& Kaulfuss.

Nov. Act. Acad. Caes. Leop-Car., vol 15, pt. 2, I83I, p. 224, pl. lxv, fig. 2. D. White: Bull. Geol. Soc. Am.,vol. I I, I900, p. I63. [Pottsville group; Kanawha formation.]

Sphenopteris sp. $c f$. Hildreti Lesquereux.

Second Geol. Surv. Pa., Rept. Progr. P (Coal F1.), vol. I, I880, p. 283. D. White: Bull. Geol. Soc. Am., vol. I I, I900, p. I63. [Pottsville group; Kanawha formation.]

SPHENOPTERIS $c f$. TRICHOMANOIDES Brongniart.

Hist. Veg. Foss., Paris, vol. I, I829, p. I82, pl. xlviii, fig 3. D. White: Bull. Geol. Surv. Am., vol. II, I900, p. I69 [Pottsville group; Kanawha formation.] 
Sphenopteris LinkiI (Goeppert) Presl.

In Sternberg, Flora d. Vorwelt, vol. 2 (Versuch), pts. 7 $\& 8,1838$, p. I32. D. White: Bull. Geol. Soc. Am., vol. I I, I900, p. I6I. [Pottsville group; Kanawha formation.]

SPHENOPTERIS TRACYANA Lesquereux?

Second Geol. Surv. Pa., Rept. Progr. P (Coal F1.), vol. 3, I884, p. 766, pl. ci, fig. 2. D. White: Bull. Geol. Soc. Am., vol. I I, I900, p. 16I. [Pottsville group; Kanawha formation.]

Sphenopteris tenella Brongniart.

Hist. Veg. Foss., Paris, vol. I, I829, p. I86, pl. xlix, fig. I. D. White: Bull. Geol. Soc. Am., vol. I I, I900, p. I69. [Pottsville group; Kanawha formation.]

Sphenopteris (Zeilleria) delicatula Sternberg.

Flora d. Vorwelt (Tentamen), I825, p. xvi, pl. xxvi, fig. 5.

D. White: Bull. Geol. Soc. Am., vol. I I, I900, p. I63.

[Pottsville group; Kanawha formation.]

SPHENOPTERIS FLEXICAULIS Lesquereux.

Second Geol. Surv. Pa., Rept. Prog. P (Coal Fl.), vol. I, I880, p. 284. D. White: Bull. Geol. Soc. Am., vol. 6, I895, p. 317. [Pottsville group; Sewell formation.]

Sphenopteris $c f$. Larischil (Stur) Lesquereux.

Second Geol. Surv. Pa., Rept. Prog. P (Coal Fl.), vol. I, I880, p. 288, pl. 1v, figs. 7, 7a. D. White: Bull. Geol. Soc. Am., vol. 6, I895, p. 317. [Pottsville group; Sewell formation.]

Sphenopteris hymenophylloides Brongniart.

Dict. Sci. Nat., vol. 57 (Prodrome), I828, p. 60 (5I). D. White: Bull Geol. Soc. Am., vol. I I, I900, p. I69. [Pottsville group; Kanawha formation.]

Sphenopteris Pinnatifida (Lesquereux) D. White.

Bull. Geol. Soc. Am., vol. 8, I896 [1897], p. 291. D. White: Bull. Geol. Soc. Am., vol. I I, I900, p. I7I. [Allegheny formation.]

SpHENOPTERIS DICKSONIOIDES (Goeppert) Schuetze, form.

Abh. Geol. Specialk. Preuss., vol. 4, No. 4, I882, p. 22. D. White: Bull. Geol. Soc. Am., vol. 6, I895, p. 315. [Pottsville group; Quinnimont formation.]

SPHENOPTERIS KARWINENSIS Stur.

Verh. K.-K. Geol. Reichsanst., I874, p. 299. D. White: Bull. Geol. Soc. Am., vol. I I, I900, p. I69. [Pottsville group; Kanawha formation.]

SPHENOPTERIS $c f$. DUBUISSONIS Brongniart.

Dict. Sci. Nat., vol. 57 (Prodrome), I828, p. 60 (5I). D. White: Bull. Geol. Soc. Am., vol. I I, I900, p. I6I. [Pottsville group; Kanawha formation.] 
Sphenopteris minuti-secta Fontaine \& I. C. White.

Second Geol. Surv. Pa., Rept. Progr. PP, I88o, p. 43, pl. v, figs. I-4. [Conemaugh formation; and Dunkard formation (Permian).]

SpHENopteris (Renaultia) MICROCARPA Lesquereux.

Second Geol. Surv. Pa., Rept. Progr. P (Coal F1.), vol. I, I88o, p. 28o, pl. xlvii, figs. 2-2b. D. White: Bull. Geol. Soc. Am., vol. 6, I895, p. 315. [Pottsville group; Quinnimont, Sewell, and Kanawha formations.]

SPHENOPTERIS SCHATZLARENSIS Stur.

Abh. K.-K. Geol. Reichsanst., vol. 8, No. 2 (Culm. F1., pt. 2), I877, p. 321 (427). D. White: Bull. Geol. Soc. Am., vol. I I, I900, pp. I6I, I63. [Pottsville group; Kanawha formation.]

Sphenopteris chaerophylloides (Brongniart) Presl.

In Sternberg, Flora d. Vorwelt, vol. 2 (Versuch), pts. 7 \& 8, I838, p. I3I. D. White: Bull. Geol. Soc. Am., vol. I I, I900, p. I70. [Allegheny formation.]

Sphenopteris breviloba (Fontaine \& I. C. White) D. White.

Second Geol. Surv. Pa., Rept. Prog. PP, r88o, p. 86, p1. xxxi, fig. 3. [Dunkard formation (Permian).]

Sphenopteris formosa (Fontaine \& I. C. White) D. White.

Second Geol. Surv. Pa., Rept. Progr. PP, I880, p. 86, pl. xxxi, figs. I-2. [Dunkard formation (Permian).]

Sphenopteris lobata (Fontaine \& I. C. White) D. White.

Second Geol. Surv. Pa., Rept. Progr. PP, r880, p. 87, pl. xxxi, fig. 4. [Dunkard formation (Permian).]

Sphenopteris obtusiloba (Fontaine \& I. C. White) D. White.

Second Geol. Surv. Pa., Rept. Prog. PP, I88o, p. 85, pl. xxxi, figs. 5-6. [Dunkard formation (Permian).]

Sphenopteris $c f$. Broadheadi D. White.

Mon. U. S. Geol. Survey, vol. 37, I899, p. 4I, pl. xiii, figs. I, 2. D. White: Bull. Geol. Soc. Am., vol. I I, I900, p. I69. [Pottsville group; Kanawha formation.]

Sphenopteris Lescuriana Fontaine \& I. C. White.

Second Geol. Survey Pa., Rept. Prog. PP, I88o, p. 44, pl. vi, fig: I ; pl. vii, figs. I, 2. [Dunkard formation (Permian).]

Sphenopteris dentata Fontaine \& I. C. White.

Second Geol. Surv. Pa., Rept. Prog. PP, I88o, p. 42, pl. v, figs. 7-8. [Dunkard formation (Permian).]

Sphenopteris auriculata Fontaine \& I. C. White.

Second Geol. Surv. Pa., Rept. Prog. PP, I880, p. 42, pl. vii, figs. 3-4. [Dunkard formation (Permian).]

Sphenopteris foliosa Fontaine \& I. C. White.

Second Geol. Surv. Pa., Rept. Prog. PP, 1880, p. 44, pl. v, figs. 9-II. [Dunkard formation (Permian).] 
Sphenopteris pachynervis Fontaine \& I. C. White.

Second Geol. Surv. Pa., Rept. Prog. PP, i88o, p. 46, pl. vii, figs. 5-6. [Dunkard formation (Permian).]

Sphenopteris hastata Fontaine \& I. C. White.

Second Geol. Surv. Pa., Rept. Prog. PP, r88o, p. 46, pl. vii, fig. 7. [Dunkard formation (Permian).]

Sphenopteris acrocarpa Fontaine \& I. C. White.

Second Geol. Surv. Pa., Rept. Prog. PP, I88o, p. 40, pl. iii, figs. I-3; pl. iv, figs. I-5. [Dunkard formation (Permian).]

Sphenopteris SP.? Fontaine \& I. C. White.

Second Geol. Surv. Pa., Rept. Prog. PP, I88o, p. 42, pl. xi, figs. 5-7. [Dunkard formation (Permian).]

SPHENOPTERIS $c f$. CANNELTONENSIS D. White.

Mon. U. S. Geol. Surv., vol. 37, I899, p. 55, pl. xv, fig. 2. D. White: Bull. Geol. Soc. Am., vol. I I, I900, p. I63. [Pottsville group; Kanawha formation.]

SPHENOPTERIS MIXTA Schimper.

Pal. Veg., vol. I, I869, p. 382. D. White: Bull. Geol. Soc. Am., vol. II, I900, p. I69. [Pottsville group; Kanawha formation.] [Also in Allegheny formation.]

Sphenopteris $c f$. STIPUlata Gutbier.

In Reichenbach, Gaa von Sachs. (Verst. Obersachsen), I843, p. 74 . D. White: Bull. Geol. Soc. Am., vol. I I, I90o, p. I7I. [Allegheny formation.]

SPHENOPTERIS COMMUNIS Lesquereux.

Second Geol. Surv. Pa., Rept. Prog. P (Coal F1.), vol. 3, I884, p. 762, pl. civ, figs. I, Ia. D. White: Bull. Geol. Soc. Am., vol. 6, I895, p. 317. [Pottsville group; Sewell formation.]

Sphenopteris SP. D. White.

Bull. Geol. Soc. Am., vol. 6, I895, p. 313. [Princeton conglomerate (Mississippian).]

Sphenopteris N. SP. D. White.

Bull. Geol. Soc. Am., vol. 6, I895, p. 3I4. Near. Pocahontas coal. [Pottsville group; Clark formation.]

Oligocarpia alabamensis Lesquereux?

Second Geol. Surv. Pa., Rept. Progr. P (Coal F1.), vol. I, I880, p. 266, pl. xlvii, figs. I-Ib. D. White: Bull. Geol. Soc. Am., vol. II, I900, p. I63. [Pottsville group; Kanawha formation.]

Oligocarpia SP. D. White.

Bull. Geol. Soc. Am., vol. i I, Igoo, p. I63. [Pottsville group; Kanawha formation.] 
Sphenopteris (Crossotheca) sagittatus (Lesquereux) D. White. Bull. Geol. Soc. Am., vol. II, Ig00, p. I7I. [Allegheny formation.]

Sphenopterís (Crossotheca) ophioglossoides (Lesquereux) D. White.

Bull. Geol. Soc. Am., vol. 8, I896 [1897], p. 300. D. White: Bull. Geol. Soc. Am., vol. I I, I900, p. I69. [Pottsville group; Kanawha formation.]. [Also in Allegheny formation.]

Sphenopteris (Crossotheca) N. SP. $c f$. CRepini Zeiller.

Bull. Soc. Geol. de France, 3rd ser., vol. 12, I884, p. I94.

D. White: Bull. Geol. Soc. Am., vol. I I, I900, p. I63. [Pottsville group; Kanawha formation.]

Pecopteris Pluckeneti Sternberg.

Flora d. Vorwelt (Tentamen), I825, p. xix. Fontaine \& I. C. White: Second Geol. Surv. Pa., Rept. Progr. PP, p. 67, pl. xxi, figs. 4-5. [Conemaugh formation; and Dunkard formation (Permian).]

Pecopteris Pluckeneti var. constricta Fontaine \& I. C. White.

Second Geol. Surv. Pa., Rept. Progr. PP, I880, p. 68, pl. xxi, fig. 8. [Dunkard formation (Permian).]

Pecopteris Germari (Weiss) Fontaine \& I. C. White.

Second Geol. Surv. Pa., Rept. Progr. PP, i88o, p. 68, pl. xix, figs. I-7. [Dunkard formation (Permian).]

Pecopteris Germari var. crassinervis Fontaine \& I. C. White.

Second Geol. Surv. Pa., Rept. Progr. PP, i880, p. 70, pl. $\mathrm{xx}$, fig. 5. [Dunkard formation (Permian).]

Pecopteris Germari var. cuspidata Fontaine \& I. C. White.

Second Geol. Surv. Pa., Rept. Progr. PP, I880, p. 70, pl. $\mathrm{xx}$, fig. 4. [Dunkard formation (Permian).]

Pecopteris plumosa (Artis) Sternberg?

Flora, Regensburg, vol. I, I827, p. I37. D. White: Bull. Geol. Soc. Am., vol. I I, I900, p. I63. [Pottsville group; Kanawha formation, and Nuttall sandstone lentil of the Sewell formation.]

Pecopteris (?) serrulata (Dawson) Hartt, Dawson. Acad. Geol., London, 2d Ed., I868: p. 553, text-fig. I92K. D. White: Bull. Geol. Soc. Am., vol. 6, I895, p. 317. [Pottsville group; Sewell formation.]

Pecopteris dentata Brongniart.

Dict. Sci. Nat., vol. 57 (Prodrome), I828, p. 67 (58). D. White: Bull. Geol. Soc. Am., vol. I I, I900, p. I7I. [Allegheny formation.] 
Pecopteris dentata var. Parva Fontaine \& I. C. White.

Second Geol. Survey Pa., Rept. Progr. PP, I880, p. 67, pl. xxii, fig. 2. Millspaugh: Bull. No. 24, W. Va. Agric. Exp. Sta., Morgantown, I892, p. 524. [Dunkard formation (Permian).]

Pecopteris dentata var. crenata Fontaine \& I. C. White.

Fontaine \& I. C. White: Second Geol. Surv. Pa., Rept. Progr. PP, I880, p. 66, pl. xxii, figs. I-5. [Dunkard formation (Permian).]

PeCopteris ASPERA Brongniart.

Dict. Sci. Nat., vol. 57 (Prodrome), 1828, p. 67 (58). D. White: Bull. Geol. Soc. Am., vol. I I, I900, p. I63. [Pottsville group; Kanawha formation.]

Pecopteris pachypteroides Fontaine \& I. C. White.

Second Geol. Surv. Pa., Rept. Progr. PP, i88o, p. 76, pl. xxvi, figs. I-4. [Dunkard formation (Permian).]

Pecopteris unita Brongniart (not P. unita (F. \& I. C. W.) D. W.)

Dict. Sci. Nat., vol. 57 (Prodrome), I828, p. 67 (58). D. White: Bull. Geol. Soc. Am., vol. I I, I900, p. I70. [Allegheny and Conemaugh formations.]

Pecopteris emarginata (Goeppert) Presl.

In Sternberg, Flora d. Vorwelt, vol. 2 (Versuch), pts. 7 \& 8, 1838, p. I 58. D. White: Bull. Geol. Soc. Am., vol. I I, I900, p. I70, Fontaine \& I. C. White: Second Geol. Surv. Pa., Rept. Progr. PP, I880, p. 82. [Allegheny formation.] [Dunkard formation (Permian).]

Pecopteris (Goniopteris) oblonga (Fontaine \& I. C. White) Miller.

Am. Pal. Foss., Supplement, I883, p. 255. [Dunkard formation (Permian).]

Pecopteris (Goniopteris) Newberryana (Fontaine \& I. C. White) Miller.

Am. Pal. Foss., Supplement, I883, p. 255. Identified by Zeiller as P. foeminaeformis (Schloth.) (Zeill.) var. diplazioides. [Dunkaard formation (Permian).]

Pecopteris (Goniopteris) LONGIFolia (Fontaine \& I. C. White) D. White.

Fontaine \& I. C. White: Second Geol. Surv. Pa., Rept. Progr. PP, I88o, p. 82. [Dunkard formation (Permian).] Pecopteris (Goniopteris) elliptica (Fontaine \& I. C. White) D. White.

Fontaine \& I. C. White: Second Geol. Surv. Pa., Rept. Progr. PP, I880, p. 83, pl. xxx, fig. I. [Dunkard formation (Permian).] 
Pecopteris (Goniopteris) sp? Fontaine \& I. C. White.

Second Geol. Surv. Pa., Rept. Progr. PP, i88o, p. 83, pl. xvii, fig. 6. [Dunkard formation (Permian).]

Pecopteris (Goniopteris) arguta (Brongn.) Schimp.

Pal. Veg., vol. I, I869, p. 543. Fontaine \& I. C. White: Second Geol. Surv. Pa., Rept. Progr. PP, I880, p. 82.

[Dunkard formation (Permian).]

Pecopteris goniopteroides Fontaine \& I. C. White.

Second Geol. Surv. Pa., Rept. Prog. PP, ı88o, p. 8o, pl. $\mathrm{xxv}$, fig. 2. [Dunkard formation (Permian).]

Pecopteris (Goniopteris) elegans (Germar) Schimper.

Pal. Veg., vol. I, I869, p. 542. Fontaine \& I. C. White: Second Geol. Surv. Pa., Rept. Prog. PP, I88o, p. 82. [Dunkard formation (Permian).]

PeCOPteris ARborescens (Schlotheim) Brongniart.

Dict. Sci. Nat., vol. 57 (Prodrome), I828, p. 65 (56).

Fontaine \& I. C. White: Second Geol. Surv. Pa., Rept. Progr.

PP, I88o, p. 62. [Dunkard formation (Permian).]

Pecopteris arborescens var. integripinna Fontaine \& I. C.

White.

Second Geol. Surv. Pa., Rept. Progr. PP, I880, p. 63, pl. xxvii, fig. 6. [Dunkard formation (Permian).]

Pecopteris microphylla Brongniart.

Dict. Sci. Nat., vol. 57 (Prodrome), I828, p. 67 (58).

Lacoe in Millspaugh : Bull. No. 24, W. Va. Agric. Exp. Sta., Morgantown, I892, p. 524. [Dunkard formation (Permian).] Pecopteris nodosa (Goeppert) Schimper.

Pal. Veg., vol. I, I869, p. 500, pl. xli, fig. I4. Lesquereux:

Second Geol. Survey Pa., Rept. Progr. P (Coal F1.), vol. 3, I884, p. 872. [Conemaugh formation.]

Pecopteris vestita Lesquereux.

Second Geol. Surv. Pa., Rept. Progr. P (Coal F1.), vol. I, I88o, p. 252, pl. xliii, figs. I-7. D. White: Bull. Geol. Soc.

Am., vol. I I, Igoo, p. I7O. [Allegheny formation.]

Pecopteris villosa Brongniart?

Hist. Veg. Foss., Paris, vol. I, I834, p. 315, pl. civ, fig. 3.

D. White: Bull. Geol. Soc. Am., vol. I I, I900, p. I69. [Allegheny and Conemaugh formations.]

Pecopteris pennaeformis var. Latifolia Fontaine \& I. C. White.

Second Geol. Surv. Pa., Rept. Progr. PP, I88o, p. 65, pl. xvii, figs. 4, 5. [Dunkard formation (Permian).]

Pecopteris Candolleana Brongn.

Dict. Sci. Nat, vol. 57 (Prodrome), 1828, p. 65 (56). Fontaine \& I. C. White: Second Geol. Surv. Pa., Rept. Progr. PP, I880, p. 63, pl. xx, figs. I, 2, 3. [Dunkard formation (Permian).] 
Pecopteris OREOPteridia (Schlotheim) Sternberg.

Flora d. Vorwelt (Tentamen), I825, p. xix. D. White: Bull. Geol. Soc. Am., vol. I I, I900, p. I70. Fontaine \& I. C. White: Second Geol. Surv. Pa., Rept. Progr. PP, I88o, p. 64. [Dunkard formation (Permian).] [Allegheny and Conemaugh formations.]

Pecopteris rarinervis Fontaine \& I. C. White.

Second Geol. Surv. Pa., Rept. Progr. PP, I880, p. 7I, pl. $\mathrm{xx}$, figs. $6,7,8$. [Dunkard formation (Permian).]

Pecopteris imbricata Fontaine \& I. C. White.

Second Geol. Surv. Pa., Rept. Progr. PP, I880, p. 72, pl. xxiii, fig. I. [Dunkard formation (Permian).]

Pecopteris imbricata Fontaine \& I. C. White.

Second Geol. Surv. Pa., Rept. Progr. PP, I880, p. 72, pl. xxiii, fig. I. [Dunkard formation (Permian).]

Pecopteris platynervis Fontaine \& I. C. White.

Second Geol. Surv. Pa., Rept. Progr. PP, I880, p. 73, pl. xviii, figs. I-6. [Dunkard formation (Permian).]

Pecopteris asplenioides Fontaine \& I. C. White.

Second Geol. Surv. Pa., Rept. Progr. PP, r88o, p. 72, pl. xxv, fig. I. [Dunkard formation (Permian).]

Pecopteris rotundiloba Fontaine \& I. C. White.

Second Geol. Surv. Pa., Rept. Progr. PP, I88o, p. 74, pl. xvii, fig. 2. [Dunkard formation (Permian).]

Pecopteris angustipinna Fontaine \& I. C. White.

Second Geol. Surv. Pa., Rept. Progr. PP, r880, p. 76, pl. xxvii, figs. I-3. [Dunkard formation (Permian!).]

Pecopteris tenuinervis Fontaine \& I. C. White.

Second Geol. Surv. Pa., Rept. Progr. PP, I880, p. 77, pl. xxviii, figs. $\mathrm{I}^{-4}$. [Dunkard formation (Permian).]

Pecopteris sub-falcata Fontaine \& I. C. White.

Second Geol. Surv. Pa., Rept. Progr. PP, I880, p. 70, pl. xxi, figs. I-2. [Dunkard formation (Permian).]

Pecopteris Heeriana Fontaine \& I. C. White.

Second Geol. Surv. Pa., Rept. Progr. PP, I88o, p. 77, pl. xxv, figs. 3-7. [Dunkard formation (Permian).]

Pecopteris Schimperiana Fontaine \& I. C. White.

Second Geol. Surv. Pa., Rept. Progr. PP, I880, p. 75, pl. xxiv, figs I-5. [Dunkard formation (Permian) ]

Pecopteris lanceolata Fontaine \& I. C. Whit:

Second Geol. Surv. Pa., Rept. Progr. PP, I88o, p. 79, pl. xxix, figs. 7, 8, 9. [Dunkard formation (Permian).]

Pecopteris inclinata Fontaine \& I. C. White.

Second Geol. Surv. Pa., Rept. Progr. PP, I88o, p. 80, pl. xxix, fig. 4. [Dunkard formation (Permian).] 
Pecopteris notata Lesquereux.

Boston Jour. Nat. Hist., vol. 6 (New Spec. Foss. Plts Anthr.), I854, p. 424. Fontaine \& I. C. White: Second Geol Surv. Pa., Rept. Progr. PP, I880, p. 68. [Monongahela formation.]

Pecopteris Merianiopteroines Fontaine \& I. C. White.

Second Geol. Surv. Pa., Rept. Progr. PP, I880, p. 78, pl. xxix, figs. I-2. [Dunkard formation (Permian).]

Pecopteris rotundifolia Fontaine \& I. C. White.

Second Geol. Surv. Pa., Rept. Progr. PP, I880, p. 73, pl. xxiv, fig. 6. [Dunkard formation (Permian).]

Pecopteris Sp.? Fontaine \& I. C. White.

Second Geol. Surv. Pa., Rept. Progr. PP, I880, p. 80, pl. xxvii, figs. 4, 5, 7. [Dunkard formation (Permian).]

Pecopteris sp. $c f$. INTEgra (Andrae) Schimper.

Pal. Veg., vol. I, I869, p. 530. D. White: Bu1l. Geol. Soc. Am., vol. II, I900, p. I6r. [Pottsville group; Kanawha formation.]

Pecopteris n. SP. $c f$. Crenulata Brongniart.

Dict. Sci. Nat., vol. 57 (Prodrome), I828, p. 66 (57). D. White: Bull. Geol. Soc. Am., vol. I I, I900, p. I63. [Pottsville group; Kanawha formation.]

Pecopteris ovoldes Fontaine \& I. C. White.

Second Geol. Surv. Pa., Rept. Progr. PP, I88o, p. 79, pl. xxix, fig. 3. [Dunkard formation (Permian).]

Pecopteris latifolia Fontaine \& I. C. White.

Second Geol. Surv. Pa., Rept. Progr. PP, I88o, p. 79, pl. xxix, figs. 5-6. [Dunkard formation (Permian).]

Pecopteris $c f$. Jenneyi D. White.

Mon. U. S. Geol. Surv., vol. 37 (Fl. Low. Coal. Meas. Mo.), I899, p. 80, pl. xxxvi, figs. I, 2. D. White: Bull. Geol. Soc. Am., vol. II, Ig00, p. I70. [Conemaugh formation.]

PeCopteris pteroides Brongniart.

Dict. Sci. Nat., vol. 57 (Prodrome), I828, p. 65 (57). Fontaine \& I. C. White: Second Geol. Surv. Pa., Rept. Progr. PP, I880, p. 67. [Dunkard formation (Permian).]

Pecopteris Miltoni (Artis) Sternberg.

Flora, Regensburg, vol. I, I827, p. I37. Fontaine \& I. C. White: Second Geol. Surv. Pa., Rept. Progr. PP, I880, p. 65, pl. xxiii, figs. 2, 3. D. White : Bull. Geol. Soc. Am., vol. II, I900, p. I70. [Allegheny \& Conemaugh formations.] [Dunkard formation (Permian).]

PECOPTERIS POLYMORPHA Brongniart.

Dict. Sci. Nat., vol. 57 (Prodrome), I828, p. 65 (56). D. White: Bull. Geol. Soc. Am., vol. I I, I900, p. I72. [Conemaugh formation.] [Dunkard formation (Permian).] 
Pecopteris elliptica Bunbury.

Quart. Jour. Geol. Soc. London, vol. 2, I845, p. 84, pl. vii. Fontaine \& I. C. White: Second Geol. Surv. Pa., Rept. Progr. PP, I880, p. 64, pl. xvii, fig. I. [Dunkard formation (Permian).]

PeCOPTERIS (Callipteridium) GRANDifolia (Fontaine \&. I. C. White) D. White.

Second Geol. Surv. Pa., Rept. Progr. PP, I88o, p. 58, p1. xv, figs. I-4; pl. xvi, figs. 2-4. D. White: Bull. Geol. Soc. Am., vol. I4, I904, p. 540. [Dunkard formation (Permian).] Pecopteris (Callipteridium) oblongifolia (Fontaine \& I. C. White) D. White.

Second Geol. Surv. Pa., Rept. Progr. PP, i88o, p. 56, pl. xii, figs. $\mathbf{I}^{-} 5$.[Dunkard formation (Permian).]

Pecopteris (Callipteridium) odontopteroides (Fontaine \& I. C. White) D. White.

Second Geol. Surv. Pa., Rept. Progr. PP, I88o, p. 59, pl. xvi, fig. I. D. White: Bull. Geol. Soc. Am., vol. I4, I904, p. 539. [Dunkard formation (Permian).]

Pecopteris (Callipteridium) unita (Fontaine \& I. C. White) D. White.

Second Geol. Surv. Pa., Rept. Progr. PP, I88o, p. 6o, pl. xiv, figs. 2,3 . [Dunkard formation (Permian).]

Pecopteris (Callipteridium) Dawsonianiana (Fontaine \& I. C. White) D. White.

Second Geol. Surv. Pa., Rept. Progr. PP, I880, p. 56, pl. xiii, figs. I-2; pl. xiv, fig. I. D. White: Bull. Geol. Soc. Am., vol. I4, I904, p. 539. [Dunkard formation (Permian).]

Pecopteris SP. D. White.

Bull. Geol. Soc. Am., vol. I I, I900, p. I72. [Conemaugh formation).

Alethopteris Decurrens (Artis) Sternberg.

Flora, Regensburg, vol: I, I827, p. I38. D. White: Bull. Geol. Soc. Am., vol. I I, I900, p. 161. [Pottsville group: Kanawha formation.] Characteristic of the middle and upper Pottsville.

Alethopteris AQUilina (Schlotheim) Goeppert.

Nov. Acta Acad. Caes. Leop. Car., vol. I7, Suppl. (Syst. Fil. Foss.), 1836, p. 298. Lacoe in Millspaugh: Bull. No. 24, W. Va. Agric. Exp. Sta., Morgantown, I8g2, p. 519. [Conemaugh formation.]

Alethopteris Lonchitica (Schlotheim) Sternberg.

Flora d. Vorwelt (Tentamen), I825, p. xxi. D. White: Bull. Geol. Soc. Am., vol. I I, I900, p. I63. [Pottsville group; Sewell? and Kanawha formation].

Characteristic of the upper Pottsville. The typical form is unknown in the Allegheny or later beds. 


\section{Alethopteris Serli (Brongniart) Goeppert.}

Nov. Act. Acad. Caes. Leop. Car., vol. I7, Suppl. (Sys. Fil. Foss.) r836, p. 301, pl. xxi, figs. 6-7. D. White: Bull. Geol. Soc. Am., vol. I I, Ig0o, p. I6I.

Species mainly confined to the Allegheny and upper Pottsville formations. [Pottsville group; Kanawha formation]. [Conemaugh formation].

Alethopteris Serlin var. europaea (Brongniart) Goeppert.

Nov. Act. Acad. Caes. Leop. Car., volv I7, I874, Suppl. (Sys. Fil. Foss.), 1836, p. 301. Fontaine: Amer. Jour. Sci., 3rd ser., vol. 7, 1874, p. 574; ibid, vol. II, 1876, p. 379. [Pottsville group; Sewell formation].

This species is near Alethopteris helenae, Lesquereux (Geol Surv. Alabama, Rept. Prog., 1875 [1876́], p. 77), a type known only in the Pottsville. It is probably the plant listed by Fontaine as Alethopteris Serlii.

Alethopteris Serli var. americana (Brongniart) Goeppert

Nov. Act. Acad. Caes. Leop. Car., vol. I7, Suppl. (Sys. Fil. Foss.), 1836, p. 30I. Fontaine: Amer. Jour. Sci., 3rd ser., vol. 7, i874, p. 574. [Pottsville group; Sewell formation].

Alethopteris Massilionis (Lesquereux) D. White.

Second Geol. Surv. Pa., Rept. Prog. P, I880, p. I73. Lacoe, in Millspaugh: Bull. No. 24, W. Va. Agric. Exp. Sta, Morgantown, I892, p. 520. [Pottsville group; Kanawha formation].

Alethopteris $c f$. GRANDIFOlia Newberry.

Ann. Sci., Cleveland, vol. I, 1853, p. 107. Fontaine: Amer. Jour. Sci., 3rd ser., vol. I I, 1876, p. 379. [Pottsville group: Clark formation].

Alethopteris Evansi Lesquereux.

Second. Geol. Surv. Pa., Rept. Prog. P, (Coal Fl.), vol 3, I884, p. 834. D. White: Bull. Geol. Soc. Amer., vol. 6, I895, p. 317. [Pottsville group; Sewell formation].

Alethopteris $c f$. ambigua Lesquereux.

In I. C. White: Geol. Surv. Pa., Rept. Prog. Q, I875 [1878], p. 54. D. White: Bull. Geol. Soc. Am., vol. 6, I895, p. 318. [Pottsville group; Nuttall sandstone lentil of Sewell formation].

Alethopteris pennsylvanica Lesquereux.

Boston Jour. Nat. Hist., vol. 6, 1854 , p. 433. D. White: Bull. Geol. Soc. Am., vol. I I, I900, p. I72. [Allegheny formation].

Alethopteris virginiana Fontaine \& I. C. White.

Second Geol. Surv. Pa., Rept. Prog. PP, I88o, p. 88, pl. xxxii, figs. I-5; pl. xxxiii, figs. I-4. [Dunkard formation (Permian).] 
Alethopteris gigas (Gutbier) Geinitz.

Leitpfl. Rothl. u. Zechst. Sachsen, 1858, p. 12, pl. i, figs. 2-3. Fontaine \& I. C. White: Second Geol. Surv. Pa., Rept. Prog. PP, I880, p. 89, pl. xxxiii, figs. 5-6. [Dunkard formation (Permian).]

Alethopteris SP. D. White.

Bull. Geol. Soc. Am., vol. 6, I895, p. 315. [Pottsville group; Quinnimont formation].

Alethopteris SP. D. White.

Bull. Geol. Soc. Am., vol. 6, I895, p. 3I5. [Pottsville group; Clark formation.]

Callipteridium SP. D. White.

Bull. Geol. Soc. Am., vol. 6, I895, p. 317. [Pottsville group; Sewell formation.]

CAllipteridium, inAeQUale Lesquereux.

Second Ǵeol. Surv. Pa., Rept. Prog. P, i88o, p. I68, pl. xxxiii, figs. 2-5. D. White: Bull. Geol. Soc. Am., vol. II, Igoo, p. I72. [Allegheny formation].

Callipteris CONFerta (Sternberg) Brongniart.

Dict. Univ. Hist. Nat., vol. I3 (Tableau), r849, p. 66 ( I7). Fontaine \& I. C. White: Second Geol. Surv. Pa., Rept. Prog. PP, I88o, p. 54, pl. xi, figs. I-4. [Dunkard formation (Permian).]

Callipteris lyratifolia var. coriacea (Fontaine \& I. C. White) D. White.

This is the Sphenopteris coriacea of Fontaine and I. C. White: Second Geol. Surv. Pa., Rept. Prog. PP, I88o, p. 4I, pl. v, figs. 5-6. D. White: Bull. Geol. Soc. Am., vol. I4, I904, p. 539. [Dunkard formation (Permian).]

Callipteris CURRetiensis Zeiller.

In Mouret: Bass. Houill. Perm. Brive (Stratigr., pt. I), I89i, p. 83. D. White: Bull. Geol. Soc. Am., vol. I4, I903, p. 539; W. Va., Geol. Surv., vol. 2, I903, p. I20. [Dunkard formation (Permian).]

Cardiopteris frondosa (Goeppert) Schimper?

Pal. Veg., vol. I, I869, p. 453, pl. xxv. Lacoe in Millspaugh: Bull. No. 24, W. Va. Agric. Exp. Sta., Morgantown, I892, p. 52I. The identification of this species is very doubtful. [Pocono sandstone].

Megalopteris SP. D. White.

Am. Jour. Sci., 3rd ser., vol. I I, I876, p. 383. [Pottsville group; Kanawha formation].

Megalopteris $c f$. Dawsoni (Hartt) Andrews.

Rept. Geol. Surv. Ohio, vol. 2, pt. 2, Palaeont., I875, p. 4I5. D. White: Bull. Geol. Soc. Am., vol. 6, I895, p. 315. [Pottsville group; Quinnimont formation]. 
Megalopteris Sewellensis Fontaine.

Am. Jour. Sci., 3rd ser., vol. I I, I876, p. 383. [Pottsville group; Sewell formation].

Megalopteris SEWELLENSIS Fontaine.

Am. Jour. Sci., 3rd ser., vol. I I, I876, p. 383. D. White:

Bull. Geol. Soc. Am., vol. 6, I895, p. 3I5. [Pottsville group;

Quinnimont formation].

Megalopteris Harti Andrews.

Rept. Geol. Surv. Ohio, vol. 2, pt. 2, Palaeont., 1875, p. 4I6, pl. xlvi, figs. I, Ia. Fontaine: Amer.Jour. Sci., 3rd ser., vol. I I, I876, p. 382 . Probably the species earlier listed by Fontaine as M. Dawsoni (Amer. Jour. Sci., vol. 7, 1874, p. 574. [Pottsville group; Sewell formation.]

Taeniopteris Lescuriana Fontaine \& I. C. White.

Second Geol. Surv. Pa., Rept. Prog. PP, I88o, p. 9I, pl. xxxiv, fig. 9. [Dunkard formation (Permian).]

Taeniopteris Newberriana Fontaine \& I. C. White.

Second Geol. Surv. Pa., Rept. Prog. PP, I880, p. 9I, pl. xxxiv, figs. I-8. [Dunkard formation (Permian).]

Taeniopteris Newberriana var. angusta Fontaine \& I. C. White.

Second Geol. Surv. Pa., Rept. Prog. PP, I880, pl. xxxiv, fig. 8. [Dunkard formation (Permian).]

Neuropteris Pocahontas D. White.

Twentieth Ann. Rept. U. S. Geol. Surv., I898-'99, pt. 2, I900, p. 888, pl. clxxxix figs. 4, 4a; pl. cxci, figs. 5, 5a. [Pottsville group; Clark and Thurmond formations].

Type characteristic of the Pocahontas or lowest Pottsville. It gave risé to numerous variations, several of which are distinct species. In the upper part (Quinnimont formation) of the lower Pottsville it is replaced by a group of forms, generally listed as N. Smithii Lesq. (see below), while in the middle Pottsville it is followed by forms probably indistinguishable from N. Schlehani Stur.

Neuropteris Smithi Lesquereux.

Geol. Surv. Alabama, Rept. Prog. I875 [1876], p. 76. D. White: Bull. Geol. Soc. Am., vol. 6, I895, p. 314. [Pottsville group; Clark and Quinnimont formations].

This species, everywhere present in the lower Pottsville, and represented by derivatives in the middle Pottsville, belongs to the group referred by European palecbotanists to Neuropteris Schlehani.

Neuropteris Schlehani Stur. var. D. White.

Abh. K. K. Geol. Reichsanst., vol. 8, No. 2 (Culm. Fl., pt. 2), I877, p. 183 (289), pl. xi, figs. 7, 8a-c. [Pottsville group; Sewell formation]. 
Neuropteris bIformis Lesquereux (form) D. White.

Bull. Geol. Soc. Am., vol. 6, 1895, p. 317. [Pottsville group; Sewell formation].

Neuropteris Elrodi Lesquereux (form) D. White.

Bull. Geol. Soc. Am., vol. 6, 1895, p. 317. [Pottsville group; Sewell formation].

Neuropteris tenuifolia (Schlotheim) Sternberg.

Flora d. Vorwelt (Tentamen), I825, p. xvii. [Pottsville group; Clark formation].

Species characteristic of the upper Pottsville, especially the Mercer group. The plant referred by Fontaine (Amer. Jour. Sci., New Haven, vol. I I, I876, p. 380), to this species is undoubtedly referable to the $N$. Smithii type.

Neuropteris flexuosa Sternberg.

Flora d. Vorwelt (Tentamen), I825, p. xvi, pl. xxxii, fig. 2, D. White: Bull. Geol. Soc. Am., vol. I I, Ig00, p. I64. [Pottsville group; Kanawha formation.]

Abundant in the Kanawha formation and the Mercer coal group. The species from the Dunkard formation listed (Second Geol. Surv. Pa., Rept. Prog. PP, p. 49), under this name is quite distinct.

Neuropteris Plancitardi var. longifolia (Fontaine \& 1. C. White) D. White.

Second Geol. Surv. Pa., Rept. Prog. PP, i88o, p. 49, pl. viii, fig. I. D. White: Bull. Geol. Soc. Am., vol. I4, 1903 [I904], p. 540. [Dunkard formation (Permian).]

This variety is specifically distinct from $N$. Alexuosa.

Neuropteris Lindleyana Sternb., var. Fontaine.

Am. Jour. Sci., 3rd ser., vol. I I, I876, p. 38r. [Pottsville group; Quinnimont formation].

The species listed under this name is probably the N.Pocahontas D. White.

NEUROPTERIS RARINERVIS Bunbury.

Quart. Jour. Geol. Soc., London, vol. 3, I847, ,p. 425, pl. xxii. D. White: Bull. Geol. Soc. Am., vol. I I, I900, p. I69. [Pottsville group; Kanawha formation]. [Also in Allegheny formation].

Plant unknown below the "Mercer Coal group" and upper Kanawha.

Neuropteris sp. $c f$. gigantea Sternberg.

Flora d. Vorwelt (Tentamen), I825, p. xvii, pl. xxii. D. White: Bull. Geol. Soc. Am., vol. I I, I900, p. I63. [Pottsville group; Kanawha formation.]

Type is strictly Pottsville.

Neuropteris ovata Hoffman.

Teutschl. geogn. geol. dargest., Weimar, vol. 4, 1826, p. I 59, pl. lb, figs. 5-8. D. White: Bull. Geol. Soc. Am., vol 
II, I900, p. I69. [Pottsville group; Kanawha formation.] [Also in the Allegheny and Conemaugh formations.] [Dunkard formation (Permian).]

In West Virginia this species is not known below the highest horizons of the Kanawha.

Neuropteris gibbosa Lesquereux.

Boston Jour. Nat. Hist., vol. 6, I854, p. 4I8. Lacoe, in Millspaugh: Bull. No. 24, W. Va., Agric. Exp. Sta., Morgantown, I892, p. 522. [Dunkard formation (Permian).]

Neuropteris plicata Sternberg.

Flora d. Vorwelt (Tentamen), I825, p. xvi. Lacoe, in Millspaugh: Bull. No. 24, W. Va. Agric. Exp. Sta. Morgantown, I892, p. 523. Probably identified with the preceeding species. [Dunkard formation (Permian).]

Neuropteris Vermicularis Lesquereux.

Second Rept. Geol. Reconn. Arkansas, 1860, p. 315. D. White: Bull. Geol. Soc. Am., vol. I I, I900, p. I7I. [Allegheny formation].

So far as known this species is confined to the lower part of the Allegheny.

Neuropteris $c f$. Carri Lesquereux.

Second Geol. Surv. Pa., Rept. Prog. P, (Coal F1.), vol. 3, I884, p. 73I, pl. xciv, figs. 4-7. D. White: Buil. Geol. Soc. Am., vol. II, I900, p. I69. [Pottsville group; Kanawha formation].

This plant belongs to a group unknown below the highest horizon of the Pottsville.

Neuropteris Cisti Brongniart.

Dict. Sci. Nat., vol. 57 (Prodrome), I828, p. 52 (53). D. White: Bull. Geol. Soc. Am., vol. I I, I900, p. I64. [Pottsville group; Kanawha formation].

Neuropteris Grangeri Brongniart.

Dict. Sci. Nat., vol. 57 (Prodrome), 1828, p. 62, (53), Lacoes in Millspaugh: Bull. No. 24, W. Va. Agric. Exp. Sta. Morgantown, I892, p. 522. [Conemaugh formation].

NeUropteris callosa Lesquereux.

Second Geol. Surv. Pa., Rept. Prog. P (Coal F1.), vol. I. I880, p. I I 5, pl. xvi, figs. I-8. [Conemaugh formation].

Neuropteris Dictyopteroides Fontaine \& I. C. White.

Second Geol. Surv. Pa., Rept. Prog. PP, I88c, p. 49, pl. viii, figs. 3-5. [Dunkard formation (Permian).]

NeURopteris FImbriata Lesquereux.

Third Rept. Geol. Surv. Kentucky, Frankfort, I857, p. 536. D. White: Bull. Geol. Soc. Am., vol. I I, I900, p. I7I. [Allegheny and Conemaugh formations.] [Dunkard formation (Permian).] 
Neuropteris $c f$. Zeilleri Potonie.

Jahrb. K. Preuss. Geol. Landesanst., Berlin, vol. I2, I89I [1893], p. 22, text-fig. 5. D. White: Bull. Geol. Soc. Am., vol. II, I900, p. I6I. [Pottsville group; Kanawha formation].

Neuropteris Scheuch zeri Hoffman.

Teutschl. geogn. geol. dargest., Weimar, vol. 4, I826, p. I 57, pl. 1b, figs. I-4. D. White: Bull. Geol. Soc. Am., vol. I I, I900, p. I69.

This fossil, long known as $N$. hirsuta Lesq., is very abundant in the Allegheny and higher beds, but not known below the Mercer group and topmost Kanawha. [Pottsville group; Kanawha formation.] [Also in Conemaugh and Allegheny formations.]

Neuropteris odontopteroides Fontaine \& I. C. White.

Second Geol. Surv. Pa., Rept. Prog. PP, i880, p. 50, pl. ix, figs. I-6. [Dunkard formation (Permian).]

Neuropteris Cordata Brongniart.

Hist. Veg. Foss., Paris, vol. I, I830, p. 229, pl. 1xiv, fig. 5. Fontaine \& I. C. White: Second Geol. Surv. Pa., Rept. Prog. PP, I880, p. 5I. [Dunkard formation (Permian).]

Neuropteris auriculata Brongniart.

Hist. Veg. Foss., Paris, vol. I, I830, p. 236, pl. 1xvi. Fontaine \& I. C. White: Second Geol. Surv. Pa., Rept. Progr. PP, I880, p. 5O. [Dunkard formation (Permian).]

Neuropteris crenulata Brongniart.

Hist. Veg. Foss., Paris, vol. I, I830, p. 234, pl. 1xiv, fig. 2. Lesquereux: Second Geol. Surv. Pa., Rept. Prog. P (Coal F1.), vol. 3, 1884, p. 871. [Conemaugh formation.]

Neuropteris Agassizi Lesquereux.

Second Geol. Surv. Pa., Rept. Prog. P (Coal F1.), vol. I, I88o, p. I I7, pl. xvii, figs. I-4. D. White: Buil. Geol. Soc. Am., vol. I I, Ig0o, p. I72. [Allegheny formation.]

Doubtful identification.

Neuropteris N. SP. No. I, D. White.

Bull. Geol. Soc. Am., vol. I I, I900, p. I64. [Allegheny formation].

Neuropteris SP. D. White.

Bull. Geol. Soc. Am., vol. 6, I895, p. 318. [Pottsville group; Nuttall sandstone lentil of the Sewell formation.] Neuropteris sp. ? Fontaine.

Amer. Jour. Sci., 3rd ser., vol. I I, I876, p. 378 . [Potts-ville group; Sewell formation]. 
LinOPTERIS OBLIQUA (Bunbury) Zeiller.

Mem. Soc. Geol. France, vol. 8, Mem. 21, I899, p. 46, pl. $\mathrm{xx}$ (iv), figs. I4-I7. D. White: Bull. Geol. Soc. Am., vol. II, I900, p. I72. [Allegheny formation].

A plant characteristic of middle and upper Allegheny. LESCUROPTERIS ADIANTITES Lesquereux.

Second Geol. Surv. Pa., Rept. Prog. P, I88o, p. 163, pl. xxvi, fig. 4. Millspaugh: Bull. No. 24, W. Va. Agric. Exp. Sta., Morgantown, I892, p. 522. [?Dunkard formation (Permian).]

Lescuropteris MooriI (Lesquereux) Schimper.

Pal. Veg., vol. I, I869, p. 465. Millspaugh: Bull. No. 24, W. Va. Agric. Exp. Sta., Morgantown, I892, p. 522. [Conemaugh formation].

ODONTOPTERIS NEUROPTEROIDES Newberry.

Ann. Sci., Cleveland, vol. I, 1853, p. 106. Fontaine: Amer. Jour. Sci., 3rd ser., vol. I I, I876, p. 383. [Pottsville group; Quinnimont formation].

Probably wrongly identified and may be $N$. Pocahontas, D. White.

Odontopteris obtusiloba VAR. RARINervis Fontaine \& I. C. White.

Second Geol. Surv. Pa., Rept. Prog. PP, 1880, p. 52. [Dunkard formation (Permian).]

Odontopteris nervosa Fontaine \& I. C. White.

Second Geol. Surv. Pa., Rept. Prog. PP, r88o, p. 52, pl. x, figs. I-2. [Dunkard formation (Permian).]

ODONTOPTERIS WORTHENI Lesquereux.

Geol. Surv. Illinois, vol. 2, I866, Pal., p. 432, pl. xxxvi, figs. I, Ib. D. White: Bull. Geol. Soc. Am., vol. I I, I900, p. I72. [Allegheny formation.]

ODONTOPTERIS SUBCUNEATA Bunbury.

Quart. Jour. Geo!. Soc., London, vol. 3, I847, p. 427, pl. xxiii, figs. IA, IB. D. White: Bull. Geol. Soc. Am., vol. I I, I900, p. I7I. [Allegheny formation.]

Odontopteris densifolia Fontaine \& I. C. White.

Second Geol. Surv. Pa., Rept. Progr. PP, I88o, p. 54, pl. $\mathrm{x}$, fig. 3. Dunkard formation (Permian).]

ODONTOPTERIS AEQUALIS Lesquereux.

Geol. Surv. Illinois, vol 2, I866, Pal., p. 434, pl. xxxvi, fig.

2. D. White: Bull. Geol. Soc. Am., vol. I I, I900, p. I7I. [Allegheny formation.]

OdONTOPTERIS NEwbERRYi Lesquereux.

Second Geol. Surv. Pa. Rept. Progr. P, I880, p. I27. Millspaugh: Bull. No. 24, W. Va. Agric. Exp. Sta., Morgantown, I892, p. 523. [Pottsville group; Sewell formation.] 
Odontopteris Reichiana Gutbier.

Abdr. Verst. Zwick, Schwarzk., Zwickau, I835, p. 65, pl. ix, figs. I-3, 5, 7; pl. x, fig. 13. Millspaugh: Bull. No. 24,

W. Va. Agric. Exp. Sta., Morgantown, I892, p. 523. [Dunkard formation (Permian).]

ODONTOPTERIS GRACILLIMA Newberry.

Rept. Geol. Surv. Ohio, vol. I, pt. 2, Palaeont., 1873, p. 378, pl. xlvi, figs. I-3a. Fontaine: Amer. Jour. Sci., 3rd ser., vol. I I, I876, p. 378. [Pottsville group; Sewell formation and Nuttall sandstone lentil in the Sewell.]

Caulopteris gigantea Fontaine \& I. C. White.

Second Geol. Surv. Pa., Rept. Progr. PP, i88o, p. 95, pl. xxxvi, fig. I ; pl. xxxviii, fig. 5. [Dunkard formation (Permian).]

Caulopteris elliptica Fontaine \& I. C. White.

Second Geol. Surv. Pa., Rept. Progr. PP, I880, p. 95, pl. xxxv, figs. 4, 5. [Dunkard formation (Permian).]

ApHlebia spinosa (Goeppert) D. White.

Bull. Geol. Soc. Am., vol. 8, I896 [1897], p. 292 [Sphenopteris]. [Pottsville group; Clark formation.]

Aphlebia (Rhacophyllum) laciniata (Fontaine \& I. C. White) Sellards.

Second Geol. Surv. Pa., Rept. Progr. PP, I880, p. 94, pl. xxxv, fig. 2. Sellards: Univ. Geol. Surv. Kansas, vol. 9, Igo8, p. 454. [Dunkard formation (Permian).]

ApHLEBIA FILICIFORMis (Gutbier) Schimper.

Pal. Veg., vol. I, I869, p. 685 , pl. xlviii, figs. 3-6. Lacoe, in Millspaugh: Bull. No. 24, W. Va. Agric. Exp. Sta., Morgantown, I892, p. 526. [Conemaugh formation.]

Aphlebia lactuca (Presl) Sterzel.

Erlaut. Geol. Specialk. Konigr. Sachsen, Leipzig, Blatt II3, I88I, p. 104. Fontaine: \& I. C. White: Second Geol. Surv. Pa.. Rept. Progr. PP, I880, p. 94. [Dunkard formation (Permian).]

Aphlebia speciosissima (Schimper) D. White.

Pal. Veg., vol. I, I869, p. 685. Fontaine \& I. C. White: Second Geol. Surv. Pa., Rept. Progr. PP, I880, p. 94. [Dunkard formation (Permian).]

Aphlebia filiciformis var. majus (Fontaine \& I. C. White) D. White.

Second Geol. Surv. Pa., Rept. Progr. PP, I880, p. 93, pl. xxxv, fig. I. [Dunkard formation (Permian).]

EQUisetites RUgosus Schimper.

Pal. Veg., vol. I, I869, p. 287 , pl. xvii, figs. I-3. Fontaine \& I. C. White: Second Geol. Surv. Pa., Rept. Progr. PP, I88o, p. 33, pl. i, fig. 6. [Dunkard formation (Permian).]. 
Equisetites striatus Fontaine \& I. C. White.

Second Geol. Surv. Pa., Rept. Progr. PP, I880, p. 34, pl.

i, fig. 5. [Dunkard formation (Permian).]

Equisetites elongatus Fontaine \& I. C. White.

Second Geol. Surv. Pa., Rept. Progr. PP, I880, p. 33, pl.

i, figs. I-4. [Dunkard formation (Permian).]

EQUISETITES OCCIDENTALIS Lesquereux.

Geol. Surv. Illinois, vol. 4, Pal. pt. 2, I87o, p. 425, pl. $\mathrm{xx}$,

fig. 5. D. White: Bull. Geol. Soc. Am., vol. 6, I895, p. 3I5.

[Pottsville group; Clark and Quinnimont formations.]

Known only in lower Pottsville of West Virginia, Tennessee and Georgia. It may be the same as the fossil described by Fontaine as Equisetites sp.? (Amer. Jour. Sci., 3rd ser., vol. I I, I876, p. 38I ).

Asterocalamites scrobiculatus (Schlotheim) Zeiller.

Expl. Carte Geol. France, Paris, vol. 4, Atlas, 1878, pl. clix, fig. 2 I ; text I879, p. I6. [Pöttsville group; Clark and Quinnimont formations.]

This species is generally known in our American literature as Bornia radiata (Brongniart) Schimper (Pal. Veg., vol. I, I869, p. 335, pl. xxiv, figs. I-IO). D. White: Bull. Geol. Soc. Am., vol. 6, I895, p. 3I5. Species generally characteristic of the upper Mississippian. The identification of this plant in the Pennsylvanian is questionable.

Calamites Suckowi Brongiart.

Hist. Veg. Foss., Paris, vol. I, I828, p. I24, pl. xiv, fig. 6; pl. xv, figs. I-6; pl. xvi. D. White: Bull. Geol. Soc. Am., vol. II, I900, p. I64. [Pottsville group; Kanawha formation.]

Calamites Suckowi Brongniart.

Hist. Veg. Foss., Paris, vol. I, I828, p. I24, pl. xiv, fig. 6; pl. xv, figs. I-6; pl. xvi. Fontaine: \& I. C. White: Second Geol. Surv. Pa., Rept. Progr. PP, ı880, p. 35. [Dunkard formation (Permian).]

Calamites Cisti Brongniart.

Hist. Veg. Foss., Paris, vol. I, I828, p. I29, pl. xx. D. White: Bull. Geol. Soc. Am., vol. I I, Igoo, p. I64. [Pottsville group; Kanawha formation.]. [Also in Allegheny formation.]

Calamites cannaeformis Schlotheim.

Petrefactenkunde, Gotha, I820, p. 398, pl. xx, fig. I. Fontaine: Amer. Jour. Sci., 3rd ser., vol. II, I876, p. 379. [Pottsville group; Quinnimont formation.]

Calamites Roemeri Goppert?

Zeitscher. Deutsch. Geol. Ges., vol. 3, I851, p. I90. Fontaine: Amer. Jour. Sci., 3rd. ser., vol. I I, I876, p. 378. [Pottsville group; Sewell formation.] 
Calamites ramosus Artis.

Antediluvian Phytology, London, 1825, p. 2, p1. ii. D. White: Bull. Geol. Soc. Am., vol. I I, Ig00, p. I6I. [Pottsville group; Kanawha formation.]

Calamites approximatus Schlotheim.

Petrefactenkunde, Gotha, I820, p. 399. Fontaine: Amer. Jour. Sci., 3rd ser., vol. II, I876, p. 383. D. White: Bull: Geol. Soc. Am., vol. I I, I900, p. I64. [Pottsville group; Sewell, including Nuttall sandstone lentil, and Kanawha formations.]

Calamites sp. D. White.

Bull. Geol. Soc. Am., vol. 6, I895, p. 315. [Pottsville group; Quinnimont formation.]

Calamitina sp. D. White.

Bull. Geol. Soc. Am., vol. I I, I900, p. I64. [Pottsville group; Kanawha formation.]

Calamodendron sp. D. White.

Bull. Geol. Soc. Am., vol. I I, I900, p. I64. [Pottsville group ; Kanawha formation.]

Asterophyllites minutus Andrews.

Rept. Geol. Surv., Ohio, vol. 2, pt. 2, Palaeont., I875, p. 424, pl. li, figs. 4, 4a. D. White: Bull. Geol. Soc. Am., vo!. 6, I895, p. 315 ; Bull. Geol. Soc. Am., vol. I I, I900, p. I6I and I64. Species in general characteristic of the Pottsville group. [Princeton conglomerate?? (Mississippian).] [Pottsville group; Quinnimont and Kanawha formations and Nuttall sandstone lentil of Sewell formation.]

AsterophyLlites GRACILIS Lesquereux.

Second Rept. Geol. Recom. Arkansas, I860, p. 310, pl. ii, fig. 4. D. White: Bull. Geol. Soc. Am., vol. 6, I895, p. 317. [Pottsville group; Sewell formation.]

ASTEROPHYLLITES EQUISETIFORMIS (Schlotheim) Brongniart.

Dict. Sci. Nat., vol. 57 (Prodrome), I828, p. I56 (I 59). D. White: Bull. Geol. Soc. Am., vol. I I, I900, p. I69. [Pottsville group; Kanawha formation.] mian.

Plant ranges from Mercer group upwards into the PerAsterophyllites LyCOPODIOIDES Zeiller.

F1. foss. Bass. Houill. Valenciennes, Paris, Atlas, ı886, pl. lix, figs. I, 2 ; text I888, p. 380 . D. White: Bull. Geol. Soc. Am., vol. II, I900, p. I64. [Pottsville group; Kanawha formation.]

Asterophyllites sp. indet. D. White.

Bull. Geol. Soc. Am., vol. 6, I895, p. 313. [Princeton conglomerate (Mississippian).] 
Asterophyllites erectifolius Andrews.

Rept. Geol. Surv. Ohio, vol. 2, pt. 2, Palaeont., 1875, p. 425, pl. xlix, fig. 3. D. White: Bull. Geol. Soc. Am., vol. 6,

I895, p. 317. [Pottsville group; Sewell formation.]

Asterophyllites RIGIDUS (Sternberg) Brongniart.

Dict. Sci. Nat., vol. 57 (Prodrome), I828, p. I 57 (I 59).

D. White: Bull. Geol. Soc. Am., vol. I I, I900, p. I6I. [Potts-

ville group; Kanawha formation.]

Nematophyllum angustum Fontaine \& I. C. White.

Second Geol. Surv. Pa., Rept. Progr. PP, r88o, p. 35, pl.

ii, figs. I-5. [Dunkard formation (Permian).]

Annularia ACICUlaris (Dawson) Renault.

Cours. de Bot. Foss., vol. 2, I882, p. I7I. D. White: Bull.

Geol. Soc. Am., vol. II, I900, p. I6r. [Pottsville group;

Kanawha formation.]

ANNUlaria RAmosa (Brongniart) Weiss.

Neues Jahrb. f. Min., vol. 2, I881, p. 273. D. White:

Bull. Geol. Soc. Am., vol. 6. 1895, p. 315; Bull. Geol. Soc.

Am., vol. I I, I900, p. I6I. [Pottsville group; Quinnimont?,

and Kanawha formations.] [Also in Conemaugh formation.]

AnNUlaria CUSPIDATa Lesqueretix.

Second Geol. Surv. Pa., Rept. Progr. P, I884, p. 725, pl. xcii, figs. 7, 7a. D. White: Bull. Geol. Soc. Am., vol. I I, I900, p. I62. [Pottsville group; Kanawha formation, and

Nuttall sandstone lentil of Sewell formation.]

Annularia Radiata (Brongniart) Sternberg.

Flora d. Vorwelt (Tentamen), I825, p. xxxi. D. White: Bull. Geol. Soc. Am., vol. I I, I900, p. I64. Fontaine \& I. C.

White: Second Geol. Surv. Pa, Rept. Progr. PP, I880, p. 39.

[Pottsville group; Sewell formation.] [Dunkard formation

(Permian) ?]

Annularia stellata (Schlotheim) Wood.

Proc. Acad. Nat. Sci., Philadelphia, vol. I2, I860, p. 236.

D. White: Bull. Geol. Soc. Am., vol. I I, I900, p. I69. [Pottsville group; Kanawha formation.] [Also in Coremaugh and Allegheny formations; and Dunkard formation (Permian).] AnNUlaria SPHeNOPHylloides (Zenker) Gutbier.

Isis von Oken, Leipzig, vol. 30, I837, p. 436. Fontaine \& I. C. White: Second Geol. Surv. Pa., Rept. Progr. PP, I880, p. 39. [Pottsville group; Kanawha formation.] [Also in Allegheny and Conemaugh formations, and in Dunkard formation (Permian).]

Has same wide range as the preceding species.

ANNULARIA SPHENOPHYLLOIDES var. INTERMEDIA LESquereux.

Second Geol. Surv. Pa., Rept. Progr. P, I884, p. 724. [Allegheny and Conemaugh formations.] 
AnNUlaria CARINATA Gutbier.

Isis von Oken, Leipzig, vol. 30, I837, p. 436. Fontaine \&

I. C. White: Second Geol. Surv. Pa., Rept. Progr. PP, I88o,

p. 38. [Dunkard formation (Permian).]

ANNULARIA MINUTA Brongiart.

Dict. Sci. Nat., vol. 57 (Prodrome), I828, p. I53 (I55).

Fontaine \& I. C. White: Second Geol. Surv. Pa., Rept. Progr

PP, I88o, p. 39. [Dunkard formation (Permian).]

Annularia sp. D. White.

Bull. Geol. Soc., Am., vol. 6, I895, p. 315. [Pottsville group ; Quinnimont formation.]

Calamostachys lanceolata Lesquereux.

Second Geol. Surv. Pa., Rept. Progr. P, (Coal F1.), vol. 3, I884, p. 7I 5, pl. xci, figs. I, 2; pl. xciii. D. White: Bull. Geol. Soc. Am., vol. 6, I895, p. 315. [Pottsville group; Quinnimont formation.]

Calamostachys ramosus (Artis) Weiss.

Abh. Geol. Specialk. Preuss, vol. 5, No. 2 (Steink.-Calam. pt. 2), I884, p. I93 (I07), pl. xx, figs. I, 2. D. White: Bull. Geol. Soc. Am., vol. I I, I900, p. I6I. [Pottsville group; Kanawha formation.]

Macrostachya sp. D. White.

Bull. Geol. Soc. Am., vol. 6, I895, p. 3I7. [Pottsville group; Sewell formation.]

Expl. Carte Geol. France, vol. 4, Atlas, I878, pl. clxi, figs. I, 2 ; text I879, p. 30. D. White: Bull. Geol. Soc. Am., vol. I I, I900, p. I6I. [Pottsville group; Kanawha formation and Nuttall sandstone lentil of Sewell formation.]

Plant characteristic of the middle and upper Pottsville formations. Typical form is unknown in Allegheny or higher beds.

Sphenoph yllum antiguum Dawson.

Canadian Naturalist, Montreal, vol. 6, I86I, p. I70, text fig. 7. Fontaine: Amer. Jour.'Sci., 3rd ser., vo!. 7, I874, p. 574. [Pottsville group; Sewell formation.]

Sphenophyllum lescurianum D. White.

Mon. U. S. Geol. Surv., vol. 37, I899, p. I82, pl. 1, fig. 6b; pl. li, fig. b; pl. xxiv, fig. 3c. D. White: Bull. Geol. Soc. Am., vol. II, I900, p. I69. [Pottsville group; Kanawha formation.]

Sphenophyllum emarginatum (Brongniart) Koenig.

Icones Foss. Sect., London, I825(?), pl. xii, fig. I49. D. White: Bull. Geol. Soc. Am., vol. I I, I900, p. I69. [Pottsville group; Kanawha formation.] [Also in Allegheny and Conemaugh formations.]

Plant unknown below "Mercer coal group." 
Sphenophyllum majus (Bronn in Bischoff) Bronn.

Letheae Geogn., Stuttgart, vol. I, I834, p. 32, pl. viii, fig. 9, a, b. D. White: Bull. Geol. Soc. Am., vol. II, I900, p. I7I. [Allegheny and Conemaugh formations.]

Sphenopirylum furcatum (Lindley \& Hutton) Geinitz.

Furstl. Jablon. Ges. Preisschr., No. 5 (F1. Hain.-Ebers. \& Floeh. Kohl.), Leipzig, 1854, p. 36, pl. i, figs. Iо-12; pl. ii, figs. 2, I. D. White: Bull. Geol. Soc. Am., vol. I I, Ig00, p. I6I. [Pottsville group; Kanawha formation.]

Sphenophyllum tenUe D. White.

Twentieth Ann. Rept. U. S. Geol. Surv., I8c8-'99, pt. 2, I900, p. 900, pl. cxci, figs. 6, 7. D. White: Bull. Geol. Soc. Am., vol. 6, I895, p. 315. [Pottsville group; Quinnimont formation.]

Species characteristic of lower Pottsville and basal middle Pottsville in the central and northern Appalachian regions. Sphenophyllum angustifolium (Germat) Goppert.

In Bronn, Naturgeschte d. drei Reiche, vol. 3 (Nomencl. Palaeont.), 1848, p. I I66. Millspaugh: Bull. No. 24, W. Va. Agric. Exp. Sta., Morgantown, I892, p. 526. [Dunkard formation (Permian).]

Sphenophyllum oblongifolium (Germar \& Kaulfuss) Unger.

Gen. et Spec. P1. Foss., I850, p. 70. Fontaine \& I. C. White: Second Geol. Surv. Pa., Rept. Progr. PP, I880, p. 38. [Dunkard formation (Permian).]

Sphenophyllum longifolium (Germar) Geinitz \& Gutbier.

In Reichenbach, Gaa von Sachs. (Verst. Obersachsen), I843, p. 72. Fontaine \& I. C. White: Second Geol. Surv. Pa., Rept. Progr. PP, I88o, p. 38. [Dunkard formation (Permian).]

Sphenophyllum Thoni Mahr.

Zeitschr. Deutsch. Geol. Ges., vol. 20, I868, p. 433, pl. viii.

D. White: Bull. Geol. Soc. Am., vol. II, I900, p. I72. [Dunkard formation (Permian).]

Sphenophyllum Fontaineaum S. A. Miller.

North American Geol. \& Palaeont., Cincinnati, I889, p. I4I. [Dunkard formation (Permian).]

This is is the plant described as Sphenophyllum latifolium

Fontaine \& I. C. White, a preoccupied name, in the Second Geol. Surv. Pa., Rept. Progr. PP, I88o, p. 36, pl. i, figs. Io-1 I. Sphenophyllum filiculme Lesquereux.

Boston Jour. Nat. Hist., voi. 6, I854, p. 415. Fontaine \&

I. C. White: Second Geol. Surv. Pa., Rept. Progr. PP, I880,

p. 37, pl. i, fig. 8. [Dunkard formation (Permian).]

Sphenophyllum tenuifolium Fontaine \& I. C. White.

Second Geol. Surv. Pa., Rept. Progr. PP, i88o, p. 38, pl. i, fig. 9. [Dunkard formation (Permian).] 
Sphenophyllum densifolium Fontaine \& I. C. White.

Second Geol. Surv. Pa., Rept. Progr. PP, I880, p. 37, p1. i, fig. 7. [Dunkard formation (Permian).]

Lycopodites Meekir Lesquereux.

Geol. Surv. Illinois, vol. 4, Geol. \& Pal., pt. 2, 1870, p. 426, pl. xxvi, figs. 6, 6a. D. White: Bull. Geol. Soc. Am., vol. I I, I900, p. I69. [Pottsville group; Kanawha formation.]

LyCOPODITES PENDULUS Lesquereux.

Second Geol. Surv. Pa., Rept. Progr. P (Coal F1.), vol. 2, I880, p. 357, pl. 1xii, figs. 2, 2a. D. White: Bull. Geol. Soc. Am., vol. II, I900, p. I7I. [Conemaugh formation.]

Lycopodites SIMPLEX Lesquereux?

Second Geol. Surv. Pa., Rept. of Progr., G7, Harrisburg, I882 [I883], p. 40. D. White: Bull. Geol. Soc. Am., vol. I I, I900, p. I64. [Pottsville group; Kanawha formation.]

Lycopodites n. sp. D. White.

Bull. Geol. Soc. Am., vol. 6, I895, p. 315. [Pottsville group; Quinnimont formation.]

Bothrodendron sp. $c f$. Minutifolium (Boulay) Zeiller.

Expl. Carte Geol. France, Paris, vol. 4, text I879, p. II7.

D. White: Bull. Geol. Soc. Am., vol. I , I900, p. I65. [Pottsville group; Kanawha formation.]

Bothrodendron n. sp. D. White.

Bull. Geol. Soc. Am., vol. I I, I900, p. I6I. [Pottsville group; Kanawha formation.]

LEPIDODENDRON sp. $c f$. BRITTSII Lesquereux.

Second Geol. Surv. Pa., Rept. Progr. P (Coal Fl.), vol. 2, I880, p. 368, pl. 1xiii, figs. I-2. D. White: Bull. Geol. Soc. Am., vol. II, I900, p. I64. [Pottsville group; Kanawha formation.]

LEPIDODENIRON RUSHVILlense Andrews.

Rept. Geol. Surv., Ohio, vol. 2, pt. 2, Paleont., I875, p 423, pl. liii, fig. 4. D. White: Bull. Geol. Soc. Am., vol. I I, I900, p. 162. [Pottsville group; Kanawha formation.]

LEPIDODENDRON ' SELAGINOIDES Sternberg.

Flora d. Vorwelt, vol. I (Versuch), pt. 2, I822, p. 3I, pl. xvi, fig. 3 ; pl. xvii, fig. I. Fontaine: Amer. Jour. Sci., 3rd ser., vol. I I, I876, p. 378. [Pottsville group; Clark formation.]

LEPIDODENDRON SCOBINIFORME Meek.

Bull. Phil. Soc. Washington, vol. I, Appendix VIII, I874,

p. xiii, pl. i, fig. I. [Pocono sandstone.]

This species is cited generally as L. corrugatum Dawson. Fontaine: Amer. Jour. Sci., 3rd ser., vol. 7, 1874, p. 578. 
LEPIDODENDRON ACUMINATUM (Goeppert) Unger.

Gen. et. Spec. P1. Foss., I850, p. 26r. D. White: Bull. Geol. Soc. Am., vol. II, I900, p. I64. [Pottsville group;

Quinnimont(?) and Kanawha formations.]

Lepidodendron Sternbergit (form.) D. White.

Bull. Geol. Soc. Am., vol. 6, I895, p. 315. [Pottsville group; Quinnimont and Sewell formations.]

Lepidodendron Veltheimianum Sternberg.

Flora d. Vorwelt (Tentamen), I825, p. xii, pl. lii, fig. 3. D. White: Bull. Geol. Soc. Am., vol. 6, I895, p. 315. D. White: Bull. Geol. Soc. Am., Rochester, vol. II, I900, p. I64. [Pottsville group; Quinnimont and Kanawha formations.]

LEPIDODENDRON LANCEOLATUM Lesquereux.

Second Geol. Surv. Pa., Rept. Progr. P (Coal F1.), vol. 2, I880, p. 369, pl. 1xiii, figs. 3-5a. D. White: Bull. Geol. Soc. Am., vol. II, I900, p. I69. [Pottsville group; Kanawha formation.]

LEPIDODENDRON sp. $c f$. DICHотомum Sternberg.

Flora d. Vorwelt, vol. I (Versuch), pt. I, I820, p. 23, pls. i, ii, iii ; pl. lxviii, fig. I. D. White: Bull. Geol. Soc. Am., vol. I I, Ig00, p. I6I. [Pottsville group; Kanawha formation.] LEPIDODENDRON CLYPEATUM Lesquereux.

Boston Jour. Nat. Hist., vo1. 6, I854, p. 429. D. White: Bull. Geol. Soc. Am., vol. I I, I900, p. I64. [Pottsville group; Kanawha formation.]

Characteristic of the upper and middle Pottsville.

LEPIDODENDRON OBOVATUM Sternberg.

Flora d. Vorwelt, vol. I (Versuch), pt. I, I820, p. 23, pl. vi, fig. 2 ; pl. viii, fig. I $\mathrm{B}, \mathrm{a}-\mathrm{b}$; pl. $\mathrm{xv}$, fig. 5 . D. White : Bull. Geol. Soc. Am., vol. I I, I900, p. I6I. [Pottsville group. Kanawha formation.]

LEPIDODENDRON $c f$. MAGNUM Wood.

Proc. Acad. Nat. Sci., Philadelphia, vol. I2, I860, pl. vi, fig. 4. D. White : Bull. Geol. Soc. Am., Rochester, vol. I I, I900, p. I64. [Pottsville group; Kanawha formation.]

LEPIDODENDRON MODULATUM Lesquereux.

Boston Jour. Nat. Hist., vol. 6, I854, p. 428. D. White Bull. Geol. Soc. Am., vol. I I, I900, p. I69. Plant generally characteristic of the Allegheny and not known below the "Mercer coal group." [Pottsville group; Kanawha formation.] [Also in Allegheny formation.]

LEPIDOPHLOIOS sp. cf. LARICINUS Sternberg.

Flora d. Vorwelt (Tentamen), I825, p. xiii, pl. xi, figs. 2, 3, 4. D. White: Bull. Geol. Soc. Am., vol. II, I900, p. I64. [Pottsville group; Kanawha formation.] 
Lepidophloios sp. D. White.

Bull. Geol. Soc. Am., vol. I I, Igoo, p. I72. [Allegheny formation.]

ULODENDRON MAJUS Lindley \& Hutton.

Foss. Fl. Great Britain, vol. I, I831, p. 22, pl. v. D. White: Bull. Geol. Soc. Am., vol. I I, I900, p. I65. [Pottsville group; Kanawha formation.]

UlODENDRON sp. D. White.

Bull. Geol. Soc. Am., vol. 6, I895, p. 3I5. [Pottsville group; Quinnimont farmation.]

Halonia sp. D. White.

Bull. Geol. Soc. Am., vol. 6, I895, p. 315. [Pottsville group; Quinnimont formation.]

LEPIDOSTROBUS VARIABILIS Lindley \& Hutton.

Foss. F1. Great Britain, vol. I, I831, p. 31, pls. x, xi. D. White: Bull. Geol. Soc. Am., vol. 6, I895, p. 3I5; ibid., vol. I I, I900, p. I6I, I69. [Pottsville group; Quinnimont and Kanawha formations, and Nuttall sandstone lentil of Sewell formation.]

LEPIDOSTROBUS ORNATUS Brongniart.

Dict. Sci. Nat., vol. 57 (Prodrome), I828, p. 93 (88). D. White: Bull. Geol. Soc. Am., vol. I I, I900, p. I65. [Pottsville group; Kanawha formation.]

Lepidostrobus SAlisburyi Lesquereux.

Second Geol. Surv. Pa., Rept. Progr. P, I88o, p. 443, pl. lxix, figs. I, 2. Millspaugh: Bull No. 24, W. Va. Agric. Exp. Sta., I892, p. 521. [Pottsville group: Kanawha formation.] Lepidostrobus sp. nov.? D. White.

Bull. Geol. Soc. Am., vol. I I, I900, p. I65. [Pottsville group; Kanawha formation.]

LEPIDOPHYLLUM BREVIFOLIUM Lesquereux.

Boston Jour. Nat. Hist., vol. 6, 1854 , p. 430. D. White: Bull. Geol. Soc. Am., vol. I I, Ig0o, p. I7I. [Allegheny formation.]

Lepidophyllum Jenneyi D. White.

Mon. U. S. Geol. Surv., vol. 37, r899, p. 214, pl. lix, figs. I-3; pl. lxiii, fig. 6. D. White: Bull. Geol. Soc. Am., vol. I I, Igoo, p. I72. [Allegheny formation.]

LEPIDOPHYLLUM ACUMINATUM Lesquereux.

Boston Jour. Nat. Hist., vol. 6, I854, p. 430. D. White: Bull. Geol. Soc. Am., vol. I I, I900, p. I65. [Pottsville group; Kanawha formation.]

LEPIDOPHYLLUM OBLONGIFOLIUM Lesquereux.

Second Geol. Surv. Pa., Rept. Progr. P (Coa! F1.), vol. 3, I884, p. 868. D. White: Bull. Geol. Soc. Am., vol. I , I900, p. I7I. [Allegheny formation.] 
LePidophyllum LANCEOLATUm Lindley \& Hutton.

Foss. Fl. Great Britain, vol. I, I83I, p. 28, pl. vii, figs. 3, 4 . D. White : Bull. Geol. Soc. Am., vol. I I, I900, p. 169. [Pottsville group; Kanawha formation.]

LEPIDOPHyLLUM CAMPBELLIANUM Lesquereux.

Second Geol. Surv. Pa., Rept. of Progr., G ${ }^{7}$, Harrisburg, I882 ([ I883], p. 40. D. White: Bull. Geol. Soc. Am., vol. 6, I895, p. 3 I 5 ; ibid., Bull. Geol. Soc. Am., vol. I I, I900, pp. I6I, I65. [Pottsville group; Kanawha formation.]

LEPIDOPHYLLUM $c f$. CULTRIFORME Lesquereux.

Second Geol. Surv. Pd., Rept. Progr. P (Coal Fl.), vol. 3, I884, p. 785, pl. cvii, figs. I3, I4; pl. cviii, fig. 2. D. White: Bull. Geol. Soc. Am., vol. II, I900, p. I65. [Pottsville group; Kanawha formation.]

LEPIDOPHyllum hastatum Lesquereux.

Boston Jour. Nat. Hist., vol. 6, I854, p. 430. D. White: Bull. Geol. Soc. Am., vol. II, Ig0o, p. I72. ([Allegheny formation.]

Lepidophyllum n. sp. D. White.

Bull. Geol. Soc. Am., vol. 6, I895, p. 315. [Pottsville group ; Quinnimont and Sewell formations.]

LEPIDOCystis vesicularis Lesquereux.

Second Geol. Surv. Pa., Rept. Progr. P, I88o, p. 457, pl. lxix, figs. I8-20. [Allegheny and Conemaugh formations.]

Sigillaria camptotaenia (Wood) Wood.

Trans. Am. Phil. Soc.,Philadelphia, vol. 13, I869, p. 342.

D. White: Bull. Geol. Soc. Am., vol. I I, I900, p. I7I. [Allegheny formation.]

This species is in general characteristic of the Allegheny formation, though rare in the "Mercer coal group."

Sigillaria fissa Lesquereux.

Boston Jour. Nat. Hist., vol. 6, I854, p. 426. D. White: Bull. Geol. Soc. Am., vol. I I, I900, pp. I69, I7I. [Pottsville group; Kanawha formation.]

Sigillaria $c f$. Reticulata Lesquereux. Non (Steinhauer) Miller.]

Second Rept. Geol. Reconn. Arkansas, Philadelphia, I86o, p. 310, pl. iii, fig. 2. D. White: Bull. Geol. Soc. Am., vol. 6, 1895, p. 317. [Pottsville group; Sewell formation.]

Sigillaria Menardi Brongniart.

Dict. Sci. Nat., vol. 57 (Prodrome), I828, p. 74 (65). Lesquereux: Second Geol. Surv. Pa., Rept. Progr. P (Coal F1.), vol. 3, 1884, p. 87i. [Conemaugh formation.] 
Sigillaria Brardi Brongniart.

Dict. Sci. Nat., vol. 57 (Prodrome), I828, p. 74 (65). Fontaine \& I. C. White: Second Geol. Surv. Pa., Rept. Progr. PP, I880, p. 97, pl. xxxii, fig. I0. [Dunkard formation (Permian).]

Sigillaria $c f$. DeNTATA Newberry.

Ann. Sci., Cleveland, vol. I, 1853, p. 165, text fig. 4. D. White: Bull. Geol. Soc. Am., vol. 6, 1895, p. 315. [Pottsville group; Quinnimont formation.]

Sigillaria $c f$. Reticulata Lesquereux.

Second Rept. Geol. Reconn. Arkansas, Philadelphia, I860, p. 3 Io, pl. iii, fig. 2. D. White: Bull. Geol. Soc. Am., vol. II, I900, p. I65. [Pottsville group; Kanawha formation.]

Sigillaria sp. $c f$. IChtyolepis (Sternberg) Corda.

Beitr. Fl. Vorwelt, I845, p. 29, pl. ix, fig. I9. D. White: Bull. Geol. Soc. Am., vol. I I, I900, p. I65. [Pottsville group; Kanawha formation.]

Sigillaria Approximata Fontaine \& I. C. White.

Second Geol. Surv. Pa., Rept. Progr. PP, i880, p. 96, pl. xxxvii, fig. 3. [Dunkard formation (Permian).]

Sigillaria sp. D. White.

Bull. Geol. Soc. Am., vol. 6, I895, p. 315. [Pottsville group; Quinnimont formation.]

Triletes sp. D. White.

Bull. Geol. Soc. Am., vol. 6, I895, p. 318. [Pottsville group; Nuttall sandstone lentil of Sewell formation.]

CORDAITES BORASSIFoliUs (Sternberg) Unger.

Gen. et. Spec. P1. Foss., 1850, p. 277. D. White: Bull. Geol. Soc. Am., vol. I I, I900, p. I65. [Pottsville group; Kanawha formation.]

Cordaites Robbii Dawson.

Canadian Naturalist, Montreal, vol. 6, I861, p. I68. Fontaine: Amer. Jour. Sci., 3rd ser., vol. I I, 1876, p. 379. [Pottsville group; Clark, Quinnimont and Sewell formations.]

Cordaites CRAssinervis Fontaine \& I. C. White.

Second Geol. Surv. Pa., Rept. Progr. PP, I880, p. 97, pl. xxxvii, fig. IO. [Dunkard formation (Permian).]

Poacordaites sp. D. White.

Bull. Geol. Soc. Am., vol. I I, I900, p. 169. [Pottsville group; Kanawha formation.]

Baiera virginiana Fontaine \& I. C. White.

Second Geol. Surv. Pa., Rept. Progr. PP, I880, p. IO3, pl. xxxvii, figs. II, I2. [Dunkard formation (Permian).] 
Whittleseya elegans Newberry.

Ann. Sci., Cleveland, vol. I, I853, p. I06. D. White: Bull. Geol. Soc. Am., vol. 6, I895, p. 317. [Pottsville'group; Sewell formation.]

SaportaeA gRANDIfolia Fontaine \& I. C. White.

Second Geol. Surv. Pa., Rept. Progr. PP, I88o, p. IOI, pl. xxxviii, fig. 4. [Dunkard formation (Permian).]

Saportaea salisburioides Fontaine \& I. C. White.

Second Geol. Surv. Pa., Rept: Progr. PP, I880, p. 102, pl. xxxiii, figs. I-3. [Dunkard formation (Permian).]

Cordaicarpon Gutbieri (Geinitz) Grand 'Eury.

F1. Carb. Dept. Loire, Paris, I877, p. 236, pl. xxvi, fig. I9. D. White: Bull. Geol. Soc. Am., vol. I I, I900, p. I7I. [Conemaugh formation.]

CORDAICARPon CIRCUlaris (Lesquereux) D. White.

Bull. Geol. Soc. Am., vol. I I, I900, p. I69. [Pottsville group; Kanawha formation.]

CoRDAICARPON CINCTUM Lesquereux.

Second Geol. Surv. Pa., Rept. Progr. P (Coal Fl.), vol. 3, I884, p. 804, pl. cix, figs. 5, 6. D. White: Bull. Geol. Soc. Am., vol. II, I900, p. I69. [Pottsville group; Kanawha formation.]

Cardiocarpon Cornutum Dawson.

Quart. Jour. Geol. Soc., London, vol. I8, I862, p. 324, pl. xiii, figs. 23, 24. D. White: Bull. Geol. Soc. Am., vol. I I, I900, p. I62. [Pottsville group; Kanawha formation.]

Cardiocarpon bicuspidatum (Sternberg) Newberry.

Ann. Sci., Cleveland, vol. I, I853, p. I53, text-fig. 7. D.

White: Bull. Geol. Soc. Am., vol. 6, 1895, p. 318. [Pottsville group; Nuttall sandstone lentil of Sewell formation.] CardiocarPon minor Newberry.

Ann. Sci., Cleveland, vol. I, I853, p. I53, text-fig. 4. D. White: Bull. Geol. Soc. Am., vol. I I, I900, p. I65. [Pottsville group; Sewell and Kanawha formations.]

RHABDOCARPOS TENAX Lesquereux.

Second Geol. Surv. Pa., Rept. Progr. P, I884, p. 818, pl. cxi, figs. 25, 26. Lacoe, in Millspaugh: Bull. No. 24, W. Va. Agric. Exp. Sta., Morgantown, I892, p. 526. [Pottsville group; Kanawha formation.]

Cardiocarpon elongatum Newberry.

Ann. Sci., Cleveland, vol. I, I853, p. I53, text-fig. 6. D. White: Bull. Geol. Soc. Am., vol. 6, I895, p. 317. [Pottsville group; Sewell formation.]

RhabDocarpos multistriatus (Presl) Lesquereux.

Second Geol. Surv. Pa., Rept. Progr. P (Coal F1.), vol. 2, I88o, p. 578, pl. 1xxxv, figs. 22, 23. D. White: Bull. Geol. 
Soc. Am., vol. I I, I900, p. I65. [Pottsville group; Kanawha formation.]

Probably the fruit of Neuropteris flexuosa.

Rhabdocarpos sulcatus (Lindley \& Hutton) Schimper.

Pal. Veg., vol. 2, I870, p. 220. D. White: Bull. Geol. Soc. Am., vol. II, I900, p. I6I. [Pottsville group; Kanawha formation.]

RhabdocARPos AMygdalaEFormis (Goeppert) Goeppert \&

Berger, Fruc. Semin. Form. Lith., Breslau, I848, p. 21 , pl. i, fig. I2. D. White : Bull. Geol. Soc. Am., vol. I I, I900, p. I65. [Pottsville group; Kanawha formation.]

RhaBdocARPos oblongatus Fontaine \& I. C. White.

Second Geol. Surv. Pa., Rept. Progr. PP, I880, p. 98, pl. xxxvii, figs. 8, 9. [Dunkard formation (Permian).]

RhaBdocaRpos Bockschianus Goeppert \& Berger.

Fruct. Semin. Form. Lith., Breslau, 1848, p. 21, pl. i, figs. I3, I4. Lacoe, in Millspaugh: Bull. No. 24, W. Va. Agric. Exp. Sta., Morgantown, I892, p. 525. [Pottsville group; Kanawha formation.]

Rhabdocarpos sp. D. White.

Bull. Geol. Soc. Am., vol. 6, I895, p. 313. [Princeton conglomerate (Mississippian).]

RhaBdocarpos sp. D. White.

Bull. Geol. Soc. Am., vol. 6, I895, p. 3I4. Near Pocahontas coal. [Pottsville group; Clark formation.]

RHABDOCARPOS n. sp. D. White.

Bull. Geol. Soc. Am., vol. 6, I895, p. 315. [Pottsville group; Quinnimont and Sewell formations.]

TRIgONOCARPUM TRILOCUlaRe (Hildreth) Newberry.

Rept. Geol. Surv. Ohio, vol. I, pt. 2, Palaeont., I873, p. 367 , pl. xliii, figs. I, I3, I3a; pl. xliii, fig. 13. Fontaine. Amer. Jour. Sci., 3rd ser., vol. I I, I876, p. 382. [Pottsville group; Sewell formation.]

Trigonocarpum oliviaeforme Lindley \& Hutton?

Foss. F1. Gt. Brit., vol. 3, I837, p. I83,pl. ccxxii, fig. I-3. D. White: Bull. Geol. Soc. Am., vol. 6, I895, p. 317. [Pottsville group; Sewell formation.]

Trigonocarpum clavatum (Sternberg) D. White.

Bull. Geol. Soc. Am., vol. 6, I895, p. 3I5. [Pottsville group; Quinnimont formation.]

TRIGONOCARPUM AMPULLAEFORME Lesquereux.

Second Geol. Surv. Pa., Rept. Progr. P., I884, p. 823, pl. cix, figs. I8-20, 21 ? Lacoe, in Millspaugh: Bull. No. 24, W. Va. Agric. Exp. Sta., Morgantown, I892, p. 527. [Pottsville group; Kanawha formation.] 
Trigonocarpum Noeggerathil (Sternberg) Brongniart.

Dict. Sci. Nat., vol. 57 (Prodrome), 1828, p. 137. Lacoe, in Millspaugh, Bull. No. 24, W. Va. Agric. Exp. Sta., Morgantown, 1892 , p. 527. [Pottsville group; Kanawha formation.]

Carpolithes bi-Carpus Fontaine \& I. C. White.

Second Geol. Surv. Pa., Rept. Progr. PP, i88o, p. 98, p1. xxxvii, figs. 6, 7. [Dunkard formation (Permian).]

Carpolithes ellipticus Sternberg.

Flora d. Vorwelt (Tentamen), r825, p. xl, pl. vii, fig. I. D. White: Bull. Geol. Soc. Am., vol. I I, I900, p. I7I. [Allegheny formation.]

Carpolithes marginatus Fontaine \& I. C. White.

Second Geol. Surv. Pa., Rept. Progr. PP, I880, p. 98, pl. xxxvii, fig. I. [Dunkard formation (Permian).]

Carpolithes sp. D. White.

Bull. Geol. Soc. Amer., vol. 6, I895, p. 315. [Pottsville group; Quinnimont formation.]

Carpolithes sp. D. White.

Bull. Geol. Soc. Am., vol. 6, I895, p. 313. [Princeton conglomerate (Mississippian).]

Carpolithes? Fragarioides Newberry.

Rept. Geol. Surv., Ohio, vol. I, pt. 2, Palaeont., I873, p. 370, pl. xliii, figs. 2, 2a. D. White: Bull. Geol. Soc. Amer., vol. II, I900, p. 165. [Pottsville group; Kanawha formation.]

GuIlielmites orbicularis Fontaine \& I. C. White.

Second Geol. Surv. Pa., Rept. Progr. PP, I880, p. 99, pl. xxxii, fig. 2. [Dunkard formation (Permian).]

The fossil described under the above name is possibly a Dolerophyllum.

PAlaeoxyris appendiculata Lesquereux.

Geol. Surv. Illinois, vol. 4, Geol. \& Pal., pt. 2, I870, p. 546, pl. xxvii, fig. Ir. D. White: Bull. Geol. Soc. Am., vol. I I, I900, p. I69. [Pottsville group; Kanawha formation.]

The species of this genus, though described as plants, are thought by many to be egg-capsules of a fossil shark or allied type of animal life. There is, at all events, little doubt that they are the envelopes of some type of fructification.

EQUISETUM ARVENSE L.

Knowlton, Amer. Geologist, Minneapolis, vol. 2, I896, p. 371. Pleistocene (Interglacial). [Carmichaels clays.]

Cyperus sp. Knowlton.

Amer. Geologist, Minneapolis, vol. 2, I896, p. 371. Pleistocene (Interglacial) : [Carmichaels clay.] 
Potamogeton Robbinsi Oakes.

Knowlton, Amer. Geologist, Minneapolis, vol. 2, 1896, p.

371. Pleistocene (Interglacial): [Carmichaels clay.]

Fagus ferruginea Ait.

Knowlton: Amer. Geologist, Minneapolis, vol. 2, p. 37 I.

Pleistocene (Interglacial): [Carmichaels clay.]

Castanea pumila Mill.

Knowlton, Amer. Geologist, Minneapolis, vol. I8, I8g6, p.

37I. Pleistocene (Interglacial) : [Carmichaels clay.]

Quercus falcata Mich.

Knowlton, Amer. Geologist, Minneapolis, vol. 2, ı896, p.

371. Pleistocene (Interglacial): [Carmichaels clay.] BetUla NIGRA L.

Knowlton, Amer. Geologist, Minneapolis, vol. 2, I896, p.

37 I. Pleistocene (Interglacial): [Carmichaels clay.]

Platanus occidentalis L.

Knowlton, Amer. Geologist, Minneapolis, vol. 2, 1896, p.

371. Pleistocene (Interglacial) : [Carmichaels clay.]

Ulmus RACEMOSA Thomas.

Knowlton: Amer. Geologist, Minneapolis, vol. 2, ı896, p.

371. Pleistocene (Interglacial) : [Carmichaels clay.]

LIQUIDAMBAR STYRACIFLUA.

Knowlton: Amer. Geologist, Minneapolis, vol. 2, I896, p.

37 I. Pleistocene (Interglacial): [Carmichaels clay.]

PLANTS REPORTED FROM THE POCONO (MISSISSIPPIAN) AT LEWIS TUNNEL, VA., SIX MILES

EAST OF WHITE SULPHUR, W. VA.

Archaeopteris alleghaniensis (Meek) Fontaine \& I. C. White. Archaeopteris Bockschianus Lesquereux.

Triphyliopteris Lescuriana (Meek) Fontaine.*

TRIPHYLLOPTERIS VIRGINIANA (Meek) Meek.

LEPIDODENDRON SCOBINIFORME Meek.

Lepidocystis SILIQUA (Dawson) D. White.

*The plants listed by Prof. Fontaine (Amer. Jour. Sci. (3), vols. 7 and II, I874 and I876) as Cyclopteris Jacksoni, Palaeopteris Jacksoni, and $P$. cf. obtusa, probably are forms closely related to, if not indentical with, Triphyllopteris virginiana and $T$. Lescuriana. 
PLANTS REPORTED FROM THE PRINCETON CONGLOMERATE (MISSISSIPPIAN) ON PINEY CREEK ROAD, RALEIGH COUNTY.

Aneimites sp.

EREMOPTERIS cf. DECOM POSITA (Kidston) D. White.

SPHENOPTERIS DISTANS Sternberg.

Sphenopteris subgeniculata (Stur) Rothpletz.

SPHENOPTERIS sp.

CARdiopteris frondosa (Goepp.) Schimp.

Asterophyllites minutus Andrews?

ASTEROPHYLLITES sp.

RHABDOCARPOS n. sp.

Carpolithes sp.

PLANTS REPORTED FROM THE CLARK FORMATION (PENNSYLVANIAN : POTTSVILLE GROUP).

Aneimites adiantoides (Lindley \& Hutton) Bailey.

Lower Piney Creek plant bed.

Aneimites (Wardia) fertilis D. White.

Keeney Creek Branch railway near Nuttall.

ERemopteris macilenta (Lindley \& Hutton) D. White.

Lower Piney Creek plant bed.

Sphenopteris Hoeninghausi Brongniart.

Lower Piney Creek plant bed.

SPHENOPTERIS RARINERVIS Fontaine.

Lower Piney Creek plant bed.

Sphenopteris sp. D. White.

Near Pocahontas coal, mouth of Arbuckle Creek.

Alethopteris cf.GRANDIFOLIA Newberry.

Lower Piney Creek plant bed.

Alethopteris sp. D. White.

Near Pocahontas coal, mouth of Arbuckle Creek.

Neuropteris Pocahontas D. White.

Present nearly everywhere.

Neuropteris Smithir Lesquereux.

Clark Gap.

Neuropteris tenuifolia (Sclotheim) Sternberg*?

Lower Piney Creek plant bed.

*Undoubtedly wrongly identified. Probably a derivative of $N$. Pocahontas comparable to $N$. biformis Lesquereux. 
Aphlebia spinosa (Goeppert) D. White.

Lower Piney Creek plant bed.

EQUISETITES OCCIDENTALIS Lesquereux.

Lower Piney Creek plant bed.

Asterocalamites scrobiculatus (Schlotheim) Zeiller.

Lower Piney Creek plant bed.

LEPIDODENDRON SELAGINOIDES Sternberg.

Lower Piney Creek plant bed.

Cordaites Robbir Dawson?

Lower Piney Creek plant bed.

RHABDOCARPoS sp.

Near Pocahontas coal at mouth of Arbuckle Creek and near Rush Run.

PLANTS REPORTED FROM THE QUINNIMONT FORMATION (PENNSYLVANIAN : POTTS-

VILLE GROUP).

Aneimites tenuifolius (Brongniart) D. White.

Quinnimont coal at Princes, Rush Run, Dimmock, Fayette, etc.

EREMopteris cf. elegans (Ett.) Lx.

EREMOPTERIS MICROPHYLLA Lx.?

Quinnimont coal at Rush Run, Fayette, Harveys.

Quinnimont coal near Nuttall.

Mariopteris potTSVilleA D. White.

Quinnimont coal at Beechwood, Red Ash, Princes, Rush Run. Dimmock, etc.

MARIOPTERIS LATIFOLIA (Brongn.) Zeill.?

Quinnimont coal at Dimmock, Rush Run, Harveys.

MARIOPTERIS DIMORPHA (Lesquereux) D. White.

Quinnimont coal at Princes, near Fayette station and

Nuttall.

Sphenopteris divaricata Geinitz \& Gutbier.

Quinnimont coal at Princes and Fire Creek.

SphenOPteris cf. Goepperti (Ett.) Schimp.

Quinnimont coal at Fire Creek and Nuttall.

Sphenopteris Hoeninghausi Brogn.

Quinnimont coal at Quinnimont, Princes, Rush Run,

Beechwood and Harveys.

Sphenopteris patentissima (Ett.) Schimp.

Quinnimont coal at Princes, Red Ash, and Fayette.

SphenOPTERIS DICKSONOIDES (Goeppert) Weiss.

Quinnimont coal at Fire Creek. 
Sphenopteris (Renaultia) microcarpa Lesq.

Quinnimont coal at Quinnimont, Fayette, Beurys(?) and Rush Run.

Megalopteris cf. Dawsoni Andrews.

Quinnimont coal at Quinnimont.

Megalopteris Sewellensis Font.?

Quinnimont coal at Dimmock, Rush Run and Harveys.

Alethopteris sp. D. White.

Quinnimont coal at Fire Creek, Nuttall, Harveys, and Princes.

Neuropteris Smithin Lesq.

Quinnimont coal at Fire Creek, Nuttall, Princes, Rush Run, etc.

Neuropteris Lindleyana Sternb. var. Font.

Quinnimont coal at Sewell station.

OdONTOPTERIS NEUROPTEROIDES Newberry*.

Quinnimont coal at Quinnimont.

EQUiseTites occidentalis Lesq.

Quinnimont coal at Quinnimont.

Asterocalamites scrobiculatus (Scloth.) Zeill.?

Quinnimont coal at Princes and Rush Run.

Calamites cannaeformis Schloth.

Quinnimont coal at Quinnimont.

Calamites sp. D. White.

Quinnimont coal at Quinnimont and Dimmock.

Asterophyllites minutus Andrews.

Quinnimont coal at Rush Run, Dimmock and Harveys.

Annularia Ramosa (Brong.) Weiss.

Quinnimont coal at Dimmock, Rush Run and Harveys.

AnNularia n. sp. D. White.

Quinnimont coal at Rush Run.

Calamostachys lanceolata Lesq. (form.).

Quinnimont coal at Rush Run.

Sphenophyllum tenue D. White.

Quinnimont coal at Dimmock.

Lycopodites n. sp. D. White.

Quinnimont coal at Princes, Fire Creek, Dimmock and Rush Run.

LePidodendron acuminatum (Goepp.) Unger?

Quinnimont coal at Rush Run, Red Ash, Fire Creek and

Quinnimont.

Lepidodendron Sternbergit Brongn. (form.).

Quinnimont coal at Dimmock and Princes.

Lepidodendron Veltheimianum Sternb.

Quinnimont coal at Rush Run:

*Probably wrong identification. 
UlodendRon sp. D. White.

Quinnimont coal at Quinnimont, Dimmock, Beechwood. HALONIA sp. D. White.

Quinnimont coal at Rush Run.

LePIDOSTROBUS VARIABILIS Lindl. \& Hutt.

Quinnimont coal at Fire Creek.

Lepidophyllum Campbellianum Lesq.

Quinnimont coal at Dimmock and Rush Run.

LEPIDOPHYLLUM sp. D. White.

Quinnimont coal at Dimmock and Rush Run.

Sigillaria cf. Dentata Newberry.

Quinnamont coal at Beurys and Beechwood.

Sigillaria sp. D. White.

Quinnimont coal at Beurys.

CoRdaites RobBiI Dawson?

Quinnimont coal at Quinnimont.

RhabdocARpos sp. D. White.

Quinnimont coal at Fire Creek, Rush Run, Dimmock.

Trigonocarpum clavatum (Sternb.) D. White.

Quinnimont coal at Quinnimont and Dimmock.

Carpolithes sp. D. White.

Quinnimont coal at Fire Creek.

PLANTS RECORDED FROM THE SEWELL FORMATION (PENNSYLVANIAN : POTTSVILLE GROUP).

EREMopteris cf. elegans (Ett.) Lesq.

Sewell coal at Harveys.

Eremopteris Cheathami Lesq.

Sewell coal at Harveys.

ERemopteris macilenta (Lesq.) D. W. form.

Sewell coal at Thurmond, Nuttall and Sewell.

Mariopteris muricata (Schloth.) Zeill. form.

Sewell coal at Thurmond, Harveys, and Cunard.

Mariopteris pottsvillea D. W., var. Nov.

Sewell coal, Thurmond and Harveys.

Sphenopteris cf. Royi Lesq.

Sewell coal at Nuttall.

SPHENOPTERIS FLEXICAULIS Lesq.

Sewell coal, Harveys.

Sphenopteris cf. LARISCHII (Stur) Lesq.

Sewell coal, Harveys.

SPHENOPTERIS MICROCARPA Lesq.

Sewell coal, Harveys.

Sphenopteris communis Lesq. 
Sewell coal at Nuttall.

Pecopteris(?) SERrulata (Daws.) Hartt.

Sewell coal at Thurmond.

MEgalopteris SEWELLENSIS Font.

Sewell coal, Sewell

Megalopteris Hartil Andrews.

Sewell coal, Sewell

Alethopteris lonchitica (Schloth.) Stb. (?)

Sewell coal at Thurmond, Macdonald and Nuttall.

Alethopteris Serli var. Europea (Brongn.) Goepp.

Sewell coal, Sewell

Alethopteris Serli var. americana (Brongn.) Goepp.

Sewell coal, Sewell

Altehopteris Evansil Lesq.

Sewell coal at Cunard and Nuttall.

Callipteridium sp.

Sewell coal at Cunard and Nuttall.

Neuropteris Schlehani Stur. var.

Sewell coal in all of New River Region.

NeURopteris BIFormis Lesq. form.

Sewell coal, Macdonald and Cunard.

Neuropteris? sp. Fontaine.

Sewell coal, Sewell

Neuropteris Elrodi Lesq.

Sewell coal: Macdonald, Thurmond and Cunard.

Onontopteris NewberRyi Lesq. (?)

Sewell coal at Macdonald.

ODONTOPTERIS GRACILLIMA Newberry.

Sewell coal, Sewell

Calamites Roemeri Gopp.?

Sewell coal, Sewell

Calamites approximatus -Schlotheim.

Sewell coal, Sewell

AsterophyLlites GRACILIS Lesq.

Sewell coal at Macdonald.

Asterophyllites ERECTIFOLIUS Andr.

Sewell coal at Macdonald, Turkey Knob, Harveys and

Thurmond.

Annularia Radiata (Brongn.) Stb.

Sewell coal at Thurmond.

Macrostachya sp.

Sewell coal at Cunard.

Sphenophyllum antigudm Dawson.

Sewell coal, Sewell

Lepidodendron Sternbergi Brongn.

Sewell coal at Stone Cliff. 
LEPIDOPHYLLUM sp.

Sewell coal at Thurmond.

Sigillaria cf. Reticulata Lesq.

Sewell coal at Cunard.

Cordaites Robbil Dawson?

Sewell coal at Sewell.

Whittleseya elegans Newby.

Sewell coal at Macdonald.

Cardiocarpon $c f$. bicuspidatum (Sternb.) Newby

Sewell coal at Stone Cliff.

Cardiocarpon minor Newby.

Sewell coal at Turkey Knob.

Cardiocarpon elongatum Newby.

Sewell coal at Macdonald and Turkey Knob.

RHABDOCARPOS sp.

Sewell coal at Macdonald, Turkey Knob, Harveys, Stone

Cliff, and Thurmond.

Trigonocarpum triloculare (Hildreth) Newberry.

Sewell coal at Sewell.

TRIgONOCARPUM OLIVIAEFORME L. \& H.

Sewell coal at Turkey Knob, Harveys, Thurmond and (?)

Macdonald.

PLANTS REPORTED FROM THE NUTTALL SANDSTONE LENTIL OF THE SEWELL FORMATION NEAR ANSTED.

Archaeopteris stricta Andrews.

Archaeopteris sp. D. White.

EREMOPTERIS sp. D. White.

MARIOPTERIS MURICATA (Schlotheim) Zeiller.

Mariopteris pygmaea D. White.

MARIOPTERIS ACUTa (Brongniart) Zeiller.

Sphenopteris Furcata Brongniart.

Sphenopteris $c f$. Linearis Sternburg.

Alethopteris $c f$. AMBigua Lesquereux.

Pecopteris plumosa Sternberg.

Megalopteris sp. D. White.

Neuropteris sp. D. White.

OdONTOPTERIS GRACILLIMA Newberry.

Calamites approximatus Sternberg.

AsteropHyllites minutus Andrews.

Annularia cuspidata Lesquereux.

Calamostace ys sp. D. White.

Spienophyllum cuneifolium (Sternberg) Zeiller. 
LEPIDODENDRON RUSHVILLENSE Andrews.

LEPIDOSTROBUS VARIABILIS Lindley \& Hutton.

LEPIDOPHYLlum sp. D. White.

Triletes sp. D. White.

CARdiocarpon bicuspidatum (Sternberg) Newberry.

Cardiocarpon CORnUtum Dawson.

PLANTS REPORTED FROM THE KANAWHA FORMATION (PENNSYLVANIAN : POTTSVILLE GROUP).

Eremopteris $c f$. Sauveuri (Crepin) D. White.

Cedar Grove coal at East Bank.

Eremopteris artemisiaefolia (Sternb.) Schimp.

Coalmont.

EREmopteris cf. Lincolniana D. White.

Eagle coal at St. Clair mine.

EREmopteris n. sp. D. White.

Gas coal at Ansted; Peerless coal at Peerless, Cedar Grove and Handley; Cedar Grove coal at East Bank and Riverside mine.

Cheilanthites trifoliolatus (Artis) Goepp.

Gas-Tunnel coal at Forest Hill mine near Edgewater; Peerless coal at Peerless, Slaughters Creek, Black Diamond, Monarch, Handley and Black Cat mines.

Cheilanthites obtusilobus var. dilatus (Lesquereux) D.

White.

Cedar Grove coal at East Bank, Black Cat Mine, and Cotton Hill? Also at Short Creek and Peytona.

Cheilanthites cf. nummularius (Gutbier) D. White.

Stockton coal at Belmont, and on Buffalo Lick Fork and Spanglers Fork of Blue Creek.

Mariopteris inflata (Newberry) D. White.

Eagle coal at Eagle. Gas coal at Ansted; Diamond.

MARIOPTERIS cf. JacQUoti (Zeiller) D. White.

Cedar Grove coal at Cedar Grove.

MARIopteris muricata (Schlotheim) Zeiller.

Eagle coal at Eagle and St. Clair; Stockton coal on Hurricane Branch.

MARIOPTERIS LATIFOLIA (Brongniart) Zeiller.

Coalmont.

MARIopteris ACUTA (Brongniart) Zeiller.

Eagle coal at Eagle, Crescent and St. Clair. Peerless coal, Cedar Grove, Peerless, Black Diamond and Slaughters Creek. 
Mariopteris Andraeana (v. Roehl) D. White.

Gas coal at Ansted; Peerless coal at Peerless; Cedar Grove coal at East Bank, Black Cat. Also at Blacksburg and Coalmont.

MARIOPTERIS SPHENOPTEROIDES (Lesquereux) Zeiller var. nov. D. White.

Cedar Grove coal at East Bank, Blacksburg.

MARIOPTERIS NERVOSA (Brongniart) Zeiller.

Eagle coal at Eagle; Gas coal at Ansted and Diamond;

Stockton coal at Belmont.

Sphenopteris spinosa Gopp.

Eagle coal at Eagle and St. Clair; Peerless coal: Cedar Grove, Black Cat, and Slaughter Creek.

SpHenOPTERIS FURCATA Brongn.

Eagle coal at Crescent.

Sphenopteris elegans (Brongn.) Sternb.

Malden.

Sphenopteris geniculata Germar \& Kaulfuss.

Cedar Grove coal at East Bank and Black Cat mines.

Sphenopteris Hildrethi Lesquereux.

Cedar Grove coal at East Bank; also at Cotton Hill.

SPHENOPTERIS cf. TRICHOMANOIDES Brongn.

Stockton coal on Buffalo Lick Fork near Cannelton.

Sphenopteris LinkiI (Goeppert) Presl.

Eagle coal at Crescent.

SPHENOPTERIS TRACYANA Lesquereux?

Eagle coal at Eagle.

Sphenopteris tenella Brongniart

Stockton coal on Buffalo Lick Fork, near Cannelton and at Pond Gap.

Sphenopteris (Zeilleria) delicatula Sternberg.

Gas coal at Ansted.

SPHENOPTERIS HYMENOPHYLlOIDES Brongn.

Stockton coal at Stanton mine, Pond Gap, and near mouth of Hurricane Creek.

Sphenopteris Karwinensis Stur.

Stockton coal, Pond Gap, and Buffalo Lick Fork near

Cannelton.

Sphenopteris cf. DUbUissonis Brongn.

Eagle coal at St. Clair; Gas coal at Forest Hill mine;

Peerless coal at Peerless, Handley and Black Cat mines.

Sphenopteris (Renaultia) microcarpa Lesquereux?

Eagle coal at Eagle and St. Clair.

Sphenopteris schatzlarensis Stur.

Eagle coal at Crescent and St. Clair; Peerless coal at

Peerless and Monarch mines. 
Sphenopteris $c f$. Broadheadi D. White.

Stockton coal at Pond Gap.

Sphenopteris $c f$. CAN Neltonensis D. White.

Peerless coal, Monarch mine.

Sphenopteris mixta Schimp.

Stockton coal, Belmont mine, and Pond Gap.

Sphenopteris (Crossotheca) ophioglossoides (Lesq.) D.

White.

Stockton coal on Buffalo Lick Fork near Cannelton.

Sphenopteris (Crossotheca) $c f$. Crepini Zeiller.

Peerless coal at Peerless; Gas coal at Ansted; Stockton

coal on Buffalo Lick near Cannelton and at Stanton mine.

Oligocarpia alabamensis Lesquereux?

Cedar Grove coal, Cedar Grove.

Oligocarpia sp. D. White.

Peerless coal, Handley.

Pecopteris plumosa Artis.

Gas coal, at Crescent, Diamond, Morris Creek and Ansted.

Pecopteris Aspera Brongn.

Gas coal? Cotton Hill.

Pecopteris sp. $c f$. INTEgRa (Andrae) Schimper.

Eagle coal at Crescent.

Pecopteris villosa Brongniart.

Stockton coal ; Pond Gap.

Pecopteris sp. $c f$. Crenulata Brongn.

Tunnel coal and Gas coal, Ansted, Diamond, Morris

Creek, Crescent, Kelly's Creek and Carbon Hill.

ALETHOPTERIS DECURRENS (Artis) Sternb.

Eagle coal at Crescent.

Alethopteris massilionis Lx.

Short Creek.

AleTHOPTERIS LONCHITICA (Schlotheim) Sternberg.

Gas coal, Ansted: Peerless coal at Cedar Grove; Cedar

Grove coal at Cedar Grove, East Bank, and Black Cat mines.

Alethopteris Serlir (Brongn.) Goepp.

Eagle coal at Eagle and St. Clair; Gas-Tunnel coal at Diamond, Forest Hill, Morris Creek; Peerless coal at Peerless, Monarch, Handley, Black Cat and on Slaughter Creek; Cedar Grove coal at East Bank, Riverside mine and on Hurricane and Kelly creeks; Stockton coal at Buffalo Lick Fork and Spanglers Fork of Blue Creek.

Neuropteris flexuosa Sternb.

Eagle coal at Eagle; Gas-Tunnel coal at Forest Hill, Diamond, Crescent, Morris Creek and Ansted; Peerless coal at Peerless, Cedar Grove, Black Diamond, Monarch, Black Cat, Handley, and on Slaughter Creek; Cedar Grove coal at Cedar Grove, East Bank and Black Cat. 
NeURopteris RARINERVIS Bunbury.

Stockton coal at Buffalo Lick Fork, Belmont, Pond Gap.

Stanton mine on Kelly's Creek, and on Hurricane Creek.

Neuropteris cf. Gìgantea Sternb.

Gas coal at Ansted; Cedar Grove coal at East Bank.

Neuropteris ovata Hoffm.

Stockton coal, Pond Gap.

Neuropteris cf. CarriI Lesq.

Stockton coal on Spangler's Fork of Blue Creek.

Neuropteris Cisti Brongn.

Cedar Grove coal at Eastbank and Riverside.

Neuropteris $c f$. Zeilleri Potonie.

Eagle coal at Crescent.

Neuropteris Scheuchzeri Hoffm.

Stockton coal at Belmont, Staunton mine on Hurricane

Branch, and on Slaughter's Fork of Blue Creek.

Calamites Suckowi Brongn.

Gas coal: Ansted and Forest Hill mines; Cedar Grove coal at Cedar Grove and Black Cat mines.

Calamites Cisti Brongn.

Gas coal: Ansted; Cedar Ġrove coal at Eastbank.

Calamites ramosus Artis.

Eagle coal, St. Clair mine; Gas coal at Forest Hill ; Peerless coal at Peerless; Cedar Grove coal at Eastbank; Stocktonl coal at Belmont.

Calamites approximatus Schlotheim.

Cotton Hill.

Calamitina sp. D. White.

Gas coal at Morris Creek.

Calamodendron sp. D. White.

Peerless coal : Peerless.

Asterophyllites minutus Andrews.

Eagle coal, Crescent; Peerless coal, Cedar Grove and

Handley.

ASTEROPHYLLITES EQUISETIFORMIS (Schlotheim) Brongn:

Stockton coal, Belmont.

Asterophyllites LyCOPODIOIDES Zeiller.

Cedar Grove coal at Eastbank.

Asterophyllites RIGIDUs (Sternberg) Brongn.

Eagle coal: Eagle. Gas coal: Morris Creek. Cedar Grove coal: Hurricane Creek, Kelley Creek and Eastbank. Annularia acicularis (Dawson) Renault.

Eagle coal: Eagle and Crescent. AnNularia RAdiata (Brongniart) Sternberg.

Gas coal: Ansted, Cotton Hill; Peerless coal: Peerless, Cedar Grove, on Slaughters Fork of Blue Creek; Cedar Grove coal at Eastbank. 
Annularia stellata (Schlotheim) Wood.

Stockton coal: Belmont, Stanton mine and Pond Gap.

ANNULARIA SPHENOPHYLLOIDES (Zenker) Gutbier.

Stockton coal on Buffalo Lick Fork, at Stanton mine and Pond Gap.

Annularta ramosa (Artis) Weiss.

Eagle coal at St. Clair and Crescent; Gas coal: Ansted; Cedar Grove coal: Eastbank; Peerless coal: Peerless, Cedar Grove, Slaughter Creek, and Handley; Stockton coal: Belmont and Stanton mines.

Calamostachys ramosus. Weiss.

Eagle coal at St. Clair; Peerless coal at Peerless.

Sphenophyllum cuneifolium (Sternberg) Zeiller.

Eagle coal: Eagle and St. Clair; Gas coal: Forest Hill, Morris Creek, Black Cat and Handley; Cedar Grove coal: Cedar Grove, Hurricane Creek; Stockton coal: Buffalo Lick Fork; Spanglers Fork of Blue Creek, Stanton mine and at Pond Gap.

Sphenophyllum Lescurianum D. White.

Stockton coal: Buffalo Lick Fork and Pond Gap.

Sphenophyllum emarginatum (Brongniart) Koenig.

Stockton coal: Spanglers Fork of Blue Creek.

Sphenophyllum furcatum (Lindl. \& Hutt.) Gein. ley.

Eagle coal at Crescent and Eagle: Peerless coal at Hand-

Lycopodites MeEkir Lesquereux.

Stockton coal at Stanton mine and on Hurricane Creek. Lycopodites SIMPLEX Lesquereux.

Peerless coal: Peerless; Cedar Grove coal: Eastbank, Cotton Hill?

Bothrodendron cf. minUtiFolium (Boulay) Zeiller.

Gas coal: Morris Creek, Ansted and Forest Hill mine; Peerless coal: Peerless, Cedar Grove, Monarch, Handley, Black Cat and on Slaughter Creek; Cedar Grove coal: Cedar Grove, Eastbank, and Kelleys Creek; Stockton coal: Stanton mine.

BothrodEndRon sp. D. White.

Eagle coal: St. Clair.

LEPIDODENDRON $c f$. BRITTSII Lesq.

Gas coal : Forest Hill mine; Peerless coal: Monarch.

LePIDODENDRON ACUMINATUM (Goepp.) Ung.

Gas coal: Crescent.

Lepidodendron Veltheimianum Sternb.

Gas coal: Morris Creek; Peerless coal at Handley.

LEPIDODENDRON LANCEOLATUM Lesq.

Stockton coal: Stanton mine and on Hurricane Creek. 
LEPIDODENDRON $c f$. DICHOTOMUm Sternb.

Eagle coal: Eagle and Crescent.

LEPIDODENDRON CLYPEATUM Lesq.

Cedar Grove coal on Kelley's Creek below mouth of Hurricane Creek.

LEPIDODENDRON OBOVATUM Sternb.

Eagle coal: Eagle; Gas coal : Diamond mine.

LEPIDODENDRON MODULA'TUM Lesq.

Stockton coal at Pond Gap.

LEPIDOPHLOIOS $c f$. LARICINUS Sternb.

Gas coal: Morris Creek and Ansted; Peerless coal at Handley.

ULODENDRON MAJUS Lindl. \& Hutt.

Gas coal at Ansted; Cedar Grove coal at Eastbank.

LePIDOSTRobus VARIABILIS Lindl. \& Hutt.

Eagle coal: Eagle and St. Clair; Gas coal: Forest Hill,

Diamond and Ansted; Peerless coal: Cedar Grove, Monarch and Handley; Cedar Grove coal: Hurricane Branch; Stockton coal: Belmont and Stanton mines.

LEPIDOSTROBUS ORNATUS Brongn.?

Gas coal: Tunnel Mine, Kelley Creek.

Lepidostrobus Salisburyi Lesq.

Coal River.

Lepidostrobus sp. D. White.

Gas coal: Kelleys Creek.

LePidophyllum aCUMinAtum Lesq.

Peerless coal: Peerless.

Lepidophyllum LANCEOlatum Lindl. \& Hutt. ?

Stockton coal: Sta nton mine.

Lepidophyllum Campbellianum Lesq.

Eagle coal: Eagle and St. Clair; Gas coal: Diamond, Morris Creek, Forest Hill and Ansted; Peerless coal: Peerless; Cedar Grove coal : Kelley Creek below month of Hurricane Branch.

Lepidophyllum $c f$. CULTRIFORME Lesq.

Gas coal : Diamond, Morris Creek and Kelley Creek. Lepidocystis obtusus Lesq.

Gas coal: Morris Creek and Kelleys Creek.

LEPIDOCYSTIS VESICULARIS Lesq.

Stockton coal: Buffalo Lick Fork and Stanton mine.

Sigillaria fissa Lesq.

Stockton coal: Hurricane Branch.

Sigillaria $\mathrm{cf}$. Reticulata Lesq.

Gas coal: Morris Creek.

Sigillaria cf. ichtyolepis (Sternb.) Corda.

Gas coal: Tunnel mine.

CoRdaites borassifolius (Sternb.) Ung. 
Gas coal: Tunnel mine. Poacordaites sp. D. White.

Stockton coal; Pond Gap.

Cordaicarpon circulare (Lesq.) D. White.

Stockton coal: Stockton.

Cordaicarpon Cinctum Lesq.

Stockton coal: Pond Gap.

CARDIOCARPON MiNor Newberry.

Gas coal: Tunnel mine.

Rhabdocarpos Bockschianus Goepp. \& Berg.

Campbells Creek.

Rhabdocarpos multistriatus (Presl) Lesq.

Gas coal: Ansted.

RHABDOCARPOS TENAX Lesq.

Campbells Creek.

Rhabdocarpos sulcatus (Lindl. \& Hutt.) Schimp.

Eagle coal: Eagle; Gas coal: Forest Hill and Morris Creek.

Rhabdocarpos amygdalaeformis Goepp. \& Berger.

Peerless coal: Monarch.

Carpolithes? Fragarioides Newberry.

Gas coal: Ansted; Peerless coal: Peerless.

Palaeoxyris appendiculata Lesq.

Stockton coal: Stanton mine.

Trigonocarpum ampUllaeforme Lesq.

Malden.

Trigonocarpum Noeggerathin (Sternb.) Brongn.

Campbells Creek and Malden.

PLANTS REPORTED FROM THE ALLEGHENY FORMATION (PENNSYLVANIAN).

EREMopteris solida (Lesq.) D. White.

Kittanning coal group near Clendennin.

Cheilanthites squamosus (Lesq.) D. White.

Granny Branch of Indian Creek near Mason; Kittanning coal group near Clendennin.

Mariopteris Sillimanni (Brongn.) D. White.

Granny Branch of Indian Creek near Mason; Kittanning coal group: Graham mine near Clendennin.

Mariopteris Newberryi (Lesq.) D. White.

Kittanning coal group near Clendennin.

MARIOPTERIS NERVOSA (Brongn.) Zeill.

Kittanning coal group on Belva-Lizemore road; Furnace Hollow, Wayne Co. 
Mariopteris sphenopteroides (Lesq.) Zeill.

Granny Branch of Indian Creek near Mason.

Sphenopteris pinnatifida (Lesq.) D. White.

Kittanning coal group near Clay Court House.

Sphenopteris chaerophylloides (Brongn.) Presl.

Kittanning coal group near Clendennin.

Sphenopteris $c f$. STipulata Gutb.

Granny Branch of Indian Creek near Mason.

Sphenopteris OPHIOGLOSSOIDES (Lesq.) D. White.

Kittanning coal group: Belva-Lizemore road and near

Clendennin.

Sphenopteris (Crossotheca) sagittatus (Lesq.) D. White.

Kittanning coal group near Clay Court House.

Pecopteris dentata Brongn.

Kittanning coal group near Clay Court House; also in

the Allegheny at Furnace Hollow, Wayne Co.

Pecopteris Unita Brongn.

Kittanning coal group near Clendennin and on the Belva-

Lizemore road.

Pecopteris emarginata (Goepp.) Presl.

Kittanning coal group at Pleasant Retreat and Clendennin

(Cobb mine).

Pecopteris vestita Lesq.

Kittanning coal group, Cobb mine near Clendennin.

Pecopteris villosa Brongn.?

Kittanning coal group near Clendennin, and Graham mine near Mason, and along Belva-Lizemore road and near Clay Court House; also in the Allegheny at Furnace Hollow.

Wayne County; also present on Granny Branch of Indian

Creek near Mason.

Pecopteris OREopteridia (Schloth.) Sternb.

Kittanning coal group: Graham mine near Mason; also in beds said to be Lower Freeport at Furnace Hollow, Wayne County.

Pecopteris Miltoni (Artis) Sternb.

Kittanning coal group at Pleasant Retreat and near Clay Court House.

Alethopteris pennsylvanica Lesq.

Bed said by Dr. I. C. White to be near the Lower Freeport coal, near Furnace Hollow, Wayne County.

Callipteridium? inaequale Lesq.

Bed said by Dr. I. C. White to be near the Lower Freeport coal, near Furnace Hollow; Wayne County.

Neuropteris Rarinervis Bunb.

Kittanning coal group near Clay Court House; also in beds said to be Lower Freeport at Furnace Hollow, Wayne County. 
Neuropteris vermicularis Lesq.

Gunter Hollow near Mason.

Neuropteris ovata Hoffm.

Granny Branch of Indian Creek near Mason; also in Kittanning coal group near Clay Court House, Pleasant Retreat and near Clendennin.

NeURopteris fimbriata Lesq.

Kittanning coal group near Clay Court House.

Neuropteris Scheuchzeri Hoffm.

Furnace Hollow, Wayne County, and Granny Branch of Indian Creek near Mason; also from Kittanning coal group near Clay Court House, Graham mine, Pleasant Retreat and near Clendennin.

Neuropteris Agassizi Lesq.?

Supposed Lower Freeport horizon in Furnace Hollow, Wayne County.

LINOPTERIS OBLIQUA (Bunbury) Zeill.

Kittanning coal group near Clay Court House.

ODONTOPTERIS WORTHENI Lesq.

Granny Branch of Indian Creek near Mason.

ODONTOPTERIS SUBCUNEATA Bunbury.

Kittanning coal group at Graham mine near Mason.

ODONTOPTERIS AEQUALIS Lesq.

Kittanning coal group, Belva-Lizemore road.

Calamites Cistui Brongn.

Kittanning coal group near Clendennin.

Annularia stellata (Schloth.) Wood.

Kittanning coal group: Graham mine, Cob mine near Clendennin; near Clay Court House, and Pleasant Retreat. Also reported on Granny Branch of Indian Creek near Mason.

Annularia sphenophylloides (Zenk.) Gutb.

Kittanning coal group: Graham mine.

ANNULARIA SPHENOPHYLLOIDES var. INTERMEDIA Lesq.

Furnace Hollow, Wayne County.

Sphenophyllum emarginatum (Brongn.) Koenig.

Kittanning coal group; Cob mine near Clendennin; near Clay Court House, and on Belva-Lizemore road. Also reported on Granny Branch of Indian Creek near Mason. Beds said to be Lower Freeport age, near Furnace Hollow, Wayne County.

Sphenophyllum majus Bronn.

Kittanning coal group; near Clendennin.

LEPIDODENDRON MODULATUM Lesq.

Granny Branch of Indian Creek near Mason; also over "Lower Freeport" coal, at Furnace Hollow, Wayne County. 
Sphenophyllum majus Bronn.

Kittanning coal group near Clendennin.

LEPIDOPHLOIOS sp. D. White.

Furnace Hollow, near Huntington pike ("Lower Freeport").

LEPIDOPHYLLUM BREVIFOLIUM Lesq.

Kittanning coal group near Clendennin.

LePIDOPHYLLUM JenNyi D. White.

Granny Branch of Indian Creek near Mason.

LEPIDOPHYLLUM OBLONGIFOLIUM Lesq.

Kittanning coal group near Clendennin and on Belva-Lizemore road; also over "Lower Freeport" coal in Furnace

Hollow, Wayne County.

LEPIDOPHYLLUM HASTATUM Lesq.

Kittanning coal group near Clay Court House.

LEPIDOCYSTIS VESCICULARIS Lesq.

Kittanning coal group: Belva-Lizemore road.

Sigillaria camptotaenia (Wood) Zeill.

Kittanning coal group: Belva-Lizemore road, Clendennin

near Clay Court House.

Carpolithes ellipticus Sternb.

Kittanning group: Cobb mine near Clendennin; also reported from the formation on Granny Branch of Indian

Creek near Mason.

\section{PLANTS RECORDED FROM THE CONEMAUGH FORMATION (PENNSYLVANIAN).}

Cheilanthites (Sphenopteris) solidus (Lesq.) D. White.

Left Fork of Mill Creek, Wayne. Co.

Cheilanthites obtusilobus (Brongn.) Gopp.

Left Fork of Mill Creek, Wayne. Co.

Cheilanthites squamosus (Lesq.) D. White.

Left Fork of Mill Creek, Wayne. Co.

Mariopteris Sillimanni (Brongn.) D. White.

Left Fork of Mill Creek, Wayne Co.

MARIOPTERIS Nervosa (Brongn.) Zeill.

Left Fork of Mill Creek, Wayne. Co.

Sphenopteris minutisecta Font. \& I. C. White.

Left Fork of Mill Creek, Wayne. Co.

Sphenopteris (CrossothecA) ophioglossoides Lesq.

Left Fork of Mill Creek, Wayne. Co.

Alethopteris Serlir. (Brongn). Gopp.

Left Fork of Mill Creek, Wayne. Co.

Pecopteris unita Brongn.

Left Fork of Mill Creek, Wayne. Co. 
Pecopteris villosa Brongn.?

Left Fork of Mill Creek, Wayne. Co.

Pecopteris $c f$. Jenneyi D. White.

Left Fork of Mill Creek, Wayne. Co.

Pecopteris OREOPTERIdiA (Schloth.) Sternb.

Left Fork of Mill Creek, Wayne Co.; near Lavalette.

Pecopteris Miltoni (Artis) Sternb.

Left Fork of Mill Creek, Wayne Co.

PECOPTERIS POLYMORPHA Brongn.

Near Lavalette.

Pecopteris sp. D. White.

Near Lavalette.

Neuropteris ovata Hoffm.

Left Fork of Mill Creek, Wayne Co.

Neuropteris fimbriata Lesq.

Left Fork of Mill Creek, Wayne Co.

Neuropteris Scheuchzeri Hoffm.

Wheeling, and 20 feet below the Pittsburgh coal at

Bellaire, Ohio.

Sphenophyllum majus Bronn.

Left Fork of Mill Creek, Wayne Co.

LyCOPODITES PENDULUS Lesq.

Left Fork of Mill Creek, Wayne Co.

Sigillaria FisSa Lesq.

Left Fork of Mill Creek, Wayne Co.

LEPIDOCYSTIS VESICULARIS Lesq.

Left Fork of Mill Creek, Wayne Co.

Cordaicarpon Gutbieri (Gein.) Gr. 'Ery.

Left Fork of Mill Creek, Wayne Co.

PLANTS RECORDED FROM THE MONONGAHELA FORMATION (PENNSYLVANIAN).

Mariopteris? spinulosa (Lesq.) D. White.

Wheeling, W. Va.

Alethopteris aquilina (Schlcth.) Goepp.

Wheeling, $\dot{W}$. Va.

Pecopteris unita Brogn.

Wheeling, W. Va.

Pecopteris villosa Brongn.?

Wheeling, W. Va.

Pecopteris $c f$. Jenneyi D. White.

Wheeling, W. Va.

Pecopteris notata Lesq.

Redstone coal, Wheeling, W. Va. 
Pecopteris nodosa (Goepp.) Schimp.

Marietta, Ohio.

Dicksonites Pluckeneti (Schloth.)

Wheeling, W. Va.

Neuropteris callosa Lesq.

Pomeroy, Ohio.

NeUropteris CRENUlata Brongn.

Pomeroy, Ohio.

Neuropteris Grangeri Brongn.

Wheeling, W. Va., and Bellaire, Ohio.

NeUROPTERIS ScheUCHZERI Hoffm.

Wheeling, W. Va., and Bellaire, Ohio, 20 feet below Pittsburgh coal.

Lescuropteris Moori (Lesq.) Schimp.

Wheeling, W. Va.

Aphlebia filiciformis (Gutb.) Schimp.

Wheeling, W. Va.

Sigillaria Menardi Brongn.

Pomeroy, Ohio.

\section{LIST OF FOSSIL PLANTS REPORTED FROM THE DUNKARD FORMATION* (PERMIAN).}

Diplothmema pachyderma (Font. \& I. C. White) D. White.

Waynesburg coal, Cassville, and Dents Run; also 500 feet above the same coal.

Sphenopteris minutisecta Font. \& I. C. White.

Waynesburg coal at West Union.

Sphenopteris (Cymoglossa) breviloba (Font. \& I. C. W.)

D. White.

Waynesburg coal at Cassville.

Sphenopteris (Cymoglossa) formosa (Font. \& I. C. White)

D. White.

Waynesburg coal at Cassville.

Sphenopteris (Cymoglossa) lobata (Font. \& I. C. White)

D. White.

Waynesburg coal at Cassville.

Sphenopteris (Cymoglossa) obtusifolia (Font. \& I. C. White)

D. White.

Waynesburg coal at Cassville.

*For notes on the Permian-Monongahela boundary see Bull. Geol. Soc., Amer., vol. xiv, I903, p. 538; Geol. Survey W. Va., vol. II, p. I20: and the U. S. Geol. Survey, Waynesburg and Rogersville Folios. 
Sphenopteris Lescuriana Meek.

Waynesburg coal at West Union.

Sphenopteris dentata Font. \& I. C. White.

Waynesburg coal at Cassville.

Sphenopteris auriculata Font. \& I. C. White.

Waynesburg coal at Cassville.

Sphenopteris foliosa Font. \& I. C. White.

Waynesburg coal at Cassville.

Sphenopteris hastata Font. \& I. C. White

Waynesburg coal at West Union.

Sphenopteris hastata Font. \& I. C. White.

Waynesburg coal at Cassville.

Sphenopteris acrocarpa Font. \& I. C. White.

Waynesburg coal at Cassville.

Sphenopteris sp.? Font. \& I. C. White.

Waynesburg coal at Cassville.

Pecopteris Pluckeneti (Schloth.) Sternb.

Waynesburg coal at West Union.

Pecopteris Pluckeneti var. constricta Font. \& I. C. White.

Waynesburg coal at West Union.

Pecopteris Germari (Weiss) Font. \& I. C. White.

Waynesburg coal at West Union and Cassville.

Pecopteris Germari var. Crassinervis Font. \& I. C. White.

Waynesburg coal at West Union.

Pecopteris Germari var. cuspidata Font. \& I. C. White.

Waynesburg coal at West Union.

Pecopteris dentata var. crenata Font. \& I. C. White.

Waynesburg coal, Cassville.

Pecopteris dentata var. parva Lesq.

Waynesburg coal, Cassville.

Pecopteris pachypteroides Font. \& I. C. White.

Waynesburg coal, Cassville.

Pecopteris (Goniopteris) emarginata (Gopp.) D. White.

Waynesburg coal, Cassville and 800 feet higher at Bellton.

Pecopteris (Goniopteris) oblonga (Font. \& I. C. White)

Miller.

Waynesburg coal, West Union.

Pecopteris (Goniopteris) Newberriana (Font. \& I. C. W.)

Miller.

Waynesburg coal, West Union.

Pecopteris (Goniopteris) longifolia (Font. \& I. C. W.), D. White.

Waynesburg coal, Cassville.

Pecopteris (Goniopteris) elliptica (Font. \& I. C. White) D. White.

Waynesburg coal, Cassville. 
Pecopteris (Goniopteris) sp.? Font. \& I. C. White.

Waynesburg coal, Cassville.

PECOPTERIS (GoNiopteris) ARGUTA (Brongn.).

Waynesburg coal, Cassville and West Union.

Pecopteris goniopteroides Font. \& I. C. White.

Waynesburg coal, Cassville.

Pecopteris (Goniopteris) elegans (Germ.).

Waynesburg coal, Cassville.

Pecopteris arborescens (Schloth.) Brongn.

Waynesburg coal at Cassville, West Union, etc.; 400 feet above that coal at Bellton.

Pecopteris arborescens var. Integripinna Font. \& I. C. White.

400-600 feet above the Waynesburg coal in Monongalia,

Tyler and Marshall counties.

Pecopteris pennaeformis var. latifolia Font. \& I. C. White.

Waynesburg coal, Cassville.

Pecopteris Candolleana Brongn.

Waynesburg coal, West Union.

Pecopteris OREOPteridia (Schloth.) Sternb.

400 feet above Waynesburg coal at Bellton.

Pecopteris rarinervis Font. \& I. C. White.

Waynesburg coal, Cassville.

Pecopteris imbricata Font. \& I. C. White.

Waynesburg coal, Cassville.

Pecopteris platynervis Font. \& I. C. White.

Waynesburg coal, Cassville.

Pecopteris asplenioides Font. \& I. C. White.

Waynesburg coal, Cassville.

Pecopteris rotundiloba Font. \& I. C. White.

Waynesburg coal, Cassville.

PeCopteris Microphylla Brongn.

Waynesburg coal, Cassville.

Pecopteris angustipinna Font. \& I. C. White.。

Waynesburg coal, West Union.

Pecopteris tenuinervis Font. \& I. C. White.

Waynesburg coal, Cassville.

Pecopteris subfalcata Font. \& I. C. White.

Waynesburg coal, Cassville.

Pecopteris Heeriana Font. \& I. C. White.

Waynesburg coal, Cassville.

Pecopteris Schimperiana Font. \& I. C. White.

Waynesburg coal, West Union.

Pecopteris lanceolata Font. \& I. C. White.

Waynesburg coal at Moundsville, and 400 feet higher at

Bellton.

Pecopteris inclinata Font. \& I. C. White.

Waynesburg coal, Cassville. 
Pecopteris merianopteroides Font. \& I. C. White.

\section{Penna.}

Waynesburg coal, Cassville, W. Va., and Carmichaels.

Pecopteris rotundifolia Font. \& I. C. White.

Waynesburg coal, Cassville.

Pecopteris sp.? D. White.

Waynesburg coal, Cassville.

Pecopteris ovoides Font. \& I. C. White.

400 feet above Waynesburg coal at Bellton.

Pecopteris latifolia Font. \& I. C. White.

Waynesburg coal, Cassville and at Bellton.

PECOPTERIS PTEROIDES Brongn.

Waynesburg coal near Arnettsville.

Pecopteris Miltoni (Artis) Sternb.

Waynesburg coal, West Union.

PeCOPTERIS POLYMORPHA Brongn.

Cassville?

Pecopteris elliptica Bunbury.

Waynesburg coal, Cassville.

Pecopteris (Callipteridium) grandifolia (F. \& I. C. W.).

D. White.

Waynesburg coal: West Union, Dents Run, and Georgetown.

Pecopteris (Callipteridium) oblongifolia (F. \& I. C. W.). D. White.

Waynesburg coal at Dents Run, Georgtown and Cassville, and 400 feet higher at Bellton.

Pecopteris (Callipteridium) odontopteroides (F. \& I. C. W.)

D. White.

Waynesburg coal at Cassville, Georgetown and Arnettsville.

Pecopteris (Callipteridium) unitum (Font. \& I. C. White.)

D. White. •

Waynesburg coal at Cassville, Georgetown and Arnettsville.

Pecopteris (Callipteridium) Dawsonianum (F. \& I. C. W.)

D. White.

Waynesburg coal, West Union, Cassville and Dents Run Alethopteris virginiana Font. \& I. C. White.

Waynesburg coal, Cassville.

Alethopteris gigas (Gutb.) Gein.

500 feet above Waynesburg coal at Bellton.

Callipteris Conferta (Sternb.) Brongn.

Washington coal near Brown's Bridge, Monongalia County. 
Callipteris lyratifolia (Grand 'Eury) var. Coriacea (F. \& I. C. W.) D. White.

Washington coal group near Jollytown, $\mathrm{Pa}$.

Callipteris curretiensis Zeill. (F \& I. C. W.) D. White.

Washington coal group near Jollytown, $\mathrm{Pa}$.

Taeniopteris Lescuriana Font. \& I. C. White.

Waynesburg coal, Cassville.

Taeniopteris Newberriana Font. \& I: C. White.

Waynesburg coal, Cassville.

Taeniopteris Newberriana var. angusta Font. \& I. C.White

Waynesburg coal, Cassville.

Neuropteris ovata Hoffm. (variety).

Waynesburg coal, Cassville and Brown's Mills.

Neuropteris Gibbosa Lesq.

Waynesburg coal at Brown's Mills.

Neuropteris Planchardi Zeill. var. longifolia (F. \& I. C. W.)

D. White.

Waynesburg coal at West Union.

Neuropteris dictyopteroides F \& I. C. White.

Waynesburg coal at West Union and 400 feet above

Waynesburg coal at Bellton.

NeUropteris fimbriata Lesq.

Waynesburg coal at Carmichaels, $\mathrm{Pa}$.

Neuropteris cordata Brongn.

Waynesburg coal at Cassville and West Union.

Neuropteris auriculata Brongn.

Waynesburg coal at Cassville and West Union, and 400

feet higher at Bellton.

LESCUROPTERIS ADIANTOIDES Lesq.

Waynesburg coal at Georgetown.

Odontopteris Reichiana Gutb.

Waynesburg coal at Brown's Mills.

ODONTOPTERIS OBTUSILOBA var. RARINERVISFont. \& I. C. White.

400 feet above Waynesburg coal at Bellton.

Odontopteris nervosa Font. \& I. C. White.

Waynesburg coal at Cassville and West Union.

Odontopteris densifolia Font. \& I. C. White.

Waynesburg coal at Cassville.

Caulopteris gigantea Font. \& I. C. White.

Waynesburg coal, Cassville.

Caulopteris elliptica Font. \& I. C. White.

Waynesburg coal, Cassville.

Aphlebia (Rhacophyllum) laciniatum (F. \& I. C. White). Sellards.

Waynesburg coal, Cassville. 
Aphlebia lactuca (Presl) Sterzel.

Waynesburg coal, Cassville; West Union; also Carmichels, $\mathrm{Pa}$.

Aphlebia (Rhacophyllum) speciosissima (Schimp.) D.White. Waynesburg coal, West Union, W. Va., and Carmichaels, $\mathrm{Pa}$.

Aphlebia (Rhacophyllum) Filiciformis var. majus (F \& I

C. White) D. White.

Waynesburg coal, Cassville.

EQuisetites RUgosus Schimp.

Waynesburg coal, West Union.

Equisetites striatus Font. \& I. C. White.

Waynesburg coal, West Union.

Equisetites elongatus Font. \& I. C. White.

Waynesburg coal, West Union.

Calamites Suckowi Brongn.

Waynesburg coal, West Union and Cassville.

Nematophyllum angustum Font. \& I. C. White.

Waynesburg coal, Cassville and West Union.

Annularia Radiata (Brongn.) Sternb.*

Waynesburg coal, Cassville.

Annularia stellata (Schloth.) Wood.

Waynesburg coal, Cassville and West Union.

Annularia sphenophylloides (Zenk.) Gutb.

Waynesburg coal, Cassville.

Annularia CARINATA Gutb.

Waynesburg coal : Cassville, West Union and Bellton.

ANNULARIA MINUTA Brongn.

Washington coal, Little Washington.

Sphenophyllum oblongifolium (Germ. \& Kauf.) Ung.

Waynesburg coal, Cassville.

Sphenophyllum longifolium (Germ.) Gein. \& Gutb.

Waynesburg coal, Cassville and West Union.

Sphenophyllum Thoni Mahr.

Sphenophyllum Fontaineanum S. A. Mill.

Washington, $\mathrm{Pa}$.

Waynesburg coal, Cassville and West Union.

Sphenophyllum filiculme Lesq.

Waynesburg coal, Cassville and West Union; also at Carmichaels, $\mathrm{Pa}$.

Sphenophyllum tenuifolium Font. \& I. C. White.

Waynesburg coal, Cassville and West Union.

Sphenophyllum angustifolium Gutb.

Waynesburg coal at Dents Run and Wadestown.

Sphenophyllum densifolium Font. \& I. C. White.

Waynesburg coal, Cassville.

* Probably not this species. 
Sigillaria Brardi Brongn.

Waynesburg coal, Cassville.

Sigillaria approximata Font. \& I. C. White.

Waynesburg coal, Arnettsville.

Cordaites Crassinervis Font. \& I. C. White.

Waynesburg coal, Cassville.

Baiera virginiana Font. \& I. C. White.

Waynesburg coal, Cassville.

Saportaea salisburioides Font. \& I. C. White.

Waynesburg coal, Cassville.

Rhabdocarpos oblongatus Font. \& I. C. White.

Waynesburg coal, Cassville.

Carpolithes bicarpus Font. \& I. C. White.

Waynesburg coal, Cassville.

Carpolithes marginatus Font. \& I. C. White.

Waynesburg coal, Cassville.

GuIlielmites orbicularis Font. \& I. C. White.

Waynesburg coal, Cassville.

FOSSIL PLANTS REPORTED FROM THE CARMICH-. AELS CLAY (PLEISTOCENE) NEAR MORGANTOWN.

EQUisetum ARVENSE L.

Potamogeton Robbinsii Oakes.

Cyperus sp. Knowlton.

Castanea pumila Mill.

FAGUS fERRUGinea Ait.

BetUla NIGRA L.

Quercus falcata Mich.

Ulmus Racemosa Thomas.

Platanus occidentalis L.

LIQUIDAMBAR STYRACIFLUA L. 


\section{INDEX TO PART I.}

\begin{tabular}{|c|c|c|c|c|c|}
\hline BIES & 01 & ADICEA & 243 & alba aristata & \\
\hline Canadensis ......... & 201 & pumila $\ldots \ldots \ldots \ldots$ & 24 & $\operatorname{sima} \ldots \ldots \ldots \ldots$ & \\
\hline Fraseri $\ldots \ldots \ldots \ldots$ & & DLUMia $\quad \ldots \ldots \ldots \ldots$ & 262 & $2 x^{2}$ & \\
\hline BUTILON & 304 & fungosa $\ldots .$. & 262 & ralis ...... & \\
\hline Abutilon...$\ldots \ldots$ & 304 & DOPOGON $\ldots \ldots \ldots \ldots$ & 366 & nedia $\ldots$ & \\
\hline Avicennae $\quad . .$. & 304 & amplexicaulis $\ldots . .$. & 366 & nnans .. & \\
\hline CAL & 295 & lion & 366 & GYRIUM & \\
\hline Virginica . & 295 & m $\quad \ldots \cdots$ & 00 & . & \\
\hline hedia & 295 & $\cdots \cdots \cdots$ & $\begin{array}{l}85 \\
78\end{array}$ & ulosa & \\
\hline CEAE $\ldots$. & 56 & $\operatorname{um} \ldots \ldots \ldots$ & 85 & CEAE $\ldots \ldots \ldots$ & 248 \\
\hline MA $\ldots \ldots$ & 101 & ntis .. & 85 & $\cdots$ & \\
\hline $\mathrm{m} \ldots \ldots \ldots$ & 101 & ae $\ldots \ldots$ & 85 & $\cdots$ & $229+3$ \\
\hline CER $\cdots \cdots \cdots \cdots \cdots \cdots$ & 299 & ginis $\ldots \ldots \ldots$ & 85 & . & 0 \\
\hline dasycarpum $\ldots \ldots \ldots$ & 300 & us $\cdots$. & 300 & datica $\ldots$ & \\
\hline ando $\ldots \ldots \ldots$ & 3 & $\ldots \ldots$ & 301 & ALISMACEAE $\ldots \ldots$ & \\
\hline$\cdots \ldots \ldots \ldots$ & 300 & 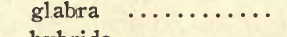 & 300 & $\ldots \ldots \ldots \ldots$ & 227 \\
\hline ranicum $\ldots$. & 9 & da $\ldots \ldots \ldots \cdots$ & 301 & ense $\ldots \ldots \ldots$ & \\
\hline$\ldots \ldots \ldots \ldots$ & 300 & Ira $\ldots$ & 30 & 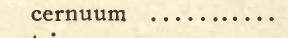 & 228 \\
\hline rinum $L \ldots . .$. & & $O C$ & & - & \\
\hline irinum Wang.. & & cens $\ldots$ & 30 & • & \\
\hline 1arum $\quad \ldots \cdots \cdots$ & 300 & 0.00 & 30 & $\ldots \ldots \ldots$ & 240 \\
\hline ........ & & 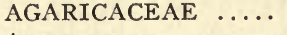 & & (n) & \\
\hline CEAE $\cdots$ & & $s \quad \ldots \ldots \ldots \ldots$ & 13 & $a \ldots \ldots \ldots \ldots$ & 240 \\
\hline s $\ldots \ldots \ldots$ & 3 & is $\ldots \ldots \ldots \ldots$ & 13 & $a \cdots \cdots \cdots \cdots$ & 240 \\
\hline . & 3 & ster $\ldots$ & 13 & lata & 24 \\
\hline$m$ & 385 & s $\ldots \ldots \ldots$ & 12 & $s \ldots$ & 40 \\
\hline $\operatorname{lium} \ldots \ldots \ldots$ & 38 & us $\ldots \ldots \ldots$ & 131 & $E \ldots \ldots \ldots \ldots$ & 250 \\
\hline HES $\ldots \ldots \ldots$ & 2 & $\operatorname{ces} \ldots \ldots \ldots$ & 13 & n........... & 250 \\
\hline lata $\ldots \ldots \ldots$ & 29 & i $\quad \ldots \ldots \ldots$ & 13 & olia & 250 \\
\hline VTHES $\ldots \ldots \ldots$ & 2 & us $\ldots$ & 13 & $\ldots \ldots$ & 250 \\
\hline ia $\quad \ldots \ldots \ldots \ldots$ & 36 & $\cdots$ & 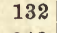 & is.. & 50 \\
\hline $\mathrm{UM} \quad \cdots \cdots \cdots \cdots$ & 2 & E $\quad \cdots$ & 342 & • & \\
\hline $\operatorname{tum} \ldots \ldots$ & 2 & $s$. & 342 & .. & \\
\hline tum $\ldots \ldots \ldots$ & & . & 342 & Altr & 303 \\
\hline$\ldots \ldots \ldots \ldots$ & 224 & IA $\quad \cdots \cdots \cdots$ & 277 & a $\ldots$ & \\
\hline us $\ldots \ldots \ldots \ldots$. & 2 & ana $\ldots \ldots$ & 278 & $A \ldots \ldots$ & 11 \\
\hline$N A E \quad \ldots \ldots$ & 1 & ria...... & 277 & rea $\ldots$ & 12 \\
\hline$O G Y N A E \quad \ldots$ & 164 & epala...... & 27 & $a \quad \ldots \ldots \ldots \ldots$ & 12 \\
\hline EA $\quad \cdots \cdots \cdots \cdots \cdots$ & 2 & ta $\ldots \ldots \ldots \ldots$ & 277 & $s a \ldots \ldots \ldots$ & 121 \\
\hline alba $\ldots \ldots \ldots \ldots \ldots$ & 255 & $\mathrm{mc}$ & 27 & rubens & 11 \\
\hline$\cdots$ & & lora.... & 278 & ii $\quad \ldots \ldots \ldots \ldots$ & 120 \\
\hline ta rubra.... & 2 & 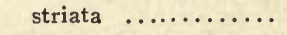 & 27 & caria & 119 \\
\hline MERIS & 385 & RON $\ldots \ldots \ldots$ & 215 & $\ldots \ldots$ & 119 \\
\hline folia.... & & ... & & des .. & 12 \\
\hline NEMA & 6 & MMA & 249 & ramis & 119 \\
\hline 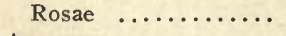 & 65 & o $\ldots \ldots \ldots$ & 249 & is $\ldots \ldots \ldots$. & 120 \\
\hline$\cdots \ldots \ldots$ & & $s \quad \ldots \ldots$ & 2 & 12. & 120 \\
\hline$\ldots \ldots \ldots$ & 2 & & 21 & & 12 \\
\hline
\end{tabular}




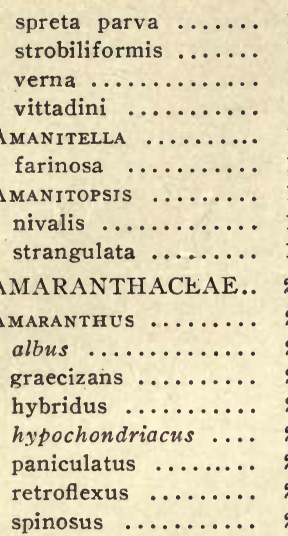

AMARYLLIDACEAE. 232

Амвцумтеgium $\ldots \ldots \ldots \quad 185$

adnatum ......... 185

fluviatile ......... 186

irreguum ......... 186

juratskanum ....... 186

orthocladon ....... 186

radicale .......... 186

riparium ......... 186

serpens $\ldots \ldots \ldots \ldots .186$

serpens orthocladon 186

varium ........... 186

Ambrosia ......... 369

artemisiaefolia ..... 369

bidentata ......... 369

trifida $\ldots . \ldots \ldots \ldots .369$

trifida integrifolia $\ldots \quad 369$

AMBROSIACE.AE ....

AMELANCHIER .........

Botryapium ........

Canadensis .........

Canadensis oblongi-

folia ............

Amianthium .........2226

muscaetoxicum ......

Ampelopsis ..........

cordata ...........

quinquefolia .......

tricuspidata ........

AmpHICARPA ........

monoica ...........

AMPHISPHAERIA ......

pinicola ...........

Amygdalus ..........

Persica ......... 282

Anacamptodon $\ldots . . .183$

apiculatus $\ldots \ldots \ldots \ldots 183$

splachnoides $\ldots \ldots$.

ANACARDIACEAE . . \begin{tabular}{r|}
20 \\
120
\end{tabular}

119

20

21

21.

21

248

48

48

48

48

48

8

Canadensis $\ldots \ldots \ldots$
cylindrica $\ldots \ldots \ldots$

nemorosa .........

Pennsylvanica ......

quinquefolia .......

trifolia ...........

Virginianz $\ldots \ldots \ldots$.

Aneura...$\ldots \ldots \ldots .164$

latifolia .......... 164

Angelica $\ldots \ldots \ldots \ldots .315$

atropurpurea ...... $3: 5$

Curtisii ......... 315

hirsuta ......... 315

villosa $\ldots \ldots \ldots \ldots \ldots .315$

Anomodon $\ldots \ldots \ldots \ldots .185$

attenuatus $\ldots \ldots \ldots .185$

obtusifolius ....... 185

rostratus ........ 185

ANONACEAE $\ldots \ldots . .253$

Antennaria ....... 378

fallax ......... 378

neodioica ......... 378

Parlinii .......... 378

plantaginifolia ..... 378

propinqua $\ldots \ldots \ldots \ldots \quad 378$

solitaria ......... 378

ANтнемis ......... 385

arvensis ........ 385

Cotula ......... 385

ANTHOCERATA-

CEAE $\ldots \ldots \ldots \ldots 171$

Anthoceros $\ldots \ldots \ldots .172$

laevis $\ldots \ldots \ldots \ldots \ldots 172$

punctatus ....... 172

Anthostoma $\ldots . . .999$ discincola ....... 90 microecium ....... 93

microplacum $\ldots \ldots .99$

183 Anthoxanthum $\ldots . .209$

296 odoratum ........ 209
Anychia .......... 251

Canadensis ....... 251

capillacea ......... 251

dichotoma ........ 251

divaricata ........ 251

polygonoides ...... 251

ApHANORRHEGMA $\ldots \ldots 178$

serratum $\ldots \ldots \ldots \ldots .178$

APHYLLON $\ldots \ldots \ldots \ldots .355$

uniflorum $\ldots \ldots \ldots .355$

APIACEAE $\ldots \ldots \ldots .315$

ApIos ............ 290

Apios .......... 290

tuberosa ......... 290

APISPORIUM $\ldots \ldots \ldots .89$

pelliculosum ..... 89

Aplectrum $\ldots \ldots \ldots \ldots .236$

hyemale ......... 236

spicatum ........ 236

Aplosia $\ldots \ldots \ldots \ldots .165$

autumnale ........ 165

APOCYNACEAE $\ldots 331$

APOCYNUM $\ldots \ldots \ldots \ldots 331$

album $\ldots \ldots \ldots \ldots .331$

androsaemifolium .. 331

cannabinum ....... 331

pubescens ........ 331

Aponectria $\ldots \ldots \ldots \ldots 104$

inaurata $\ldots \ldots \ldots \ldots .104$

Aposphaeria $\ldots \ldots \ldots .56$

pezizoides $\ldots \ldots \ldots \ldots \quad 56$

AQUIFOLIACEAE $\quad . \quad 298$

AQuillegia $\ldots \ldots \ldots .255$

Canadensis ....... 255

Arabis ........... 267

Canadensis ....... 267

laevigata ........ $26 \%$

lyrata .......... 267

patena .......... 267

ARACEAE $\ldots \ldots \ldots \ldots 223$

Aralia ............ 314

hispida $\ldots \ldots \ldots \ldots \ldots \quad 314$

nudicaulis $\ldots \ldots \ldots .314$

racemosa $\ldots \ldots \ldots \ldots .314$

spinosa .......... 314

trifolia $\ldots \ldots \ldots \ldots \ldots 314$

ARALIACEAE $\ldots \ldots .314$

ARchangelica ...... 315

atropurpurea ...... 315

Archilejeunea ...... 171

calypeata ......... 171

ArCtium ......... 387

Lappa ......... 387

minus .......... 387

Arcyria $\ldots \ldots \ldots \ldots . \quad 80$

cinerea $\ldots \ldots \ldots \ldots .30$

nutans $\ldots \ldots \ldots \ldots \ldots .31$

punicea ......... 30 

serpyllifolia .......

Arisaema...$\ldots \ldots \ldots$

Dracontium .......

triphyllum .......

Aristida $\quad \ldots \ldots \ldots \ldots$.

dichotoma ......... gracilis ...........

lanata $. . . . \ldots \ldots .$.

lanosa ............

oligantha .........

Aristolochia ........

macrophylla ......

serpentaria...$\ldots \ldots$

Sipho ............

ARISTOLOCHIACEAE.

ARMILLARIA $\ldots \ldots \ldots$. Mellea ...........

Aronia ............. arbutifolia...$\ldots \ldots$

nigra...$\ldots \ldots \ldots$

Arrhenantherum $\ldots$... avenaceum ........

elatius ............

Artemisia $\ldots \ldots \ldots \ldots$

annua...$\ldots \ldots \ldots$

Arthonia ...........

aleuromela......

astroidea..$\ldots \ldots \ldots$

cinnabarina...$\ldots$.

dispersa ..........

punctiformis ......

spectabilis..$\ldots \ldots \ldots$

taediosa ..........

Aruncus ...........

Aruncus .........

Asarum $\ldots \ldots \ldots \ldots$.

Canadense ........

Shuttleworthii .....

Virginicum $\ldots \ldots \ldots$

Aschersonia ........

ASCLEPIADACEAE. .

Asclepias .........

amplexicaulis .....

Cornuti ...........

exaltata ...........

incarnata .........

obtusifolia ........

phytolaccoides ......

pulchra ...........

purpurascens ......

quadrifolia ........

rubra $. . . \ldots \ldots \ldots .$.

Syriaca ......... 332

tuberosa ......... 331

variegata $\ldots \ldots \ldots \ldots \quad 332$

ASCOBOLACEAE .... 114

211

211

211

386

386

161

161

161

161

161

161

161

161

273

273

244

244

244

244

70

331

331

332

332

332

332

332

332

332

332

331

32
251 Ascoвolus ...........

251 carbonarius ....... 114

223 furfuraceus ....... 114

223 Ascochyта ........ 65

223 Clematidina ...... 65

209 Ascophanus ........ 114

209 carneus .......... 115

209 granuliformis ..... 114

209 Ascyrum .......... 304

209 Crux-Andreae ..... 304

209 hypericoides ...... 304

245 Asimina $\ldots \ldots \ldots \ldots \ldots 253$

245 triloba ......... 253

245 Asparagus ........ 229

245 officinalis ....... 229

Aspergillus ........ 32

flavus ........... 33

glaucus ......... 32

glaucus oblongisporus. $\quad 32$

279 Aspidium .......... 194

280 acrostichoides ...... 195 acrostichoides

Schweinitzii $\ldots \ldots .195$

cristatum ........ 195

Filix-mas ....... 195

fragrans ......... 194

Goldieanum ....... 195

marginale ......... 195

Novaeboracense ..... 194

spinulosum ........ 194

spinulosum interme-

dium ...........

spinulosum dilatatum. 195

Thelypteris ...... 194

Asplenium $\ldots \ldots \ldots .192$

angustifolium ...... 193

ebenum ......... 193

Filix-foemina ...... 193

montanum ........ 193

pinnatifidum ...... 192

platyneuron ....... 193

thelypteroides ...... 193

Trichomanes ....... 193

Trichomanes incisum 193

Asprella $\ldots \ldots \ldots \ldots 216$

Hystrix $\ldots \ldots \ldots \ldots 216$

Aster $\ldots \ldots \ldots \ldots \ldots \quad 375$

acuminatus ....... 377

Claytoni ......... 375

cordifolius ....... 375

corymbosus ....... 376

depauperatus pusillus 376

diffusus .......... 376

divaricatus $\ldots \ldots \ldots .376$

dumosus $\ldots \ldots \ldots \ldots 376$

ericoides ........ 376

ericoides depauperatus ericoides pilosus.... 376

ericoides pusillus.... 376

ericoides villosus.... 376

Faxoni ........ 376

hirsuticaulis ...... 376

infirmus ....... 378

laevis .......... 375

lateriflorus $\ldots \ldots \ldots \ldots \quad 376$

linariifolius ...... 378

Lowrieanus ...... 375

Lowrieanus lancifol-

ius ........... 375

macrophyllus ..... 375

miser $\ldots \ldots \ldots \ldots \ldots 376$

multiflorus ....... 376

Novae-Angliae ..... 377

Novi-Belgii ....... 377

paniculatus ....... 377

patens $\ldots \ldots \ldots \ldots \ldots \quad 375$

phlogifolius ...... 375

polyphyllus $\ldots \ldots \ldots \ldots \quad 376$

prenanthoides $\ldots \ldots .377$

puniceus ........ 377

puniceus firmus.... 377

purpuratus ...... 376

sagittifolius $\ldots \ldots \ldots .375$

salicifolius ...... 377

Shortii ........ 377

simplex ........ 377

Tradescanti ...... 376

umbellatus ........ 378

undulatus ....... 375

vimineus $\ldots \ldots \ldots \ldots 37 \hat{n}$

vimineus foliosus.... 376

virgatus ......... 376

Asterina .......... 89

LeEmingir $\ldots \ldots \ldots . \quad 89$

Asterostoma ........ 148

cervicolor ......... 148

corticolum ........ 148

Astilbe .......... 269

biternata ......... 269

decandra ........ 269

Astragalus ........ 287

Canadensis ........ 287

Carolinianus ...... 287

distortus ........ 287

ASTROPHYLLUM $\ldots \ldots \ldots 180$

hornum ......... 180

punctatum ........ 180

rostratum ......... 180

sylvaticum ....... 180

Athyrium $\ldots \ldots \ldots \ldots .193$

Filix-foemina ..... 193

Filix-foemina pe-

ctinatum ...... 193

thelypteroides ..... 193 
Atragene ..........

Americana ........

ATRICHUM ......... 182

angustatum ....... 182

undulatum ........ 182

AULACOMNIACEAE. 180

Aulacomnium ....... 180

heterostichum ..... 180

Aurantiporus $\ldots \ldots \ldots .136$

Pilotae .......... 136

Auricularia $\ldots \ldots \ldots \ldots \quad 149$

Auricula ...........

Avena ..............

fatua $\ldots \ldots \ldots \ldots \ldots$.

Azalea $\ldots \ldots \ldots \ldots \ldots$

arborescens ........

canescens ${ }^{\circ} . \ldots \ldots \ldots$.

lutea .............

nudiflora $\ldots \ldots \ldots \ldots$.

viscosa $\ldots \ldots \ldots \ldots \ldots$

viscosa glauca.....

viscosa nitida ......

Baccillus .......... acidi-1actici...$\ldots \ldots$. amylivorus $\ldots \ldots \ldots$. subtilis $\ldots \ldots \ldots \ldots$. tuberculosis ........ ulna $\ldots \ldots \ldots \ldots \ldots$

Bacterium ..........

Lineola ............

Termo ............

Bacteridium ........ flavum ...........

Baeomyces ......... aeruginosus ........ roseus $\ldots \ldots \ldots \ldots$.

BALSAMINACEAE ..

Baptisia .......... australis $\ldots \ldots \ldots \ldots$. tinctoria ........... villosa $\ldots \ldots \ldots \ldots$.

Barbarea $\ldots \ldots \ldots \ldots$

Barbarea ..........

praecox ...........

vulgaris.$\ldots \ldots \ldots$.

BarbUla $\ldots \ldots \ldots \ldots \ldots 177$

caespitosa ........ 177

humilis ......... 177

muralis ......... 177

tortuosa ......... 177

unguiculata $\ldots \ldots \ldots \quad 177$

BARTONIA ......... 330

Virginica ........ 330

BARTRAMIA ......... 180

pomiformis ....... 180

pomiformis crispa..., 181

BARTRAMIACEAE .., 180
Bazzania ......... 168

deflexa .......... 168

triangularis ....... 168

trilobata ........ 168

Bellincinia ....... 170

platyphylla ....... 170

Porella ......... 170

Bellis .......... 375

perennis $\ldots \ldots \ldots \ldots 375$

Belonidium $\ldots \ldots \ldots . . . . .114$

aurelia $\ldots \ldots \ldots \ldots .114$

Benzoin $\ldots \ldots \ldots \ldots .261$

Benzoin ........ 261

BERBERIDACEAE .. 260

Berberis ......... 260

Canadensis $\ldots \ldots \ldots 260$

vulgaris $\ldots \ldots \ldots \ldots$.

Betula ......... 239

lenta $\ldots \ldots \ldots \ldots \ldots 239$

lenta X lucida..... 239

lutea .......... 240

nigra $\ldots \ldots \ldots \ldots \ldots 240$

populifolia $\ldots \ldots \ldots .240$

BETULACEAE $\ldots \ldots .239$

Biatora ......... 158

atrogrisea $\ldots \ldots \ldots \ldots \quad 159$

fuliginosa $\ldots \ldots \ldots \ldots 159$

granulosa $\ldots \ldots \ldots \ldots \quad 159$

hypnophila $\ldots \ldots \ldots .159$

inundata $\ldots \ldots \ldots \ldots \quad 1.59$

Laureri ........ 159

rubella $\ldots \ldots \ldots \ldots \ldots 159$

russula $\ldots \ldots \ldots \ldots .158$

Russellii ....... 159

Schweinitzii $\ldots \ldots \ldots 159$

suffusa $\ldots \ldots \ldots \ldots \ldots \quad 159$

uliginosa $\ldots \ldots \ldots \ldots \quad 159$

varians $\ldots \ldots \ldots \ldots .159$

vernalis $\ldots \ldots \ldots \ldots .159$

Bicuculla $\ldots \ldots \ldots \ldots 262$

Canadensis ...... 262

Cucullaria ....... 26,2

eximina ........ 262

Bidens .......... 384

bipinnata $\ldots \ldots \ldots .384$

cernua ......... 384

chrysanthemoides ... 384

comosa ......... 384

connata ........ 384

frondosa L........ 384

frondosa Torr...... 384

laevis .......... 384

melanocarpa ...... 384

trichosperma $\ldots \ldots \ldots 384$

Bignonia .......... 356

capreolatal $\ldots \ldots \ldots .356$

erucigera ........ 356

BIGNONIACEAE ... 355
BJERKANDERA $\ldots \ldots \ldots \quad 135$

adusta $\ldots \ldots \ldots \ldots .135$

Blephariglottis $\ldots . .234$

ciliaris ......... 234

lacera ........... 234

peramoena ....... 234

psycodes ........ 234

Blepharostoma ..... 165

trichophyllum $\ldots \ldots . \quad 165$

Blephilia $\ldots \ldots \ldots \ldots .344$

ciliata $\ldots \ldots \ldots \ldots .345$

hirsuta ......... 344

Boehmeria $\ldots \ldots \ldots \ldots 243$

cylindrica ........ 243

cylindrica scabra.... 243

Boletus .......... 133

affinis $\ldots \ldots \ldots \ldots \ldots 134$

auriflammeus ..... 133

bicolor ........... 134

chromapes ........ 133

chrysentron ....... 134

cyanescens ....... 133

edulis .......... 134

eximius ......... 134

gracilis ........ 133

nigrellus ........ 133

parasiticus ........ 134

Ravenelii ........ 134

separans ......... 134

speciosus ........ 134

Bombardia $\ldots \ldots \ldots \ldots 9$

fasciculata ....... 93

BORAGINACEAE $\ldots 337$

BotrychIUM ....... 190

Matricariae ........ 190

obliquum ........ 190

silaifolium $\ldots \ldots \ldots . \quad 190$

ternatum ........ 190

ternatum australe... 190

Virginianum ...... 190

BotRyodiplodia ..... 65

Acerina ......... 65

BotryosphaERIA $\quad \ldots . .96$

Araliae ......... 97

fuliginosa $\ldots \ldots \ldots .63$

Hibisci ......... 97

pyriospora $\ldots \ldots \ldots .97$

Quercuum ........ 96

BOTRYOSPORIUM $\ldots \ldots .32$

pulchrum $\ldots \ldots \ldots . \quad 32$

Botrytis ......... 33

olivacea $\ldots \ldots \ldots \ldots \quad 33$

torta $\ldots \ldots \ldots \ldots \ldots .33$

vulgaris $\ldots \ldots \ldots \ldots .33$

Bovista .......... 25

lepidophora $\ldots \ldots \ldots$ 25

pila $\ldots \ldots \ldots \ldots \ldots 25$ 
Bovistella ........ 25

Ohioense ........ 25

BoYKINIA .......... 269

aconitifolia ....... 269

Brachychaeta ....... 374

cordata .......... 374

sphacelata $\ldots . \ldots . .374$

Brachyelytrum ..... 210

aristatum ......... 210

erectum ......... 210

erectum glabratum.. 210

BRACHYTHECIACEAE

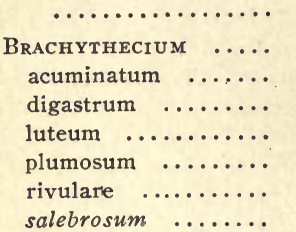

Brassica ............

arvensis $\ldots \ldots \ldots \ldots$.

nigra $\ldots . \ldots \ldots \ldots$

Sinapistrum .......

BRASSICACEAE ....

Braunerta ........... purpurea........

BREMIA.........

Lactucae ..........

BRomus .............

ciliatus ......... 215

hordaceus ....... 215

intermis .......... 215

mollis '........... 215

racemosus ..........

secalinus $\ldots . . . \ldots$.

Broomelia .......... 105

Ravenelii ........ 105

Broussonetia ....... 243

papyrifera ....... 243

Brunelta .............

vulgaris ............

vulgaris albiflora....

BRYACEAE ........

BRYUM ............

argenteum ........

bimum ............

caespiticium ........

capillare ...........

intermedium ........

proliferum .........

pseudotriquetrum ....

pyriforme ..........

roseum ...........

Buchnera ...........

Americana ........

179

179

179

179

179

180

179

354
354

${ }^{B}$ B

Buellia $\ldots \ldots \ldots \ldots$
colludens $\ldots \ldots \ldots \ldots$
myriocarpa $\ldots \ldots \ldots$
petraea $\ldots \ldots \ldots$
. $\ldots \ldots$

Bulgaria .............

inquinans ........ 116

BULGARIACEAE $\ldots . . \quad 116$

Bursa ........... 266

Bursa-pastoris ..... 266

Butneria .......... 261

fertilis .......... 261

florida .......... 261

laevigatus ....... 261

BUXACEAE ........ 296

Buxbaumia ......... 181

aphylla .......... 181

BUXBAUMIACEAE... 181

Cacalia ............ 386

atriplicifolia ....... 386

reniformis ........ 386

suaveolens ........ 387

CACTACEAE ....... 309

Cakoma $\ldots \ldots \ldots \ldots \ldots .6 \%$

nitens $\ldots \ldots \ldots \ldots \ldots .86$

CAESALPINIACEAE.. 282

Calamagrostis ...... 211

cinnoides $\ldots . \ldots \ldots .211$

Calamintha $\ldots . . . .3 \%$

$\mathrm{C}$

Clinopodium .......

vulgare .......... 345

CALICIACEAE ..... 161

Calicium ............ 161

subtile ........... 161

tigillare $\ldots . \ldots \ldots \ldots, 161$

CALLITRICHACEAE. . 296

Callitriche ....... 296

heterophylla ....... 296

Cat.ocera ........... 149

viscosa $\ldots \ldots \ldots \ldots .6 \quad 149$

Calopogon .......... 236

pulchellus ......... 236

Calospora ........... 102

aculeans ......... 102

Rhoina ......... 102

Caltha .......... 254

palustris ........ 254

CALYCANTHACEAE. . 261

Calycanthus ....... 261 fertilis .............

floridus ............

Calypogeia $\ldots . . . \ldots$.

trichomanis .........

Camarosporium ......

Linderae ...........

Camassia $\ldots . . . \ldots \ldots$

Fraseri ............
Camelina $\ldots \ldots \ldots \ldots .266$

microcarpa ....... 266

sativa .......... 266

Campunula .......... 364

Americana ........ 364

aparinoides ........ 364

divaricata ........ 364

rotundifolia ....... 364

CAMPANULACEAE... 364

Camptosorus ......... 193

rhizophyllus ....... 193

Campylium .......... 186

chrysophyllum ..... 186

hispidulum ....... 189

tenellum ......... 186

Campylopus $\ldots \ldots \ldots \ldots \quad 175$

flexuousus ......... 175

Cannabis $\ldots \ldots \ldots \ldots \ldots .243$

sativa $\ldots \ldots \ldots \ldots .243$

Cantharellus $\ldots \ldots .126$

aurantiacus ....... 126

cibarius .......... 126

cinnabarinus $\ldots \ldots \ldots \quad 126$

floccosus $\ldots \ldots \ldots \ldots, 126$

infundibuliformis.... 126

minor ........... 126

tubaeformis ....... 126

Wrightii ......... 126

Capnotdes ........... 262

aureum .......... 263

flavulum ......... 263

sempervirens ...... 262

Capnodium ......... 89

pelliculosum ....... 89

CAPPARIDACEAE.... 267

CAPRIFOLIACEAE. . . 360

Capsella $\ldots \ldots \ldots \ldots .266$

Bursa-pastoris ..... 266

Cardamine ......... 265

bulbosa $\ldots \ldots \ldots \ldots .265$

hirsuta .......... 265

parviflora ......... 265

Pennsylvanica ..... 265

pratensis ......... $\quad 265$

purpurea ......... 265

rhomboidea ........ 265

rhomboidea purpurea. 265

rotundifolia ....... 265

CARDUACEAE ..... 370

CarduUs ............ 388

altissimus ........ 388

arvensis ......... 388

discolor ......... 388

lanceolatus ........ 388

Muticus .......... 388

odoratus ......... 388

Virginianus ....... 388 


\begin{tabular}{|c|c|c|c|c|}
\hline$\cdots$ & 19 & & 0 & IGELLA \\
\hline phibola $\ldots \ldots \ldots$ & 221 & $\cdots \cdots \cdots$ & 223 & Aceris $\quad \ldots \ldots \ldots \ldots$ \\
\hline -Grayi $\quad \ldots \ldots \ldots$ & 219 & uloides turbata... & 223 & Cenchrus $\ldots \ldots \ldots \ldots$ \\
\hline aileyi $\ldots \ldots \ldots \ldots$ & 220 & ps $\ldots$ & 220 & tribuloides $\quad \ldots \ldots \ldots$ \\
\hline lellii $\ldots \ldots \ldots$ & 223 & roides & 220 & Centaurea $\quad \ldots \ldots \ldots$ \\
\hline scens $\ldots \ldots \ldots$ & 223 & lata & $221>-2$ & nigra \\
\hline escens gracilior. & 223 & aria $\cdots$ & 221 & Cephalanthus $\ldots \ldots$ \\
\hline ens vulgar & 223 & sta $m$ & 221 & occidentalis $\ldots \ldots$ \\
\hline ophora $\ldots \ldots$ & 223 & cens & 220 & Cephalothecium $\quad \cdots .$. \\
\hline unis $\ldots \ldots \ldots$ & 222 & scens costata.... & 220 & $\cdots$ \\
\hline ata $\ldots \ldots \ldots$ & 220 & . & 22 & Cephalozia \\
\hline ta $\ldots \ldots \ldots \ldots$ & 220 & 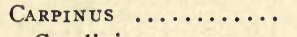 & 239 & olia $\ldots \ldots \ldots$...... \\
\hline ta $\mathrm{X}$ prasina... & 220 & . & 239 & $\ldots \ldots$ \\
\hline debilis Rudgei...... & 221 & $\ldots \ldots \ldots \ldots$ & 237 & ra $\ldots \ldots \ldots$ \\
\hline is $\ldots \ldots$ & 221 & $a l b a$ & 237 & $a \quad \ldots \ldots \ldots$ \\
\hline lata & & 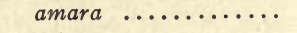 & 237 & na $\ldots .$. \\
\hline rii $\quad \cdots$ & & microcarpa $\quad \ldots \ldots \ldots$ & 237 & $J_{M} \quad \ldots \ldots \ldots \cdots$ \\
\hline i $\cdots$ & & porcina $\ldots \ldots \ldots$ & 237 & 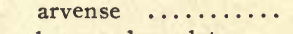 \\
\hline dea $\quad \ldots \ldots \ldots$ & & sulcata $\ldots \ldots \ldots \ldots$ & 237 & 10 \\
\hline ima $\ldots \ldots \cdots$ & 221 & ntosa $\quad \ldots \ldots$ & 237 & $\ldots \ldots \ldots$ \\
\hline $\begin{array}{l}\text { ularis } \ldots \\
\text { vii ...... }\end{array}$ & & CARYOPHYLIAACEAE & 249 & 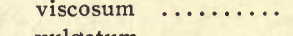 \\
\hline ii $\ldots$ angust & 221 & Not & 102 & $\cdots \cdots$ \\
\hline $\begin{array}{l}\text { stifolia... } \\
\text { na } \ldots . . .\end{array}$ & 221 & $\mathrm{um} \quad \ldots \ldots$ & 02 & $x_{A} \quad \cdots \cdots \cdots$ \\
\hline $\begin{array}{l}\text { cockiana } \ldots \ldots \\
\text { escens } \quad . . . \cdots\end{array}$ & 219 & 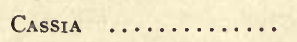 & & sa' \\
\hline $\begin{array}{l}\text { escens } \ldots \ldots \ldots \\
\text { ii } \quad \ldots \ldots \ldots \ldots\end{array}$ & 222 & aecrista $\ldots$ & 283 & CER \\
\hline $\lim _{\text {is }} \ldots \ldots \ldots \ldots$ & 221 & $\begin{array}{l}\text { laecrista } \\
\text { landica }\end{array}$ & 283 & $\ldots \ldots \ldots$ \\
\hline $\operatorname{mis} \ldots \ldots \ldots \ldots$ & 221 & $\begin{array}{l}\text { ndica } \ldots \\
\text { eri } \ldots \ldots\end{array}$ & 282 & $\ldots \ldots$ \\
\hline ra latifolia.... & 221 & eri & 28 & us $\ldots \ldots \ldots \ldots$ \\
\hline $\begin{array}{ll}\text { ora } & \text { latifolia.... } \\
\text { ora } & \text { patuliflora.. }\end{array}$ & & to & 283 & .......... \\
\hline $\begin{array}{ll}\text { pra } & \text { patu } \\
\text { ea } & \ldots\end{array}$ & & (5) & 3 & 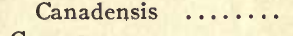 \\
\hline $\begin{array}{ll}\text { ea } & \ldots \\
\text { ina } & \ldots\end{array}$ & & $A \quad \ldots$. & 252 & $\ldots \ldots \ldots$ \\
\hline a. & & ta $\ldots \ldots \cdots$ & 252 & $\ldots \cdots \cdots$ \\
\hline ia var... & & EA $\quad \ldots \ldots \ldots$ & 240 & $\cdots \cdots \cdots$ \\
\hline $\begin{array}{l}\text { ia var... } \\
\text { ia Xalapen }\end{array}$ & & ta & 240 & dis $\ldots \ldots$ \\
\hline $\begin{array}{l}\text { ia Xalapen } \\
\ldots \ldots \ldots \ldots\end{array}$ & 223 & la $\quad \cdots \cdots \cdots$ & 240 & ntica $\ldots \ldots$ \\
\hline$\cdots \cdots \cdots \cdots$ & 221 & Americana. & 240 & ae $\quad \cdots \cdots \cdots$ \\
\hline pa $\ldots \ldots \ldots$ & 221 & EJA $\cdots \cdots \cdots \cdots$ & 354 & $\cdots \cdots$ \\
\hline $\begin{array}{ll}\text { pa } & \ldots \\
\text { ata } & \ldots\end{array}$ & 222 & ea & 354 & $\cdots \cdots$ \\
\hline lata $\ldots \ldots \ldots$ & 221 & $\because \ldots \ldots$ & 356 & ae $\quad \cdots \cdots$ \\
\hline $\begin{array}{l}\text { lvanica } \ldots \ldots \\
\text { tinea } \ldots \ldots \ldots\end{array}$ & 221 & ioides & 356 & li $\ldots \ldots \ldots$ \\
\hline $\begin{array}{l}\text { inea } \quad \ldots \ldots \ldots \\
\text { ylla } \ldots \ldots \ldots\end{array}$ & 221 & pa $\ldots . .$. & 356 & hi $\quad \ldots \ldots \ldots$ \\
\hline $\begin{array}{c}\text { ylla } \ldots . . . \\
\text { choides } \ldots \ldots\end{array}$ & 221 & sa $\ldots \ldots \ldots$ & 356 & $\cdots \cdots \cdots$ \\
\hline $\begin{array}{c}\text { choides } \ldots \ldots \\
\ldots \ldots \ldots\end{array}$ & 220 & NAEA & 182 & is $\ldots \ldots \cdots$ \\
\hline $\operatorname{exa} \ldots \ldots$ & 223 & ata & 182 & es $\ldots \ldots \ldots$ \\
\hline $\begin{array}{c}\operatorname{Hexa} \quad \ldots \ldots \ldots \\
\ldots \ldots \ldots\end{array}$ & 22 & 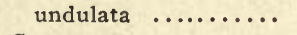 & 18 & $\cdots \cdots \cdots$ \\
\hline radiata........ & 222 & YLLUM. & 26 & $\because \ldots \cdots$ \\
\hline $\begin{array}{l}\text { radiata. } \\
\text { ata } \ldots . .\end{array}$ & 223 & Ch & 260 & rmis $\ldots$ \\
\hline ta & 223 & us $\ldots . .$. & 30 & $\ldots$ \\
\hline $\begin{array}{l}\text { ia } \ldots \ldots \\
\text { ana } \ldots\end{array}$ & 220 & canus $\ldots$ & 302 & ermi \\
\hline noides $\ldots \ldots \ldots$ & 223 & Cedronelia $\cdots$ & & $\ldots \ldots \ldots$ \\
\hline $\begin{array}{l}\text { noides } \ldots \\
\text { osa } \ldots .\end{array}$ & 220 & $\overline{1}$ & & erae $\ldots \ldots \ldots$ \\
\hline $\begin{array}{l}\text { osa } \\
\text { epis }\end{array}$ & 220 & $\mathrm{RAC}$ & & des $\ldots \ldots \ldots$ \\
\hline epis ... & 2 & $\ldots \ldots$ & 29 & $\ldots \ldots \ldots \ldots$ \\
\hline$\ddot{e a}$ & & $\begin{array}{l}\ldots \ldots \\
\ldots \ldots\end{array}$ & 29 & ides $\quad \ldots \ldots \ldots$ \\
\hline ea & & s $\ldots \ldots \ldots \ldots$ & 25 & 1a $\quad \ldots \ldots \ldots$ \\
\hline & $\begin{array}{l}220 \\
220\end{array}$ & $\ldots \ldots \ldots$ & 24 & s $\ldots \ldots \ldots \ldots$ \\
\hline$a$ & 22 & ntalis $\ldots$ & 24 & cae $\quad \ldots \ldots \ldots$ \\
\hline & 221 & & 2 & 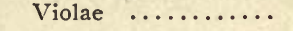 \\
\hline
\end{tabular}




\begin{tabular}{|c|c|c|c|c|c|}
\hline & 33 & $\mathrm{am}$ & 71 & & \\
\hline ... & 134 & n........ & 247 & vulgaris & \\
\hline auriflammeus $\quad \ldots \ldots$ & 33 & urbicum $\ldots \ldots \ldots$ & 247 & Ladonia $\ldots$ & 57 \\
\hline ......... & 34 & HILOCYPHUS $\ldots \ldots \ldots$ & 166 & piticia. & \\
\hline$\ldots \ldots$ & 33 & ens $\ldots . . . \cdots$ & 166 & sa $\ldots$ & 157 \\
\hline nis $\ldots \ldots \ldots$. & 34 & hus...... & 166 & ra pleur & 157 \\
\hline ........... & 4 & ILA $\ldots$ & 321 & ella $\ldots$. & 158 \\
\hline is separans.... & 34 & $\cdots$ & 322 & is $\ldots$ & 57 \\
\hline n......... & 4 & ata $\ldots$ & 21 & 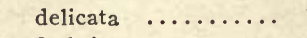 & \\
\hline us $\ldots \ldots \ldots$ & 4 & s $\ldots .$. & 20 & a . & \\
\hline$\ldots \ldots \ldots$ & 4 & a $\ldots \ldots \ldots . .$. & 326 & ta tub & 57. \\
\hline$\ldots \ldots \ldots \ldots$ & 140 & Hus $\ldots \ldots \ldots$ & 329 & a racer & 58 \\
\hline$\ldots \ldots \ldots \ldots$ & 140 & $\ldots \ldots \ldots$ & 329 & 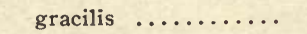 & 157 \\
\hline$\ldots \ldots \ldots \ldots$ & 151 & ENIUM $\ldots$ & & $s$ verti & 157 \\
\hline$s \ldots \ldots \ldots$ & 151 & um $\ldots$. & 112 & ta $\ldots$. & 158 \\
\hline ........... & 15 & tzii $\ldots .$. & 1 & ..... & \\
\hline ........... & 151 & ne & 11 & a $\ldots$ & \\
\hline$\ldots \ldots \ldots \ldots$ & 151 & A $\cdots$ & 3 & a epip & 157 \\
\hline a spinulosa. & 151 & ...... & 36 & a $\ldots$. & 58 \\
\hline LUM & 318 & & 22 & 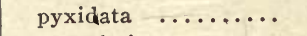 & 57 \\
\hline is $\ldots \ldots$. & & icum $\ldots$ & 2 & na & 158 \\
\hline LOA $\ldots \ldots \ldots$ & 208 & EMUM .. & 3 & ens $\ldots$. & 158 \\
\hline$\ldots \ldots .$. & 2 & mum $\ldots$ & 385 & a $\ldots$. & 158 \\
\hline n........ & 208 & $\mathbf{M} \ldots \ldots$ & 380 & denticollis.. & 158 \\
\hline$\ldots \ldots \ldots \ldots$ & 20 & $\mathrm{~m} \quad \ldots \ldots$ & 3 & $\ldots \ldots$ & 57 \\
\hline . & 208 & N $\ldots . .$. & 205 & a $\ldots$ & 157 \\
\hline RISTA & 283 & eum $\ldots . .$. & 205 & laxiu & 157 \\
\hline iixta.. & 2 & .......... & Noe & is $\ldots$. & 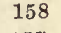 \\
\hline OM $\quad \ldots$ & 2 & s $\ldots \ldots$. & & $\cdots \cdots$ & 57 \\
\hline 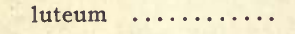 & 2 & .......... & & $\ldots \ldots$ & 158 \\
\hline .... & 3 & ENIUM . & ${ }^{9}$ & ata & 157 \\
\hline 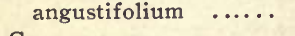 & 3 & aum $\ldots$. & 1 & ACEAE $\ldots$ & 157 \\
\hline ma & 29 & & & $M \quad \ldots \ldots$ & 36 \\
\hline 年 & 294 & EAE & 366 & chum $\ldots$ & \\
\hline$\ldots \ldots \ldots$ & 4 & $\cdots$ & $\begin{array}{l}300 \\
366\end{array}$ & $\ldots \ldots \ldots$ & 36 \\
\hline$\ldots \ldots \ldots \ldots$ & & $\cdots$ & $36 t$ & $\operatorname{lm} \ldots$. & 36 \\
\hline HES $\ldots \ldots \ldots$ & 192 & $\cdots$ & $\begin{array}{l}30 \mathrm{c} \\
31\end{array}$ & $m \quad \ldots$. & 36 \\
\hline$\ldots \ldots \ldots \ldots$ & 19 & $\begin{array}{l}\cdots \\
\cdots\end{array}$ & 31 & im fasciculare & 36 \\
\hline .......... & 192 & . & 319 & $m \quad \ldots . \cdots$ & \\
\hline $\cos a \ldots . .$. & 192 & A $\quad \ldots \ldots$ & 255 & $\ldots \ldots$ & 66 \\
\hline t & 192 & na $\cdots$ & 25 & PORIUM & 37 \\
\hline & & sana & 25 & $\ldots \ldots$ & 37 \\
\hline UNEA $\ldots .$. & 170 & a...... & 210 & um $\ldots$ & 37 \\
\hline$\ldots \ldots$ & 170 & nacea $\ldots$ & 210 & ........ & 130 \\
\hline IUM & 262 & $\begin{array}{l}\text { inacea } \\
\text { ia } \quad \ldots \ldots \ldots\end{array}$ & 21 & us $\ldots$. & 130 \\
\hline & 262 & $\begin{array}{l}\text { ula } \\
\text { ula }\end{array}$ & 21 & $\cdots$ & 30 \\
\hline ZHELONB & 351 & $A^{n} \quad \ldots \ldots \ldots \ldots$ & 31 & Le & 148 \\
\hline$\ldots \ldots$ & 35 & $\cdots \cdots \cdots$ & 31 & na & 14 \\
\hline$\cdots \cdots$ & & a ..... & 31 & & \\
\hline ODIACEAE & 2 & Ina $\ldots$. & & . & \\
\hline IUM & 247 & LLUM $\ldots$. & 19 & mis . & \\
\hline & & ..... & 38 & & \\
\hline & 24 & & & & \\
\hline & 247 & $\cdots \cdots$ & 3 & & \\
\hline intic & 247 & $\mathrm{AE}$ & 3 & LACEAE & 148 \\
\hline & 247 & $\begin{array}{l}\text { TACEAE } \ldots \ldots \\
\text { RULLUS } \ldots \ldots\end{array}$ & $\begin{array}{l}306 \\
363\end{array}$ & & \\
\hline & & & & Oliniana & \\
\hline
\end{tabular}




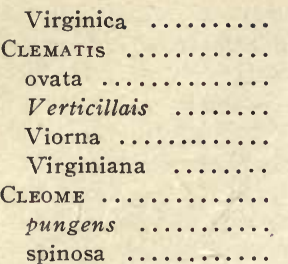

Clethra........... acuminata .......... CLETHRACEAE .... CLIMACIACEAE .... Climacium $\ldots . . . \ldots$. Americanum ....... Americanum Kindbergii ...........

Clinopodium ......... Calamintha ......... vulgare $\ldots . . . \ldots . .$.

Clintonia $\ldots . . . . . .$. borealis ........... umbellulata ........

Clitocybe ........... candicans $\ldots . . . .$. . cerussata .......... cyathiformis ....... dealbata ........... illudens ............ infundibuliformis ... laccata ............ nebularis ........... odora ..............

Clitopilus ........... abortivus ........... orcella ............ prunulus ...........

Coenogonium ........ interpositum .......

Coleosporium ........ Campanulae ........ Helianthi .......... Ipomoeae........ Senecionis ......... Soliđaginis ......... Sonchi ............ Vernoniae ......... Collema........... flaccidum .......... myochroum ........

COLlEMACEAE ....

Colletotrichum $\ldots . .$. lagenarium ......... Lindemuthianum .... lineola

Collinsia......... verna
249
Collinsonia .

Canadensis ........

Collybia ..............

butyracea ..........

cirrhata ...........

confluens ...........

conigenoides ........

dryophila .........

maculata ..........

platyphylla .........

radicata...$\cdots \cdots$.

stipitaria $. . . \ldots \ldots .$.

velutipes........

Collybidium $\ldots . . . .$.

velutipes $\ldots . . . \ldots$.

Collybiopsis ......... ramealis ..........

Cololejeunea......

Biddlecomiae ......

Comatricha $\ldots . . . .$.

Friesiana ..........

Commelina........

Virginica ..........

COMMELINACEAE ..

COMPOSITAE .......

Comptonia $\ldots . . . . .$.

peregrina.......

Cóntophora $\ldots . . .$.

olivacea ............

Coniosporium ........

Harknesioides ......

Coniothyrium ........

concentricum .......

Fuckelii ............

Pirina ............

Conocephalum .......

conicum ...........

Conocephalus ........

conicus .......... 163

Conocybe .......... 130

hypnorum ......... 130

tener ............ 130

CoNOPHOLIS ........ 355

Americana ........ 355

Convallaria $\ldots \ldots \ldots .231$

majalis .......... 231

CONVALLARIACEAE 229

CONVOLVULACEAE. 333

Convolvulus ......... 334

repens..$\ldots \ldots \ldots \ldots .334$

Sepium .......... 334

spithameus $\ldots \ldots \ldots .334$

Coprinus ........... 132

angulatus $\ldots \ldots \ldots \ldots \quad 133$

domesticus ........ 132

ephemerus ........ 133

micaceus.$\ldots \ldots \ldots .132$ semilanatus $\ldots \ldots \ldots \quad 133$

variegatus $\ldots \ldots \ldots \quad 132$

Coptrs ............ 254

trifolia $\ldots \ldots \ldots \ldots .254$

Corallorhiza $\ldots \ldots \ldots .236$

Corallorhiza ....... 236

innata .......... 236

multiflora ........ 236

odontorrhiza $\ldots \ldots \ldots \quad 236$

CoRDYCEPS .......... 105

militaris ......... 105

ophioglossoides .... 106

parasitica ........ 106

Corema $\ldots \ldots \ldots \ldots .296$

Conradi .......... 296

Coreopsis ......... 383

auriculata ........ 383

lanceolata villosa.... 383

major ........... 383

major stellata ...... 383

pubescens ......... 383

senifolia ......... 383

stellata $\ldots \ldots \ldots \ldots . \quad 383$

trichosperma $\ldots . \ldots . \quad 384$

tripteris ......... 384

Coriolellus ........ 139

Sepium .......... 139

Coriolus ........... 138

abietinus ......... 138

nigromarginatus .... 139

prolificans ........ 138

pubescens ........ 138

veriscolor ........ 138

CORNACEAE $\ldots \ldots \ldots \quad 320$

Cornus ............. 320

alternifolia ........ 320

Amomum ......... 320

Canadensis ........ 320

candidissima ..... 320

circinata ......... 320

florida ......... 320

paniculata ........ 320

sericea .......... 320

stolonifera ........ 320

Corticium $\ldots \ldots \ldots \ldots .146$

$\begin{array}{lll}\text { alboflavescens } & \ldots \ldots & 146\end{array}$

$\begin{array}{lll}\text { arachnoideum } \ldots \ldots & 146\end{array}$

colliculosum ....... 147

globiferum ........ 146

incarnatum ....... 147

leptaleum ........ 147

leve $\ldots \ldots \ldots \ldots \ldots, 146$

lilacino-fuscum $\ldots \ldots \quad 147$

lilacinum $\ldots, \ldots \ldots \quad 147$

Martianum ........ 147

Oakes:i ......... 146

$\begin{array}{llll}\text { ochraceum } & \ldots \ldots \ldots & 147\end{array}$ 


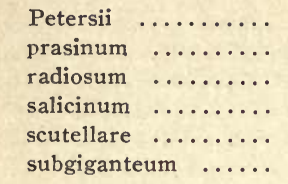

Cortinarius .........

armihatus .........

caerulescens ........

cinnamomeus .......

pholideus ..........

purpurascens .......

rodes $\ldots \ldots \ldots \ldots \ldots$

torvus ............

violaceus ...........

Corylus ........... 239

Americana .........

rostrata ...........

Coryne ..............

sarcoides

urnalis ............

Coryneum $\quad . . . \ldots \ldots$

cupulatum ...........

pustulatum .........

Cotinus .............

cotinoides ..........

Cracca $\ldots . . . \cdots \cdots \cdot$

Virginiana......

CRASSUlaceaE ....

Crataegus ...........

amara ............

apiifolia

Brownii

coccinea ...........

coccinea mollis.....

cordata ............

Crus-galli ..........

flava .............

foetida .............

Holmesiana .......

macrantha .........

macrosperma forma .

Margaretta .........

mollis ............

Oxyacantha ........

parviflora ..........

pausiaca ...........

pruniosa ..........

punctata ...........

Roanensis ..........

rotundifolia ........

spathulata ..........

straminea ..........

succulenta ..........

tomentosa .........

uniflora ...........

Craterocolla ........
147 clavus ........... 149

Creonectria ........ 103

coccinea ......... 104

purpurea ......... 103

Crepidotus ......... 130

applanatus $\ldots \ldots \ldots .131$

calotepis ......... 131

fulvotomentosus .... 131

herbarum ........ 131

malachius ......... 131

putrigenus $\ldots \ldots \ldots \ldots, 131$

versutus .......... 131

CREPIS ............. 368

capillaris ......... 368

Cribaria ............ 30

aurantiaca ......... 30

Crinipellus $\ldots \ldots \ldots .122$

stipitaria ......... 122

Crucibulum ......... 24

vulgare $\ldots \ldots \ldots \ldots .24$

CRUCIFERAE ..... 263

Cryptosporella $\ldots \ldots . \quad 97$

compacta $\ldots . \ldots \ldots .97$

CRYPTOSPORA .......... 103

cinctula .......... 103

femoralis ........ 103

trichospora $\ldots \ldots \ldots \quad 103$

Cryptotaenia ........ 319

Canadensis ........ 319

Cubelium ........... 309

concolor ......... 309

Cucumis ............ 363

Melo ............. 363

Cucurbita $\ldots \ldots \ldots \ldots .363$

ovifera .......... 363

CUCURBITACEAE .. 363

Cúcurbitaria ....... 103

elongata $\ldots \ldots \ldots \ldots, 103$

Fraxini ......... 103

Cunila ............. 346

Mariana ......... 346

origanoides $\ldots \ldots \ldots \ldots \quad 346$

Cuphaea ........... 310

viscosissima ........

Cuscuta .............

arvensis........

Epithymum .........

glomerata .........

Gronovii ..........

paradoxa ...........

trifolii .............

CUSCUTACEAE ....

Cyathicula .......... quisquillaris .......

Cylindrocolla .......

dendroctonii ........

flagellaris lactea ............ 46

CyLindRosPorium ..... 74

Crategi .......... $\quad \mathbf{7 4}$

Toxicodendri ...... 71

CylindRothecium $\ldots . .184$

cladorrhizans ....... 184

seductrix ......... 184

Cymbalaria ........ 351

Cymbalaria ........ 351

Cymbella .......... 28

gastroides ......... 28

turgida .......... 28

Cynoctonum $\ldots \ldots \ldots .329$

Mitreola ......... 329

Cynoglossum ....... 337

officinale ........ 337

Virginicum ........ 337

Cynosurus .......... 214

cristatus ......... 21』

CYPERACEAE ..... 217

Cyperus ........... 217

diandrus $\ldots \ldots \ldots \ldots, 217$

esculentus ........ 217

filiculmis ........ 217

flavescens ........ 217

Lancastriensis ..... 217

ovularis ........ 21i

refractus $\ldots \ldots \ldots \ldots 217$

retrof ractus $\ldots \ldots \ldots 217$

rivularis $\ldots \ldots \ldots \ldots, 217$

strigosus ........ 217

CYPRIPEDIUM $\ldots \ldots \ldots .233$

acaule $\ldots . \ldots \ldots \ldots .233$

hirsutum ........ 233

parviflorum ....... 233

pubescens ......... 233

Cystopteris ......... 196

bulbifera ......... 196

fragilis .......... 196

Cystopus ........... 26

Bliti ............. 26

candidus $\ldots \ldots \ldots .26$

Ipomoeae-panduratae. $\quad 26$

Portulacae ........ 26

Tragopogonis $\ldots \ldots .26$

Cytisus ............ 284

scoparius ......... 284

Cytospora .......... 59

Betulina .......... 61

carphosperma ..... 61

caryigena $\ldots \ldots \ldots .60$

Celtidis .......... 59

ceratophora...$\ldots .61$

coccinea .......... 61

Curryi .......... 61

exasperans...$\ldots .65$

Halesiae ........ 59 
leucosperma

leucostoma

orthospora $\ldots \ldots \ldots$.

Persicae ...........

Platani ............

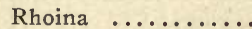

Rhois-hirtae .......

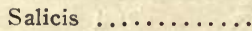

Sassafras ..........

tumulosa ..........

Cytosporella ........

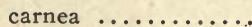

DACRyomyces .......

corticioides $\ldots \ldots \ldots$.

deliquescens...$\ldots$.

minor ............

stellatus $\ldots \ldots \ldots \ldots$.

Syringae ..........

Dactruis .......... glomerata...$\ldots \ldots$

Dactylium ..........

dendroides .........

Dactyloctenium .....

Aegyptium ........

Daedalia ..........

Aesculi $\ldots \ldots \ldots \ldots$

ambigua ..........

confragosa .........

unicolor ...........

Dalibarda $\ldots \ldots \ldots \ldots$

repens $\ldots \ldots \ldots \ldots$.

Daldinia $\ldots \ldots \ldots \ldots$

concentrica $\ldots \ldots \ldots$.

vernicosa $\ldots \ldots \ldots \ldots$

Danthonia $\ldots \ldots \ldots \ldots$.

compressa .........

spicata ...........

DAsYscypha ........

Ellisiana ..........

luteola ...........

virginea $\ldots \ldots \ldots \ldots$

Dasystoma $\ldots \ldots \ldots \ldots$.

flava .............

laevigata ..........

Pedicularia .......

Virginica .........

Datura ....................

Stramonium ..... 349

Tatula ......... 349

Daucus ........... 315

Carota .......... 315

Carota rosea ...... 315

Delphintum ........ 255

Ajacis .......... 255

consolida ........ 256

tricorne ......... 955

tricorne albiflora ... 255
61 DEMATIACEAE ...

DENDRODOCHIUM ...... affine...$\ldots \ldots \ldots$

rubellum microsporum

61. DENDROPHOMA .......

60 Therryana ........

60 Dennstaedtia .......

61 punctilobula .......

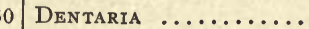

\section{DIAPORTHE .................}

Araliae $\ldots \ldots \ldots . . . . .$.

Aceris ...........

aculeata .........

cercophora .........

Gladioli

Halesiae ..........

Hydrangae

obscura

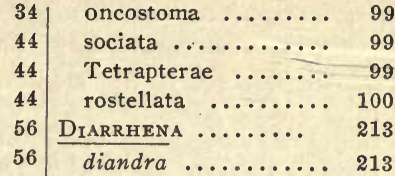

196

196

265

265

266

266

266

266

319

319

115

115

115

115

115

114

114

177

177

287

287

288

288

288

288

288

288

289

289

287

288

288

287

288

288

30

30

356

356

250

250

327

98

98

99

100

99

98

98

99

100

DIATOMACEAE .... 28

DiAtrype .......... 92

platystoma ........ 93

Stigma...$\ldots \ldots \ldots .62$

virescens $\ldots . \ldots \ldots .93$

Diatrypella ........ 93

verruciformis ...... 93

DichRoPHYLLUM ..... 294

marginatum ....... 294

Dicksonia $\ldots \ldots \ldots \ldots \quad 196$

punctilobula ...... 196

DICRANACEAE .... 173

Dicranella ....... 173

heteromala ....... 173

rufescens ......... 173

varia $\ldots \ldots \ldots \ldots \ldots \quad 173$

Dicranodontium $\ldots . .174$

denudatum $\ldots \ldots \ldots .174$

Millspaughii ..... 175

Virginicus ....... 174

Dicranum $\ldots \ldots \ldots \ldots .174$

Drummondii ...... 174

flagellare $\ldots \ldots \ldots \ldots \quad 174$

fulvum $\ldots \ldots \ldots \ldots \ldots 174$

fuscesens $\ldots \ldots \ldots \ldots \quad 174$

longifolium $\ldots \ldots \ldots .174$

montanum ....... 174

scoparium ........ 174

Didymella ........ 97

Physocarpi ....... 97

Didymum ........ 29

squamulosum ...... 29

Diervilla ......... 362

Diervilla ......... 362

trifida $\ldots \ldots \ldots \ldots \ldots \quad 362$

Digitalis ......... 354

purpurea ......... 354

DimerosPoRIUM $\ldots \ldots .89$

Collinsii ......... 89

Galactis ......... 89

DINEMASPORIUM $\ldots \ldots \quad 71$

hispidulum ....... 71

Diodia .......... 359

teres $\ldots \ldots \ldots \ldots \ldots .359$

Dioscorea .......... 232

glauca .......... 232

villosa .......... 232

DIOSCOREACEAE .. 232

Diospyros ......... 328

Virginiana ...... 328

\section{年}

9
, ${ }_{232}^{232}$

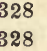

$-2$

3

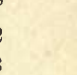

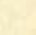

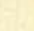

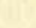

6

3

3

4

74

74

4

174

74

7

9

62

4


coloratum ........ 311

lineare...$\ldots \ldots \ldots$.

spicatum ...........

EQUISETACEAE ....

EQUisetuM ........... 197

arvense $\ldots \ldots \ldots \ldots$ 19

hyemale ......... 197

laevigatum $\ldots \ldots \ldots \ldots 197$

sylvaticum ....... 197

ERAGRostis ......... 213

capillaris ........ 213

Eragrostis ....... 213

Frankii ......... 213

hypnoides ........ 219

major ......... 213

minor .......... 213

pectinacea ....... 213

pilosa .......... 213

Purshii .......... 213

reptans ......... 213

ERechtites $\ldots \ldots \ldots \ldots \quad 386$

hieracifolia $\ldots \ldots \ldots .386$

ERICACEAE $\ldots \ldots \ldots \quad 322$

ERIGENIA $\ldots \ldots \ldots \ldots .320$

bulbosa ......... 320

Erigeron $\ldots \ldots \ldots \ldots .377$

annuus $\ldots \ldots \ldots \ldots .377$

bellidifolius ....... 377

Philadelphicus ..... 377

pulchellus ........ 377

ramosus ........ 377

strigosus $\ldots \ldots \ldots \ldots \ldots \quad 377$

ERINELLA ...........

minioposis $\ldots \ldots \ldots$.

ERIOGONUM $\ldots \ldots \ldots \ldots$

Alleni...$\ldots \ldots \ldots$

ERIOPHORUM $\ldots \ldots \ldots$

Virginicum $\ldots \ldots \ldots$.

ERYNGIUM $\ldots \ldots \ldots \ldots$

aquaticum $\ldots \ldots \ldots$.

yuccaefolium .......

ERYsimum .......... cheiranthoides ......

ERysiphe $\ldots \ldots \ldots \ldots$ aggregata $\ldots \ldots \ldots$

Cichoriacearum ..... communis $\ldots \ldots \ldots \ldots$ graminis .......... Liriodendri ........ Polygon $\ldots . . . \ldots$.

ERTthRONIUM $\ldots \ldots \ldots$ albidum ...........

Americanum ........

Euonymus ..........

Americanus ........ Americanus obovatus atropurpureus .....

88

89 obovatus

Eupatorium .........

ageratoides $\quad \ldots \ldots \ldots$

altissimum ........

aromaticum .......

coelestinum .......

hyssopifolium ......

maculatum

maculatum amoenum.

perfoliatum

pubescens

.........

purpureum ........

sessilifolium .......

trifoliatum ........

Euphorbia $\ldots \ldots \ldots$

commutata ........

corollata ..........

Cyparissias ........

Darlingtonii .......

dentata ............

glyptosperma pubes-

cens ...........

Lathyrus .........

maculata ..........

marginata .........

obtusata ..........

Preslii ............

EUPHORBIACEAE .

Eurotium $\ldots \ldots \ldots \ldots$

herbariorum .......

Euthamia .........

Caroliniana...$\ldots$.

graminifolia...$\ldots$

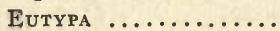

milliaria...$\ldots \ldots$.

spinosa $\ldots \ldots \ldots \ldots$

Eutypella .........

densissima $\ldots \ldots \ldots$.

Platani ............

rugiella $\ldots \ldots \ldots \ldots$.

stellulata $\ldots \ldots \ldots \ldots$

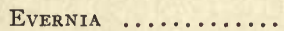

furfuracea ....... 152
299 Fagus ............ 240

Americana ........ 240

371 ferruginea ....... 240

371 Falcata .......... 290

371 comosa .......... 290

372 Favolus ........... 141

371 Europaeus ........ 141

371 Festuca ........... 215

371 elatior ........... 215

371 nutans .......... 215

371 octoflora ........ 215

371 ovina .......... 215

371 pratensis ........ 215

371 tenella .......... 215

294 Ficus ............ 242

295 carica .......... 242

294 FILICES ......... 191

295 FILIX ............ 196

295 bulbifera ......... 196

295 fragilis .......... 196

FIMBRISTYLIS ........ 218

294 autumnalis ....... 218

295 Fissidens .......... 176

294 adiantoides ........ 176

294 decipiens ......... 176

295 minutulus ........ 176

294 obtusiolius $\ldots \ldots \ldots . .176$

294 subbasilaris ....... 176

89 FISSIDENTACEAE .. 176

89 Fistulina ........ 134

374 hepatica ......... 134

374 Flammula $\ldots \ldots \ldots \ldots 130$

374 flavida .......... 130

92 FloerkeA .......... 296

92 proserpinacoides .... 296

92 Fomes ............ 137

92 applanatus ....... 137

92 carneus .......... 138

92 fomentarius ....... 137

92 lucidus $\ldots \ldots \ldots \ldots .137$

92 pinicola .......... 137

152 rimosus $\ldots \ldots \ldots \ldots . \ldots 137$

152 roseus $\ldots \ldots \ldots \ldots \ldots 138$

EXCIPULACEAE .... 71 ungulatus $\ldots \ldots \ldots \ldots 137$

Exidia $\ldots \ldots \ldots \ldots \ldots 149$ volvatus $14 . \ldots \ldots \ldots 137$ alba $\ldots \ldots \ldots \ldots \ldots .149$ FONTINALACEAE .. 182

glandulosa ....... 149 Fontinalis ......... 182

Exoascus ................... 118 Dalecarlica 182

deformans ............... 118 Fossombronia 165

Pruni .......... 118 Wondraczeckii ..... 165

Exobasidium $\ldots \ldots \ldots .148$ Fragaria ......... 276

Rhododendri ..... 148 Indica $\ldots \ldots \ldots \ldots .276$

FABRONIACEAE ... 183 vesca ........... 276

Virginiana $\ldots \ldots \ldots 276$

Fraxinus .............. 328

Americana ....... 328

lanceolata ....... 328 


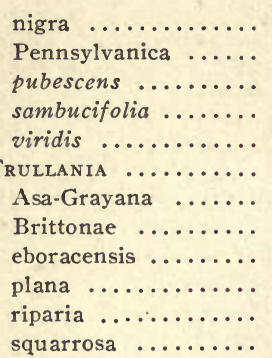

Fumaria ............. officinalis $\ldots . \ldots .$.

Funaria ............. flavicans....... .

hygrometrica ...... hygrometrica patula.. FUNARIACEAE .....

Fusarium $\ldots \ldots \ldots \ldots$. aleurinum....... culmorum .......... Oxydendri .......... roseum........ sarcochroum ....... Solani .............

Fucosporia $\ldots \ldots \ldots$. viticola $\ldots . . . \ldots \ldots$

Fusicladium .......... dendriticum...... Virginiense .........

Fusicoccum ......... Ilicinum $\ldots . . . \ldots$. nervicolum ..........

Galactia $\ldots . . . \cdots \cdots$ regularis.......

Galax $\ldots . . . \ldots \ldots$ aphylla ...........

GaLEOPSis ............ Tetrahit ...........

Galeorchis .......... spectabilis .........

GALERA $\cdots \cdots \cdots \cdots$

hypnoruns $\ldots . . . \cdots$

tener ..............

GALIN SOGA ........ 384 parviflora ........ 384

Galium ........... 359

Aparine ......... 359

asprellum ........ 360

circaezans ........ 359

Claytoni ........ 359

concinnum ...... 360

lanceolatum ....... 359

latifolium ........ 360

pilosum .......... 359

trifidum $\ldots \ldots \ldots \ldots .360$ triflorum

359

Ganoderma $\ldots \ldots \ldots \ldots, 137$ $\begin{array}{lll}\text { Tsugae } \ldots \ldots \ldots \ldots & 137\end{array}$

Gaultheria ....... 325 probumbens ...... 325

Gaura $\ldots \ldots \ldots \ldots \ldots .313$

biennis ............

Gaylussacia .......... dumosa ............

frondosa $\ldots . . . . .$.

resinosa ...........

Geaster $\ldots \ldots \ldots \ldots$. hygrometricus ...... mammosus ........

Gelatinosporum $\ldots .$. Betulinum .........

Gentiana ............ Andrewsii ......... flavida......... linearis ............ quinqueflora ....... quinquefolia ....... Saponaria ......... GENTIANACEAE $\ldots \quad 330$ Geocalyx .......... 167 graveolens ........ 167

Geoglossum a...... 110 glabrum ......... 110

GEOPYXIS .......... 108 carbonaria ........ 108 cupularis ........ 108 nebulosa ......... 108

Georgia ................ 181 pellucida $\ldots \ldots \ldots \ldots, 181$

GEORGIACEAE $\ldots . .181$

GERANIACEAE $\ldots . \quad 290$

Geranium $\ldots \ldots \ldots \ldots$.... 290

Carolinianum ..... 291 maculatum ........

Robertianum .......

Gerardia ............ auriculata ......... flava .............. loevigata .......... paupercula...... . pedicularia .......... quercifolia ......... tenuifolia ..........

Geum ............... album ............

Canadense ......... flavum......... rivale.......... vernum $\ldots . . . \ldots .$. Virginianum ....... Gibberella $\ldots \ldots \ldots \ldots$ pulicaris...$\ldots \ldots$.
Saubinetii ........ 105

Gifola $\ldots \ldots \ldots \ldots \ldots 378$

Germanica ....... 378

Gili.enia $\ldots \ldots \ldots \ldots \quad 273$

$\begin{array}{llll} & \text { stipulacea } \ldots \ldots \ldots & 273\end{array}$

trifoliata ........ 273

Glaucium .......... 262

Glaucium ........ 262

Glecoma $\ldots \ldots \ldots \ldots .342$

hederacea ........ 342

Gleditsia $\ldots \ldots \ldots \ldots \quad 283$

triacanthos ....... 283

Gloeophyleum ...... 128

hirsutum $\ldots \ldots \ldots \ldots \quad 128$

trabeum ......... 128

Glokoporus $\ldots \ldots \ldots \ldots$. 141

conchoides ........ 141

Gloeosporium ....... 71

Alni ........... 72

aridum $\ldots \ldots \ldots \ldots .6 \% \quad 72$

betularum ........ 72

cylindrospermum ... 72

nervisequum ...... 72

Robergi ......... 72

rubicolum $\ldots \ldots \ldots . \quad 72$

Rumicis ......... 72

Sanguinariae $\ldots . .6 \quad 71$

venetum ........ 72

Gromerella ........ 66

rufomaculans $\ldots \ldots .66$

Gronium ............ 118

parvulum ......... 118

simulans ........ 118

stellatum ......... 118

Glyceria $\ldots \ldots \ldots \ldots .214$

Canadensis ....... 214

nervata ......... 215

Gnaphalium $\ldots \ldots \ldots . \quad 379$

decurrens ....... 379

Germanicum ...... 378

obtusifolium ...... 379

polycephalum ..... 379

purpureum ....... 379

uliginosum $\ldots \ldots \ldots, 379$

GNOMONIA .......... 96

Ulmea .......... 96

Gnomoniella $\ldots \ldots \ldots .96$

Coryli ........... 96

fimbriata ........ 96

Gonatobotkyum ..... 35

maculicolum ...... 35

Gonolobus ......... 333

laevis ........... 333

Goodyera ........... 235

105 pubescens ........ 235

105 repens .......... 235 
Gorgoniceps $\ldots \ldots \ldots .110$

Guernisaci ....... 110

GRAMINEAE $\ldots \ldots \ldots 203$

Grandinia $\ldots \ldots \ldots \ldots .144$ crustosa $\ldots \ldots \ldots \ldots .144$

granulosa ......... 144

GRAPHIDACEAE $\ldots 160$

GRAPHIOLA ........ 77

Phoenicis $\ldots \ldots \ldots .77$

Graphis .......... 161

dendritica ........ 161

elegans .......... 161

scripta $\ldots \ldots \ldots \ldots \ldots 161$

scripta recta $\ldots \ldots \ldots 161$

Gratiola ..........

sphaerocarpa...$\ldots$

Virginiana $\ldots \ldots \ldots$

Grifola $\ldots \ldots \ldots \ldots \ldots$

Berkeleyi ........

ramosissima $\ldots \ldots \ldots \quad 136$

Grimmia $\ldots \ldots \ldots \ldots .177$ apocarpa $\ldots \ldots \ldots \ldots \quad 177$

GRIMMIACEAE $\ldots . \quad 177$

GROSSULARIACEAE 270

Guepinia ......... 150

spathularia ........

Gymnadeniopsis ......

clavellata ........ 234

GYMNOASACEAE .. 118

Gymnocladus ....... 283

Canadensis ....... 283

dioica .......... 283

Gymnoconia ........

interstitialis $\ldots \ldots \ldots$

GyMnOPUs ........ 123

radicata ......... 123

Gymnosporangium $\ldots$ clavariiforme.... corniculans ........ germinale...$\ldots \ldots$ globosum ......... Juniperinum ....... Juniperi-Virginianae. macropus ......... Nidus-avis $\ldots \ldots \ldots$.

Gyromitra ........ 107 curtipes $\ldots \ldots \ldots \ldots 107$

GYROPORUS .......... 133 cyanesuens $\ldots \ldots \ldots .133$

Gyrostachys $\ldots \ldots \ldots .235$ cernua $\ldots \ldots \ldots \ldots .235$ gracilis ......... 235

Habenaria $\ldots \ldots \ldots \ldots 234$

ciliaris ......... 234

flava ........... 234

lacera ............ 234

orbicularia ........ 234 peramoena ........ 234

psycodes ......... 234

tridentata $\ldots \ldots \ldots \ldots 234$

HaINESIA t...........

Rubi ......... 71

Halesia $\ldots \ldots \ldots \ldots \ldots .328$

tctraptera ........ 328

HAMAMELIDACEAE 271

Hamamelis ....... 271

Virginiana $\ldots \ldots \ldots .271$

Haplopilus ........ 136

gilvus $\ldots \ldots \ldots \ldots \ldots 136$

licnoides ......... 136

rutilans ......... 136

Haplosporella ..... 63

Araliae ......... 63

Celtidis ........ 63

Harpalejeunea $\ldots \ldots .170$

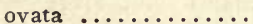

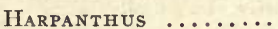
scutatus

Harperetlla ........

vivipara $\ldots \ldots \ldots \ldots$

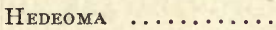
pulegioides...$\ldots$.

Hedwigra $\ldots \ldots \ldots \ldots$. ciliata ...........

ciliata secunda .....

HEDW IGIACEAE ...

Helenium ......... autumnale .........

Helianthemum ..... majus...$\ldots \ldots \ldots$

Helianthus $\ldots \ldots \ldots$. decapetalus...$\ldots$. divaricatus ........ doronicoides ....... giganteus ........ grosseserratus ..... hirsutus $\ldots \ldots \ldots \ldots$. laetiflorus..$\ldots \ldots$. laevigatus ......... microcephalus ...... occidentalis ....... occidentalis Dowellianus ........... parviflorus ......... strumosus $\ldots \ldots \ldots$. tomentosus ....... tracheliifolius ......

Heliopsis .......... helianthoides.... . laevis .......... 381 scabra ......... 381

Helminthosporium .. 37 attenuatum $\ldots \ldots \ldots \quad 37$ brachypus $\ldots \ldots \ldots \ldots 37$ folliculatum ...... 37

fusciforme ...... 38

macrocarpon ...... 37

persistens $\ldots \ldots \ldots .37$

septemseptatum $\ldots . \quad 37$

Heliotiella ....... 113

Nuttallii ........ 113

Helotium ........ 110

castaneum $\ldots \ldots \ldots .111$

citrinum ........ 111

confluens $\ldots \ldots \ldots \ldots 110$

epiphyllum ...... 111

fraternum ....... 111

fructigenum $\ldots \ldots \ldots \quad 111$

herbarum ....... 110

lacteum ......... 110

phyllophilum ...... 111

HELIOTROPIUM $\ldots \ldots .337$

Indicum $\ldots \ldots \ldots \ldots 337$

Hediscus .......... 46

Lugdunensis ..... 46

Helleborus ........ 254

viridis $\ldots \ldots \ldots \ldots .254$

Helvella ........ 107

crispa ......... 107

macropus ....... 107

HELVELLACEAE ... 107

Hemerocallis ...... 227

fulva ......... 227

Hemiarcyria ....... 31

clavata ......... 31

rubiformis $\ldots \ldots \ldots \ldots \quad 31$

stipata ......... 31

Hendersonia $\ldots \ldots \ldots .65$

Desmazieri ........ 65

Lirella ......... 65

Hepatica $\ldots \ldots \ldots \ldots .257$

acuta .......... 257

Hepatica ........ 257

HEPATICAE ...... 163

Heracleum $\ldots \ldots \ldots \ldots 317$

lanatum ......... 317

Herbertia ........ 169

adunca $\ldots \ldots \ldots \ldots \ldots \quad 169$

Hercospora ........ 98

Tiliae .......... 98

Hericium .......... 142

coralloides $\ldots \ldots \ldots \ldots \quad 142$

croceum ......... 142

erinaceus $\ldots \ldots \ldots \ldots \quad 142$

HeRPotrichia ...... 102

diffusa .......... 102

Hesperis $\ldots \ldots \ldots \ldots \ldots 267$

matronalis ........ 267

Heuchera ........ 270

Americana ....... 270

parviflora ........ 270

pubescens ....... 270 


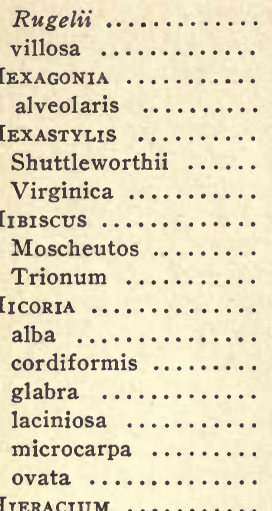

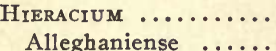

Canadense ..........

Gronovii ...........

longipilum .........

paniculątum .........

scabrum ...........

venosum $\ldots . . . \ldots$.

HiRMEOLA........

Auricula-Judae ......

HoLcus .............

lanatus ..............

Holwaya ............

ophiobolus ..........

Homalocenchrus .....

oryzoides ...........

Virginicus .........

HomalotheCiella .... subcapillata .........

Hookeria ............ Sullivantii .......... HOOKERIACEAE ...

HoRoMyces .......... fragiformis .........

Houstonia ...........

ciliolata ...........

coerulea ...........

coerulea albiflora.....

longifolia ...........

purpurea ..........

purpurea calycosa.... serpyllifolia ......... tenuifolia ...........

Humaria ............

avaneosa ..........

cestrica ...........

echinosperma .......

Gerardii ........... 109

Humulus .......... 243

Lupulus .......... 243

HYDNACEAE ..... 142

43

\begin{tabular}{|c|c|c|c|}
\hline & & & \\
\hline Nuttallii $\ldots \ldots \ldots \ldots$ & 43 & ratum & \\
\hline velutinum $\ldots .$. & 13 & cum .. & \\
\hline YYDNUM $\ldots \ldots \ldots$ & 12 & tatum .. & \\
\hline$m \ldots \ldots \ldots$ & 42 & Sarothra .... & \\
\hline rinum $\ldots \ldots$ & 2 & tum acutif & \\
\hline ides .......... & 142 & Virginicum $\quad . .$. & \\
\hline . $\ldots \ldots \ldots$ & 42 & LIA $\quad \ldots \ldots \ldots$ & \\
\hline$u m \ldots \ldots \ldots$ & 142 & ris $\ldots \ldots \ldots$ & \\
\hline$u m \ldots \ldots$ & 143 & MA $\ldots \ldots \ldots$ & \\
\hline$u m \ldots \ldots$. & 142 & :ulatum .... & \\
\hline $\operatorname{am} \ldots \ldots \ldots$ & 42 & lleanum ..... & \\
\hline$\iota m \ldots \ldots \ldots$ & 43 & .......... & \\
\hline$A \ldots \ldots \ldots$ & 70 & aris $\ldots \ldots \ldots$ & \\
\hline ns ns Kanawha- $^{\prime}$ & 270 & $\begin{array}{l}\text { labunda } \ldots \ldots \\
\text { ritium } \ldots \ldots\end{array}$ & \\
\hline & 270 & MYCETAC & \\
\hline$\because \ldots \ldots \ldots$ & 254 & AE $\ldots$ & \\
\hline sis $\ldots \ldots \ldots$ & 254 & $\ldots \ldots \ldots \ldots$ & \\
\hline ARITA- & & castrense... . & \\
\hline$\cdots \cdots \cdots \cdots$ & 203 & siforme ....... & \\
\hline LE $\ldots \ldots \ldots$ & 19 & $\operatorname{lium} \ldots \ldots$ & \\
\hline a & 319 & $m$ & \\
\hline AE & 36 & atum $\ldots \ldots$ & \\
\hline & 336 & nianum $\ldots$ & \\
\hline um $\ldots .$. & 3 & $\ldots \ldots \ldots \ldots$ & \\
\hline e $\ldots \ldots \ldots$ & 337 & $\operatorname{slum} \ldots \ldots \ldots$ & \\
\hline $\operatorname{lum} . . . .$. & 3 & $s \ldots \ldots \ldots$ & \\
\hline m $\ldots \ldots \ldots$ & & arpum $\ldots$. & \\
\hline RUS $\ldots \ldots \ldots$ & 1 & ........... & \\
\hline & & tre $\ldots \ldots \ldots$ & \\
\hline a $\ldots \ldots \ldots \ldots$ & 124 & tae $\ldots \ldots \ldots$. & \\
\hline$\ldots \ldots \ldots \ldots$ & 24 & um $\ldots \ldots \ldots$ & \\
\hline & 1 & $\mathrm{um} \ldots \ldots \ldots$ & \\
\hline$\ldots \ldots \ldots \ldots$ & 124 & ans $\ldots . . . \ldots$ & \\
\hline ............ & & ............. & \\
\hline$\cdots \cdots$ & 124 & $m$ m $\quad \ldots \ldots$ & \\
\hline is ... & & i $\ldots \ldots \ldots \ldots$ & \\
\hline$\ldots \ldots \ldots$ & 4 & $n s \quad \ldots \ldots \ldots$ & \\
\hline $\mathbf{M} \ldots \ldots \ldots$ & & $1 \mathrm{~m} \quad \ldots \ldots \ldots$ & \\
\hline trum $\ldots \ldots$ & 7 & um $\ldots \ldots \ldots$ & \\
\hline$u m \ldots . . .$. & 7 & IERIS $\ldots \ldots$ & \\
\hline n....... & 7 & ta $\ldots . . . \ldots$. & \\
\hline HAEte $\ldots \ldots$. & 146 & EA $\cdots$ & \\
\hline a $\ldots \ldots \ldots$. & 1 & $\ldots \ldots \ldots$ & \\
\hline sa $\ldots \ldots \ldots$ & 1 & (6) & \\
\hline a $\ldots \ldots \ldots$. & & nitzii $\ldots .$. & \\
\hline$\ldots \ldots \ldots$ & 6 & & \\
\hline$A \ldots \ldots \ldots$ & 4 & nsis $\ldots \ldots \ldots$ & \\
\hline & 45 & EACEAE $\ldots$ & \\
\hline ACEAE .... & 304 & sis $\ldots \ldots \ldots$ & \\
\hline$M \quad \ldots \ldots \ldots$ & 3 & ides $\ldots . . . \ldots$ & \\
\hline$m \quad \ldots \ldots \ldots$ & 30 & $\ldots \ldots$ & \\
\hline nse $\ldots \ldots \ldots$ & 305 & MA $\quad \ldots \ldots \ldots$ & \\
\hline $\operatorname{m} \quad \ldots \ldots \ldots$ & 30 & $\ldots \ldots$ & \\
\hline$\ldots \ldots \ldots$ & 305 & ieri $\ldots \ldots \ldots$ & \\
\hline $\mathrm{m} \quad \cdots$ & & $\operatorname{rum} \ldots \ldots$ & \\
\hline$\cdots$ & 05 & $\cdots \cdots$ & \\
\hline
\end{tabular}


aurantius $\ldots \ldots \ldots \ldots$.

Hypopitys ........ 32

Hypopitys ....... 322

Hypoxis .......... 232

erecta $\ldots \ldots \ldots \ldots \ldots 232$

hirsuta $\ldots \ldots \ldots \ldots .232$

HyPoxylon ..........

atroviride $\ldots \ldots \ldots \ldots$

coccineum ..........

colliculosum..... .

fuscum $\ldots \ldots \ldots \ldots$.

Howeianum .......

multiforme ........

Nuttallii .........

perforatum ........

rubiginosum .......

Sassafras ........

stigmateum $\ldots \ldots \ldots$

HYPSILOPHORA $\ldots \ldots$.

fragiformis .........

HYSTERIACEAE ....

Hysterium $\ldots \ldots \ldots$.

Kalmiae .........

HysterographIUM $\ldots$. insidens $\ldots \ldots \ldots \ldots$.

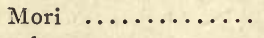

subrugosum .......

Hystrix ..........

Hystrix .........

ILEX $\ldots \ldots \ldots \ldots \ldots$

mollis ...........

monticola .........

opaca ............

verticillata ........

ILICIOIDES ......... mucronata $\ldots \ldots \ldots$

ILLOSPORIUM $\ldots \ldots \ldots$ caespitosum ....... malifoliorum ......

ILYSANTHES $\ldots \ldots \ldots \ldots$ dubia ............ gratioloides ........

Impatiens ......... aurea ............ biflora...$\ldots \ldots \ldots$. fulva......... pallida ...........

IMPERATORIA $\ldots \ldots \ldots$. Ostruthium $\ldots \ldots \ldots$.

InULA $\ldots \ldots \ldots \ldots \ldots$ Helenium .........

Iodanthus .......... pinnatifidus...$\ldots$.

Ionactss .......... linariifolius $\ldots \ldots \ldots$.

Ionotus ........... perplexus \begin{tabular}{l|}
103 \\
103 \\
322 \\
322 \\
232 \\
232 \\
232
\end{tabular}

94
94

95

95

95

95

95

95

95

95

95

95

150

150

118

118

118

118

118

118

118

216

216

298

298

298

298

298

298

298

45

45

45

352

352

352

301

301

301

301

301

317

317

380

380

264

264

378

378

135

135
Iтomoea $\ldots \ldots \ldots \ldots \ldots$

coccinea ......... 333

hederacea ........ 333

lacunosa ......... 333

pandurata ....... 333

purpurea ......... 333

IRIDACEAE $\ldots \ldots \ldots .233$

IrIS ............ 233

cristata $\ldots \ldots \ldots \ldots .232$

verna ........... 233

versicolor ........ 233

IrPEX .......... 143

lacteus ......... 143

obliquus $\ldots \ldots \ldots \ldots .143$

sinuosus $\ldots \ldots \ldots \ldots .143$

IRPICIPORUS $\ldots \ldots \ldots \ldots 135$

lacteus .......... 135

mollis .......... 135

Isanthus $\ldots \ldots \ldots \ldots$

brachnatus ........

coeruleus ......... 340

IsArta $\ldots \ldots \ldots \ldots \ldots$

clavata $\ldots \ldots \ldots \ldots$.

Virginiensis .......

Ischnoderma $\ldots \ldots \ldots$

fuliginosum $\ldots \ldots \ldots$.

ISNARDIA $\ldots \ldots \ldots \ldots$

palustris $\ldots \ldots \ldots \ldots$

IsOPYRUM $\ldots \ldots \ldots \ldots$.

trifolium ..........

Isotria $\ldots \ldots \ldots \ldots \ldots$

verticillata $\ldots \ldots \ldots \ldots 235$

ITHYPHALLUS ....... 24

impudicus ........ 24

JAMESONELLA $\ldots \ldots \ldots 165$

autumnalis ........ 165

JefFersonia ........ 260

diphylla ......... 260

Jubula ........... 171

Hutchinsiae Sullivan-

tii ........... 171

Pennsylvanica ...... 171

JUGLANDACEAE $\ldots \quad 237$

Juglans ......... 237

cinerea $\ldots \ldots \ldots \ldots .237$

nigra $\ldots \ldots \ldots \ldots \ldots 237$

JUNCACEAE ...... 225

JuncoIdes ......... 226

campestre $\ldots \ldots \ldots \ldots 226$

pilosum .......... 226

saltuensis $\ldots \ldots \ldots \ldots 226$

Juncus ........... 225

acuminatus $\ldots \ldots \ldots 225$

brevicaudatus ..... 225

Canadensis ....... 225

Canadensis subcordatus

effusus marginatus

nodosus

setaceus .............. 225

tenuis .......... 225

tenuis anthelatus.... 225

JungERMANNIA $\ldots \ldots \ldots 165$

exsecta ......... 167

lanceolata ........ 165

pumila .......... 165

Schraderi ........ 165

JUNGERMANNIA-

CEAE ........ 164

Juniperus $\ldots \ldots \ldots \ldots .199$

communis ....... 199

Virginiana ....... 199

Kalmia ........... 324

angustifolia $\ldots \ldots \ldots .324$

latifolia .......... 324

Kantia .......... 168

trichomanis $\ldots \ldots \ldots \quad 168$

KnEIFFIA ......... 312

fruticosa ........ 312

fruticosa differta.... 312

linearis ......... 313

longipedicillata .... 313

pumila .......... 312

setigera ......... 144

Koellia ........... 345

clinopodioides ..... 345

flexuosa ......... 345

incana ........ 346

montana ........ 346

mutica .......... 346

pycnanthemoides .... 345

verticillata ....... 345

Virginiana ....... 346

Korycarpus ........ 213

Americana ....... 213

diandrus ......... 213

KrIGIA .......... 366

amplexicaulis ...... 366

Dandelion ......... 366

KUEHNEOLA ........ 84

obtusa ......... 84

Uredinis ........ 84

KuHNiA .......... 372

eupatorioides ...... 372

KYLLINGA ........ 217

pumila ......... 217

LABIATAE $\ldots \ldots \ldots \ldots 340$

LACHNEA .......... 109

erinacea $\ldots \ldots \ldots \ldots 110$

hirta .......... 110

scutellata ........ 109

umbrarum ....... 110

vitellina $\ldots \ldots \ldots \ldots .110$

LACHNELLA ........ 113

Virginica $\ldots \ldots \ldots \ldots 113$ 
LACHNOCLADIUM ...... 149 semivestitum ...... 149

LACINIARIA $\ldots \ldots \ldots \ldots .372$ spicata ......... 372 scariosa squarrulosa. 372

LACTARIA .......... 124

cilicioides ........ 124

deliciosa ......... 125

glaucescens $\ldots \ldots \ldots 125$

hydropiperoides ..... 125

Indigo $\ldots \ldots \ldots \ldots \ldots \quad 125$

insulsa ......... 125

lactiflua ......... 125

pergamena ........ 124

piperata $\ldots \ldots \ldots \ldots \quad 124$

plinthogala ....... 125

subdulcis ......... 125

trivalis ........ 125

vellera .......... 125

LACTARJUS ......... 125

fuliginosus ....... 125

glaucescens ....... 125

volemus ......... 125

Lactuca ......... 367

Canadensis ....... 367

Floridana ....... 367

hirsuta ......... 367

integrifolia ....... 367

leucophaea ....... 367

sagittifolia $\ldots \ldots \ldots .367$

Scariola ........ 367

spicata ......... 367

villosa $\ldots \ldots \ldots \ldots \ldots \quad 367$

LAETIPORUS ......... 136

speciosus $\ldots \ldots \ldots \ldots 136$

LAMIUM .......... 343

amplexicaule $\ldots \ldots \ldots \quad 343$

maculatum ....... 343

purpureum ....... 343

LAMPRODERMA ....... violaceum

LAPortea ........... Canadensis

LAPPUla .......... Virginiana ........

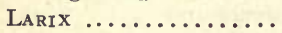
Americana .........

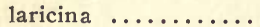

LAsiosphaeria ....... ovina

LATHYRUS .......... latifolius .......... venosus ...........

LAURACEAE ......

Lecanidion $\ldots \ldots \ldots \ldots$ atratum ..........

Hamammelidis .....

LECANORA

243

243

338

338

200

200

200

101

101

290

290

290

261

118

118

118

155 atra $\ldots \ldots \ldots \ldots \ldots \quad 155$

cervina $\ldots \ldots \ldots \ldots \ldots 156$

coarctata ........ 155

deplanans ......... 155

muralis versicolor... 156

pallescens ........ 156

pallida .......... 156

privigena $\ldots \ldots \ldots \ldots .156$

subfusca $\ldots \ldots \ldots \ldots \quad 155$

tartarea ......... 156

varia $\ldots \ldots \ldots \ldots \ldots \quad 156$

LECANORACEAE ... 155

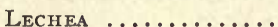

Leggettii ..........

major .............

minor $\mathrm{L} . . . . . . .$. .

minor $\operatorname{Lam} . . . \ldots \ldots$

Lecidea .....................

albocoerulescens ....

crustulata .........

ericina ............

fuliginea $\ldots \ldots \ldots \ldots \quad 159$

Nuttallii ........ 159

platycarpa steriza.... 160

speirea .......... 160

Tennesseensis ..... 159

Virginiensis ...... 160

LECIDACEAE ...... 158

Leersia .......... 209

oryzoides ........ 209

streptocarpa $\ldots \ldots \ldots . \quad 176$

Virginicus .........

Lejeunea ..........

calypcata ..........

cavifolia ..........

cucullata ..........

Lemna ...........

minor ............

LEMNACEAE ......

LENTIBULARIACEAE
Lentinus ..........

crinitus.$\ldots \ldots \ldots \ldots$

lepideus ...........

strigosts ..........

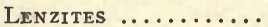

abietina ...........

betulina...$\ldots \ldots \ldots$

corrugata $\ldots \ldots \ldots$.

sepiaria ...........

Leocarpus .........

fragilis

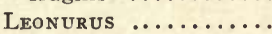

Cardiaca ...........

Leotia ............

lubrica .......... 116

LePidium ......... 263

campestre ........ 263

Virginicum ........
LePidozia $\ldots \ldots \ldots \ldots . \ldots 168$

reptans $\ldots \ldots \ldots \ldots \ldots 168$

sylvatica $\ldots \ldots \ldots \ldots .168$

Lepiota .......... 120

acutisquamosa ..... 121

Americana ........ 121

Amianthina ....... 121

cristata .......... 121

clypeolaria ........ 121

metulaespora ...... 121

naucina ......... 120

procera .......... 120

Leptamnium ....... 355

Virginianum ...... 355

LeptandRa $\ldots \ldots \ldots \ldots 353$

Virginica $\ldots \ldots \ldots \ldots 353$

Leptilon ......... 378

Canadense ....... 378

LEPTOBRYUM ........ 180

py riforme ........ 180

LEPTODON ........... 183

trichom:trion $\ldots \ldots \ldots 183$

Leptogrum ......... 155

chloromelum stellans. 155

pulchellum ....... 155

tremelloides ...... 155

Leptonia ......... 129

asperella ........ 129

serrulata ........ 129

LEPTORCHIS ........ 236

liliifolia ......... 236

Leptosphaeria $\ldots \ldots \ldots \quad 100$

clavigera ......... 101

Doliolum ......... 100

Ogilviensis ....... 101

orthogramma ...... 101

vagabunda $\ldots \ldots \ldots \ldots 101$

LEPTOSTROMACEAE 70

Leptostromella ..... 71

filicina ......... 71

LEPTOTHYRIUM ...... 70

Castaneae ........ 70

dryinum ......... 70

foraminulatum ..... 70

Liriodendri ....... 70

petiolorum ....... 70

Pyri ........... 70

vulgare $\ldots \ldots \ldots \ldots \ldots \quad 70$

Leptotrichum ...... 173

pallidum .......... 173

tortile .......... 173

Leskea ........... 184

gracilescens $\ldots \ldots \ldots .184$

obscura .......... 184

polycarpa ........ 184

LESKEACEAE ..... 184

Lespedeza $\ldots \ldots \ldots \ldots .289$

capitata ......... 289 
frutescens $\ldots \ldots \ldots$.

polystachya $\ldots . . .$.

repens...$\ldots \ldots \ldots$.

striata $\ldots \ldots \ldots \ldots$

Stuvei ............

Stuvei intermedia....

Stuvei reticulata.....

violacea ..........

Virginica .........

LEUCOBRYACEAE ..

LEU COBRYuM ........ glaucum .........

Leucodon .......... brachypus ......... julaceus ..........

LEUCODONTACEAE.

LIATRIS

scariosa squarrulosa.. spicata ...........

Li Bertella ......... acerina $\ldots \ldots \ldots \ldots$.

faginea $\ldots \ldots \ldots \ldots$.

LICHENES ........

Ligusticum $\ldots \ldots \ldots \ldots$

Canadense ........

Ligustrum ......... vulgare $\ldots \ldots \ldots \ldots$.

LILIACEAE .......

Lilium $\ldots \ldots \ldots \ldots \ldots$

Canadense ........

Philadelphicum .....

superbum ........

tigrinum $\ldots \ldots \ldots \ldots$

LIMNANTHACEAE...

LIMODORUM $\ldots \ldots \ldots \ldots$ tuberosum $\ldots \ldots \ldots$.

LINACEAE .......

LinARIA .......... Linaria ..........

LindBLAdia $\ldots \ldots \ldots \ldots$ effusa $\ldots \ldots \ldots \ldots$.

I.INDERA $\ldots \ldots \ldots \ldots$. Benzoin ...........

Linnaea $\ldots \ldots \ldots \ldots$. Americana ........

Linum ........... striatum ......... usitatissimum ..... Virginianım .......

LiPARIS .......... liliifolia $\ldots \ldots \ldots \ldots$.

LIPPIA .......... lanceolata .........

LIQUidAM BAR $\ldots \ldots \ldots$. styraciflua $\ldots \ldots \ldots$.

LIRIODENDRON $\ldots \ldots \ldots$. Tulipifera
289
289
289
289
289
289
289
289
289
289
176
176
176
183
183
183
183
372

372 372

74

74

74

151

317

317

329

329

227

228

228

228

228

228

296

236

292

350

350

30

30
261

261

361

361

292

292

292

292

236

236

340

340

271

253

253
Lithospermum $\ldots \ldots .338$

arvense ......... 338

canescens ........ 339

laifolium ........ 338

Lobaria .......... 153

amplissima ....... 153

LobeLIA ........... 364

amoena glandulifera. 365

cardinalis ........ 364

inflata ......... 365

inflata simplex..... 365

leptostachys ...... 365

puberula ........ 365

spicata ......... 365

spicata parviflora.... 365

syphilitica ........ 365

syphilitica albiflora...

LOGANIACEAE

Lolium . ..........

Italicum .........

perenne ...........

Lonicera ..........

Canadensis ........

dioica ............

glauca ...........

Japonica ..........

sempervirens .......

Lophanthus .........342

nepetoides ......... 342

Lophidium .......... 106

compressum ....... 105

nitidum ......... 106

LOPHIOSTOMACEAE. 106

Lophiotrema ........ 106

nucula ......... 106

Lophocolea ........ 166

bidentata ........ 166

heterophylla ...... 166

minor .......... 166

LOPHODERMIUM $\ldots \ldots \ldots .119$

culmigenum ...... 119

pinastri .......... 119

Rhododendri ....... 119

LopHozIA .........

Marchica .........

ventricosa .........

LORANTHACEAE ...

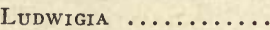

alternifolia .........

alternifolia linearifo-

lia .......... 310

Lunularia $\ldots \ldots \ldots \ldots 163$

cruciata ......... 163

Lupinus .......... 284

perennis $\ldots \ldots \ldots \ldots 284$

Luzula .............. 226

campestris ....... 226

pilosa .......... 226 saltuensis ........ 226

vernalis ......... 226

LYCHNIS ......... 249

Githago ......... 249

Lycrum . ....... 349

vulgare $\ldots \ldots \ldots \ldots . \quad 349$

Lycogala $\ldots \ldots \ldots \ldots .31$

conicum .......... 31

epidendron $\ldots \ldots \ldots \ldots \quad 31$

LYCOPERDACEAE .. 24

Ly COPERDON ....... 25

furfuraceum $\ldots \ldots \ldots \quad 25$

gemmatum ....... 25

pedicellatum ...... 25

pyriforme ........ 25

Lycopersicon $\ldots \ldots \ldots \quad 349$

Lycopersicon ..... 349

LYCOPODIACEAE $\quad \ldots \quad 197$

Lycopodrum $\ldots \ldots \ldots .197$

annotinum ....... 197

clavatum ........ 197

complanatum ...... 198

dendroldeum ...... 197

lucidulum ....... 197

obscurum ........ 197

tristachyum $\ldots \ldots \ldots \quad 197$

Lycopus .......... 346

Americanus ...... 346

communis ........ 346

membranaceus ..... 346

sinuatus ........ 346

Virginicus $\ldots \ldots \ldots \ldots 346$

Lysias .......... 234

orbiculata ........ 234

Lysimachia $\ldots \ldots \ldots \ldots .327$

Nummularia ..... 327

quadrifolia ....... 327

stricta $\ldots \ldots \ldots \ldots \ldots \quad 327$

terrestris ........ 327

thyrsiflora $\ldots \ldots \ldots .327$

LYTHRACEAE .... 309

Maclura $\ldots \ldots \ldots \ldots .242$

aurantiaca $\ldots \ldots \ldots .242$

Macrosporium $\ldots \ldots .40$

Abutilonis ........ 41

antennaeforme $\ldots . .41$

asclepiadeum ...... 40

caudatum ........ 41

commune ....... 40

Maydis ......... 41

olivaceum ....... 41

Saponariae ....... $4 \mathrm{P}$

Tomato ......... 4i

Magnolia ......... 252.

acuminata ....... 252

Fraseri ......... 253

tripetala ........ 252

Umbrella ........ 252 
MAGNOLIACEAE ...

Malus ............ angustifolia $\ldots \ldots \ldots$ coronaria .......... glaucescens .......

Malva ............. moschata...$\ldots \ldots$ rotundifolia .......

MALVACEAE ......

Marasmius ..........

androsaceus ....... campanulatus ...... erythropus ......... foetidus...$\ldots \ldots$. instititus..$\ldots \ldots \ldots$. opacus $\ldots \ldots \ldots \ldots$. oreades ........... perforans .......... ramealis ........... rotula...$\ldots \ldots \ldots$. scorodonius ....... semihirtipes ....... splanchnoides ......

Marchantia ........ polymorpha...$\ldots$. MARCHANTIACEAE.

Markubium $\ldots \ldots \ldots \ldots$ vulgare $\ldots \ldots \ldots \ldots$.

Marshallia ......... grandiflora ........ obovata platyphylla..

Marsonia ........... Juglandis $\ldots \ldots \ldots$. Martini ..........

Marsupella ......... emarginata ........

Massaria ........... Magnoliae ........

Matricaria ......... discoidea ........... matricarioides ......

Medeola ........... Vilrginiana $\quad \ldots \ldots \ldots$.

Medicago .......... lupulina $\ldots \ldots \ldots \ldots$. sativa $\ldots \ldots \ldots \ldots \ldots$

Meehania .......... cordata $\ldots \ldots \ldots \ldots$.

Meíomia .......... arenicola $\ldots \ldots \ldots$. bracteosa ......... Canadensis ........ canescens ......... Dillenii ........... grandiflora...$\ldots$. laevigata ......... Marylandica ....... Michauxii nudiflora $\ldots \ldots \ldots \ldots 287$

obtusa $\ldots \ldots \ldots \ldots \ldots 288$

ochroleuca ....... 288

paniculata ....... 288

pauciflora ........ 287

rigida ......... 288

Melampsora $\ldots \ldots \ldots \ldots \quad 79$

farinosa $\ldots \ldots \ldots \ldots \ldots \quad 79$

MELAMPYRUM $\ldots \ldots \ldots .354$

Americanum .......

lineare...$\ldots \ldots \ldots$

MELANCONIACEAE.

Melanconis ...........

modonia ..........

Tiliacea...$\ldots \ldots$.

Melanconium $\ldots . .$.

bicolor ............

oblongum ..........

pallidum ..........

melanomma $\quad . \ldots \ldots \ldots$ pulvis-pyrius...$\ldots$

MELANTHACEAE ..

Melanthium .......

parviflorum .......

Virginicum ........

MELASTOMACEAE...

Melica .............

mutica ...........

Melilotus ...........

alba..$\ldots \ldots \ldots \ldots$

officinalis .........

Melissa ...........

officinalis $\ldots \ldots \ldots$.

MENISTERMACEAE.

Menispermum $\ldots \ldots \ldots$

Canadense...$\ldots \ldots$

Mentha ............... arvensis glabrata.... Canadensis ........ piperita .......... sativa

spicata..$\ldots \ldots \ldots$.

viridis $\ldots \ldots \ldots \ldots$.

MENYANTHACEAE .

Menyanthes $\ldots \ldots \ldots$. trifoliata $\ldots \ldots \ldots \ldots$

Menziesia $\ldots \ldots \ldots \ldots$ globularis .......... pilosa $\ldots \ldots \ldots \ldots$.

Mertensia ..........

Virginica...$\ldots \ldots$

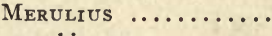

ambiguus $\ldots \ldots \ldots$

Corinum ...........

lachrymans ........

molluscus .........

pulverentus .......

rubellus tremellosus ....... 141

Mesadenia ......... 386

atriplicifolia ....... 386

reniformis $\ldots \ldots \ldots .386$

Metzgeria ......... 164

conjugata $\ldots \ldots \ldots \ldots \quad 164$

crassipilis ........ 164

Micrampelis ....... 363

echinata ........ 363

lobata .......... 363

Microcera ........ 48

erumpens $\ldots \ldots \ldots \ldots .48$

98 Mrcrococcus ........ 28

98 aurantiacus ...... 28

98 crepusculum ....... 28

74 luteus .......... 28

75 septicus .......... 28

74 ureae ............ 28

74 Microlejeunea ...... 170

101 lucens .......... 170

101 Microsphaera ....... 87

$226 \quad$ Alni $\ldots \ldots \ldots \ldots \ldots . . .67$

226 Alni-Vaccinii ...... 88

226 diffusa $\ldots \ldots \ldots \ldots \ldots . . .68$

226 elevata ......... 88

310 erineophila ....... 87

213 Euphorbiae ....... 88

213 Grossulariae ...... 88

285 Quercina ........ 88

285 Russellii ........ 88

285 Vaccinii ........ 88

345 Microstylis ....... 236

345 unifolia ......... 236

260 MICROTHYRIACEAE. 106

260 Milium .......... 209

260 effusum ......... 209

346 Mimulus $\ldots \ldots \ldots \ldots \ldots .352$

346 alatus .......... 352

346 ringens $\ldots \ldots \ldots \ldots .352$

346 Mitchella ......... 358

346 repens $\ldots \ldots \ldots \ldots \ldots 358$

346 Mitella .......... 269

346 diphylla .......... 269

331 Mitremyces ......... 24

331 lutescens ......... 24

331 Mitreola ......... 239

323 petiolata ........ 329

323 Mitrula .......... 107

323 lutescens ......... 107

838 phalloides ........ 107

338 MNIACEAE ........ 180

141 MNIOBRYUM $\ldots \ldots \ldots \ldots .179$

141 albicans ......... 179

141 MNIUM ........... 180

141 cuspidatum ....... 180

141 affine ........... 180

142 hornum ......... 180

141 marginatum ...... 180 
punctatum ........ 180

rostratum ........ 180

MOHRODENDRON ...... 328

Carolinum ........ 328

Molita .......... 177

viridula $\ldots \ldots \ldots \ldots .177$

Mollisia .......... 112

cinerea $\ldots \ldots \ldots \ldots \ldots \quad 112$

Dehnii .......... 112

pinastri ......... 112

Mollugo ........... 248

verticillata $\ldots \ldots \ldots \ldots 248$

Monarda......... 344

Clinopodia ....... 344

didyma $\ldots \ldots \ldots \ldots .344$

fistulosa ......... 344

media .......... 344

scabra ......... 344

Moneses ............

grandiflora .........

uniflora ...........

Monilia ...........

aureo-fulva ........

fructigena.$\ldots \ldots \ldots$.

Monomyces .........

rutilens ...........

Monotropa ..........

Hypopitys ........

uniflora ...........

MONOTROPACEAE .

Monotropsis ........ odorata ...........

MORACEAE ........

MORCHELLA ..........

angusticeps $\ldots \ldots \ldots$.

esculenta .........

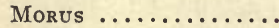

alba.$\ldots \ldots \ldots \ldots \ldots$

rubra ............

Mucor .............

mucedo ............

MUCORACEAE ......

MUCRONOPORUS .......

Everhartii ..........

ferruginosus .......

Gilvus ............

Muhlen bergia ....... diffusa...$\ldots \ldots \ldots$

Mexicana ..........

sobolifera $\ldots \ldots \ldots$.

sylvatica .........

Mulgedium $\ldots \ldots \ldots$......

acuminatum ........

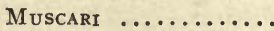

botryoides ..........

racemosum ........

MUSCI ...........

MYCENA $\ldots \ldots \ldots \ldots \ldots$

229 corticola ......... 123

epiterygia..$\ldots \ldots \ldots$.

filopes..$\ldots \ldots \ldots \ldots$

galericulata ........

galopa .............

haematopa...$\ldots$.

pura ..............

rubro-marginata .....

tenerrima .........

vulgaris . ..........

MYICOPORON ...........

Smilacis ..........

Mrosotis ......... 338

arvensis ........ 338

laxa ........... 338

palustris ........ 338

Myriadoporus ....... 141

induratus $\ldots \ldots \ldots \ldots 141$

MyriangIUM ....... 157

Duriaei ........ 157

MYrica ........... 239

asplenifolia ....... 239

MYRICACEAE ..... 239

MyuRella $\ldots \ldots \ldots \ldots .184$

gracilis ......... 184

MYXOMYCETACEAE.

MYxosporium ........

luteum .............

platanicolum ........

Rhois ............

seriatum..$\ldots \ldots \ldots$

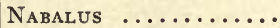

albus..$\ldots \ldots \ldots \ldots$.

altissimus .........

serpentarius .......

NaEmatelia .........

nucleata ...........

Nardia ............

crenulata .........

crenuliformis .......

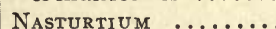

Armoracia .........

obtusum ..........

officinale ..........

palustre ............

palustre hispidum....

sylvestre ..........

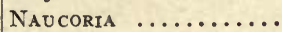

festiva ..........

pediades ..........

semiorbicularis ......

Naumbergia .........

367

2:29

229

173

123

nobilis ...........

rhomboides ........ trinodis .......... 29

viridis $\ldots \ldots \ldots \ldots \ldots 28$

Neckera ........... 183

pennata ......... 183

NECKERACEAE .... 183

Neckeria ......... 262

aurea ........... 263

flavula .......... 263

glauca ........... 262

Nectria ............ 104

cinnabarina ....... 103

coccinea .......... 104

ditissima ...........104

episphaeria ....... 104

Peziza .......... 104

Rexiana .......... 105

vulpina .......... 104

Nemopanthes ....... 298

fascicularis ........ 298

NeOKnEIFfia ....... 144

setigera .......... 144

Nepeta .......... 342

Cataria .......... 342

hederacea ........ 342

NePHROMA ......... 154

Helveticum ....... 154

NicandRa ......... 349

physalodes ........ 349

NIDULARIACEAE .. 24

NigREDO ............ 77

appendiculata $\ldots \ldots \ldots \quad 78$

Caladii .......... 79

caryophyllina ...... 79

fallens .......... 78

Hedysari-paniculati . 78

Houstoniata ...... 78

Howei .......... 79

Hyperici-frondosa ... 79

Junci-effusi $\ldots \ldots \ldots \quad \mathbf{7 7}$

Lespedezae-procumbentis $\ldots \ldots \ldots \ldots \ldots 78$

Medicaginis ....... 78

pedatata ........ 77

plumbaria ........ 79

Polygoni ......... 78

proeminens ....... 78

Rhyncosporae ...... 79

Silphii ......... 77

Spermacoces ....... 79

Trifolii .......... 78

Nitzschia ......... 29

Amphioxys intermedia 29

Nolanea .......... 129

mammosa ........ 129

pascua .......... 129

Notothylas ......... 171

orbicularis ....... 171

Nowella ........... 
curvifolia ..........

Nummularia ........

discreta ..........

punctulata .........

Nyctalis ...........

asterophora ........

Nymphaea .........

advena ............

odorata ...........

NYMPHAEACEAE ..

Nyssa ............ sylvatica $\ldots \ldots \ldots \ldots$

OAKEsia $\ldots \ldots \ldots \ldots$.

sessilifolia

Obolarta

Virginica ..........

Odontra $\ldots \ldots \ldots \ldots$.

farinacea ...........

Odontochisma .......

denudatum ........

prostratum ........

Sphagni .........

Oenothera $\cdots \ldots \ldots$.

biennis ...........

fruticosa ..........

linearis ...........

pumila ............

Ordium ............

erysiphoides .......

leucoconium ........

monilioides ........

OLEACEAE ........

OMBROPHILA $\ldots \ldots \ldots$

clavus .............

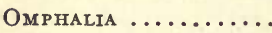

campanella ........

chrysophylla .......

fibula $\ldots \ldots \ldots \ldots \ldots$

stellata $\ldots \ldots \ldots \ldots$.

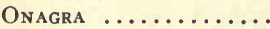

argillicola...$\ldots \ldots$

biennis ...........

biennis grandiflora...

ONAGRACEAE .....

ONOCLEA ............

sensibilis $\ldots \ldots \ldots \ldots$

Onosmodium ........

Carolinianum .......

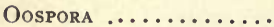
fasciculata $\ldots \ldots \ldots$.

Opegrapha ......... atra..$\ldots \ldots \ldots \ldots$

minutula ..........

varia $\ldots \ldots \ldots \ldots \ldots$

acuminatus ........ 103

fulgidus $\ldots \ldots \ldots \ldots .103$

porphyrogonus ......
167

96
96
96

96
126

126

252

252

252

252

321

321
227

227

330

330
144

144

168

168

168
168

311

311

312

313

312

32

32

32

328

149

149

123

123

123

124

123

311

312

311

311

310

196

196

338

338

31

31

160

160

160

160

103

103

103
OPHIOGLOSSACEAE.

OPHIOGLOSSUM $\ldots \ldots$. vulgatum $\ldots \ldots \ldots \ldots$

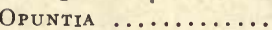

Opuntia ..........

vulgaris ...........

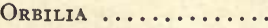

coccinella .........

luteo-rubella ........

occulta ...........

rubella $\ldots \ldots \ldots \ldots$

vinosa $\ldots \ldots \ldots \ldots$.

xanthostigma ......

ORCHIDACEAE ....

Orchis $\ldots \ldots \ldots \ldots$

spectabilis $\ldots \ldots \ldots$.

Ornithogalum $\ldots . .$.

nutans $\ldots \ldots \ldots \ldots$.

umbellatum .......

OROBANCHACEAE

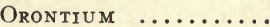

aquaticum .........

ORTHOTRICHACEAE

ORTHOTRICHUM ......

Braunii ............

Ohioense .........

Porteri ...........

strangulatum .......

OSMORRHIZA $\ldots \ldots \ldots$

brevistylis .........

longistylis $\ldots \ldots \ldots$.

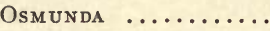

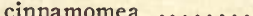

Claytoniana ....... 197

regalis .......... 196

Ostropa ........... 119

cinerea ......... 119

Ostrya ........... 239

Virginiana $\ldots \ldots \ldots \ldots 239$

Otidea ............ 109

onotica ochracea.... 109

OXALIDACEAE .... 291

Oxalis ........... 291

Acetosella ........ 291

cymosa ......... 291

filipes $\ldots \ldots \ldots \ldots \ldots 291$

grandis ......... 291

recurva $\ldots \ldots \ldots \ldots \ldots 291$

stricta ......... 291

violacea ......... 291

Oxycoccus ......... 326

erythrocarpus $\ldots \ldots .326$

macrocarpus ....... 326

Oxycoccus ....... 326

OXYDENDRUM $\ldots \ldots \ldots .324$

arboreum $\ldots \ldots \ldots \ldots 324$

Oxypols $\ldots \ldots \ldots \ldots .315$

rigidior $\ldots \ldots \ldots \ldots .315$ rigidus .......... 315

Ozonium .......... 140

auricomum ....... 140

PACHYSANDRA ....... 296

procumbens ...... 297

Pachystima $\ldots \ldots \ldots .299$

Canbyi ......... 299

Pallavicinia ....... 164

Lyellii .......... 164

Panaeolus .......... 133

campanulatus ...... 133

solidipes ........ 133

Panax .......... 314

quinquefolium ..... 314

trifolium ......... 314

Panicularia ........ 214

Canadensis ........ 214

elongata ......... 215

melicaria ........ 215

nervata .......... 215

nervata major...... 215

Torreyana ....... 215

Panicum ........... 205

agrostoides ....... 206

anceps .......... 206

Ashei .......... 208

barbulatum ...... 207

Boscii ......... 208

capillare ......... 206

capillare campestre.. 206

clandestinum ...... 208

commutatum ...... 208

Crus.Galli ........ 208

depauperatum ..... 206

dichotomiflorum .... 205

dichotomuin ...... 207

dichotomum depaupe-

rata ......... 207

fasciculatum ..... 207

flexile .......... 206

Gattingeri ....... 206

geniculatum ....... 205

gracile ......... 207

Huachucac silvicola.. 207

latifolium ........ 208

Lindheimeri ...... 207

linearifolium ..... 206

lucidum ......... 207

microcarpon ....... 207

nitidum ........ 207

Philadelphicum .... 206

polyanthes ....... 207

Porterianum ...... 205

proliferum ........ 205

pubescens ......... 207

sanguinale ...... 205

stipitatum ....... 206

Tennesseense ..... 207 
tsugetorum $\ldots \ldots \ldots 207$ villosissimum ....... virgatum $\ldots \ldots \ldots \ldots 20$

Pannaria .......... 154 leucosticta $\ldots \ldots \ldots \ldots \quad 154$ nigra $\ldots \ldots \ldots \ldots \ldots 154$ rubiginosa $\ldots \ldots \ldots \ldots 154$

PANNARIACEAE $\ldots 154$

PANUS .......... 127 conchatus ....... 128 dorsalis .......... 128 stipticus $\ldots \ldots \ldots \ldots 127$

PAPAVER...$\ldots \ldots \ldots$. dubium $\ldots \ldots \ldots \ldots$.

PAPAVERACEAE ... 261 PAPILIONACEAE $\ldots \quad 284$

Parietaria .......... Pennsylvanica ......

Parmelia ......... 152 Borreri ......... 152 Borreri rudecta..... 152 caperata ........ 152 cetrata ......... 152 colpodes ........ 152 conspersa ........ 152 exasperata ....... 15 olivacea $\ldots \ldots \ldots \ldots$ 152 olivaria ........ 152 perlata .......... 152 physodes ........ 152 rudecta .......... 152 tiliacea ......... 152

PARMELIACEAE ... 152

Parnassia ......... 268 asarifolia $\ldots \ldots \ldots .268$ grandiflora .........

PARNASSIACEAE ..

PARONYCHIA ......... dichotoma .........

Parsonsta .......... petiolata $\ldots \ldots \ldots \ldots$

Parthenium ........ integrifolium .......

Parthenocissus ...... quinquefolia $\ldots \ldots$.

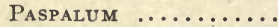
laeve...$\ldots \ldots \ldots$.

laeve pilosum....... mucronatum ....... Muhlenbergii ....... setaceum .........

Passiflora $\ldots \ldots \ldots \ldots$ incarnata $\ldots \ldots \ldots$. lutea .............

PASSIFLORACEAE .

Pastinaca .......... sativa ............. PATELLARIACEAE. . 310

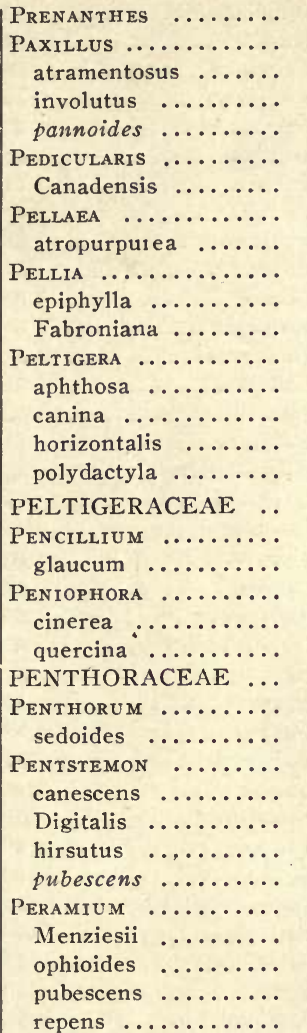

Perichaena .........

flavida ............

Périconia ........... pycnospora ........

310 Perider mium ........

368

131

131

131

127

354

354

192

192

164

164

164

154

154

154

154

154

153

33

33

148

148

148

268

268

268

351

351

351

351

351

235

235

235

235

235

31

31
35

35

85

Balsameum ........

Peckii ...........

Perilla $\ldots \ldots \ldots \ldots \ldots$

frutescens .........

t: utescens Nankinensis

ocymoides crispa.....

PERISPORACEAE ...

Peronospora .........

Arthuri ..........

Corydalis .........

obovata ...........

, parasitica .........

PERONOSPORACEAE

Perularia ..........

flava ............ 23

Pertusaria ......... 156

corallina ....... 156

leioplaca $\ldots \ldots \ldots \ldots .156$ inultipuncta $\ldots \ldots \ldots .156$

pustulata ........ 156

velata $\ldots \ldots \ldots \ldots \ldots 156$

Pestalozzia $\ldots \ldots \ldots \ldots$ 7j

funerea $\ldots \ldots \ldots \ldots \ldots \quad 75$

Guepini ......... 75

Jefferisii ........ 75

toxica .......... 75

Petunia .......... 350

violacea ......... 350

Peziza ........... 108

aurelia .......... 114

aurantia ......... 108

badia .......... 108

cinerea ......... 112

repanda ........... 10 s

vesiculosa ........ 108

PEZIZACEAE ..... 10s

Phacelia ......... 337

bipinnatifida $\ldots \ldots \ldots \quad 337$

dubia .......... 337

parviflora $\ldots . . . \ldots .337$

Purshii ......... 337

PHACIDIACEAE .... 117

Phagozus ......... 136

sistotremoides ..... 136

Phaeopeziza ....... 100

scabrosa .......... 109

Phalaris ......... 209

arundinacea ...... 209

Canariensis ....... 209

PHALLACEAE ..... 24

Phaseolus ......... 290

helvolus ......... 290

perennis ......... 290

pulystachyus ....... 290

Phegnpteris $\ldots \ldots \ldots . \quad 194$

Dryopteris ....... 195

hexagonoptera ..... 195

Phegopteris ....... 195

polypodioides ..... 195

Philalea ......... 111

cyathoides ........ 111

scutula ......... 111

virgultorum ...... 111

Philonotis ......... 181

fontana $\ldots \ldots \ldots \ldots \quad 181$

Philotria ......... 203

Canadensis ....... 203

Phlebia ........... 144

merismoides ....... 144

radiata $\ldots \ldots \ldots \ldots \ldots \quad 14:$

Pinlettar . .......... 210

prateise ......... 210

Phlox ........... 335

amoena $\ldots \ldots \ldots \ldots .335$

Brittonii ........ 336

divaricata $\ldots \ldots \ldots .335$

maculata ........ 335 
paniculata ......... paniculata acuminata. reptaiss subulata ...........

Phlyctaena .........

arcuata ............

Ipomoeae .......... septorioides ....... vagabunda $\ldots \ldots \ldots$.

Pholiota .......... adiposa ........... candicans..$\ldots \ldots \ldots$ caperala . ......... erebia ............ nututabilis ..........

Phoma ..............

Asclepiadea .......

herbarum ........

ilicicola ..........

leucostoma ........

nielaleuca .........

mixta ............ negundicole ramicola. Nyssaecarpa ....... obscurans ......... pedunculi ......... Pennsylvanica ...... Phytolaccae ....... samararum ........ scabra ........... Smilacina ......... Spiraeae ......... uvicola ...........

Phoradendron ....... flavescens .........

Phragmidium .......

Americanum ....... disciflorum ........ Potentillae ........ Rubi-odorati ........ subcorticium .......

Phragmites ......... Phragmites ........

Phryma .............. Leptostachya ......

PHRYMACEAE .....

Phyllachora ........ Caricis ........... Graminis ......... pomagena .........

Phyllactinia ....... corylea ........... suffulta ..........

Phyllanthus ........ Carolinensis .......

Phyllosticta ....... acericola .......... althaeina
Ampelopsidis ......

Araliae ..........

Asiminae ..........

caryigena .........

Castaneae .........

Catalpae ..........

Celastri ...........

Celtidis ..........

cercidicola .........

Chionanthi ........

Cookei ............

cornicola ..........

Dioscoreae ........

Galicis ...........

gentianicola ........

globifera ..........

Hamamelidis .......

Haynaldi ..........

Ipomoeae ........

Labruscae .........

Lappae ...........

lindericola .........

Liriodendrica .......

macrospora ........

Negundinis...$\ldots$.

Opaca

Oxydendri .........

Paviae ............

phomiformis .......

Pirina ...........

pirina .............

Quercus-prini .......

rhoicola ..........

Ribis ...........

Rosae ...........

Sambuci .........

Sanguinariae ......

Sassafras ..........

Smilacis subeffusa... solitaria ..........

Tiliae ...........

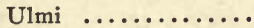

Xanthorrhizae ......

\section{Physalis}

angulata $\ldots \ldots \ldots \ldots$

heterophylla ........

lanceolata .........

Philadelphica ......

pubescens ..........

Virginiana $\ldots \ldots \ldots$.

viscosa ......... 349

Physalodes ......... 349

Physalodes ....... 349

Physalospora .......

Dracaenae .........

Ilicis .............

Physarum $\ldots \ldots \ldots \ldots$

citrinum...$\ldots \ldots$.
$50 \mid$ psittacinum ....... 29

pulcherrimum ..... 29

rufipes .......... 29

sinuosum ........ 29

Physcia $\ldots \ldots \ldots \ldots \ldots 153$

aquila $\ldots \ldots \ldots \ldots \ldots \quad 153$

ciliaris ......... 153

ciliaris crinalis...... 153

comosa .......... 153

leucomela ........ 153

leucomelaena ...... 153

speciosa ......... 153

stellaris ......... 153

stellaris apiolia..... 133

PHYSCIACEAE .... 153

Physcomitrium $\quad \ldots . . .179$

priforme ........ 179

Physma .......... 154

luridum ......... 154

Physocarpa ........ 272

opulifolia ........ 272

Physostegia ....... 343

Virginiana ....... 343

Phytolacca ........ 248

decandra ......... 248

PHYTOLACCACEAE. 248

PhytophthoRA ...... 26

infestans ........ 26

Picea ............. 200

Mariana ......... 200

rubens ......... 200

Pieris ............ 324

floribunda ....... 324

Mariana ......... 324

Pilacre ........... 43

gracillipes $\ldots \ldots \ldots \ldots \quad 43$

Petersii ......... 43

Pilea $\ldots \ldots \ldots \ldots \ldots 242$

pumila .......... 243

Pileolaria ......... 86

Toxicodendri ..... 86

Pimpinella ....... 318

integerrima ...... 318

PINACEAE ........ 199

Pinus ............ 199

echinata ......... 200

inops ........... 200

mitis ........... 200

pungens ......... 200

resinosa .......... 199

rigida $\ldots \ldots \ldots \ldots \ldots 200$

Strobus ......... 199

Taeda .......... 200

Virginiana ....... 200

96 Placodium ......... 155

96 camptidium ...... 155

29 cerinum $\ldots \ldots \ldots \ldots .155$

29 cinnabarinum ...... 155 
elegans $\ldots \ldots \ldots \ldots .155$

ferrugineum ...... 155

rupestre $\ldots \ldots \ldots \ldots \quad 155$

Plagiochila ........ 166

porelloides ....... 166

Sullivantii $\ldots \ldots \ldots \quad 166$

Virginica $\ldots \ldots \ldots \ldots \quad 166$

Plagiothecium $\ldots . . .186$

delicatulum densum.. 186

denticulatum ...... 186

micans ......... 187

striatellum $\ldots \ldots \ldots \ldots \quad 187$

Sullivantiae ...... 187

sylvaticum $\ldots \ldots \ldots . \quad 187$

turfaceum $\ldots \ldots \ldots \ldots \quad 187$

PLANTAGINACEAE .

Plantago ...........

aristata $\ldots \ldots \ldots \ldots$

lanceolata .........

major ...........

Rugelii ...........

Virginiana $\ldots \ldots \ldots$.

Plasmopara .........

Cubensis ...........

Geranii ...........

Halstedii .........

obducens ...........

ribicola ...........

viticola .............

PLATANACEAE ....

Platanus ......................

occidentalis $\ldots \ldots \ldots$.

Platygyrium ........

repens .......... 183

Pleonectria ....... 105

denigrata ........ 105

Missouriensis ..... 105

Pleosphaeria ....... 103

corticola ......... 103

Pleuridium $\ldots \ldots \ldots \ldots .173$

alternifolum ...... 173

Pleurotus $\quad \ldots \ldots \ldots . .124$

corticatus ........ 124

cyphelliformis ..... 124

niger ........... 124

petaloides ........ 124

sapidus ......... 124

serotinus ........ 124

Pluteus ........... 129

admirabilis ....... 129

cervinus ........ 129

longistriatus $\ldots \ldots \ldots \quad 129$

Plicariella ......... 109

miniata ........ 109

scabrosa ......... 109

Plowrightia $\ldots \ldots \ldots .106$

morbosa ......... 106

Pot $\ldots \ldots \ldots \ldots \ldots .214$ alsodes $\ldots \ldots \ldots \ldots \ldots$

annua ........... 214

a utumnalis ....... 214

brevifolia ........ 214

compressa ......... 214

compressa depauperata 214

flexuosa .......... 214

pratensis ........ 214

sylvestris ....... 214

trivialis ......... 214

Podophyllum ....... 260

peltatum ........ 260

Podosphaera $\ldots \ldots \ldots . \quad 86$

leucotricha ....... 86

Oxyacanthae $\ldots . . .86$

tridactyla ........ 86

Podostemon ......... 267

Ceratophyllum ..... 267

PODOSTEMONACEAE 267

Podostroma .......... 105

alutaceum ........ 105

Pogonatum .......... 181

brevicaule ......... 181

Pogonia ........... 235

ophioglossoides .... 235

trianthophora ...... 236

verticillata ....... 235

Pohlia ........... 179

nutans ......... 179

Poinsetria ......... 295

dentata ......... 295

POLEMONIACEAE ..

Polemonium ........

coeruleum .........

reptans ...........

Van Bruniae........

Polycodium ........

stamineum $\ldots \ldots \ldots$.

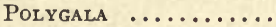

ambigua..$\ldots \ldots \ldots$

cruciata...$\ldots \ldots$.

Curtissii ..........

fastigiata...$\ldots \ldots$

Mariana ..........

Nuttallii .........

paucifolia .........

sanguinea .........

Senega ...........

verticillata $\ldots \ldots \ldots$.

viridescens $\ldots \ldots \ldots$.

viridescens albiflora.

POLYGALACEAE ...

POLYGONACEAE ...

Polygonatum .......

biflorum ..........

commutatum .......

giganteum .........

Polygonum ......... acre $\ldots \ldots \ldots \ldots \ldots 246$

arifolium $\ldots \ldots \ldots .247$

aviculare $\ldots \ldots \ldots \ldots \quad 246$

cilinode ......... 246

Convolvulus ...... 247

cristatum ........ 247

erectum ........ 246

Hydropiper ....... 246

hydropiperoides .... 246

orientale $\ldots \ldots \ldots \ldots 246$

Pennsylvanicum .... 246

Persicaria ........ 246

Persicaria albiflora.. 246

punctatum ....... 246

sagittatum $\ldots \ldots \ldots 247$

scandens $\ldots \ldots \ldots \ldots 247$

tenue .......... 246

Virginianum $\quad . \ldots \ldots 246$

Polymnia ......... 380

Canadensis ...... 380

Canadensis radiata... 380

Uvedalia ........ 380

Polymyces ......... 121

cinereus $\ldots \ldots \ldots \ldots .121$

Polypodium $\ldots . . . . .191$

incanum ......... 192

polypodiodes ...... 192

vulgare .......... 191

vulgare biserrata.... 191

vulgare deceptum ... 191

POLYPORACEAE ... 133

POLYPORUS ......... 131

adustus ......... 135

arcularius ........ 134

Berkeleyi ......... 136

brumalis ......... 134

elegans ......... 135

fissus $\ldots \ldots \ldots \ldots \ldots 134$

lactifluus ........ 136

nidulans ......... 136

Pilotae .......... 136

pocula ........... 135

Polyporus ........ 134

prolificans ........ 138

pubescens ........ 138

resinous ......... 135

sulphureus ....... 136

umbellatus ....... 136

rarius .......... 135

Polyscytalum ...... 32

sericeum ........ 32

Polystichum $\ldots \ldots \ldots .195$

acrostichioides .... 195

acrostichioides

Schweinitzii ..... 195

Polystictus ........ 138

abietinus ......... 138

cinnabąrinus $\ldots \ldots \ldots 138$ 


\begin{tabular}{|c|c|c|c|c|c|}
\hline tus & 138 & & 76 & cis & \\
\hline amenus & 138 & Canadensis & 277 & Caricis-Asteris & 79 \\
\hline versicolor & 138 & Canadensis simplex.. & 277 & Chrysanthemi.... . & 80 \\
\hline OLYTHELIS & 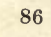 & Monspeliensis ...... & 277 & Circaeae........ & \\
\hline$\ldots \ldots \ldots \ldots \ldots$ & 86 & rvegica....... & 277 & Conoclinii $\quad \ldots \ldots \ldots$ & 2 \\
\hline DLYTHRINCIUM $\ldots .$. & 36 & $\ldots \ldots \ldots$ & 277 & Convolvuli $\ldots \ldots \ldots$ & 31 \\
\hline lii $\quad \ldots . . . \ldots$. & 6 & & 277 & n....... & 81 \\
\hline POLYTRICHACEAE.. & 181 & POTERIU M & 278 & n........ & 0 \\
\hline POLYTRICHUM $\ldots \ldots$ & 81 & ense & 278 & Dayi $\ldots \ldots \ldots \ldots$ & 80 \\
\hline nune $\ldots . . . \cdots$ & 181 & EAE $\ldots \ldots$ & 177 & oniae $\ldots \ldots \ldots \ldots$ & 80 \\
\hline$\ldots \ldots \ldots$ & 31 & PREN & 368 & ridis $\ldots \ldots \ldots$ & 80 \\
\hline ense........ & & $a l b$ & 36 & ta $\ldots \ldots \ldots$ & 80 \\
\hline$u m \quad \ldots \ldots \ldots$ & & $\ldots \ldots \ldots$ & 36 & $s \ldots \ldots \ldots \ldots$ & 30 \\
\hline ......... & 181 & aria $\quad . . . . .$. & 369 & i $\ldots \ldots \cdots$ & 80 \\
\hline $\mathrm{EAE} \quad \ldots \ldots \ldots$ & 279 & PRIMULACEAE .... & 327 & e $\quad \cdots \cdots \cdots$ & 82 \\
\hline . $\ldots \ldots \ldots \ldots$ & 237 & $\ldots \ldots$ & 167 & ntis $\ldots \ldots \ldots$ & $\begin{array}{l}81 \\
80\end{array}$ \\
\hline$\cdots \ldots \ldots \ldots \cdots$ & 237 & ianus $\quad . . . . .$. & 167 & ita $\ldots . . . \ldots \ldots$ & 80 \\
\hline ns $\ldots \ldots \ldots$. & 238 & n........ & 117 & ipes $\quad \ldots \ldots \ldots \ldots$ & 80 \\
\hline …....... & & & 117 & iae $\ldots . . . . .$. & 80 \\
\hline itáta..... & 25 & Le & 117 & malvacearum ..... & 02 \\
\hline$\ldots \ldots \ldots$ & & Prosartes & 230 & Mariae-Wilsoni ..... & 82 \\
\hline tre & & $\operatorname{lan}$ & 230 & ae $\ldots \ldots \ldots \ldots$ & 81 \\
\hline OREL & & LA $\quad \cdots \cdots \cdots$ & 342 & ergiae.... . & 82 \\
\hline w....... & & $\ldots \ldots \ldots \ldots$ & 34 & $\ldots \ldots \ldots \ldots$ & 82 \\
\hline $\begin{array}{l}\cdots \cdots \cdots \\
\cdots \ldots \cdots \cdots\end{array}$ & 1 & is albiflora & 3 & ........... & 82 \\
\hline ns & 1 & $\ldots \ldots \ldots \ldots$ & 28 & $a \ldots \ldots \ldots$ & 82 \\
\hline $\begin{array}{l}\text { ens } \\
\text { la } \ldots \ldots \ldots . . .\end{array}$ & & . $\ldots \ldots \ldots$ & 28 & ellae....... & 81 \\
\hline ella $\ldots \ldots \ldots \ldots \ldots$ & 1 & $\cdots \cdots \cdots$ & & ......... & 83 \\
\hline ns $\quad \ldots \ldots \ldots \ldots \ldots$ & 1 & $\because \cdots \cdots \cdots$ & & li $\ldots \ldots \ldots$ & 80 \\
\hline$\cdots \cdots \cdots$ & 1 & a $\ldots \cdots$ & 28 & -amphibii ... & 80 \\
\hline ns & 1 & & & $\ldots \ldots \ldots \ldots$ & 80 \\
\hline & 1 & 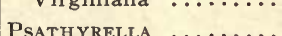 & & era $\ldots \ldots$ & 81 \\
\hline icta $\ldots .$. & 1 & ta $\ldots \ldots \ldots$ & 13 & e $\quad \ldots \ldots \ldots$ & 82 \\
\hline ae $\ldots \ldots \ldots$ & 1 & TIUM $\ldots . .$. & 111 & $\ldots \ldots \ldots \ldots$ & \\
\hline & 13 & & 111 & s $\ldots . . . .$. & 82 \\
\hline 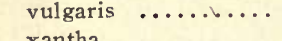 & & $\mathrm{ZIZA} \quad \ldots$. & $11:$ & olens $\ldots . . . .$. . & 81 \\
\hline & & ginis...... & 1 & $\ldots \ldots \ldots$ & 80 \\
\hline $\begin{array}{c}\text { us } \ldots \ldots \ldots \\
\ldots \ldots \ldots \ldots\end{array}$ & 1 & i $\quad . . .6 \ldots$ & 11 & oniae........ & 82 \\
\hline IUM $\ldots \ldots \ldots$ & 1 & IENIDIA $\ldots \ldots$ & 31 & Veratri $\quad \ldots \ldots \ldots \ldots$ & 82 \\
\hline IUM & 1 & $\cdots$ & 3 & . & 81 \\
\hline THus $\ldots \ldots \ldots$ & 27 & ALSA $\quad \ldots \ldots$ & 10 & riae $\ldots$. & 82 \\
\hline & & $\cdots$ & 10 & n........ & 82 \\
\hline$\ldots \ldots \ldots$ & 27 & dea & 65 & TRUM & 83 \\
\hline oRTUlaca $\ldots \ldots \ldots$ & 24 & Ptelea & 292 & Agrimoniae $\ldots . . .$. & 83 \\
\hline$\ldots \ldots \ldots$ & 24 & $\cdots \cdots$ & 29 & Hydrangeae ........ & 83 \\
\hline LACACEAE .. & 248 & PTE & 19 & im $\ldots .$. & 83 \\
\hline on & 20 & Pr & 169 & LETUS $\ldots \ldots$ & 34 \\
\hline ... & & & 169 & .. & 134 \\
\hline$\cdots$ & 2 & ......... & 188 & IEMUM & 345 \\
\hline$f l$ & 2 & renge & 188 & dioides ...... & 345 \\
\hline es & 20 & ........ & 79 & ........... & 346 \\
\hline & & & & ium & 35 \\
\hline$\cdots \cdots \cdots$ & 2 & 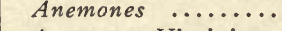 & & $u m$ & 46 \\
\hline GETO & & iniana. & 82 & n........ & 346 \\
\hline & & 31 & 79 & $y i \quad \ldots$. & 345 \\
\hline Potentilla & 277 & Asteris & 80 & ullia & 345 \\
\hline
\end{tabular}




\begin{tabular}{|c|c|c|c|c|c|}
\hline & & & & Carolınıana & \\
\hline us $\ldots \ldots \ldots$. & & ata & & ata & \\
\hline inus $\ldots . .$. & & nctoria $\ldots \ldots \ldots \ldots$ & 11 & RHeX1A & \\
\hline YLAISIA $\ldots \ldots$. & 34 & velutina & & Virginica $\quad \ldots \ldots \ldots$ & \\
\hline ta $\ldots \ldots \ldots \ldots$ & 84 & .... & 99 & HIZINA $\ldots$ & \\
\hline velutina $\quad \ldots \ldots \ldots \ldots$ & 84 & lanta $\ldots \ldots \ldots$ & 0 & .... & \\
\hline YRENOPEZIZA $\ldots \ldots \ldots$ & 12 & $x \ldots \ldots$ & 9 & DFNDRON $\ldots \ldots$ & \\
\hline lacerata $\ldots \ldots \ldots$. & 12 & ensis ... & 9 & wbiense & \\
\hline ORA $\quad \ldots \ldots \ldots$ & 2 & $\ldots \ldots \ldots \ldots$ & & $\mathrm{am}$ & \\
\hline cens $\ldots \ldots \ldots$ & 2 & liae $\ldots \ldots \ldots$ & 14 & $\ldots \ldots \ldots \ldots$ & \\
\hline LA $\ldots \ldots \ldots \ldots$ & 32 & are $\ldots . .$. & & $\ldots \ldots \ldots$ & \\
\hline$a \quad \ldots .$. & 32 & $\ldots \ldots \ldots$ & & $\cdots \cdots \cdots$ & \\
\hline$\cdots$ & 2 & is fastigiata.. & & $\ldots \ldots \ldots$ & \\
\hline$\ldots \ldots \ldots$ & 62 & ula $\ldots . .$. . & & les ......... & \\
\hline$\ldots \ldots \ldots$ & 2 & $\ldots \ldots \ldots \ldots$ & 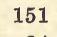 & $\ldots \ldots \ldots \ldots$ & \\
\hline$\ldots \ldots \ldots$ & 2 & A & & $\ldots \ldots \ldots \ldots$ & \\
\hline ACEAE $\ldots$ & & ri $\ldots \ldots \ldots \ldots$ & 4 & $s \quad \ldots \ldots \ldots$ & \\
\hline$\ldots \ldots \ldots \ldots$ & & ci $\quad \ldots \ldots \ldots \ldots$ & & endron $\ldots .$. & \\
\hline$\ldots \ldots \ldots \ldots$ & & i $\ldots \ldots \ldots$ & & $\ldots \ldots \ldots$ & \\
\hline & 1 & & & $t a \ldots \ldots \ldots$ & \\
\hline EAE $\ldots \ldots$ & & s $\ldots \ldots \ldots$ & & $\ldots \ldots \ldots \ldots$ & \\
\hline RUS $\ldots \ldots$. & 7 & is $\ldots \ldots \ldots$ & & STEGIUM $\ldots$. & \\
\hline as $\ldots \ldots$. & & $\ldots \ldots \ldots \ldots$ & & $m \ldots \ldots$ & \\
\hline s $\ldots \ldots \ldots$ & & 1aniensis $\ldots \ldots$ & & me $\ldots \ldots \ldots$ & \\
\hline ae $\ldots \ldots \ldots$ & & $n s \quad \ldots \ldots \ldots$ & & $1 \mathrm{~m} \ldots \ldots$ & \\
\hline$A \quad \therefore \ldots \ldots \ldots$ & & is $\ldots \ldots \ldots \ldots$ & & $\ldots \ldots \ldots \ldots$ & \\
\hline$\cdots$ & & is $\quad \ldots$ & & $n \quad \ldots \ldots \ldots$ & \\
\hline$\cdots \cdots$ & & $\ldots \ldots$ & & orans $\ldots \ldots \ldots$ & \\
\hline$a$. & & s $\ldots \ldots \ldots$ & & $\ldots \ldots \ldots \ldots$ & \\
\hline $\operatorname{lin}$ & & us ... & & $\ldots \ldots \ldots$ & \\
\hline$i a \quad \ldots \ldots \ldots$ & 279 & icus .... & & $\ldots \ldots \ldots$ & \\
\hline$a r b$ & & 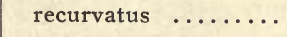 & & $\mathrm{ti} \ldots \ldots \ldots$ & \\
\hline$\ldots \ldots \ldots$ & & $\ldots \ldots \ldots$ & & $\ldots \ldots \ldots$ & \\
\hline$i a \quad \ldots$ & & & & $\operatorname{um} \ldots$ & \\
\hline$\ldots \ldots \ldots \ldots$ & 1 & lis & & $\operatorname{lium} \ldots$ & \\
\hline a $\ldots$ & & & & $\cdots \cdots$ & \\
\hline$\cdots \cdots$ & & $\ldots \ldots \ldots$ & & $s \cdots \ldots$ & \\
\hline na. & & ECIUM & & la & \\
\hline IT $\quad \ldots \ldots \ldots \ldots$ & & & 189 & $\cdots \cdots$ & \\
\hline & & num admix- & & & \\
\hline s $\ldots$. & & $\ldots \ldots \ldots \ldots$ & 1 & ara $\ldots .$. & \\
\hline & & um .. & $1 \xi$ & $\mathrm{AE}$ & \\
\hline & & dicum $\ldots \ldots$ & & $\mathrm{A} \cdots \cdots \cdots \cdots$ & \\
\hline & & $A \quad \ldots \ldots \ldots \ldots$ & 86 & $s \quad \ldots \ldots \ldots \ldots$ & \\
\hline ........ & & • & & A $\ldots$ & \\
\hline$\cdots$ & & LIA $\ldots \ldots \ldots$ & & $\ldots \ldots \ldots$ & \\
\hline$\ldots \ldots \ldots \ldots$ & & & & es $\ldots$ & \\
\hline & & A $\ldots$ & & $\ldots \ldots \ldots$ & \\
\hline$a \quad \ldots . .$. & & 11 . & & & \\
\hline pa ........ & & RA $\ldots$ & & Acacia..... & \\
\hline & & 11. & & & \\
\hline & & OWEISIA .. & & . & \\
\hline & & & & ia $\ldots$. & \\
\hline$\cdots \cdots \cdots$ & & TRIUM . & 17 & & \\
\hline & & & & ium $\quad \ldots \ldots \ldots$ & \\
\hline & & CEAE . & & $\ldots \ldots \ldots$ & 2 \\
\hline . & & s $\quad \ldots \ldots \ldots$ & & .......... & \\
\hline A. $12 \pi n c 0$ & $2 \pm 1$ & ifolia $\ldots \ldots \ldots$ & 301 & ylvestris & $20=$ \\
\hline
\end{tabular}


INDEX, LIVING FLORA.

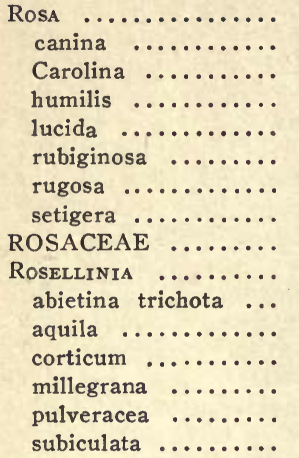

Rotala ............. ramosior ..........

Rozites $. . . . . \ldots .$. caperata ........... RUBIASEAE ....... Rubus ............ Ayllegheniensis .... Americanus ........ argutus ............ Baileyanus ......... Baileyanus frondosus Canadensis ......... Canadensis roribaccus Columbianus ....... hispidus .......... Millspaughii ....... nigrobaccus...... . occidentalis ........ odoratus ........... strigosus........ triflorus ........... trivialis........ . villosus ............ villosus humifusus..

RUDBECKIA ........... fulgida $\ldots \ldots \ldots \ldots$. hirta.......... laciniata .......... laciniata humilis ... speciosa ........... triloba.........

Ruellia ............. ciliosa .............

Rumex $\ldots \ldots \ldots \ldots . .$. Acetosella ......... Brittanica ......... conglomeratus ...... crispus ........... crispus $x$ obtusifolius. . obtusifolius ........ sanguineus ........ Russula

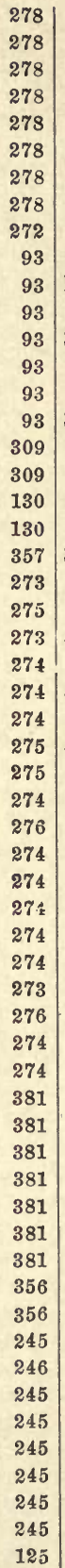

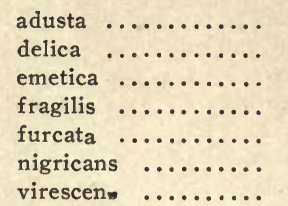

Ruta ...............

graveolens .........

RUTACEAE .......

RYNCHOSPORA $\ldots \ldots$...

glomerata ..........

Sabbatia ............

angularis...$\ldots \ldots$.

lanceolata .........

Saccharom Yces ...... cerevisae....... . mycoderma ........

SACCHAROMYCETACEAE

SAccidium ............ viticolum .......... vitis $. . . \ldots . . . . .$.

Saccogyna ........... graveolens .........

Sagina.......... procumbens ........

Sagittaria ........... angustifolia ........ arifolia ........... graminea .......... SALICACEAE ....... Salix ............... alba-vitellina.... . amygdaloides ........ Babylonica ........ cordata ........... discolor ............ humilis ........... nigra ............. nigra falcata ....... sericea ...........

SALOMONIA........ biflora......... . commutata ......... Salsola $\ldots . . . \ldots \ldots$. Tragus...$\ldots \ldots$...

Salvia $\ldots . . . \ldots \ldots$. lyrata..........

Sambucus ........... Canadensis ......... pubens ............ pubens albicocca ... racemosus .........

Samolus ............. floribundus ........ Sanguinaria ......... Canadensis .........

\begin{tabular}{|c|c|c|}
\hline & Av & \\
\hline & Canadensis . & \\
\hline & ANICULA $\ldots \ldots$ & \\
\hline & Canadensis $\quad . . .$. & \\
\hline & a $\ldots$. & \\
\hline & Idica $\ldots . .$. & \\
\hline & $\ldots \ldots \ldots$ & \\
\hline & SANTALACEAE $\ldots$ & \\
\hline & SAPINDACEAE $\ldots$. & \\
\hline & SAPONARIA $\ldots \ldots \ldots$ & 249 \\
\hline & officinalis $\ldots \ldots \ldots$. & 249 \\
\hline & SARCINELLA $\ldots \ldots \ldots$ & 49 \\
\hline & ospora..... . & \\
\hline & DON $\ldots \ldots \ldots \ldots$ & 43 \\
\hline & us $\ldots \ldots \ldots$ & 143 \\
\hline & SARCOSCYPHA $\ldots \ldots \ldots$ & 09 \\
\hline & $a \quad \ldots \ldots \ldots$ & 09 \\
\hline & $\ldots \ldots \ldots \ldots$ & 09 \\
\hline & entalis $\ldots \ldots$ & 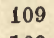 \\
\hline & $n \ldots \ldots \ldots \ldots$ & 09 \\
\hline & MA $\ldots \ldots \ldots \ldots$ & 16 \\
\hline & n........ & 16 \\
\hline & $\ldots \ldots \ldots$ & 06 \\
\hline & oides ........ & 06 \\
\hline & Sassafras $\quad \ldots \ldots \ldots \ldots$ & 61 \\
\hline & nale $\ldots . .$. & 61 \\
\hline & s $\ldots \ldots \ldots$ & 61 \\
\hline & ACEAE $\ldots$. & 37 \\
\hline & $\ldots \ldots \ldots \ldots$ & 37 \\
\hline & us $\quad \ldots \ldots \ldots$ & 37 \\
\hline & Saxifraga $\quad \ldots \ldots \ldots$ & 69 \\
\hline & 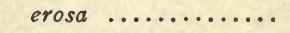 & 69 \\
\hline & nthidifolia $\quad \ldots$. & 39 \\
\hline & Ivanica $\quad \ldots \ldots$ & 69 \\
\hline & nsis $\ldots \ldots \ldots$ & 69 \\
\hline & GACEAE .. & 69 \\
\hline & $\ldots \ldots \ldots \ldots$ & 169 \\
\hline & a $\ldots \ldots \ldots$ & 69 \\
\hline & $\ldots \ldots \ldots$ & 69 \\
\hline & ERIA $\ldots \ldots$ & 202 \\
\hline & $\ldots \ldots \ldots \ldots$ & 02 \\
\hline & HZERIA- & \\
\hline & $\ldots \ldots \ldots$ & 02 \\
\hline & ETA- & \\
\hline & ........... & 27 \\
\hline & HYLLUM $\ldots .$. & 128 \\
\hline & $\ldots \ldots \ldots \ldots$ & 128 \\
\hline & ne $\ldots \ldots \ldots$ & 128 \\
\hline & ss $\ldots \ldots \ldots \ldots$ & 218 \\
\hline & ricanus $\ldots \ldots$. & 218 \\
\hline & ns $\ldots \ldots \ldots$ & 219 \\
\hline & nus $\ldots \ldots \ldots$. & 219 \\
\hline & & '218 \\
\hline & rum $\ldots$ & 219 \\
\hline & is $\ldots \ldots \ldots \ldots$ & 218 \\
\hline & s $\quad \ldots \ldots \ldots$ & 219 \\
\hline & lus $\ldots$. & 219 \\
\hline & pungens & 218 \\
\hline
\end{tabular}


sylvaticus $\ldots \ldots \ldots \ldots$.

ScLERoderma $\ldots \ldots \ldots$.

Bovista $\ldots \ldots \ldots$.

vulgare $\ldots \ldots \ldots \ldots$.

ScleroderRIS $\ldots \ldots \ldots$.

pallidula ...........

Rhabarbarina ......

Sclerotinia ...........

fructigena $\ldots \ldots \ldots$.

trifoliorum $\ldots \ldots \ldots$.

tuberosa $\ldots \ldots \ldots \ldots$.

Scoleonectria ....... 105

scoleosperma ...... 105

Scorias ...........

spongiosa $\ldots \ldots \ldots \ldots$

Scoriomycrs ........

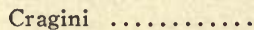

Scorteus .......... oreades $\ldots \ldots \ldots \ldots$.

Scrophularia $\ldots \ldots \ldots$ leporella ..........

Marilandica ........

SCROPHULAR-

IACEAE

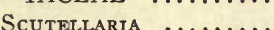

Canescens ..........

cordifolia .........

galericulata .......

galericulata albiflora

incana ...........

integrifolia......

lateriflora $. . . \ldots .$.

nervosa ..........

parkula ..........

pilosa ...........

pilosa hirsuta ......

saxatilis $\ldots \ldots \ldots \ldots$.

serrata ...........

versicolor minor ...

SEDUM $\ldots \ldots \ldots \ldots \ldots$

Nevii...$\ldots \ldots \ldots$.

pulchellum ........

telephiodes......

Telephium ........

ternatum...$\ldots \ldots$

SELAGINELLA $\ldots \ldots \ldots$.

apus $\ldots \ldots \ldots \ldots \ldots$

rupestris $\ldots \ldots \ldots \ldots$

SELAGINELLACEAE.

SEMATOPHYLLA-

CEAE

SENicio

...........

antennariifolius $\ldots$.

aureus ............

Balsamitae ....... 387

obovatus ........ 387

vulgaris $\ldots \ldots \ldots \ldots$.
SEPTORIA

atro-purpurea ......

aciculosa $\ldots \ldots \ldots \ldots$.

Brassicae .........

cornicula ........

Cornus-Maris .....

Helianthi .........

hyalina ..........

kalmiaecola .......

Leptostachyae .....

Lobeliae

microsperma ......

Nabali ...........

Nolitangerae ......

ochroleuca .........

Polygonorum .......

Polymniae ........

psilostega .........

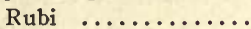

Saxifragae .......

Scrophulariae ......

Smilacinae .......

Tecomae ..........

Trautvetteriae .....

Verbenae .........

Violae ...........

Septosporium $\ldots \ldots \ldots$

Equiseti $\ldots \ldots \ldots \ldots$

Sericocarpus $\ldots \ldots \ldots$

asteroides $\ldots \ldots \ldots$.

linifolius $\ldots \ldots \ldots \ldots$

Setaria $\ldots \ldots \ldots \ldots$

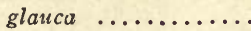

imberbis ..........

Italica $\ldots \ldots \ldots \ldots \ldots$

viridis ...........

Siherardia $\ldots \ldots \ldots$.

arvensis $\ldots \ldots \ldots \ldots$

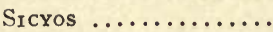

angulatus $\ldots \ldots \ldots$.

SIDA

hermaphrodita ......

Napaea ..........

spinosa ...........

Silene ...........

alba $\ldots \ldots \ldots \ldots$

antirrhina...$\ldots \ldots$

Caroliniana ........

nivea.........

Pennsylvanica .....

stellata ...........

Virginica $\ldots \ldots \ldots$.

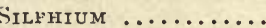

Asteriscus $\ldots \ldots \ldots$.

perfoliatum $\ldots \ldots \ldots$

trifoliatum ........

SIMARUBACEAE ...

Sirococcus
66

69

68

67

67

67

68

68

67

68

68

67

69

68

67

69

67

69

67

68

68

69

66

67

68

68

42

42

374

374

374

208

208

208

208

208

359

359

364

364

304

304

304

304

249

249

249

249

243

249

249

249

380

380

380

380

293

57
Ialesiae ........ 57

SisYMERIUM $\ldots \ldots \ldots .263$

altissimum ........ 263

officinale ........ 263

Thalianum ....... 266

SISYRINCHIVM ....... 233

anceps .......... 233

angustifolium ..... 233

Bermudianum ..... 233

graminoicies ...... 233

mucronatun $\ldots \ldots \ldots 233$

Srum $\ldots \ldots \ldots \ldots \ldots . \ldots \ldots$

cicutaefolium $\ldots \ldots .315$

SMIILACEAE ...... 232

Smilacina ......... 230

bifolia Canadensis .. 230

Smilax .......... 232

glauca .......... 239

herbacea ........ 232

hispida $\ldots \ldots \ldots \ldots .232$

Pseudn-China ..... 232

rotundifolia ...... 232

tamnifolia ........ 232

SOLANACEAE ..... 347

Solanum ......... 347

Carolinense ...... 347

Dulcamara ....... 347

nigrum $\ldots \ldots \ldots \ldots 347$

rostratum ....... 347

tuberosum $\ldots \ldots \ldots \ldots \quad 347$

Solea .......... 309

Concoler ......... 309

Solenia .......... 142

ochracea ........ 142

villosa $\ldots \ldots \ldots \ldots \ldots \quad 142$

Solenostoma $\ldots \ldots \ldots .165$

lanceolata $\ldots \ldots \ldots .165$

Solidago $\ldots \ldots \ldots \ldots .372$

arguta $\ldots \ldots \ldots \ldots \ldots 373$

bicolor ........ 373

Boottii ......... 373

caesia .......... 372

Canadensts ........ 374

Curtisii ......... 372

flexicaulis ....... 372

juncea $\ldots \ldots \ldots \ldots .373$

juncea ramosa ..... 374

juncea scabrella.... 373

lanccolata ........ 374

latifolia ......... 372

monticola ........ 373

neglecta $\ldots \ldots \ldots \ldots .373$

nemoralis ....... 374

odora .......... 373

puberula ........ 373

rugosa $\ldots \ldots \ldots \ldots \ldots \quad 373$

rupestris ........ 374

serotina $\ldots \ldots \ldots \ldots \quad 374$ 


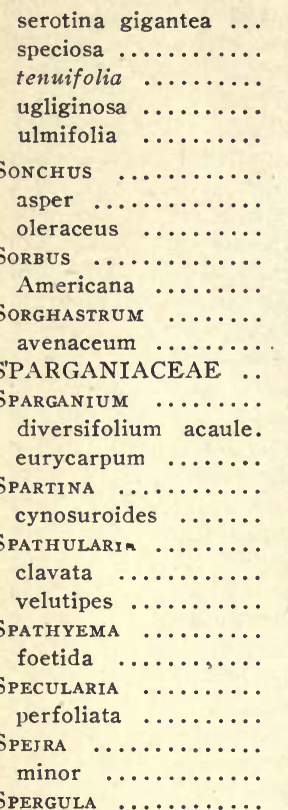

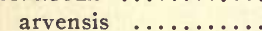

Sphacelotheca $\ldots \ldots$ Hydropiperis ......

ShaErella $\ldots \ldots \ldots \ldots$ conicola ........... Gaultheriae ....... infuscens...$\ldots \ldots$. maculiformis ...... nyssaecola ........

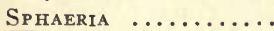

Kalmianum .......

SphaErocephalus ....

heterostichus ......

Sphaerographum .... Fraxini .......... hystericinum ...... SPHAEOPSIDEAE . acerinum $\ldots \ldots \ldots \ldots$ corneum ........... infuscans $\ldots \ldots \ldots \ldots$ Magnoliae ......... Physocarpi ........ NHAEOPSIDEAE .. Sphaeropsis $\ldots \ldots \ldots$. Ampelopsidis ...... Asiminae ......... Asiminae fructigena. Caryae ............ Celastrina
Cerasina

62 iStACHYLIDIUM ...... 35

Ipomoeae

62

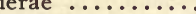

Malorum ..........

Menispermi $\quad . . \ldots \ldots$

Oenotherae .........

phomatella ........

Physocarpi ........

Sassafras .........

Sumachi .........

Sphaerotheca .......

Castagne1 .........

Humuli .........

Humuli-fuliginea ....

Mali ............

Mors-Uvae ........

pannosa ...........

SPHAGNACEAE $\ldots . .172$

Sphagnum ........ 172

cymbifolium ...... 172

cymbifolium squar-

rulosum ....... 172

Girgensohnii ...... 172

imbricatum affine ... 172

Magellanicum ..... 172

quinquefarium ..... 172

recurvum ........ 172

Sphenolobus ....... 167

excectus ........ 167

Michauxii ....... 167

minutus $\ldots \ldots \ldots \ldots 167$

SPIRAEA ........ 272

Aruncus .......... 273

betulifolia ....... 272

corymbosa ....... 272

lobata .......... 276

salicifolia $\ldots \ldots \ldots \ldots 273$

tomentosa $\ldots \ldots \ldots .273$

Ulmaria $\ldots \ldots \ldots \ldots \quad 276$

Virginiana $\ldots \ldots \ldots 272$

Sptranthes $\ldots \ldots \ldots \ldots 235$

cernua ......... 235

gracilts ......... 235

Spirillum $\ldots \ldots \ldots \ldots 28$

undula $\ldots \ldots \ldots \ldots \ldots 28$

Sporobolus ........ 210

neglectus ........ 210

vaginaeflorus $\ldots \ldots 210$

Sporocybe ......... 43

Azaleae ......... 43

Rhois ......... 43

SPORODESMIUM $\ldots \ldots \ldots \quad 40$ aurantiacum $\ldots \ldots .40$ concinnum ...... 40 moriforme ....... 40 toruloides ........ 40

SPOROdINIA $\ldots \ldots \ldots \ldots .26$

Aspergillus $\ldots \ldots \ldots .26$

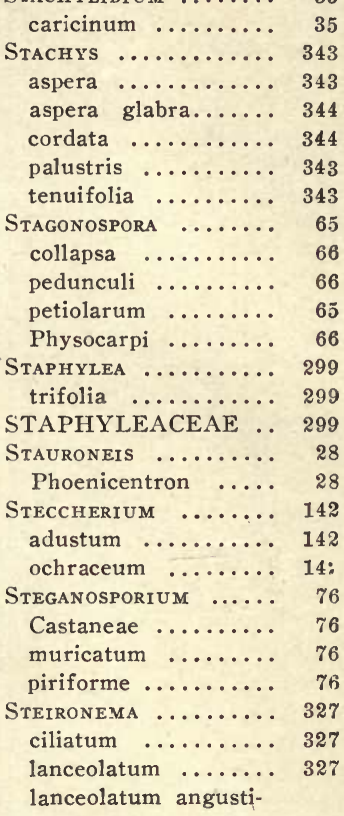

folium $\ldots \ldots \ldots \ldots .327$

Stellaria $\ldots \ldots \ldots \ldots .250$

media ........... 250

longifolia ........ 250

pubera .......... 250

Stenophragma ...... 266

Thaliana ........ 266

Stephania ........ 170

complanata ....... 170

Stereodon .......... 188

cupressiformis ..... 188

curvifolius ....... 188

imponens ........ 188

recurvans $\ldots \ldots \ldots \ldots .189$

Stereum .......... 144

acerinum $\ldots \ldots \ldots \ldots \quad 146$

albo-badium $\ldots \ldots \ldots \quad 145$

complicatum ..... 145

fasciatum $\ldots \ldots \ldots \ldots \quad 145$

frustulosum ...... 145

hirsutum $\ldots \ldots \ldots \ldots \quad 145$

lobatum ........ 145

ochraceo-flavum .... 145

Pini ........... 145

purpureum ....... 145

sanguinolentum $\ldots . \quad 145$

sericeum ........ 145

sulphuratum $\ldots \ldots \ldots \quad 145$

triste $\ldots \ldots \ldots \ldots \ldots \quad 145$ 


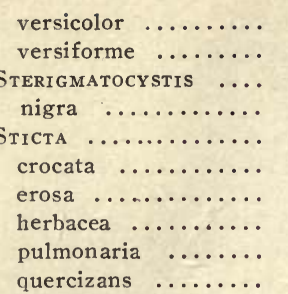

STICTACEAE .......

Stictis .............

fimbriata

STILBACEAE ........

Stilbum $\ldots . . . \cdots \ldots$.

erythrocephalum ....

flavipes $\ldots \ldots \ldots \ldots$.

magnum ..........

vulgare.........

Stremontis $\ldots . . . \cdots$

ferruginea $\ldots \ldots \ldots$.

fusca...$\ldots \ldots \ldots$

Smithii ............

Streptococcus ........

pyogenes...$\ldots \ldots$

ureae...........

StREPtopus ...........

roseus $\ldots \ldots \ldots \ldots$.

Streptothrix $\ldots \ldots \ldots$

atra $\ldots \ldots \ldots \ldots$

STROBILOMYCES .......... strobilaceus ........

Stropharia $\ldots . . . \cdots$ semiglobata...$\ldots$. stercoraria .........

Strophostyles ....... helvola ...........

StYlophorum ......... diphyllum ..........

Stylosanthes ........ biflora ............ elatiar ............ procumbens ........ riparia ............

STYRACACEAE .....

SYMPHORICARPOS ...... orbiculata .......... Symphoricarpos .... 362

Symphytum $\ldots . \ldots \ldots .338$ officinale ......... 338

SyMPLOCARPUS $\ldots \ldots \ldots \quad 223$

foetidus .......... 223

SynANDRA $\ldots \ldots \ldots \ldots \ldots, 343$ hispidula $\ldots \ldots \ldots \ldots \quad 343$

SYNCHYTRIUM $\ldots . . . .$. decipiens $\ldots \ldots \ldots .$.

Syndesmon $\ldots \ldots \ldots \ldots \quad 257$ thalictroides $\ldots \ldots \ldots \quad 257$ thalictroides rosea... 257

Synedra $\ldots \ldots \ldots \ldots$.

ulna ..............

Synosma .............

suaveolens $\ldots \ldots \ldots$.

Syntherisma $\ldots . . .$.

linearis ............

sanguinalis......

TaEnidia $\ldots \ldots \ldots \ldots$

integerrima .........

TAGETES $\ldots \ldots \ldots \ldots$

patula .............

Tanacetum ...........

vulgare $\ldots \ldots \ldots \ldots$.

TAPESIA $\ldots \ldots \ldots \ldots$

fusca $\ldots \ldots \ldots \ldots \ldots \quad 112$

sanguinea ........ 112

subiculata ........ 113

Taphrina ................. 118

deformans ........ 118

Pruni ........... 118

TaPinia ........... 127

panuoides $\ldots \ldots \ldots \ldots \quad 127$

Taraxacum $\ldots \ldots \ldots \ldots .366$

erythrospermum $\ldots . \quad 367$

officinale .......... 366

Taraxacum ........ 366

TAXACEAE ........ 201

Taxus ..............

baccata Canadensis..

Canadensis .........

Tecoma ...............

radicans $\ldots . . . \ldots$.

Teichospora ...........

nitida ..............

TEPHRosia $\cdots \cdots \ldots .$.

Virginiana ..........

Tethraphis .............

pellucida ...........

Teucrium ............

Canadense .........

Thalesia $\ldots \ldots \ldots \ldots$

uniflora ............

Thalictrum ..........

anemonoides .......

clavatum ...........

dioicum ...........

polygamum......

purpurascens .......

Thamnium ...........

Allegheniense.... .

Thaspium $\ldots \ldots \ldots \ldots$

barbinode ......... 317

trifoliatum $\ldots \ldots \ldots . \quad 317$

trifoliatum aureum.. 317

THELEPHORA $\ldots \ldots \ldots .144$

pedicellata ....... 144

sebacea
THELEPHORACEAE. 144

Thelia ............ 184

asprella $\ldots \ldots \ldots \ldots \ldots$. 185

hirtella .......... 184

Thelochistes $\ldots \ldots \ldots \quad 151$

concolor .......... 152

concolor effusa..... $\quad 152$

parietinus ......... 151

Thelotrema $\ldots \ldots \ldots \ldots \quad 156$

subtile ........... 156

THERofon $\ldots \ldots \ldots \ldots .269$

aconitifolium ....... 269

Thuidium $\ldots \ldots \ldots \ldots .185$

delicatulum ....... 185

microphyllum ..... 185

minutulum ........ 185

recognitum ........ 185

scitum ........... 185

Virginianum ...... 185

Tнuja ............. 199

occidentalis ...... 199

THYMELEACEAE $\quad \ldots \quad 309$

Thyronectria ....... 105

denigrata ........ 105

Missouriensis ...... 105

ThYRSIDIUM $\ldots \ldots \ldots .75$

hedericolum Carpini. 75

TIARELla $\ldots \ldots \ldots \ldots .269$

cordifolia $\ldots \ldots \ldots .269$

Tredemannia $\ldots \ldots \ldots .315$

rigida $\ldots \ldots \ldots \ldots .315$

TILIA .............. 303

Americana ....... 303

heterophylla ...... 303

Michauxii ........ 303

TILIACEAE $\ldots \ldots \ldots .303$

Tilletia $\ldots \ldots \ldots \ldots .77$

striaeformis $\ldots \ldots \ldots \quad 77$

Tritici .......... $7 \boldsymbol{7}$

Tilmadoche ........ 29

gyrocephala ....... 29

nutans $\ldots \ldots \ldots \ldots \ldots, 29$

viridis $\ldots \ldots \ldots \ldots \ldots \quad 29$

Tipularia $\ldots \ldots \ldots \ldots .235$

unifolia .......... 235

TithYMALOPSIS $\ldots \ldots \ldots \quad 294$

corollata ......... 294

Tithymalus ........ 295

commutatus ....... 295

Cyparissias ........ 295

Darlingtonii ....... 295

Lathyrus ......... 295

obtusatus ........ 295

Tortella $\ldots \ldots \ldots \ldots$. 177

humilis $\ldots \ldots \ldots \ldots, 177$

tortuosa $\ldots \ldots \ldots \ldots \quad 177$

unguiculata $\ldots \ldots \ldots \quad 177$

144 Tortula $\ldots \ldots \ldots \ldots .177$ 
muralis

Torula $\ldots \ldots \ldots \ldots \ldots$

dimidiata

herbarum

TOXYLCN

pomiterum ...........

Tradescantia $\ldots \ldots \ldots$

pilosa...$\ldots \ldots \ldots$

Virginiana $\ldots \ldots \ldots$.

Tragupogon $\ldots \ldots \ldots \ldots$

porrifolius ........

Trametes ........... mollis ........... 140

odorata ......... 140

Ohioensis ........ 140

robineophila ....... 140

Sepium .......... 139

Tranzschelia $\ldots \ldots \ldots \quad 86$ punctata ........ 86

Trautvetteria ...... 258

Carolinensis ...... 258

palmata .......... 258

Trematosphaeria $\ldots \ldots \quad 101$

vitigena $\ldots \ldots \ldots \ldots \ldots 101$

Tremella ......... 149

aurantia ......... 149

frondosa $\ldots \ldots \ldots \ldots \quad 149$

intumescens ...... 149

virens .......... 150

Tremellodon $\ldots \ldots \ldots 143$

gelatinosum ....... 143

TrIADENUM $\ldots \ldots \ldots \ldots 306$

Virginicum $\ldots \ldots \ldots 306$

Trichaegum $\ldots \ldots \ldots$. nodulosum

TRICHIA

chrysosperma

fallax

proximella

Trichocolea .........

tomentella $\ldots \ldots \ldots$.

Trichoderma $\ldots \ldots \ldots$

lignorum .........

Tricholoma .........

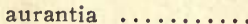

fumoso-luteum ....

grave...$\ldots \ldots \ldots$.

Peckii ............

rutilens.......

TrichopezizA .......

albo-lutea .........

capitata ..........

Trichosphaieria .... pulchrista

Trichostema ........ dichotomum ........

TRICHOSTOMUM ....... pallidum ..........
TRICHOTHECIUM .....

roseum $\ldots \ldots \ldots \ldots \ldots$
Tricuspis $\ldots \ldots \ldots \ldots \ldots$
seslerioides $\ldots \ldots \ldots \ldots$

Trientalis..$\ldots \ldots \ldots$

Americana ........

TRIFOLIUM .........

agrarium ..........

arvense ...........

aureum ...........

hybridum..$\ldots \ldots \ldots$.

pratense ..........

procumbens .......

repens ...........

stoloniferum $\ldots \ldots \ldots$.

Virginicum .......

Trillium $\ldots \ldots \ldots \ldots$

cernuum .........

erectum ..........

erectum album.....

erectum declinatum..

erythrocarpum ......

grandiflorum ......

nivale

sessile..$\ldots \ldots \ldots \ldots$

undulatum ........

Trimmatostroma ....

Americana ........

TRIODIA

cuprea ............

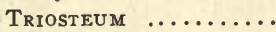

perfoliatum,$\ldots \ldots \ldots$

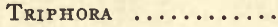

trianthophora $\ldots \ldots$.

Tripsacum ..........

dactyloides $\ldots \ldots \ldots$.

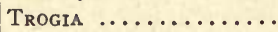

crispa $\ldots \ldots \ldots \ldots \ldots$

TRYPETHELIACEAE.

Trypethelium ....... 162

exocanthum ...... 162

virens $\ldots \ldots \ldots \ldots \ldots$ : 162
34 gracilis .......... 133

34 Tyдostoma ......... 24

212 mammosum ....... 24

212 TyMpanis ......... 115

328 conspersa ........ 115

328 Oxydendri ........ 115

285 TYрна ........... 201

286 latifolia ......... 201

285 TYPHACEAE $\ldots \ldots \ldots \quad 201$

286 TYROMYCES .......... 135

286 chioneus ........ 135

285 undosus ......... 135

286 Ulex ............ 284

285 Europaeus ....... 284

286 ULMACEAE $\ldots \ldots \ldots .242$

285 Ulmaria .......... 276

231 rubra $\ldots \ldots \ldots \ldots \ldots . \ldots 276$

231 Ulmaria ......... 276

231 Ulmus ........... 242

231 Americana ....... 242

«31 fulva .......... 242

231 racemosa $\ldots \ldots \ldots \ldots 242$

231 Ulocolla .......... 149

231 foliacea $\ldots \ldots \ldots \ldots ., 149$

231 Ulota ............ 178

231 Americana ....... 178

48 Hutchinsiae ....... 178

48 Ludwegii ......... 178

212 ulophylla ......... 178

212 UMBELLIFERAE ... 325

361 UMBILICARIA ........ 153

361 Dillenii .......... 153

236 Muhlenbergii ..... 15.3

236 papulosa ......... 153

3 Pennsylvanica .... 153

203 pustulata ........ 153

128 UMBILICARIACEAE. 153

128 UNCINULA .......... 87

Americana ......... 87

Ampelopsidis ...... 87

circinata $\ldots \ldots \ldots \ldots .6 \%$

geniculata $\ldots \ldots \ldots . \quad 87$

macrospora $\ldots \ldots \ldots . \quad 87$

necator $\ldots \ldots \ldots \ldots . \quad 87$

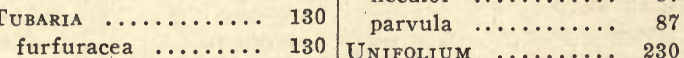

Tubercularia $\ldots \ldots \ldots .44$ Canadense $4 . \ldots \ldots .230$

Celastri ......... 44 racemosum ....... 230

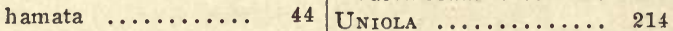

Sambuci ........ $44 \quad$ latifolia $\ldots \ldots \ldots \ldots . . \ldots 214$

vulgaris $\ldots \ldots \ldots \ldots \cdot 44$ UREDINACEAE $\ldots . .77$

TUBERCULARIACEAE 44 UREDO $\ldots \ldots \ldots \ldots \ldots . . . . . . .65$

Tuberculina $\ldots \ldots \ldots .45$ Agrimoniae..$\ldots \ldots .85$

persicina $\ldots \ldots \ldots \ldots .45 \quad$ Bigelowif $\ldots \ldots \ldots \ldots .85$

Tubulina .......... 30 Medusae ......... 85

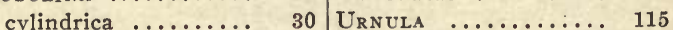

TYlopilus $\ldots \ldots \ldots \ldots .133$ craterium $\ldots \ldots \ldots .115$

alboaster $\ldots \ldots \ldots \ldots 133$ Urocystis $13 . \ldots \ldots \ldots .77$ 


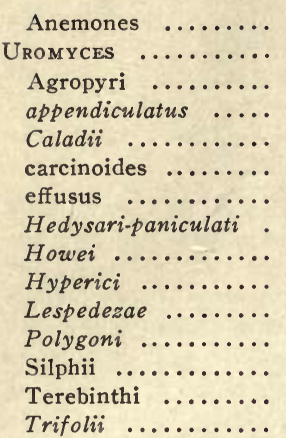

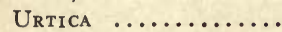

gracilis $\ldots \ldots \ldots \ldots$.

urens $\ldots \ldots \ldots \ldots \ldots$.

URTICACEAE $\ldots \ldots$.

URTICASTRUM $\ldots \ldots \ldots$.

divaricatum $\ldots \ldots \ldots$

UsNeA $\ldots \ldots \ldots \ldots \ldots$

barbata ......... 151

barbata florida..... 151

trichodes ........ 151

USNEACEAE ....... 151

USTILAGINACEAE .

UstiLAGo $\ldots \ldots \ldots \ldots$.

anomala ...........

Avenae ...........

Avenalaevis .......

cesati ............

laevis ............

Maydis ...........

perennans .........

segetum $\ldots \ldots \ldots \ldots$.

tricuspidis $\ldots \ldots \ldots$.

Tritici $\ldots \ldots \ldots \ldots \ldots$

Tritici ..........

utriculosa $\ldots \ldots \ldots \ldots$

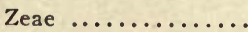

Ustulina $\ldots \ldots \ldots \ldots$

vulgaris $\ldots \ldots \ldots \ldots$

UTRICULARIA $\ldots \ldots \ldots$

biflora ......... 355

cornuta ......... 355

Uvularia ......... 226

grandiflora $\ldots \ldots \ldots 227$

perfoliata ........ 226

puberula ......... 227

sessilifolia $\ldots \ldots \ldots .227$

VACCARIA $\ldots \ldots \ldots \ldots .250$

Vaccaria ......... 250

VACCINIACEAE .... 325

VACCINIUM $\ldots \ldots \ldots \ldots .326$

Canadense ....... 326

corymbosum ....... 326

erythrocarpum ..... 326 macrocarpon ...... 326

mucronatum ....... 298

nigrum $\ldots \ldots \ldots \ldots .326$

pallidum ........ 326

Pennsylvanicum ... 326

stamineum ....... 325

vacillans $\ldots \ldots \ldots \ldots .326$

VAGNERA,$\ldots \ldots \ldots \ldots$

racemosa .........

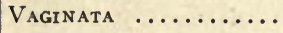

Vaginata...$\ldots \ldots$

VALERIANa ...........

pauciflora ..........

VALERIANACEAE ..

VALERIANELla ....... chenopodifolia ......

Vallisneria ........ spiralis $\ldots \ldots \ldots \ldots$.

VALIISNERIACEAE.

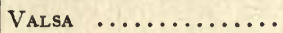

Abietis...$\ldots \ldots \ldots$

albopuncta

ambiens ...........

caryigena .......66, 64

ceratophora ...... 90

Chionanthi ........ 91

coronata...$\ldots \ldots$.

Diospyri ..........

etherialis

goniotoma .........

leucostoma ........

Linderae .........

Nyssae...$\ldots \ldots \ldots$.

pauperata ..........

praestans .........

subclypeata .......

Vitis...$\ldots \ldots \ldots$

Valsaria .............

exasperans $\ldots \ldots \ldots$.

Venenarius $\ldots \ldots \ldots$.

muscarius .........

Veratrum $\ldots \ldots \ldots \ldots$

viride $\ldots \ldots \ldots \ldots$.

Verbascum $\ldots \ldots \ldots$

Blattaria .........

Lychinitis...$\ldots$.

Thapsus ..........

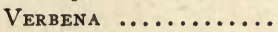

angustifolia $\ldots \ldots \ldots$.

hastata $. . . \ldots \ldots .$.

officinalis $\ldots \ldots \ldots$.

urticaefolia $\ldots \ldots \ldots .340$

VERBENACEAE $\ldots .340$

Verbesina $\ldots \ldots \ldots \ldots .383$

alternifolia ....... 383

occidentalis ...... 383

Vermicutaria ........

Arctii

57
57

compacta dematium ....... 5 r

liliacearum ....... 58

petiolarum ....... 57

phlogina ........ 57

subeffigurata $\ldots \ldots \ldots \quad 57$

subeffigurata scapincola $\ldots \ldots \ldots \ldots \ldots \quad 57$

Toxica ......... 57

Trautvetteriae ..... 57

VERNONIA $\ldots \ldots \ldots \ldots 370$

altissima $\ldots \ldots \ldots \ldots \quad 370$

gigantea $\ldots \ldots \ldots \ldots \quad 370$

maxima ........ 370

maxima pubescens... 370

Noveboracensis ..... 370

Noveboracensis latifo-

lia ........... 370

Veronica $\ldots \ldots \ldots \ldots .352$

Americana ....... 352

Anagallis-aquatica .. 352

arvensis ........ 352

LEPTANDRA ........ 353

officinalis $\ldots \ldots \ldots \ldots \quad 352$

peregrina $\ldots \ldots \ldots \ldots 352$

serpyllifolia ...... 352

Virginica $\ldots \ldots \ldots \ldots \quad 353$

Verrucaria $\ldots \ldots \ldots \ldots 162$

fuscella $\ldots \ldots \ldots \ldots \ldots \quad 162$

punctella ........ 162

VERRUCARIACEAE . 162

Verticillium $\ldots \ldots \ldots .33$

osteophilum ...... 33

puniceum ........ 34

Vibrissea ......... 108

truncorum ....... 108

VIBURNUM $\ldots \ldots \ldots \ldots .360$

acerifolium $\ldots \ldots \ldots 360$

alnifolium ....... 360

cassinoides..$\ldots \ldots .361$

dentatum ........ 361

lantanoides ...... 360

Lentago ........ 361

Opulus Americanum. 361

nudum ......... 361

prunifolium ....... 361

Vicia ........... 289

Caroliniana ...... 289

Viola ........... 306

affinis ......... 307

affinis $x$ cucullata... 308

alsophila ........ 307

amoena .......... 307

blanda .......... 307

Canadensis ........ 308

canina Muhlenbergii. 308

conspersa ........ 308

cucullata ........ 306

fimbriatula ...... 307

hastata ........ 308 
INDEX, LIVING FLORA.

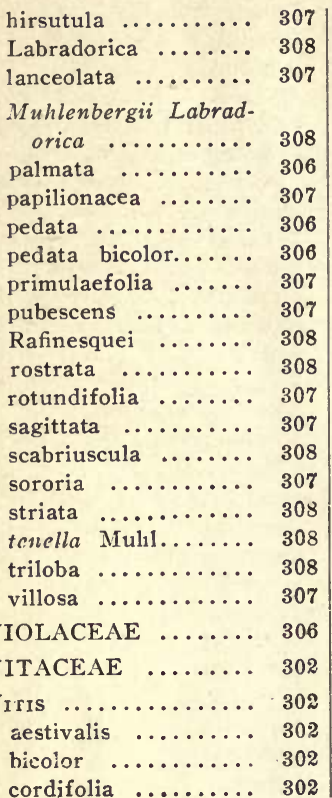

\begin{tabular}{|c|c|}
\hline & \\
\hline a & 02 \\
\hline olia .. & 302 \\
\hline ris $\ldots \ldots \ldots \ldots$. & 302 \\
\hline vulpina $\ldots . \ldots \ldots \ldots$ & 302 \\
\hline olutella $\ldots \ldots \ldots \ldots$ & 43 \\
\hline n.......... & 46 \\
\hline$\cdots \cdots \cdots$ & 128 \\
\hline ...... & $12 \mathrm{~s}$ \\
\hline ta $\ldots \ldots \ldots$ & 129 \\
\hline NIA $\ldots$. & 27 \\
\hline es $\ldots$ & $7 \%$ \\
\hline ONIA $\ldots$ & 318 \\
\hline i $\ldots . . . .$. & 318 \\
\hline iongistyliśs $\ldots \ldots \ldots$ & 319 \\
\hline Weberera $\ldots \ldots \ldots \ldots$ & 181 \\
\hline $\mathrm{pr}$ & 181 \\
\hline sessilis $\ldots \ldots \ldots \ldots$ & 181 \\
\hline WEBERACEAE ... & 181 \\
\hline (....... & 177 \\
\hline Americane ... & 178 \\
\hline ulophylla....... & 178 \\
\hline viridula $\ldots \ldots \ldots \ldots$ & 177 \\
\hline Woodsia $\ldots \ldots \ldots$ & 196 \\
\hline obtusa & 196 \\
\hline A & 6 \\
\hline
\end{tabular}

commune ........ 369

glabratum ......... 369

$\begin{array}{lll}\text { strumarium } & \ldots \ldots & 369\end{array}$

spinosum ......... 369

Xanthorrhiza $\ldots \ldots \ldots \quad 254$

apiifolia ......... 254

XanthoXylum ...... 292

Americanum ...... 292

Xolisma ........... 324

ligustrina ........ 324

ligustrina pubescens. 324

Xylaria ............ 94

corniformis ....... 94

Cornu-Damae ..... 94

flabelliformis ...... 94

Hypoxylon ........ 94

polymorpha ....... 94

Zizia ............. 319

aurea ............ 319

aurea Bebbii....... 319

Bebbii .......... 319

cordata ......... 319

Zygodesmus ..........

graminicola ....... 35

pannosus ......... 35

tiliaceus ......... 35 


\section{SUMMARY OF PART I.}

Genera. Species.

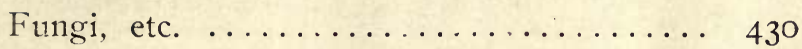

Lichenes .................... 39

Thallephyta, etc. ........... 469

Hepaticae .................... 48

Musci ...................... 68

Sphagnaceae $\ldots \ldots \ldots \ldots \ldots \ldots \ldots \ldots \ldots 2$

Bryophyta $\ldots \ldots \ldots \ldots \ldots \ldots \ldots$ I 18

Equisitae $\ldots \ldots \ldots \ldots \ldots \ldots \ldots \ldots \ldots \ldots$ I

Filices .................... I6

Ophioglossaceae .................. 2

Lycopodiaceae ................. I

Selaginellaceae $\ldots \ldots \ldots \ldots \ldots \ldots \ldots$ I

Pteridophyta

Gymmospermae ................ 8

Monocotyledonae ................ I I

Dicotyledonae $\ldots \ldots \ldots \ldots \ldots \ldots \ldots \ldots .476$

Anthophyta ............... 602

Total number of species, varieties and forms detected in the

State to the date of this Flora.............. 34 I I 


\section{INDEX TO PART II.}

A

\section{ALETHOPTERIS}

aquilina, 407,446

ambigua, 408, 435

decurrens, 407, 438

Evansii, 408, 434

gigas, 409,450

grandifolia, 408, 430

lonchitica, 407, 434, 438

Massilionis, 408, 438

pennsylvanica, 408,443

Serlii (Brongn), 408, 438, 445

Serlii var. americana, 408, 434

Serlii var. europeae, 408, 434

sp.?, 409, 430, 432

virginiana, 408,450

ALLEGHENY FO R M A TION, Plants from, 442

\section{ANEIMITES}

Adiantites, 393

adiantoides, 393,430

fertilis, 393,430

sp., 430

sp.?, 393

tenuifolius, 393, 431

\section{ANNULARIA}

acicularis, 418, 439

carinata, 419,452

cuspidata, 418,435

minuta, 419, 452

radiata, $418,434,439,452$

ramosa, 418, 432, 440

sphenophylloides, 418, 440, 444, 452

sp.?, 419, 432

stellata, 418, 440, 444, 452

\section{APHLEBIA}

filiciformis, $415,447,452$

laciniata, 415

laciniatum, 451

lactuca, 415, 452

speciosissima, 415, 452

spinosa, 415,431

\section{ARCHAEOPTERIS}

alleghaniensis, 392, 429

Bockschiana, 393

Bockschianus, 429

n. sp.?, 393,435

stricta, 393, 435

ASTEROCALAMITES

scrobiculatus, 416, 431, 432

ASTEROPHYLLITES

equisetiformis 417,439

erectifolius, 418,434

gracilis, 417,434

lycopodioides, 417, 439

minutus, 417, 430, 432, 435, 439

rigidus, 418,439

sp., 430

sp.?, 417

\section{BAIERA}

virginiana, 425,453

BETULA

nigra, 429, 453

BOTHRODENDRON

minutifolium, 421, 440

sp.?, 421, 440

\section{C}

\section{CALAMITES}

approximatus, 417, 434, 435,439

cannaeformis, 416, 432

Cistii, 416, 439, 444

ramosus, 417, 439

Roemeri, 416, 434

sp.?, 417,432

Suckowi, 416, 439, 452

CALAMITINA

sp.?, 417, 439

CALAMODENDRON

sp.?, 417, 439

\section{CALAMOSTACHYS}

lanceolata, 419, 432

ramosus, 419, 440

sp. ?, 435
CALLIPTERIDIUM

inaequale, 409,443

sp., 434

sp.?, 409

\section{CALLIPTERIS}

conferta, 409, 450

currentiensis, 409,451

frondosa, 409

lyratifolia, 451

lyratifolia var. coriacea, 409

\section{CARDIOCARPON}

bicuspidatum, 426, 435, 436

cornutum, 426,436

elongatum, 426, 435

minor. $426,435,442$

CARDIOPTERIS

frondosa, 430

CARMICHAELS CLAY,

Fossils from, 453

\section{CARPOLITHES}

bicarpus, 428,453

ellipticus, 428,445

fragarioides, 428,442

marginatus, 428, 453

sp., 430

sp.?, 428, 433

CASTANEA

pumila, 429, 453

CAULOPTERIS

elliptica 415, 451

gigantea, 415,451

CHEILANTHITES

nummularius, 395, 436

obtusilobus, 395,445

obtusilobus var. dilatata, 395,436

solidus, 395,445

squamosus, $395,442,445$

trifoliolatus, 394, 436

CLARK FORMATION,

Plants from, 430

CONEMAUGH F O R M A

TION; Plants from, 445 
CORDAICARPON

cinctum, 426, 442

circulare, 442

circularis, 426

Gutbieri, 426, 446

CORDAITES

borassifolius, 425,441 crassinervis, 425,453

Robbii, 425, 431, 435

CYPERUS

sp.?, 428,453

\section{D}

\section{DICKSONITES}

Pluckeneti, 447

DIPLOTHMEMA

Jacquoti, 395

pachyderma, 395, 447

DUNKARD FORMATION,

Plants from, 447

\section{E}

\section{EQUISETITES}

elongatus, 416, 452

occidentalis, $416,431,432$

rugosus, 415,452

striatus, 416,452

EQUISETUM

arvense L., 428, 453

EREMOPTERIS

artemisiaefolia, 393, 436

Cheathami, 394, 433

decomposita, 394, 433

elegans, 394, 431, 433

lincolniana, 394, 436

macilenta, 394, 430, 433

microphylla, 394, 431

n. sp., 436

Sauveuri, 394, 436

solida, 442

sp.?, 394,435

\section{F}

FAGUS

ferruginea, 429,453

\section{G}

\section{GUILIELMITES}

orbicularis, 428,453

H

\section{HALONIA}

sp.?, 423,433
K

KANAWHA FORMATION, Plants from, 436

L

\section{LEPIDOCYSTIS}

obtusus, 441

siliqua, 429

vesicularis, $424,441,445$, 446

\section{LEPIDODENDRON}

acuminatum, $422,432,440$

Brittsii, 421, 440

clypeatum, 422,441

dichotomum, 422,441

lanceolatum, 422, 440

magnum, 422

modulatum, 422, 441, 444

obovatum, 422, 441

rushvillense, 421,436

scrobiniforme, 421,429

selaginoides, 421,431

Sternbergii, 422, 432, 434

Veltheimianum, 422, 432,

440

\section{LEPIDOPHLOIOS}

laricinus, 422, 441

sp.?, 423, 445

\section{LEPIDOPHYLLUM}

acuminatum 423,441

brevifolium, 423, 445

Campbellianum, 424, 433, 441

cultriforme, 424,441

hastatum, 424,445

Jenneyi, 423, 445

lanceolatum, 424, 441

oblongifolium, 423,445

sp.?, 424, 433, 435, 436

LEPIDOSTROBUS

ornatus, 423,441

Salisburyi, 423, 441

sp.?, 441

sp. nov., 423

variabilis, $423,433,436$,

441

\section{LESCUROPTERIS}

adiantites, 414

adiantoides, 451

Moorii, 414, 447

LINOPTERIS

obliqua, 414, 444

LIQUIDAMBAR styraciflua, 429, 453

LYCOPODITES

Meekii, 421, 440 n. sp., 421,432

pendulus, 421,446

simplex, 421,440

\section{M}

\section{MACROSTACHYA}

sp., 434

sp. ?, 419

\section{MARIOPTERIS}

acuta, 396, 435, 436

Andraena, 396, 437

dimorpha, 396, 431

inflata, 396, 436

Jacquoti, 436

latifolia, 396, 431, 436

muricata, 396, 433, 435, 436

nervosa, 396, 437, 442, 445

Newberryi, 397, 442

pottsvillea, 395, 431, 433

pottsvillea var., 396

pygmaea, 396,435

Sillimanni, 396, 442, 445

sphenopteroides, 397,437 ,

443

spinulosa, 397, 446

MEGALOPTERIS

Dawsoni, 409, 432

Hartii, 410, 434

sewellensis, $410,432,434$

sp. ?, 409, 435

MONONGAHELA FORMA-

TION, Plants from, 446

\section{N}

\section{NEMATOPHYLLUM}

angustum, 418, 452

\section{NEUROPTERIS}

Agassizi, 413, 444

auriculata, 413,451

biformis, 411, 434

callosa, 412, 447

Carrii, 412, 439

Cistii, 412, 439

cordata, 413,451

crenulata, 413, 447

dictyopteroides, 412, 451

Elrodi, 411, 431

fimbriata, 412, 444, 446, 451

flexuosa, 411, 438

Gibbosa, 412, 451

gigantea, 411, 439

Grangeri, 412, 447

Lindleyana, 411, 432 
n. sp. No. 1, 413

odontopteroides, 413

ovata, 411, 439, 444, 446, 451

Planchardi var. longifolia, 411,451

plicata, 412

Pocahontas, 410, 430

rarinervis, $411,439,443$

Scheuchzeri, 413, 439, 444, 446, 447

Schlehani, 410, 434

Smithii, 410, 430, 432

sp. ?, 413, 434, 435

tenuifolia, 411, 430

vermicularis, 412,444

Zeilleri, 413, 439

NUTTALL SANDSTONE,

Plants from, 435

\section{O}

\section{ODONTOPTERIS}

aequalis, 414,444

densifolia, 414, 451

gracillima, 415, 434, 435

nervosa, 414, 451

neuropteroides, 414, 432

Newberryi, 414, 434

obtusiloba var. rarinervis, 414,451

Reichiana, 415, 451

subcuneata, 414, 444

Wortheni, 414, 444

OLIGOCARPIA

alabamensis, 401, 438

sp.?, 401,438

\section{P}

\section{PALAEOXYRIS}

alppendiculata, 428, 442

\section{PECOPTERIS}

angustipinna, 405, 449

arborescens, 404, 449

arborescens var. integripin-

na, 404

arguta, 404, 449

aspera, 403, 438

asplenioides, 405,449

Callipteridium, 407, 450

Candolleana, 404, 449

crenulata, 406,438

Dawsonianiana, 407,450

dentata, 402, 443

dentata var. parva, 405,448

dentata var. crenata, 403 , 448

elegans, 404,449 elliptica, 403, 407, 448, |POACORDAITES

450 sp.?, 425, 442

emarginata, $403,443,448$ P O C O N O FORMATION,

Germari, 402, 448

Germari var. crassinervis, 402,448

402,448 var. cuspidata, PRINCETON CONGLOM-

Goniopteris, 403, 404, 448, 449

Goniopteroides, 404, 449

grandifolia, 407,450

Heeriana, 405,449

imbricata, 405, 449

inclinata, 405, 449

integra, 406, 438

Jenneyi, 406, 446

lanceolata, 405, 449

latifolia, 406, 450

longifolia, 403, 448

Merianopteroides, 406, 450

microphylla, 404, 449

Miltoni, 406, 443, 446, 450

Newberryana, 403, 448

nodosa, 404, 447

notata, 406,446

oblonga, 403, 448

oblongifolia, 407,450

odontopteroides, 407,450

oreopteridia, 405,443, 446, 449

ovoides, 406, 450

pachypteroides, 403,448

pennaeformis var. latifolia, 404, 449

platynervis, 405,449

plumosa, 402, 435, 438

Pluckeneti, 402, 448

Pluckeneti var. constricta, 402,448

polymorpha, 406, 446, 450

pteroides, 406,450

rarinervis, 405,449

rotundifolia, 406,450

rotundiloba,. 405,449

Schimperiana, 405, 449

serrulata, 402, 434

sp.?, 406, 407, 446, 449, 450

subfalcata, 405,449

tenuinervis, 405,449

unita, 403, 407, 443, 445,

$$
446
$$

unitum, 450

vestita, 404,443

villosa, 404, 438,443, 446

\section{PLATANUS}

occidentalis, 429,453 430

\section{QUERCUS}

falcata, 429,453

QUINNIMONT FORMA-

TION, Plants from, 431

\section{$\mathrm{R}$}

\section{RHABDOCARPOS}

amygdalaeformis, 427,442

Bockschianus, 427,442

multistriatus, 426, 442

n. sp., 43 n

oblongatus. 427,453

sp.?, 427,433

sp.. 431,435

sulcatus, 427,442

tenax, $426 \quad 442$

\section{$S$}

SAPORTAEA

grandifolia, 426

salisburioides, 426, 453

S E E E L FORMATION, Plants from, 433

\section{SIGILLARIA}

approximata, 425,453

Brardii, 425, 453

camptotaenia, 424, 445

dentata, 425,433

fissa, 424, 441, 446

ichtyolepis, 425,441

Menardi, 424, 447

reticulata, $424,435,441$

sp.?, 425, 433

\section{SPHENOPHYLLUM}

angustifolium, 420, 452

antiquum, 419,434

cuneifolium, 435, 440

densifolium, 421, 452

emarginatum, 419, 44G, 444

filiculme, 420,452

Fontaineaum, 420, 452

furcatum, 420,440

Lescurianum, 419, 440

longifolium, 420, 452

majus, $420,444,445,446$

oblongifolium, 420,452

tenue, 420,432

tenuifolium, 420,452

Thoni, 420, 452 


\section{SPHENOPTERIS}

acrocarpa, 401,448

auriculata, 400,448

breviloba, 400, 447

Broadheadi, 400, 438

canneltonensis, 401, 438

chaerophylloides, $400,44 \%$

communis, 401, 433

Crepini, 402, 438

Crossotheca, 397, 402, 438, 443,445

Cymoglossa, . 447

delicatula, 399,437

dentata, 400, 447

dicksonoides, 399, 431

distans, 398, 430

divaricata, 398, 431

dubuissonis, 399,437

elegans, 397, 437

flexicaulis, 399,433

foliosa, 400, 448

formosa, 400,447

furcata, 397, 435, 437

geniculata, 398,437

Goepperti, 398, 431

hastata, 401, 448

Hildrethi, 398, 437

Hoeninghausi, 398, 430, 431

hymenophylloides, 399,437 karwinensis, 399,437

Larischii, 399, 433

Lescuriana, 400, 448

linearis, 398, 435

Linkii, 399, 437

lobata, 400,447

microcarpa, 400, 432, 433, 437

minutisecta, 400, 445, 447

mixta, 401, 438

obtusifolia, 447

obtusiloba, 400

ophioglossoides, 397,402 , $438,443,445$

pachynervis, 401

patentissima, 397, 431

pinnatifida, 399, 443

rarinervis, 398,430

Renaultia, 400, 432, 437

Royi, 397, 433

sagittatus, 402,443

schatzlarensis, 400,437

spinosa, 397,437

sp.?, 401, 430,448

stipulata, 401,443

subgeniculata, 398,430

tenella, 399, 437

tracyana, 399,437

trichomanoides, 398,437

zeilleria, 399,437
$T$

TAENIOPTERIS

Lescuriana, 410, 451

Newberriana, 410, 451

Newberriana var. angusta, 410, 451

TRIGONOCARPUM

ampullaeforme, 427,442

clavatum, 427

Noeggerathii, 428, 442

oliviaef orme, 427,435

triloculare, 427,435

TRILETES

sp.?, 425, 436

TRIPHYLLOPTERIS

Lescuriana, 393, 429

virginiana, 393,429

U

\section{ULMUS}

racemosa, 429,453

ULODENDRON

majus, 423, 441

sp.?, 423,433

W

WHITTLESEYA

elegans, 426, 435 


\section{DAY USE \\ RETURN TO DESK FROM WHICH BORROWED \\ EARTH SCIENCES LIBRARY}

This book is due on the last date stamped below, or on the date to which renewed.

Renewed books are subject to immediate recall.

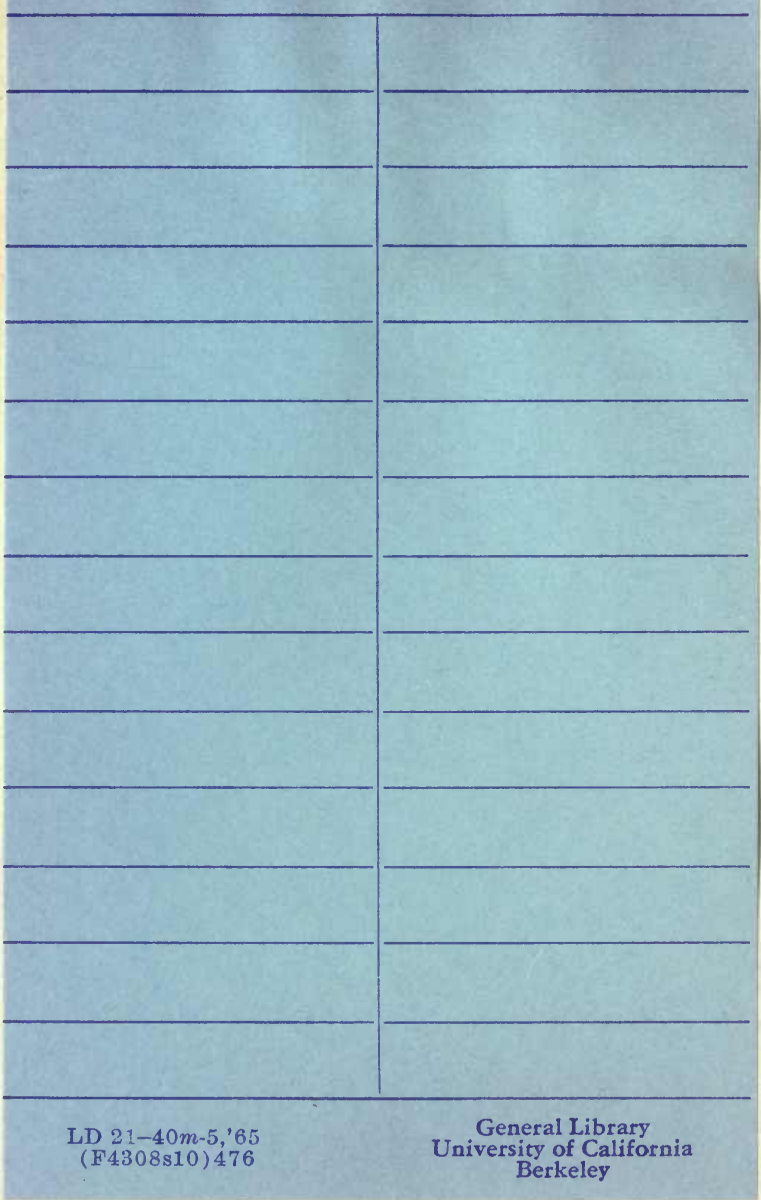


IUIU U.C. BERKELEY LIBRARIES

$\operatorname{co3} 3852795$

108 
W

1

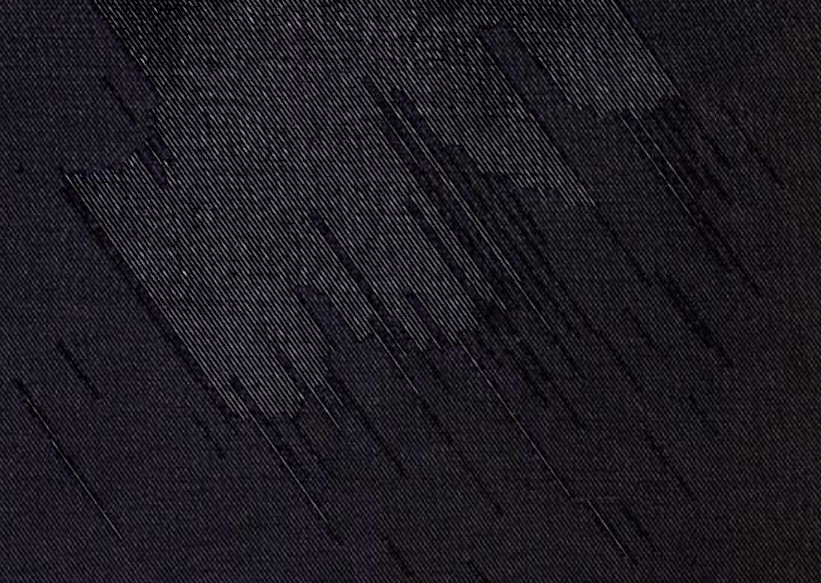

